\title{
A Formal Study on Backward Compatible Dynamic Software Updates
}

\author{
Jun Shen \\ Arizona State University \\ jun.shen.1@asu.edu
}

\author{
Rida A. Bazzi \\ Arizona State University \\ bazzi@asu.edu
}

\begin{abstract}
We study the dynamic software update problem for programs interacting with an environment that is not necessarily updated. We argue that such updates should be backward compatible. We propose a general definition of backward compatibility and cases of backward compatible program update. Based on our detailed study of real world program evolution, we propose classes of backward compatible update for interactive programs, which are included at an average of $32 \%$ of all studied program changes. The definitions of update classes are parameterized by our novel framework of program equivalence, which generalizes existing results on program equivalence to non-terminating executions. Our study of backward compatible updates is based on a typed extension of $W$ language.
\end{abstract}

Categories and Subject Descriptors D.3.1 [Formal Definitions and Theory]: Semantics, Syntax; D.2.4 [Software/Program Verification]: Correctness Proof, Formal Methods; F.3.2 [Semantics of Programming Languages]: Operational Semantics, Program Analysis; D.3.3 [Language Constructs and Features]: Input/output, Procedures, functions, and subroutines

\section{General Terms Theory}

Keywords dynamic software update, backward compatibility, program equivalence, proof rule, operational semantic

\section{Contents}

1 Introduction

2 Backward compatibility

2.1 Programs and Specifications . . . . . . . . . .

2.2 Hybrid executions and state mapping . . . . . . .

2.3 Backward compatibility . . . . . . . . . . . .

2.4 Backward compatible program behavior changes .

3 Real world backward compatible update classes: brief description

3.1 Observational equivalence: the old behavior . . . .

3.2 Enum. type extension: old behavior for old input and allowing new input . . . . . . . . .

3.3 Variable type weakening: more output when the old program terminates .............

3.4 Exit on errors: stopping execution while the old program produces more output . . . . . . . .

3.5 Improved prompt messages: functionally equivalent outputs . . . . . . . . . . .

3.6 Missing variable initialization: enforcing restrictions on program states $\ldots \ldots \ldots$

4 Formal programming language
4.1 Syntax of the formal language . . . . . . . . .

4.2 Small-step operational semantics of the formal lan-

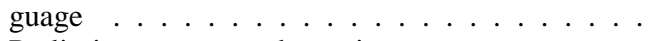

4.3 Preliminary terms and notations . . . . . . . .

5 Program equivalence

5.1 Definitions of execution . . . . . . . . . .

5.2 Equivalent computation for terminating programs .

5.2.1 Proof rule for equivalent computation for terminating programs .........

5.2.2 Soundness of the proof rule for equivalent computation for terminating programs . .

5.2.3 Supporting lemmas for the soundness proof of equivalent computation for terminating

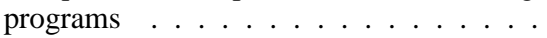

5.3 Termination in the same way . . . . . . . .

5.3.1 Proof rule for termination in the same way

5.3.2 Soundness of the proof rule for termination in the same way . . . . . . . . . .

5.3.3 Supporting lemmas for the soundness proof of termination in the same way . . . . . .

5.4 Behavioral equivalence . . . . . . . . . .

5.4.1 Proof rule for behavioral equivalence . . .

5.4.2 Soundness of the proof rule for behavioral equivalence ............

5.4.3 Supporting lemmas for the soundness proof of behavioral equivalence ........

5.5 Backward compatible DSU based on program

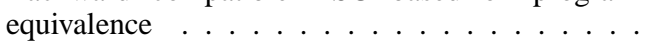

6 Real world backward compatible update classes: proof rules

6.1 Proof rule for specializing new configuration variables ....................

6.2 Proof rule for enumeration type extension . . . . .

6.3 Proof rule for variable type weakening . . . . . .

6.4 Proof rule for exit on errors . . . . . . . . . .

6.5 Proof rule for improved prompt message . . . . .

6.6 Proof rule for missing variable initializations . . .

7 Related Work

8 Conclusion

A Type system 67

B Syntactic definitions 67

C Properties of imported variables 68 


\section{E Properties of remaining execution}

\section{Introduction}

Dynamic software update (DSU) allows programs to be updated in the middle of their execution by mapping a state of an old version of the program to that of a newer version. The ability to update programs without having to restart them is useful for high-availability applications that cannot afford the downtime incurred by offline updates [16]. DSU has been an active area of research[5, 16, 22, 25] with much of the published work emphasizing the update mechanism that implements a state mapping which maps the execution state of an old version of the program to that of a new version. DSU safety has not yet been successfully studied. Existing studies on DSU safety are lacking in one way or another: high-level studies are concerned with change management for system components [9, 19] and lower-level studies typically require significant programmer annotations [15, 24, 32] or have a restricted class of applications to which they apply (e.g., controller systems [28]).

In this paper, we consider the safety of DSU when applied to possibly non-terminating programs interacting with an environment that is not necessarily updated. For such updates, the new version of the program must be able to interact with the old environment, which means that it should be, in some sense, backward compatible with the old version. A strict definition of backward compatibility would require the new version to exhibit the same I/O behavior as the old version; in other words the two programs are observationally equivalent. It should be immediately clear that a more nuanced definition is needed because observational-equivalence does not allow changes which one would want to allow as backward compatible such as bug fixes, new functionalities, or usability improvement (e.g., improved user messages). Allowing for such differences would be needed in any practical definition of backward compatibility. One contribution of this work is a general definition of backward compatibility, a classification of common backward compatible program behavior changes, as well as classes of program change from real world program evolution.

Determining backward compatibility, which allows for differences between two program versions, is requiring one to solve the semantic equivalence problem which has been extensively studied [6, 13, 17, 18, 20, 21, 23, 31]. Unfortunately, existing results turned out to be lacking in one or more aspects which rules out retrofitting them for our setting. In fact, existing work on program equivalence typically guarantees equivalence at the end of an execution. Such equivalence is not adequate for our purposes because it does not allow us to express that a point in the middle of a loop execution of one program corresponds (in a well defined sense) to a point in the middle of a loop execution of another program. The ability to express such correspondences is desirable for dynamic software update. Besides, existing formulations of the program equivalence problem either do not use formal semantics [7, 17, 18], only apply to terminating programs [6, 20], severely restrict the programming model [13, 18, 31], or rely on some form of model checking [21, 23] (which is not appropriate for non-terminating programs with infinite states). Our goal for program equivalence is to establish compile-time conditions ensuring that two programs have the same I/O behavior in all executions. In particular, if one program enters an infinite loop and does not produce a certain output, the other program should not produce that output either. This is different from much of the literature on program equivalence which only guarantees same behavior in terminating executions.

The closest work that aims to establish program equivalence for nonterminating programs is that of Godlin and Strichman [13] who give sufficient conditions for semantic equivalence for a language that includes recursive functions, but does not allow loops (loops are extracted as recursive functions). That and the fact that equiv- alence is enforced on corresponding functions severely limits the applicability of the work to general transformations affecting loops such as loop-invariant code motion, loop fission/fusion. So, as a major component of our formal treatment of backward compatible updates, we set out to develop sufficient conditions for semantic equivalence for programs in a typed extension of the $W$ languages [11] with small-step operational semantics. The syntax of language is extended with arrays and enumeration types and the semantics take into consideration the execution environment to allow various classes of updates.

In summary, the paper makes the following contributions:

1. We formally define backward compatibility and identify cases of backward compatible program behavior for typical program update motivation.

2. We identify and formally define classes of program changes that result in backward compatible program update based on empirical study of real world program evolution.

3. We give a formal treatment of the semantic equivalence for nonterminating imperative programs.

The rest of the paper is organized as follows. Section 2 proposes the general backward compatibility and cases of backward compatible new program behavior. Then we describe real world update classes that result in backward compatible update in Section 3 . Section 4 formally defines our extension of the $\mathbf{W}$ language to study backward compatible updates. Section 4.3 shows terms, notations and definitions (e.g., execution) heavily used in the technical result. The technical results on semantic equivalence are presented in Section 5 We propose our formal treatment of real world update classes in Section 6 A more detailed comparison to related work is given in Section 7 . Section 8 concludes the paper.

\section{Backward compatibility}

\subsection{Programs and Specifications}

Programs are designed to satisfy specifications. Specification can be explicitly provided or implicitly defined by the behavior of a program. Programs interact with their environment by receiving inputs and producing outputs. In this section we introduce enough of a computing model to describe the input/output behavior of programs; In the next section we introduce a specific programming language to reason about specific software updates.

An execution of a program consists of a sequence of steps from a finite set of steps, $\mathcal{S}=\mathcal{S}_{\text {in }} \cup \mathcal{S}_{\text {internal }} \cup \mathcal{S}_{\text {out }} \cup\{$ halt $\}$. A step of a program can either be an input step in which input is received, an internal step in which the state of the program is modified, an output step in which output is produced, or a halt.

We make a distinction between internal state of a program and external state (e.g., application settings) of the local environment in which the program executes. Such external state can include the state of a file system that program can access; we include both as part of the program state. The state of a program is an element of a set $\mathcal{M} \times \mathcal{I}$, where the set $\mathcal{M}=\mathcal{M}_{\text {int }} \times \mathcal{M}_{\text {ext }}, \mathcal{M}_{\text {int }}=\prod_{k=0}^{n_{\text {int }}} V_{k}$ is a cartesian product of $n_{\text {int }}$ sets of values, one for each internal memory location, and $\mathcal{M}_{\text {ext }}=\prod_{k=0}^{n_{e x t}} V_{k}$ is a cartesian product of $n_{\text {ext }}$ sets of values, one for each external location. The input value last received is an element of the set $\mathcal{I}$ of input values.

A program executes in an execution environment. An execution environment $\left(M_{\text {ext }}, I\right)$ specifies an initial value for the external program state $M_{e x t_{0}}$ and a possibly infinite sequence of input values $I$. The input sequence is assumed to be produced by users that we do not model explicitly.

A step of a program $P$ is a mapping that specifies the next program state and the next step to execute. For an internal step $s_{\text {internal }} \in \mathcal{S}_{\text {internal }}$, the mapping is $s_{\text {internal }}: \mathcal{M} \times \mathcal{I} \mapsto$ 
$\mathcal{S} \times \mathcal{M} \times\{\perp\}$, which specifies the next step and how the state is modified. The internal steps clear input in the state if any. For an output step $s_{\text {out }} \in \mathcal{S}_{\text {out }}$, the mapping $s_{\text {out }}: \mathcal{M} \mapsto \mathcal{S} \times \mathcal{O}$ which specifies the next step to execute and the output value produced. $\mathcal{O}$ is the set of output values produced by the program. An input step $s_{i n} \in \mathcal{S}_{i n}$ is simply an element of $\mathcal{S} \times \mathcal{I}$ and specifies the next step to execute and the input obtained from the environment. (We simply write $s_{i n}()$ to denote the next step and the input received.) Because the input value is received by the program, we do not restrict the next step to execute. We allow the input value to be ignored by the program by two consecutive input steps. When the step is halt, there is no further action as if halt were mapped to itself.

Definition 1. (Program) A program $P$ is a tuple $\left(\mathcal{S}, \mathcal{M}, M_{i n t_{0}}, s_{0}\right.$ $\mathcal{I}, \mathcal{O})$, where $\mathcal{S}$ is the set of steps as defined above, $\mathcal{M}$ is the set of program states, $M_{\text {int }_{0}}$ is the initial internal state, $s_{0}$ is the initial step, and $\mathcal{I}$ and $\mathcal{O}$ are disjoint sets of input and output values.

We do not include the initial external state $M_{\text {ext }}$ in the program definition; we include it in the execution environment of $P$.

Definition 2. (Execution) An execution of a program $P=$ $\left(\mathcal{S}, \mathcal{M}, M_{\text {int }_{0}}, s_{0}, \mathcal{I}, \mathcal{O}\right)$ in execution environment $\left(M_{\text {ext }}, I\right)$, where $I$ is a possibly infinite sequence of input values from $\mathcal{I}$, is a sequence of configurations $C$ from the infinite set $\left\{\left(M, s, i, I_{r}, I O\right)\right\}$. A configuration $c$ has the form $c=\left(M, s, i, I_{r}, I O\right)$, where $M$ is a state, $s$ is a step, $i$ is the last input received, $I_{r}$ is a sequence of remaining input values and $I O$ is the input/output sequence produced so far. The kth configuration $c_{k}$ in an execution is obtained from the $(k-1)$ th configuration $c_{k-1}=\left(M, s, i, I_{r}, I O\right)$ where $s \neq$ halt in one of the following cases:

1. The first configuration $c_{0}$ is of the form $\left(M_{0}, s_{0}, \perp, I, \varnothing\right)$, where $M_{0}=\left(M_{\text {int }_{0}}, M_{\text {ext }}\right)$;

2. $s \in \mathcal{S}_{\text {internal }}: c_{k}=\left(M^{\prime}, s^{\prime}, \perp, I_{r}, I O\right)$, where $\left(s^{\prime}, M^{\prime}, \perp\right)=$ $s(M, i)$

3. $s \in \mathcal{S}_{i n}$ and the remaining inputs $I_{r}$ is not empty: $c_{k}=$ $\left(M, s^{\prime}\right.$, head $\left(I_{r}\right)$, tail $\left(I_{r}\right)$, IO head $\left.\left(I_{r}\right)\right)$ where $\left(s^{\prime}\right.$, head $\left.\left(I_{r}\right)\right)$ $=s\left(I_{r}\right)$;

4. $s \in \mathcal{S}_{i n}$ and the remaining inputs $I_{r}$ is empty: $c_{k}=c_{k-1}$;

5. $s \in \mathcal{S}_{\text {out }}: c_{k}=\left(M, s^{\prime}, i, I_{r}, I O \cdot o^{\prime}\right)$, where $\left(s^{\prime}, o^{\prime}\right)=s(M)$;

In the definition, head $(I)$ denotes the head (leftmost) element in the sequence $I$ and $\operatorname{tail}(I)$ denotes the remaining sequence without the head. The input value in $i$ is either consumed by the next internal step or updated by another input from the next input step. Execution is stuck if an input step is attempted in state in which there are no remaining inputs. In what follows, we include the execution environment in the execution and we abuse notation to say $\left(M_{\text {ext }}, I, C\right)$ is an execution of a program $P$.

Specifications We consider specifications that define the input/output behavior of programs. Specifications are not concerned with how fast an output is produced or about the internal state of the program.

Definition 3. (Specification) Given a set $\mathcal{M}_{\text {ext }}$ of external states, a set $\operatorname{seq}(\mathcal{I})$ of input sequences, and a set $\operatorname{seq}(\mathcal{I} \cup \mathcal{O})$ of $I / O$ sequences, specification $\Sigma$ is a predicate: $\mathcal{M}_{\text {ext }} \times \operatorname{seq}(\mathcal{I}) \times \operatorname{seq}(\mathcal{I} \cup$ $\mathcal{O}) \times \mapsto\{$ true, false $\}$.

We define the I/O sequence of a sequence of configurations $C$ to be a sequence $I O(C)$ of values from $\mathcal{I} \cup \mathcal{O}$ such that every finite prefix of $I O(C)$ is the IO sequence of some configuration $c \in C$ and every $\mathrm{I} / \mathrm{O}$ sequence of a configuration $c \in C$ is a finite prefix of $I O(C)$.

An execution $\left(M_{\text {ext }}, I, C\right)$ of program $P$ satisfies a specification $\Sigma$ if $\Sigma\left(M_{e x t_{0}}, I, I O(C)\right)=$ true. A specification distin- guishes executions into those that satisfy the specification and those that do not.

A specification defines the external behavior of a program that is observed by a user. The input sequence and I/O sequence are obviously part of external behavior. We also include $\mathcal{M}_{\text {ext }}$ in specification domain because a user can have information about the external state. For example, a user who has data stored in the file system considers the program's refusal to access the stored data a violation of the service specification; this is not the case if the user has no stored data.

\subsection{Hybrid executions and state mapping}

DSU is a process of updating software while it is running. This results in a hybrid execution in which part of the execution is that of the old program and part of the execution is for the new program.

State mapping is a function $\delta$ mapping an internal state and a non-halt step of one program $P$ to an internal state and a step of another program $P^{\prime}, \delta: \mathcal{M}_{\text {int }}^{P} \times\left(\mathcal{S}^{P} \backslash\{\right.$ halt $\left.\}\right) \mapsto \mathcal{M}_{\text {int }}^{P^{\prime}} \times \mathcal{S}^{P^{\prime}}$. The external state is not mapped because the environment is not necessarily updated. In addition, we cannot change input and output that already occurred and that $\mathrm{I} / \mathrm{O}$ must be part of the hybrid execution.

Definition 4. (Hybrid execution) A hybrid execution $\left(M_{\text {ext }}, I\right.$, $\left.C_{P} ; C_{P^{\prime}}\right)$, produced by DSU using state mapping $\delta$ from program $P$ to program $P^{\prime}$, is an execution $\left(M_{\text {ext }}, I, C_{P}\right)$ of $P$ concatenated with an execution $\left(M_{e x t}^{\prime}, I_{r}^{\prime}, C_{P^{\prime}}\right)$ of $P^{\prime}$ where the first configuration $c_{P^{\prime}}=\left(\left(M_{\text {int }}^{\prime}, M_{\text {ext }}^{\prime}\right), s^{\prime}, i^{\prime}, I_{r}^{\prime}, I O^{\prime}\right)$ in $C_{P^{\prime}}$ is obtained by applying the state mapping to the last configuration $c_{P}=\left(\left(M_{\text {int }}, M_{\text {ext }}\right), s(\neq\right.$ halt $\left.), i, I_{r}, I O\right)$ in $C_{P}$ as follows:

- $\left(M_{\text {int }}^{\prime}, s^{\prime}\right)=\delta\left(M_{\text {int }}, s\right)$;

- $\left(i^{\prime}=i\right) \wedge\left(I_{r}^{\prime}=I_{r}\right) \wedge\left(I O^{\prime}=I O\right) \wedge\left(M_{e x t} \subseteq M_{\text {ext }}^{\prime}\right)$.

\subsection{Backward compatibility}

In this paper, we consider updates in which the environment is not necessarily updated. It follows that in order for the hybrid execution to be meaningful, the new program should provide functionality expected by both old and new users of the system.

In practice, specifications are not explicitly available. Instead, the program is its own specification. This means that the specification that the program satisfies can only be inferred by the external behavior of the program. Bug fixes create a dilemma for dynamic software updates. When a program has a bug, its external behavior does not captures its implicit specification and the update will change the behavior of the program. In what follows, we first discuss what flexibility we can be afforded for a backward compatible update and then we give formal definitions of backward compatibility and state our assumptions for allowing bug fixes.

We consider a hybrid execution starting from a program $P=$ $\left(\mathcal{S}, \mathcal{M}_{\text {int }} \times \mathcal{M}_{\text {ext }}, M_{\text {int }_{0}}, s_{0}, \mathcal{I}, \mathcal{O}\right)$ and being updated to a program $P^{\prime}=\left(\mathcal{S}^{\prime}, \mathcal{M}_{i n t}^{\prime} \times \mathcal{M}_{\text {ext }}^{\prime}, M_{i n t_{0}}^{\prime}, s_{0}^{\prime}, \mathcal{I}^{\prime}, \mathcal{O}^{\prime}\right)$. We examine how the two programs should be related for a meaningful hybrid execution.

1. (Inputs) Input set $\mathcal{I}^{\prime}$ of $P^{\prime}$ should be a superset of that $\mathcal{I}$ of $P$ to allow for old users to interact with $P^{\prime}$ after the update. It is possible to allow for new input values in $\mathcal{I}^{\prime}$ to accommodate new functionality under the assumption that old users do not generate new input values. Such new input values should be expected to produce erroneous output by old users as they are not part of $P$ 's specification.

2. (Outputs) Output produced by $P^{\prime}$ should be identical to output produced by $P$ if all the input in an execution comes from the input set of $P$. This is needed to ensure that interactions between old users and the program $P^{\prime}$ can make sense from the 
perspective of old users. This is true in the case that the update does not involve a bug fix, but what should be done if the update indeed involves a bug fix and the output produced by the old program was not correct to start with? As far as syntax, a bug fix should not introduce new output values. As far as semantics, we should allow the bug fix to change what output is produced for a given input. We discuss this further under the bug fix heading. In summary, if we ignore bug fixes, the new program should behave as the old program when provided with input meant for the old program.

3. (Bugfix) Handling bug fixes is problematic. If the produced output already violates the fix, then there is no way for the hybrid execution to satisfy the implicit semantics of the program or the semantics of the new program. Some bug fixes can be handled. For example, a bug that causes a program to crash for some input can be fixed to allow the program to continue executing. Applying the fix to a program that has not encountered the bug should not be problematic. Another case is when the program should terminate for some input sequence, but the old program does not terminate. A bug fix that allows the program to terminate should not present a semantic difficulty for old users.

In general, we assume that there are valid executions and invalid executions of the old program. I/O sequences produced in invalid executions are not in specification of the program. We assume that an invalid execution will lead to an error configuration not explicitly handled by the program developers. We do not expect the state mapping to change an error configuration into an non-error configuration just as static updating does not fix occurred errors. Besides, we do not attempt to determine if a particular configuration is an error configuration. Such determination is not possible in general and very hard in practice. We simply assume that the configuration at the time of the update is not an error configuration. (which is equivalent to assuming the existence of an oracle $\mathcal{J}_{P}$ to determine if a particular configuration is erroneous, $\mathcal{J}_{P}\left(C_{P}\right)=$ true if the configuration $C_{P}$ is not erroneous).

4. (New functionality) New functionality is usually accompanied by new inputs/outputs and the expansion of external state. We assume that new functionality is independent of existing functionality in the sense that programs $P$ and $P^{\prime}$ produce the same I/O sequence when receiving inputs in $\mathcal{I}$ only. We therefore assume all new inputs $\mathcal{I}^{\prime} \backslash \mathcal{I}$ are introduced by new functionality.

Every external state of $P$ is part of some external state of program $P^{\prime}$ because of the definition of the specification of $P$. We only consider expansion of the external state of $P$ for new functionalities in $P^{\prime}$ where the expansion of external state is independent of values in existing external state. One of the motivating examples is to add application settings for new program feature.

In light of the discussion above we give the following definition of backward compatibility in the absence of bug fixes.

Definition 5. (Backward compatible hybrid executions) Let $P=\left(\mathcal{S}, \mathcal{M}_{\text {int }} \times \mathcal{M}_{\text {ext }}, M_{\text {int }_{0}}, s_{0}, \mathcal{I}, \mathcal{O}\right)$ be a program satisfying a specification $\Sigma$. We say that a hybrid execution $\left(M_{\text {ext }}, I, C_{P} ; C_{P}^{\prime}\right)$ from $P$ to a program $P^{\prime}=\left(\mathcal{S}^{\prime}, \mathcal{M}_{\text {int }}^{\prime} \times \mathcal{M}_{\text {ext }}^{\prime}, M_{\text {int }}^{\prime}, s_{0}^{\prime}, \mathcal{I}^{\prime}, \mathcal{O}^{\prime}\right)$ is backward compatible with implicit specification of $P$ if all of the following hold:

- The last configuration in $C_{P}$ is not an error configuration, $C_{P}=$ "C $C^{\prime} ;\left(M, s^{\prime}, i, I_{r}, I O\right) ": \mathcal{J}_{P}\left(C_{P}\right)=$ true.

- The hybrid execution satisfies the specification $\Sigma$ of $P$, $\Sigma\left(M_{\text {ext }}, I, I O\left(C_{P} ; C_{P}^{\prime}\right)\right)=$ true;
- Inputs/outputs/external states of $P$ are a subset of those of $P^{\prime}: \mathcal{I} \subseteq \mathcal{I}^{\prime}, \mathcal{O} \subseteq \mathcal{O}^{\prime}$ and $\mathcal{M}_{\text {ext }} \subseteq \mathcal{M}_{\text {ext }}^{\prime}$;

If there is bug fix between programs $P^{\prime}$ and $P$, we need to adapt Definition 5 to allow for some executions on input sequences from $\mathcal{I}$ to violate the specification of $P$. Above we identified two cases in which bug fixes are safe (replacing a response with no response or replacing a no response with a correct response without introducing new output values). We omit the definition.

We have the backward compatible updates by extending the definition of a backward compatible hybrid execution to all possible hybrid executions.

Definition 6. (Backward compatible updates) We say an updated program $P^{\prime}$ is backward compatible with a program $P$ in configuration $C$ if there is hybrid execution, from configuration $C$ of $P$ to $P^{\prime}$ that is backward compatible with specification of $P$.

\subsection{Backward compatible program behavior changes}

With the formal definition of backward compatibility, it is desirable to check what behavior changes of an updated program help ensure a safe update. Backward compatibility is essentially a relation between I/O sequences produced by an old program and those produced by an updated program. We summarized typical possibilities of the relation into six cases in Figure 1 by considering consequence of major update motivation (i.e., new functionality, bug fix and program perfective/preventive needs [1]). According to David Parnas [29], a program is updated to adapt to changing needs. In other words, program changes are to produce more or less or different output according to changing needs. These changes are captured by case 2, 3, 4, 5 and 6 in Figure 1 We also capture outputpreserving changes which are most likely motivated by the program developer's own needs (e.g., software maintainability), which is case 1 in Figure 1

Furthermore, we find that an update is backward compatible if in every execution the new program behavior is one of the six cases in Figure 1 Cases 1 and 2 are obviously backward compatible because an old client is guaranteed to get old responses. Cases 3 , 4, 5, and 6 are not obviously backward compatible. Unlike case 1 and 2, case 3, 4 and 5 are backward compatible under specific assumptions on program semantics while case 6 is different. Case 3 is backward compatible because we assume the change is either adding new functionality, or fixing a bug in which the old program hanged or crashed. Similarly, case 4 is backward compatible. Case 5 is backward compatible because different I/O interaction could express the same application semantics. For example, a greeting message could be changed from "hi" to "hello". Case 6 is backward compatible in that the new program makes implicit specification of the program explicit by enforcing restrictions on program state and therefore eliminating undesired I/O sequence.

The six cases in Fig. 1 have covered the changes of output, including more or less or different output. There exists more specific cases of backward compatible program behavior changes under various specific assumptions. However, these more specific cases could be attributed to one of the six cases as far as the changes of output are concerned. In conclusion, it is not possible to go much beyond the six cases of backward compatibility in Fig. 1

\section{Real world backward compatible update classes: brief description}

We have studied evolution of three real world programs (i.e., vsftpd, sshd and icecast) to identify real world changes that are backward compatible. We chose these three programs because the programs are widely used in practice [2, 3] and are widely studied in the DSU community [26, 27]. We have studied several years of re- 


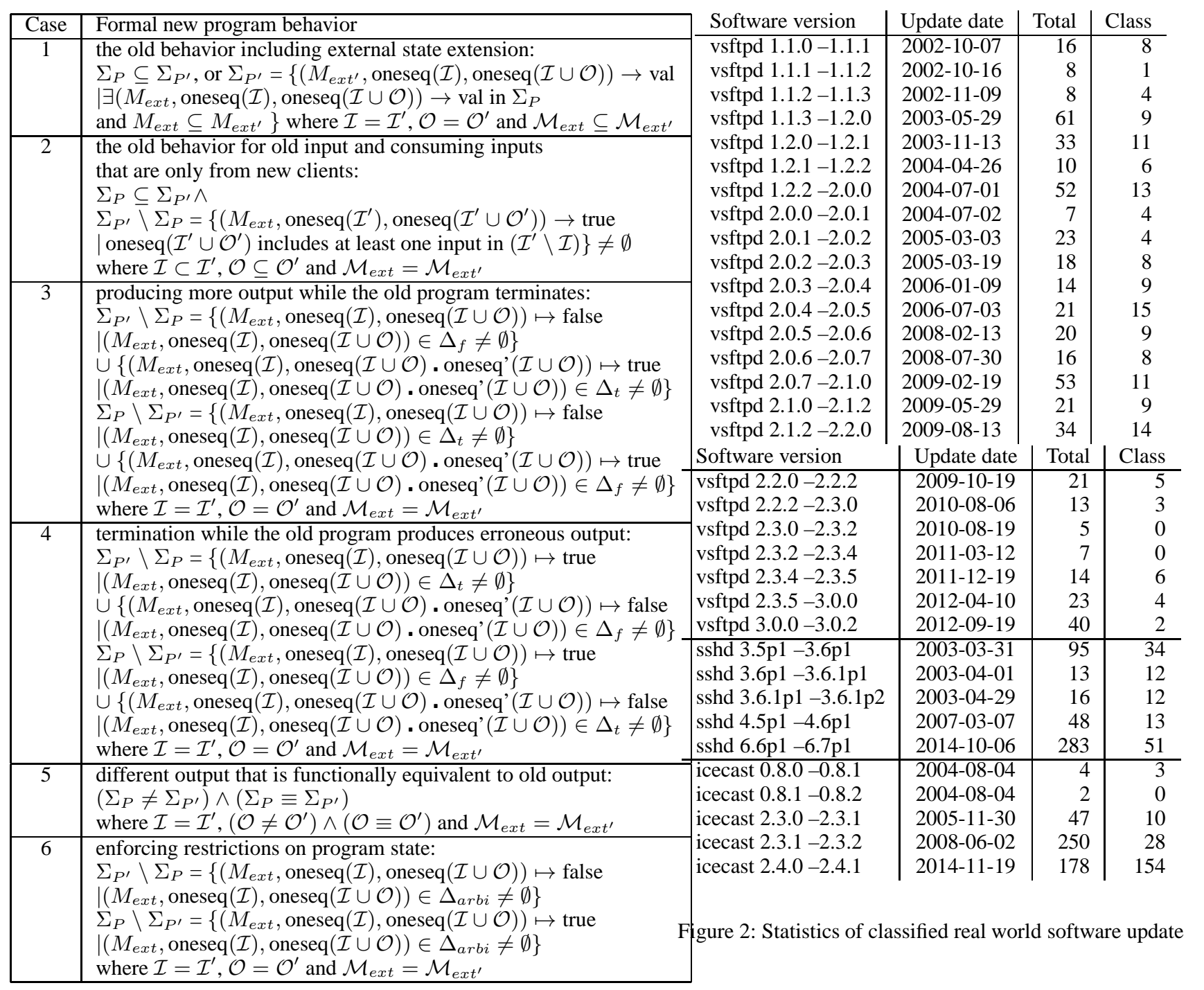

Figure 1: Six cases of formalized general new program behavior

leases of vsftpd and consecutive updates of sshd and icecast. This is because vsftpd is more widely studied by the DSU community [2527].

Our study of real world program evolution is carried out as follows. We examined every changed function manually to classify updates. For every individual change, we first identified the motivation of the change, then the assumptions under which the change could be considered backward compatible. If the assumption under which the change is considered backward compatible is reasonable, we recorded the change into one particular update class. Finally we summarized common update classes observed in the evolution of studied programs.

Fig. 2] shows the statistics from our study of real world program evolution where "total" refers to the number of all updated functions, "class" refers to the number of updated functions with at least one classified update pattern. In summary, $32 \%$ of all updated functions include at least one classified program update; the unclassified updates are mostly bug fix that are related to specific program logic. We summarized seven most common real world update classes from all the studied updates in Fig. 3 and we believe that these update classes are also widespread in other program evolution. Each of the six real world update classes falls in one of the five cases of backward compatibility in Fig. 1 We present informal descriptions of all update classes including required assumptions for the two programs to produce same or equivalent output sequence which guarantees backward compatible DSU.

\subsection{Observational equivalence: the old behavior}

In case 1 in Fig. 1 two programs are backward compatible because the new program keeps all old behaviors ("observational equivalence"). In our study, we differentiate two types of "observational equivalence" based on if assumptions are required.

Program equivalence We consider several types of program changes that are allowed by "observational equivalence" without user assumptions. These changes include: loop fission or fusion, statement reordering or duplication, and extra statements unrelated to output(e.g., logging related changes). We incorporate these changes in our framework of program equivalence which ensures two programs produce the same output regardless of whether the programs terminate or not. The details of the formal treatment is in Section 5

Specializing new configuration variables Another update class of "observational equivalence" is "specializing new configuration variables", which is backward compatible under user assumptions. 


\begin{tabular}{|c|l|}
\hline Update class (Case) & $\begin{array}{l}\text { Required assumptions for backward } \\
\text { compatible update }\end{array}$ \\
\hline program equivalence (1) & none \\
\hline new config. variables (1) & $\begin{array}{l}\text { no redefinitions of new config } \\
\text { variables after initialization }\end{array}$ \\
\hline enum type extension (2) & $\begin{array}{l}\text { no inputs from old clients match } \\
\text { the extended enum labels }\end{array}$ \\
\hline var. type weakening (3) & $\begin{array}{l}\text { no intentional use of value type } \\
\text { mismatch and array out of bound }\end{array}$ \\
\hline exit on error (4) & correct error check before exit \\
\hline improved prompt msgs (5) & $\begin{array}{l}\text { changing prompt messages for } \\
\text { more effective communication }\end{array}$ \\
\hline missing var. init. (6) & $\begin{array}{l}\text { no intentional use of undefined } \\
\text { variables }\end{array}$ \\
\hline
\end{tabular}

Figure 3: Required assumptions for real world backward compatible update classes

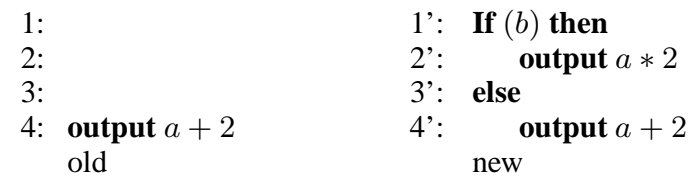

Figure 4: Specializing new configuration variables

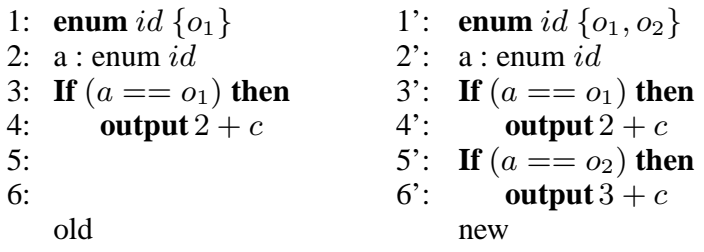

Figure 5: Enumeration type extension

In this update class, new configuration variables are introduced to generalize functionality. For example, in Fig. 15 a new configuration variable $b$ is used to introduce new code. The two statement sequences in Fig. 15 are equivalent when the new variable $b$ is specialized to 0 . In general, if all new code is introduced in a way that is similar to that in Fig. 15 where there is a valuation of new configuration variables under which new code is not executed, and new configuration variables are not redefined after initialization, then the new program and the old program produce the same output sequence. The point is that new functionality is not introduced abruptly in interaction with an old client. Instead new functionality could be enabled for a new client when old clients are not a concern.

\subsection{Enum. type extension: old behavior for old input and allowing new input}

Enumeration types allow developers to list similar items. New code is usually accompanied with the introduction of new enumeration labels. Fig. 16 shows an example of the update. The new enum label $o_{2}$ gives a new option for matching the value of the variable $a$, which introduces the new code "output $3+c$ ". To show enumeration type extensions to be backward compatible, we assume that values of enum variables, used in the If-predicate introducing the new code, are only from inputs that cannot be translated to new enum labels. This is case 2 of the backward compatibility.

$\begin{array}{lcl}\text { 1: } & 1^{\prime}: & \text { If }(1 /(a-5)) \text { then } \\ \text { 2: } & 2^{\prime}: & \text { skip } \\ \text { 3: output } a & 3^{\prime}: & \text { output } a \\ & \text { old } & \text { new }\end{array}$

Figure 6: Exit-on-error

\subsection{Variable type weakening: more output when the old program terminates}

In program updates, variable types are changed either to allow for larger ranges (weakening) or smaller ranges to save space (strengthening). For example, an integer variable might be changed to become a long variable to avoid integer overflow or a long variable might be changed to an integer variable because the larger range of long is not needed. Type weakening also includes adding a new enumeration value and increasing array size. The kinds of strengthening or weakening that should be allowed are application dependent and would need to be defined by the user in general. The type weakening considered is either changes from type int to long or increase of array size. These updates fix integer overflow or array index out of bound respectively, the case 3 of backward compatibility. Implicitly, we assume that there is no intentional use of integer overflow and array out of bound as program semantics.

\subsection{Exit on errors: stopping execution while the old program produces more output}

One kind of bug fix, which we call exit on error, causes a program to exit in observation of errors that depend on application semantic. Fig. 17 shows an example of exit-on-error update. In the example, the fixed bugs refer to the program semantic error that $a=5$. Instead of using an "exit" statement, we rely on the crash from expression evaluations to model the "exit". When errors do not occur, the two programs in Fig. 17 produce the same output sequence. This is case 4 of backward compatibility. Naturally, we assume that all error checks are correct.

\subsection{Improved prompt messages: functionally equivalent outputs}

In practice, outputs could be classified into prompt outputs and actual outputs. Prompt outputs are those asking clients for inputs, which are constants hardcoded in output statements. Actual outputs are dynamic messages produced by evaluation of non-constant expressions in execution. If the differences between two programs are only the prompt messages that a client receives, we consider that the two programs are equivalent. The prompt messages are the replaceable part of program semantics. We observe cases of improving prompt messages in program evolution for effective communication. The changes of prompt outputs do not matter only for human clients. This is case 5 of backward compatibility.

\subsection{Missing variable initialization: enforcing restrictions on program states}

Another kind of bug fix, which we call missing variable initialization, includes initializations for variables whose arbitrary initial values can affect the output sequence in the old program. Fig. 18 shows an example of missing variable initialization. The initialization $b:=2$ ensures the value used in "output $b+c$ " not to be undefined. Despite of initialization statements, the two programs are same. In general, initializations of variables only affect rare buggy executions of the old program, where undefined variables affect the output sequence. This update class is case 6 of backward compatibility and we assume that there is no intentional use of undefined variable in the program. When there are no uses of variables with 


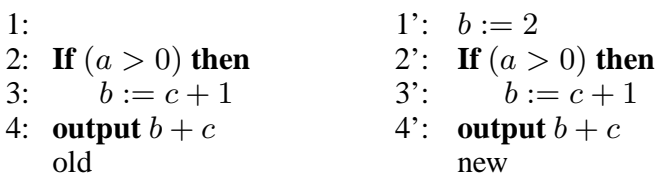

Figure 7: Missing initialization

\begin{tabular}{|c|c|c|c|}
\hline Identifier & Constant & & Label \\
\hline Enum Items & el & $::=$ & $l \mid e l_{1}, e l_{2}$ \\
\hline Enumeration & $E N$ & $::=$ & $\varnothing \mid$ enum $i d\{e l\} \mid E N_{1}, E N_{2}$ \\
\hline Prompt Msg & $m s g$ & $::=$ & $l: n \mid m s g_{1}, \mathrm{msg}_{2}$ \\
\hline Prompts & Pmpt & $t::=$ & $\varnothing \mid\{m s g\}$ \\
\hline Base type & $\tau$ & $::=$ & Int $\mid$ Long $\mid$ pmpt | enum $i d$ \\
\hline Variables & $V$ & $::=$ & $\varnothing|\tau i d| \tau i d[n] \mid V_{1}, V_{2}$ \\
\hline Left value & lval & $::=$ & $i d\left|i d_{1}\left[i d_{2}\right]\right| i d[n]$ \\
\hline Expression & $e$ & $::=$ & $i d==l \mid$ lval $\mid$ other \\
\hline Statement & $s$ & $::=$ & $\begin{array}{l}\text { lval }:=e \mid \text { input } i d \mid \text { output } e \mid \text { skip } \\
\mid \text { while }(e)\{S\}\end{array}$ \\
\hline Stmt Seq. & $S$ & $::=$ & $s_{1} ; \ldots ; s_{k}$ for $k \geq 1$ \\
\hline Program & $P$ & $::=$ & Pmpt $; E N ; V ; \bar{S}_{\text {entry }}$ \\
\hline
\end{tabular}

Figure 8: Abstract syntax

undefined variables in executions of the old program, the two programs produce the same output sequence.

\section{Formal programming language}

We present the formal programming language based on which we prove our semantic equivalence results and describe categories of backward compatible changes. We first explain the language syntax, then the language semantics.

\subsection{Syntax of the formal language}

The language syntax is in Figure. 8 We use $i d$ to range over the set of identifiers, $n$ to range over integers, $l$ to range over labels. We assume unique identifiers across all syntactic categories, unique labels across all enumeration types and the prompt type. We have base type Int and Long for integer values. The integers defined in type Int are also defined in type Long. Every label defined in the prompt type is related with an integer constant as the actual value used in output statement. We differentiate type Long and Int to define the bug fix of type relaxation from Int to Long to prevent overflow in calculation (e.g., $\mathrm{a}+\mathrm{b}$ can cause an error with Int but not with Long). The type Int is necessary reflecting the concern of space and time efficiency in practical computation. We also have user-defined enumeration type, prompt type and array type.

We explicitly have " $i d==l$ " and lval as expressions for convenience of the definition of specific updates. To make our programming language general and to separate the concern of expression evaluation, we parameterize the language by "other" expressions which are unspecified.

We have explicit input and output statement because we model the program behavior as the $\mathrm{I} / \mathrm{O}$ sequence which is the observational behavior of a program. The I/O statement makes it convenient for the argument of program behavior correspondence. In this paper, every I/O value is an integer value which is a common I/O representation [14]. A Statement sequence is defined as $s_{1} ; \ldots ; s_{k}$ where $k>0$ for the convenience of syntax-direct definition from both ends of the sequence.

A program is composed of a possibly empty prompt type Pmpt, a possibly empty sequence of enumeration types $E N$, a possibly empty sequence of global variables $V$ and a sequence of entry

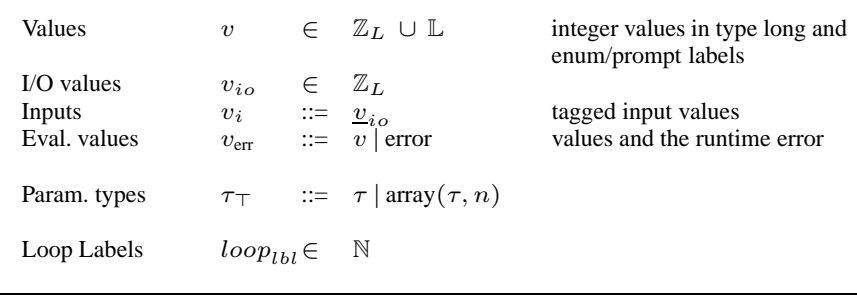

Figure 9: Values, types and domains

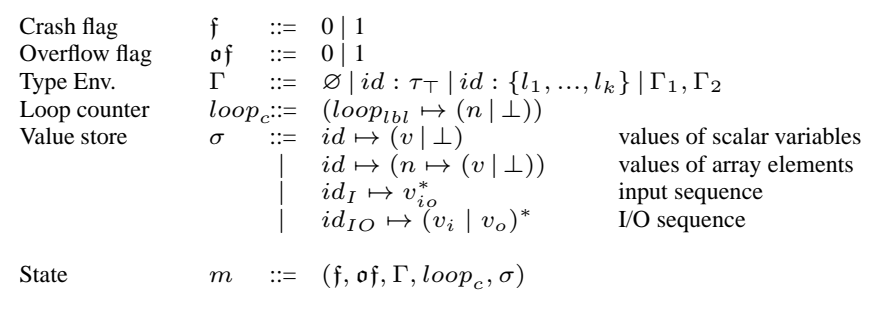

Figure 10: Elements of an execution state

statements $S_{\text {entry }}$. Finally, we have a standard type system based on our syntax.

\subsection{Small-step operational semantics of the formal language}

Figure 9 shows semantic categories of our language. We consider values to be either labels $\mathbb{L}$ or integer numbers $\mathbb{Z}_{L}$ defined in type Long. The integer numbers defined $\mathbb{Z}_{I}$ of type Int are a proper subset of those in type Long, $\mathbb{Z}_{I} \subset \mathbb{Z}_{L}$. We use the notation $\mathbb{Z}_{L+}$ for the positive integers defined in type Long. We use the notation udf $\llbracket \tau \rrbracket$ for an undefined value of type $\tau$. Unlike the "undef" in Clight [8], we need to parameterize the undefined value with a type $\tau$ because we do not have an underlining memory model that can interpret any block content according to a type. An individual value in I/O sequence is an integer number with tag differentiating inputs and outputs, our tags for inputs and outputs are standard notations [14]. The value from expression evaluation is a pair. One of the pair is either a value $v$ or "error" for runtime errors(e.g., division by zero); the other is the overflow flag (i.e., 0 for no overflow).

We use notation $\tau_{\top}$ for all types that are defined in syntax, including array types.

Every loop statement in a program is with a unique label $l o o p_{l b l}$ of a natural number in order to differentiate their executions.

The composition of an execution state is in Figure 10

1. The crash flag $\mathfrak{f}$ is initially zero and is set to one whenever an exception occurs. Once the crash flag is set, it is not cleared. We only consider unrecoverable crashes. The crash flag is used to make sure that updates do not occur in error states.

2. The overflow flag of is initially zero and is set to one whenever an integer overflow in expression evaluation occurs. Overflow flag is sticky in the sense that once it is set, the flag is not cleared. According to [12], integer overflows are common in mature programs.

3. $\Gamma$ is the type environment mapping enumeration type identifers and variable identifiers to their types. Type environment is necessary for checking array index out of bound or checking value mismatch in execution of input/assignment statement.

4. Loop counters $l o o p_{c}$ are to record the number of iterations for one instance of a loop statement. The loop counters $l o o p_{c}$ is 


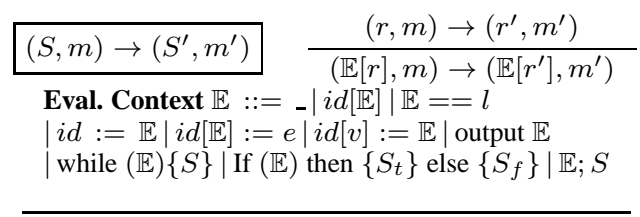

Figure 11: Contextual semantic rule

$$
\begin{aligned}
& (r, m) \rightarrow\left(r^{\prime}, m^{\prime}\right) \\
& \mathcal{E}: \text { other } \rightarrow \sigma \rightarrow\left(v_{\text {err }} \times\{0,1\}\right) \\
& \text { Err : other } \rightarrow\{i d\} \quad \text { (unspecified) } \\
& \operatorname{Var} \frac{\mathfrak{f}=0 \quad \sigma(i d)=v}{(i d, m(\mathfrak{f}, \sigma)) \rightarrow(v, m)} \\
& \operatorname{Arr}-1 \frac{\mathfrak{f}=0 \quad \sigma\left(i d, v_{1}\right)=v_{2}}{\left(i d\left[v_{1}\right], m(\mathfrak{f}, \sigma)\right) \rightarrow\left(v_{2}, m\right)} \\
& \operatorname{Arr}-2 \frac{\mathfrak{f}=0 \quad(\Gamma \vdash i d: \operatorname{array}(\tau, n)) \wedge \neg\left(1 \leq v_{1} \leq n\right)}{\left(i d\left[v_{1}\right], m(\mathfrak{f}, \Gamma)\right) \rightarrow\left(i d\left[v_{1}\right], m(1 / \mathfrak{f})\right)} \\
& \mathrm{Eq}-\mathrm{T} \frac{\mathfrak{f}=0}{(l==l, m(\mathfrak{f})) \rightarrow(1, m)} \\
& \text { Eq-F } \frac{\mathfrak{f}=0 \quad l_{1} \neq l_{2}}{\left(l_{1}==l_{2}, m(\mathfrak{f})\right) \rightarrow(0, m)} \\
& \text { EEval } \frac{\mathfrak{f}=0 \quad e=\text { other }}{(e, m(\mathfrak{f}, \sigma)) \rightarrow(\mathcal{E} \llbracket e \rrbracket \sigma, m)} \\
& \text { ECrash } \frac{\mathfrak{f}=0}{\left(\left(\text { error, } v_{\mathfrak{o f}}\right), m(\mathfrak{f})\right) \rightarrow(0, m(1 / \mathfrak{f}))} \\
& \text { EOflow-1 } \frac{\mathfrak{f}=0 \quad \mathfrak{o f}=0}{\left(\left(v, v_{\mathfrak{o} \mathfrak{f}}\right), m(\mathfrak{f}, \mathfrak{o f})\right) \rightarrow\left(v, m\left(v_{\mathfrak{o} \mathfrak{f}} / \mathfrak{o f}\right)\right)} \\
& \text { EOflow- } 2 \frac{\mathfrak{f}=0 \quad \text { of }=1}{\left(\left(v, v_{\mathfrak{o f}}\right), m(\mathfrak{f}, \mathfrak{o f})\right) \rightarrow(v, m)}
\end{aligned}
$$

Figure 12: SOS rules for expressions

not necessary for program executions but are needed for our reasoning of the execution of loops. When a counter entry for loop label $n$ is not defined in loop counters $l o o p_{c}$, we write $\operatorname{loop}_{c}(n)=\perp$. Otherwise, we write $\operatorname{loop}_{c}(n) \neq \perp$.

5. The value store $\sigma$ is a valuation for scalar variables, array elements, the input sequence variable, and the $\mathrm{I} / \mathrm{O}$ sequence variable.

Execution state $m$ is a composition of elements discussed above. In our SOS rules, we only show components of a state $m$ when necessary (e.g., $m(\Gamma, \sigma)$ ).

Figure 11, shows typical contextual rule and Figure 12,13 and 14 show all SOS rules.

Figure 12 shows rules for expression evaluation. We use the expression meaning function $\mathcal{E}$ : other $\rightarrow \sigma \rightarrow\left(v_{\text {err }} \times\{0,1\}\right)$ to evaluate "other" expressions. In evaluation of expression "other" against a value store $\sigma$, the expression meaning function $\mathcal{E}$ returns a pair $\left(v_{\text {err }}, \mathfrak{o f}\right)$ where the value $v_{\text {err }}$ is either a value $v$ or an "error", of is a flag indicating if there is integer overflow in the evaluation (e.g., 1 if there is overflow). The meaning function $\mathcal{E}$ interprets "other" expressions deterministically. In addition, there is a function Use :

$$
\begin{aligned}
& (r, m) \rightarrow\left(r^{\prime}, m^{\prime}\right) \\
& \text { As-Scl } \frac{\mathfrak{f}=0 \quad \sigma(i d) \neq \perp}{(i d:=v, m(\mathfrak{f}, \sigma)) \rightarrow(\text { skip }, m(\sigma[v / i d]))} \\
& \text { As-Arr } \frac{\mathfrak{f}=0 \quad \sigma\left(i d, v_{1}\right) \neq \perp}{\left(i d\left[v_{1}\right]:=v_{2}, m(\mathfrak{f}, \sigma)\right) \rightarrow\left(\text { skip, } m\left(\sigma\left[v_{2} /\left(i d, v_{1}\right)\right]\right)\right)} \\
& \text { As-Err1 } \frac{\mathfrak{f}=0 \quad(\Gamma \vdash i d: \operatorname{array}(\tau, n)) \wedge \neg\left(1 \leq v_{1} \leq n\right)}{\left(i d\left[v_{1}\right]:=v_{2}, m(\mathfrak{f}, \Gamma)\right) \rightarrow\left(i d\left[v_{1}\right]:=v_{2}, m(1 / \mathfrak{f})\right)} \\
& \mathfrak{f}=0 \quad \sigma(i d) \neq \perp \\
& \text { As-Err2 } \frac{(\Gamma \vdash i d: \text { Int }) \wedge\left(v \in\left(\mathbb{Z}_{L} \backslash \mathbb{Z}_{I}\right)\right)}{(i d:=v, m(\mathfrak{f}, \Gamma, \sigma)) \rightarrow(i d:=v, m(1 / \mathfrak{f}))} \\
& \mathfrak{f}=0 \quad \sigma\left(i d, v_{1}\right) \neq \perp \\
& \text { As-Err3 } \frac{(\Gamma \vdash i d: \operatorname{array}(\operatorname{Int}, n)) \wedge\left(v_{2} \in\left(\mathbb{Z}_{L} \backslash \mathbb{Z}_{I}\right)\right)}{\left(i d\left[v_{1}\right]:=v_{2}, m(\mathfrak{f}, \Gamma, \sigma)\right) \rightarrow\left(i d\left[v_{1}\right]:=v_{2}, m(1 / \mathfrak{f})\right)} \\
& \text { If-T } \frac{\mathfrak{f}=0 \quad\left(v \in \mathbb{Z}_{L}\right) \wedge(v \neq 0)}{\left(\text { If }(v) \text { then }\left\{S_{t}\right\} \text { else }\left\{S_{f}\right\}, m(\mathfrak{f})\right) \rightarrow\left(S_{t}, m\right)} \\
& \text { If-F } \frac{\mathfrak{f}=0}{\left(\text { If }(0) \text { then }\left\{S_{t}\right\} \text { else }\left\{S_{f}\right\}, m(\mathfrak{f})\right) \rightarrow\left(S_{f}, m\right)} \\
& \text { Wh-T } \frac{\mathfrak{f}=0 \quad\left(v \in \mathbb{Z}_{L}\right) \wedge(v \neq 0) \quad \operatorname{loop}_{c}(n)=k}{\left(\text { while }_{\langle n\rangle}(v)\{S\}, m\left(\mathfrak{f}, \operatorname{loop}_{c}\right)\right) \rightarrow} \\
& \text { Wh-F } \frac{\mathfrak{f}=0 \quad \operatorname{loop}_{c}(n) \neq \perp}{\left(\text { while }_{\langle n\rangle}(0)\{S\}, m\left(\mathfrak{f}, \operatorname{loop}_{c}\right)\right) \rightarrow\left(\text { skip }, m\left(\operatorname{loop}_{c}[0 / n]\right)\right)} \\
& \text { Seq } \frac{\mathfrak{f}=0}{(\text { skip; } S, m(\mathfrak{f})) \rightarrow(S, m)} \quad \operatorname{Crash} \frac{\mathfrak{f}=1}{(s, m(\mathfrak{f})) \rightarrow(s, m)}
\end{aligned}
$$

Figure 13: SOS rules for Assignment, If, and While statements

other $\rightarrow\{i d\}$ maps an "other" expression to a set of variables used in the expression; there is a function Err : other $\rightarrow\{i d\}$ maps an expression to a set of variables whose values decide if the evaluation of expression leads to crash. We assume function Use and Err available. The value returned by the expression meaning function only depends on the values of variables in the use set of the expression and the error evaluation only depends on the variables in the error set.

As to integer overflow, there are two ways of handling overflow in practice one is to wrap around overflow using twoscomplement representation (e.g., the gcc option -fwrapv); the other is to generates traps for overflow (e.g., the gcc option -ftrapv). We adopt a combination of the two handling of overflow: the meaning function $\mathcal{E}$ wraps the overflow in some representation (e.g., two-complement) and notifies the overflow in return value. Rule EOflow-1 and EOflow-2 update the sticky overflow flag. The evaluation of $l \mathrm{val}$ or $i d==l$ is shown by respective rules in Figure 12

Figure 13 shows SOS rules for assignment, If, while statements, statement sequence, and crash, which are almost standard. There are four particular crash in execution of assignment statements. One is array out of bound for array access for l-value (e.g., rule As-Err1); the second is assigning a value defined in type Long but not type Int to an Int-typed variable (e.g., rule As-Err2); the third is value mismatch in input statement; the last is expression evaluation exception. As to loop statement, if the predicate expression evaluates to a nonzero integer, corresponding loop counter value increments by one; otherwise, the loop counter value is reset to zero. We 


$$
\begin{aligned}
& (r, m) \rightarrow\left(r^{\prime}, m^{\prime}\right) \\
& \text { In-1 } \frac{\mathfrak{f}=0 \quad \sigma(i d) \neq \perp \quad \operatorname{hd}\left(\sigma\left(i d_{I}\right)\right)=v_{i o} \quad \Gamma \vdash i d: \text { Long }}{\text { (input } i d, m(\mathfrak{f}, \Gamma, \sigma)) \rightarrow} \\
& \text { (skip, } m\left(\sigma\left[v_{i o} / i d\right]\left[\operatorname{tl}\left(\sigma\left(i d_{I}\right)\right) / i d_{I}\right]\left[\text { " } \sigma\left(i d_{I O}\right) \cdot \underline{v}_{i o} " / i d_{I O}\right]\right) \\
& \mathfrak{f}=0 \\
& \sigma(i d) \neq \perp \\
& \text { In-2 } \frac{\operatorname{hd}\left(\sigma\left(i d_{I}\right)\right)=v_{i o} \quad(\Gamma \vdash i d: \text { Int }) \wedge\left(v_{i o} \in \mathbb{Z}_{I}\right)}{(\text { input } i d, m(\mathfrak{f}, \Gamma, \sigma)) \rightarrow(\text { skip, }} \\
& m\left(\sigma\left[v_{i o} / i d\right]\left[\operatorname{tl}\left(\sigma\left(i d_{I}\right)\right) / i d_{I}\right]\left[" \sigma\left(i d_{I O}\right) \cdot \underline{v}_{i o} " / i d_{I O}\right]\right) \\
& \mathfrak{f}=0 \\
& \sigma(i d) \neq \perp \\
& \text { In-3 } \frac{\operatorname{hd}\left(\sigma\left(i d_{I}\right)\right)=v_{i o} \quad(\Gamma \vdash i d: \text { Int }) \wedge\left(v_{i o} \notin \mathbb{Z}_{I}\right)}{(\text { input } i d, m(\mathfrak{f}, \Gamma, \sigma)) \rightarrow(\text { input } i d, m(1 / \mathfrak{f}))} \\
& \mathfrak{f}=0 \quad \sigma(i d) \neq \perp \quad \operatorname{hd}\left(\sigma\left(i d_{I}\right)\right)=v_{i o} \\
& \text { In-4 } \frac{\left(\Gamma \vdash i d: \text { enum } i d^{\prime}\right) \wedge\left(\Gamma \vdash i d^{\prime}:\left\{l_{1}, \ldots, l_{k}\right\}\right) \wedge\left(1 \leq v_{i o} \leq k\right)}{(\text { input } i d, m(\mathfrak{f}, \Gamma, \sigma)) \rightarrow(\operatorname{skip},} \\
& \mathfrak{f}=0 \quad \sigma(i d) \neq \perp \quad \operatorname{hd}\left(\sigma\left(i d_{I}\right)\right)=v_{\text {io }} \\
& \text { In-5 } \frac{\left(\Gamma \vdash i d: \text { enum } i d^{\prime}\right) \wedge\left(\Gamma \vdash i d^{\prime}:\left\{l_{1}, \ldots, l_{k}\right\}\right) \wedge \neg\left(1 \leq v_{i o} \leq k\right)}{(\text { input } i d, m(\mathfrak{f}, \Gamma, \sigma)) \rightarrow(\text { input } i d, m(1 / \mathfrak{f}))} \\
& \text { In-6 } \frac{\mathfrak{f}=0 \quad \sigma\left(i d_{I}\right)=\varnothing}{(\text { input } i d, m(\mathfrak{f}, \sigma)) \rightarrow(\text { input } i d, m(1 / \mathfrak{f}))} \\
& \text { Out-1 } \frac{\mathfrak{f}=0 \quad v \in \mathbb{Z}_{L}}{(\text { output } v, m(\mathfrak{f}, \sigma)) \rightarrow\left(\operatorname{skip}, m\left(\sigma\left[" \sigma\left(i d_{I O}\right) \cdot \bar{v} " / i d_{I O}\right]\right)\right)} \\
& \text { Out-2 } \frac{\mathfrak{f}=0 \quad \Gamma \vdash i d:\left\{l_{1}, \ldots, l_{k}\right\} \wedge v=l_{i} \in\left\{l_{1}, \ldots, l_{k}\right\}}{(\text { output } v, m(\mathfrak{f}, \Gamma, \sigma)) \rightarrow\left(\operatorname{skip}, m\left(\sigma\left[" \sigma\left(i d_{I O}\right) \cdot \bar{i} " / i d_{I O}\right]\right)\right)} \\
& \text { Out-3 } \frac{\mathfrak{f}=0 \quad \Gamma \vdash p m p t:\left\{l_{1}: n_{1}, \ldots, l_{k}: n_{k}\right\}}{\text { " } l: n " \in\left\{l_{1}: n_{1}, \ldots, l_{k}: n_{k}\right\}}
\end{aligned}
$$

Figure 14: SOS rules for input/output statements

use rule Crash to treat crash as non-terminating execution, telling apart normally terminating executions and others.

Figure 14 shows rules for the execution of input/output statements. As to input, there are conversion from values of type Long to those of Int or enumeration types but not the prompt type. For an enumeration type, the Long-typed value is transformed to the label with index of that value if possible. There is crash when value conversion is impossible. Besides, there is crash when executing input statement with empty input sequence. We use standard list operation hd and tl for fetching the list head(leftmost element) or the list tail(the list by removing its head) respectively [30].

Last, we construct initial state in following steps: First, crash flag $\mathfrak{f}$, overflow flag of are zero. Second, type environment is obtained after parsing of the program. Third, every loop counter value in loop $_{c}$ is initially zero. Fourth, every scalar variable or array element has an entry in value store with some initial value if specified. Last, there is initial input sequence and empty I/O sequence.

\subsection{Preliminary terms and notations}

We present terms, notations and definitions for program equivalence and backward compatible update classes.

We use Use $(e)$ or Use $(S)$ to denote used variables in an expression $e$ or a statement sequence $S ; \operatorname{Def}(S)$ denotes the set of defined variables in a statement sequence $S$. The full definitions of Use and Def are in appendix B

We use symbol $\in$ for two different purposes: $x \in X$ denotes one variable to be in a set of variables, $s \in S$ denotes a statement to be in a statement sequence. We use the symbol $\subset$ to refer to proper subset relation.

We call an "If" statement or a "while" statement as a compound statement; all other statements are simple statements. We introduce terms referring to a part of a compound statement. Let $s=$ "If $(e)$ then $\left\{S_{t}\right\}$ else $\left\{S_{f}\right\}$ " be an "If" statement, we call $e$ in $s$ the predicate expression, $S_{t} / S_{f}$ the true/false branch of $s$.

\section{Program equivalence}

We consider several types of program changes that are allowed by "observational equivalence" without user assumptions. These changes include: statement reordering or duplication, extra statements unrelated to output(e.g., logging related changes), loop fission or fusion, and extra statements unrelated to output. Our program equivalence ensures two programs produce the same output, which means two programs produce same I/O sequence till any output. The program equivalence is established upon two other kinds of equivalence, namely equivalent terminating computation of a variable and equivalent termination behavior.

We first define terminating and nonterminating execution. Then we present the framework of program equivalence in three steps in which every later step relies on prior ones. We first propose a proof rule ensuring two programs to compute a variable in the same way. We then suggest a condition ensuring two programs to either both terminate or both do not terminate. Finally we describe a condition ensuring two programs to produce the same output sequence. Our proof rule of program equivalence gives program point mapping as well as program state mapping. Though we express the program equivalence as a whole program relation, it is easy to apply the equivalence check for local changes using our framework under user's various assumptions for equivalence.

\subsection{Definitions of execution}

We define an execution to be a sequence of configurations which are pairs $(S, m)$ where $S$ is a statement sequence and $m$ is a execution state shown in Figure 10 Let $\left(S_{1}, m_{1}\right),\left(S_{2}, m_{2}\right)$ be two consecutive configurations in an execution, the later configuration $\left(S_{2}, m_{2}\right)$ is obtained by applying one semantic rule w.r.t to the configuration $\left(S_{1}, m_{1}\right)$, denoted $\left(S_{1}, m_{1}\right) \rightarrow\left(S_{2}, m_{2}\right)$, called one step (of execution). For our convenience, we use the notation $(S, m) \stackrel{k}{\rightarrow}\left(S^{\prime}, m^{\prime}\right)$ for $k$ steps execution where $k>0$. When we do not care the exact (finite) number of steps, we write the execution as $(S, m) \stackrel{*}{\rightarrow}\left(S^{\prime}, m^{\prime}\right)$. We express terminating executions, nonterminating executions including crash in Definition 7 and 8

Definition 7. (Termination) A statement sequence $S$ normally terminates when started in a state $m$ iff $(S, m) \stackrel{*}{\rightarrow}\left(\right.$ skip, $\left.m^{\prime}(\mathfrak{f})\right)$ where $\mathfrak{f}=0$.

Definition 8. (Nontermination) A statement sequence $S$ does not terminate when started in a state $m$ iff, $\forall k>0:(S, m) \stackrel{k}{\rightarrow}$ $\left(S_{k}, m_{k}\right)$ where $S_{k} \neq$ skip.

\subsection{Equivalent computation for terminating programs}

We propose a proof rule under which two terminating programs are computing a variable in the same way. We start by giving the definition of equivalent computation for terminating programs right after this paragraph. Then we present the proof rule of equivalent computation in the same way. We prove that the proof rule ensures equivalent computation for terminating programs by induction on the program size of the two programs in the proof rule. We also list auxiliary lemmas required by the soundness proof for the proof rule for equivalent computation for terminating programs. 
Definition 9. (Equivalent computation for terminating programs) Two statement sequences $S_{1}$ and $S_{2}$ compute a variable $x$ equivalently when started in states $m_{1}$ and $m_{2}$ respectively, written $\left(S_{1}, m_{1}\right) \equiv_{x}\left(S_{2}, m_{2}\right)$, iff $\left(S_{1}, m_{1}\right) \stackrel{*}{\rightarrow}\left(\right.$ skip, $\left.m_{1}^{\prime}\left(\sigma_{1^{\prime}}\right)\right)$ and $\left(S_{2}, m_{2}\right) \stackrel{*}{\rightarrow}\left(\right.$ skip, $\left.m_{2}^{\prime}\left(\sigma_{2^{\prime}}\right)\right)$ imply $\sigma_{1^{\prime}}(x)=\sigma_{2^{\prime}}(x)$.

\subsubsection{Proof rule for equivalent computation for terminating programs}

We define a proof rule under which $\left(S_{1}, m_{1}\right) \equiv_{x}\left(S_{2}, m_{2}\right)$ holds for generally constructed initial states $m_{1}$ and $m_{2}$, written $S_{1} \equiv_{x}^{S}$ $S_{2}$. Our proof rule for equivalent computation for terminating programs allows updates including statement reordering or duplication, loop fission or fusion, additional statements unrelated to the computation and statements movement across if-branch.

Definition 12 includes the recursive proof rule of equivalent computing for terminating programs. The base case is the condition for two simple statements in Definition 11 Definition 10 of imported variables captures the variable def-use chain which is the essence of our equivalence. In Definition 10 the Def and Use refer to variables defined or used in a statement (sequence) or an expression similar to those in the optimization chapter in the dragon book [4]; $S^{i}$ refers to $i$ consecutive copies of a statement sequence $S$.

Definition 10. (Imported variables) The imported variables in a sequence of statements $S$ relative to variables $X$, written $\operatorname{Imp}(S, X)$, are defined in one of the following cases:

1. $\operatorname{Def}(S) \cap X=\emptyset: \operatorname{Imp}(S, X)=X$;

2. $S=$ "id:=e" or "input id" or "output e" and $\operatorname{Def}(S) \cap X \neq$ $\emptyset:$

$\operatorname{Imp}(S, X)=\operatorname{Use}(S) \cup(X \backslash \operatorname{Def}(S))$;

3. $S=$ "If $(e)$ then $\left\{S_{t}\right\}$ else $\left\{S_{f}\right\}$ " and $\operatorname{Def}(S) \cap X \neq \emptyset$ : $\operatorname{Imp}(S, X)=U \operatorname{se}(e) \cup \bigcup_{y \in X}\left(\operatorname{Imp}\left(S_{t},\{y\}\right) \cup \operatorname{Imp}\left(S_{f},\{y\}\right)\right) ;$

4. $S=$ “while(e) $\left\{S^{\prime}\right\}$ ” where $\left.\left(\operatorname{Def}\left(S^{\prime}\right) \cap X\right) \neq \emptyset\right): \operatorname{Imp}(S, X)$ $=\bigcup_{i>0} \operatorname{Imp}\left(S^{\prime}\right.$, Use $\left.(e) \cup X\right)$;

5. For $k>0, S=s_{1} ; \ldots ; s_{k+1}$ :

$\operatorname{Imp}(S, X)=\operatorname{Imp}\left(s_{1} ; \ldots ; s_{k}, \operatorname{Imp}\left(s_{k+1}, X\right)\right)$

Definition 11. (Base cases of the proof rule for equivalent computation for terminating programs) Two simple statements $s_{1}$ and $s_{2}$ satisfy the proof rule of equivalent computation of a variable $x$, written $s_{1} \equiv_{x}^{S} s_{2}$, iff one of the following holds:

1. $s_{1}=s_{2}$

2. $s_{1} \neq s_{2}$ and one of the following holds:

(a) $s_{1}=$ "input $i d_{1}$ ", $s_{2}=$ "input $i d_{2}$ ", $x \notin\left\{i d_{1}, i d_{2}\right\}$;

(b) Case a) does not hold and $x \notin \operatorname{Def}\left(s_{1}\right) \cup \operatorname{Def}\left(s_{2}\right)$;

Definition 12. (Proof rule of equivalent computation for terminating programs) Two statement sequences $S_{1}$ and $S_{2}$ satisfy the proof rule of equivalent computation of a variable $x$, written $S_{1} \equiv{ }_{x}^{S} S_{2}$, iff one of the following holds:

1. $S_{1}$ and $S_{2}$ are one statement and one of the following holds:

(a) $S_{1}$ and $S_{2}$ are simple statement: $s_{1} \equiv_{x}^{S} s_{2}$;

(b) $S_{1}=$ "If $(e)$ then $\left\{S_{1}^{t}\right\}$ else $\left\{S_{1}^{f}\right\}$ ", $S_{2}=$ "If $(e)$ then $\left\{S_{2}^{t}\right\}$ else

$\left\{S_{2}^{f}\right\}$ " such that all of the following hold:

- $x \in \operatorname{Def}\left(S_{1}\right) \cap \operatorname{Def}\left(S_{2}\right)$;

- $\left(S_{1}^{t} \equiv_{x}^{S} S_{2}^{t}\right) \wedge\left(S_{1}^{f} \equiv_{x}^{S} S_{2}^{f}\right)$;

(c) $S_{1}=$ "while ${ }_{\left\langle n_{1}\right\rangle}(e)\left\{S_{1}^{\prime \prime}\right\} ", S_{2}=$ "while ${ }_{\left\langle n_{2}\right\rangle}(e)\left\{S_{2}^{\prime \prime}\right\} "$ such that both of the following hold:

- $x \in \operatorname{Def}\left(S_{1}\right) \cap \operatorname{Def}\left(S_{2}\right)$;

- $\forall y \in \operatorname{Imp}\left(S_{1},\{x\}\right) \cup \operatorname{Imp}\left(S_{2},\{x\}\right): S_{1}^{\prime \prime} \equiv_{y}^{S} S_{2}^{\prime \prime}$; (d) $S_{1}$ and $S_{2}$ do not define the variable $x: x \notin \operatorname{Def}\left(S_{1}\right) \cup$ $\operatorname{Def}\left(S_{2}\right)$.

2. $S_{1}$ and $S_{2}$ are not both one statement and one of the following holds:

(a) $S_{1}=S_{1}^{\prime} ; s_{1}, S_{2}=S_{2}^{\prime} ; s_{2}$ and last statements both define the variable $x$ such that both of the following hold:

- $\forall y \in \operatorname{Imp}\left(s_{1},\{x\}\right) \cup \operatorname{Imp}\left(s_{2},\{x\}\right): S_{1}^{\prime} \equiv_{y}^{S} S_{2}^{\prime}$;

- $s_{1} \equiv_{x}^{S} s_{2}$ where $x \in \operatorname{Def}\left(s_{1}\right) \cap \operatorname{Def}\left(s_{2}\right)$;

(b) Last statement in $S_{1}$ or $S_{2}$ does not define the variable $x$ : $\left(x \notin \operatorname{Def}\left(s_{2}\right) \wedge\left(S_{1} \equiv_{x}^{S} S_{2}^{\prime}\right)\right) \vee\left(x \notin \operatorname{Def}\left(s_{1}\right) \wedge\left(S_{1}^{\prime} \equiv_{x}^{S}\right.\right.$ $\left.S_{2}\right)$ );

(c) $S_{1}=S_{1}^{\prime} ; s_{1}, S_{2}=S_{2}^{\prime} ; s_{2}$ and there are statements moving in/out of If statement: $s_{1}=$ "If $(e)$ then $\left\{S_{1}^{t}\right\}$ else $\left\{S_{1}^{f}\right\}$ ", $s_{2}=$ "If $(e)$ then $\left\{S_{2}^{t}\right\}$ else $\left\{S_{2}^{f}\right\}$ " such that none of the above cases hold and all of the following hold:

- $\forall y \in U \operatorname{se}(e): S_{1}^{\prime} \equiv_{y}^{S} S_{2}^{\prime}$;

- $\left(S_{1}^{\prime} ; S_{1}^{t} \equiv_{x}^{S} S_{2}^{\prime} ; S_{2}^{t}\right) \wedge\left(S_{1}^{\prime} ; S_{1}^{f} \equiv_{x}^{S} S_{2}^{\prime} ; S_{2}^{f}\right)$;

The generalization of definition $S_{1} \equiv_{x}^{S} S_{2}$ to a set of variables is as follows.

Definition 13. Two statement sequences $S_{1}$ and $S_{2}$ have equivalent computation of variables $X$, written $S_{1} \equiv_{X}^{S} S_{2}$, iff $\forall x \in X$ : $S_{1} \equiv{ }_{x}^{S} S_{2}$.

\subsubsection{Soundness of the proof rule for equivalent computation for terminating programs}

We show that if two programs satisfy the proof rule of equivalent computation of a variable $x$ (Definition 12) and their value stores in initial states agree on values of the imported variables relative to $x$, then the two programs compute the same value of $x$ if they terminate. We start by proving the theorem for the base cases of terminating computation equivalently.

Theorem 1. If $s_{1}$ and $s_{2}$ are simple statements that satisfy the proof rule for equivalent computation of $x, s_{1} \equiv_{x}^{S} s_{2}$, and their initial states $m_{1}\left(\sigma_{1}\right)$ and $m_{2}\left(\sigma_{2}\right)$ agree on the values of the imported variables relative to $x, \forall y \in \operatorname{Imp}\left(s_{1},\{x\}\right) \cup \operatorname{Imp}\left(s_{2},\{x\}\right)$ : $\sigma_{s_{1}}(y)=\sigma_{s_{2}}(y)$, then $s_{1}$ and $s_{2}$ equivalently compute $x$ when started in states $m_{1}$ and $m_{2}$ respectively, $\left(s_{1}, m_{1}\right) \equiv_{x}\left(s_{2}, m_{2}\right)$.

Proof. The proof is a case analysis according to the cases in the definition of the proof rule for equivalent computation (i.e., Definition 11.

1. $s_{1}=s_{2}$

Since the two statements are identical, they have the same imported variables. By assumption, the imported variables of $s_{1}$ and $s_{2}$ have the same initial values, so it is enough to show that the value of $x$ at the end of the computation only depends on the initial values of the imported variables.

(a) $s_{1}=s_{2}=$ "skip". In this case, the states before and after the execution of skip are the same and $\operatorname{Imp}($ skip, $\{x\})=$ $\{x\}$.

(b) $s_{1}=s_{2}=$ "lval $:=e "$.

i. lval $=x$.

$s_{1}=s_{2}=$ " $x:=e$ ". By the definition of imported variables, $\operatorname{Imp}(x:=e,\{x\})=\operatorname{Use}(e)$. The execution of $s_{1}$ proceeds as follows.

$(x:=e, m(\sigma))$

$\rightarrow\left(x:=\mathcal{E}^{\prime} \llbracket e \rrbracket \sigma, m(\sigma)\right)$ by the EEval' rule

$\rightarrow\left(\right.$ skip, $\left.m\left(\sigma\left[\mathcal{E}^{\prime} \llbracket e \rrbracket \sigma / x\right]\right)\right)$ by the Assign rule.

The value of $x$ after the full execution is $\sigma[(\mathcal{E} \llbracket e \rrbracket \sigma) / x](x)$ which only depend on the initial values of the imported 
variables by the property of the expression meaning function.

ii. $l v a l \neq i d$.

By the definition of imported variables, $\operatorname{Imp}\left(s_{1},\{x\}\right)=$ $\operatorname{Imp}\left(s_{2},\{x\}\right)=\{x\}$. It follows, by assumption, that $\sigma_{1}(x)=\sigma_{2}(x)$ and also $s_{1}$ terminate, $\left(s_{1}, m_{1}\left(\sigma_{1}\right)\right) \stackrel{*}{\rightarrow}$ (skip, $\left.m_{1}^{\prime}\left(\sigma_{1}^{\prime}\right)\right)$. Hence, $\sigma_{1}^{\prime}(x)=\sigma_{1}(x)$ by Corollary E.2 Similarly, $s_{2}$ terminates, $\left(s_{2}, m_{2}\left(\sigma_{2}\right)\right) \stackrel{*}{\rightarrow}$ (skip, $m_{2}^{\prime}\left(\sigma_{2}^{\prime}\right)$ ) and $\sigma_{2}^{\prime}(x)=\sigma_{2}(x)$. Therefore, $\sigma_{2}^{\prime}(x)=$ $\sigma_{2}(x)=\sigma_{1}(x)=\sigma_{1}^{\prime}(x)$ and the theorem holds.

(c) $s_{1}=s_{2}=$ "input $i d$ ".

i. $x \in \operatorname{Def}($ input $i d)=\left\{i d, i d_{I}, i d_{I O}\right\}$.

By the In rule, the execution of input $i d$ is the following.

$$
\begin{aligned}
& (\text { input } i d, m(\sigma)) \\
\rightarrow & \left(\operatorname{skip}, m\left(\sigma\left[\operatorname{tl}\left(\sigma\left(i d_{I}\right)\right) / i d_{I}\right]\right.\right. \\
& {\left.\left.\left[\text { " } \sigma\left(i d_{I O}\right) \cdot \operatorname{hd}\left(\sigma\left(i d_{I}\right)\right) " / i d_{I O}\right]\left[\operatorname{hd}\left(\sigma\left(i d_{I}\right)\right) / i d\right]\right)\right) . }
\end{aligned}
$$

The value of $x$ after the execution of "input $i d$ " is one of the following:
A. $\operatorname{tl}\left(\sigma\left(i d_{I}\right)\right)$ if $x=i d_{I}$.
B. $\sigma_{1}\left(i d_{I O}\right) \cdot \operatorname{hd}\left(\sigma\left(i d_{I}\right)\right)$ if $x=i d_{I O}$.
C. $\operatorname{hd}\left(\sigma\left(i d_{I}\right)\right)$ if $x=i d$.

By the definition of imported variables, $\operatorname{Imp}($ input $i d,\{x\})$ $=\left\{i d_{I O}, i d_{I}\right\}$. So, in all cases, the value of $x$ only depends on the initial values of the imported variables $i d_{I}$ and $i d_{I O}$.

ii. $x \notin \operatorname{Def}($ input $i d)=\left\{i d, i d_{I}, i d_{I O}\right\}$.

By same argument in the subcase $i d \neq x$ of case $s_{1}=$ $s_{2}=$ " $i d:=e "$, the theorem holds.

(d) $s_{1}=s_{2}=$ "output $e$ ".

\section{i. $x=i d_{I O}$}

By the definition of imported variables, $\operatorname{Imp}($ output $e,\{x\})=$ $\left\{i d_{I O}\right\} \cup \operatorname{Use}(e)$. The execution of $s_{1}$ proceeds as follows.

$$
\begin{aligned}
& \text { (output } e, m(\sigma)) \\
\rightarrow & (\text { output } \mathcal{E} \llbracket e \rrbracket \sigma, m(\sigma)) \\
\rightarrow & \left(\text { skip, } m\left(\sigma\left[" \sigma\left(i d_{I O}\right) \cdot \mathcal{E} \llbracket e \rrbracket \sigma " / i d_{I O}\right]\right)\right) .
\end{aligned}
$$

The value of $x$ after the execution is " $\sigma\left(i d_{I O}\right) \cdot \mathcal{E} \llbracket \bar{e} \rrbracket \sigma$ ", which only depends on the initial value of the imported variables of the statement "output $e$ " by the expression meaning function.

ii. $x \neq i d_{I O}$

By same argument in the subcase $i d \neq x$ of case $s_{1}=$ $s_{2}=$ "id $:=e "$, the theorem holds.

2. $s_{1} \neq s_{2}$

(a) $s_{1}=$ "input $i d_{1}$ ", $s_{2}=$ "input $i d_{2} ", x \notin\left\{i d_{1}, i d_{2}\right\}$.

\section{i. $x \in\left\{i d_{I}, i d_{I O}\right\}$.}

By the definition of imported variables, $\operatorname{Imp}\left(s_{1},\{x\}\right)=$ $\operatorname{Imp}\left(s_{2},\{x\}\right)=\left\{i d_{I O}, i d_{I}\right\}$. It follows, by assumption, that $\sigma_{1}(y)=\sigma_{2}(y), \forall y \in\left\{i d_{I O}, i d_{I}\right\}$. The execution of $s_{1}$ proceeds as follows.

$$
\begin{aligned}
& \left(s_{1}, m_{1}\right) \\
= & \left(\text { input } i d_{1}, m_{1}\left(\sigma_{1}\right)\right) \\
\rightarrow & \left(\operatorname{skip}, m_{1}\left(\sigma_{1}\left[\operatorname{tl}\left(\sigma_{1}\left(i d_{I}\right)\right) / i d_{I}\right]\right.\right. \\
& {\left.\left.\left[\text { " } \sigma_{1}\left(i d_{I O}\right) \cdot \underline{\operatorname{hd}\left(\sigma_{1}\left(i d_{I}\right)\right) "} / i d_{I O}\right]\left[\operatorname{hd}\left(\sigma_{1}\left(i d_{I}\right)\right) / i d_{1}\right]\right)\right) }
\end{aligned}
$$

Let $\sigma_{1}^{\prime}=\sigma_{1}\left[\operatorname{tl}(\vec{v}) / i d_{I}, " \sigma_{1}\left(i d_{I O}\right) \cdot \operatorname{hd}(\vec{v}) " / i d_{I O}, \operatorname{hd}(\vec{v}) / i d_{1}\right]$. The value of $x$ after the execution of $\overline{s_{1}}$ is one of the following:
A. $\sigma_{1}^{\prime}(x)=\operatorname{tl}\left(\sigma_{1}\left(i d_{I}\right)\right)$ if $x=i d_{I}$.
B. $\sigma_{1}^{\prime}(x)=\sigma_{1}\left(i d_{I O}\right) \cdot \operatorname{hd}\left(\sigma_{1}\left(i d_{I}\right)\right)$ if $x=i d_{I O}$.

Similarly, $\left(s_{2}, m_{2}\right) \rightarrow$ (skip, $m_{2}\left(\sigma_{2}\left[\operatorname{tl}\left(\sigma_{2}\left(i d_{I}\right)\right) / i d_{2}\right]\right.$ $\left[" \sigma_{2}\left(i d_{I O}\right) \cdot \operatorname{hd}\left(\sigma_{2}\left(i d_{I}\right)\right)\right.$ " $\left.\left.\left./ i d_{I O}\right]\left[\mathrm{hd}\left(\sigma_{2}\left(i d_{I}\right)\right) / i d_{2}\right]\right)\right)$. Let $\sigma_{2}^{\prime}=\sigma_{2}\left[\overline{\mathrm{tl}\left(\sigma_{2}\left(i d_{I}\right)\right) / i} d_{I}\right]\left[\right.$ " $\left.\sigma_{2}\left(i d_{I O}\right) \cdot \mathrm{hd}\left(\sigma_{2}\left(i d_{I}\right)\right) " / i d_{I O}\right]$ [hd $\left.\left(\sigma_{2}\left(i d_{I}\right)\right) / i d_{2}\right]$. Then the value of $x$ after the execution of $s_{2}$ is one of the following:

A. $\sigma_{2}^{\prime}(x)=\operatorname{tl}\left(\sigma_{2}\left(i d_{I}\right)\right)$ if $x=i d_{I}$

B. $\sigma_{2}^{\prime}(x)=\sigma_{2}\left(i d_{I O}\right) \cdot \operatorname{hd}\left(\sigma_{2}\left(i d_{I}\right)\right)$ if $x=i d_{I O}$

Repeatedly, $\sigma_{2}\left(i d_{I}\right)=\overline{\sigma_{1}\left(i d_{I}\right)}$ and $\sigma_{2}\left(i d_{I O}\right)=$ $\sigma_{1}\left(i d_{I O}\right)$. Therefore, the theorem holds.

ii. $x \notin\left\{i d_{I}, i d_{I O}\right\}$

Repeatedly, $x \notin\left\{i d_{1}, i d_{2}\right\}$. By same argument in the subcase $i d \neq x$ of case $s_{1}=s_{2}=$ "id :=e", the theorem holds.

(b) all the above cases do not hold and $x \notin \operatorname{Def}\left(s_{1}\right) \cup \operatorname{Def}\left(s_{2}\right)$ By same argument in the subcase $i d \neq x$ of case $s_{1}=s_{2}=$ " $i d:=e$ ", the theorem holds.

Theorem 2. If statement sequence $S_{1}$ and $S_{2}$ satisfy the proof rule of equivalent computation of a variable $x, S_{1} \equiv_{x}^{S} S_{2}$, and their initial states $m_{1}\left(\sigma_{1}\right)$ and $m_{2}\left(\sigma_{2}\right)$ agree on the initial values of the imported variables relative to $x, \forall y \in \operatorname{Imp}\left(S_{1},\{x\}\right) \cup$ $\operatorname{Imp}\left(S_{2},\{x\}\right): \sigma_{1}(y)=\sigma_{2}(y)$, then $S_{1}$ and $S_{2}$ equivalently compute the variable $x$ when started in state $m_{1}$ and $m_{2}$ respectively, $\left(S_{1}, m_{1}\right) \equiv_{x}\left(S_{2}, m_{2}\right)$.

Proof. By induction on $\operatorname{size}\left(S_{1}\right)+\operatorname{size}\left(S_{2}\right)$, the sum of the program size of $S_{1}$ and $S_{2}$.

Base case.

$S_{1} \equiv{ }_{x}^{S} \quad S_{2}$ where $S_{1}$ and $S_{2}$ are two simple statements. This theorem holds by theorem 1

\section{Induction step}

The hypothesis $\mathrm{IH}$ is that Theorem 2 holds when $\operatorname{size}\left(S_{1}\right)+$ $\operatorname{size}\left(S_{2}\right)=k \geq 2$.

Then we show that the Theorem holds when $\operatorname{size}\left(S_{1}\right)+\operatorname{size}\left(S_{2}\right)=$ $k+1$. The proof is a case analysis according to the cases in the definition of the proof rule of terminating computation of statement sequence. the two big categories enum

1. $S_{1}$ and $S_{2}$ are one statement such that one of the following holds:

(a) $S_{1}$ and $S_{2}$ are If statement that define the variable $x$ :

$S_{1}=$ "If $(e)$ then $\left\{S_{1}^{t}\right\}$ else $\left\{S_{1}^{f}\right\}$ ", $S_{2}=$ "If $(e)$ then $\left\{S_{2}^{t}\right\}$ else $\left\{S_{2}^{f}\right\}$ " such that all of the following hold:

- $x \in \operatorname{Def}\left(S_{1}\right) \cap \operatorname{Def}\left(S_{2}\right)$;

- $S_{1}^{t} \equiv_{x}^{S} S_{2}^{t}$;

- $S_{1}^{f} \equiv_{x}^{S} S_{2}^{f}$;

We first show that the evaluations of the predicate expression of $S_{1}$ and $S_{2}$ produce the same value when started from state $m_{1}\left(\sigma_{1}\right)$ and $m_{2}\left(\sigma_{2}\right)$, w.l.o.g. say zero. Next, we show that $S_{1}^{f}$ started in the state $m_{1}$ and $S_{2}^{f}$ in the state $m_{2}$ equivalently compute the variable $x$.

In order to show that the evaluations of predicate expression of $S_{1}$ and $S_{2}$ produce same value when started from state $m_{1}\left(\sigma_{1}\right)$ and $m_{2}\left(\sigma_{2}\right)$, we show that the variables used in predicate expression of $S_{1}$ and $S_{2}$ are a subset of imported variables in $S_{1}$ and $S_{2}$ relative to $x$. This is true by the definition of imported variables, $\operatorname{Use}(e) \subseteq \operatorname{Imp}\left(S_{1},\{x\}\right), \operatorname{Use}(e) \subseteq \operatorname{Imp}\left(S_{2},\{x\}\right)$. By assumption, the value stores $\sigma_{1}$ and $\sigma_{2}$ agree on the values of the variables used in predicate expression of $S_{1}$ and $S_{2}, \sigma_{1}(y)=\sigma_{2}(y), \forall y \in \operatorname{Use}(e)$. By the property of 
expression meaning function $\mathcal{E}$, the predicate expression of $S_{1}$ and $S_{2}$ evaluate to the same value when started in states $m_{1}\left(\sigma_{1}\right)$ and $m_{2}\left(\sigma_{2}\right), \mathcal{E} \llbracket e \rrbracket \sigma_{1}=\mathcal{E} \llbracket e \rrbracket \sigma_{2}$, w.l.o.g, $\mathcal{E} \llbracket e \rrbracket \sigma_{1}=\mathcal{E} \llbracket e \rrbracket \sigma_{2}=\left(0, v_{\mathfrak{o f}}\right)$. Then the execution of $S_{1}$ proceeds as follows.

$$
\begin{aligned}
& \left(S_{1}, m_{1}\left(\sigma_{1}\right)\right) \\
= & \left(\text { If }(e) \text { then }\left\{S_{1}^{t}\right\} \text { else }\left\{S_{1}^{f}\right\}, m_{1}\left(\sigma_{1}\right)\right) \\
\rightarrow & \left(\text { If }\left(\left(0, v_{\text {of }}\right)\right) \text { then }\left\{S_{1}^{t}\right\} \text { else }\left\{S_{1}^{f}\right\}, m_{1}\left(\sigma_{1}\right)\right) \\
& \text { by the EEval' rule } \\
\rightarrow & \left(\text { If }(0) \text { then }\left\{S_{1}^{t}\right\} \text { else }\left\{S_{1}^{f}\right\}, m_{1}\left(\sigma_{1}\right)\right) \\
& \text { by the E-Oflow1 or E-Oflow } 2 \text { rule } \\
\rightarrow & \left(S_{1}^{f}, m_{1}\left(\sigma_{1}\right)\right) \text { by the If-F rule. }
\end{aligned}
$$

Similarly, the execution from $\left(s_{2}, m_{2}\left(\sigma_{2}\right)\right)$ gets to $\left(S_{2}^{f}, m_{2}\left(\sigma_{2}\right)\right)$. By the hypothesis IH, we show that $S_{1}^{f}$ and $S_{2}^{f}$ compute the variable $x$ equivalently when started in state $m_{1}\left(\sigma_{1}\right)$ and $m_{2}\left(\sigma_{2}\right)$ respectively. To do that, we show that all required conditions are satisfied for the application of hypothesis IH.

- $\operatorname{size}\left(S_{1}^{f}\right)+\operatorname{size}\left(S_{2}^{f}\right)<k$.

Because size $\left(S_{1}\right)=1+\operatorname{size}\left(S_{1}^{t}\right)+\operatorname{size}\left(S_{1}^{f}\right), \operatorname{size}\left(S_{2}\right)=$ $1+\operatorname{size}\left(S_{2}^{t}\right)+\operatorname{size}\left(S_{2}^{f}\right)$.

- the value stores $\sigma_{1}$ and $\sigma_{2}$ agree on the values of the imported variables in $S_{1}^{f}$ and $S_{2}^{f}$ relative to $x, \sigma_{1}(y)=$ $\sigma_{2}(y), \forall y \in \operatorname{Imp}\left(S_{1}^{f},\{x\}\right) \cup \operatorname{Imp}\left(S_{2}^{f},\{x\}\right)$.

By the definition of imported variables,

$\operatorname{Imp}\left(S_{1}^{f},\{x\}\right) \subseteq \operatorname{Imp}\left(S_{1},\{x\}\right), \operatorname{Imp}\left(S_{2}^{f},\{x\}\right) \subseteq \operatorname{Imp}\left(S_{2},\{x\}\right)$.

By the hypothesis IH, $S_{1}^{f}$ and $S_{2}^{f}$ compute the variable $x$ equivalently when started in state $m_{1}\left(\sigma_{1}\right)$ and $m_{2}\left(\sigma_{2}\right)$ respectively. Therefore, the theorem holds.

(b) $S_{1}$ and $S_{2}$ are while statement that define the variable $x$ : $S_{1}=$ "while ${ }_{\left\langle n_{1}\right\rangle}(e)\left\{S_{1}^{\prime \prime}\right\} ", S_{2}=$ "while ${ }_{\left\langle n_{2}\right\rangle}(e)\left\{S_{2}^{\prime \prime}\right\} "$ such that both of the following hold:

- $x \in \operatorname{Def}\left(S_{1}\right) \cap \operatorname{Def}\left(S_{2}\right)$;

- $S_{1}^{\prime \prime} \equiv_{y}^{S} S_{2}^{\prime \prime}$ for $\forall y \in \operatorname{Imp}\left(S_{1},\{x\}\right) \cup \operatorname{Imp}\left(S_{2},\{x\}\right)$;

By Lemma 5.2 we show $S_{1}$ and $S_{2}$ compute the variable $x$ equivalently when started from state $m_{1}\left(m_{c}^{1}, \sigma_{1}\right)$ and $m_{2}\left(m_{c}^{2}, \sigma_{2}\right)$ respectively. The point is to show that all required conditions are satisfied for the application of lemma 5.2

- loop counter value of $S_{1}$ and $S_{2}$ are zero.

By our assumption, the loop counter value of $S_{1}$ and $S_{2}$ are initially zero.

- $S_{1}$ and $S_{2}$ have same imported variables relative to $x$, $\operatorname{Imp}\left(S_{1},\{x\}\right)=\operatorname{Imp}\left(S_{2},\{x\}\right)=\operatorname{Imp}(\Delta)$.

This is obtained by Lemma 5.3

- the initial value store $\sigma_{1}$ and $\sigma_{2}$ agree on the values of the imported variables in $S_{1}$ and $S_{2}$ relative to $x$, $\sigma_{1}(y)=\sigma_{2}(y), \forall y \in \operatorname{Imp}\left(S_{1},\{x\}\right) \cup \operatorname{Imp}\left(S_{2},\{x\}\right)$. By assumption, this holds.

- $S_{1}^{\prime \prime}$ and $S_{2}^{\prime \prime}$ compute the imported variables in $S_{1}$ and $S_{2}$ relative to $x$ equivalently, $\left(S_{1}^{\prime \prime}, m_{S_{1}^{\prime \prime}}\left(\sigma_{S_{1}^{\prime \prime}}\right)\right) \equiv_{y}$ $\left(S_{2}^{\prime \prime}, m_{S_{2}^{\prime \prime}}\left(\sigma_{S_{2}^{\prime \prime}}\right)\right), \forall y \in \operatorname{Imp}(\Delta)$ with value stores $\sigma_{S_{1}^{\prime \prime}}$ and $\sigma_{S_{2}^{\prime \prime}}$ agreeing on the values of the imported variables in $S_{1}^{\prime \prime}$ and $S_{2}^{\prime \prime}$ relative to $\operatorname{Imp}(\Delta), \sigma_{S_{1}^{\prime \prime}}(z)=$ $\sigma_{S_{2}^{\prime \prime}}(z), \forall z \in \operatorname{Imp}\left(S_{1}^{\prime \prime}, \operatorname{Imp}(\Delta)\right) \cup \operatorname{Imp}\left(S_{2}^{\prime \prime}, \operatorname{Imp}(\Delta)\right)$. By the definition of program size, the sum of the program size of $S_{1}^{\prime}$ and $S_{2}^{\prime}$ is less than $k$, size $\left(S_{1}^{\prime \prime}\right)+$ $\operatorname{size}\left(S_{2}^{\prime \prime}\right)<k$. By the hypothesis IH, $S_{1}^{\prime \prime}$ and $S_{2}^{\prime \prime}$ compute the imported variables in $S_{1}$ and $S_{2}$ relative to $x$ equivalently when started in states $m_{S_{1}^{\prime \prime}}\left(\sigma_{S_{1}^{\prime \prime}}\right)$ and $m_{S_{2}^{\prime \prime}}\left(\sigma_{S_{2}^{\prime \prime}}\right)$ with value store $\sigma_{S_{1}^{\prime \prime}}$ and $\sigma_{S_{2}^{\prime \prime}}$ agreeing on the values of the imported variables in $S_{1}^{\prime \prime}$ and $S_{2}^{\prime \prime}$ relative to the variables $\operatorname{Imp}(\Delta)$.

By Lemma 5.2 we show $S_{1}$ and $S_{2}$ compute the variable $x$ equivalently when started from state $m_{1}\left(m_{c}^{1}, \sigma_{1}\right)$ and $m_{2}\left(m_{c}^{2}, \sigma_{2}\right)$ respectively. The theorem holds.

(c) $S_{1}$ and $S_{2}$ do not define the variable $x: x \notin \operatorname{Def}\left(S_{1}\right) \cup$ $\operatorname{Def}\left(S_{2}\right)$.

By the definition of imported variable, the imported variables in $S_{1}$ and $S_{2}$ relative to $x$ are both $x, \operatorname{Imp}\left(S_{1},\{x\}\right)=$ $\operatorname{Imp}\left(S_{2},\{x\}\right)=\{x\}$. By assumption, the initial values $\sigma_{1}$ and $\sigma_{2}$ agree on the value of the variable $x, \sigma_{1}(x)=$ $\sigma_{2}(x)$. In addition, by assumption, execution of $S_{1}$ and $S_{2}$ when started in state $m_{1}\left(\sigma_{1}\right)$ and $m_{2}\left(\sigma_{2}\right)$ terminate, $\left(S_{1}, m_{1}\left(\sigma_{1}\right)\right) \stackrel{*}{\rightarrow}\left(\operatorname{skip}, m_{1}^{\prime}\left(\sigma_{1}^{\prime}\right)\right),\left(S_{2}, m_{2}\left(\sigma_{2}\right)\right) \stackrel{*}{\rightarrow}\left(\operatorname{skip}, m_{2}^{\prime}\left(\sigma_{2}^{\prime}\right)\right)$. Finally, by Corollary E.2, the value of $x$ is not changed in execution of $S_{1}$ and $S_{2}, \sigma_{1}^{\prime}(x)=\sigma_{1}(x)=\sigma_{2}(x)=\sigma_{2}^{\prime}(x)$. The theorem holds.

2. $S_{1}$ and $S_{2}$ are not both one statement such that one of the following holds:

(a) Last statements both define the variable $x$ such that all of the following hold:

- $S_{1}^{\prime} \equiv{ }_{y}^{S} S_{2}^{\prime}, \forall y \in \operatorname{Imp}\left(s_{1},\{x\}\right) \cup \operatorname{Imp}\left(s_{2},\{x\}\right)$;

- $x \in \operatorname{Def}\left(s_{1}\right) \cap \operatorname{Def}\left(s_{2}\right)$;

- $s_{1} \equiv{ }_{x}^{S} s_{2}$;

We show that $S_{1}^{\prime}$ and $S_{2}^{\prime}$ compute the imported variables in $s_{1}$ and $s_{2}$ relative to the variable $x$ equivalently when started in state $m_{1}\left(\sigma_{1}\right)$ and $m_{2}\left(\sigma_{2}\right)$ respectively by the hypothesis $\mathrm{IH}$. To do that, we show the required conditions are satisfied for applying the hypothesis IH.

- $\operatorname{size}\left(S_{1}^{\prime}\right)+\operatorname{size}\left(S_{2}^{\prime}\right)<k$.

By the definition of program size, $\operatorname{size}\left(s_{1}\right) \geq 1$, $\operatorname{size}\left(s_{2}\right) \geq 1$. Hence, $\operatorname{size}\left(S_{1}^{\prime}\right)+\operatorname{size}\left(S_{2}^{\prime}\right)<k$.

- the executions from $\left(S_{1}, m_{1}\left(\sigma_{1}\right)\right)$ and $\left(S_{2}, m_{2}\left(\sigma_{2}\right)\right)$ terminate respectively,

$\left(S_{1}^{\prime}, m_{1}\left(\sigma_{1}\right)\right) \stackrel{*}{\rightarrow}\left(\right.$ skip, $\left.m_{1}^{\prime \prime}\left(\sigma_{1}^{\prime \prime}\right)\right),\left(S_{2}^{\prime}, m_{2}\left(\sigma_{2}\right)\right) \stackrel{*}{\rightarrow}$ (skip, $m_{2}^{\prime \prime}\left(\sigma_{2}^{\prime \prime}\right)$ ).

By assumption, the execution from $\left(S_{1}, m_{1}\left(\sigma_{1}\right)\right)$ and $\left(S_{2}, m_{2}\left(\sigma_{2}\right)\right)$ terminate, then the execution of $S_{1}^{\prime}$ and $S_{2}^{\prime}$ from state $m_{1}\left(\sigma_{1}\right)$ and $m_{2}\left(\sigma_{2}\right)$ terminate, $\left(S_{1}^{\prime}, m_{1}\left(\sigma_{1}\right)\right) \stackrel{*}{\rightarrow}$ (skip, $\left.m_{1}^{\prime \prime}\left(\sigma_{1}^{\prime \prime}\right)\right),\left(S_{2}^{\prime}, m_{2}\left(\sigma_{2}\right)\right) \stackrel{*}{\rightarrow}$ (skip, $\left.m_{2}^{\prime \prime}\left(\sigma_{2}^{\prime \prime}\right)\right)$.

- the initial value stores agree on the values of the variables:

$\operatorname{Imp}\left(S_{1}^{\prime}, \operatorname{Imp}\left(s_{1},\{x\}\right)\right) \cup \operatorname{Imp}\left(S_{2}^{\prime}, \operatorname{Imp}\left(s_{2},\{x\}\right)\right)$

By Lemma 5.3 $s_{1}$ and $s_{2}$ have the same imported variables relative to $x, \operatorname{Imp}\left(s_{1},\{x\}\right)=\operatorname{Imp}\left(s_{2},\{x\}\right)$ $=\operatorname{Imp}(x)$. By the definition of imported variables, imported variables in $S_{1}^{\prime}$ relative to $\operatorname{Imp}(x)$ are same as the imported variables in $S_{1}$ relative to $x, \operatorname{Imp}\left(S_{1}^{\prime}, \operatorname{Imp}\left(s_{1},\{x\}\right)\right)=$ $\operatorname{Imp}\left(S_{1},\{x\}\right)$. Similarly, $\operatorname{Imp}\left(S_{2}^{\prime}, \operatorname{Imp}\left(s_{2},\{x\}\right)\right)=$ $\operatorname{Imp}\left(S_{2},\{x\}\right)$. Then, by assumption, the initial value stores agree on the values of the variables $\operatorname{Imp}\left(S_{1}^{\prime}, \operatorname{Imp}\left(s_{1},\{x\}\right)\right)$ and $\forall y \in \operatorname{Imp}\left(S_{1}^{\prime}, \operatorname{Imp}\left(s_{1},\{x\}\right)\right) \cup \operatorname{Imp}\left(S_{2}^{\prime}, \operatorname{Imp}\left(s_{2},\{x\}\right)\right)$, $\operatorname{Imp}\left(S_{2}^{\prime}, \operatorname{Imp}\left(s_{2},\{x\}\right)\right), \sigma_{1}(y)=\sigma_{2}(y)$.

By the hypothesis $\mathrm{IH}$, after the full execution of $S_{1}^{\prime}$ from state $m_{1}\left(\sigma_{1}\right)$ and the execution of $S_{2}^{\prime}$ from state $m_{2}\left(\sigma_{2}\right)$, the value stores agree on the values of the imported variables in $s_{1}$ and $s_{2}$ relative to $x, \sigma_{1}^{\prime \prime}(y)=\sigma_{2}^{\prime \prime}(y), \forall y \in \operatorname{Imp}(x)=$ $\operatorname{Imp}\left(s_{1},\{x\}\right)=\operatorname{Imp}\left(s_{2},\{x\}\right)$.

Then, we show $s_{1}$ and $s_{2}$ compute $x$ equivalently. By Corollary E.1 $s_{1}$ and $s_{2}$ continue execution after the full execution of $S_{1}^{\prime}$ and $S_{2}^{\prime}$ respectively, $\left(S_{1}^{\prime} ; s_{1}, m_{1}\left(\sigma_{1}\right)\right) \stackrel{*}{\rightarrow}$ $\left(s_{1}, m_{1}^{\prime \prime}\left(\sigma_{1}^{\prime \prime}\right)\right),\left(S_{2}^{\prime} ; s_{2}, m_{2}\left(\sigma_{2}\right)\right) \stackrel{*}{\rightarrow}\left(s_{2}, m_{2}^{\prime \prime}\left(\sigma_{2}^{\prime \prime}\right)\right)$. When $s_{1}$ and $s_{2}$ are while statements, by our assumption of unique 
loop labels, $s_{1}$ is not in $S_{1}^{\prime}$. By Corollary E.4 the loop counter value of $s_{1}$ is not redefined in the execution of $S_{1}^{\prime}$. Similarly, the loop counter value of $s_{2}$ is not redefined in the execution of $S_{2}^{\prime}$. By the hypothesis $\mathrm{IH}$ again, after the full execution of $s_{1}$ and $s_{2}$, the value stores agree on the value of $x,\left(s_{1}, m_{1}^{\prime \prime}\left(\sigma_{1}^{\prime \prime}\right)\right) \stackrel{*}{\rightarrow}\left(\operatorname{skip}, m_{1}^{\prime}\left(\sigma_{1}^{\prime}\right)\right),\left(s_{2}, m_{2}^{\prime \prime}\left(\sigma_{2}^{\prime \prime}\right)\right) \stackrel{*}{\rightarrow}$ (skip, $\left.m_{2}^{\prime}\left(\sigma_{2}^{\prime}\right)\right)$ such that $\sigma_{1}^{\prime}(x)=\sigma_{2}^{\prime}(x)$. The theorem holds.

(b) One last statement does not define the variable $x$ : W.1.o.g., $\left(x \notin \operatorname{Def}\left(s_{2}\right)\right) \wedge\left(S_{1} \equiv_{x}^{S} S_{2}^{\prime}\right)$.

We show that $S_{1}$ and $S_{2}^{\prime}$ compute the variable $x$ equivalently when started from state $m_{1}\left(\sigma_{1}\right)$ and $m_{2}\left(\sigma_{2}\right)$ by the hypothesis IH. First, by the definition of program size, $\operatorname{size}\left(s_{2}\right) \geq 1$. Hence, $\operatorname{size}\left(S_{1}\right)+\operatorname{size}\left(S_{2}^{\prime}\right) \leq k$. Next, by the definition of imported variables, $\operatorname{Imp}\left(S_{2}^{\prime},\{x\}\right) \subseteq$ $\operatorname{Imp}\left(S_{2},\{x\}\right)$. By assumption, $\sigma_{1}(y)=\sigma_{2}(y)$ for $\forall y \in$ $\operatorname{Imp}\left(S_{2}^{\prime},\{x\}\right) \cup \operatorname{Imp}\left(S_{1},\{x\}\right)$. By the hypothesis IH, $S_{1}$ and $S_{2}^{\prime}$ compute the variable $x$ equivalently when started in state $m_{1}\left(\sigma_{1}\right)$ and $m_{2}\left(\sigma_{2}\right)$ respectively, $\left(S_{2}^{\prime}, m_{2}\left(\sigma_{2}\right)\right) \stackrel{*}{\rightarrow}$ (skip, $\left.m_{2}^{\prime \prime}\left(\sigma_{2}^{\prime \prime}\right)\right),\left(S_{1}, m_{1}\left(\sigma_{1}\right)\right) \stackrel{*}{\rightarrow}$ (skip, $\left.m_{1}^{\prime}\left(\sigma_{1}^{\prime}\right)\right)$ such that $\sigma_{1}^{\prime}(x)=\sigma_{2}^{\prime \prime}(x)$.

Then, we show that $S_{1}$ and $S_{2}$ compute the variable $x$ equivalently after the full execution of $s_{2}$. By Corollary E.1. $s_{2}$ continues execution immediately after the full execution of $S_{2}^{\prime},\left(S_{2}^{\prime} ; s_{2}, m_{2}\right) \stackrel{*}{\rightarrow}\left(s_{2}, m_{2}^{\prime \prime}\right)$. By assumption, the execution from $\left(S_{2}^{\prime} ; s_{2}, m_{2}\right)$ terminates, $\left(s_{2}, m_{2}^{\prime \prime}\left(\sigma_{2}^{\prime \prime}\right)\right) \stackrel{*}{\rightarrow}$ (skip, $\left.m_{2}^{\prime}\left(\sigma_{2}^{\prime}\right)\right)$. By Corollary E.2 the value of $x$ is not changed in the execution of $s_{2}, \sigma_{2}^{\prime}(x)=\sigma_{2}^{\prime \prime}(x)$. Hence, $\sigma_{1}^{\prime}(x)=\sigma_{2}^{\prime}(x)$. The theorem holds.

(c) There are statements moving in/out of If statement: $s_{1}=$ "If $(e)$ then $\left\{S_{1}^{t}\right\}$ else $\left\{S_{1}^{f}\right\}$ ", $s_{2}=$ "If $(e)$ then $\left\{S_{2}^{t}\right\}$ else $\left\{S_{2}^{f}\right\}$ " such that none of the above cases hold and all of the following hold:

- $S_{1}^{\prime} \equiv_{y}^{S} S_{2}^{\prime}$ for $\forall y \in \operatorname{Use}(e)$;

- $S_{1}^{\prime} ; S_{1}^{t} \equiv_{x}^{S} S_{2}^{\prime} ; S_{2}^{t}$;

- $S_{1}^{\prime} ; S_{1}^{f} \equiv_{x}^{S} S_{2}^{\prime} ; S_{2}^{f}$;

- $x \in \operatorname{Def}\left(s_{1}\right) \cap \operatorname{Def}\left(s_{2}\right)$;

Repeatedly $S_{1}=S_{1}^{\prime} ; s_{1}, S_{2}=S_{2}^{\prime} ; s_{2}$. We first show that, after the full execution of $S_{1}^{\prime}$ and $S_{2}^{\prime}$ started in state $m_{1}$ and $m_{2}$, the predicate expression of $s_{1}$ and $s_{2}$ evaluate to the same value, w.l.o.g, zero. Next we show that $S_{1}$ and $S_{1}^{\prime} ; S_{1}^{f}$ compute the variable $x$ equivalently when (1) both started in state $m_{1}$ and (2) the predicate expression of $s_{1}$ evaluates to zero after the full execution of $S_{1}^{\prime}$ started in state $m_{1}$, similarly $S_{2}$ and $S_{2}^{\prime} ; S_{2}^{f}$ compute the variable $x$ equivalently when (1) both started in the state $m_{2}$ and (2) the predicate expression of $s_{2}$ evaluates to zero after the full execution of $S_{1}^{\prime}$ when started in state $m_{2}$. Last we prove the theorem by showing that $S_{1}^{\prime} ; S_{1}^{f}$ started in state $m_{1}$ and $S_{2}^{\prime} ; S_{2}^{f}$ started in state $m_{2}$ compute the variable $x$ equivalently.

In order to show that $S_{1}^{\prime}$ and $S_{2}^{\prime}$ compute the variables used in predicate expression of $s_{1}$ and $s_{2}$ equivalently by the hypothesis $\mathrm{IH}$, we show that all required conditions are satisfied for the application of hypothesis IH.

- $\operatorname{size}\left(S_{1}^{\prime}\right)+\operatorname{size}\left(S_{2}^{\prime}\right)<k$.

The sum of program size of $S_{1}^{\prime}$ and $S_{2}^{\prime}$ are less than $k$ by the definition of program size for $s_{1}$ and $s_{2}, \operatorname{size}\left(S_{1}^{\prime}\right)+$ $\operatorname{size}\left(S_{2}^{\prime}\right)<k$.

- the execution of $S_{1}^{\prime}$ and $S_{2}^{\prime}$ terminate, $\left(S_{1}^{\prime}, m_{1}\right) \stackrel{*}{\rightarrow}$ (skip, $\left.m_{1}^{\prime \prime}\left(\sigma_{1}^{\prime \prime}\right)\right)$, and $\left(S_{2}^{\prime}, m_{2}\right) \stackrel{*}{\rightarrow}\left(\right.$ skip, $\left.m_{2}^{\prime \prime}\left(\sigma_{2}^{\prime \prime}\right)\right)$.

By assumption, the execution of $S_{1}$ and $S_{2}$ from the state $m_{1}$ and $m_{2}$ respectively terminate, then the exe- cution of $S_{1}^{\prime}$ and $S_{2}^{\prime}$ terminate when started in state $m_{1}$ and $m_{2}$ respectively.

- the initial value stores $\sigma_{1}$ and $\sigma_{2}$ agree on the values of the imported variables in $S_{1}^{\prime}$ and $S_{2}^{\prime}$ relative to the variables used in the predicate expression of $s_{1}$ and $s_{2}$. By Lemma5.3, the imported variables in $S_{1}^{\prime}$ and $S_{2}^{\prime}$ relative to the variables used in predicate expression of $s_{1}$ and $s_{2}$ are same, $\operatorname{Imp}\left(S_{1}^{\prime}, \operatorname{Use}(e)\right)=\operatorname{Imp}\left(S_{2}^{\prime}, \operatorname{Use}(e)\right)=$ $\operatorname{Imp}(e)$. By the definition of imported variable, the imported variables in $S_{1}^{\prime}$ relative to the variables used in predicate expression of $s_{1}$ are a subset of the imported variables in $S_{1}$ relative to $x$ respectively, $\operatorname{Imp}\left(S_{1}^{\prime}, \operatorname{Use}(e)\right) \subseteq$ $\operatorname{Imp}\left(S_{1}^{\prime}, \operatorname{Imp}\left(s_{1},\{x\}\right)\right)=\operatorname{Imp}\left(S_{1},\{x\}\right)$. Similarly $\operatorname{Imp}\left(S_{2}^{\prime}, \operatorname{Use}(e)\right) \subseteq \operatorname{Imp}\left(S_{2},\{x\}\right)$. Then, by assumption, the initial value stores agree on the values of the imported variables in $S_{1}^{\prime}$ and $S_{2}^{\prime}$ relative to the variables used in the predicate expression of $s_{1}$ and $s_{2}$, $\sigma_{1}(y)=\sigma_{2}(y), \forall y \in \operatorname{Imp}(e)=\operatorname{Imp}\left(S_{1}^{\prime}, \operatorname{Use}(e)\right)=$ $\operatorname{Imp}\left(S_{2}^{\prime}, \operatorname{Use}(e)\right)$.

By the hypothesis $\mathrm{IH}$, after the full execution of $S_{1}^{\prime}$ and $S_{2}^{\prime}$, the value stores agree on the values of the variables used in the predicate expression of $s_{1}$ and $s_{2}, \sigma_{1}^{\prime \prime}(y)=$ $\sigma_{2}^{\prime \prime}(y), \forall y \in \operatorname{Use}(e)$. By Corollary E.1, $s_{1}$ and $s_{2}$ continue execution after the full execution of $S_{1}^{\prime}$ and $S_{2}^{\prime}$ respectively, $\left(S_{1}^{\prime} ; s_{1}, m_{1}\right) \stackrel{*}{\rightarrow}\left(s_{1}, m_{1}^{\prime \prime}\left(\sigma_{1}^{\prime \prime}\right)\right)$, and $\left(S_{2}^{\prime} ; s_{2}, m_{2}\right) \stackrel{*}{\rightarrow}$ $\left(s_{2}, m_{2}^{\prime \prime}\left(\sigma_{2}^{\prime \prime}\right)\right)$.

By the property of expression meaning function $\mathcal{E}$, expression $e$ evaluates to the same value w.r.t value stores $\sigma_{1}^{\prime \prime}$ and $\sigma_{2}^{\prime \prime}$, w.l.o.g., zero, $\mathcal{E} \llbracket e \rrbracket \sigma_{1}^{\prime \prime}=\mathcal{E} \llbracket e \rrbracket \sigma_{2}^{\prime \prime}=0$. Then the execution of $s_{1}$ proceeds as follows.

$$
\begin{aligned}
& \left(s_{1}, m_{1}^{\prime \prime}\left(\sigma_{1}^{\prime \prime}\right)\right) \\
= & \left(\text { If }(e) \text { then }\left\{S_{1}^{t}\right\} \text { else }\left\{S_{1}^{f}\right\}, m_{1}^{\prime \prime}\left(\sigma_{1}^{\prime \prime}\right)\right) \\
\rightarrow & \left(\text { If }(0) \text { then }\left\{S_{1}^{t}\right\} \text { else }\left\{S_{1}^{f}\right\}, m_{1}^{\prime \prime}\left(\sigma_{1}^{\prime \prime}\right)\right) \text { by the EEval rule. } \\
\rightarrow & \left(S_{1}^{f}, m_{1}^{\prime \prime}\left(\sigma_{1}^{\prime \prime}\right)\right) \text { by the If-F rule. }
\end{aligned}
$$

Similarly, the execution from $\left(s_{2}, m_{2}^{\prime \prime}\left(\sigma_{2}^{\prime \prime}\right)\right)$ gets to $\left(S_{2}^{f}, m_{2}^{\prime \prime}\left(\sigma_{2}^{\prime \prime}\right)\right)$.

Then, we show that $S_{1}$ and $S_{1}^{\prime} ; S_{1}^{f}$ compute the variable $x$ equivalently when both started from state $m_{1}\left(\sigma_{1}\right)$. The execution of $S_{1}^{\prime} ; S_{1}^{f}$ started from state $m_{1}$ also gets to configuration $\left(S_{1}^{f}, m_{1}^{\prime \prime}\left(\sigma_{1}^{\prime \prime}\right)\right)$ because execution of $S_{1}=S_{1}^{\prime} ; s_{1}$ and $S_{1}^{\prime} ; S_{1}^{f}$ share the common execution $\left(S_{1}^{\prime}, m_{1}\right) \stackrel{*}{\rightarrow}$ (skip, $m_{1}^{\prime \prime}\left(\sigma_{1}^{\prime \prime}\right)$ ). By Corollary E.1 $S_{1}^{f}$ continues execution after the full execution of $S_{1}^{\prime},\left(S_{1}^{\prime} ; S_{1}^{f}, m_{1}\right) \stackrel{*}{\rightarrow}\left(S_{1}^{f}, m_{1}^{\prime \prime}\right)$. Therefore, the execution of $S_{1}$ and $S_{1}^{\prime} ; S_{1}^{f}$ from state $m_{1}$ compute the variable $x$ equivalently because both executions get to same intermediate configuration. Similarly, $S_{2}$ and $S_{2}^{\prime} ; S_{2}^{f}$ compute the variable $x$ equivalently when both started from state $m_{2}\left(\sigma_{2}\right)$.

Lastly, we show that $S_{1}^{\prime} ; S_{1}^{f}$ and $S_{2}^{\prime} ; S_{2}^{f}$ compute the variable $x$ equivalently when started in states $m_{1}\left(\sigma_{1}\right)$ and $m_{2}\left(\sigma_{2}\right)$ respectively by the hypothesis IH. To do that, we show that all required conditions are satisfied for the application of hypothesis IH.

- $\operatorname{size}\left(S_{1}^{\prime} ; S_{1}^{f}\right)+\operatorname{size}\left(S_{2}^{\prime} ; S_{2}^{f}\right)<k$.

This is obtained by the definition of program size.

- execution of $S_{1}^{\prime} ; S_{1}^{f}$ and $S_{2}^{\prime} ; S_{2}^{f}$ terminate when started in state $m_{1}\left(\sigma_{1}\right)$ and $m_{2}\left(\sigma_{2}\right)$ respectively.

This is obtained by above argument.

- $\sigma_{1}(y)=\sigma_{2}(y), \forall y \in \operatorname{Imp}\left(S_{1}^{\prime} ; S_{1}^{f},\{x\}\right) \cup \operatorname{Imp}\left(S_{2}^{\prime} ; S_{2}^{f},\{x\}\right)$. We show that $\operatorname{Imp}\left(S_{1}^{\prime} ; S_{1}^{f},\{x\}\right) \subseteq \operatorname{Imp}\left(S_{1},\{x\}\right)$ as follows.

$$
\operatorname{Imp}\left(S_{1}^{f},\{x\}\right)
$$


$\subseteq \operatorname{Imp}\left(s_{1},\{x\}\right)$ (1) by the definition of imported variables.

$$
\begin{aligned}
& \operatorname{Imp}\left(S_{1}^{\prime} ; S_{1}^{f},\{x\}\right) \\
= & \operatorname{Imp}\left(S_{1}^{\prime}, \operatorname{Imp}\left(S_{1}^{f},\{x\}\right)\right) \text { by LemmaC.1 } \\
\subseteq & \operatorname{Imp}\left(S_{1}^{\prime}, \operatorname{Imp}\left(s_{1},\{x\}\right)\right) \text { by }(1) \\
= & \operatorname{Imp}\left(S_{1},\{x\}\right) \text { by the definition of imported variables. }
\end{aligned}
$$

Similarly, $\operatorname{Imp}\left(S_{2}^{\prime} ; S_{2}^{f},\{x\}\right) \subseteq \operatorname{Imp}\left(S_{2},\{x\}\right)$. Then, by assumption, the initial value stores agree on the values of the imported variables in $S_{1}^{\prime} ; S_{1}^{f}$ and $S_{2}^{\prime} ; S_{2}^{f}$ relative to $x$.

Then, by the hypothesis IH, after the full execution of $S_{1}^{\prime} ; S_{1}^{f}$ and $S_{2}^{\prime} ; S_{2}^{f}$, the value stores agree on the value of $x,\left(S_{1}^{\prime} ; S_{1}^{f}, m_{1}\right) \stackrel{*}{\rightarrow}$ (skip, $\left.m_{1}^{\prime}\left(\sigma_{1}^{\prime}\right)\right),\left(S_{2}^{\prime} ; S_{2}^{f}, m_{2}\right) \stackrel{*}{\rightarrow}$ (skip, $m_{2}^{\prime}\left(\sigma_{2}^{\prime}\right)$ ) such that $\sigma_{1}^{\prime}(x)=\sigma_{2}^{\prime}(x)$.

In conclusion, after execution of $S_{1}$ and $S_{2}$, the value stores agree on the value of $x$. Therefore, the theorem holds.

\subsubsection{Supporting lemmas for the soundness proof of equivalent computation for terminating programs}

The lemmas include the proof of two while statements computing a variable equivalently used in the proof of Theorem 2 and the property that two programs have same imported variables relative to a variable $x$ if the two programs satisfy the proof rule of equivalent computation of the variable $x$. From the proof rule of terminating computation of a variable $x$ equivalently, we have the two programs either both define $x$ or both do not.

Lemma 5.1. Let $s_{1}=$ "while ${ }_{\left\langle n_{1}\right\rangle}(e)\left\{S_{1}\right\}$ " and $s_{2}=$ "while ${ }_{\left\langle n_{2}\right\rangle}(e)$ $\left\{S_{2}\right\}$ " be two while statements with the same set of imported variables relative to a variable $x$ (defined in $s_{1}$ and $s_{2}$ ), $\operatorname{Imp}(x)$, and whose loop bodies $S_{1}$ and $S_{2}$ terminatingly compute the variables in $\operatorname{Imp}(x)$ equivalently when started in states that agree on the values of the variables imported by $S_{1}$ or $S_{2}$ relative to $\operatorname{Imp}(x)$ :

- $x \in \operatorname{Def}\left(s_{1}\right) \cap \operatorname{Def}\left(s_{2}\right)$;

- $\operatorname{Imp}\left(s_{1},\{x\}\right)=\operatorname{Imp}\left(s_{2},\{x\}\right)=\operatorname{Imp}(x)$;

- $\forall y \in \operatorname{Imp}(x), \forall m_{S_{1}}\left(\sigma_{S_{1}}\right), m_{S_{2}}\left(\sigma_{S_{2}}\right)$ :

$\left(\left(\forall z \in \operatorname{Imp}\left(S_{1}, \operatorname{Imp}(x)\right) \cup \operatorname{Imp}\left(S_{2}, \operatorname{Imp}(x)\right): \sigma_{S_{1}}(z)=\right.\right.$ $\left.\left.\sigma_{S_{2}}(z)\right) \Rightarrow\left(S_{1}, m_{S_{1}}\left(\sigma_{S_{1}}\right)\right) \equiv_{y}\left(S_{2}, m_{S_{2}}\left(\sigma_{S_{2}}\right)\right)\right)$.

If the executions of $s_{1}$ and $s_{2}$ terminate when started in states $m_{1}\left(\operatorname{loop}_{c}^{1}, \sigma_{1}\right)$ and $m_{2}\left(\operatorname{loop}_{c}^{2}, \sigma_{2}\right)$ in which $s_{1}$ and $s_{2}$ have not already executed (loop counter initially $0: \operatorname{loop}_{c}^{1}\left(n_{1}\right)=$ $\left.\operatorname{loop}_{c}^{2}\left(n_{2}\right)=0\right)$, and whose value stores $\sigma_{1}$ and $\sigma_{2}$ agree on the values of the variables in $\operatorname{Imp}(x), \forall y \in \operatorname{Imp}(x), \sigma_{1}(y)=\sigma_{2}(y)$, then, for any positive integer $i$, one of the following holds:

1. The loop counters for $s_{1}$ and $s_{2}$ are always less than $i$ :

$\forall m_{1}^{\prime}, m_{2}^{\prime}$ such that $\left(s_{1}, m_{1}\right) \stackrel{*}{\rightarrow}\left(S_{1}^{\prime}, m_{1}^{\prime}\left(\right.\right.$ loop $\left._{c}^{1^{\prime}}\right)$ and $\left(s_{2}, m_{2}\right)$

$\stackrel{*}{\rightarrow}\left(S_{2}^{\prime}, m_{2}^{\prime}\left(\operatorname{loop}_{c}^{2^{\prime}}\right)\right)$,

$\operatorname{loop}_{c}^{1^{\prime}}\left(n_{1}\right)<i$ and loop $_{c}^{2^{\prime}}\left(n_{2}\right)<i$;

2. There are two configurations $\left(s_{1}, m_{1_{i}}\right)$ and $\left(s_{2}, m_{2_{i}}\right)$ reachable from $\left(s_{1}, m_{1}\right)$ and $\left(s_{2}, m_{2}\right)$, respectively, in which the loop counters of $s_{1}$ and $s_{2}$ are equal to $i$ and value stores agree on the values of imported variables relative to $x$ and, for every state in execution, $\left(s_{1}, m_{1}\right) \stackrel{*}{\rightarrow}\left(s_{1}, m_{1_{i}}\right)$ or $\left(s_{2}, m_{2}\right) \stackrel{*}{\rightarrow}$ $\left(s_{2}, m_{2_{i}}\right)$ the loop counters for $s_{1}$ and $s_{2}$ are less than or equal to $i$ respectively:

$\exists\left(s_{1}, m_{1_{i}}\right),\left(s_{2}, m_{2_{i}}\right):\left(s_{1}, m_{1}\right) \stackrel{*}{\rightarrow}\left(s_{1}, m_{1_{i}}\left(\right.\right.$ loop $\left.\left._{c}^{1_{i}}, \sigma_{1_{i}}\right)\right) \wedge$

$\left(s_{2}, m_{2}\right) \stackrel{*}{\rightarrow}\left(s_{2}, m_{2_{i}}\left(\right.\right.$ loop $\left.\left._{c}^{2_{i}}, \sigma_{2_{i}}\right)\right)$ where

- $\operatorname{loop}_{c}^{1_{i}}\left(n_{1}\right)=\operatorname{loop}_{c}^{2_{i}}\left(n_{2}\right)=i$; and

- $\forall y \in \operatorname{Imp}(x): \sigma_{1_{i}}(y)=\sigma_{2_{i}}(y)$ and
- $\forall m_{1}^{\prime}:\left(s_{1}, m_{1}\right) \stackrel{*}{\rightarrow}\left(S_{1}^{\prime}, m_{1}^{\prime}\left(\operatorname{loop}_{c}^{1^{\prime}}\right)\right) \stackrel{*}{\rightarrow}$ $\left(s_{1}, m_{1_{i}}\left(\operatorname{loop}_{c}^{1_{i}}, \sigma_{1_{i}}\right)\right)$, $\operatorname{loop}_{c}^{1^{\prime}}\left(n_{1}\right) \leq i$; and

- $\forall m_{2}^{\prime}:\left(s_{2}, m_{2}\right) \stackrel{*}{\rightarrow}\left(S_{2}^{\prime}, m_{2}^{\prime}\left(\right.\right.$ loop $\left.\left._{c}^{2^{\prime}}\right)\right) \stackrel{*}{\rightarrow}$ $\left(s_{2}, m_{2_{i}}\left(\operatorname{loop}_{c}^{2 i}, \sigma_{2_{i}}\right)\right), \operatorname{loop}_{c}^{2^{\prime}}\left(n_{2}\right) \leq i$;

Proof. By induction on $i$.

Base case. $i=1$.

By assumption, initial loop counters of $s_{1}$ and $s_{2}$ are of value zero. Initial value stores $\sigma_{1}$ and $\sigma_{2}$ agree on the values of the variables in $\operatorname{Imp}(x)$. Then we show one of the following cases hold:

1. The loop counters for $s_{1}$ and $s_{2}$ are always less than 1: $\forall m_{1}^{\prime}, m_{2}^{\prime}$ such that $\left(s_{1}, m_{1}\right) \stackrel{*}{\rightarrow}\left(S_{1}^{\prime}, m_{1}^{\prime}\left(\operatorname{loop}_{c}^{1^{\prime}}\right)\right)$ and $\left(s_{2}, m_{2}\right)$ $\stackrel{*}{\rightarrow}\left(S_{2}^{\prime}, m_{2}^{\prime}\left(\operatorname{loop}_{c}^{2^{\prime}}\right)\right), \operatorname{loop}_{c}^{1^{\prime}}\left(n_{1}\right)<1$ and $\operatorname{loop}_{c}^{2^{\prime}}\left(n_{2}\right)<1$;

2. There are two configurations $\left(s_{1}, m_{1_{1}}\right)$ and $\left(s_{2}, m_{2_{1}}\right)$ reachable from $\left(s_{1}, m_{1}\right)$ and $\left(s_{2}, m_{2}\right)$, respectively, in which the loop counters of $s_{1}$ and $s_{2}$ are equal to 1 and value stores agree on the values of imported variables relative to $x$ and, for every state in execution, $\left(s_{1}, m_{1}\right) \stackrel{*}{\rightarrow}\left(s_{1}, m_{1_{1}}\right)$ or $\left(s_{2}, m_{2}\right) \stackrel{*}{\rightarrow}$ $\left(s_{2}, m_{2_{1}}\right)$ the loop counters for $s_{1}$ and $s_{2}$ are less than or equal to one respectively:

$$
\begin{aligned}
& \exists\left(s_{1}, m_{1_{1}}\right),\left(s_{2}, m_{2_{1}}\right):\left(s_{1}, m_{1}\right) \stackrel{*}{\rightarrow}\left(s_{1}, m_{1_{1}}\left(\operatorname{loop}_{c}^{1_{1}}, \sigma_{1_{1}}\right)\right) \wedge \\
& \left(s_{2}, m_{2}\right) \stackrel{*}{\rightarrow}\left(s_{2}, m_{2_{1}}\left(\operatorname{loop}_{c}^{2_{1}}, \sigma_{2_{1}}\right)\right) \text { where } \\
& \text { - } \operatorname{loop}_{c}^{1_{1}}\left(n_{1}\right)=\operatorname{loop}_{c}^{2_{1}}\left(n_{2}\right)=1 \text {; and } \\
& \text { - } \forall y \in \operatorname{Imp}(x): \sigma_{1_{1}}(y)=\sigma_{2_{1}}(y) ; \text { and } \\
& \text { - } \forall m_{1}^{\prime}:\left(s_{1}, m_{1}\right) \stackrel{*}{\rightarrow}\left(S_{1}^{\prime}, m_{1}^{\prime}\left(\operatorname{loop}_{c}^{1^{\prime}}\right)\right) \stackrel{*}{\rightarrow}\left(s_{1}, m_{1_{1}}\left(\operatorname{loop}_{c}^{1_{1}}, \sigma_{1_{1}}\right)\right), \\
& \quad \operatorname{loop}_{c}^{1^{\prime}}\left(n_{1}\right) \leq 1 ; \text { and } \\
& \text { - } \forall m_{2}^{\prime}:\left(s_{2}, m_{2}\right) \stackrel{*}{\rightarrow}\left(S_{2}^{\prime}, m_{2}^{\prime}\left(\operatorname{loop}_{c}^{2^{\prime}}\right)\right) \stackrel{*}{\rightarrow}\left(s_{2}, m_{2_{1}}\left(\operatorname{loop}_{c}^{2_{1}}, \sigma_{2_{1}}\right)\right), \\
& \operatorname{loop}_{c}^{2^{\prime}}\left(n_{2}\right) \leq 1 .
\end{aligned}
$$

We show evaluations of the predicate expression of $s_{1}$ and $s_{2}$ w.r.t value stores $\sigma_{1}$ and $\sigma_{2}$ produce same value. By the definition of imported variables, $\operatorname{Imp}\left(s_{1},\{x\}\right)=\bigcup_{j \geq 0} \operatorname{Imp}\left(S_{1}^{j},\{x\} \cup\right.$ Use $(e))$. By our notation of $S^{0}, S_{1}^{0}=$ skip. By the definition of imported variables, $\operatorname{Imp}\left(S_{1}^{0},\{x\} \cup \operatorname{Use}(e)\right)=\{x\} \cup \operatorname{Use}(e)$. Then Use $(e) \subseteq \operatorname{Imp}(x)$. By assumption, value stores $\sigma_{1}$ and $\sigma_{2}$ agree on the values of the variables in $\operatorname{Use}(e)$. By Lemma D.1 the predicate expression $e$ of $s_{1}$ and $s_{2}$ evaluates to same value $v$ w.r.t value stores $\sigma_{1}, \sigma_{2}, \mathcal{E}^{\prime} \llbracket e \rrbracket \sigma_{1}=\mathcal{E}^{\prime} \llbracket e \rrbracket \sigma_{2}=v$. Then there are two possibilities to consider.

1. $\mathcal{E}^{\prime} \llbracket e \rrbracket \sigma_{1}=\mathcal{E}^{\prime} \llbracket e \rrbracket \sigma_{2}=v=0$

The execution from $\left(s_{1}, m_{1}\left(\operatorname{loop}_{c}^{1}, \sigma_{1}\right)\right)$ proceeds as follows.

$$
\begin{aligned}
& \left(s_{1}, m_{1}\left(\operatorname{loop}_{c}^{1}, \sigma_{1}\right)\right) \\
= & \left(\text { while }_{\left\langle n_{1}\right\rangle}(e)\left\{S_{1}\right\}, m_{1}\left(\operatorname{loop}_{c}^{1}\right)\right) \\
\rightarrow & \left(\text { while }_{\left\langle n_{1}\right\rangle}(0)\left\{S_{1}\right\}, m_{1}\left(\operatorname{loop}_{c}^{1}\right)\right) \text { by the EEval' rule } \\
\rightarrow & \left(\text { skip, } m_{1}\left(\operatorname{loop}_{c}^{1}\left[0 / n_{1}\right]\right)\right) \text { by the Wh-F rule. }
\end{aligned}
$$

Similarly, $\left(s_{2}, m_{2}\left(\operatorname{loop}_{c}^{2}, \sigma_{2}\right)\right) \stackrel{2}{\rightarrow}$ (skip, $\left.m_{2}\left(\operatorname{loop}_{c}^{2}\left[0 / n_{2}\right]\right)\right)$. In conclusion, the loop counters of $s_{1}$ and $s_{2}$ in any states of the execution from $\left(s_{1}, m_{1}\right)$ and $\left(s_{2}, m_{2}\right)$ respectively are less than $1, \forall m_{1}^{\prime}, m_{2}^{\prime}$ such that $\left(s_{1}, m_{1}\right) \stackrel{*}{\rightarrow}\left(S_{1}{ }^{\prime}, m_{1}^{\prime}\left(\operatorname{loop}_{c}^{1^{\prime}}\right)\right.$ and $\left(s_{2}, m_{2}\right) \stackrel{*}{\rightarrow}\left(S_{2}{ }^{\prime}, m_{2}^{\prime}\left(\operatorname{loop}_{c}^{2^{\prime}}\right)\right), \operatorname{loop}_{c}^{1^{\prime}}\left(n_{1}\right)<1, \operatorname{loop}_{c}^{2^{\prime}}\left(n_{2}\right)<$ 1 .

2. $\mathcal{E}^{\prime} \llbracket e \rrbracket \sigma_{1}=\mathcal{E}^{\prime} \llbracket e \rrbracket \sigma_{2}=v \neq 0$

The execution from $\left(s_{1}, m_{1}\left(\operatorname{loop}_{c}^{1}, \sigma_{1}\right)\right)$ proceeds as follows.

$$
\begin{aligned}
& \left(s_{1}, m_{1}\left(\operatorname{loop}_{c}^{1}, \sigma_{1}\right)\right) \\
= & \left(\text { while }_{\left\langle n_{1}\right\rangle}(e)\left\{S_{1}\right\}, m_{1}\left(\operatorname{loop}_{c}^{1}, \sigma_{1}\right)\right) \\
\rightarrow & \left(\text { while }_{\left\langle n_{1}\right\rangle}(v)\left\{S_{1}\right\}, m_{1}\left(\operatorname{loop}_{c}^{1}, \sigma_{1}\right)\right) \text { by the EEval' rule } \\
\rightarrow & \left(S_{1} ; \text { while }_{\left\langle n_{1}\right\rangle}(e)\left\{S_{1}\right\}, m_{1}\left(\operatorname{loop}_{c}^{1}\left[1 /\left(n_{1}\right)\right], \sigma_{1}\right)\right) \\
& \text { by the Wh-T rule. }
\end{aligned}
$$


Similarly, $\left(s_{2}, m_{2}\left(\operatorname{loop}_{c}^{2}, \sigma_{2}\right)\right) \stackrel{2}{\rightarrow}\left(S_{2} ;\right.$ while $_{\left\langle n_{2}\right\rangle}(e)\left\{S_{2}\right\}, m_{2}$ $\left.\left(\operatorname{loop}_{c}^{2}\left[1 /\left(n_{2}\right)\right], \sigma_{2}\right)\right)$. Then, the loop counters of $s_{1}$ and $s_{2}$ are 1 , value stores $\sigma_{1_{1}}$ and $\sigma_{2_{1}}$ agree on values of variables in $\operatorname{Imp}(x): \operatorname{loop}_{c}^{1}\left[1 / n_{1}\right]\left(n_{1}\right)=$

$\operatorname{loop}_{c}^{2}\left[1 /\left(n_{2}\right)\right]\left(n_{2}\right)=1$; and $\forall y \in \operatorname{Imp}(x), \sigma_{1_{1}}(y)=\sigma_{2_{1}}(y)$. By assumption, the execution of $s_{1}$ terminates when started in the state $m_{1}\left(, \sigma_{1}\right)$, then the execution of $S_{1}$ terminates when started in the state $m_{1}\left(\operatorname{loop}_{c}^{1}\left[1 /\left(n_{1}\right)\right], \sigma_{1}\right),\left(s_{1}, m_{1}\right) \stackrel{*}{\rightarrow}$ $\left(S_{1} ;\right.$ while $\left._{\left\langle n_{1}\right\rangle}(e)\left\{S_{1}\right\}, m_{1}\left(\operatorname{loop}_{c}^{1}\left[1 /\left(n_{1}\right)\right], \sigma_{1}\right)\right) \stackrel{*}{\rightarrow}$ (skip, $\left.m_{1}^{\prime}\right) \Rightarrow$ $\left(S_{1}, m_{1}\left(\operatorname{loop}_{c}^{1}\left[1 /\left(n_{1}\right)\right], \sigma_{1}\right)\right) \stackrel{*}{\rightarrow}\left(\operatorname{skip}, m_{1_{1}}\left(\operatorname{loop}_{c}^{1_{1}}, \sigma_{1_{1}}\right)\right)$. Similarly, the execution of $S_{2}$ terminates when started in the state $m_{2}\left(\operatorname{loop}_{c}^{2}\left[1 /\left(n_{2}\right)\right], \sigma_{2}\right)$,

$\left(S_{2}, m_{2}\left(\operatorname{loop}_{c}^{2}\left[1 /\left(n_{2}\right)\right], \sigma_{2}\right)\right) \stackrel{*}{\rightarrow}\left(\right.$ skip, $\left.m_{2_{1}}\left(\operatorname{loop}_{c}^{2_{1}}, \sigma_{2_{1}}\right)\right)$.

We show that, after the full execution of $S_{1}$ and $S_{2}$, the following four properties hold.

- The loop counters of $s_{1}$ and $s_{2}$ are of value $1, \operatorname{loop}_{c}^{1_{1}}\left(n_{1}\right)=$ $\operatorname{loop}_{c}^{2_{1}}\left(n_{2}\right)=1$.

By assumption of unique loop labels, $s_{1} \notin S_{1}$. Then, the loop counter value of $n_{1}$ is not redefined in the execution of $S_{1}$ by corollary E.4 $\operatorname{loop}_{c}^{1}\left[1 /\left(n_{1}\right)\right]\left(n_{1}\right)=\operatorname{loop}_{c}^{1_{1}}\left(n_{1}\right)=1$. Similarly, the loop counter value of $n_{2}$ is not redefined in the execution of $S_{2}$, $\operatorname{loop}_{c}^{2}\left[1 /\left(n_{2}\right)\right]\left(n_{2}\right)=\operatorname{loop}_{c}^{21}\left(n_{2}\right)=1$.

- In any state in the execution $\left(s_{1}, m_{1}\right) \stackrel{*}{\rightarrow}\left(s_{1}, m_{1_{1}}\left(\operatorname{loop}_{c}^{1_{1}}, \sigma_{1_{1}}\right)\right)$, the loop counter of $s_{1}$ is less than or equal to 1 .

The loop counter of $s_{1}$ is zero in any of the two states in the one step execution $\left(s_{1}, m_{1}\right) \rightarrow\left(\right.$ while $_{\left\langle n_{1}\right\rangle}(v)\left\{S_{1}\right\}$, $\left.m_{1}\left(\operatorname{loop}_{c}^{1},, \sigma_{1}\right)\right)$, and the loop counter of $s_{1}$ is 1 in any states in the execution

$$
\left(S_{1} ; \text { while }_{\left\langle n_{1}\right\rangle}(e)\left\{S_{1}\right\}, m_{1}\left(\operatorname{loop}_{c}^{1}\left[i /\left(n_{1}\right)\right], \sigma_{1}\right)\right) \stackrel{*}{\rightarrow}
$$$$
\left(s_{1}, m_{1_{1}}\left(\operatorname{loop}_{c}^{1_{1}}, \sigma_{1_{1}}\right)\right) \text {. }
$$

- In any state in the executions $\left(s_{2}, m_{2}\right) \stackrel{*}{\rightarrow}\left(s_{2}, m_{2_{1}}\left(\operatorname{loop}_{c}^{21}, \sigma_{2_{1}}\right)\right)$, the loop counter of $s_{2}$ is less than or equal to 1 .

By similar argument for the loop counter of $s_{1}$.

- The value stores $\sigma_{1_{1}}$ and $\sigma_{2_{1}}$ agree on the values of the imported variables in $s_{1}$ and $s_{2}$ relative to the variable $x$ : $\forall y \in \operatorname{Imp}(x), \sigma_{1_{1}}(y)=\sigma_{2_{1}}(y)$.

We show that the imported variables in $S_{1}$ relative to those in $\operatorname{Imp}(x)$ are a subset of $\operatorname{Imp}(x)$.

$$
\operatorname{Imp}\left(S_{1}, \operatorname{Imp}(x)\right)
$$

$=\operatorname{Imp}\left(S_{1}, \operatorname{Imp}\left(s_{1},\{x\} \cup \operatorname{Use}(e)\right)\right)$ by the definition of $\operatorname{Imp}(x)$

$=\operatorname{Imp}\left(S_{1}, \bigcup_{j \geq 0} \operatorname{Imp}\left(S_{1}^{j},\{x\} \cup \operatorname{Use}(e)\right)\right)$

by the definition of imported variables

$=\bigcup_{j \geq 0} \operatorname{Imp}\left(S_{1}, \operatorname{Imp}\left(S_{1}^{j},\{x\} \cup \operatorname{Use}(e)\right)\right)$ by Lemma C.2

$=\bigcup_{j>0} \operatorname{Imp}\left(S_{1}^{j},\{x\} \cup \operatorname{Use}(e)\right)$ by LemmaC.1

$\subseteq \bigcup_{j \geq 0} \operatorname{Imp}\left(S_{1}^{j},\{x\} \cup \operatorname{Use}(e)\right)$

$=\operatorname{Imp}\left(s_{1},\{x\} \cup \operatorname{Use}(e)\right)=\operatorname{Imp}(x)$.

Similarly, $\operatorname{Imp}\left(S_{2}, \operatorname{Imp}(x)\right) \subseteq \operatorname{Imp}(x)$. Consequently, the value stores $\sigma_{1_{1}}$ and $\sigma_{2_{1}}$ agree on the values of the imported variables in $S_{1}$ and $S_{2}$ relative to those in $\operatorname{Imp}(x)$, $\forall y \in \operatorname{Imp}\left(S_{1}, \operatorname{Imp}(x)\right) \cup \operatorname{Imp}\left(S_{2}, \operatorname{Imp}(x)\right), \sigma_{1}(y)=\sigma_{2}(y)$. Because $S_{1}$ and $S_{2}$ have computation of every variable in $\operatorname{Imp}(x)$ equivalently when started in states agreeing on the values of the imported variables relative to $\operatorname{Imp}(x)$, then value store $\sigma_{1_{1}}$ and $\sigma_{2_{1}}$ agree on the values of the variables $\operatorname{Imp}(x), \forall y \in \operatorname{Imp}(x), \sigma_{1_{1}}(y)=\sigma_{2_{1}}(y)$.

It follows that, by corollary E.1

$\left(S_{1} ; s_{1}, m_{1}\left(\operatorname{loop}_{c}^{1}\left[1 / n_{1}\right], \sigma_{1}\right)\right) \stackrel{*}{\rightarrow}\left(s_{1}, m_{1_{1}}\left(\operatorname{loop}_{c}^{1_{1}}, \sigma_{1_{1}}\right)\right)$ and $\left(S_{2} ; s_{2}, m_{2}\left(\operatorname{loop}_{c}^{2}\left[1 / n_{2}\right], \sigma_{2}\right)\right) \stackrel{*}{\rightarrow}\left(s_{2}, m_{2_{1}}\left(\operatorname{loop}_{c}^{2}, \sigma_{2_{1}}\right)\right)$.

\section{Induction Step.}

The induction hypothesis $\mathrm{IH}$ is that, for a positive integer $i$, one of the following holds:

1. The loop counters for $s_{1}$ and $s_{2}$ are always less than $i$ : $\forall m_{1}^{\prime}, m_{2}^{\prime}$ such that $\left(s_{1}, m_{1}\right) \stackrel{*}{\rightarrow}\left(S_{1}^{\prime}, m_{1}^{\prime}\left(\operatorname{loop}_{c}^{1^{\prime}}\right)\right.$ and $\left(s_{2}, m_{2}\right) \stackrel{*}{\rightarrow}$ $\left(S_{2}^{\prime}, m_{2}^{\prime}\left(\operatorname{loop}_{c}^{2^{\prime}}\right)\right)$,

$\operatorname{loop}_{c}^{1^{\prime}}\left(n_{1}\right)<i$ and loop $_{c}^{2^{\prime}}\left(n_{2}\right)<i$;

2. There are two configurations $\left(s_{1}, m_{1_{i}}\right)$ and $\left(s_{2}, m_{2_{i}}\right)$ reachable from $\left(s_{1}, m_{1}\right)$ and $\left(s_{2}, m_{2}\right)$, respectively, in which the loop counters of $s_{1}$ and $s_{2}$ are equal to $i$ and value stores agree on the values of imported variables relative to $x$ and, for every state in execution, $\left(s_{1}, m_{1}\right) \stackrel{*}{\rightarrow}\left(s_{1}, m_{1_{i}}\right)$ and $\left(s_{2}, m_{2}\right) \stackrel{*}{\rightarrow}\left(s_{2}, m_{2_{i}}\right)$ the loop counters for $s_{1}$ and $s_{2}$ are less than or equal to $i$ respectively:

$$
\begin{aligned}
& \exists\left(s_{1}, m_{1_{i}}\right),\left(s_{2}, m_{2_{i}}\right):\left(s_{1}, m_{1}\right) \stackrel{*}{\rightarrow}\left(s_{1}, m_{1_{i}}\left(\operatorname{loop}_{c}^{1_{i}}, \sigma_{1_{i}}\right)\right) \wedge \\
& \left(s_{2}, m_{2}\right) \stackrel{*}{\rightarrow}\left(s_{2}, m_{2_{i}}\left(\operatorname{loop}_{c}^{2_{i}}, \sigma_{2_{i}}\right)\right) \text { where } \\
& \text { - } \operatorname{loop}_{c}^{1_{i}}\left(n_{1}\right)=\operatorname{loop}_{c}^{2_{i}}\left(n_{2}\right)=i \text {; and } \\
& \text { - } \forall y \in \operatorname{Imp}(x), \sigma_{1_{i}}(y)=\sigma_{2_{i}}(y) ; \text { and } \\
& \text { - } \forall m_{1}^{\prime}:\left(s_{1}, m_{1}\right) \stackrel{*}{\rightarrow}\left(S_{1}^{\prime}, m_{1}^{\prime}\left(\operatorname{loop}_{c}^{1^{\prime}}\right) \stackrel{*}{\rightarrow}\right. \\
& \quad\left(s_{1}, m_{1_{i}}\left(\operatorname{loop}_{c}^{1_{i}}, \sigma_{1_{i}}\right)\right), \operatorname{loop}_{c}^{1^{\prime}}\left(n_{1}\right) \leq i \text {; and } \\
& \text { - } \forall m_{2}^{\prime}:\left(s_{2}, m_{2}\right) \stackrel{*}{\rightarrow}\left(S_{2}^{\prime}, m_{2}^{\prime}\left(\operatorname{loop}_{c}^{2^{\prime}}\right)\right) \stackrel{*}{\rightarrow} \\
& \quad\left(s_{2}, m_{2_{i}}\left(\operatorname{loop}_{c}^{2_{i}}, \sigma_{2_{i}}\right)\right), \operatorname{loop}_{c}^{2^{\prime}}\left(n_{2}\right) \leq i .
\end{aligned}
$$

Then we show that, for the positive integer $i+1$, one of the following holds:

1. The loop counters for $s_{1}$ and $s_{2}$ are always less than $i+1$ : $\forall m_{1}^{\prime}, m_{2}^{\prime}$ such that $\left(s_{1}, m_{1}\right) \stackrel{*}{\rightarrow}\left(S_{1}^{\prime}, m_{1}^{\prime}\left(\operatorname{loop}_{c}^{1^{\prime}}\right)\right)$ and $\left(s_{2}, m_{2}\right)$ $\stackrel{*}{\rightarrow}\left(S_{2}^{\prime}, m_{2}^{\prime}\left(\operatorname{loop}_{c}^{2^{\prime}}\right)\right)$, $\operatorname{loop}_{c}^{1^{\prime}}\left(n_{1}\right)<i+1$ and $\operatorname{loop}_{c}^{2^{\prime}}\left(n_{2}\right)<i+1$;

2. There are two configurations $\left(s_{1}, m_{1_{i+1}}\right)$ and $\left(s_{2}, m_{2_{i+1}}\right)$ reachable from $\left(s_{1}, m_{1}\right)$ and $\left(s_{2}, m_{2}\right)$, respectively, in which the loop counters of $s_{1}$ and $s_{2}$ are equal to $i+1$ and value stores agree on the values of imported variables relative to $x$ and, for every state in executions $\left(s_{1}, m_{1}\right) \stackrel{*}{\rightarrow}\left(s_{1}, m_{1_{i+1}}\right)$ and $\left(s_{2}, m_{2}\right) \stackrel{*}{\rightarrow}\left(s_{2}, m_{2_{i+1}}\right)$ the loop counters for $s_{1}$ and $s_{2}$ are less than or equal to $i+1$ respectively:

$\exists\left(s_{1}, m_{1_{i+1}}\right),\left(s_{2}, m_{2_{i+1}}\right):\left(s_{1}, m_{1}\right) \stackrel{*}{\rightarrow}\left(s_{1}, m_{1_{i+1}}\left(\operatorname{loop}_{c}^{1_{i+1}}\right.\right.$, $\left.\left.\sigma_{1_{i+1}}\right)\right) \wedge\left(s_{2}, m_{2}\right) \stackrel{*}{\rightarrow}\left(s_{2}, m_{2_{i+1}}\left(\operatorname{loop}_{c}^{2_{i+1}}, \sigma_{2_{i+1}}\right)\right)$ where

- $\operatorname{loop}_{c}^{1_{i+1}}\left(n_{1}\right)=\operatorname{loop}_{c}^{2_{i+1}}\left(n_{2}\right)=i+1$; and

- $\forall y \in \operatorname{Imp}(x), \sigma_{1_{i+1}}(y)=\sigma_{2_{i+1}}(y)$; and

- $\forall m_{1}^{\prime}:\left(s_{1}, m_{1}\right) \stackrel{*}{\rightarrow}\left(S_{1}^{\prime}, m_{1}^{\prime}\left(\operatorname{loop}_{c}^{1^{\prime}}\right)\right) \stackrel{*}{\rightarrow}$

$\left(s_{1}, m_{1_{i+1}}\left(\operatorname{loop}_{c}^{1_{i+1}}, \sigma_{1_{i+1}}\right)\right)$, $\operatorname{loop}_{c}^{1^{\prime}}\left(n_{1}\right) \leq i+1$; and

- $\forall m_{2}^{\prime}:\left(s_{2}, m_{2}\right) \stackrel{*}{\rightarrow}\left(S_{2}^{\prime}, m_{2}^{\prime}\left(\operatorname{loop}_{c}^{2^{\prime}}\right)\right) \stackrel{*}{\rightarrow}$

$\left(s_{2}, m_{2_{i+1}}\left(\operatorname{loop}_{c}^{2_{i+1}}, \sigma_{2_{i+1}}\right)\right)$, $\operatorname{loop}_{c}^{2^{\prime}}\left(n_{2}\right) \leq i+1$.

By the hypothesis $\mathrm{IH}$, one of the following holds:

1. The loop counters for $s_{1}$ and $s_{2}$ are always less than $i$ :

$\forall m_{1}^{\prime}, m_{2}^{\prime}$ such that $\left(s_{1}, m_{1}\right) \stackrel{*}{\rightarrow}\left(S_{1}^{\prime}, m_{1}^{\prime}\left(\operatorname{loop}_{c}^{1^{\prime}}\right)\right.$ and $\left(s_{2}, m_{2}\right)$ $\stackrel{*}{\rightarrow}\left(S_{2}^{\prime}, m_{2}^{\prime}\left(\operatorname{loop}_{c}^{2^{\prime}}\right)\right)$,

loop $_{c}^{1^{\prime}}\left(n_{1}\right)<i$ and loop ${ }_{c}^{2^{\prime}}\left(n_{2}\right)<i$;

When this case holds, then we have the loop counters for $s_{1}$ and $s_{2}$ are always less than $i+1$ :

$\forall m_{1}^{\prime}, m_{2}^{\prime}$ such that $\left(s_{1}, m_{1}\right) \stackrel{*}{\rightarrow}\left(S_{1}^{\prime}, m_{1}^{\prime}\left(\operatorname{loop}_{c}^{1^{\prime}}\right)\right.$ and $\left(s_{2}, m_{2}\right)$ $\stackrel{*}{\rightarrow}\left(S_{2}^{\prime}, m_{2}^{\prime}\left(\operatorname{loop}_{c}^{2^{\prime}}\right)\right)$,

loop $_{c}^{1^{\prime}}\left(n_{1}\right)<i+1$ and $\operatorname{loop}_{c}^{2^{\prime}}\left(n_{2}\right)<i+1$.

2. There are two configurations $\left(s_{1}, m_{1_{i}}\right)$ and $\left(s_{2}, m_{2_{i}}\right)$ reachable from $\left(s_{1}, m_{1}\right)$ and $\left(s_{2}, m_{2}\right)$, respectively, in which the loop counters of $s_{1}$ and $s_{2}$ are equal to $i$ and value stores agree on the 
values of imported variables relative to $x$ and, for every state in executions $\left(s_{1}, m_{1}\right) \stackrel{*}{\rightarrow}\left(s_{1}, m_{1_{i}}\right)$ and $\left(s_{2}, m_{2}\right) \stackrel{*}{\rightarrow}\left(s_{2}, m_{2_{i}}\right)$ the loop counters for $s_{1}$ and $s_{2}$ are less than or equal to $i$ respectively:

$$
\begin{aligned}
& \exists\left(s_{1}, m_{1_{i}}\right),\left(s_{2}, m_{2_{i}}\right):\left(s_{1}, m_{1}\right) \stackrel{*}{\rightarrow}\left(s_{1}, m_{1_{i}}\left(\operatorname{loop}_{c}^{1_{i}}, \sigma_{1_{i}}\right)\right) \wedge \\
& \left(s_{2}, m_{2}\right) \stackrel{*}{\rightarrow}\left(s_{2}, m_{2_{i}}\left(\operatorname{loop}_{c}^{2_{i}}, \sigma_{2_{i}}\right)\right) \text { where } \\
& \text { - } \operatorname{loop}_{c}^{1_{i}}\left(n_{1}\right)=\operatorname{loop}_{c}^{2_{i}}\left(n_{2}\right)=i \text {; and } \\
& \text { - } \forall y \in \operatorname{Imp}(x), \sigma_{1_{i}}(y)=\sigma_{2_{i}}(y) ; \text { and } \\
& \text { - } \forall m_{1}^{\prime}:\left(s_{1}, m_{1}\right) \stackrel{*}{\rightarrow}\left(S_{1}^{\prime}, m_{1}^{\prime}\left(\operatorname{loop}_{c}^{1^{\prime}}\right) \stackrel{*}{\rightarrow}\left(s_{1}, m_{1_{i}}\left(\operatorname{loop}_{c}^{1_{i}},\right.\right.\right. \\
& \left.\left.\quad, \sigma_{1_{i}}\right)\right), \operatorname{loop}_{c}^{1^{\prime}}\left(n_{1}\right) \leq i \text {; and } \\
& \text { - } \forall m_{2}^{\prime}:\left(s_{2}, m_{2}\right) \stackrel{*}{\rightarrow}\left(S_{2}^{\prime}, m_{2}^{\prime}\left(\operatorname{loop}_{c}^{2^{\prime}}\right)\right) \stackrel{*}{\rightarrow}\left(s_{2}, m_{2_{i}}\left(\operatorname{loop}_{c}^{2_{i}},\right.\right. \\
& \left.\left.\quad), \sigma_{2_{i}}\right)\right), \operatorname{loop}_{c}^{2^{\prime}}\left(n_{2}\right) \leq i .
\end{aligned}
$$

By similar argument in base case, evaluations of the predicate expression of $s_{1}$ and $s_{2}$ w.r.t value stores $\sigma_{1_{i}}$ and $\sigma_{2_{i}}$ produce same value. Then there are two possibilities:

(a) $\mathcal{E}^{\prime} \llbracket e \rrbracket \sigma_{1_{i}}=\mathcal{E}^{\prime} \llbracket e \rrbracket \sigma_{2_{i}}=\left(0, v_{\mathfrak{o f}}\right)$

The execution from $\left(s_{1}, m_{1}\left(\operatorname{loop}_{c}^{1}, \sigma_{1}\right)\right)$ proceeds as follows.

$$
\left(s_{1}, m_{1_{i}}\left(\operatorname{loop}_{c}^{1_{i}}, \sigma_{1_{i}}\right)\right)
$$

$=\left(\right.$ while $\left._{\left\langle n_{1}\right\rangle}(e)\left\{S_{1}\right\}, m_{1_{i}}\left(\operatorname{loop}_{c}^{1_{i}}, \sigma_{1_{i}}\right)\right)$

$\rightarrow\left(\right.$ while $\left._{\left\langle n_{1}\right\rangle}\left(\left(0, v_{\text {of }}\right)\right)\left\{S_{1}\right\}, m_{1_{i}}\left(\operatorname{loop}_{c}^{1_{i}}, \sigma_{1_{i}}\right)\right)$ by the EEval' rule

$\rightarrow\left(\right.$ while $\left._{\left\langle n_{1}\right\rangle}(0)\left\{S_{1}\right\}, m_{1_{i}}\left(\operatorname{loop}_{c}^{1_{i}}, \sigma_{1_{i}}\right)\right)$

by the E-Oflow1 and E-Oflow2 rule

$\rightarrow\left(\right.$ skip, $\left.m_{1_{i}}\left(\operatorname{loop}_{c}^{1_{i}}\left[0 / n_{1}\right], \sigma_{1_{i}}\right)\right)$ by the Wh-F rule.

By the hypothesis $\mathrm{IH}$, the loop counter of $s_{1}$ and $s_{2}$ in any configuration in executions $\left(s_{1}, m_{1}\right) \stackrel{*}{\rightarrow}\left(s_{1}, m_{1_{i}}\left(\operatorname{loop}_{c}^{1_{i}}, \sigma_{1_{i}}\right)\right)$ and $\left(s_{2}, m_{2}\right) \stackrel{*}{\rightarrow}\left(s_{2}, m_{2_{i}}\left(\operatorname{loop}_{c}^{2_{i}}, \sigma_{2_{i}}\right)\right)$ respectively are less than or equal to $i$,

$\forall m_{1}^{\prime}:\left(s_{1}, m_{1}\right) \stackrel{*}{\rightarrow}\left(S_{1}^{\prime}, m_{1}^{\prime}\left(\operatorname{loop}_{c}^{1^{\prime}}\right) \stackrel{*}{\rightarrow}\left(s_{1}, m_{1_{i}}\left(\operatorname{loop}_{c}^{1_{i}}\right.\right.\right.$, ,$\left.\left.\sigma_{1_{i}}\right)\right)$, $\operatorname{loop}_{c}^{1^{\prime}}\left(n_{1}\right) \leq i$; and

$\forall m_{2}^{\prime}:\left(s_{2}, m_{2}\right) \stackrel{*}{\rightarrow}\left(S_{2}^{\prime}, m_{2}^{\prime}\left(\operatorname{loop}_{c}^{2^{\prime}}\right)\right) \stackrel{*}{\rightarrow}\left(s_{2}, m_{2_{i}}\left(\right.\right.$ loop $_{c}^{2_{i}}$, ),$\left.\left.\sigma_{2_{i}}\right)\right), \operatorname{loop}_{c}^{2^{\prime}}\left(n_{2}\right) \leq i$.

Therefore, the loop counter of $s_{1}$ and $s_{2}$ in any configuration in executions

$$
\left(s_{1}, m_{1}\right) \stackrel{*}{\rightarrow}\left(\text { skip, } m_{1_{i}}\left(\operatorname{loop}_{c}^{1_{i}} \backslash\left\{\left(n_{1}\right)\right\}, \sigma_{1_{i}}\right)\right) \text { and }
$$

$\left(s_{2}, m_{2}\right) \stackrel{*}{\rightarrow}$ (skip, $\left.m_{2_{i}}\left(\operatorname{loop}_{c}^{2_{i}}\left[0 / n_{2}\right], \sigma_{2_{i}}\right)\right)$ respectively are less than $i+1$.

(b) $\mathcal{E}^{\prime} \llbracket e \rrbracket \sigma_{1_{i}}=\mathcal{E}^{\prime} \llbracket e \rrbracket \sigma_{2_{i}}=v \neq 0$

The execution from $\left(s_{1}, m_{1_{i}}\left(\operatorname{loop}_{c}^{1_{i}}, \sigma_{1_{i}}\right)\right)$ proceeds as follows.

$\left(s_{1}, m_{1_{i}}\left(\operatorname{loop}_{c}^{1_{i}}, \sigma_{1_{i}}\right)\right)$

$=\left(\right.$ while $\left._{\left\langle n_{1}\right\rangle}(e)\left\{S_{1}\right\}, m_{1_{i}}\left(\operatorname{loop}_{c}^{1_{i}}, \sigma_{1_{i}}\right)\right)$

$\rightarrow\left(\right.$ while $\left._{\left\langle n_{1}\right\rangle}(v)\left\{S_{1}\right\}, m_{1_{i}}\left(\operatorname{loop}_{c}^{1_{i}}, \sigma_{1_{i}}\right)\right)$ by the EEval' rule

$\rightarrow\left(S_{1} ;\right.$ while $_{\left\langle n_{1}\right\rangle}(e)\left\{S_{1}\right\}, m_{1_{i}}\left(\operatorname{loop}_{c}^{1_{i}}\left[i+1 /\left(n_{1}\right)\right]\right.$

,$\left.\left.\sigma_{1_{i}}\right)\right)$ by the Wh-T rule.

Similarly, $\left(s_{2}, m_{2_{i}}\left(\operatorname{loop}_{c}^{2_{i}}, \sigma_{2_{i}}\right)\right) \stackrel{2}{\rightarrow}\left(S_{2}\right.$; while $_{\left\langle n_{2}\right\rangle}(e)\left\{S_{2}\right\}$, $\left.m_{2_{i}}\left(\operatorname{loop}_{c}^{2_{i}}\left[i+1 /\left(n_{2}\right)\right], \sigma_{2_{i}}\right)\right)$.

By similar argument in base case, the executions of $S_{1}$ and $S_{2}$ terminate when started in states $m_{1_{i}}\left(\operatorname{loop}_{c}^{1_{i}}[i+\right.$ $\left.\left.1 /\left(n_{1}\right)\right],, \sigma_{1_{i}}\right)$ and $m_{2_{i}}\left(\operatorname{loop}_{c}^{2_{i}}\left[i+1 /\left(n_{2}\right)\right], \sigma_{2_{i}}\right)$ respectively, $\left(S_{1} ; s_{1}, m_{1_{i}}\left(\operatorname{loop}_{c}^{1_{i}}\left[i+1 /\left(n_{1}\right)\right], \sigma_{1_{i}}\right)\right) \stackrel{*}{\rightarrow}$

$\left(s_{1}, m_{1_{i+1}}\left(\operatorname{loop}_{c}^{1_{i+1}}, \sigma_{1_{i+1}}\right)\right)$ and $\left(S_{2} ; s_{1}, m_{2_{i}}\left(\operatorname{loop}_{c}^{2_{i}}[i+\right.\right.$ $\left.\left.\left.1 /\left(n_{2}\right)\right], \sigma_{2_{i}}\right)\right) \stackrel{*}{\rightarrow}\left(s_{2}, m_{2_{i+1}}\left(\operatorname{loop}_{c}^{2_{i+1}}, \sigma_{2_{i+1}}\right)\right)$ such that all of the following holds:

- $\operatorname{loop}_{c}^{1_{i+1}}\left(n_{1}\right)=\operatorname{loop}_{c}^{2_{i+1}}\left(n_{2}\right)=i+1$; and

- $\forall y \in \operatorname{Imp}(x), \sigma_{1_{i+1}}(y)=\sigma_{2_{i+1}}(y)$, and

- in any state in the execution

$\left(s_{1}, m_{1_{i}}\right) \stackrel{*}{\rightarrow}\left(s_{1}, m_{1_{i+1}}\left(\operatorname{loop}_{c}^{1_{i+1}}, \sigma_{1_{i+1}}\right)\right)$, the loop counter of $s_{1}$ is less than or equal to $i+1$.
- in any state in the executions

$\left(s_{2}, m_{2_{i}}\right) \stackrel{*}{\rightarrow}\left(s_{2}, m_{2_{i+1}}\left(\operatorname{loop}_{c}^{2_{i+1}}, \sigma_{2_{i+1}}\right)\right)$, the loop counter of $s_{2}$ is less than or equal to $i+1$.

With the hypothesis $\mathrm{IH}$, there are two configurations $\left(s_{1}, m_{1_{i+1}}\right)$ and $\left(s_{2}, m_{2_{i+1}}\right)$ reachable from $\left(s_{1}, m_{1}\right)$ and $\left(s_{2}, m_{2}\right)$, respectively, in which the loop counters of $s_{1}$ and $s_{2}$ are equal to $i+1$ and value stores agree on the values of imported variables relative to $x$ and, for every state in executions $\left(s_{1}, m_{1}\right) \stackrel{*}{\rightarrow}\left(s_{1}, m_{1_{i+1}}\right)$ and $\left(s_{2}, m_{2}\right) \stackrel{*}{\rightarrow}\left(s_{2}, m_{2_{i+1}}\right)$ the loop counters for $s_{1}$ and $s_{2}$ are less than or equal to $i+1$ respectively:

$$
\begin{aligned}
& \exists\left(s_{1}, m_{1_{i+1}}\right),\left(s_{2}, m_{2_{i+1}}\right): \\
& \left(s_{1}, m_{1}\right) \stackrel{*}{\rightarrow}\left(s_{1}, m_{1_{i+1}}\left(\operatorname{loop}_{c}^{1_{i+1}}, \sigma_{1_{i+1}}\right)\right) \wedge \\
& \left(s_{2}, m_{2}\right) \stackrel{*}{\rightarrow}\left(s_{2}, m_{2_{i+1}}\left(\operatorname{loop}_{c}^{2_{i+1}}, \sigma_{2_{i+1}}\right)\right) \text { where } \\
& \text { - } \operatorname{loop}_{c}^{1_{i+1}}\left(n_{1}\right)=\operatorname{loop}_{c}^{2_{i+1}}\left(n_{2}\right)=i+1 \text {; and } \\
& \text { - } \forall y \in \operatorname{Imp}(x), \sigma_{1_{i+1}}(y)=\sigma_{2_{i+1}}(y) \text {; and } \\
& \text { - } \forall m_{1}^{\prime}:\left(s_{1}, m_{1}\right) \stackrel{*}{\rightarrow}\left(S_{1}^{\prime}, m_{1}^{\prime}\left(\operatorname{loop}_{c}^{1^{\prime}}\right)\right) \stackrel{*}{\rightarrow} \\
& \quad\left(s_{1}, m_{1_{i+1}}\left(\operatorname{loop}_{c}^{1_{i+1}}, \sigma_{1_{i+1}}\right)\right), \operatorname{loop}_{c}^{1^{\prime}}\left(n_{1}\right) \leq i+1 ; \text { and } \\
& \text { - } \forall m_{2}^{\prime}:\left(s_{2}, m_{2}\right) \stackrel{*}{\rightarrow}\left(S_{2}^{\prime}, m_{2}^{\prime}\left(\operatorname{loop}_{c}^{2^{\prime}}\right)\right) \stackrel{*}{\rightarrow} \\
& \quad\left(s_{2}, m_{2_{i+1}}\left(\operatorname{loop}_{c}^{2_{i+1}}, \sigma_{2_{i+1}}\right)\right), \operatorname{loop}_{c}^{2^{\prime}}\left(n_{2}\right) \leq i+1 .
\end{aligned}
$$

Lemma 5.2. Let $s_{1}=$ "while ${ }_{\left\langle n_{1}\right\rangle}(e)\left\{S_{1}\right\}$ " and $s_{2}=$ "while ${ }_{\left\langle n_{2}\right\rangle}(e)$ $\left\{S_{2}\right\}$ " be two while statements with the same set of imported variables relative to a variable $x$ (defined in $s_{1}$ and $s_{2}$ ), and whose loop bodies $S_{1}$ and $S_{2}$ terminatingly compute the variables in $\operatorname{Imp}(x)$ equivalently when started in states that agree on the values of the variables imported by $S_{1}$ or $S_{2}$ relative to $\operatorname{Imp}(x)$ :

- $x \in \operatorname{Def}\left(s_{1}\right) \cap \operatorname{Def}\left(s_{2}\right)$;

- $\operatorname{Imp}\left(s_{1},\{x\}\right)=\operatorname{Imp}\left(s_{2},\{x\}\right)=\operatorname{Imp}(x)$;

- $\forall y \in \operatorname{Imp}(x) \forall m_{S_{1}}\left(\sigma_{S_{1}}\right) m_{S_{2}}\left(\sigma_{S_{2}}\right)$ :

$\left(\left(\forall z \in \operatorname{Imp}\left(S_{1}, \operatorname{Imp}(x)\right) \cup \operatorname{Imp}\left(S_{2}, \operatorname{Imp}(x)\right), \sigma_{S_{1}}(z)=\sigma_{S_{2}}(z)\right) \Rightarrow\right.$ $\left(\left(S_{1}, m_{S_{1}}\left(\sigma_{S_{1}}\right)\right) \equiv_{y}\left(S_{2}, m_{S_{2}}\left(\sigma_{S_{2}}\right)\right)\right)$.

If the executions of $s_{1}$ and $s_{2}$ terminate when started in states $m_{1}\left(\right.$ loop $\left._{c}^{1}, \sigma_{1}\right)$ and $m_{2}\left(\right.$ loop $\left._{c}^{2}, \sigma_{2}\right)$ in which $s_{1}$ and $s_{2}$ have not already executed (loop counter initially $0: \operatorname{loop}_{c}^{1}\left(n_{1}\right)=$ loop $\left._{c}^{2}\left(n_{2}\right)=0\right)$, and whose value stores $\sigma_{1}$ and $\sigma_{2}$ agree on the values of the variables in $\operatorname{Imp}(x), \forall y \in \operatorname{Imp}(x) \sigma_{1}(y)=$ $\sigma_{2}(y)$, when $s_{1}$ and $s_{2}$ terminate, $\left(s_{1}, m_{1}\right) \stackrel{*}{\rightarrow}\left(\right.$ skip, $\left.m_{1_{i}}\left(\sigma_{1}^{\prime}\right)\right)$ and $\left(s_{2}, m_{2}\right) \stackrel{*}{\rightarrow}\left(\right.$ skip, $\left.m_{2_{i}}\left(\sigma_{2}^{\prime}\right)\right)$, value stores $\sigma_{1}^{\prime}$ and $\sigma_{2}^{\prime}$ agree on the value of $x, \sigma_{1}^{\prime}(x)=\sigma_{2}^{\prime}(x)$.

Proof. We show that there must exist a finite integer $k$ such that the loop counters of $s_{1}$ and $s_{2}$ in executions started in states $m_{1}$ and $m_{2}$ is always less than $k$. By the definition of terminating execution, there are only finite number of steps in executions of $s_{1}$ and $s_{2}$ started in states $m_{1}$ and $m_{2}$ respectively. Then, by LemmaE.8 there must be a finite integer $k$ such that the loop counter of $s_{1}$ and $s_{2}$ is always less than $k$. In the following, we consider $k$ be the smallest positive integer such that the loop counter of $s_{1}$ and $s_{2}$ in executions started in states $m_{1}$ and $m_{2}$ is always less than $k$.

By Lemma 5.1 there are two possibilities:

1. The loop counters for $s_{1}$ and $s_{2}$ are always less than $1(k=1)$ : $\forall m_{1}^{\prime}, m_{2}^{\prime}$ such that $\left(s_{1}, m_{1}\right) \stackrel{*}{\rightarrow}\left(S_{1}^{\prime}, m_{1}^{\prime}\left(\operatorname{loop}_{c}^{1^{\prime}}\right)\right)$ and $\left(s_{2}, m_{2}\right)$ $\stackrel{*}{\rightarrow}\left(S_{2}^{\prime}, m_{2}^{\prime}\left(\operatorname{loop}_{c}^{2^{\prime}}\right)\right)$,

$\operatorname{loop}_{c}^{1^{\prime}}\left(n_{1}\right)<1$ and $\operatorname{loop}_{c}^{2^{\prime}}\left(n_{2}\right)<1$;

By the proof in base case of Lemma 5.1 the execution of $s_{1}$ proceeds as follows: 
$\left(s_{1}, m_{1}\right)$

$=\left(\right.$ while $\left._{\left\langle n_{1}\right\rangle}(e)\left\{S_{1}\right\}, m_{1}\left(\operatorname{loop}_{c}^{1}, \sigma_{1}\right)\right)$

$\rightarrow\left(\right.$ while $\left._{\left\langle n_{1}\right\rangle}(0)\left\{S_{1}\right\}, m_{1}\left(\operatorname{loop}_{c}^{1}, \sigma_{1}\right)\right)$ by the EEval' rule

$\rightarrow$ (skip, $\left.m_{1}\left(\operatorname{loop}_{c}^{1}\left[0 /\left(n_{1}\right)\right], \sigma_{1}\right)\right)$ by the Wh-F rule.

Similarly, the execution of $s_{2}$ proceeds to

(skip, $\left.m_{2}\left(\operatorname{loop}_{c}^{2}\left[0 / n_{2}\right], \sigma_{2}\right)\right)$. Therefore, $\sigma_{1}^{\prime}=\sigma_{1}$ and $\sigma_{2}^{\prime}=$ $\sigma_{2}$.

By the definition of imported variables, $x \in \operatorname{Imp}\left(s_{1},\{x\}\right)$. By assumption, value stores $\sigma_{1}$ and $\sigma_{2}$ agree on the value of $x$, $\sigma_{1}^{\prime}(x)=\sigma_{1}(x)=\sigma_{2}(x)=\sigma_{2}^{\prime}(x)$. The lemma holds.

2. For some finite positive $k(>1)$, both of the following hold:

- The loop counters for $s_{1}$ and $s_{2}$ are always less than $k$ :

$\forall m_{1}^{\prime}, m_{2}^{\prime}$ such that $\left(s_{1}, m_{1}\right) \stackrel{*}{\rightarrow}\left(S_{1}^{\prime}, m_{1}^{\prime}\left(\operatorname{loop}_{c}^{1^{\prime}}\right)\right.$ and $\left(s_{2}, m_{2}\right) \stackrel{*}{\rightarrow}\left(S_{2}^{\prime}, m_{2}^{\prime}\left(\operatorname{loop}_{c}^{2^{\prime}}\right)\right)$,

$\operatorname{loop}_{c}^{1^{\prime}}\left(n_{1}\right)<k$ and $\operatorname{loop}_{c}^{2^{\prime}}\left(n_{2}\right)<k$;

- There are two configuration $\left(s_{1}, m_{1_{k-1}}\right)$ and $\left(s_{2}, m_{2_{k-1}}\right)$ reachable from $\left(s_{1}, m_{1}\right)$ and $\left(s_{2}, m_{2}\right)$, respectively, in which the loop counters of $s_{1}$ and $s_{2}$ are equal to $k-1$ and value stores agree on the values of imported variables relative to $x$ and, for every state in execution, $\left(s_{1}, m_{1}\right) \stackrel{*}{\rightarrow}$ $\left(s_{1}, m_{1_{k-1}}\right)$ or $\left(s_{2}, m_{2}\right) \stackrel{*}{\rightarrow}\left(s_{2}, m_{2_{k-1}}\right)$ the loop counters for $s_{1}$ and $s_{2}$ are less than or equal to $k-1$ respectively:

$\exists\left(s_{1}, m_{1_{k-1}}\right),\left(s_{2}, m_{2_{k-1}}\right)$ :

$\left(s_{1}, m_{1}\right) \stackrel{*}{\rightarrow}\left(s_{1}, m_{1_{k-1}}\left(\operatorname{loop}_{c}^{1_{k-1}}, \sigma_{1_{k-1}}\right)\right) \wedge$

$\left(s_{2}, m_{2}\right) \stackrel{*}{\rightarrow}\left(s_{2}, m_{2_{k-1}}\left(\operatorname{loop}_{c}^{2} 2_{k-1}, \sigma_{2_{k-1}}\right)\right)$ where

- $\operatorname{loop}_{c} 1_{k-1}\left(n_{1}\right)=\operatorname{loop}_{c}^{2_{k-1}}\left(n_{2}\right)=k-1$; and

- $\forall y \in \operatorname{Imp}(x): \sigma_{1_{k-1}}(y)=\sigma_{2_{k-1}}(y)$; and

- $\forall m_{1}^{\prime}:\left(s_{1}, m_{1}\right) \stackrel{*}{\rightarrow}\left(S_{1}^{\prime}, m_{1}^{\prime}\left(\operatorname{loop}_{c}^{1^{\prime}}\right) \stackrel{*}{\rightarrow}\right.$

$\left(s_{1}, m_{1_{k-1}}\left(\operatorname{loop}_{c}^{1_{k-1}}, \sigma_{1_{k-1}}\right)\right), \operatorname{loop}_{c}^{1^{\prime}}\left(n_{1}\right) \leq k-1$; and

- $\forall m_{2}^{\prime}:\left(s_{2}, m_{2}\right) \stackrel{*}{\rightarrow}\left(S_{2}^{\prime}, m_{2}^{\prime}\left(\operatorname{loop}_{c}^{2^{\prime}}\right)\right) \stackrel{*}{\rightarrow}$ $\left(s_{2}, m_{2_{k-1}}\left(\operatorname{loop}_{c}^{2_{k-1}}, \sigma_{2_{k-1}}\right)\right), \operatorname{loop}_{c}^{2^{\prime}}\left(n_{2}\right) \leq k-1$;

By proof of Lemma 5.1 value stores $\sigma_{1_{k-1}}$ and $\sigma_{2_{k-1}}$ agree on the values of the variables in Use $(e)$. By Lemma D.1. $\mathcal{E}^{\prime} \llbracket e \rrbracket \sigma_{1_{k-1}}=\mathcal{E}^{\prime} \llbracket e \rrbracket \sigma_{2_{k-1}}=v$. Because the loop counter of $s_{1}$ and $s_{2}$ is less than $k$ in executions of $s_{1}$ and $s_{2}$ when started in states $m_{1}$ and $m_{2}$, then by our semantic rules, the predicate expression of $s_{1}$ and $s_{2}$ must evaluate to zero w.r.t value stores $\sigma_{1_{k-1}}$ and $\sigma_{2_{k-1}}, \mathcal{E}^{\prime} \llbracket e \rrbracket \sigma_{1_{k-1}}=\mathcal{E}^{\prime} \llbracket e \rrbracket \sigma_{2_{k-1}}=\left(0, v_{\mathfrak{o f}}\right)$. Then the execution of $s_{1}$ proceeds as follows.

$$
\begin{aligned}
& \left(\text { while }_{\left\langle n_{1}\right\rangle}(e)\left\{S_{1}\right\}, m_{1_{k-1}}\left(\operatorname{loop}_{c}^{1_{k-1}}, \sigma_{1_{k-1}}\right)\right) \\
\rightarrow & \left(\text { while }_{\left\langle n_{1}\right\rangle}\left(\left(0, v_{\mathfrak{o f}}\right)\right)\left\{S_{1}\right\}, m_{1_{k-1}}\left(\operatorname{loop}_{c}{ }^{1_{k-1}}, \sigma_{1_{k-1}}\right)\right) \\
& \text { by the EEval' rule } \\
\rightarrow & \left(\text { while }_{\left\langle n_{1}\right\rangle}(0)\left\{S_{1}\right\}, m_{1_{k-1}}\left(\operatorname{loop}_{c}{ }^{1_{k-1}}, \sigma_{1_{k-1}}\right)\right) \\
& \text { by the E-Oflow1 or E-Oflow } 2 \text { rule } \\
\rightarrow & \left(\text { skip, } m_{1_{k-1}}\left(\operatorname{loop}_{c} 1_{k-1}\left[0 / n_{1}\right], \sigma_{1_{k-1}}\right)\right) \\
& \text { by the Wh-F rule. }
\end{aligned}
$$

Similarly, the execution of $s_{2}$ proceeds to (skip, $\left.m_{2_{k-1}}\left(\operatorname{loop}_{c}^{2_{k-1}}\left[0 / n_{2}\right], \sigma_{2_{k-1}}\right)\right)$. Therefore, $\sigma_{2}^{\prime}=\sigma_{2_{k-1}}$, $\sigma_{1}^{\prime}=\sigma_{1_{k-1}}$. By the definition of imported variables, $x \in$ $\operatorname{Imp}(x)$. In conclusion, $\sigma_{2}^{\prime}(x)=\sigma_{2_{k-1}}(x)=\sigma_{1_{k-1}}(x)=$ $\sigma_{1}^{\prime}(x)$.

Lemma 5.3. If two statement sequences $S_{1}$ and $S_{2}$ satisfy the proof rule of terminating computation of a variable $x$ equivalently, then $S_{1}$ and $S_{2}$ have same imported variables relative to $x:\left(S_{1} \equiv_{x}^{S}\right.$ $\left.S_{2}\right) \Rightarrow\left(\operatorname{Imp}\left(S_{1},\{x\}\right)=\operatorname{Imp}\left(S_{2},\{x\}\right)\right)$.
Proof. By induction on size $\left(S_{1}\right)+\operatorname{size}\left(S_{2}\right)$, the sum of the program size of $S_{1}$ and $S_{2}$.

\section{Base case.}

$S_{1}$ and $S_{2}$ are simple statement. Then the proof is a case analysis according to the cases in the definition of the proof rule of computation equivalently for simple statements.

\section{1. $S_{1}=S_{2}$}

By the definition of imported variables, same statement have same imported variables relative to same $x$.

\section{2. $S_{1} \neq S_{2}$}

There are two further cases:

- $S_{1}=$ "input $i d_{1}$ ", $S_{2}=$ "input $i d_{2}$ " and $x \notin\left\{i d_{1}, i d_{2}\right\}$. When $x=i d_{I}$, by the definition of imported variables, $\operatorname{Imp}\left(S_{1},\left\{i d_{I}\right\}\right)=\operatorname{Imp}\left(S_{2},\left\{i d_{I}\right\}\right)=\left\{i d_{I}\right\}$. When $x=$ $i d_{I O}$, by the definition of imported variables, $\operatorname{Imp}\left(S_{1},\left\{i d_{I O}\right\}\right)=$ $\operatorname{Imp}\left(S_{2},\left\{i d_{I O}\right\}\right)=\left\{i d_{I}, i d_{I O}\right\}$.

When $x \notin\left\{i d_{I}, i d_{I O}\right\}$, by the definition of imported variables, $\operatorname{Imp}\left(S_{1},\{x\}\right)=\operatorname{Imp}\left(S_{2},\{x\}\right)=\{x\}$.

- the above cases do not hold and $x \notin \operatorname{Def}\left(S_{1}\right) \cup \operatorname{Def}\left(S_{2}\right)$. By the definition of imported variables, $\operatorname{Imp}\left(S_{1},\{x\}\right)=$ $\operatorname{Imp}\left(S_{2},\{x\}\right)=\{x\}$.

\section{Induction Step}

The hypothesis $\mathrm{IH}$ is that the lemma holds when $\operatorname{size}\left(S_{1}\right)+$ $\operatorname{size}\left(S_{2}\right)=k \geq 2$.

Then we show the lemma holds when $\operatorname{size}\left(S_{1}\right)+\operatorname{size}\left(S_{2}\right)=k+1$. The proof is a case analysis based on the cases in the definition of the proof rule of computation equivalently for statement sequence, $S_{1} \equiv{ }_{x}^{S} S_{2}$ :

1. $S_{1}$ and $S_{2}$ are one statement such that one of the following holds:

(a) $S_{1}$ and $S_{2}$ are If statement that define the variable $x$ :

$S_{1}=$ "If $(e)$ then $\left\{S_{1}^{t}\right\}$ else $\left\{S_{1}^{f}\right\}$ ", $S_{2}=$ "If $(e)$ then $\left\{S_{2}^{t}\right\}$ else $\left\{S_{2}^{f}\right\}$ " such that all of the following hold:

- $x \in \operatorname{Def}\left(S_{1}\right) \cap \operatorname{Def}\left(S_{2}\right)$;

- $S_{1}^{t} \equiv_{x}^{S} S_{2}^{t}$;

- $S_{1}^{f} \equiv{ }_{x}^{S} S_{2}^{f}$;

By the hypothesis IH, the imported variables in $S_{1}^{t}$ and $S_{2}^{t}$ relative to $x$ are same, $\operatorname{Imp}\left(S_{1}^{t},\{x\}\right)=\operatorname{Imp}\left(S_{2}^{t},\{x\}\right)$. Similarly, $\operatorname{Imp}\left(S_{1}^{f},\{x\}\right)=\operatorname{Imp}\left(S_{2}^{f},\{x\}\right)$. By the definition of imported variables, $\operatorname{Imp}\left(S_{1},\{x\}\right)=\mathrm{Use}(e) \cup$ $\operatorname{Imp}\left(S_{1}^{t},\{x\}\right) \cup \operatorname{Imp}\left(S_{1}^{f},\{x\}\right)$. Similarly, $\operatorname{Imp}\left(S_{2},\{x\}\right)$ $=\operatorname{Use}(e) \cup \operatorname{Imp}\left(S_{2}^{t},\{x\}\right) \cup \operatorname{Imp}\left(S_{2}^{f},\{x\}\right)$. Then, the lemma holds.

(b) $S_{1}$ and $S_{2}$ are while statement that define the variable $x$ : $S_{1}=$ "while ${ }_{\left\langle n_{1}\right\rangle}(e)\left\{S_{1}^{\prime \prime}\right\} ", S_{2}=$ "while ${ }_{\left\langle n_{2}\right\rangle}(e)\left\{S_{2}^{\prime \prime}\right\} "$ such that both of the following hold:

- $x \in \operatorname{Def}\left(S_{1}\right) \cap \operatorname{Def}\left(S_{2}\right)$;

- $\forall y \in \operatorname{Imp}\left(S_{1},\{x\}\right) \cup \operatorname{Imp}\left(S_{2},\{x\}\right), S_{1}^{\prime \prime} \equiv_{y}^{S} S_{2}^{\prime \prime}$; By the definition of imported variables, $\operatorname{Imp}\left(S_{1},\{x\}\right)=$ $\bigcup_{i \geq 0} \operatorname{Imp}\left(S_{1}^{\prime \prime} i,\{x\} \cup \operatorname{Use}(e)\right)$. Similarly, $\operatorname{Imp}\left(S_{2},\{x\}\right)=$ $\bigcup_{i>0} \operatorname{Imp}\left(S_{2}^{\prime \prime} i,\{x\} \cup U \operatorname{Use}(e)\right)$. Then, we show that $\operatorname{Imp}\left(S_{1}^{\prime \prime} i,\{x\} \cup\right.$ $\mathrm{Use}(e))=\operatorname{Imp}\left(S_{2}^{\prime \prime}{ }^{i},\{x\} \cup \operatorname{Use}(e)\right)$ by induction on $i$. Base case.

By our assumption of the notation $S^{0}, S_{1}^{\prime \prime O}=$ skip, $S_{2}^{\prime \prime}{ }^{\circ}=$ skip.

Then, $\operatorname{Imp}\left(S_{1}^{\prime \prime 0},\{x\} \cup \operatorname{Use}(e)\right)=\{x\} \cup \operatorname{Use}(e), \operatorname{Imp}\left(S_{2}^{\prime \prime} 0,\{x\} \cup\right.$ $\operatorname{Use}(e))=\{x\} \cup \operatorname{Use}(e)$.

Hence, $\operatorname{Imp}\left(S_{1}^{\prime \prime} 0,\{x\} \cup U \operatorname{se}(e)\right)=\operatorname{Imp}\left(S_{2}^{\prime \prime} 0,\{x\} \cup \operatorname{Use}(e)\right)$. Induction step. 
The hypothesis $\mathrm{IH} 2$ is that $\operatorname{Imp}\left(S_{1}^{\prime \prime},\{x\} \cup \operatorname{Use}(e)\right)=$ $\operatorname{Imp}\left(S_{2}^{\prime \prime} i,\{x\} \cup \operatorname{Use}(e)\right)$ for $i \geq 0$.

Then we show that $\operatorname{Imp}\left(S_{1}^{\prime \prime} i+1,\{x\} \cup \operatorname{Use}(e)\right)=\operatorname{Imp}\left(S_{2}^{\prime \prime} i+1,\{x\} \cup\right.$ Use $(e))$.

$$
\begin{aligned}
& \operatorname{Imp}\left(S_{1}^{\prime \prime} i+1,\{x\} \cup \operatorname{Use}(e)\right) \\
&= \operatorname{Imp}\left(S_{1}^{\prime \prime}, \operatorname{Imp}\left(S_{1}^{\prime \prime} i,\{x\} \cup \operatorname{Use}(e)\right)\right)(1) \text { by corollaryC.1 } \\
& \operatorname{Imp}\left(S_{2}^{\prime \prime} i+1,\{x\} \cup \operatorname{Use}(e)\right) \\
&= \operatorname{Imp}\left(S_{2}^{\prime \prime}, \operatorname{Imp}\left(S_{2}^{\prime \prime} i,\{x\} \cup \operatorname{Use}(e)\right)\right)(2) \text { by corollaryC.1 } \\
& \operatorname{Imp}\left(S_{1}^{\prime \prime} i,\{x\} \cup \operatorname{Use}(e)\right)=\operatorname{Imp}\left(S_{2}^{\prime \prime}{ }^{i},\{x\} \cup \operatorname{Use}(e)\right) \\
& \text { by the hypothesis IH } 2 \\
& \operatorname{Imp}\left(S_{1}^{\prime \prime}, \operatorname{Imp}\left(S_{1}^{\prime \prime} i_{i},\{x\} \cup \operatorname{Use}(e)\right)\right) \\
&=\operatorname{Imp}\left(S_{2}^{\prime \prime}, \operatorname{Imp}\left(S_{2}^{\prime \prime} i,\{x\} \cup \operatorname{Use}(e)\right)\right) \\
& \text { by the hypothesis IH } \\
& \operatorname{Imp}\left(S_{1}^{\prime \prime} i+1,\{x\} \cup \operatorname{Use}(e)\right) \\
&=\operatorname{Imp}\left(S_{2}^{\prime \prime} i+1,\{x\} \cup \operatorname{Use}(e)\right) \text { by (1),(2) }
\end{aligned}
$$

Therefore, $\operatorname{Imp}\left(S_{1}^{\prime \prime}{ }^{i+1},\{x\} \cup \operatorname{Use}(e)\right)=\operatorname{Imp}\left(S_{2}^{\prime \prime}{ }^{i+1},\{x\} \cup\right.$ $\operatorname{Use}(e))$.

In conclusion, $\operatorname{Imp}\left(S_{1},\{x\}\right)=\operatorname{Imp}\left(S_{2},\{x\}\right)$. The lemma holds.

(c) $S_{1}$ and $S_{2}$ do not define the variable $x: x \notin \operatorname{Def}\left(S_{1}\right) \cup$ $\operatorname{Def}\left(S_{2}\right)$.

By the definition of imported variable, the imported variables in $S_{1}$ and $S_{2}$ relative to $x$ is $x, \operatorname{Imp}\left(S_{1},\{x\}\right)=$ $\operatorname{Imp}\left(S_{2},\{x\}\right)=\{x\}$. The lemma holds.

2. $S_{1}$ and $S_{2}$ are not both one statement such that one of the following holds:

(a) Last statements both define the variable $x$ such that all of the following hold:

- $\forall y \in \operatorname{Imp}\left(s_{1},\{x\}\right) \cup \operatorname{Imp}\left(s_{2},\{x\}\right), S_{1}^{\prime} \equiv{ }_{y}^{S} S_{2}^{\prime}$;

- $x \in \operatorname{Def}\left(s_{1}\right) \cap \operatorname{Def}\left(s_{2}\right)$;

- $s_{1} \equiv{ }_{x}^{S} s_{2}$;

By the hypothesis IH, we have $\operatorname{Imp}\left(s_{1},\{x\}\right)=\operatorname{Imp}\left(s_{2},\{x\}\right)=$ $\operatorname{Imp}(\Delta)$. Then, by the hypothesis IH again, we have that $\forall y \in \operatorname{Imp}(\Delta)=\operatorname{Imp}\left(s_{1},\{x\}\right)=\operatorname{Imp}\left(s_{2},\{x\}\right), \operatorname{Imp}\left(S_{1}^{\prime},\{y\}\right)$ $=\operatorname{Imp}\left(S_{2}^{\prime},\{y\}\right)$. By taking the union of all $\forall y \in \operatorname{Imp}(\Delta)$, $\operatorname{Imp}\left(S_{1}^{\prime},\{y\}\right)$ and $\operatorname{Imp}\left(S_{2}^{\prime},\{y\}\right)$, by the $\operatorname{LemmaC.2} \operatorname{Imp}\left(S_{1}^{\prime}\right.$, $\operatorname{Imp}(\Delta))=\operatorname{Imp}\left(S_{2}^{\prime}, \operatorname{Imp}(\Delta)\right)$. By the definition of imported variables, $\operatorname{Imp}\left(S_{1},\{x\}\right)=\operatorname{Imp}\left(S_{1}^{\prime}, \operatorname{Imp}\left(s_{1},\{x\}\right)\right), \operatorname{Imp}\left(S_{2},\{x\}\right)=$ $\operatorname{Imp}\left(S_{2}^{\prime}, \operatorname{Imp}\left(s_{2},\{x\}\right)\right)$. Therefore, the lemma holds.

(b) One last statement does not define the variable $x$ : w.l.o.g., $\left(x \notin \operatorname{Def}\left(s_{1}\right) \wedge\left(S_{1}^{\prime} \equiv_{x}^{S} S_{2}\right)\right)$;

By the definition of imported variables, we have $\operatorname{Imp}\left(s_{1},\{x\}\right)$ $=\{x\}$. By the hypothesis $\operatorname{IH}, \operatorname{Imp}\left(S_{1}^{\prime},\{x\}\right)=\operatorname{Imp}\left(S_{2},\{x\}\right)$ Therefore, by the definition of imported variables, $\operatorname{Imp}\left(S_{1},\{x\}\right)$ $=\operatorname{Imp}\left(S_{1}^{\prime}, \operatorname{Imp}\left(s_{1},\{x\}\right)\right)=\operatorname{Imp}\left(S_{1}^{\prime},\{x\}\right)=\operatorname{Imp}\left(S_{2},\{x\}\right)$.

(c) There are statements moving in/out of If statement:

$s_{1}=$ "If $(e)$ then $\left\{S_{1}^{t}\right\}$ else $\left\{S_{1}^{f}\right\} ", s_{2}=$ "If $(e)$ then $\left\{S_{2}^{t}\right\}$ else $\left\{S_{2}^{f}\right\}$ " such that none of the above cases hold and all of the following hold:

- $\forall y \in \operatorname{Use}(e), S_{1}^{\prime} \equiv{ }_{y}^{S} S_{2}^{\prime}$;

- $S_{1}^{\prime} ; S_{1}^{t} \equiv_{x}^{S} S_{2}^{\prime} ; S_{2}^{t}$;

- $S_{1}^{\prime} ; S_{1}^{f} \equiv_{x}^{S} S_{2}^{\prime} ; S_{2}^{f}$;

- $x \in \operatorname{Def}\left(s_{1}\right) \cap \operatorname{Def}\left(s_{2}\right)$;

We show all of the following hold.

i. $\operatorname{Imp}\left(S_{1}^{\prime}, \operatorname{Use}(e)\right)=\operatorname{Imp}\left(S_{2}^{\prime}, \operatorname{Use}(e)\right)$.

By the hypothesis IH and the assumption that $\forall y \in$ Use $(e), S_{1}^{\prime} \equiv_{y}^{S} S_{2}^{\prime}$. Then, by LemmaC.2.

$\operatorname{Imp}\left(S_{1}^{\prime}, \operatorname{Use}(e)\right)=\operatorname{Imp}\left(S_{2}^{\prime}, \operatorname{Use}(e)\right)$. ii. $\operatorname{Imp}\left(S_{1}^{\prime}, \operatorname{Imp}\left(S_{1}^{t},\{x\}\right)\right)=\operatorname{Imp}\left(S_{2}^{\prime}, \operatorname{Imp}\left(S_{2}^{t},\{x\}\right)\right)$. Because size("If $(e)$ then $\left\{S_{t}\right\}$ else $\left.\left\{S_{f}\right\} "\right)=1+$ $\operatorname{size}\left(S_{t}\right)+\operatorname{size}\left(S_{f}\right)$, then $\operatorname{size}\left(S_{1}^{\prime} ; S_{1}^{t}\right)+\operatorname{size}\left(S_{2}^{\prime} ; S_{2}^{t}\right)<k$. By the hypothesis IH, $\operatorname{Imp}\left(S_{1}^{\prime} ; S_{1}^{t},\{x\}\right)=\operatorname{Imp}\left(S_{2}^{\prime} ; S_{2}^{t},\{x\}\right)$.

Besides, by Lemma C.1

$\operatorname{Imp}\left(S_{1}^{\prime}, \operatorname{Imp}\left(S_{1}^{t},\{x\}\right)\right)=\operatorname{Imp}\left(S_{1}^{\prime} ; S_{1}^{t},\{x\}\right)$

$=\operatorname{Imp}\left(S_{2}^{\prime} ; S_{2}^{t},\{x\}\right)=\operatorname{Imp}\left(S_{2}^{\prime}, \operatorname{Imp}\left(S_{2}^{t},\{x\}\right)\right)$.

iii. $\operatorname{Imp}\left(S_{1}^{\prime}, \operatorname{Imp}\left(S_{1}^{f},\{x\}\right)\right)=\operatorname{Imp}\left(S_{2}^{\prime}, \operatorname{Imp}\left(S_{2}^{f},\{x\}\right)\right)$. By similar argument in the case that

$\operatorname{Imp}\left(S_{1}^{\prime}, \operatorname{Imp}\left(S_{1}^{t},\{x\}\right)\right)=\operatorname{Imp}\left(S_{2}^{\prime}, \operatorname{Imp}\left(S_{2}^{t},\{x\}\right)\right)$.

Then, by combining things together,

$\operatorname{Imp}\left(S_{1},\{x\}\right)$

$=\operatorname{Imp}\left(S_{1}^{\prime}, \operatorname{Imp}\left(s_{1},\{x\}\right)\right)$

by the definition of imported variables

$=\operatorname{Imp}\left(S_{1}^{\prime}, \operatorname{Imp}\left(S_{1}^{t},\{x\}\right) \cup \operatorname{Imp}\left(S_{1}^{f},\{x\}\right) \cup \operatorname{Use}(e)\right)$

by the definition of imported variables

$=\operatorname{Imp}\left(S_{1}^{\prime}, \operatorname{Imp}\left(S_{1}^{t},\{x\}\right)\right) \cup \operatorname{Imp}\left(S_{1}^{\prime}, \operatorname{Imp}\left(S_{1}^{f},\{x\}\right)\right)$

$\cup \operatorname{Imp}\left(S_{1}^{\prime}, \operatorname{Use}(e)\right)$ by LemmaC.2

$=\operatorname{Imp}\left(S_{2}^{\prime}, \operatorname{Imp}\left(S_{2}^{t},\{x\}\right)\right) \cup \operatorname{Imp}\left(S_{2}^{\prime}, \operatorname{Imp}\left(S_{2}^{f},\{x\}\right)\right)$ $\cup \operatorname{Imp}\left(S_{2}^{\prime}, \operatorname{Use}(e)\right)$ by i, ii, iii

$=\operatorname{Imp}\left(S_{2}^{\prime}, \operatorname{Imp}\left(S_{2}^{t},\{x\}\right) \cup \operatorname{Imp}\left(S_{2}^{f},\{x\}\right) \cup \operatorname{Use}(e)\right)$ by Lemma C.2

$=\operatorname{Imp}\left(S_{2}^{\prime}, \operatorname{Imp}\left(S_{2}^{t},\{x\}\right) \cup \operatorname{Imp}\left(S_{2}^{f},\{x\}\right) \cup \operatorname{Use}(e)\right)$ by the definition of imported variables

$=\operatorname{Imp}\left(S_{2}^{\prime}, \operatorname{Imp}\left(s_{2},\{x\}\right)\right)$

by the definition of imported variables

$=\operatorname{Imp}\left(S_{2},\{x\}\right)$.

Hence, the lemma holds.

\subsection{Termination in the same way}

We proceed to propose a proof rule under which two statement sequences either both terminate or both do not terminate. We start by giving the definition of termination in the same way. Then we present the proof rule of termination in the same way. Our proof rule of termination in the same way allows updates such as statement duplication or reordering, loop fission or fusion and additional terminating statements. We prove that the proof rule ensures terminating in the same way by induction on the program size of the two programs in the proof rule. We also list auxiliary lemmas required by the proof of termination in the same way.

Definition 14. (Termination in the same way) Two statement sequences $S_{1}$ and $S_{2}$ terminate in the same way when started in states $m_{1}$ and $m_{2}$ respectively, written $\left(S_{1}, m_{1}\right) \equiv_{H}\left(S_{2}, m_{2}\right)$, iff one of the following holds:

1. $\left(S_{1}, m_{1}\right) \stackrel{*}{\rightarrow}\left(\right.$ skip, $\left.m_{1}^{\prime}\right)$ and $\left(S_{2}, m_{2}\right) \stackrel{*}{\rightarrow}\left(\right.$ skip, $\left.m_{2}^{\prime}\right)$;

2. $\forall i \geq 0,\left(S_{1}, m_{1}\right) \stackrel{i}{\rightarrow}\left(S_{1}^{i}, m_{1}^{i}\right)$ and $\left(S_{2}, m_{2}\right) \stackrel{i}{\rightarrow}\left(S_{2}^{i}, m_{2}^{i}\right)$ where $S_{1}^{i} \neq$ skip, $S_{2}^{i} \neq$ skip.

\subsubsection{Proof rule for termination in the same way}

We define proof rules under which two statement sequences $S_{1}$ and $S_{2}$ terminate in the same way. We summarize the cause of nonterminating execution and then give the proof rule.

We consider two causes of nonterminating executions: crash and infinite iterations of loop statements. As to crash [?], we consider four common causes based on our language: expression evaluation exceptions, the lack of input value, input/assignment value type mismatch and array index out of bound. In essence, the causes of nontermination are partly due to the values of some particular variables during executions. We capture variables affecting each 
source of nontermination; loop deciding variables $\operatorname{LVar}(S)$ are variables affecting the evaluation of a loop statements in the statement sequence $S$, crash deciding variables $C \operatorname{Var}(S)$ are variables whose values decide if a crash occurs in $S$. We list the definitions of $\operatorname{Lar}(S)$ and $C \operatorname{Var}(S)$ in Definition 15 and 16 Definition 17 summarizes the variables whose values decide if one program terminates.

Definition 15. (Loop deciding variables) The loop deciding variables of a statement sequence $S$, written LVar $(S)$, are defined as follows:

1. $\operatorname{LVar}(S)=\emptyset$ if $\nexists s=$ "while $(e)\left\{S^{\prime}\right\} "$ and $s \in S$;

2. LVar("If $(e)$ then $\left\{S_{t}\right\}$ else $\left.\left\{S_{f}\right\} "\right)=U$ se $(e) \cup \operatorname{LVar}\left(S_{t}\right) \cup$ $\operatorname{LVar}\left(S_{f}\right)$ if "while $(e)\left\{S^{\prime}\right\} " \in S$;

3. $\operatorname{LVar}\left(" w h i l e(e)\left\{S^{\prime}\right\} "\right)=\operatorname{Imp}\left(S\right.$, Use $\left.(e) \cup \operatorname{LVar}\left(S^{\prime}\right)\right)$;

4. For $k>0, \operatorname{LVar}\left(s_{1} ; \ldots ; s_{k} ; s_{k+1}\right)=\operatorname{LVar}\left(s_{1} ; \ldots ; s_{k}\right) \cup$ $\operatorname{Imp}\left(s_{1} ; \ldots ; s_{k}, \operatorname{LVar}\left(s_{k+1}\right)\right)$

Definition 16. (Crash deciding variables) The crash deciding variables of a statement sequence $S$, written $C \operatorname{Var}(S)$, are defined as follows:

1. $C \operatorname{Var}($ skip $)=\emptyset$;

2. $C \operatorname{Var}($ lval $:=e)=I d x($ lval $) \cup$ Use $(e)$ if $(\Gamma \vdash$ lval : Int $) \wedge(\Gamma \vdash e:$ Long $)$;

3. $C \operatorname{Var}($ lval $:=e)=I d x($ lval $) \cup \operatorname{Err}(e)$ if $(\Gamma \vdash \operatorname{lval}:$ Int $) \wedge(\Gamma \vdash$ $e$ : Long) does not hold;

4. $C \operatorname{Var}($ input $i d)=\left\{i d_{I}\right\}$;

5. $C \operatorname{Var}($ output e) $=\operatorname{Err}(e)$;

6. $C \operatorname{Var}\left(" I f(e)\right.$ then $\left\{S_{t}\right\}$ else $\left.\left\{S_{f}\right\} "\right)=\operatorname{Err}(e)$, if $C \operatorname{Var}\left(S_{t}\right)$ $\cup \operatorname{CVar}\left(S_{f}\right)=\emptyset$;

7. $C \operatorname{Var}\left(" I f(e)\right.$ then $\left\{S_{t}\right\}$ else $\left.\left\{S_{f}\right\} "\right)=U$ se $(e) \cup \operatorname{CVar}\left(S_{t}\right)$ $\cup C \operatorname{Var}\left(S_{f}\right)$, if $C \operatorname{Var}\left(S_{t}\right) \cup C \operatorname{Var}\left(S_{f}\right) \neq \emptyset$;

8. CVar ("while $\left.\langle n\rangle(e)\left\{S^{\prime}\right\} "\right)=\operatorname{Imp}\left(\right.$ "while $\langle n\rangle(e)\left\{S^{\prime}\right\} "$, Use $(e) \cup$ $\left.C \operatorname{Var}\left(S^{\prime}\right)\right)$

9. For $k>0, C \operatorname{Var}\left(s_{1} ; \ldots ; s_{k+1}\right)=C \operatorname{Var}\left(s_{1} ; \ldots ; s_{k}\right)$ $\cup \operatorname{Imp}\left(s_{1} ; \ldots ; s_{k}, C \operatorname{Var}\left(s_{k+1}\right)\right)$;

Definition 17. (Termination deciding variables) The termination deciding variables of statement sequence $S$ are $C \operatorname{Var}(S) \cup L \operatorname{Var}(S)$, written $T \operatorname{Var}(S)$.

Definition 18. (Base cases of the proof rule of termination in the same way) Two simple statements $s_{1}$ and $s_{2}$ satisfy the proof rule of termination in the same way, written $s_{1} \equiv{ }_{H}^{S} s_{2}$, iff one of the following holds:

1. $s_{1}$ and $s_{2}$ are same, $s_{1}=s_{2}$;

2. $s_{1}$ and $s_{2}$ are input statement with same type variable: $s_{1}=$ "input $i d_{1}$ ", $s_{2}=$ "input $i d_{2}$ " where $\left(\Gamma_{s_{1}} \vdash i d_{1}: \tau\right) \wedge\left(\Gamma_{s_{2}} \vdash\right.$ $\left.i d_{2}: \tau\right)$

3. $s_{1}=$ "output e" or "id $1:=e ", s_{2}=$ "outpute" or " $i d_{2}:=e$ " where both of the following hold:

- There is no possible value mismatch in " $i d_{1}:=e$ ", $\neg\left(\Gamma_{s_{1}} \vdash i d_{1}:\right.$ Int $) \vee \neg\left(\Gamma_{s_{1}} \vdash e:\right.$ Long $) \vee\left(\Gamma_{s_{1}} \vdash e:\right.$ Int $)$.

- There is no possible value mismatch in " $i d_{2}:=e$ ", $\neg\left(\Gamma_{s_{2}} \vdash i d_{2}:\right.$ Int $) \vee \neg\left(\Gamma_{s_{2}} \vdash e:\right.$ Long $) \vee\left(\Gamma_{s_{2}} \vdash e:\right.$ Int $)$.

Definition 19. (proof rule of termination in the same way) Two statement sequences $S_{1}$ and $S_{2}$ satisfy the proof rule of termination in the same way, written $S_{1} \equiv{ }_{H}^{S} S_{2}$, iff one of the following holds:

1. $S_{1}$ and $S_{2}$ are both one statement and one of the following holds.

(a) $S_{1}$ and $S_{2}$ are simple statements: $s_{1} \equiv_{H}^{S} s_{2}$;

(b) $S_{1}=$ "If(e) then $\left\{S_{1}^{t}\right\}$ else $\left\{S_{1}^{f}\right\}$ ”, $S_{2}=$ "If $(e)$ then $\left\{S_{2}^{t}\right\}$ else $\left\{S_{2}^{f}\right\}$ " and one of the following holds: i. $S_{1}^{t}, S_{1}^{f}, S_{2}^{t}, S_{2}^{f}$ are all sequences of "skip";

ii. At least one of $S_{1}^{t}, S_{1}^{f}, S_{2}^{t}, S_{2}^{f}$ is not a sequence of "skip" such that: $\left(S_{1}^{t} \equiv_{H}^{S} S_{2}^{t}\right) \wedge\left(S_{1}^{f} \equiv_{H}^{S} S_{2}^{f}\right)$;

(c) $S_{1}=$ "while ${ }_{\left\langle n_{1}\right\rangle}(e)\left\{S_{1}^{\prime \prime}\right\}$ ", $S_{2}=$ "while ${ }_{\left\langle n_{2}\right\rangle}(e)\left\{S_{2}^{\prime \prime}\right\}$ " and both of the following hold:

- $S_{1}^{\prime \prime} \equiv_{H}^{S} S_{2}^{\prime \prime}$;

- $S_{1}^{\prime \prime}$ and $S_{2}^{\prime \prime}$ have equivalent computation of $T \operatorname{Var}\left(S_{1}\right) \cup$ $T \operatorname{Var}\left(S_{2}\right)$

2. $S_{1}$ and $S_{2}$ are not both one statement and one of the following holds:

(a) $S_{1}=S_{1}^{\prime} ; s_{1}$ and $S_{2}=S_{2}^{\prime} ; s_{2}$ and all of the following hold:

- $S_{1}^{\prime} \equiv_{H}^{S} S_{2}^{\prime}$;

- $S_{1}^{\prime}$ and $S_{2}^{\prime}$ have equivalent computation of $T \operatorname{Var}\left(s_{1}\right) \cup$ $T \operatorname{Var}\left(s_{2}\right)$;

- $s_{1} \equiv_{H}^{S} s_{2}$ where $s_{1}$ and $s_{2}$ are not "skip";

(b) One last statement is "skip":

$\left(\left(S_{1}=S_{1}^{\prime} ;\right.\right.$ "skip" $\left.) \wedge\left(S_{1}^{\prime} \equiv_{H}^{S} S_{2}\right)\right) \vee\left(\left(S_{2}=S_{2}^{\prime} ;\right.\right.$ "skip" $) \wedge$ $\left.\left(S_{1} \equiv_{H}^{S} S_{2}^{\prime}\right)\right)$.

(c) One last statement is a "duplicate" statement and one of the following holds:

i. $S_{1}=S_{1}^{\prime} ; s_{1}^{\prime} ; S_{1}^{\prime \prime} ; s_{1}$ and all of the following hold:

- $S_{1}^{\prime} ; s_{1}^{\prime} ; S_{1}^{\prime \prime} \equiv_{H}^{S} S_{2}$;

- $\left(s_{1}^{\prime} \equiv{ }_{H}^{S} s_{1}\right) \wedge\left(s_{1} \neq\right.$ "skip" $)$,

- $\operatorname{Def}\left(s_{1}^{\prime} ; S_{1}^{\prime \prime}\right) \cap \operatorname{TVar}\left(s_{1}\right)=\emptyset$;

ii. $S_{2}=S_{2}^{\prime} ; s_{2}^{\prime} ; S_{2}^{\prime \prime} ; s_{2}$ and all of the following hold:

- $S_{1} \equiv_{H}^{S} S_{2}^{\prime} ; s_{2}^{\prime} ; S_{2}^{\prime \prime}$;

- $\left(s_{2}^{\prime} \equiv_{H}^{S} s_{2}\right) \wedge\left(s_{2} \neq\right.$ "skip" $)$;

- $\operatorname{Def}\left(s_{2}^{\prime} ; S_{2}^{\prime \prime}\right) \cap \operatorname{TVar}\left(s_{2}\right)=\emptyset$;

(d) $S_{1}=S_{1}^{\prime} ; s_{1} ; s_{1}^{\prime}$ and $S_{2}=S_{2}^{\prime} ; s_{2} ; s_{2}^{\prime}$ where $s_{1}$ and $s_{2}$ are reordered and all of the following hold:

- $S_{1}^{\prime} \equiv{ }_{H}^{S} S_{2}^{\prime}$;

- $S_{1}^{\prime}$ and $S_{2}^{\prime}$ have equivalent computation of $T \operatorname{Var}\left(s_{1} ; s_{1}^{\prime}\right) \cup$ $T \operatorname{Var}\left(s_{2} ; s_{2}^{\prime}\right)$.

- $s_{1} \equiv{ }_{H}^{S} s_{2}^{\prime}$;

- $s_{1}^{\prime} \equiv{ }_{H}^{S} s_{2}$;

- $\operatorname{Def}\left(s_{1}\right) \cap \operatorname{TVar}\left(s_{1}^{\prime}\right)=\emptyset$;

- $\operatorname{Def}\left(s_{2}\right) \cap \operatorname{TVar}\left(s_{2}^{\prime}\right)=\emptyset$;

\subsubsection{Soundness of the proof rule for termination in the same} way

We show that two statement sequences satisfy the proof rules of termination in the same way, and their initial states agree on the values of their termination deciding variables, then they either both terminate or both do not terminate.

Theorem 3. If two simple statements $s_{1}$ and $s_{2}$ satisfy the proof rule of termination in the same way, $s_{1} \equiv_{H}^{s} s_{2}$, and their initial states $m_{1}\left(\mathfrak{f}_{1}, \sigma_{1}\right)$ and $m_{2}\left(\mathfrak{f}_{2}, \sigma_{2}\right)$ with crash flags not set, $\mathfrak{f}_{1}=$ $\mathfrak{f}_{2}=0$, and whose value stores agree on values of the termination deciding variables of $s_{1}$ and $s_{2}, \forall x \in T \operatorname{Tar}\left(s_{1}\right) \cup \operatorname{TVar}\left(s_{2}\right)$ : $\sigma_{1}(x)=\sigma_{2}(x)$, when executions of $s_{1}$ and $s_{2}$ start in states $m_{1}$ and $m_{2}$ respectively, then $s_{1}$ and $s_{2}$ terminate in the same way when started in states $m_{1}$ and $m_{2}$ respectively: $\left(s_{1}, m_{1}\right) \equiv_{H}$ $\left(s_{2}, m_{2}\right)$.

Proof. The proof is a case analysis of those cases in the definition of $s_{1} \equiv_{H}^{s} s_{2}$. Because $s_{1}$ is a simple statement and $s_{1}$ 's execution is without function call, we only care the crash variables of $s_{1}$ in the termination deciding variables of $s_{1}, \mathrm{CVar}\left(s_{1}\right)$. Similarly, we only care CVar $\left(s_{2}\right)$. 
First $s_{1}$ and $s_{2}$ are same: $s_{1}=s_{2}$;

We show the theorem by induction on abstract syntax of $s_{1}$ and $s_{2}$.

1. $s_{1}=s_{2}=$ skip

By definition of termination in the same way, both $s_{1}$ and $s_{2}$ terminate. The theorem holds.

2. $s_{1}=s_{2}=$ "lval $:=e "$.

There are further cases regarding what lval is.

(a) $l v a l=i d$.

By definition, $\mathrm{C} \operatorname{Var}\left(s_{1}\right)=\mathrm{C} \operatorname{Var}\left(s_{2}\right)=\operatorname{Err}(e)$ or Use $(e)$ based on if there is possible value mismatch (e.g., assigning value defined only in type Long to a variable of type Int). There are two subcases.

- Left value $i d$ is of type Int and expression $e$ is of type Long but not type Int, $(\Gamma \vdash i d:$ Int $) \wedge(\Gamma \vdash e$ : Long) $\wedge \neg(\Gamma \vdash e:$ Int $)$.

By definition, $\mathrm{C} \operatorname{Var}\left(s_{1}\right)=\mathrm{CVar}\left(s_{2}\right)=\mathrm{Use}(e)$. By assumption, $\forall x \in \operatorname{Use}(e), \sigma_{1}(x)=\sigma_{2}(x)$. By Lemma D.1 the expression evaluates to the same value w.r.t two pairs of value stores $\sigma_{1}$ and $\sigma_{2}$ respectively,

- Both evaluations of expression lead to crash,

$\mathcal{E} \llbracket e \rrbracket \sigma_{1}=\mathcal{E} \llbracket e \rrbracket \sigma_{2}=$ (error, $\left.v_{\mathfrak{o f}}\right)$.

Then the execution of $s_{1}$ is as follows:

$$
\begin{aligned}
& \left(s_{1}, m_{1}\right) \\
= & \left(i d:=e, m_{1}\left(\sigma_{1}\right)\right) \\
\rightarrow & \left(i d:=(\text { error, } *), m_{1}\left(\sigma_{1}\right)\right) \text { by rule EEval' } \\
\rightarrow & \left(i d:=0, m_{1}(1 / \mathfrak{f})\right) \text { by rule ECrash. } \\
& \stackrel{i}{\rightarrow}\left(i d:=0, m_{1}(1 / \mathfrak{f})\right) \text { for any } i>0 \text { by rule Crash. }
\end{aligned}
$$

Similarly, $s_{2}$ does not terminate. The theorem holds.

- Both evaluations of expression lead to no crash, $\mathcal{E} \llbracket e \rrbracket \sigma_{1}=\mathcal{E} \llbracket e \rrbracket \sigma_{2}=\left(v, v_{\mathfrak{o f}}\right)$.

Then there are cases regarding if value mismatch occurs.

$\sqrt{ }$ The value $v$ is only defined in type Long, $(\Gamma \vdash$ $v$ : Long $) \wedge \neg(\Gamma \vdash v$ : Int $)$.

The execution of $s_{1}$ is as follows:

$$
\begin{aligned}
& \left(s_{1}, m_{1}\right) \\
= & \left(i d:=e, m_{1}\left(\sigma_{1}\right)\right) \\
\rightarrow & \left(i d:=\left(v, v_{\mathfrak{o f}}\right), m_{1}\left(\sigma_{1}\right)\right) \text { by rule EEval } \\
\rightarrow & \left(i d:=v, m_{1}\left(\sigma_{1}\right)\right) \text { by rule EOflow- } 1 \text { or EOflow- } 2 . \\
\rightarrow & \left(i d:=v, m_{1}(1 / \mathfrak{f})\right) \text { by rule Assign-Err. } \\
& \rightarrow\left(i d:=v, m_{1}(1 / \mathfrak{f})\right) \text { for any } i>0 \text { by rule Crash. }
\end{aligned}
$$

Similarly, $s_{2}$ does not terminate. The theorem holds.

$\sqrt{ }$ The value $v$ is defined in type Int, $\Gamma \vdash v:$ Int. Assuming that the variable $i d$ is a global one, the execution of $s_{1}$ is as follows:

$$
\left(s_{1}, m_{1}\right)
$$$$
=\left(i d:=e, m_{1}\left(\sigma_{1}\right)\right)
$$$$
\rightarrow\left(i d:=\left(v, v_{\mathfrak{o f}}\right), m_{1}\left(\sigma_{1}\right)\right) \text { by rule EEval }
$$$$
\rightarrow\left(i d:=v, m_{1}\left(\sigma_{1}\right)\right) \text { by rule EOflow-1 or EOflow-2. }
$$$$
\left.\rightarrow \text { (skip, } m_{1}\left(\sigma_{1}\left(\sigma_{1}[v / i d]\right)\right)\right) \text { by rule Assign. }
$$

Similarly, $s_{2}$ terminate. The theorem holds.

When the variable $i d$ is a local variable, by similar argument for the global variable, we can show that $s_{1}$ and $s_{2}$ terminate. Then the theorem holds.

- It is not the case that left value $i d$ is of type Int and the expression $e$ is of type Long only, $\neg((\Gamma \vdash i d$ : Int $) \wedge(\Gamma \vdash e$ : Long $) \wedge \neg(\Gamma \vdash e:$ Int $))$. There are two cases based on if there is crash in evaluation of expression $e$. $\sqrt{ }$ Both evaluations of expression lead to crash, $\mathcal{E} \llbracket e \rrbracket \sigma_{1}=\mathcal{E} \llbracket e \rrbracket \sigma_{2}=\left(\right.$ error, $\left.v_{\mathfrak{o f}}\right)$.

By the same argument in case where left value $i d$ is of type Int and the expression $e$ is of type Long only, this theorem holds.

$\sqrt{ }$ Both evaluations of expression lead to no crash, $\mathcal{E} \llbracket e \rrbracket \sigma_{1}=\mathcal{E} \llbracket e \rrbracket \sigma_{2}=\left(v, v_{\mathfrak{o f}}\right)$.

By the same argument in subcase of no value mismatch in case where left value $i d$ is of type Int and the expression $e$ is of type Long only, this theorem holds.

(b) $l v a l=i d[n]$.

There are two subcases based on if $n$ is within the array bound of $i d$. By our assumption, array variable $i d$ is of the same bound in two programs. W.l.o.g., we assume $i d$ is local variable.

i. $n$ is out of bound of array variable $i d,((i d, n) \mapsto$ $\left.v_{1}\right) \notin \sigma_{1}$ and $\left((i d, n) \mapsto v_{2}\right) \notin \sigma_{2}$;

Then the execution of $s_{1}$ continues as follows:

$$
\begin{aligned}
&\left(s_{1}, m_{1}\right) \\
&=\left(i d[n]:=e, m_{1}\left(\sigma_{1}\right)\right) \\
& \rightarrow\left(i d[n]:=e, m_{1}(1 / \mathfrak{f})\right. \text { by rule Arr-3 } \\
& \stackrel{i}{\rightarrow}\left(i d[n]:=e, m_{1}(1 / \mathfrak{f})\right) \text { by rule Crash. }
\end{aligned}
$$

Similarly, $s_{2}$ does not terminate. The theorem holds.

ii. $n$ is within the bound of array variable $i d,((i d, n) \mapsto$ $\left.v_{1}\right) \in \sigma_{1}$ and $\left((i d, n) \mapsto v_{2}\right) \in \sigma_{2}$;

There are cases of $C \operatorname{Var}\left(s_{1}\right)$ and $C \operatorname{Var}\left(s_{2}\right)$ based on if there is possible value mismatch exception in $s_{1}$ and $s_{2}$.

- Left value $i d[n]$ is of type Int and expression $e$ is of type Long but not type Int, $(\Gamma \vdash i d[n]:$ Int $) \wedge(\Gamma \vdash$ $e$ : Long $) \wedge \neg(\Gamma \vdash e$ : Int $)$.

By definition, $\mathrm{CVar}\left(s_{1}\right)=\mathrm{C} \operatorname{Var}\left(s_{2}\right)=\mathrm{Use}(e)$. By assumption, $\forall x \in \operatorname{Use}(e), \sigma_{1}(x)=\sigma_{2}(x)$. By Lemma D.1 the expression evaluates to the same value w.r.t two value stores $\sigma_{1}$ and $\sigma_{2}$ respectively,

- Both evaluations of expression lead to crash,

$\mathcal{E} \llbracket e \rrbracket \sigma_{1}=\mathcal{E} \llbracket e \rrbracket \sigma_{2}=\left(\right.$ error, $\left.v_{\mathfrak{o f f}}\right)$.

Then the execution of $s_{1}$ is as follows:

$$
\begin{aligned}
& \left(s_{1}, m_{1}\right) \\
= & \left(i d[n]:=e, m_{1}\left(\sigma_{1}\right)\right) \\
\rightarrow & \left(i d[n]:=(\text { error }, *), m_{1}\left(\sigma_{1}\right)\right) \text { by rule EEval' } \\
\rightarrow & \left(i d[n]:=0, m_{1}(1 / \mathfrak{f})\right) \text { by rule ECrash. } \\
& \stackrel{i}{\rightarrow}\left(i d[n]:=0, m_{1}(1 / \mathfrak{f})\right) \text { for any } i>0 \\
& \text { by rule Crash. }
\end{aligned}
$$

Similarly, $s_{2}$ does not terminate. The theorem holds.

- Both evaluations of expression lead to no crash, $\mathcal{E} \llbracket e \rrbracket \sigma_{1}=\mathcal{E} \llbracket e \rrbracket \sigma_{2}=\left(v, v_{\mathfrak{o f}}\right)$.

Then there are cases regarding if value mismatch occurs.

$\sqrt{ }$ The value $v$ is only defined in type Long, $(\Gamma \vdash$ $v$ : Long $) \wedge \neg(\Gamma \vdash v$ : Int $)$.

The execution of $s_{1}$ is as follows:

$$
\begin{aligned}
& \left(s_{1}, m_{1}\right) \\
= & \left(i d[n]:=e, m_{1}\left(\sigma_{1}\right)\right) \\
\rightarrow & \left(i d[n]:=\left(v, v_{\mathfrak{o f}}\right), m_{1}\left(\sigma_{1}\right)\right) \text { by rule EEval' } \\
\rightarrow & \left(i d[n]:=v, m_{1}\left(\sigma_{1}\right)\right) \\
& \text { by rule EOflow-1 or EOflow- } 2 . \\
\rightarrow & \left(i d[n]:=v, m_{1}(1 / \mathfrak{f})\right) \text { by rule Assign-Err. } \\
& \stackrel{i}{\rightarrow}\left(i d[n]:=v, m_{1}(1 / \mathfrak{f})\right) \text { for any } i>0 \\
& \text { by rule Crash. }
\end{aligned}
$$


Similarly, $s_{2}$ does not terminate. The theorem holds.

$\sqrt{ }$ The value $v$ is defined in type Int, $\Gamma \vdash v:$ Int.

The execution of $s_{1}$ is as follows:

$$
\begin{aligned}
& \left(s_{1}, m_{1}\right) \\
= & \left(i d[n]:=e, m_{1}\left(\sigma_{1}\right)\right) \\
\rightarrow & \left(i d[n]:=\left(v, v_{\mathfrak{o f}}\right), m_{1}\left(\sigma_{1}\right)\right) \text { by } \\
\rightarrow & \left(i d[n]:=v, m_{1}\left(\sigma_{1}\right)\right) \\
& \text { by rule EOflow-1 or EOflow- } 2 . \\
\rightarrow & \left(\text { skip, } m_{1}\left(\sigma_{1}\left(\sigma_{1}[v /(i d, n)]\right)\right)\right) \\
& \text { by rule Assign-A. }
\end{aligned}
$$$$
\rightarrow\left(i d[n]:=\left(v, v_{\mathfrak{o f}}\right), m_{1}\left(\sigma_{1}\right)\right) \text { by rule EEval' }
$$

Similarly, $s_{2}$ terminate. The theorem holds.

When the variable $i d$ is a global variable, by similar argument for the global variable, we can show that $s_{1}$ and $s_{2}$ terminate. Then the theorem holds.

- It is not the case that left value $i d$ is of type Int and the expression $e$ is of type Long only,

$\neg((\Gamma \vdash i d$ : Int $) \wedge(\Gamma \vdash e$ : Long $) \wedge \neg(\Gamma \vdash e$ : Int)).

There are two cases based on if there is crash in evaluation of expression $e$

- Both evaluations of expression lead to crash, $\mathcal{E} \llbracket e \rrbracket \sigma_{1}=\mathcal{E} \llbracket e \rrbracket \sigma_{2}=\left(\right.$ error,$\left.v_{\mathfrak{o f f}}\right)$.

By the same argument in case where left value $i d$ is of type Int and the expression $e$ is of type Long only, this theorem holds.

- Both evaluations of expression lead to no crash, $\mathcal{E} \llbracket e \rrbracket \sigma_{1}=\mathcal{E} \llbracket e \rrbracket \sigma_{2}=\left(v, v_{\text {of }}\right)$.

By the same argument in subcase of no value mismatch in case where left value $i d$ is of type Int and the expression $e$ is of type Long only, this theorem holds.

If array variable $i d$ is a global variable, by similar argument above, the theorem holds.

(c) $l v a l=i d_{1}\left[i d_{2}\right]$.

By definition, $\operatorname{Idx}\left(s_{1}\right)=\operatorname{Idx}\left(s_{2}\right)=\left\{i d_{2}\right\} \subseteq \operatorname{CVar}\left(s_{1}\right)=$ CVar $\left(s_{2}\right)$. By assumption, $\sigma_{1}\left(i d_{2}\right)=\sigma_{2}\left(i \bar{d}_{2}\right)=n$. By the same argument in the case where lval $=i d[n]$, the theorem holds.

3. $s_{1}=s_{2}=$ "input $i d "$,

By definition, $C \operatorname{Var}\left(s_{1}\right)=C \operatorname{Var}\left(s_{2}\right)=\left\{i d_{I}\right\}$. By assumption $\sigma_{1}\left(i d_{I}\right)=\sigma_{2}\left(i d_{I}\right)$. There are cases regarding if input sequence is empty or not.

(a) There is empty input sequence, $\sigma_{1}\left(i d_{I}\right)=\sigma_{2}\left(i d_{I}\right)=$

$\varnothing$.

Then the execution of $s_{1}$ continues as follows:

$\left(s_{1}, m_{1}\right)$

$=\left(\right.$ input $\left.i d, m_{1}\left(\sigma_{1}\right)\right)$

$\rightarrow\left(\right.$ input $\left.i d, m_{1}(1 / \mathfrak{f})\right)$ by rule In-7

$\stackrel{i}{\rightarrow}\left(\right.$ input $\left.i d, m_{1}(1 / \mathfrak{f})\right)$ by rule Crash.

Similarly, $s_{2}$ does not terminate. The theorem holds.

(b) There is nonempty input sequence, $\sigma_{1}\left(i d_{I}\right)=\sigma_{2}\left(i d_{I}\right) \neq$ $\varnothing$

There are cases regarding if type of the variable $i d$ is Long or not.

i. $i d$ is of type Long, $\Gamma \vdash i d$ : Long;

Assuming $i d$ is a local variable, then the execution of $s_{1}$ continues as follows:

$$
\begin{aligned}
& \left(s_{1}, m_{1}\right) \\
= & \left(\text { input } i d, m_{1}\left(\sigma_{1}\right)\right) \\
\rightarrow & \left(\operatorname{skip}, m_{1}\left(\sigma _ { 1 } \left[v_{i o} / i d, \operatorname{tl}\left(\sigma_{1}\left(i d_{I}\right)\right) / i d_{I},\right.\right.\right. \\
& \text { " } \left.\left.\left.\sigma_{1}\left(i d_{I O}\right) \cdot \underline{v}_{i o} " / i d_{I O}\right]\right)\right) \text { by rule In-3. }
\end{aligned}
$$

Similarly, $s_{2}$ terminates. The theorem holds.
When the variable $i d$ is a global variable, by similar argument, the theorem holds.

ii. $i d$ is of type Int or enumeration, $\Gamma \vdash i d$ : Int or enum $i d^{\prime}$;

There are cases regarding if the head of input sequence can be transformed to type of $i d$. Let $v_{i o}=$ $\operatorname{hd}\left(\sigma_{1}\left(i d_{I}\right)\right)$.

- $i d$ is of type Int.

If $v_{i o}$ is not of type Int, $\Gamma \vdash v_{i o}$ : Long and $\neg(\Gamma \vdash$ $v_{i o}$ : Int), then the execution of $s_{1}$ continues as follows:

$\left(s_{1}, m_{1}\right)$

$=\left(\right.$ input $\left.i d, m_{1}\left(\sigma_{1}\right)\right)$

$\rightarrow$ (input $\left.i d, m_{1}(1 / \mathfrak{f})\right)$ by Rule In- 4 .

$\stackrel{i}{\rightarrow}\left(\right.$ input $\left.i d, m_{1}(1 / \mathfrak{f})\right)$ by Rule crash.

Similarly, $s_{2}$ does not terminate. The theorem holds.

If $v_{i o}$ is of type Int, $\Gamma \vdash v_{i o}:$ Long and $\Gamma \vdash$ $v_{i o}$ : Int, assuming $i d$ is a local variable, then the execution of $s_{1}$ continues as follows:

$\left(s_{1}, m_{1}\right)$

$=\left(\right.$ input $\left.i d, m_{1}\left(\sigma_{1}\right)\right)$

$\rightarrow$ (skip, $m_{1}\left(\sigma_{1}\left[v_{i o} / i d, \operatorname{tl}\left(\sigma_{1}\left(i d_{I}\right)\right) / i d_{I}\right.\right.$,

" $\left.\left.\left.\sigma_{1}\left(i d_{I O}\right) \cdot \underline{v}_{i o} " / i d_{I O}\right]\right)\right)$ by Rule In-8.

Similarly, $s_{2}$ terminates. The theorem holds.

When $i d$ is a global variable, by similar argument, the theorem holds.

- If $i d$ is of type enum $i d^{\prime}=\left\{l_{1}, \ldots, l_{k}\right\}$.

If $\left(v_{i o}<1\right) \vee\left(v_{i o}>k\right)$, then the execution of $s_{1}$ continues as follows:

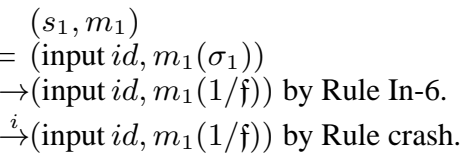

Similarly, $s_{2}$ does not terminate. The theorem holds. When $i d$ is a global variable, by similar argument, the theorem holds.

If $1 \leq v_{i o} \leq k$, assuming $i d$ is a local variable, then the execution of $s_{1}$ continues as follows:

$$
\begin{aligned}
& \left(s_{1}, m_{1}\right) \\
= & \left(\text { input } i d, m_{1}\left(\sigma_{1}\right)\right) \\
\rightarrow & \left(\operatorname{skip}, m_{1}\left(\sigma _ { 1 } \left[l_{v_{i o}} / i d, \operatorname{tl}\left(\sigma_{1}\left(i d_{I}\right)\right) / i d_{I},\right.\right.\right. \\
& \text { " } \left.\left.\left.\sigma_{1}\left(i d_{I O}\right) \cdot \underline{v}_{i o} " / i d_{I O}\right]\right)\right) \text { by Rule In-5. }
\end{aligned}
$$

Similarly, $s_{2}$ terminates. The theorem holds. When $i d$ is a global variable, by similar argument, the theorem holds.

(c) $s_{1}=s_{2}=$ "output $e$ ";

There are two cases based on if evaluation of expression $e$ crashes. By definition, CVar $\left(s_{1}\right)=\mathrm{CVar}\left(s_{2}\right)=$ $\operatorname{Err}(e)$. By assumption, $\forall x \in \operatorname{Err}(e), \sigma_{1}(x)=\sigma_{2}(x)$. By Lemma D.2 evaluation of the expression $e$ w.r.t two value stores $\sigma_{1}$ and $\sigma_{2}$ either both crash or both do not crash.

i. There is crash in evaluation of the expression $e$ w.r.t two value stores $\sigma_{1}$ and $\sigma_{2}, \mathcal{E} \llbracket e \rrbracket \sigma_{1}=\left(\right.$ error, $\left.v_{\mathfrak{o f}}^{1}\right)$ and $\mathcal{E} \llbracket e \rrbracket \sigma_{2}=$ (error, $v_{\mathfrak{o f}}^{2}$ )

The execution of $s_{1}$ continues as follows:

$$
\begin{aligned}
& \left(s_{1}, m_{1}\right) \\
= & \left(\text { output } e, m_{1}\left(\sigma_{1}\right)\right) \\
\rightarrow & \left(\text { output }\left(\text { error, } v_{\text {of }}^{1}\right), m_{1}(1 / \mathfrak{f})\right) \text { by Rule EEval' } \\
\rightarrow & \left(\text { output } 0, m_{1}(1 / \mathfrak{f})\right) \text { by Rule ECrash. } \\
& \stackrel{i}{\rightarrow}\left(\text { output } 0, m_{1}(1 / \mathfrak{f})\right) \text { by Rule crash. }
\end{aligned}
$$


Similarly, $s_{2}$ does not terminate. The theorem holds.

ii. There is no crash in evaluation of the expression $e$ w.r.t two value stores $\sigma_{1}$ and $\sigma_{2}, \mathcal{E} \llbracket e \rrbracket \sigma_{1}=\left(v_{1}, v_{\text {of }}^{1}\right)$ and $\mathcal{E} \llbracket e \rrbracket \sigma_{2}=\left(v_{2}, v_{\mathfrak{o f}}^{2}\right)$.

According to rule Out-1 and Out-2, there is no exception in transformation of different typed output value. We therefore only show the execution for output value of Int type. The execution of $s_{1}$ continues as follows:

$$
\begin{aligned}
& \left(s_{1}, m_{1}\right) \\
= & \left(\text { output } e, m_{1}\left(\sigma_{1}\right)\right) \\
\rightarrow & \left(\text { output }\left(v_{1}, v_{\mathfrak{o f}}^{1}\right), m_{1}(1 / \mathfrak{f})\right) \text { by Rule EEval' } \\
\rightarrow & \left(\text { output } v_{1}, m_{1}\left(v_{\mathfrak{o f}}^{1} / \mathfrak{o f}\right)\right) \\
& \text { by Rule EOflow-1 or EOflow- } 2 . \\
\rightarrow & \left(\text { skip, } m_{1}\left(\sigma_{1}\left[{ }^{\prime} \sigma\left(i d_{I O}\right) \cdot \bar{v}_{1} " / i d_{I O}\right]\right)\right) \\
& \text { by Rule Out-1. }
\end{aligned}
$$

Similarly, $s_{2}$ terminates. Theorem holds.

Second $s_{1}$ and $s_{2}$ are input statement with same type variable: $s_{1}=$ "input $i d_{1}$ ", $s_{2}=$ "input $i d_{2}$ " where $\left(\Gamma_{s_{1}} \vdash i d_{1}\right.$ : $t) \wedge\left(\Gamma_{s_{2}} \vdash i d_{2}: t\right)$;

The theorem holds by similar argument for the case $s_{1}=s_{2}=$ input $i d$.

Third $s_{1}=$ "output $e$ " or " $i d_{1}:=e$ ", $s_{2}=$ "output $e$ " or " $i d_{2}:=$ $e$ " where both of the following hold:

- There is no possible value mismatch in " $i d_{1}:=e$ ", $\neg\left(\Gamma_{s_{1}} \vdash i d_{1}\right.$ : Int $) \vee \neg\left(\Gamma_{s_{1}} \vdash e\right.$ : Long $) \vee\left(\Gamma_{s_{1}} \vdash e\right.$ : Int $)$.

- There is no possible value mismatch in " $i d_{2}:=e$ ", $\neg\left(\Gamma_{s_{2}} \vdash i d_{2}\right.$ : Int $) \vee \neg\left(\Gamma_{s_{2}} \vdash e\right.$ : Long $) \vee\left(\Gamma_{s_{2}} \vdash e\right.$ : Int $)$.

We show that the evaluations of the expression $e$ w.r.t the value stores $\sigma_{1}$ and $\sigma_{2}$ either both raise an exception or both do not. By the definition of crash variables, the crash variables of $s_{1}$ are those obtained by the function $\operatorname{Err}(e), C \operatorname{Var}\left(s_{1}\right)=\operatorname{Err}(e)$. Similarly, the termination deciding variables of $s_{2}$ are $\operatorname{Err}(e)$. By assumption, the initial value stores $\sigma_{1}$ and $\sigma_{2}$ agree on values of those in $C \operatorname{Var}\left(s_{1}\right)$ and $C \operatorname{Var}\left(s_{2}\right), \forall x \in \operatorname{Err}(e)=$ $\left(\mathrm{C} \operatorname{Var}\left(s_{1}\right) \cup \mathrm{C} \operatorname{Var}\left(s_{2}\right)\right): \sigma_{1}(x)=\sigma_{2}(x)$. By LemmaD.2 the evaluations of expression $e$ w.r.t two value stores, $\sigma_{1}$ and $\sigma_{2}$, either both raise an exception or both do not raise an exception.

1. The evaluations of the expression $e$ raise an exception w.r.t two value stores $\sigma_{1}$ and $\sigma_{2}, \mathcal{E}^{\prime} \llbracket e \rrbracket \sigma_{1}=\left(\right.$ error, $\left.v_{\mathfrak{o f}}^{1}\right), \mathcal{E}^{\prime} \llbracket e \rrbracket \sigma_{2}=$ (error, $v_{\text {of }}^{2}$ ):

We show the execution of $s_{1}$ proceeds to an configuration where the crash flag is set and then does not terminate.

When $s_{1}=$ "output $e$ ", the execution of "output $e$ " proceeds as follows.

$$
\text { (output } e, m_{1}\left(\sigma_{1}\right) \text { ) }
$$

$\rightarrow\left(\right.$ output (error, $\left.\left.v_{\mathfrak{o f}}^{1}\right), m_{1}\left(\sigma_{1}\right)\right)$ by rule EEval'

$\rightarrow$ (output $\left.0, m_{1}(1 / \mathfrak{f})\right)$ by rule ECrash

$\stackrel{i}{\rightarrow}\left(\right.$ output $\left.0, m_{1}(1 / \mathfrak{f})\right)$ for any $i \geq 0$, by rule Crash.

When $s_{1}=$ " $i d_{1}:=e$ ", the execution of " $i d_{1}:=e$ " proceeds as follows.

$$
\begin{aligned}
\left(i d_{1}:=e, m_{1}\left(\sigma_{1}\right)\right) \\
\rightarrow\left(i d_{1}:=\left(\text { error, } v_{\mathfrak{o f}}^{1}\right), m_{1}\left(\sigma_{1}\right)\right) \text { by rule EEval } \\
\rightarrow\left(i d_{1}:=0, m_{1}(1 / \mathfrak{f})\right) \text { by rule ECrash } \\
\stackrel{i}{\rightarrow}\left(i d_{1}:=0, m_{1}(1 / \mathfrak{f})\right) \text { for any } i \geq 0, \text { by rule Crash. }
\end{aligned}
$$

Similarly, the execution of $s_{2}$ proceeds to a configuration where the crash flag is set. Then, by the crash rule, the execution of $s_{2}$ does not terminate. The theorem 3 holds.

2. the evaluations of expression $e$ do not raise an exception w.r.t two value stores, $\sigma_{1}$ and $\sigma_{2}, \mathcal{E}^{\prime} \llbracket e \rrbracket \sigma_{1}=\left(v_{1}, v_{\mathfrak{o f}}^{1}\right), \mathcal{E}^{\prime} \llbracket e \rrbracket \sigma_{2}=$ $\left(v_{2}, v_{\mathfrak{o f}}^{2}\right)$ :

We show the execution of $s_{1}$ terminates.
When $s_{1}=$ output $(e)$, the execution of output $(e)$ proceeds as follows. W.l.o.g, we assume expression $e$ is of type Int. This is allowed by the condition that it does not hold that $\left(\Gamma_{s_{1}} \vdash e\right.$ : Long $) \wedge \neg\left(\Gamma_{s_{1}} \vdash e:\right.$ Int $)$. (output $e, m_{1}\left(\sigma_{1}\right)$ )

$\rightarrow$ (output $\left.\left(v_{1}, v_{\mathfrak{o f}}^{1}\right), m_{1}\left(\sigma_{1}\right)\right)$ by rule EEval'

$\rightarrow\left(\right.$ output $\left.v_{1}, m_{1}\left(v_{\mathfrak{o f}}^{1} / \mathfrak{o f}, \sigma_{1}\right)\right)$

by rule E-Oflow 1 or E-Oflow2

$\rightarrow\left(\right.$ skip, $\left.m_{1}\left(\sigma_{1}\left[" \sigma_{1}\left(i d_{I O}\right) \cdot \overline{v_{1}} " / i d_{I O}\right]\right)\right)$ by rule Out.

When $s_{1}=$ " $i d_{1}:=e$ ", by assumption, the expression $e$ is of type Int, there is no possible value mismatch in execution of " $i d_{1}:=e$ " because the only possible value mismatch occurs when assigning a value of type Long but not Int to a variable of type Int. By the condition $\neg\left(\Gamma_{s_{1}} \vdash i d_{1}\right.$ : Int $) \vee \neg\left(\Gamma_{s_{1}} \vdash e\right.$ : Long $) \vee\left(\Gamma_{s_{2}} \vdash e\right.$ : Int $)$, when expression $e$ is of type Long, then the variable $i d_{1}$ is not of type Int. In summary, there is no value mismatch.

The execution of " $i d_{1}:=e$ " proceeds as follows.

$$
\left(i d_{1}:=e, m_{1}\left(\sigma_{1}\right)\right)
$$

$\rightarrow\left(i d_{1}:=\left(v_{1}, v_{\mathfrak{o f}}^{1}\right), m_{1}\left(\sigma_{1}\right)\right)$ by rule EEval'

$\rightarrow\left(i d_{1}:=v_{1}, m_{1}\left(v_{\mathfrak{o f}}^{1} / \mathfrak{o f}, \sigma_{1}\right)\right)$ by rule EEval'

$\rightarrow$ (skip, $\left.m_{1}\left(\sigma_{1}\left[v_{1} / i d_{1}\right]\right)\right)$ by the rule Assign.

When $i d_{1}$ is a variable of enumeration or Long type, by similar argument, the theorem still holds.

Similarly, the execution of $s_{2}$ terminates when started in the state $m_{2}\left(\sigma_{2}\right)$. Theorem 3 holds.

Theorem 4. If two statement sequences $S_{1}$ and $S_{2}$ satisfy the proof rule of termination in the same way, $S_{1} \equiv_{H}^{S} S_{2}$, and their respective initial states $m_{1}\left(\mathfrak{f}_{1}, \sigma_{1}\right)$ and $m_{2}\left(\mathfrak{f}_{2}, \sigma_{2}\right)$ with crash flags not set, $\mathfrak{f}_{1}=\mathfrak{f}_{2}=0$, and whose value stores agree on values of the termination deciding variables of $S_{1}$ and $S_{2}, \forall x \in \operatorname{TVar}\left(S_{1}\right) \cup$ $T \operatorname{Var}\left(S_{2}\right): \sigma_{1}(x)=\sigma_{2}(x)$, then $S_{1}$ and $S_{2}$ terminate in the same way when started in states $m_{1}$ and $m_{2}$ respectively: $\left(S_{1}, m_{1}\right) \equiv_{H}$ $\left(S_{2}, m_{2}\right)$.

Proof. The proof is by induction on $\operatorname{size}\left(S_{1}\right)+\operatorname{size}\left(S_{2}\right)$, the sum of program size of $S_{1}$ and $S_{2}$.

Base case. $S_{1}$ and $S_{2}$ are simple statement. By Theorem 3 , Theorem 4 holds.

Induction step.

There are two hypotheses. The hypothesis $\mathrm{IH}$ is that Theorem 4 holds when $\operatorname{size}\left(S_{1}\right)+\operatorname{size}\left(S_{2}\right)=k \geq 2$.

We show Theorem 4 holds when $\operatorname{size}\left(S_{1}\right)+\operatorname{size}\left(S_{2}\right)=k+1$.

The proof of Theorem 4 is a case analysis according to the cases in the definition of the proof rule of termination in the same way, $S_{1} \equiv{ }_{H}^{S} S_{2}$.

1. $S_{1}$ and $S_{2}$ are one statement and one of the following holds.

(a) $S_{1}=$ "If $(e)$ then $\left\{S_{1}^{t}\right\}$ else $\left\{S_{1}^{f}\right\}$ ", $S_{2}=$ "If $(e)$ then $\left\{S_{2}^{t}\right\}$ else $\left\{S_{2}^{f}\right\}$ " such that one of the following holds:

i. $S_{1}^{t}, S_{1}^{f}, S_{2}^{t}, S_{2}^{f}$ are all sequences of "skip";

We show that the evaluation of expression $e$ w.r.t the value store $\sigma_{1}$ and $\sigma_{2}$ either both raise an exception or both do not. By the definition of crash/loop variables, $\mathrm{C} \operatorname{Var}\left(S_{1}^{t}\right)=\mathrm{C} \operatorname{Var}\left(S_{1}^{f}\right)=\emptyset, \operatorname{LV} \operatorname{Var}\left(S_{1}\right)=\emptyset$. By the definition of termination deciding variables, the termination deciding variables of $S_{1}$ is the crash variables of $S_{1}, \mathrm{~T} \operatorname{Var}\left(S_{1}\right)=\mathrm{C} \operatorname{Var}\left(S_{1}\right)=\operatorname{Err}(e)$. By assumption, the value stores $\sigma_{1}$ and $\sigma_{2}$ agree on the values of those in the crash variables of $S_{1}$ and $S_{2}, \forall x \in$ $\operatorname{Err}(e)=\mathrm{T} \operatorname{Var}\left(S_{1}\right)=\mathrm{T} \operatorname{Var}\left(S_{2}\right), \sigma_{1}(x)=\sigma_{2}(x)$. Вy 
the property of the expression meaning function $\mathcal{E}$, the evaluation of predicate expression $e$ of $S_{1}$ and $S_{2}$ w.r.t value store $\sigma_{1}$ and $\sigma_{2}$ either both crash or both do not crash, $\left(\mathcal{E} \llbracket e \rrbracket \sigma_{1}=\mathcal{E} \llbracket e \rrbracket \sigma_{2}=\right.$ error $) \vee\left(\left(\mathcal{E} \llbracket e \rrbracket \sigma_{1} \neq\right.\right.$ error) $\wedge\left(\mathcal{E} \llbracket e \rrbracket \sigma_{2} \neq\right.$ error $\left.)\right)$. Then we show that Theorem 4 holds in either of the two possibilities.

A. $\mathcal{E} \llbracket e \rrbracket \sigma_{1}=\mathcal{E} \llbracket e \rrbracket \sigma_{2}=$ error.

The execution of $S_{1}$ proceeds as follows:

(If $(e)$ then $\left\{S_{1}^{t}\right\}$ else $\left.\left\{S_{1}^{f}\right\}, m_{1}\left(\sigma_{1}\right)\right)$

$\rightarrow$ (If(error) then $\left\{S_{1}^{t}\right\}$ else $\left.\left\{S_{1}^{f}\right\}, m_{1}\left(\sigma_{1}\right)\right)$ by rule EEval

$\rightarrow\left(\operatorname{If}(0)\right.$ then $\left\{S_{1}^{t}\right\}$ else $\left.\left\{S_{1}^{f}\right\}, m_{1}\left(1 / \mathfrak{f}, \sigma_{1}\right)\right)$ by rule ECrash

$\stackrel{i}{\rightarrow}\left(\operatorname{If}(0)\right.$ then $\left\{S_{1}^{t}\right\}$ else $\left.\left\{S_{1}^{f}\right\}, m_{1}\left(1 / \mathfrak{f}, \sigma_{1}\right)\right)$ for any $i \geq 0$, by rule Crash.

Similarly, the execution of $S_{2}$ started in the state $m_{2}\left(\sigma_{2}\right)$ does not terminate. The theorem 4 holds.

B. $\left(\mathcal{E} \llbracket e \rrbracket \sigma_{1} \neq\right.$ error $) \wedge\left(\mathcal{E} \llbracket e \rrbracket \sigma_{2} \neq\right.$ error $)$.

W.1.o.g, $\mathcal{E} \llbracket e \rrbracket \sigma_{1}=v_{1} \neq 0, \mathcal{E} \llbracket e \rrbracket \sigma_{2}=0$. Then the execution of $S_{1}$ proceeds as follows.

(If $(e)$ then $\left\{S_{1}^{t}\right\}$ else $\left.\left\{S_{1}^{f}\right\}, m_{1}\left(\sigma_{1}\right)\right)$

$\rightarrow\left(\operatorname{If}\left(v_{1}\right)\right.$ then $\left\{S_{1}^{t}\right\}$ else $\left.\left\{S_{1}^{f}\right\}, m_{1}\left(\sigma_{1}\right)\right)$ by rule EEval

$\rightarrow\left(S_{1}^{t}, m_{1}\left(\sigma_{1}\right)\right)$ by rule If-T

$\stackrel{*}{\rightarrow}$ (skip, $\left.m_{1}^{\prime}\right)$ by rule Skip.

Similarly, the execution of $S_{2}$ started in the state $m_{2}\left(\sigma_{2}\right)$ terminates. The theorem 4 holds.

ii. At least one of $S_{1}^{t}, S_{1}^{f}, S_{2}^{t}, S_{2}^{f}$ is not a sequence of "skip" and $\left(S_{1}^{t} \equiv_{H}^{S} S_{2}^{t}\right) \wedge\left(S_{1}^{f} \equiv_{H}^{S} S_{2}^{f}\right)$;

W.l.o.g., $S_{1}^{t}$ is not of "skip" only. We show that the evaluation of the expression $e$ w.r.t the value stores $\sigma_{1}$ and $\sigma_{2}$ either both raise an exception or both produce the same integer value. Then there is either some loop statement in $S_{1}^{t}$ or the crash variables of $S_{1}^{t}$ are not $\emptyset$ or both.

A. When there is some loop statement in $S_{1}^{t}$, then, by the definition of loop variables, the loop variables of $S_{1}$ include all variables used in the predicate expression of $S_{1}, \operatorname{LVar}\left(S_{1}\right)=\mathrm{Use}(e) \cup \operatorname{LVar}\left(S_{1}^{t}\right) \cup$ $\operatorname{LVar}\left(S_{1}^{f}\right)$.

B. When the crash variables of $S_{1}^{t}$ are not $\emptyset$, then, by the definition of crash variables, the crash variables of $S_{1}$ include all variables used in the predicate expression of $S_{1}, \mathrm{CVar}\left(S_{1}\right)=\mathrm{Use}(e) \cup \mathrm{CVar}\left(S_{1}^{t}\right) \cup$ $\mathrm{C} \operatorname{Var}\left(S_{1}^{f}\right)$.

In summary, all variables used in predicate expression of $S_{1}$ is a subset of termination deciding variables of $S_{1}$, Use $(e) \subseteq \operatorname{TVar}\left(S_{1}\right)$. By assumption, the value store $\sigma_{1}$ and $\sigma_{2}$ agree on the values of those in the termination deciding variables of $S_{1}$ and $S_{2}$. It follows, by the property of expression meaning function $\mathcal{E}$, the evaluation of the predicate expression $e$ of $S_{1}$ and $S_{2}$ produce the same value w.r.t the value store $\sigma_{1}$ and $\sigma_{2}$, $\mathcal{E} \llbracket e \rrbracket \sigma_{1}=\mathcal{E} \llbracket e \rrbracket \sigma_{2}$. Then either the evaluations of the predicate expression $e$ of $S_{1}$ and $S_{2}$ both crash w.r.t the value store $\sigma_{1}$ and $\sigma_{2}$, or both evaluations produce the same integer value, $\left(\mathcal{E} \llbracket e \rrbracket \sigma_{1}=\mathcal{E} \llbracket e \rrbracket \sigma_{2}=\right.$ error $) \vee$ $\left(\mathcal{E} \llbracket e \rrbracket \sigma_{1}=\mathcal{E} \llbracket e \rrbracket \sigma_{2}=v \neq\right.$ error $)$. We show Theorem 4 holds in either of the two possibilities.

A. $\mathcal{E} \llbracket e \rrbracket \sigma_{1}=\mathcal{E} \llbracket e \rrbracket \sigma_{2}=$ error.

The execution of $S_{1}$ proceeds as follows:

(If $(e)$ then $\left\{S_{1}^{t}\right\}$ else $\left.\left\{S_{1}^{f}\right\}, m_{1}\left(\sigma_{1}\right)\right)$

$\rightarrow\left(\right.$ If(error) then $\left\{S_{1}^{t}\right\}$ else $\left.\left\{S_{1}^{f}\right\}, m_{1}\left(\sigma_{1}\right)\right)$ by rule EEval

$\rightarrow\left(\operatorname{If}(0)\right.$ then $\left\{S_{1}^{t}\right\}$ else $\left.\left\{S_{1}^{f}\right\}, m_{1}\left(1 / \mathfrak{f}, \sigma_{1}\right)\right)$ by rule ECrash
$\stackrel{i}{\rightarrow}\left(\operatorname{If}(0)\right.$ then $\left\{S_{1}^{t}\right\}$ else $\left.\left\{S_{1}^{f}\right\}, m_{1}\left(1 / \mathfrak{f}, \sigma_{1}\right)\right)$ for any $i \geq 0$, by rule Crash.

Similarly, the execution of $S_{2}$ started from state $m_{2}\left(\sigma_{2}\right)$ does not terminate. The theorem 4 holds.

B. $\mathcal{E} \llbracket e \rrbracket \sigma_{1}=\mathcal{E} \llbracket e \rrbracket \sigma_{2}=v \neq$ error, w.l.o.g., $v=0$.

Then the execution of $S_{1}$ proceeds as follows:

(If $(e)$ then $\left\{S_{1}^{t}\right\}$ else $\left.\left\{S_{1}^{f}\right\}, m_{1}\left(\sigma_{1}\right)\right)$

$\rightarrow\left(\operatorname{If}(0)\right.$ then $\left\{S_{1}^{t}\right\}$ else $\left.\left\{S_{1}^{f}\right\}, m_{1}\left(\sigma_{1}\right)\right)$ by rule EEval

$\rightarrow\left(S_{1}^{f}, m_{1}\left(\sigma_{1}\right)\right)$ by rule If-F.

Similarly, after two steps of execution, $S_{2}$ gets to the configuration $\left(S_{2}^{f}, m_{2}\left(\sigma_{2}\right)\right)$.

We show that $S_{1}^{f}$ and $S_{2}^{f}$ terminate in the same way when started in the state $m_{1}\left(\sigma_{1}\right)$ and $m_{2}\left(\sigma_{2}\right)$ respectively. Because $S_{1}^{f} \equiv_{H}^{S} S_{2}^{f}$, by Corollary 5.1 the termination deciding variables of $S_{1}^{f}$ and $S_{2}^{f}$ are same, $\operatorname{TVar}\left(S_{1}^{f}\right)=\operatorname{TVar}\left(S_{2}^{f}\right)$. By the definition of crash/loop variables, $\mathrm{CVar}\left(S_{1}^{f}\right) \subseteq \mathrm{CVar}\left(S_{1}\right)$ and $\operatorname{LVar}\left(S_{1}^{f}\right) \subseteq \operatorname{LVar}\left(S_{1}\right)$. Hence, the termination deciding variables of $S_{1}^{f}$ are a subset of the termination deciding variables of $S_{1}, \operatorname{TV} \operatorname{Var}\left(S_{1}^{f}\right) \subseteq$ $\mathrm{T} \operatorname{Var}\left(S_{1}\right)$. Similarly, $\mathrm{T} \operatorname{Var}\left(S_{2}^{f}\right) \subseteq \mathrm{T} \operatorname{Var}\left(S_{2}\right)$. Therefore, the value store $\sigma_{1}$ and $\sigma_{2}$ agree on the values of those in the termination deciding variables of $S_{1}^{f}$ and $S_{2}^{f}, \forall y \in \operatorname{TVar}\left(S_{1}^{f}\right) \cup \operatorname{TV} \operatorname{Var}\left(S_{2}^{f}\right)$ : $\sigma_{1}(y)=\sigma_{2}(y)$. In addition, the sum of program size of $S_{1}^{f}$ and $S_{2}^{f}$ is less than $k$ because program size of each of $S_{1}^{t}$ and $S_{2}^{t}$ is great than or equal to one, $\operatorname{size}\left(S_{1}^{f}\right)+\operatorname{size}\left(S_{2}^{f}\right)<k$. As is shown, crash flags are not set. Therefore, by the hypothesis IH, $S_{1}^{f}$ and $S_{2}^{f}$ terminate in the same way when started in state $m_{1}\left(\mathfrak{f}_{1}, \sigma_{1}\right)$ and $m_{2}\left(\mathfrak{f}_{2}, \sigma_{2}\right),\left(S_{1}^{f}, m_{1}\left(\mathfrak{f}_{1}, \sigma_{1}\right)\right) \equiv_{H}$ $\left(S_{2}^{f}, m_{2}\left(\mathfrak{f}_{2}, \sigma_{2}\right)\right)$. Hence, Theorem 4 holds.

(b) $S_{1}=$ "while $\left\langle n_{1}\right\rangle(e)\left\{S_{1}^{\prime \prime}\right\} ", S_{2}=$ "while ${ }_{\left\langle n_{2}\right\rangle}(e)\left\{S_{2}^{\prime \prime}\right\} "$ such that both of the following hold:

- $S_{1}^{\prime \prime} \equiv{ }_{H}^{S} S_{2}^{\prime \prime}$;

- $S_{1}^{\prime \prime}$ and $S_{2}^{\prime \prime}$ have equivalent computation of $\operatorname{TV} \operatorname{Var}\left(S_{1}\right) \cup$ $\mathrm{T} \operatorname{Var}\left(S_{2}\right)$;

By Corollary 5.3, we show $S_{1}$ and $S_{2}$ terminate in the same way when started from state $m_{1}\left(\mathfrak{f}_{1}, m_{c}^{1}, \sigma_{1}\right)$ and $m_{2}\left(\mathfrak{f}_{2}, m_{c}^{2}, \sigma_{2}\right)$ respectively. We need to show that all required conditions are satisfied.

- The crash flags are not set, $\mathfrak{f}_{1}=\mathfrak{f}_{2}=0$.

- The loop counter value of $S_{1}$ and $S_{2}$ are zero: $m_{c}^{1}\left(n_{1}\right)=$ $m_{c}^{2}\left(n_{2}\right)=0$.

- The value stores $\sigma_{1}$ and $\sigma_{2}$ agree on the values of those in the termination deciding variables of $S_{1}$ and $S_{2}, \forall x \in$ $\mathrm{T} \operatorname{Var}\left(S_{1}\right) \cap \operatorname{TV} \operatorname{Var}\left(S_{2}\right): \sigma_{1}(x)=\sigma_{2}(x)$.

The three above conditions are from assumption.

- $S_{1}$ and $S_{2}$ have same set of termination deciding variables, $\operatorname{TVar}\left(S_{1}\right)=\mathrm{T} \operatorname{Var}\left(S_{2}\right)$.

By Corollary 5.1

- The loop body $S_{1}^{\prime \prime}$ of $S_{1}$ and $S_{2}^{\prime \prime}$ of $S_{2}$ terminate in the same way when started in state $m_{S_{1}}\left(\mathfrak{f}_{S_{1}}, \sigma_{S_{1}}\right)$ and $m_{S_{2}}\left(\mathfrak{f}_{S_{2}}, \sigma_{S_{2}}\right)$ with crash flags not set and in which value stores agree on the values of those in the termination deciding variables of $S_{1}^{\prime \prime}$ and $S_{2}^{\prime \prime}:((\forall x \in$ $\left.\mathrm{T} \operatorname{Var}\left(S_{1}^{\prime \prime}\right) \cup \operatorname{TVar}\left(S_{2}^{\prime \prime}\right): \sigma_{S_{1}}(x)=\sigma_{S_{2}}(x)\right) \wedge\left(\mathfrak{f}_{S_{1}}=\right.$ $\left.\left.\mathfrak{f}_{S_{2}}=0\right)\right) \Rightarrow\left(S_{1}^{\prime \prime}, m_{S_{1}}\left(\mathfrak{f}_{S_{1}}, \sigma_{S_{1}}\right)\right) \equiv_{H}\left(S_{2}^{\prime \prime}, m_{S_{2}}\left(\mathfrak{f}_{S_{2}}, \sigma_{S_{2}}\right)\right)$. By the definition of program size, $\operatorname{size}\left(S_{1}\right)=\operatorname{size}\left(S_{1}^{\prime \prime}\right)+$ $1, \operatorname{size}\left(S_{2}\right)=\operatorname{size}\left(S_{2}^{\prime \prime}\right)+1$. Then, $\operatorname{size}\left(S_{1}^{\prime \prime}\right)+\operatorname{size}\left(S_{2}^{\prime \prime}\right)<$ 
$k$. Then, by the hypothesis IH, the loop body $S_{1}^{\prime \prime}$ of $S_{1}$ and $S_{2}^{\prime \prime}$ of $S_{2}$ terminate in the same way when started in state $m_{S_{1}}\left(\sigma_{S_{1}}\right)$ and $m_{S_{2}}\left(\sigma_{S_{2}}\right)$ with crash flags not set and whose value stores agree on values of the termination deciding variables of $S_{1}^{\prime \prime}$ and $S_{2}^{\prime \prime}$.

Then, by Corollary 5.3. $S_{1}$ and $S_{2}$ terminate in the same way when started in the states $m_{1}\left(m_{c}^{1}, \sigma_{1}\right)$ and $m_{2}\left(m_{c}^{2}, \sigma_{2}\right)$ respectively. The theorem 4 holds.

2. $S_{1}$ and $S_{2}$ are not both one statement and one of the following holds:

(a) $S_{1}=S_{1}^{\prime} ; s_{1}, S_{2}=S_{2}^{\prime} ; s_{2}$ and all of the following hold:

- $S_{1}^{\prime} \equiv{ }_{H}^{S} S_{2}^{\prime}$;

- $S_{1}^{\prime}$ and $S_{2}^{\prime}$ have equivalent computation of $\operatorname{TVar}\left(s_{1}\right) \cup$ $\mathrm{T} \operatorname{Var}\left(s_{2}\right)$;

- $s_{1} \equiv_{H}^{S} s_{2}$ where $s_{1}$ and $s_{2}$ are not sequences of "skip";

By the hypothesis IH, we show that $S_{1}^{\prime}$ and $S_{2}^{\prime}$ terminate in the same way when started in the states $m_{1}\left(\mathfrak{f}_{1}, \sigma_{1}\right), m_{2}\left(\mathfrak{f}_{2}, \sigma_{2}\right)$ respectively, $\left(S_{1}^{\prime}, m_{1}\left(\mathfrak{f}_{1}, \sigma_{1}\right)\right) \equiv_{H}\left(S_{2}^{\prime}, m_{2}\left(\mathfrak{f}_{2}, \sigma_{2}\right)\right)$. We need to show all required conditions are satisfied.

- Crash flags are not set, $\mathfrak{f}_{1}=\mathfrak{f}_{2}=0$;

By assumption.

- $\operatorname{size}\left(S_{1}^{\prime}\right)+\operatorname{size}\left(S_{2}^{\prime}\right)<k$.

By the definition, size $\left(s_{1}\right) \geq 1, \operatorname{size}\left(s_{2}\right) \geq 1$. Hence $\operatorname{size}\left(S_{1}^{\prime}\right)+\operatorname{size}\left(S_{2}^{\prime}\right)<k$.

- Value stores $\sigma_{1}$ and $\sigma_{2}$ agree on values of the termination deciding variables of $S_{1}^{\prime}$ and $S_{2}^{\prime}$.

Besides, by the definition of loop/crash variables, $\operatorname{LVar}\left(S_{1}^{\prime}\right) \subseteq$ $\operatorname{LVar}\left(S_{1}\right)$ and $\mathrm{C} \operatorname{Var}\left(S_{1}^{\prime}\right) \subseteq \mathrm{C} \operatorname{Var}\left(S_{1}\right)$. Hence, $\mathrm{T} \operatorname{Var}\left(S_{1}^{\prime}\right) \subseteq$ $\mathrm{T} \operatorname{Var}\left(S_{1}\right)$. Similarly, $\operatorname{TV} \operatorname{Var}\left(S_{2}^{\prime}\right) \subseteq \mathrm{T} \operatorname{Var}\left(S_{2}\right)$. Then, value stores $\sigma_{1}$ and $\sigma_{2}$ agree on the values of those in the termination deciding variables of $S_{1}^{\prime}$ and $S_{2}^{\prime}$, $\forall x \in \mathrm{T} \operatorname{Var}\left(S_{1}^{\prime}\right) \cup \operatorname{TV} \operatorname{Var}\left(S_{2}^{\prime}\right): \sigma_{1}(x)=\sigma_{2}(x)$.

Then, by the hypothesis IH, $S_{1}^{\prime}$ and $S_{2}^{\prime}$ terminate in the same way when started in the states $m_{1}\left(\mathfrak{f}_{1}, \sigma_{1}\right), m_{2}\left(\mathfrak{f}_{2}, \sigma_{2}\right)$ respectively, $\left(S_{1}^{\prime}, m_{1}\left(\mathfrak{f}_{1}, \sigma_{1}\right)\right) \equiv_{H}\left(S_{2}^{\prime}, m_{2}\left(\mathfrak{f}_{2}, \sigma_{2}\right)\right)$.

If the execution of $S_{1}^{\prime}$ and $S_{2}^{\prime}$ terminate when started in the states $m_{1}\left(\mathfrak{f}_{1}, \sigma_{1}\right)$ and $m_{2}\left(\mathfrak{f}_{2}, \sigma_{2}\right)$ respectively, we show that $s_{1}$ and $s_{2}$ terminate in the same way. We prove that $S_{1}^{\prime}$ and $S_{2}^{\prime}$ equivalently compute the termination deciding variables of $s_{1}$ and $s_{2}$ by Theorem 2

- Crash flags are not set, $\mathfrak{f}_{1}=\mathfrak{f}_{2}=0$;

By definition of terminating execution of $S_{1}^{\prime}$ and $S_{2}^{\prime}$ when started in states $m_{1}$ and $m_{2}$ respectively.

- The executions of $S_{1}^{\prime}$ and $S_{2}^{\prime}$ terminate when started in the states $m_{1}\left(\sigma_{1}\right)$ and $m_{2}\left(\sigma_{2}\right)$.

By assumption, $\left(S_{1}^{\prime}, m_{1}\left(\sigma_{1}\right)\right) \stackrel{*}{\rightarrow}$ (skip, $\left.m_{1}^{\prime}\left(\sigma_{1}^{\prime}\right)\right)$ and $\left(S_{2}^{\prime}, m_{2}\left(\sigma_{2}\right)\right) \stackrel{*}{\rightarrow}\left(\right.$ skip, $\left.m_{2}^{\prime}\left(\sigma_{2}^{\prime}\right)\right)$.

- $s_{1}$ and $s_{2}$ have same termination deciding variables. By Corollary 5.1 $s_{1}$ and $s_{2}$ have same termination deciding variables, $\mathrm{T} \operatorname{Var}\left(s_{1}\right)=\mathrm{T} \operatorname{Var}\left(s_{2}\right)=\mathrm{T} \operatorname{Var}(s)$.

- Value stores $\sigma_{1}$ and $\sigma_{2}$ agree on the values of variables in $\operatorname{Imp}\left(S_{1}^{\prime}, \mathrm{T} \operatorname{Var}(s)\right) \cup \operatorname{Imp}\left(S_{2}^{\prime}, \mathrm{T} \operatorname{Var}(s)\right)$.

By the definition of loop/crash variables, $\operatorname{Imp}\left(S_{1}^{\prime}, \operatorname{LVar}\left(s_{1}\right)\right) \subseteq$ $\operatorname{LVar}\left(S_{1}\right)$ and $\operatorname{Imp}\left(S_{1}^{\prime}, \mathrm{CVar}\left(s_{1}\right)\right) \subseteq \mathrm{CVar}\left(S_{1}\right)$. Hence, by Lemma C.2 the imported variables in $S_{1}^{\prime}$ relative to the termination deciding variables of $s_{1}$ is a subset of the termination deciding variables of $S_{1}, \operatorname{Imp}\left(S_{1}^{\prime}, \operatorname{TVar}(s)\right)$ $\subseteq \mathrm{T} \operatorname{Var}\left(S_{1}\right)$. Similarly, $\operatorname{Imp}\left(S_{2}^{\prime}, \mathrm{T} \operatorname{Var}(s)\right) \subseteq \mathrm{TVar}\left(S_{2}\right)$. Thus, by assumption, the value stores $\sigma_{1}$ and $\sigma_{2}$ agree on the values of the variables $\operatorname{in} \operatorname{Imp}\left(S_{1}^{\prime}, \operatorname{TVar}(s)\right) \cup$ $\operatorname{Imp}\left(S_{2}^{\prime}, \operatorname{TVar}(s)\right)$.

By Theorem $2 \forall x \in \operatorname{TV} \operatorname{Var}(s): \sigma_{1}^{\prime}(x)=\sigma_{2}^{\prime}(x)$.
By Corollary E.1. $\left(S_{1}^{\prime} ; s_{1}, m_{1}\left(\sigma_{1}\right)\right) \stackrel{*}{\rightarrow}\left(s_{1}, m_{1}^{\prime}\left(\mathfrak{f}_{1}, \sigma_{1}^{\prime}\right)\right)$ and $\left(S_{2}^{\prime} ; s_{2}, m_{2}\left(\sigma_{2}\right)\right) \stackrel{*}{\rightarrow}\left(s_{2}, m_{2}^{\prime}\left(\mathfrak{f}_{2}, \sigma_{2}^{\prime}\right)\right)$. Then, by the hypothesis IH, we show that $s_{1}$ and $s_{2}$ terminate in the same way when started in the states $m_{1}^{\prime}\left(\sigma_{1}^{\prime}\right)$ and $m_{2}^{\prime}\left(\sigma_{2}^{\prime}\right)$. We show that all required conditions are satisfied. $\operatorname{size}\left(s_{1}\right)+$ $\operatorname{size}\left(s_{2}\right)<k$ because $\operatorname{size}\left(S_{1}^{\prime}\right) \geq 1, \operatorname{size}\left(S_{2}^{\prime}\right) \geq 1$ by the definition of program size. If $s_{1}, s_{2}$ are loop statement, then, by the assumption of unique loop labels, $s_{1} \notin S_{1}^{\prime}, s_{2} \notin$ $S_{2}^{\prime}$. Then, by Corollary E.4 the loop counter value of $s_{1}$ and $s_{2}$ is not redefined in the execution of $S_{1}^{\prime}$ and $S_{2}^{\prime}$ respectively. By the hypothesis IH, $s_{1}$ and $s_{2}$ terminate in the same way when started in the states $m_{1}^{\prime}\left(\mathfrak{f}_{1}, \sigma_{1}^{\prime}\right)$ and $m_{2}^{\prime}\left(\mathfrak{f}_{2}, \sigma_{2}^{\prime}\right),\left(s_{1}, m_{1}^{\prime}\left(\mathfrak{f}_{1}, \sigma_{1}^{\prime}\right)\right) \equiv_{H} \quad\left(s_{2}, m_{2}^{\prime}\left(\mathfrak{f}_{2}, \sigma_{2}^{\prime}\right)\right)$. The theorem 4 holds.

(b) One last statement is "skip": w.l.o.g., $\left(s_{2}=\right.$ "skip" $) \wedge$ $\left(S_{1} \equiv{ }_{H}^{S} S_{2}^{\prime}\right)$.

We show that $S_{1}$ and $S_{2}^{\prime}$ terminate in the same way when started in the states $m_{1}\left(\sigma_{1}\right)$ and $m_{2}\left(\sigma_{2}\right)$ respectively by the hypothesis IH. By the definition of crash/loop variables, $\mathrm{C} \operatorname{Var}\left(S_{2}^{\prime}\right) \subseteq \mathrm{C} \operatorname{Var}\left(S_{2}\right), \operatorname{LV} \operatorname{Var}\left(S_{2}^{\prime}\right) \subseteq \operatorname{LVar}\left(S_{2}\right)$. Then, by assumption, $\forall x \in \operatorname{TVar}\left(S_{2}^{\prime}\right) \cup \operatorname{TV} \operatorname{Var}\left(S_{1}\right): \sigma_{1}(x)=$ $\sigma_{2}(x)$. Besides, size $\left(s_{2}\right) \geq 1$ by the definition of program size. Then size $\left(S_{1}\right)+\operatorname{size}\left(S_{2}^{\prime}\right) \leq k$. By the hypothesis $\mathrm{IH}, S_{1}$ and $S_{2}^{\prime}$ terminate in the same way when started in the states $m_{1}\left(\mathfrak{f}_{1}, \sigma_{1}\right), m_{2}\left(\mathfrak{f}_{2}, \sigma_{2}\right),\left(S_{1}, m_{1}\left(\mathfrak{f}_{1}, \sigma_{1}\right)\right) \equiv_{H}$ $\left(S_{2}^{\prime}, m_{2}\left(\mathfrak{f}_{2}, \sigma_{2}\right)\right)$.

When the execution of $S_{1}$ and $S_{2}^{\prime}$ terminate when started in the states $m_{1}\left(\sigma_{1}\right)$ and $m_{2}\left(\sigma_{2}\right)$ respectively, $s_{2}$ terminates after the execution of $S_{2}^{\prime}$ by the definition of terminating execution.

(c) One last statement is a "duplicate" statement such that one of the following holds:

W.l.o.g., $S_{2}=S_{2}^{\prime} ; s_{2}^{\prime} ; S_{2}^{\prime \prime} ; s_{2}$ and all of the following hold:

- $S_{1} \equiv{ }_{H}^{S} S_{2}^{\prime} ; s_{2}^{\prime} ; S_{2}^{\prime \prime}$;

- $s_{2}^{\prime} \equiv_{H}^{S} s_{2}$;

- $\operatorname{Def}\left(s_{2}^{\prime} ; S_{2}^{\prime \prime}\right) \cap \operatorname{TVar}\left(s_{2}\right)=\emptyset$;

- $s_{2} \neq$ "skip";

We show that $S_{1}$ and $S_{2}^{\prime} ; s_{2}^{\prime} ; S_{2}^{\prime \prime}$ terminate in the same way when started in the states $m_{1}\left(\mathfrak{f}_{1}, \sigma_{1}\right)$,

$m_{2}\left(\mathfrak{f}_{2}, \sigma_{2}\right)$ respectively by the hypothesis IH. The proof is same as that in case a).

We show that $s_{2}$ terminates if the execution of $S_{2}^{\prime} ; s_{2}^{\prime} ; S_{2}^{\prime \prime}$ terminates. We need to prove that $s_{2}^{\prime}$ and $s_{2}$ start in the states agreeing on the values of variables in $T \operatorname{Var}\left(s_{2}\right)$. By assumption, $S_{2}^{\prime} ; s_{2}^{\prime} ; S_{2}^{\prime \prime}$ terminates, $\left(S_{2}^{\prime} ; s_{2}^{\prime} ; S_{2}^{\prime \prime}, m_{2}\left(\mathfrak{f}_{2}, \sigma_{2}\right)\right) \stackrel{*}{\rightarrow}$ (skip, $\left.m_{2}^{\prime}\left(\mathfrak{f}_{2}, \sigma_{2}^{\prime}\right)\right)$. Then, by CorollaryE.1] $\left(S_{2}^{\prime} ; s_{2}^{\prime} ; S_{2}^{\prime \prime} ; s_{2}, m_{2}\left(\mathfrak{f}_{2}, \sigma_{2}\right)\right)$ $\stackrel{*}{\rightarrow}\left(s_{2}, m_{2}^{\prime}\left(\mathfrak{f}_{2}, \sigma_{2}^{\prime}\right)\right)$. In addition, the execution of $S_{2}^{\prime}$ and $s_{2}^{\prime}$ must terminate because the execution of $S_{2}^{\prime} ; s_{2}^{\prime} ; S_{2}^{\prime \prime}$ terminates,

$\left(S_{2}^{\prime} ; s_{2}^{\prime} ; S_{2}^{\prime \prime} ; s_{2}, m_{2}\left(\mathfrak{f}_{2}, \sigma_{2}\right)\right) \stackrel{*}{\rightarrow}\left(s_{2}^{\prime} ; S_{2}^{\prime \prime} ; s_{2}, m_{2}^{\prime \prime}\left(\mathfrak{f}_{2}, \sigma_{2}^{\prime \prime}\right)\right) \stackrel{*}{\rightarrow}$ $\left(s_{2}, m_{2}^{\prime}\left(\mathfrak{f}_{2}, \sigma_{2}^{\prime}\right)\right)$.

By assumptin, $\operatorname{Def}\left(s_{2}^{\prime} ; S_{2}^{\prime \prime}\right) \cap \operatorname{TV} \operatorname{Var}\left(s_{2}\right)=\emptyset$. Then, by CorollaryE.2 the value store $\sigma_{2}^{\prime \prime}$ and $\sigma_{2}^{\prime}$ agree on values of the termination deciding variables of $s_{2}, \forall x \in \operatorname{TVar}\left(s_{2}\right)$ : $\sigma_{2}^{\prime \prime}(x)=\sigma_{2}^{\prime}(x)$. By Corollary 5.1 $\mathrm{T} \operatorname{Var}\left(s_{2}^{\prime}\right)=\mathrm{T} \operatorname{Var}\left(s_{2}\right)$. Because the execution of $s_{2}^{\prime}$ terminates, then the execution of $s_{2}$ terminates when started in the state $m_{2}^{\prime}\left(\mathfrak{f}_{2}, \sigma_{2}^{\prime}\right)$ by the hypothesis $\mathrm{IH},\left(s_{2}, m_{2}^{\prime}\left(\mathfrak{f}_{2}, \sigma_{2}^{\prime}\right)\right) \stackrel{*}{\rightarrow}\left(\right.$ skip, $\left.m_{2}^{\prime \prime}\right)$.

In addition, we show that there is no input statement in $s_{2}$ by contradiction. Suppose there is input statement in $s_{2}$. By Lemma 5.11 $i d_{I} \in C \operatorname{Var}\left(s_{2}\right)$. Hence, the input sequence variable is in the termination deciding variables of $s_{2}, i d_{I} \in \operatorname{TVar}\left(s_{2}\right)$. By Corollary 5.1, $\operatorname{TVar}\left(s_{2}\right)=$ 
$\operatorname{TV} \operatorname{Var}\left(s_{2}^{\prime}\right)$. Then, there must be one input statement in $s_{2}^{\prime}$. Otherwise, by Lemma 5.2 the input sequence variable is not in the termination deciding variables of $s_{2}^{\prime}$. A contradiction against the result that $i d_{I} \in \operatorname{TV} \operatorname{Var}\left(s_{2}^{\prime}\right)$. Since there is one input statement in $s_{2}^{\prime}$, by Lemma 5.11 $i d_{I} \in \operatorname{Def}\left(s_{2}^{\prime}\right)$. Thus, by defintion, $i d_{I} \in \operatorname{Def}\left(s_{2}^{\prime} ; S_{2}^{\prime \prime}\right)$. Then, $\operatorname{Def}\left(s_{2}^{\prime} ; S_{2}^{\prime \prime}\right) \cap$ $\operatorname{TVar}\left(s_{2}\right) \neq \emptyset$. A contradiction. Therefore, there is no input statement in $s_{2}$.

(d) $S_{1}=S_{1}^{\prime} ; s_{1} ; s_{1}^{\prime}$; and $S_{2}=S_{2}^{\prime} ; s_{2} ; s_{2}^{\prime}$ where $s_{1}$ and $s_{2}$ are reordered and all of the following hold:

- $S_{1}^{\prime} \equiv_{H}^{S} S_{2}^{\prime}$;

- $S_{1}^{\prime}$ and $S_{2}^{\prime}$ have equivalent computation of $\operatorname{TVar}\left(s_{1} ; s_{1}^{\prime}\right) \cup$ $\mathrm{T} \operatorname{Var}\left(s_{2} ; s_{2}^{\prime}\right)$.

- $s_{1} \equiv{ }_{H}^{S} s_{2}^{\prime}$;

- $s_{1}^{\prime} \equiv{ }_{H}^{S} s_{2}$;

- $\operatorname{Def}\left(s_{1}\right) \cap \operatorname{TV} \operatorname{Var}\left(s_{1}^{\prime}\right)=\emptyset$;

- $\operatorname{Def}\left(s_{2}\right) \cap \operatorname{TV} \operatorname{Var}\left(s_{2}^{\prime}\right)=\emptyset$;

The proof is to show that if $S_{1}$ terminates when started in the state $m_{1}$, the $S_{2}$ terminates when started in the state $m_{2}$, and vice versa. Due to the symmetric conditions, it is suffice to show one direction that, w.l.o.g., $\left(S_{1}, m_{1}\right) \stackrel{*}{\rightarrow}$ (skip, $\left.m_{1}^{\prime}\right) \Rightarrow\left(S_{2}, m_{2}\right) \stackrel{*}{\rightarrow}$ (skip, $m_{2}^{\prime}$ ).

We show that the execution of $S_{2}^{\prime}$ terminates by the hypothesis IH. We need to show that all required conditions are satisfied.

- $\operatorname{size}\left(S_{1}^{\prime}\right)+\operatorname{size}\left(S_{2}^{\prime}\right)<k$.

This is because size $\left(s_{1} ; s_{1}^{\prime}\right)>1, \operatorname{size}\left(s_{2} ; s_{2}^{\prime}\right)>1$.

- Initial value stores $\sigma_{1}$ and $\sigma_{2}$ agree on values of the termination deciding variables of $S_{1}^{\prime}$ and $S_{2}^{\prime}, \forall x \in$ $\operatorname{TVar}\left(S_{1}^{\prime}\right) \cup \operatorname{TVar}\left(S_{2}^{\prime}\right): \sigma_{1}(x)=\sigma_{2}(x)$.

We show that $\operatorname{TVar}\left(S_{1}^{\prime}\right) \subseteq \mathrm{T} \operatorname{Var}\left(S_{1}\right)$. In the following, we prove that $\mathrm{C} \operatorname{Var}\left(S_{1}^{\prime}\right) \subseteq \mathrm{C} \operatorname{Var}\left(S_{1}\right)$.

$$
\mathrm{CVar}\left(S_{1}^{\prime}\right)
$$

$\subseteq \quad \mathrm{CVar}\left(S_{1}^{\prime} ; s_{1}\right)$ by the defintion of $\operatorname{CVar}\left(S_{1}^{\prime} ; s_{1}\right)$

$\subseteq \quad \mathrm{CVar}\left(S_{1}^{\prime} ; s_{1} ; s_{1}^{\prime}\right)$ by the defintion of $\mathrm{CVar}\left(S_{1}^{\prime} ; s_{1} ; s_{1}^{\prime}\right)$

Similarly, $\operatorname{LVar}\left(S_{1}^{\prime}\right) \subseteq \operatorname{LV} \operatorname{Var}\left(S_{1}\right)$. Hence, $\operatorname{TV} \operatorname{Var}\left(S_{1}^{\prime}\right) \subseteq$ $\mathrm{T} \operatorname{Var}\left(S_{1}\right)$. Similarly, $\mathrm{T} \operatorname{Var}\left(S_{2}^{\prime}\right) \subseteq \mathrm{T} \operatorname{Var}\left(S_{2}\right)$. By assumption, initial value stores $\sigma_{1}$ and $\sigma_{2}$ agree on values of the termination deciding variables of $S_{1}^{\prime}$ and $S_{2}^{\prime}$.

By the hypothesis IH, $\left(S_{1}^{\prime}, m_{1}\left(\sigma_{1}\right)\right) \equiv_{H}\left(S_{2}^{\prime}, m_{2}\left(\sigma_{2}\right)\right)$. Because the execution of $S_{1}$ terminates, then $S_{1}^{\prime}$ terminates when started in the state $m_{1}\left(\sigma_{1}\right),\left(S_{1}^{\prime}, m_{1}\left(\sigma_{1}\right)\right) \stackrel{*}{\rightarrow}$ (skip, $\left.m_{1}^{\prime}\left(\sigma_{1}^{\prime}\right)\right)$. Therefore, $S_{2}^{\prime}$ termiantes when started in the state $m_{2}\left(\sigma_{2}\right),\left(S_{2}^{\prime}, m_{2}\left(\sigma_{2}\right)\right) \stackrel{*}{\rightarrow}\left(\right.$ skip, $\left.m_{2}^{\prime}\left(\sigma_{2}^{\prime}\right)\right)$.

We show that after the execution of $S_{1}^{\prime}$ and $S_{2}^{\prime}$, value stores agree on values of the termination deciding variables of $s_{1} ; s_{1}^{\prime}$ and $s_{2} ; s_{2}^{\prime}, \forall x \in \operatorname{TVar}\left(s_{1} ; s_{1}^{\prime}\right) \cup \operatorname{TVar}\left(s_{2} ; s_{2}^{\prime}\right), \sigma_{1}^{\prime}(x)=$ $\sigma_{2}^{\prime}(x)$. We split the argument into two steps.

i. We show that $\operatorname{TVar}\left(s_{1} ; s_{1}^{\prime}\right)=\operatorname{TVar}\left(s_{2} ; s_{2}^{\prime}\right)$.

By Corollary 5.1 $\operatorname{TVar}\left(s_{1}\right)=\operatorname{TVar}\left(s_{2}^{\prime}\right)$ and $\operatorname{TVar}\left(s_{1}^{\prime}\right)=$ $\operatorname{TVar}\left(s_{2}\right)$. Then we show that $\operatorname{TV} \operatorname{Var}\left(s_{1} ; s_{1}^{\prime}\right)=\operatorname{TVar}\left(s_{1}\right) \cup$ $\operatorname{TV} \operatorname{Var}\left(s_{1}^{\prime}\right)$. To do that, we show that $\mathrm{CVar}\left(s_{1} ; s_{1}^{\prime}\right)=$ $\mathrm{C} \operatorname{Var}\left(s_{1}\right) \cup \mathrm{CVar}\left(s_{1}^{\prime}\right)$.

$\mathrm{CVar}\left(s_{1} ; s_{1}^{\prime}\right)$

$=C \operatorname{Var}\left(s_{1}\right) \cup \operatorname{Imp}\left(s_{1}, C \operatorname{Var}\left(s_{1}^{\prime}\right)\right)$ by the defintion of $\operatorname{CVar}\left(s_{1} ; s_{1}^{\prime}\right)$

$=C \operatorname{Var}\left(s_{1}\right) \cup C \operatorname{Var}\left(s_{1}^{\prime}\right)$ by $\operatorname{Def}\left(s_{1}\right) \cap \operatorname{TV} \operatorname{Var}\left(s_{1}^{\prime}\right)=\emptyset$ and the defintion of $\operatorname{Imp}(\cdot)$.

Similarly, $\operatorname{LVar}\left(s_{1} ; s_{1}^{\prime}\right)=\operatorname{LVar}\left(s_{1}\right) \cup \operatorname{LV} \operatorname{Var}\left(s_{1}^{\prime}\right)$. Thus, $\mathrm{T} \operatorname{Var}\left(s_{1} ; s_{1}^{\prime}\right)=\mathrm{TVar}\left(s_{1}\right) \cup \mathrm{TVar}\left(s_{1}^{\prime}\right)$. Similarly, $\operatorname{TVar}\left(s_{2} ; s_{2}^{\prime}\right)=$ $\operatorname{TVar}\left(s_{2}\right) \cup \operatorname{TVar}\left(s_{2}^{\prime}\right)$. In summary, $\operatorname{TVar}\left(s_{1} ; s_{1}^{\prime}\right)=$ $\operatorname{TVar}\left(s_{2} ; s_{2}^{\prime}\right)$.

ii. We show that $\operatorname{Imp}\left(S_{1}^{\prime}, \mathrm{T} \operatorname{Var}\left(s_{1} ; s_{1}^{\prime}\right)\right) \subseteq \mathrm{T} \operatorname{Var}\left(S_{1}\right)$ and
$\operatorname{Imp}\left(S_{2}^{\prime}, \mathrm{T} \operatorname{Var}\left(s_{2} ; s_{2}^{\prime}\right)\right) \subseteq \mathrm{TV} \operatorname{Var}\left(S_{2}\right)$.

W.1.o.g, we show that $\operatorname{Imp}\left(S_{1}^{\prime}, \mathrm{T} \operatorname{Var}\left(s_{1} ; s_{1}^{\prime}\right)\right) \subseteq \mathrm{TV} \operatorname{Var}\left(S_{1}\right)$. Specifically, we show $\operatorname{Imp}\left(S_{1}^{\prime}, \mathrm{CVar}\left(s_{1} ; s_{1}^{\prime}\right)\right) \subseteq \operatorname{CVar}\left(S_{1}\right)$. $\mathrm{CVar}\left(s_{1} ; s_{1}^{\prime}\right)$

$=\operatorname{CVar}\left(s_{1}\right) \cup \operatorname{Imp}\left(s_{1}, \mathrm{CVar}\left(s_{1}^{\prime}\right)\right)(1)$

by the defintion of $C \operatorname{Var}\left(s_{1} ; s_{1}^{\prime}\right)$

$\operatorname{Imp}\left(S_{1}^{\prime}, \mathrm{CVar}\left(s_{1} ; s_{1}^{\prime}\right)\right)$

$=\operatorname{Imp}\left(S_{1}^{\prime}, \mathrm{CVar}\left(s_{1}\right) \cup \operatorname{Imp}\left(s_{1}, \mathrm{CVar}\left(s_{1}^{\prime}\right)\right)\right)$ by (1)

$=\operatorname{Imp}\left(S_{1}^{\prime}, \mathrm{CVar}\left(s_{1}\right)\right) \cup \operatorname{Imp}\left(S_{1}^{\prime}, \operatorname{Imp}\left(s_{1}, \mathrm{C} \operatorname{Var}\left(s_{1}^{\prime}\right)\right)\right)$ by Lemma C.2

$\operatorname{Imp}\left(S_{1}^{\prime}, \mathrm{C} \operatorname{Var}\left(s_{1}\right)\right)$

$\subseteq \mathrm{CVar}\left(S_{1}^{\prime} ; s_{1}\right)$ by the defintion of $\mathrm{CVar}(\cdot)$

$\subseteq \mathrm{CVar}\left(S_{1}^{\prime} ; s_{1} ; s_{1}^{\prime}\right)$ by the defintion of $\mathrm{C} \operatorname{Var}(\cdot)$

$\operatorname{Imp}\left(S_{1}^{\prime}, \operatorname{Imp}\left(s_{1}, \mathrm{C} \operatorname{Var}\left(s_{1}^{\prime}\right)\right)\right)$

$=\operatorname{Imp}\left(S_{1}^{\prime} ; s_{1}, \mathrm{CVar}\left(s_{1}^{\prime}\right)\right)$ by LemmaC.1

$\subseteq \mathrm{CVar}\left(S_{1}^{\prime} ; s_{1} ; s_{1}^{\prime}\right)$ by the defintion of $\mathrm{C} \operatorname{Var}(\cdot)$.

$\operatorname{Imp}\left(S_{1}^{\prime}, \mathrm{CVar}\left(s_{1}\right)\right) \cup \operatorname{Imp}\left(S_{1}^{\prime}, \operatorname{Imp}\left(s_{1}, \mathrm{CVar}\left(s_{1}^{\prime}\right)\right)\right)$ $\subseteq \mathrm{CVar}\left(S_{1}^{\prime} ; s_{1} ; s_{1}^{\prime}\right)$ by (3) and (4).

In conclusion, $\operatorname{Imp}\left(S_{1}^{\prime}, \mathrm{CVar}\left(s_{1} ; s_{1}^{\prime}\right)\right) \subseteq \mathrm{CVar}\left(S_{1}\right)$. Similarly, $\operatorname{Imp}\left(S_{1}^{\prime}, \operatorname{LVar}\left(s_{1} ; s_{1}^{\prime}\right)\right) \subseteq \operatorname{LVar}\left(S_{1}\right)$. Thus, $\operatorname{Imp}\left(S_{1}^{\prime}, \operatorname{TVar}\left(s_{1} ; s_{1}^{\prime}\right)\right) \subseteq \operatorname{TVar}\left(S_{1}\right)$. Similarly,

$\operatorname{Imp}\left(S_{2}^{\prime}, \mathrm{T} \operatorname{Var}\left(s_{2} ; s_{2}^{\prime}\right)\right) \subseteq \mathrm{T} \operatorname{Var}\left(S_{2}\right)$.

Then, by Theorem 2, after terminating execution of $S_{1}^{\prime}$ and $S_{2}^{\prime}$, value stores $\sigma_{1}^{\prime}$ and $\sigma_{2}^{\prime}$ agree on values of the termination deciding variables of $s_{1} ; s_{1}^{\prime}$ and $s_{2} ; s_{2}^{\prime}$,

$\forall x \in \operatorname{TVar}\left(s_{1} ; s_{1}^{\prime}\right) \cup \operatorname{TVar}\left(s_{2} ; s_{2}^{\prime}\right): \sigma_{1}^{\prime}(x)=\sigma_{2}^{\prime}(x)$.

We show that the execution of $s_{2}$ terminates by the hypothesis IH. By Corollary E.1.

$\left(S_{1}^{\prime} ; s_{1} ; s_{1}^{\prime}, m_{1}\left(\sigma_{1}\right)\right) \stackrel{*}{\rightarrow}\left(s_{1} ; s_{1}^{\prime}, m_{1}^{\prime}\left(\sigma_{1}^{\prime}\right)\right)$ and $\left(S_{2}^{\prime} ; s_{2} ; s_{2}^{\prime}\right.$, $\left.m_{2}\left(\sigma_{2}\right)\right) \stackrel{*}{\rightarrow}\left(s_{2} ; s_{2}^{\prime}, m_{2}^{\prime}\left(\sigma_{2}^{\prime}\right)\right)$. By assumption, $S_{1}$ terminates, then $s_{1}$ terminates, $\left(s_{1}, m_{1}^{\prime}\left(\sigma_{1}^{\prime}\right)\right) \stackrel{*}{\rightarrow}$ (skip, $\left.m_{1}^{\prime \prime}\left(\sigma_{1}^{\prime \prime}\right)\right)$. Because $s_{1}^{\prime} \equiv{ }_{H}^{S} s_{2}$, to apply the induction hypothesis, we need to show that all required conditions hold.

- $\operatorname{size}\left(s_{2}\right)+\operatorname{size}\left(s_{1}^{\prime}\right)<k$.

By definition, $\operatorname{size}\left(S_{2}^{\prime}\right)>1, \operatorname{size}\left(S_{1}^{\prime}\right)>1, \operatorname{size}\left(s_{1}\right)>$ $1, \operatorname{size}\left(s_{2}^{\prime}\right)>1$.

- Value stores $\sigma_{1}^{\prime \prime}$ and $\sigma_{2}^{\prime}$ agree on values of the termination deciding variables of $s_{1}^{\prime}$ and $s_{2} . \forall x \in \operatorname{TV} \operatorname{Var}\left(s_{1}^{\prime}\right) \cup$ $\operatorname{TVar}\left(s_{2}\right): \sigma_{1}^{\prime \prime}(x)=\sigma_{2}^{\prime}(x)$.

By Corollary 5.1, $\mathrm{T} \operatorname{Var}\left(s_{1}^{\prime}\right)=\mathrm{T} \operatorname{Var}\left(s_{2}\right)$. Because of the condition $\operatorname{Def}\left(s_{1}\right) \cap \operatorname{TV} \operatorname{Var}\left(s_{1}^{\prime}\right)=\emptyset$, by Corollary E.2 value stores $\sigma_{1}^{\prime \prime}$ and $\sigma_{1}^{\prime}$ agree on values of the termination deciding variables of $s_{1}^{\prime}, \forall x \in \operatorname{TVar}\left(s_{1}^{\prime}\right)$ : $\sigma_{1}^{\prime \prime}(x)=\sigma_{1}^{\prime}(x)$. By the argument above, $\forall x \in \operatorname{TVar}\left(s_{2}\right)$ : $\sigma_{1}^{\prime}(x)=\sigma_{2}^{\prime}(x)$. Thus, the condition holds.

By the induction hypothesis IH, $\left(s_{1}^{\prime}, m_{1}^{\prime \prime}\left(\sigma_{1}^{\prime \prime}\right)\right) \equiv_{H}\left(s_{2}, m_{2}^{\prime}\left(\sigma_{2}^{\prime}\right)\right)$. Because the execution of $s_{1}^{\prime}$ terminates, then the exeuction of $s_{2}$ terminates when started in the state $m_{2}^{\prime}\left(\sigma_{2}^{\prime}\right)$, $\left(s_{2}, m_{2}^{\prime}\left(\sigma_{2}^{\prime}\right)\right) \stackrel{*}{\rightarrow}$ (skip, $\left.m_{2}^{\prime \prime}\left(\sigma_{2}^{\prime \prime}\right)\right)$.

We show that the execution of $s_{2}^{\prime}$ terminates. This is by the similar argument that $s_{2}$ terminates.

In conclusion, $S_{2}$ terminates when started in the state $m_{2}\left(\sigma_{2}\right)$. The theorem holds.

In addition, we show that it is impossible that $s_{1}$ and $s_{1}^{\prime}$ both include input statements by contradiction. Suppose there are input statements in both $s_{1}$ and $s_{1}^{\prime}$. By Lemma 5.11 $i d_{I} \in \operatorname{Def}\left(s_{1}\right) \cap \operatorname{TVar}\left(s_{1}^{\prime}\right)$. A contradiction against the condition that $\operatorname{Def}\left(s_{1}\right) \cap \operatorname{TV} \operatorname{Var}\left(s_{1}^{\prime}\right)=\emptyset$. Similarly, there are no input statements in both $s_{2}$ and $s_{2}^{\prime}$. 


\subsubsection{Supporting lemmas for the soundness proof of termination in the same way}

The supporting lemmas include various properties of $\operatorname{TVar}(S)$, two statement sequences satisfying the proof rule of termination in the same way consume the same number of input values when both terminate, and the proof for the case of while statement of theorem 4

\section{The properties of the termination deciding variables}

Lemma 5.4. The crash variables of $S_{1} ; S_{1}^{\prime}$ is same as the union of the crash variables of $S_{1}$ and the imported variables in $S_{1}$ relative to the crash variables of $S_{1}^{\prime}, C \operatorname{Var}\left(S_{1} ; S_{1}^{\prime}\right)=C \operatorname{Var}\left(S_{1}\right) \cup$ $\operatorname{Imp}\left(S_{1}, C \operatorname{Var}\left(S_{1}^{\prime}\right)\right)$.

Proof. Let $S_{1}^{\prime}=s_{1} ; \ldots ; s_{k}$ for $k>0$. We show the lemma by induction on $k$.

Base case.

By the definition of $C \operatorname{Var}(S)$, the lemma holds.

\section{Induction step.}

The hypothesis IH is that $\mathrm{CVar}\left(S_{1} ; s_{1} ; \ldots ; s_{k}\right)=\mathrm{CVar}\left(S_{1}\right) \cup$ $\operatorname{Imp}\left(S_{1}, \mathrm{CVar}\left(s_{1} ; \ldots ; s_{k}\right)\right)$ for $k \geq 1$.

Then we show that the lemma holds when $S_{1}^{\prime}=s_{1} ; \ldots ; s_{k+1}$.

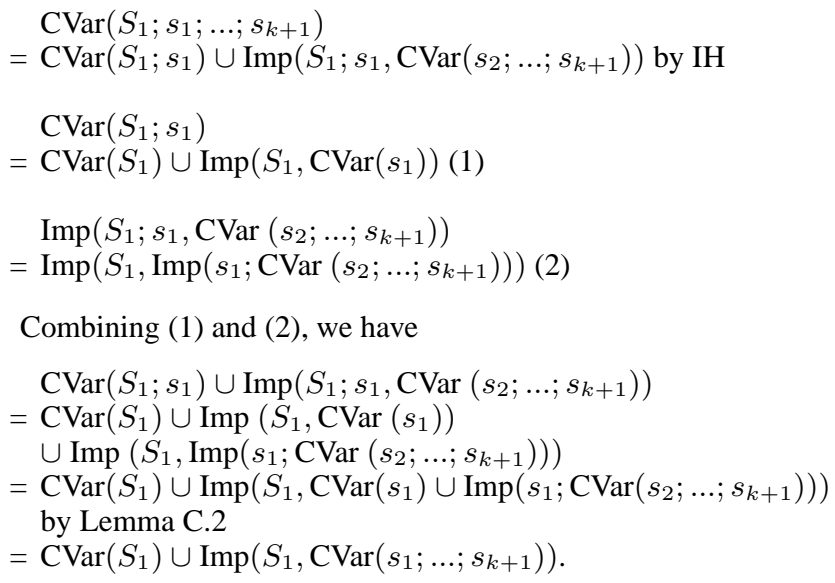

Lemma 5.5. The loop deciding variables of $S_{1} ; S_{1}^{\prime}$ is same as the union of the loop deciding variables of $S_{1}$ and the imported variables in $S_{1}$ relative to the loop deciding variables of $S_{1}^{\prime}$, $\operatorname{LVar}\left(S_{1} ; S_{1}^{\prime}\right)=\operatorname{LVar}\left(S_{1}\right) \cup \operatorname{Imp}\left(S_{1}, L \operatorname{Var}\left(S_{1}^{\prime}\right)\right)$.

By proof of Lemma 5.5 similar to that of lemma 5.4 above.

Lemma 5.6. If two statement sequences $S_{1}$ and $S_{2}$ satisfy the proof rule of termination in the same way, then $S_{1}$ and $S_{2}$ have same loop variables, $\left(S_{1} \equiv_{H}^{S} S_{2}\right) \Rightarrow\left(\operatorname{Var}\left(S_{1}\right)=\operatorname{LVar}\left(S_{2}\right)\right)$.

Proof. By induction on size $\left(S_{1}\right)+\operatorname{size}\left(S_{2}\right)$, the sum of the program size of $S_{1}$ and $S_{2}$.

Base case.

$S_{1}$ and $S_{2}$ are simple statement. There are three base cases according to the definition of $s_{1} \equiv_{H}^{S} s_{2}$.

1. two same simple statements, $S_{1}=S_{2}$;

2. $S_{1}$ and $S_{2}$ are input statement with same type variable: $S_{1}=$ "input $i d_{1}$ ", $S_{2}=$ "input $i d_{2}$ " where $\left(\Gamma_{S_{1}} \vdash i d_{1}: t\right) \wedge\left(\Gamma_{S_{2}} \vdash\right.$ $\left.i d_{2}: t\right)$;

3. $S_{1}=$ "output $e$ " or " $i d_{1}:=e$ ", $S_{2}=$ "output $e$ " or " $i d_{2}:=e$ " where both of the following hold:
- There is no possible value mismatch in " $i d_{1}:=e$ ", $\neg\left(\Gamma_{S_{1}} \vdash i d_{1}\right.$ : Int $) \vee \neg\left(\Gamma_{S_{1}} \vdash e\right.$ : Long $) \vee\left(\Gamma_{S_{1}} \vdash e\right.$ : Int $)$.

- There is no possible value mismatch in " $i d_{2}:=e$ ", $\neg\left(\Gamma_{S_{2}} \vdash i d_{2}\right.$ : Int $) \vee \neg\left(\Gamma_{S_{2}} \vdash e\right.$ : Long $) \vee\left(\Gamma_{S_{2}} \vdash e\right.$ : Int $)$.

By the definition of loop variables, $\operatorname{LVar}\left(S_{1}\right)=\operatorname{LVar}\left(S_{2}\right)=\emptyset$ in both base cases. Therefore, Lemma 5.6 holds.

Induction Step.

The hypothesis IH is that Lemma 5.6 holds when $\operatorname{size}\left(S_{1}\right)+$ $\operatorname{size}\left(S_{2}\right)=k \geq 2$.

We show that Lemma 5.6 holds when $\operatorname{size}\left(S_{1}\right)+\operatorname{size}\left(S_{2}\right)=$ $k+1$.

The proof is a case analysis according to the cases in the definition of $\left(S_{1} \equiv{ }_{H}^{S} S_{2}\right)$ :

1. $S_{1}$ and $S_{2}$ are one statement and one of the following holds.

(a) $S_{1}=$ "If $(e)$ then $\left\{S_{1}^{t}\right\}$ else $\left\{S_{1}^{f}\right\} ", S_{2}=$ "If $(e)$ then $\left\{S_{2}^{t}\right\}$ else $\left\{S_{2}^{f}\right\}$ " such that one of the following holds:

i. $S_{1}^{t}, S_{1}^{f}, S_{2}^{t}, S_{2}^{f}$ are all sequences of "skip";

By the definition of loop variables, $\operatorname{LVar}\left(S_{1}^{t}\right)=\operatorname{LVar}\left(S_{1}^{f}\right)=$ $\operatorname{LVar}\left(S_{2}^{t}\right)=\operatorname{LVar}\left(S_{2}^{f}\right)=\emptyset$. Therefore, by the definition of loop variables, $\operatorname{LVar}\left(S_{1}\right)=\operatorname{LVar}\left(S_{2}\right)=\emptyset$. The lemma 5.6 holds.

ii. At least one of $S_{1}^{t}, S_{1}^{f}, S_{2}^{t}, S_{2}^{f}$ is not a sequence of "skip" such that: $\left(S_{1}^{t} \equiv_{H}^{S} S_{2}^{t}\right) \wedge\left(S_{1}^{f} \equiv{ }_{H}^{S} S_{2}^{f}\right)$; $\operatorname{size}\left(S_{1}^{t}\right)+\operatorname{size}\left(S_{2}^{t}\right)<k, \operatorname{size}\left(S_{1}^{f}\right)+\operatorname{size}\left(S_{2}^{f}\right)<k$. Then, by the hypothesis IH1, $\operatorname{LVar}\left(S_{1}^{t}\right)=\operatorname{LVar}\left(S_{2}^{t}\right), \operatorname{LVar}\left(S_{1}^{f}\right)=$ $\operatorname{LVar}\left(S_{2}^{f}\right)$. Consequently, $\left(\operatorname{LVar}\left(S_{1}^{t}\right) \cup \operatorname{LVar}\left(S_{1}^{f}\right)\right)=$ $\left(\operatorname{LVar}\left(S_{2}^{t}\right) \cup \operatorname{LVar}\left(S_{2}^{f}\right)\right)=\operatorname{LV} \operatorname{Var}(\Delta)$. When $\operatorname{LVar}(\Delta)=$ $\emptyset$, then $\operatorname{LVar}\left(S_{1}\right)=\operatorname{LVar}\left(S_{2}\right)=\emptyset$ by the definition of loop variables. When $\operatorname{LVar}(\Delta) \neq \emptyset$, then $\operatorname{LVar}\left(S_{1}\right)=\operatorname{LVar}\left(S_{2}\right)=\operatorname{LVar}(\Delta) \cup \operatorname{Use}(e)$ by the definition of loop variables. The lemma 5.6 holds.

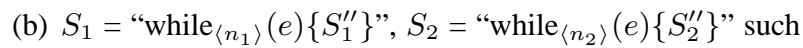
that both of the following hold:

- $S_{1}^{\prime \prime} \equiv{ }_{H}^{S} S_{2}^{\prime \prime}$;

- $S_{1}^{\prime \prime}$ and $S_{2}^{\prime \prime}$ have equivalent computation of $\operatorname{TV} \operatorname{Var}\left(S_{1}\right) \cup$ $\mathrm{TVar}\left(S_{2}\right)$;

By the hypothesis IH1, $\operatorname{LVar}\left(S_{1}^{\prime \prime}\right)=\operatorname{LVar}\left(S_{2}^{\prime \prime}\right)$. Then $\operatorname{Use}(e) \cup \operatorname{LVar}\left(S_{1}^{\prime \prime}\right)=\operatorname{Use}(e) \cup \operatorname{LVar}\left(S_{2}^{\prime \prime}\right)$. Then, we show that:

$\forall i \geq 0, \operatorname{Imp}\left(S_{1}^{\prime \prime}{ }^{i}, \operatorname{Use}(e) \cup \operatorname{LVar}\left(S_{1}^{\prime \prime}\right)\right)=\operatorname{Imp}\left(S_{2}^{\prime \prime i}, \operatorname{Use}(e) \cup\right.$ $\left.\operatorname{LVar}\left(S_{2}^{\prime \prime}\right)\right)$ by induction on $i$.

Base case

By our notation $S^{0}, S_{1}^{\prime \prime 0}=$ skip, $S_{2}^{\prime \prime}{ }^{0}=$ skip. Then, by the definition of imported variables,

$\operatorname{Imp}\left(S_{1}^{\prime \prime 0}, \operatorname{Use}(e) \cup \operatorname{LVar}\left(S_{1}^{\prime \prime}\right)\right)=\operatorname{Use}(e) \cup \operatorname{LVar}\left(S_{1}^{\prime \prime}\right)$,

$\operatorname{Imp}\left(S_{2}^{\prime \prime 0}, \operatorname{Use}(e) \cup \operatorname{LVar}\left(S_{2}^{\prime \prime}\right)\right)=\operatorname{Use}(e) \cup \operatorname{LVar}\left(S_{2}^{\prime \prime}\right)$.

Then, $\operatorname{Imp}\left(S_{1}^{\prime \prime 0}, \operatorname{Use}(e) \cup \operatorname{LVar}\left(S_{1}^{\prime \prime}\right)\right)=\operatorname{Imp}\left({S_{2}^{\prime \prime}}^{\prime \prime}, \operatorname{Use}(e) \cup\right.$ $\left.\operatorname{LVar}\left(S_{2}^{\prime \prime}\right)\right)$.

Induction step

The hypothesis IH3 is that, $\forall i \geq 0, \operatorname{Imp}\left(S_{1}^{\prime \prime}{ }^{i}, \operatorname{Use}(e) \cup\right.$ $\left.\operatorname{LVar}\left(S_{1}^{\prime \prime}\right)\right)=\operatorname{Imp}\left(S_{2}^{\prime \prime}, \operatorname{Use}(e) \cup \operatorname{LVar}\left(S_{2}^{\prime \prime}\right)\right)$.

Then we show that $\operatorname{Imp}\left(S_{1}^{\prime \prime}{ }^{i+1}, \operatorname{Use}(e) \cup \operatorname{LVar}\left(S_{1}^{\prime \prime}\right)\right)=$ $\operatorname{Imp}\left(S_{2}^{\prime \prime i+1}, \operatorname{Use}(e) \cup \operatorname{LVar}\left(S_{2}^{\prime \prime}\right)\right)$.

By Corollary C.1

$\operatorname{Imp}\left(S_{1}^{\prime \prime i+1}, \operatorname{Use}(e) \cup \operatorname{LVar}\left(S_{1}^{\prime \prime}\right)\right)=\operatorname{Imp}\left(S_{1}^{\prime \prime}, \operatorname{Imp}\left(S_{1}^{\prime \prime}{ }^{i}, \operatorname{Use}(e) \cup\right.\right.$ $\left.\left.\operatorname{LVar}\left(S_{1}^{\prime \prime}\right)\right)\right)$,

$\operatorname{Imp}\left(S_{2}^{\prime \prime i+1}, \operatorname{Use}(e) \cup \operatorname{LVar}\left(S_{2}^{\prime \prime}\right)\right)=\operatorname{Imp}\left(S_{2}^{\prime \prime}, \operatorname{Imp}\left(S_{2}^{\prime \prime}{ }^{i}, \operatorname{Use}(e) \cup\right.\right.$ $\left.\left.\operatorname{LVar}\left(S_{2}^{\prime \prime}\right)\right)\right)$.

By the hypothesis IH3, $\operatorname{Imp}\left(S_{1}^{\prime \prime}, \operatorname{Use}(e) \cup \operatorname{LVar}\left(S_{1}^{\prime \prime}\right)\right)=$ $\operatorname{Imp}\left(S_{2}^{\prime \prime i}, \operatorname{Use}(e) \cup \operatorname{LVar}\left(S_{2}^{\prime \prime}\right)\right)=\operatorname{LVar}(\Delta)$. 
Besides, by the definition of loop variables, $\operatorname{LVar}(\Delta) \subset$ $\operatorname{LVar}\left(S_{1}\right), \operatorname{LVar}(\Delta) \subseteq \operatorname{LVar}\left(S_{2}\right)$.

Then,

$\operatorname{Imp}\left(S_{1}^{\prime \prime}, \operatorname{Imp}\left(S_{1}^{\prime \prime}, \operatorname{Use}(e) \cup \operatorname{LVar}\left(S_{1}^{\prime \prime}\right)\right)\right)$

$=\operatorname{Imp}\left(S_{1}^{\prime \prime}, \operatorname{LVar}(\Delta)\right)$

$=\operatorname{Imp}\left(S_{2}^{\prime \prime}, \operatorname{LVar}(\Delta)\right)$ by Lemma 5.3

$=\operatorname{Imp}\left(S_{2}^{\prime \prime}, \operatorname{Imp}\left(S_{2}^{\prime \prime \prime}, \operatorname{Use}(e) \cup \operatorname{LVar}\left(S_{2}^{\prime \prime}\right)\right)\right)$

In summary, $\left.\operatorname{LVar}\left(S_{1}\right)\right)=\operatorname{LVar}\left(S_{2}\right)$. The lemma 5.6 holds.

2. $S_{1}$ and $S_{2}$ are not both one statement and one of the following holds:

(a) $S_{1}=S_{1}^{\prime} ; s_{1}$ and $S_{2}=S_{2}^{\prime} ; s_{2}$ such that all of the following hold:

- $S_{1}^{\prime} \equiv{ }_{H}^{S} S_{2}^{\prime}$;

- $S_{1}^{\prime}$ and $S_{2}^{\prime}$ have equivalent computation of $\operatorname{TVar}\left(s_{1}\right) \cup$ $\mathrm{TVar}\left(s_{2}\right)$;

- $s_{1} \equiv \stackrel{S}{S} s_{2}$ where $s_{1}$ and $s_{2}$ are not "skip";

By the hypothesis IH1, $\operatorname{LVar}\left(S_{1}^{\prime}\right)=\operatorname{LVar}\left(S_{2}^{\prime}\right), \operatorname{LVar}\left(s_{1}\right)=$ $\operatorname{LVar}\left(s_{2}\right)=\operatorname{LVar}(\Delta)$. Besides,

$\operatorname{Imp}\left(S_{1}^{\prime}, \operatorname{LVar}\left(s_{1}\right)\right)=\operatorname{LVar}\left(S_{2}^{\prime}, \operatorname{LVar}\left(s_{2}\right)\right)$ by Lemma 5.3 Therefore, $\operatorname{LVar}\left(S_{1}\right)=\operatorname{LVar}\left(S_{2}\right)$ by the definition of loop variables. The lemma 5.6 holds.

(b) One last statement is "skip": w.l.o.g.,

$\left(\left(S_{1} \equiv_{H}^{S} S_{2}^{\prime}\right) \wedge\left(s_{2}=\right.\right.$ "skip" $\left.)\right)$.

By the hypothesis IH1, $\operatorname{LVar}\left(S_{1}\right)=\operatorname{LVar}\left(S_{2}^{\prime}\right)$. Besides, $\operatorname{LVar}\left(s_{2}\right)=\emptyset$ by the definition of loop variables. Therefore, $\operatorname{LVar}\left(S_{1}\right)=\operatorname{LVar}\left(S_{2}\right)=\operatorname{LVar}\left(S_{2}^{\prime}\right) \cup \operatorname{Imp}\left(S_{2}^{\prime}, \emptyset\right)$. The lemma 5.6 holds.

(c) One last statement is a "duplicate" statement such that one of the following holds:

W.l.o.g. $S_{1}=S_{1}^{\prime} ; s_{1}^{\prime} ; S_{1}^{\prime \prime} ; s_{1}$ and all of the following hold:

- $S_{1}^{\prime} ; s_{1}^{\prime} ; S_{1}^{\prime \prime} \equiv_{H}^{S} S_{2}$;

- $s_{1}^{\prime} \equiv{ }_{H}^{S} s_{1}$;

- $\operatorname{Def}\left(s_{1}^{\prime} ; S_{1}^{\prime \prime}\right) \cap \operatorname{TV} \operatorname{Var}\left(s_{1}\right)=\emptyset$;

- $s_{2} \neq$ "skip";

By the hypothesis IH, LVar $\left(S_{1}^{\prime} ; s_{1}^{\prime} ; S_{1}^{\prime \prime}\right)=\operatorname{LVar}\left(S_{2}\right)$.

Then, we show that $\operatorname{Lar}\left(S_{1}^{\prime} ; s_{1}^{\prime} ; S_{1}^{\prime \prime}\right)=\operatorname{LVar}\left(S_{1}^{\prime} ; s_{1}^{\prime} ; S_{1}^{\prime \prime} ; s_{1}\right)$. By the induction hypothesis IH, LVar $\left(s_{1}^{\prime}\right)=\operatorname{LVar}\left(s_{1}\right)$.

$\operatorname{LVar}\left(S_{1}^{\prime} ; s_{1}^{\prime} ; S_{1}^{\prime \prime} ; s_{1}\right)$

$=\operatorname{LVar}\left(S_{1}^{\prime} ; s_{1}^{\prime} ; S_{1}^{\prime \prime}\right) \cup \operatorname{Imp}\left(S_{1}^{\prime} ; s_{1}^{\prime} ; S_{1}^{\prime \prime}, \operatorname{LVar}\left(s_{1}\right)\right)$ by the definition of loop variables

$\operatorname{Imp}\left(S_{1}^{\prime} ; s_{1}^{\prime} ; S_{1}^{\prime \prime}, \operatorname{LV} \operatorname{Var}\left(s_{1}\right)\right)$

$=\operatorname{Imp}\left(S_{1}^{\prime}, \operatorname{Imp}\left(s_{1}^{\prime} ; S_{1}^{\prime \prime}, \operatorname{LVar}\left(s_{1}\right)\right)\right)$ by LemmaC. 1

$=\operatorname{Imp}\left(S_{1}^{\prime}, \operatorname{LVar}\left(s_{1}\right)\right)$ by $\operatorname{Def}\left(s_{1}^{\prime} ; S_{1}^{\prime \prime}\right) \cap \operatorname{TV} \operatorname{Var}\left(s_{1}\right)=\emptyset$

$=\operatorname{Imp}\left(S_{1}^{\prime}, \operatorname{LVar}\left(s_{1}^{\prime}\right)\right)$ by $\operatorname{LVar}\left(s_{1}^{\prime}\right)=\operatorname{LVar}\left(s_{1}\right)$

$\subseteq \operatorname{LVar}\left(S_{1}^{\prime} ; s_{1}^{\prime}\right)$ by the definition of loop variables

$\subseteq \operatorname{LVar}\left(S_{1}^{\prime} ; s_{1}^{\prime} ; S_{1}^{\prime \prime}\right)$ by Lemma 5.5

In conclusion, $\operatorname{LVar}\left(S_{1}^{\prime} ; s_{1}^{\prime} ; S_{1}^{\prime \prime} ; s_{1}\right)=\operatorname{LVar}\left(S_{1}^{\prime} ; s_{1}^{\prime} ; S_{1}^{\prime \prime}\right)$. The lemma holds.

(d) $S_{1}=S_{1}^{\prime} ; s_{1} ; s_{1}^{\prime}$; and $S_{2}=S_{2}^{\prime} ; s_{2} ; s_{2}^{\prime}$ where $s_{1}$ and $s_{2}$ are reordered and all of the following hold:

- $S_{1}^{\prime} \equiv{ }_{H}^{S} S_{2}^{\prime}$;

- $S_{1}^{\prime}$ and $S_{2}^{\prime}$ have equivalent computation of $\operatorname{TVar}\left(s_{1} ; s_{1}^{\prime}\right) \cup$

$\operatorname{TVar}\left(s_{2} ; s_{2}^{\prime}\right)$.

- $s_{1} \equiv{ }_{H}^{S} s_{2}^{\prime}$;

- $s_{1}^{\prime} \equiv{ }_{H}^{S} s_{2}$;

- $\operatorname{Def}\left(s_{1}\right) \cap \operatorname{TVar}\left(s_{1}^{\prime}\right)=\emptyset$;

- $\operatorname{Def}\left(s_{2}\right) \cap \operatorname{TV} \operatorname{Var}\left(s_{2}^{\prime}\right)=\emptyset$;

By the hypothesis IH, $\operatorname{LVar}\left(S_{1}^{\prime}\right)=\operatorname{LVar}\left(S_{2}^{\prime}\right), \operatorname{LVar}\left(s_{1}\right)=$ $\operatorname{LVar}\left(s_{2}^{\prime}\right), \operatorname{LVar}\left(s_{1}^{\prime}\right)=\operatorname{LVar}\left(s_{2}\right)$.
In the following, we show $\operatorname{LVar}\left(S_{1}\right)=\operatorname{LVar}\left(S_{2}\right)$ in three steps.

i. We show $\operatorname{LVar}\left(S_{1}^{\prime} ; s_{1} ; s_{1}^{\prime}\right)=\operatorname{LVar}\left(S_{1}^{\prime}\right)$

$\cup \operatorname{Imp}\left(S_{1}^{\prime}, \operatorname{LVar}\left(s_{1}\right)\right) \cup \operatorname{Imp}\left(S_{1}^{\prime}, \operatorname{LVar}\left(s_{1}^{\prime}\right)\right)$.

$\operatorname{LVar}\left(S_{1}^{\prime} ; s_{1} ; s_{1}^{\prime}\right)$

$=\operatorname{LVar}\left(S_{1}^{\prime} ; s_{1}\right) \cup \operatorname{Imp}\left(S_{1}^{\prime} ; s_{1}, \operatorname{LVar}\left(s_{1}^{\prime}\right)\right)$

by the definition of loop variables

$\operatorname{LVar}\left(S_{1}^{\prime} ; s_{1}\right)$

$=\operatorname{LVar}\left(S_{1}^{\prime}\right) \cup \operatorname{Imp}\left(S_{1}^{\prime}, \operatorname{LVar}\left(s_{1}\right)\right)(2)$

by the definition of loop variables

$\operatorname{Imp}\left(S_{1}^{\prime} ; s_{1}, \operatorname{LV} \operatorname{Var}\left(s_{1}^{\prime}\right)\right)$

$=\operatorname{Imp}\left(S_{1}^{\prime}, \operatorname{Imp}\left(s_{1}, \operatorname{LVar}\left(s_{1}^{\prime}\right)\right)\right)$ by LemmaC.1.

$=\operatorname{Imp}\left(S_{1}^{\prime}, \operatorname{LVar}\left(s_{1}^{\prime}\right)\right)(3)$

by the condition $\operatorname{Def}\left(s_{1}\right) \cap \operatorname{TV} \operatorname{Var}\left(s_{1}^{\prime}\right)=\emptyset$

According to (2) and (3), $\operatorname{LVar}\left(S_{1}^{\prime} ; s_{1} ; s_{1}^{\prime}\right)=\operatorname{LVar}\left(S_{1}^{\prime}\right) \cup$ $\operatorname{Imp}\left(S_{1}^{\prime}, \operatorname{LVar}\left(s_{1}\right)\right) \cup \operatorname{Imp}\left(S_{1}^{\prime}, \operatorname{LVar}\left(s_{1}^{\prime}\right)\right)$.

Similarly, $\operatorname{LVar}\left(S_{2}^{\prime} ; s_{2} ; s_{2}^{\prime}\right)=\operatorname{LVar}\left(S_{2}^{\prime}\right) \cup$

$\operatorname{Imp}\left(S_{2}^{\prime}, \operatorname{LVar}\left(s_{2}\right)\right) \cup \operatorname{Imp}\left(S_{2}^{\prime}, \operatorname{LVar}\left(s_{2}^{\prime}\right)\right)$.

ii. We show that $\operatorname{Imp}\left(S_{1}^{\prime}, \operatorname{LVar}\left(s_{1}\right)\right)=\operatorname{Imp}\left(S_{2}^{\prime}, \operatorname{LVar}\left(s_{2}^{\prime}\right)\right)$. $\operatorname{Imp}\left(S_{2}^{\prime}, \operatorname{LVar}\left(s_{2}^{\prime}\right)\right)$.

We need to show that $\operatorname{LVar}\left(s_{1}\right) \subseteq \operatorname{LVar}\left(s_{1} ; s_{1}^{\prime}\right)$ and $\operatorname{LVar}\left(s_{2}^{\prime}\right) \subseteq \operatorname{LVar}\left(s_{2} ; s_{2}^{\prime}\right)$. By the definition of loop variables, $\operatorname{LVar}\left(s_{1}\right) \subseteq \operatorname{LVar}\left(s_{1} ; s_{1}^{\prime}\right)$. By the definition of loop variables again, $\operatorname{LVar}\left(s_{2} ; s_{2}^{\prime}\right)=\operatorname{LVar}\left(s_{2}\right) \cup$ $\operatorname{Imp}\left(s_{2}, \operatorname{LVar}\left(s_{2}^{\prime}\right)\right)$. Because $\operatorname{Def}\left(s_{2}\right) \cap \operatorname{TVar}\left(s_{2}^{\prime}\right)=\emptyset$, $\operatorname{Imp}\left(s_{2}, \operatorname{LVar}\left(s_{2}^{\prime}\right)\right)=\operatorname{LVar}\left(s_{2}^{\prime}\right)$.

By the induction hypothesis IH, LVar $\left(s_{1}\right)=\operatorname{LVar}\left(s_{2}^{\prime}\right)$. By Lemma 5.3, $\forall x \in \operatorname{LVar}\left(s_{1}\right)=\operatorname{LVar}\left(s_{2}^{\prime}\right), \operatorname{Imp}\left(S_{1}^{\prime},\{x\}\right)=$ $\operatorname{Imp}\left(S_{2}^{\prime},\{x\}\right)$. By Lemma C.2 $\operatorname{Imp}\left(S_{1}^{\prime}, \operatorname{LVar}\left(s_{1}\right)\right)=$ $\operatorname{Imp}\left(S_{2}^{\prime}, \operatorname{LVar}\left(s_{2}^{\prime}\right)\right)$

iii. We show that $\operatorname{Imp}\left(S_{1}^{\prime}, \operatorname{LVar}\left(s_{1}^{\prime}\right)\right)=\operatorname{Imp}\left(S_{2}^{\prime}, \operatorname{LV} \operatorname{Var}\left(s_{2}\right)\right)$. By the similar argument that $\operatorname{Imp}\left(S_{1}^{\prime}, \operatorname{LVar}\left(s_{1}\right)\right)=$ $\operatorname{Imp}\left(S_{2}^{\prime}, \operatorname{LVar}\left(s_{2}^{\prime}\right)\right)$.

In conclusion, $\operatorname{LVar}\left(S_{1}^{\prime} ; s_{1} ; s_{1}^{\prime}\right)=\operatorname{LVar}\left(S_{2}^{\prime} ; s_{2} ; s_{2}^{\prime}\right)$.

Lemma 5.7. If two statement sequences $S_{1}$ and $S_{2}$ satisfy the proof rule of termination in the same way, then $S_{1}$ and $S_{2}$ have same crash variables, $\left(S_{1} \equiv_{H}^{S} S_{2}\right) \Rightarrow\left(C \operatorname{Var}\left(S_{1}\right)=C \operatorname{Var}\left(S_{2}\right)\right)$.

By proof similar to those for Lemma 5.6

Corollary 5.1. If two statement sequences $S_{1}$ and $S_{2}$ satisfy the proof rule of termination in the same way, then $S_{1}$ and $S_{2}$ have same termination deciding variables, $\left(\begin{array}{lll}S_{1} & \equiv_{H}^{S} & S_{2}\end{array}\right) \Rightarrow$ $\left(T \operatorname{Var}\left(S_{1}\right)=T \operatorname{Var}\left(S_{2}\right)\right)$.

By Lemma 5.6 and 5.7

\section{Properties of the input sequence variable}

Lemma 5.8. If there is no input statement in a statement sequence $S$, then the input sequence variable is not in the defined variables of $S$, ( “input $x " \in S) \Rightarrow i d_{I} \notin \operatorname{Def}(S)$.

Proof. By induction on abstract syntax of $S$.

Lemma 5.9. If there is no input statement in a statement sequence $S$, then the input sequence variable is not in the crash variables of $S$, (尹"input $x " \in S) \Rightarrow\left(i d_{I} \notin C \operatorname{Var}(S)\right)$.

Proof. By induction on abstract syntax of $S$. 
Lemma 5.10. If there is no input statement in a statement sequence $S$, then the input sequence variable is in the loop variables of $S$, (Đ“input $x " \in S) \Rightarrow\left(i d_{I} \notin \operatorname{LVar}(S)\right)$.

Proof. By induction on abstract syntax of $S$.

Corollary 5.2. If there is no input statement in a statement sequence $S$, then the input sequence variable is in the termination deciding variables of $S$, ( $\nexists$ "input $x " \in S) \Rightarrow\left(i d_{I} \notin T \operatorname{Var}(S)\right.$ ).

By Lemma 5.9 and 5.10

Lemma 5.11. If there is one input statement in a statement sequence $S$, then the input sequence variable is in the crash variables and defined variables of $S,(\exists$ "input $x " \in S) \Rightarrow\left(i d_{I} \in\right.$ $C \operatorname{Var}(S)) \wedge\left(i d_{I} \in \operatorname{Def}(S)\right)$.

Proof. By induction on abstract syntax of $S$.

Lemma 5.12. If there is one input statement in a statement sequence $S$, then the imported variables in $S$ relative to the input sequence variable are a subset of the crash variables of $S$, $(\exists$ "input $x " \in S) \Rightarrow\left(\operatorname{Imp}\left(S,\left\{i d_{I}\right\}\right) \subseteq C \operatorname{Var}(S)\right)$.

Proof. By induction on abstract syntax of $S$.

1. $S=$ "input $x "$.

By the definition of $\mathrm{CVar}(\cdot)$ and $\operatorname{Imp}(\cdot), \mathrm{CVar}(S)=\operatorname{Imp}\left(S,\left\{i d_{I}\right\}\right)=$ $\left\{i d_{I}\right\}$.

2. $S=$ "If $(e)$ then $\left\{S_{t}\right\}$ else $\left\{S_{f}\right\} "$.

W.l.o.g., there is input statement in $S_{t}$, by the induction hypothesis, $\operatorname{Imp}\left(S_{t},\left\{i d_{I}\right\}\right) \subseteq \mathrm{CVar}\left(S_{t}\right)$. There are two subcases regarding if input statement is in $S_{f}$.

(a) There is input statement in $S_{f}$.

By the induction hypothesis, $\operatorname{Imp}\left(S_{f},\left\{i d_{I}\right\}\right) \subseteq \operatorname{CVar}\left(S_{f}\right)$.

Hence, the lemma holds.

(b) There is no input statement in $S_{f}$.

By the definition of imported variables, $\operatorname{Imp}\left(S_{f},\left\{i d_{I}\right\}\right)=$ $\left\{i d_{I}\right\}$. By Lemma 5.11 $i d_{I} \in \operatorname{CVar}\left(S_{t}\right)$. Therefore, the lemma holds.

3. $S=$ "while $\langle n\rangle(e)\left\{S^{\prime}\right\} "$.

By the induction hypothesis, $\operatorname{Imp}\left(S^{\prime},\left\{i d_{I}\right\}\right) \subseteq \mathrm{CVar}\left(S^{\prime}\right)$.

By the definition of $\operatorname{Imp}(\cdot), \operatorname{Imp}\left(S^{\prime},\left\{i d_{I}\right\}\right)=\bigcup_{i \geq 0} \operatorname{Imp}\left(S^{\prime i},\left\{i d_{I}\right\} \cup\right.$ Use $(e))$. By the definition of $\mathrm{C} \operatorname{Var}(\cdot), \mathrm{C} \operatorname{Var}\left(S^{\prime}\right) \stackrel{=}{=}$

$\bigcup_{i \geq 0} \operatorname{Imp}\left(S^{\prime i}, C \operatorname{Var}\left(S^{\prime}\right) \cup \operatorname{Use}(e)\right)$.

By induction on $i$, we show that, $\forall i \geq 0, \operatorname{Imp}\left(S^{\prime i},\left\{i d_{I}\right\} \cup\right.$

$\mathrm{Use}(e)) \subseteq \operatorname{Imp}\left({S^{\prime}}^{i}, \mathrm{C} \operatorname{Var}\left(S^{\prime}\right) \cup \mathrm{Use}(e)\right)$.

Base case $i=0$

By notation $S^{\prime 0}=$ skip.

$\operatorname{Imp}\left(S^{\prime 0},\left\{i d_{I}\right\} \cup \operatorname{Use}(e)\right)$

$=\left\{i d_{I}\right\} \cup \operatorname{Use}(e)$ by the definition of imported variables

$\operatorname{Imp}\left({S^{\prime}}^{0}, \mathrm{CVar}\left(S^{\prime}\right) \cup \operatorname{Use}(e)\right)$

$=\mathrm{C} \operatorname{Var}\left(S^{\prime}\right) \cup \mathrm{Use}(e)$ by the definition of imported variables

$$
\begin{aligned}
& i d_{I} \subseteq \mathrm{CVar}\left(S^{\prime}\right)(1) \text { by Lemma } 5.11 \\
& \operatorname{Imp}\left({S^{\prime}}^{0},\left\{i d_{I}\right\} \cup \operatorname{Use}(e)\right) \\
\subseteq & \operatorname{Imp}\left({S^{\prime}}^{0}, \mathrm{CVar}\left(S^{\prime}\right) \cup \operatorname{Use}(e)\right) \text { by Lemma C. } 2
\end{aligned}
$$

Induction step.

The hypothesis IH1 is that $\operatorname{Imp}\left({S^{\prime}}^{i},\left\{i d_{I}\right\} \cup \operatorname{Use}(e)\right) \subseteq$ $\operatorname{Imp}\left(S^{\prime}, C \operatorname{Var}\left(S^{\prime}\right) \cup \operatorname{Use}(e)\right)$ for $i>0$.

Then we show that $\operatorname{Imp}\left(S^{\prime i+1},\left\{i d_{I}\right\} \cup \operatorname{Use}(e)\right)$

$\subseteq \operatorname{Imp}\left(S^{\prime i+1}, \mathrm{CVar}\left(S^{\prime}\right) \cup \operatorname{Use}(e)\right)$

$$
\begin{aligned}
& \operatorname{Imp}\left(S^{\prime i},\left\{i d_{I}\right\} \cup \operatorname{Use}(e)\right) \\
\subseteq & \operatorname{Imp}\left(S^{\prime i}, \mathrm{CVar}\left(S^{\prime}\right) \cup \operatorname{Use}(e)\right)(1) \text { by the hypothesis IH1 } \\
& \operatorname{Imp}\left({S^{\prime}}^{i+1},\left\{i d_{I}\right\} \cup \operatorname{Use}(e)\right) \\
= & \operatorname{Imp}\left(S^{\prime}, \operatorname{Imp}\left(S^{\prime i},\left\{i d_{I}\right\} \cup \operatorname{Use}(e)\right)\right)(2) \text { by Corollary C.1 } \\
& \operatorname{Imp}\left({S^{\prime}}^{i+1}, \mathrm{CVar}\left(S^{\prime}\right) \cup \operatorname{Use}(e)\right) \\
= & \operatorname{Imp}\left(S^{\prime}, \operatorname{Imp}\left(S^{\prime i}, \mathrm{CVar}\left(S^{\prime}\right) \cup \operatorname{Use}(e)\right)\right) \text { (3) by Corollary C.1 }
\end{aligned}
$$

Combining (1), (2) and (3):

$$
\begin{aligned}
& \operatorname{Imp}\left(S^{\prime}, \operatorname{Imp}\left(S^{\prime i},\left\{i d_{I}\right\} \cup \operatorname{Use}(e)\right)\right) \\
\subseteq & \operatorname{Imp}\left(S^{\prime}, \operatorname{Imp}\left(S^{\prime i}, C \operatorname{Var}\left(S^{\prime}\right) \cup \operatorname{Use}(e)\right)\right) \text { by LemmaC. } 2
\end{aligned}
$$

Therefore, $\operatorname{Imp}\left({S^{\prime}}^{i+1},\left\{i d_{I}\right\} \cup U \operatorname{Use}(e)\right) \subseteq \operatorname{Imp}\left({S^{\prime}}^{i+1}, \operatorname{CVar}\left(S^{\prime}\right) \cup\right.$ Use $(e))$.

In conclusion, $\operatorname{Imp}\left(S,\left\{i d_{I}\right\}\right) \subseteq \mathrm{CVar}(S)$.

4. $S=s_{1} ; \ldots ; s_{k}$, for $k>0$.

By induction on $k$.

Base case. $k=1$.

By above cases, the lemma holds.

Induction step.

The induction hypothesis IH2 is that the lemma holds when $k>0$. We show that the lemma holds when $S=s_{1} ; \ldots ; s_{k+1}$. By the definition of crash variables, $\operatorname{CVar}\left(s_{1} ; \ldots ; s_{k+1}\right)=$ $\mathrm{C} \operatorname{Var}\left(s_{1} ; \ldots ; s_{k}\right) \cup \operatorname{Imp}\left(s_{1} ; \ldots ; s_{k}, \mathrm{CVar}\left(s_{k+1}\right)\right)$. There are two possibilities.

(a) \# "input $x " \in s_{k+1}$.

$$
\begin{aligned}
\text { By } \operatorname{Lemma} 5.8 i d_{I} \notin \operatorname{Def}(S) . \\
\\
\quad \operatorname{Imp}\left(s_{1} ; \ldots ; s_{k+1},\left\{i d_{I}\right\}\right) \\
=\operatorname{Imp}\left(s_{1} ; \ldots ; s_{k},\left\{i d_{I}\right\}\right) \text { by } i d_{I} \notin \operatorname{Def}(S) \text { and } \\
\text { the definition of imported variables } \\
\subseteq \mathrm{CVar}\left(s_{1} ; \ldots ; s_{k}\right) \text { by the hypothesis IH } 2 \\
\subseteq \mathrm{CVar}\left(s_{1} ; \ldots ; s_{k+1}\right) \text { by the definition of crash variables }
\end{aligned}
$$

(b) $\exists$ "input $x " \in s_{k+1}$.

$$
\begin{aligned}
& \operatorname{Imp}\left(s_{1} ; \ldots ; s_{k+1},\left\{i d_{I}\right\}\right) \\
= & \operatorname{Imp}\left(s_{1} ; \ldots ; s_{k}, \operatorname{Imp}\left(s_{k+1},\left\{i d_{I}\right\}\right)\right) \\
& \text { by the definition of imported variables }
\end{aligned}
$$

$$
\operatorname{Imp}\left(s_{k+1},\left\{i d_{I}\right\}\right)
$$

$\subseteq \mathrm{CVar}\left(s_{k+1}\right)$ by the hypothesis IH2

$$
\begin{aligned}
& \operatorname{Imp}\left(s_{1} ; \ldots ; s_{k}, \operatorname{Imp}\left(s_{k+1},\left\{i d_{I}\right\}\right)\right) \\
\subseteq & \operatorname{Imp}\left(s_{1} ; \ldots ; s_{k}, \mathrm{CVar}\left(s_{k+1}\right)\right) \text { by LemmaC.2 } \\
\subseteq & \operatorname{CVar}\left(s_{1} ; \ldots ; s_{k+1}\right) \text { by the definition of crash variables. }
\end{aligned}
$$

Lemma 5.13. If two programs $S_{1}$ and $S_{2}$ satisfy the proof rule of termination in the same way, then $S_{1}$ and $S_{2}$ satisfy the proof rule of terminating computation in the same way of the input sequence, $\left(S_{1} \equiv_{H}^{S} S_{2}\right) \Rightarrow\left(S_{1} \equiv_{i d_{I}}^{S} S_{2}\right)$.

Proof. By induction on size $\left(S_{1}\right)+\operatorname{size}\left(S_{2}\right)$. Base case. $S_{1}$ and $S_{2}$ are simple statements.

There are three cases.

1. $S_{1}$ and $S_{2}$ are "skip": $S_{1}=S_{2}=$ "skip";

2. $S_{1}$ and $S_{2}$ are input statement: $S_{1}=$ "input $i d_{1}$ ", $S_{2}=$ "input $i d_{2}$ "; 
3. $s_{1}$ and $s_{2}$ are with the same expression: $s_{1}=$ "output $e$ " or " $i d_{1}:=e ", s_{2}=$ "output $e$ " or " $i d_{2}:=e$ ".

By definition of the proof rule of equivalent computation, the lemma holds in above three cases.

Induction step.

The hypothesis IH is that the lemma holds when $\operatorname{size}\left(S_{1}\right)+$ $\operatorname{size}\left(S_{2}\right)=k \geq 2$.

Then, we show that the lemma holds when $\operatorname{size}\left(S_{1}\right)+\operatorname{size}\left(S_{2}\right)=$ $k+1$. The proof is a case analysis of the cases in the proof rule of termination in the same way.

1. $S_{1}$ and $S_{2}$ are one statement and one of the followings holds.

(a) $S_{1}=$ "If $(e)$ then $\left\{S_{1}^{t}\right\}$ else $\left\{S_{1}^{f}\right\} ", S_{2}=$ "If $(e)$ then $\left\{S_{2}^{t}\right\}$ else $\left\{S_{2}^{f}\right\}$ " and one of the followings holds:

i. $S_{1}^{t}, S_{1}^{f}, S_{2}^{t}, S_{2}^{f}$ are all sequences of "skip";

By Lemma 5.8, $i d_{I} \notin \operatorname{Def}\left(S_{1}\right) \cap \operatorname{Def} S_{2}$. The lemma holds.

ii. At least one of $S_{1}^{t}, S_{1}^{f}, S_{2}^{t}, S_{2}^{f}$ is not a sequence of "skip" such that:

$\left(S_{1}^{t} \equiv_{H}^{S} S_{2}^{t}\right) \wedge\left(S_{1}^{f} \equiv_{H}^{S} S_{2}^{f}\right)$;

Because size $\left(S_{1}\right)=1+\operatorname{size}\left(S_{1}^{t}\right)+\operatorname{size}\left(S_{1}^{f}\right), \operatorname{size}\left(S_{2}\right)=$ $1+\operatorname{size}\left(S_{2}^{t}\right)+\operatorname{size}\left(S_{2}^{f}\right)$. Therefore, $\operatorname{size}\left(S_{1}^{t}\right)+\operatorname{size}\left(S_{2}^{t}\right)<$ $k, \operatorname{size}\left(S_{1}^{f}\right)+\operatorname{size}\left(S_{2}^{f}\right)<k$. By the induction hypothesis IH, $\left(S_{1}^{t} \equiv_{i d_{I}}^{S} S_{2}^{t}\right) \wedge\left(S_{1}^{f} \equiv_{i d_{I}}^{S} S_{2}^{f}\right)$. Then, the lemma holds by the definition of $S_{1} \equiv{ }_{i d_{I}}^{S} S_{2}$.

(b) $S_{1}=$ "while $\left\langle n_{1}\right\rangle(e)\left\{S_{1}^{\prime \prime}\right\} ", S_{2}=$ "while ${ }_{\left\langle n_{2}\right\rangle}(e)\left\{S_{2}^{\prime \prime}\right\} "$ and both of the followings hold:

- $S_{1}^{\prime \prime} \equiv{ }_{H}^{S} S_{2}^{\prime \prime}$;

- $S_{1}^{\prime \prime}$ and $S_{2}^{\prime \prime}$ have equivalent computation of $\operatorname{TVar}\left(S_{1}\right) \cup$ $\mathrm{T} \operatorname{Var}\left(S_{2}\right)$;

By the induction hypothesis $\mathrm{IH}, S_{1}^{\prime \prime} \equiv_{i d_{I}}^{S} S_{2}^{\prime \prime}$. In addition, by Corollary 5.1. $\mathrm{T} \operatorname{Var}\left(S_{1}\right)=\mathrm{T} \operatorname{Var}\left(S_{2}\right)$. There are two cases.

i. $i d_{I} \in \mathrm{T} \operatorname{Var}\left(S_{1}\right)=\mathrm{T} \operatorname{Var}\left(S_{2}\right)$

We show that $i d_{I} \in \operatorname{Def}\left(S_{1}\right) \cap \operatorname{Def}\left(S_{2}\right)$. If there is no input statement in $S_{1}$ or $S_{2}$, then, by Corollary 5.2 $i d_{I} \notin \mathrm{T} \operatorname{Var}\left(S_{1}\right) \cap \mathrm{T} \operatorname{Var}\left(S_{2}\right)$. A contradiction. Thus, there is input statement in $S_{1}$ and $S_{2}$, by Lemma 5.11 $i d_{I} \in \operatorname{Def}\left(S_{1}\right) \cap \operatorname{Def}\left(S_{2}\right)$.

By Lemma 5.12 $\operatorname{Imp}\left(S_{1},\left\{i d_{I}\right\}\right) \subseteq \mathrm{CVar}\left(S_{1}\right)$. Similarly, $\operatorname{Imp}\left(S_{2},\left\{i d_{I}\right\}\right) \subseteq \mathrm{T} \operatorname{Var}\left(S_{2}\right)$. Hence, loop bodies of $S_{1}$ and $S_{2}$ equivalently compute every of the imported variables in $S_{1}$ and $S_{2}$ relative to the input sequence variable, $\forall x \in \operatorname{Imp}\left(S_{1},\left\{i d_{I}\right\}\right) \cup \operatorname{Imp}\left(S_{2},\left\{i d_{I}\right\}\right)$, $S_{1}^{\prime \prime} \equiv_{x}^{S} S_{2}^{\prime \prime}$. Thus, the lemma holds.

ii. $i d_{I} \notin \mathrm{T} \operatorname{Var}\left(S_{1}\right)=\mathrm{T} \operatorname{Var}\left(S_{2}\right)$.

Then there is no input statement in $S_{1}$ and $S_{2}$. Otherwise, by Lemma 5.11 $\left(i d_{I} \in \mathrm{CVar}\left(S_{1}\right)\right) \vee\left(i d_{I} \in\right.$ CVar $\left.\left(S_{2}\right)\right)$. A contradiction. Then by Lemma5.8 $i d_{I} \notin$ $\left(\operatorname{Def}\left(S_{1}\right) \cap \operatorname{Def}\left(S_{2}\right)\right)$. Hence, the lemma holds.

2. $S_{1}$ and $S_{2}$ are not both one statement and one of the followings holds:

(a) $S_{1}=S_{1}^{\prime} ; s_{1}$ and $S_{2}=S_{2}^{\prime} ; s_{2}$ and all of the followings hold:

- $S_{1}^{\prime} \equiv{ }_{H}^{S} S_{2}^{\prime}$;

- $S_{1}^{\prime}$ and $S_{2}^{\prime}$ have equivalent computation of $\operatorname{TVar}\left(s_{1}\right) \cup$ $\mathrm{T} \operatorname{Var}\left(s_{2}\right)$;

- $s_{1} \equiv \stackrel{S}{S} s_{2}$ where $s_{1}$ and $s_{2}$ are not "skip";

By the induction hypothesis IH, $s_{1} \equiv{ }_{i d_{I}}^{S} s_{2}$. By Corollary $5.1 \mathrm{~T} \operatorname{Var}\left(s_{1}\right)=\mathrm{TVar}\left(s_{2}\right)$. There are two cases. i. $i d_{I} \in \mathrm{T} \operatorname{Var}\left(s_{1}\right)=\mathrm{T} \operatorname{Var}\left(s_{2}\right)$

Then there is input statement in $s_{1}$ and $s_{2}$. Otherwise, by Lemma5.2 $i d_{I} \notin \mathrm{T} \operatorname{Var}\left(s_{1}\right)=\mathrm{T} \operatorname{Var}\left(s_{2}\right)$. A contradiction. Then, by Lemma 5.11 $i d_{I} \in \operatorname{Def}\left(s_{1}\right) \cap \operatorname{Def}\left(s_{2}\right)$. By Lemma5.3 $\operatorname{Imp}\left(s_{1},\left\{i d_{I}\right\}\right)=\operatorname{Imp}\left(s_{2},\left\{i d_{I}\right\}\right)$

By Lemma 5.12 $\operatorname{Imp}\left(s_{1},\left\{i d_{I}\right\}\right) \subseteq \mathrm{CVar}\left(s_{1},\left\{i d_{I}\right\}\right)$. Therefore, $S_{1}^{\prime}$ and $S_{2}^{\prime}$ equivalently compute $\operatorname{Imp}\left(s_{1},\left\{i d_{I}\right\}\right) \cup$ $\operatorname{Imp}\left(s_{2},\left\{i d_{I}\right\}\right)$. The lemma holds.

ii. $i d_{I} \notin \mathrm{T} \operatorname{Var}\left(s_{1}\right)=\mathrm{T} \operatorname{Var}\left(s_{2}\right)$.

Then, there is no input statement in $s_{1}$ and $s_{2}$. Otherwise, by Lemma 5.11 $i d_{I} \in \mathrm{CVar}\left(s_{1}\right)=\mathrm{CVar}\left(s_{2}\right)$. A contradiction. Then, by Lemma 5.8, $i d_{I} \notin \operatorname{Def}\left(s_{1}\right) \cup$ $\operatorname{Def}\left(s_{2}\right)$. By the induction hypothesis IH, $S_{1}^{\prime} \equiv_{i d_{I}}^{S} S_{2}^{\prime}$. The lemma holds.

(b) One last statement is "skip": W.1.o.g., $\left(\left(S_{1}^{\prime} \equiv_{H}^{S} S_{2}\right) \wedge\left(s_{1}=\right.\right.$ "skip"))

By the induction hypothesis, $S_{1}^{\prime} \equiv_{i d_{I}}^{S} S_{2}$. By definition, $i d_{I} \notin \operatorname{Def}\left(s_{1}\right)$. The lemma holds.

(c) One last statement is a "duplicate" statement such that one of the followings holds:

W.l.o.g., $S_{1}=S_{1}^{\prime} ; s_{1}^{\prime} ; S_{1}^{\prime \prime} ; s_{1}$ and all of the followings hold:

- $S_{1}^{\prime} ; s_{1}^{\prime} ; S_{1}^{\prime \prime} \equiv_{H}^{S} S_{2}$;

- $s_{1}^{\prime} \equiv{ }_{H}^{S} s_{1}$;

- $\operatorname{Def}\left(s_{1}^{\prime} ; S_{1}^{\prime \prime}\right) \cap \operatorname{TVar}\left(s_{1}\right)=\emptyset$;

- $s_{2} \neq$ "skip".

By the induction hypothesis, $S_{1}^{\prime} ; s_{1}^{\prime} ; S_{1}^{\prime \prime} \equiv_{i d_{I}}^{S} S_{2}$. In the proof of Theorem 3 , there is no input statement in $s_{2}$. Because $\forall x$ : "input $x$ " $\notin s_{2}$, by Lemma5.8, $i d_{I} \notin \operatorname{Def}\left(s_{1}\right)$. The lemma holds.

(d) $S_{1}=S_{1}^{\prime} ; s_{1} ; s_{1}^{\prime}$; and $S_{2}=S_{2}^{\prime} ; s_{2} ; s_{2}^{\prime}$ where $s_{1}$ and $s_{2}$ are reordered and all of the followings hold:

- $S_{1}^{\prime} \equiv \stackrel{S}{S} S_{2}^{\prime}$;

- $S_{1}^{\prime}$ and $S_{2}^{\prime}$ have equivalent computation of $\operatorname{TVar}\left(s_{1} ; s_{1}^{\prime}\right) \cup$ $\operatorname{TVar}\left(s_{2} ; s_{2}^{\prime}\right)$.

- $s_{1} \equiv{ }_{H}^{S} s_{2}^{\prime}$;

- $s_{1}^{\prime} \equiv{ }_{H}^{S} s_{2}$;

- $\operatorname{Def}\left(s_{1}\right) \cap \operatorname{TV} \operatorname{Var}\left(s_{1}^{\prime}\right)=\emptyset$;

- $\operatorname{Def}\left(s_{2}\right) \cap \operatorname{TVar}\left(s_{2}^{\prime}\right)=\emptyset$;

In the proof of Theorem 4 we showed that $s_{1}$ and $s_{2}$ do not both include input statement, $s_{2}$ and $s_{2}^{\prime}$ do not both include input statement. There are two subcases.

i. There are no input statements in both $s_{1}$ and $s_{1}^{\prime}$.

We show that there are no input statements in both $s_{2}$ and $s_{2}^{\prime}$. By Corollary 5.1. $\operatorname{TVar}\left(s_{1}\right)=\operatorname{TVar}\left(s_{2}^{\prime}\right)$ and $\operatorname{TVar}\left(s_{1}^{\prime}\right)=\operatorname{TVar}\left(s_{2}\right)$. By Corollary $5.2 i d_{I} \notin$ $\operatorname{TVar}\left(s_{1}\right) \cup \mathrm{T} \operatorname{Var}\left(s_{1}^{\prime}\right)$. Thus, $i d_{I} \notin \mathrm{TVar}\left(s_{2}\right) \cup \mathrm{TVar}\left(s_{2}^{\prime}\right)$. If there is input statement in $s_{2}$ or $s_{2}^{\prime}$, then, by Lemma 5.11 $i d_{I} \notin \mathrm{T} \operatorname{Var}\left(s_{2}\right) \cup \mathrm{T} \operatorname{Var}\left(s_{2}^{\prime}\right)$. A contradiction. In summary, there are no input statements in both $s_{2}$ and $s_{2}^{\prime}$.

By Lemma 5.8 $i d_{I} \notin \operatorname{Def}\left(s_{1} ; s_{1}^{\prime}\right)$ and $i d_{I} \notin \operatorname{Def}\left(s_{2} ; s_{2}^{\prime}\right)$. By the induction hypothesis, $S_{1}^{\prime} \equiv_{i d_{I}}^{S} S_{2}^{\prime}$. Therefore, the lemma holds.

ii. W.l.o.g, there are input statements in $s_{1}$ only. By similar argument in the proof of Theorem 4 that $s_{1}$ and $s_{2}$ do not both include input statements, we can show that there is no input statement in $s_{2}$ and there is input statement in $s_{2}^{\prime}$.

In the following, the proof is of two steps.

A. We show that $s_{1} ; s_{1}^{\prime} \equiv_{i d_{I}}^{S} s_{2}^{\prime}$.

By the induction hypothesis IH, $s_{1} \equiv_{i d_{I}}^{S} s_{2}^{\prime}$. Because there is no input statement in $s_{1}^{\prime}$, then by 
Lemma 5.8, $i d_{I} \notin \operatorname{Def}\left(s_{1}^{\prime}\right)$. Thus, $s_{1} ; s_{1}^{\prime} \equiv_{i d_{I}}^{S} s_{2}^{\prime}$ by definition.

B. We show that $S_{1}^{\prime}$ and $S_{2}^{\prime} ; s_{2}$ equivalently compute $\operatorname{Imp}\left(s_{1} ; s_{1}^{\prime},\left\{i d_{I}\right\}\right) \cup \operatorname{Imp}\left(s_{2}^{\prime},\left\{i d_{I}\right\}\right)$.

The argument is of two parts. First, we need to show that $\operatorname{Def}\left(s_{2}\right) \cap \operatorname{Imp}\left(s_{2}^{\prime},\left\{i d_{I}\right\}\right)=\emptyset$. By Lemma 5.12 $\operatorname{Imp}\left(s_{2}^{\prime},\left\{i d_{I}\right\}\right) \subseteq \mathrm{CVar}\left(s_{2}^{\prime}\right)$. Thus, $\operatorname{Imp}\left(s_{2}^{\prime},\left\{i d_{I}\right\}\right) \subseteq \operatorname{TVar}\left(s_{2}^{\prime}\right)$. By assumption, $\operatorname{Def}\left(s_{2}\right) \cap$ $\operatorname{TVar}\left(s_{2}^{\prime}\right)=\emptyset$. Then, $\operatorname{Def}\left(s_{2}\right) \cap \operatorname{Imp}\left(s_{2}^{\prime},\left\{i d_{I}\right\}\right)=\emptyset$. By Lemma 5.3 $\operatorname{Imp}\left(s_{1} ; s_{1}^{\prime},\left\{i d_{I}\right\}\right)=\operatorname{Imp}\left(s_{2}^{\prime},\left\{i d_{I}\right\}\right)$. Thus, $\operatorname{Def}\left(s_{2}\right) \cap \operatorname{Imp}\left(s_{1} ; s_{1}^{\prime},\left\{i d_{I}\right\}\right)=\emptyset$.

Second, we show that $\operatorname{Imp}\left(s_{2}^{\prime},\left\{i d_{I}\right\}\right) \subseteq \operatorname{TVar}\left(s_{2} ; s_{2}^{\prime}\right)$ and $\operatorname{Imp}\left(s_{1} ; s_{1}^{\prime},\left\{i d_{I}\right\}\right) \subseteq \mathrm{TVar}\left(s_{1} ; s_{1}^{\prime}\right)$. By Lemma5.12 $\operatorname{Imp}\left(s_{1} ; s_{1}^{\prime},\left\{i d_{I}\right\}\right) \subseteq \mathrm{TV} \operatorname{Var}\left(s_{1} ; s_{1}^{\prime}\right)$ and $\operatorname{Imp}\left(s_{2}^{\prime},\left\{i d_{I}\right\}\right) \subseteq$ $\mathrm{T} \operatorname{Var}\left(s_{2}^{\prime}\right)$. Then we show that $\mathrm{T} \operatorname{Var}\left(s_{2}^{\prime}\right) \subseteq \operatorname{TVar}\left(s_{2} ; s_{2}^{\prime}\right)$. We need to show that $\mathrm{C} \operatorname{Var}\left(s_{2}^{\prime}\right) \subseteq \operatorname{CVar}\left(s_{2} ; s_{2}^{\prime}\right)$ and $\operatorname{LVar}\left(s_{2}^{\prime}\right) \subseteq \operatorname{LVar}\left(s_{2} ; s_{2}^{\prime}\right)$.

$\mathrm{CVar}\left(s_{2} ; s_{2}^{\prime}\right)$

$=\mathrm{CVar}\left(s_{2}\right) \cup \operatorname{Imp}\left(s_{2}, \mathrm{C} \operatorname{Var}\left(s_{2}^{\prime}\right)\right)$

by the definition of crash variables

$\operatorname{Imp}\left(s_{2}, \mathrm{CVar}\left(s_{2}^{\prime}\right)\right)$

$=\mathrm{CVar}\left(s_{2}^{\prime}\right)(1)$ by the assumption

$\operatorname{Def}\left(s_{2}\right) \cap \operatorname{TVar}\left(s_{2}^{\prime}\right)=\emptyset$

$\operatorname{CVar}\left(s_{2}\right) \cup \operatorname{Imp}\left(s_{2}, \mathrm{CVar}\left(s_{2}^{\prime}\right)\right)$

$=\mathrm{C} \operatorname{Var}\left(s_{2}\right) \cup \mathrm{C} \operatorname{Var}\left(s_{2}^{\prime}\right)$ by $(1)$

Similarly, $\operatorname{LVar}\left(s_{2}^{\prime}\right) \subseteq \operatorname{LVar}\left(s_{2} ; s_{2}^{\prime}\right)$. Thus, $\operatorname{TVar}\left(s_{2}^{\prime}\right) \subseteq$ $\operatorname{TVar}\left(s_{2} ; s_{2}^{\prime}\right)$.

By assumption, $\forall x \in \operatorname{Imp}\left(s_{1} ; s_{1}^{\prime},\left\{i d_{I}\right\}\right) \cup \operatorname{Imp}\left(s_{2}^{\prime},\left\{i d_{I}\right\}\right)$ $S_{1}^{\prime} \equiv{ }_{x}^{S} S_{2}^{\prime}$. In addition, $\operatorname{Def}\left(s_{2}\right) \cap\left(\operatorname{Imp}\left(s_{1} ; s_{1}^{\prime},\left\{i d_{I}\right\}\right) \cup\right.$ $\left.\operatorname{Imp}\left(s_{2}^{\prime},\left\{i d_{I}\right\}\right)\right)=\emptyset$. Thus, $\forall x \in \operatorname{Imp}\left(s_{1} ; s_{1}^{\prime},\left\{i d_{I}\right\}\right) \cup$ $\operatorname{Imp}\left(s_{2}^{\prime},\left\{i d_{I}\right\}\right): S_{1}^{\prime} \equiv_{x}^{S} S_{2}^{\prime} ; s_{2}$. The lemma holds.

Lemma 5.14. If two programs $S_{1}$ and $S_{2}$ satisfy the proof rule of termination in the same way, and $S_{1}$ and $S_{2}$ both terminate when started in their initial states with crash flags not set, $\mathfrak{f}_{1}=$ $\mathfrak{f}_{2}=0$, whose value stores agree on values of variables of the termination deciding variables of $S_{1}$ and $S_{2}, \forall x \in T \operatorname{Var}\left(S_{1}\right) \cup$ $T \operatorname{Var}\left(S_{2}\right), \sigma_{1}(x)=\sigma_{2}(x)$, and $S_{1}$ and $S_{2}$ are fed with the same infinite input sequence, $\sigma_{1}\left(i d_{I}\right)=\sigma_{2}\left(i d_{I}\right),\left(S_{1}, m_{1}\left(\mathfrak{f}_{1}, \sigma_{1}\right) \stackrel{*}{\rightarrow}\right.$ (skip, $\left.m_{1}^{\prime}\left(\sigma_{1}^{\prime}\right)\right)$ and $\left(S_{2}, m_{2}\left(\mathfrak{f}_{2}, \sigma_{2}\right) \stackrel{*}{\rightarrow}\right.$ (skip, $\left.m_{2}^{\prime}\left(\sigma_{2}^{\prime}\right)\right)$, then the execution of $S_{1}$ and $S_{2}$ consume the same number of input values, $\sigma_{1}^{\prime}\left(i d_{I}\right)=\sigma_{2}^{\prime}\left(i d_{I}\right)$.

Proof. By Lemma5.13 $S_{1} \equiv_{i d_{I}}^{S} S_{2}$. By Lemma5.12 $\operatorname{Imp}\left(S_{1}, i d_{I}\right) \subseteq$ $\mathrm{CVar}\left(S_{1}\right)$ and $\operatorname{Imp}\left(S_{2}, i d_{I}\right) \subseteq \mathrm{C} \operatorname{Var}\left(S_{2}\right)$. By assumption, $\forall x \in$ $\operatorname{Imp}\left(S_{1}, i d_{I}\right) \cup \operatorname{Imp}\left(S_{2}, i d_{I}\right): \sigma_{1}(x)=\sigma_{2}(x)$. By Theorem 2 , $\sigma_{1}^{\prime}\left(i d_{I}\right)=\sigma_{2}^{\prime}\left(i d_{I}\right)$.

\section{Theorem of two loop statements terminating in the same way}

Lemma 5.15. Let $s_{1}=$ "while ${ }_{\left\langle n_{1}\right\rangle}(e)\left\{S_{1}\right\} "$ and $s_{2}=$ "while $\left\langle n_{2}\right\rangle(e)\left\{S_{2}\right\}$ " be two while statements with the same set of termination deciding variables in program $P_{1}$ and $P_{2}$ respectively, whose bodies $S_{1}$ and $S_{2}$ satisfy the proof rule of equivalently computation of variables in TVar $(s)$, and $S_{1}$ and $S_{2}$ terminate in the same way when started in states with crash flags not set and agreeing on values of variables in $\operatorname{TVar}\left(S_{1}\right) \cup \operatorname{TVar}\left(S_{2}\right)$ :

- $\operatorname{Tar}\left(s_{1}\right)=T \operatorname{Var}\left(s_{2}\right)=T \operatorname{Var}(s)$;

- $\forall x \in T \operatorname{Var}(s): S_{1} \equiv_{x}^{S} S_{2}$;
- $\forall m_{S_{1}}\left(\mathfrak{f}_{S_{1}}, \sigma_{S_{1}}\right) m_{S_{2}}\left(\mathfrak{f}_{S_{2}}, \sigma_{S_{2}}\right)$ :

$\left(\left(\left(\forall z \in \operatorname{TVar}\left(S_{1}\right) \cup \operatorname{TVar}\left(S_{2}\right)\right), \sigma_{S_{1}}(z)=\sigma_{S_{2}}(z)\right) \wedge\left(\mathfrak{f}_{S_{1}}=\right.\right.$ $\left.\left.\mathfrak{f}_{S_{2}}=0\right)\right) \Rightarrow$

$\left(S_{1}, m_{S_{1}}\left(\mathfrak{f}_{S_{1}}, \sigma_{S_{1}}\right)\right) \equiv_{H}\left(S_{2}, m_{S_{2}}\left(\mathfrak{f}_{S_{2}}, \sigma_{S_{2}}\right)\right)$.

If $s_{1}$ and $s_{2}$ start in the state $m_{1}\left(\mathfrak{f}_{1}\right.$, loop $\left._{c}^{1}, \sigma_{1}\right)$ and $m_{2}\left(\mathfrak{f}_{2}\right.$, loop $\left._{c}^{2}, \sigma_{2}\right)$ respectively in which crash flags are not set, $\mathfrak{f}_{1}=\mathfrak{f}_{2}=0, s_{1}$ and $s_{2}$ have not already executed, $\operatorname{loop}_{c}^{1}\left(n_{1}\right)=$ loop $_{c}^{2}\left(n_{2}\right)=0$, value stores $\sigma_{1}$ and $\sigma_{2}$ agree on values of variables in $T \operatorname{Var}(s), \forall x \in T \operatorname{Var}(s): \sigma_{1}(x)=\sigma_{2}(x)$, then, for any positive integer $i$, one of the following holds:

1. The loop counters for $s_{1}$ and $s_{2}$ are less than $i$ where $s_{1}$ and $s_{2}$ terminate in the same way:

$\forall m_{1}^{\prime} m_{2}^{\prime}:\left(s_{1}, m_{1}\right) \stackrel{*}{\rightarrow}\left(S_{1}^{\prime}, m_{1}^{\prime}\left(\operatorname{loop}_{c}^{1^{\prime}}\right)\right)$ and $\left(s_{2}, m_{2}\right) \stackrel{*}{\rightarrow}$ $\left(S_{2}^{\prime}, m_{2}^{\prime}\left(\right.\right.$ loop $\left.\left._{c}^{2^{\prime}}\right)\right)$, loop ${ }_{c}^{1^{\prime}}\left(n_{1}\right)<i$ and $m_{c}^{2^{\prime}}\left(n_{2}\right)<i$ and one of the following holds:

(a) $s_{1}$ and $s_{2}$ both terminate:

$$
\left(s_{1}, m_{1}\right) \stackrel{*}{\rightarrow}\left(\text { skip, } m_{1}^{\prime \prime}\right) \text { and }\left(s_{2}, m_{2}\right) \stackrel{*}{\rightarrow}\left(\text { skip, } m_{2}^{\prime \prime}\right) \text {. }
$$

(b) $s_{1}$ and $s_{2}$ both do not terminate:

$\forall k>0:\left(s_{1}, m_{1}\right) \stackrel{k}{\rightarrow}\left(S_{1_{k}}, m_{1_{k}}\right)$ and $\left(s_{2}, m_{2}\right) \stackrel{k}{\rightarrow}$ $\left(S_{2_{k}}, m_{2_{k}}\right)$ in which $S_{1_{k}} \neq$ skip, $S_{2_{k}} \neq$ skip.

2. The loop counters for $s_{1}$ and $s_{2}$ are less than or equal to $i$ where $s_{1}$ and $s_{2}$ do not terminate such that there are no configurations $\left(s_{1}, m_{1_{i}}\right)$ and $\left(s_{2}, m_{2_{i}}\right)$ reachable from $\left(s_{1}, m_{1}\right)$ and $\left(s_{2}, m_{2}\right)$, respectively, in which crash flags are not set, the loop counters of $s_{1}$ and $s_{2}$ are equal to $i$, and value stores agree on the values of variables in $T \operatorname{Var}(s)$ :

- $\forall m_{1}^{\prime} m_{2}^{\prime} \quad: \quad\left(s_{1}, m_{1}\right) \stackrel{*}{\rightarrow}\left(S_{1}, m_{1}^{\prime}\left(\operatorname{loop}_{c}^{1^{\prime}}\right)\right),\left(s_{2}, m_{2}\right) \stackrel{*}{\rightarrow}$ $\left(S_{2}, m_{2}^{\prime}\left(\right.\right.$ loop $\left.\left._{c}^{2^{\prime}}\right)\right)$ where

$\operatorname{loop}_{c}^{1^{\prime}}\left(n_{1}\right) \leq i, \operatorname{loop}_{c}^{2^{\prime}}\left(n_{2}\right) \leq i$;

- $\forall k>0$

$\left(s_{1}, m_{1}\right) \stackrel{k}{\rightarrow}\left(S_{1_{k}}, m_{1_{k}}\right),\left(s_{2}, m_{2}\right) \stackrel{k}{\rightarrow}\left(S_{2_{k}}, m_{2_{k}}\right)$ where

$S_{1_{k}} \neq$ skip, $S_{2_{k}} \neq$ skip; and

- $\nexists\left(s_{1}, m_{1_{i}}\right)\left(s_{2}, m_{2_{i}}\right)$ :

$\left(s_{1}, m_{1}\right) \stackrel{*}{\rightarrow}\left(s_{1}, m_{1_{i}}\left(\mathfrak{f}_{1}, \operatorname{loop}_{c}^{1_{i}}, \sigma_{1_{i}}\right)\right) \wedge$

$\left(s_{2}, m_{2}\right) \stackrel{*}{\rightarrow}\left(s_{2}, m_{2_{i}}\left(\mathfrak{f}_{2}\right.\right.$, loop $\left.\left._{c}^{2_{i}}, \sigma_{2_{i}}\right)\right)$ where

- $\mathfrak{f}_{1}=\mathfrak{f}_{2}=0$; and

- $\operatorname{loop}_{c}^{1_{i}}\left(n_{1}\right)=\operatorname{loop}_{c}^{2_{i}}\left(n_{2}\right)=i$; and

- $\forall x \in T \operatorname{Var}(s): \sigma_{1_{i}}(x)=\sigma_{2_{i}}(x)$.

3. There are two configurations $\left(s_{1}, m_{1_{i}}\right)$ and $\left(s_{2}, m_{2_{i}}\right)$ reachable from $\left(s_{1}, m_{1}\right)$ and $\left(s_{2}, m_{2}\right)$, respectively, in which both crash flags are not set, the loop counters of $s_{1}$ and $s_{2}$ are equal to $i$ and value stores agree on the values of variables in $\operatorname{TVar}(s)$, and for every state in execution $\left(s_{1}, m_{1}\right) \stackrel{*}{\rightarrow}\left(s_{1}, m_{1_{i}}\right)$ or $\left(s_{2}, m_{2}\right) \stackrel{*}{\rightarrow}\left(s_{2}, m_{2_{i}}\right)$, the loop counters for $s_{1}$ and $s_{2}$ are less than or equal to $i$ respectively:

$\exists\left(s_{1}, m_{1_{i}}\right)\left(s_{2}, m_{2_{i}}\right):\left(s_{1}, m_{1}\right) \stackrel{*}{\rightarrow}\left(s_{1}, m_{1_{i}}\left(\mathfrak{f}_{1}, \operatorname{loop}_{c}^{1_{i}}, \sigma_{1_{i}}\right)\right) \wedge$

$\left(s_{2}, m_{2}\right) \stackrel{*}{\rightarrow}\left(s_{2}, m_{2_{i}}\left(\mathfrak{f}_{2}\right.\right.$, loop $\left.\left._{c}^{2_{i}}, \sigma_{2_{i}}\right)\right)$ where

- $\mathfrak{f}_{1}=\mathfrak{f}_{2}=0$; and

- $\operatorname{loop}_{c}^{1_{i}}\left(n_{1}\right)=\operatorname{loop}_{c}^{2_{i}}\left(n_{2}\right)=i$; and

- $\forall x \in T \operatorname{Var}(s): \sigma_{1_{i}}(x)=\sigma_{2_{i}}(x)$; and

- $\forall m_{1}^{\prime}:\left(s_{1}, m_{1}\right) \stackrel{*}{\rightarrow}\left(S_{1}^{\prime}, m_{1}^{\prime}\left(m_{c}^{1^{\prime}}\right)\right) \stackrel{*}{\rightarrow}\left(s_{1}, m_{1_{i}}\right)$, $\operatorname{loop}_{c}^{1^{\prime}}\left(n_{1}\right) \leq i$; and

- $\forall m_{2}^{\prime}:\left(s_{2}, m_{2}\right) \stackrel{*}{\rightarrow}\left(S_{2}^{\prime}, m_{2}^{\prime}\left(m_{c}^{2^{\prime}}\right)\right) \stackrel{*}{\rightarrow}\left(s_{2}, m_{2_{i}}\right)$, $\operatorname{loop}_{c}^{2^{\prime}}\left(n_{2}\right) \leq i$;

Proof. By induction on $i$.

Base case. $i=1$. 
By assumption, initial loop counters of $s_{1}$ and $s_{2}$ are of value zero. Initial value stores $\sigma_{1}$ and $\sigma_{2}$ agree on the values of variables in $\operatorname{TVar}(s)$. Then we show one of the following cases hold:

1. The loop counters for $s_{1}$ and $s_{2}$ are less than $1, s_{1}$ and $s_{2}$ terminate in the same way:

$\forall m_{1}^{\prime} m_{2}^{\prime}$ such that $\left(s_{1}, m_{1}\right) \stackrel{*}{\rightarrow}\left(S_{1}^{\prime}, m_{1}^{\prime}\left(\operatorname{loop}_{c}^{1^{\prime}}\right)\right)$ and $\left(s_{2}, m_{2}\right) \stackrel{*}{\rightarrow}$ $\left(S_{2}^{\prime}, m_{2}^{\prime}\left(\operatorname{loop}_{c}^{2^{\prime}}\right)\right)$,

$m_{c}^{1^{\prime}}\left(n_{1}\right)<1$ and $m_{c}^{2^{\prime}}\left(n_{2}\right)<1$ and one of the following holds:

(a) $s_{1}$ and $s_{2}$ both terminate:

$\left(s_{1}, m_{1}\right) \stackrel{*}{\rightarrow}\left(\right.$ skip, $\left.m_{1}^{\prime \prime}\right)$ and $\left(s_{2}, m_{2}\right) \stackrel{*}{\rightarrow}\left(\right.$ skip, $\left.m_{2}^{\prime \prime}\right)$.

(b) $s_{1}$ and $s_{2}$ both do not terminate:

$\forall k>0,\left(s_{1}, m_{1}\right) \stackrel{k}{\rightarrow}\left(S_{1_{k}}, m_{1_{k}}\right)$ and $\left(s_{2}, m_{2}\right) \stackrel{k}{\rightarrow}\left(S_{2_{k}}, m_{2_{k}}\right)$

in which $S_{1_{k}} \neq$ skip, $S_{2_{k}} \neq$ skip.

2. The loop counters for $s_{1}$ and $s_{2}$ are less than or equal to 1 , and $s_{1}$ and $s_{2}$ do not terminate such that there are no configurations $\left(s_{1}, m_{1_{1}}\right)$ and $\left(s_{2}, m_{2_{1}}\right)$ reachable from $\left(s_{1}, m_{1}\right)$ and $\left(s_{2}, m_{2}\right)$, respectively, in which crash flags are not set, the loop counters of $s_{1}$ and $s_{2}$ are equal to 1 and value stores agree on the values of variables in $T \operatorname{Var}(s)$ :

- $\forall m_{1}^{\prime} m_{2}^{\prime}:\left(s_{1}, m_{1}\right) \stackrel{*}{\rightarrow}\left(S_{1}, m_{1}^{\prime}\left(\operatorname{loop}_{c}^{1^{\prime}}\right)\right),\left(s_{2}, m_{2}\right) \stackrel{*}{\rightarrow}$ $\left(S_{2}, m_{2}^{\prime}\left(\operatorname{loop}_{c}^{2^{\prime}}\right)\right)$ where $\operatorname{loop}_{c}^{1^{\prime}}\left(n_{1}\right) \leq i, \operatorname{loop}_{c}^{2^{\prime}}\left(n_{2}\right) \leq i$;

- $\forall k>0$ : $\left(s_{1}, m_{1}\right) \stackrel{k}{\rightarrow}\left(S_{1_{k}}, m_{1_{k}}\right),\left(s_{2}, m_{2}\right) \stackrel{k}{\rightarrow}\left(S_{2_{k}}, m_{2_{k}}\right)$ where $S_{1_{k}} \neq$ skip, $S_{2_{k}} \neq$ skip; and

$\nexists\left(s_{1}, m_{1_{1}}\right),\left(s_{2}, m_{2_{1}}\right):\left(s_{1}, m_{1}\right) \stackrel{*}{\rightarrow}\left(s_{1}, m_{1_{1}}\left(\mathfrak{f}_{1}, \operatorname{loop}_{c}^{1_{1}}, \sigma_{1_{1}}\right)\right) \wedge$

$\left(s_{2}, m_{2}\right) \stackrel{*}{\rightarrow}\left(s_{2}, m_{2_{1}}\left(\mathfrak{f}_{2}\right.\right.$, loop $\left.\left._{c}^{2_{1}}, \sigma_{2_{1}}\right)\right)$ where

- $\mathfrak{f}_{1}=\mathfrak{f}_{2}=0$; and

- $\operatorname{loop}_{c}^{1_{1}}\left(n_{1}\right)=\operatorname{loop}_{c}^{2_{1}}\left(n_{2}\right)=1$; and

- $\forall x \in \operatorname{TVar}(s): \sigma_{1_{1}}(x)=\sigma_{2_{1}}(x)$.

3 . There are two configurations $\left(s_{1}, m_{1_{1}}\right)$ and $\left(s_{2}, m_{2_{1}}\right)$ reachable from $\left(s_{1}, m_{1}\right)$ and $\left(s_{2}, m_{2}\right)$ respectively, in which the loop counters of $s_{1}$ and $s_{2}$ are equal to 1 and value stores agree on the values of variables in $\operatorname{TVar}(s)$ and, for every state in execution, $\left(s_{1}, m_{1}\right) \stackrel{*}{\rightarrow}\left(s_{1}, m_{1_{1}}\right)$ or $\left(s_{2}, m_{2}\right) \stackrel{*}{\rightarrow}\left(s_{2}, m_{2_{1}}\right)$ the loop counters for $s_{1}$ and $s_{2}$ are less than or equal to 1 respectively: $\exists\left(s_{1}, m_{1_{1}}\right)\left(s_{2}, m_{2_{1}}\right):\left(s_{1}, m_{1}\right) \stackrel{*}{\rightarrow}\left(s_{1}, m_{1_{1}}\left(\mathfrak{f}_{1}, \operatorname{loop}_{c}^{1_{1}}, \sigma_{1_{1}}\right)\right) \wedge$ $\left(s_{2}, m_{2}\right) \stackrel{*}{\rightarrow}\left(s_{2}, m_{2_{1}}\left(\mathfrak{f}_{2}\right.\right.$, loop $\left.\left._{c}^{2_{1}}, \sigma_{2_{1}}\right)\right)$ where

- $\mathfrak{f}_{1}=\mathfrak{f}_{2}=0$; and

- $\operatorname{loop}_{c}^{1_{1}}\left(n_{1}\right)=\operatorname{loop}_{c}^{2_{1}}\left(n_{1}\right)=1$; and

- $\forall x \in \operatorname{TVar}(s): \sigma_{1_{1}}(x)=\sigma_{2_{1}}(x)$; and

- $\forall m_{1}^{\prime}:\left(s_{1}, m_{1}\right) \stackrel{*}{\rightarrow}\left(S_{1}^{\prime}, m_{1}^{\prime}\left(\operatorname{loop}_{c}^{1^{\prime}}\right)\right) \stackrel{*}{\rightarrow}\left(s_{1}, m_{1_{1}}\right), \operatorname{loop}_{c}^{1^{\prime}}\left(n_{1}\right) \leq$ 1 ; and

- $\forall m_{2}^{\prime}:\left(s_{2}, m_{2}\right) \stackrel{*}{\rightarrow}\left(S_{2}^{\prime}, m_{2}^{\prime}\left(\operatorname{loop}_{c}^{2^{\prime}}\right)\right) \stackrel{*}{\rightarrow}\left(s_{2}, m_{2_{1}}\right), \operatorname{loop}_{c}^{2^{\prime}}\left(n_{2}\right) \leq$ 1 .

We show evaluations of the predicate expression of $s_{1}$ and $s_{2}$ w.r.t value stores $\sigma_{1}$ and $\sigma_{2}$ produce same value. By the definition of loop variables, $\operatorname{LVar}\left(s_{1}\right)=\bigcup_{j \geq 0} \operatorname{Imp}\left(S_{1}^{j}, \operatorname{LVar}\left(S_{1}\right) \cup \operatorname{Use}(e)\right)$. By our notation of $S^{0}, S_{1}^{0}=$ skip. By the definition of loop variables, $\operatorname{Use}(e) \subseteq \operatorname{LVar}(s)=\operatorname{LVar}\left(s_{1}\right)$. By assumption, value stores $\sigma_{1}$ and $\sigma_{2}$ agree on the values of the variables in Use $(e)$. By Lemma D.1 the predicate expression $e$ of $s_{1}$ and $s_{2}$ evaluates to same value $v$ w.r.t value stores $\sigma_{1}, \sigma_{2}, \mathcal{E}^{\prime} \llbracket e \rrbracket \sigma_{1}=\mathcal{E}^{\prime} \llbracket e \rrbracket \sigma_{2}$. Then there are three possibilities.

1. $\mathcal{E}^{\prime} \llbracket e \rrbracket \sigma_{1}=\mathcal{E}^{\prime} \llbracket e \rrbracket \sigma_{2}=($ error,$*)$

The execution from $\left(s_{1}, m_{1}\left(\mathfrak{f}_{1}, \operatorname{loop}_{c}^{1}, \sigma_{1}\right)\right)$ proceeds as follows.

$$
\begin{aligned}
& \left(s_{1}, m_{1}\left(\mathfrak{f}_{1}, \operatorname{loop}_{c}^{1}, \sigma_{1}\right)\right) \\
= & \left(\text { while }_{\left\langle n_{1}\right\rangle}(e)\left\{S_{1}\right\}, m_{1}\left(\mathfrak{f}_{1}, \operatorname{loop}_{c}^{1}, \sigma_{1}\right)\right) \\
\rightarrow & \left(\text { while }_{\left\langle n_{1}\right\rangle}((\text { error }, *))\left\{S_{1}\right\}, m_{1}\left(\mathfrak{f}_{1}, \operatorname{loop}_{c}^{1}, \sigma_{1}\right)\right) \text { by the EEval' rule }
\end{aligned}
$$

\author{
$\rightarrow\left(\right.$ while $\left._{\left\langle n_{1}\right\rangle}(0)\left\{S_{1}\right\}, m_{1}\left(1 / \mathfrak{f}_{1}\right)\right)$ by the ECrash rule \\ $\stackrel{k}{\rightarrow}\left(\right.$ while $_{\left\langle n_{1}\right\rangle}(0)\left\{S_{1}\right\}, m_{1}\left(1 / \mathfrak{f}_{1}\right.$, loop $\left.\left._{c}^{1}, \sigma_{1}\right)\right)$, \\ for any $k \geq 0$, by the Crash rule.
}

Similarly, the execution of $s_{2}$ started in the state $m_{2}\left(\mathfrak{f}_{2}, \operatorname{loop}_{c}^{2}, \sigma_{2}\right)$ does not terminate.

The loop counters of $s_{1}$ and $s_{2}$ are less than 1:

$\forall m_{1}^{\prime} m_{2}^{\prime}:\left(s_{1}, m_{1}\right) \stackrel{*}{\rightarrow}\left(S_{1}^{\prime}, m_{1}^{\prime}\left(\operatorname{loop}_{c}^{1^{\prime}}\right)\right)$ and $\left(s_{2}, m_{2}\right) \stackrel{*}{\rightarrow}$ $\left(S_{2}^{\prime}, m_{2}^{\prime}\left(\operatorname{loop}_{c}^{2^{\prime}}\right)\right)$ where $\operatorname{loop}_{c}^{1^{\prime}}\left(n_{1}\right)<1$ and loop ${ }_{c}^{2^{\prime}}\left(n_{2}\right)<1$.

Besides, $s_{1}$ and $s_{2}$ both do not terminate when started in states $m_{1}$ and $m_{2}, \forall k>0:\left(s_{1}, m_{1}\right) \stackrel{k}{\rightarrow}\left(S_{1_{k}}, m_{1_{k}}\right)$ and $\left(s_{2}, m_{2}\right)$

$\stackrel{k}{\rightarrow}\left(S_{2_{k}}, m_{2_{k}}\right)$ in which $S_{1_{k}} \neq$ skip, $S_{2_{k}} \neq$ skip.

2. $\mathcal{E}^{\prime} \llbracket e \rrbracket \sigma_{1}=\mathcal{E}^{\prime} \llbracket e \rrbracket \sigma_{2}=\left(0, v_{\mathfrak{o f}}\right)$

The execution of $s_{1}$ proceeds as follows.

$$
\begin{aligned}
& \left(s_{1}, m_{1}\left(\operatorname{loop}_{c}^{1}, \sigma_{1}\right)\right) \\
= & \left(\text { while }_{\left\langle n_{1}\right\rangle}(e)\left\{S_{1}\right\}, m_{1}\left(\operatorname{loop}_{c}^{1}\right)\right) \\
\rightarrow & \left(\text { while }_{\left\langle n_{1}\right\rangle}\left(\left(0, v_{\mathfrak{o f}}\right)\right)\left\{S_{1}\right\}, m_{1}\left(\operatorname{loop}_{c}^{1}\right)\right) \text { by the EEval' rule } \\
\rightarrow & (\text { while } \\
\left\langle n_{1}\right\rangle & \left.(0)\left\{S_{1}\right\}, m_{1}\left(\operatorname{loop}_{c}^{1}\right)\right) \text { by the E-Oflow1 or E-Oflow2 rule } \\
\rightarrow & \left(\text { skip }, m_{1}\right) \text { by the Wh-F1 rule. }
\end{aligned}
$$

Similarly, $\left(s_{2}, m_{2}\left(\right.\right.$ loop $\left.\left._{c}^{2}, \sigma_{2}\right)\right) \stackrel{2}{\rightarrow}\left(\right.$ skip, $\left.m_{2}\right)$.

The loop counters for $s_{1}$ and $s_{2}$ are less than 1:

$\forall m_{1}^{\prime} m_{2}^{\prime}:\left(s_{1}, m_{1}\right) \stackrel{*}{\rightarrow}\left(S_{1}^{\prime}, m_{1}^{\prime}\left(\operatorname{loop}_{c}^{1^{\prime}}\right)\right)$ and $\left(s_{2}, m_{2}\right) \stackrel{*}{\rightarrow}$ $\left(S_{2}^{\prime}, m_{2}^{\prime}\left(\operatorname{loop}_{c}^{2^{\prime}}\right)\right)$ where $\operatorname{loop}_{c}^{1^{\prime}}\left(n_{1}\right)<1$ and loop $2_{c}^{2^{\prime}}\left(n_{2}\right)<1$. Besides, $s_{1}$ and $s_{2}$ both terminate when started in states $m_{1}$ and $m_{2}$ :

$\left(s_{1}, m_{1}\right) \stackrel{*}{\rightarrow}$ (skip, $\left.m_{1}^{\prime \prime}\right)$ and $\left(s_{2}, m_{2}\right) \stackrel{*}{\rightarrow}\left(\right.$ skip, $\left.m_{2}^{\prime \prime}\right)$.

3. $\mathcal{E}^{\prime} \llbracket e \rrbracket \sigma_{1}=\mathcal{E}^{\prime} \llbracket e \rrbracket \sigma_{2}=\left(v, v_{\mathfrak{o f}}\right)$ where $v \notin\{0$, error $\}$;

The execution from $\left(s_{1}, m_{1}\left(\operatorname{loop}_{c}^{1}, \sigma_{1}\right)\right)$ proceeds as follows.

$$
\begin{aligned}
& \left(s_{1}, m_{1}\left(\operatorname{loop}_{c}^{1}, \sigma_{1}\right)\right) \\
= & \left(\text { while }_{\left\langle n_{1}\right\rangle}(e)\left\{S_{1}\right\}, m_{1}\left(\operatorname{loop}_{c}^{1}, \sigma_{1}\right)\right) \\
\rightarrow & \left(\text { while }_{\left\langle n_{1}\right\rangle}\left(\left(v, v_{\mathfrak{o f}}\right)\right)\left\{S_{1}\right\}, m_{1}\left(\operatorname{loop}_{c}^{1}, \sigma_{1}\right)\right) \text { by the EEval' rule } \\
\rightarrow & \left(\text { while }_{\left\langle n_{1}\right\rangle}(v)\left\{S_{1}\right\}, m_{1}\left(\operatorname{loop}_{c}^{1}, \sigma_{1}\right)\right) \\
& \text { by rule E-Oflow } 1 \text { or E-Oflow } 2 \\
\rightarrow & \left(S_{1} ;\right. \text { while } \\
\quad & \text { by the Wh-T1 rule. }
\end{aligned}
$$

Similarly, $\left(s_{2}, m_{2}\left(\operatorname{loop}_{c}^{2}, \sigma_{2}\right)\right) \stackrel{2}{\rightarrow}\left(S_{2} ;\right.$ while $_{\left\langle n_{2}\right\rangle}(e)\left\{S_{2}\right\}$, $\left.m_{2}\left(\operatorname{loop}_{c}^{2}\left[1 /\left(n_{2}\right)\right], \sigma_{2}\right)\right)$. After two steps of executions of $s_{1}$ and $s_{2}$, crash flags are not set, the loop counter value of $s_{1}$ and $s_{2}$ are 1 , value stores $\sigma_{1}$ and $\sigma_{2}$ agree on values of variables in $\mathrm{T} \operatorname{Var}(s)$.

We show that $\operatorname{TVar}\left(S_{1}\right) \subseteq \mathrm{T} \operatorname{Var}(s)$. By definition of loop variables, $\operatorname{LVar}\left(s_{1}\right)=\bigcup_{j \geq 0} \operatorname{Imp}\left(S_{1}^{j}, \operatorname{LVar}\left(S_{1}\right) \cup \operatorname{Use}(e)\right)$. By notation of $S^{0}, S^{0}=$ skip. By definition of imported variables, $\operatorname{Imp}\left(S_{1}^{0}, \operatorname{LVar}\left(S_{1}\right) \cup \operatorname{Use}(e)\right)=\operatorname{LVar}\left(S_{1}\right) \cup \operatorname{Use}(e)$. Then $\operatorname{LVar}\left(S_{1}\right) \subseteq \operatorname{LVar}(s)$. By similar argument, we have $\mathrm{C} \operatorname{Var}\left(S_{1}\right) \subseteq \mathrm{C} \operatorname{Var}(s)$. Hence, $\mathrm{T} \operatorname{Var}\left(S_{1}\right) \subseteq \mathrm{T} \operatorname{Var}(s)$. Similarly, $\mathrm{T} \operatorname{Var}\left(S_{2}\right) \subseteq \mathrm{T} \operatorname{Var}(s)$. By assumption, $S_{1}$ and $S_{2}$ either both terminate or both do not terminate when started in state $m_{1}\left(\operatorname{loop}_{c}^{1}\left[1 /\left(n_{1}\right)\right], \sigma_{1}\right)$ and $m_{2}\left(\operatorname{loop}_{c}^{2}\left[1 /\left(n_{2}\right)\right], \sigma_{2}\right)$ in which $\forall y \in \operatorname{TVar}\left(S_{1}\right) \cup \operatorname{TVar}\left(S_{2}\right), \sigma_{1}(y)=\sigma_{2}(y)$ and crash flags are not set. Then there are two possibilities:

(a) $S_{1}$ and $S_{2}$ both terminate when started in states $m_{1}$ ( $\left.\operatorname{loop}_{c}^{1}\left[1 /\left(n_{1}\right)\right], \sigma_{1}\right)$ and $m_{2}\left(\operatorname{loop}_{c}^{2}\left[1 /\left(n_{2}\right)\right], \sigma_{2}\right)$ respectively: $\left(S_{1}, m_{1}\left(\operatorname{loop}_{c}^{1}\left[1 /\left(n_{1}\right)\right], \sigma_{1}\right)\right) \stackrel{*}{\rightarrow}\left(\operatorname{skip}, m_{1_{1}}\left(\mathfrak{f}_{1}, \operatorname{loop}_{c}^{1_{1}}, \sigma_{1_{1}}\right)\right)$ and

$\left(S_{2}, m_{2}\left(\operatorname{loop}_{c}^{2}\left[1 /\left(n_{2}\right)\right], \sigma_{2}\right)\right) \stackrel{*}{\rightarrow}\left(\right.$ skip, $\left.m_{2_{1}}\left(\mathfrak{f}_{2}, \operatorname{loop}_{c}^{2}, \sigma_{2_{1}}\right)\right)$. We show that, after the full execution of $S_{1}$ and $S_{2}$, the following five properties hold. 
- The crash flags are not set.

By the definition of terminating execution, crash flags are not set, $\mathfrak{f}_{1}=\mathfrak{f}_{2}=0$.

- The loop counter of $s_{1}$ and $s_{2}$ are of value $1, \operatorname{loop}_{c}^{1_{1}}\left(n_{1}\right)=$ $\operatorname{loop}_{c}^{21}\left(n_{2}\right)=1$.

By the assumption of unique loop labels, $s_{1} \notin S_{1}$. Then, the loop counter value of $n_{1}$ is not redefined in the execution of $S_{1}$ by corollary E.2, $\operatorname{loop}_{c}^{1}\left[1 / n_{1}\right]\left(n_{1}\right)=$ loop $_{c}^{1_{1}}\left(n_{1}\right)=1$. Similarly, the loop counter value of $n_{2}$ is not redefined in the execution of $S_{2}, \operatorname{loop}_{c}^{2}\left[1 /\left(n_{2}\right)\right]\left(n_{2}\right)$ $=\operatorname{loop}_{c}^{2_{1}}\left(n_{2}\right)=1$.

- In any state in the execution $\left(s_{1}, m_{1}\right) \stackrel{*}{\rightarrow}\left(s_{1}, m_{1_{1}}\left(\operatorname{loop}_{c}^{1_{1}}, \sigma_{1_{1}}\right)\right)$, the loop counter of $s_{1}$ is less than or equal to 1 .

As is shown above, the loop counter of $s_{1}$ is zero in any of the two states in the one step execution $\left(s_{1}, m_{1}\right) \rightarrow\left(\right.$ while $\left._{\left\langle n_{1}\right\rangle}(v)\left\{S_{1}\right\}, m_{1}\left(\operatorname{loop}_{c}^{1}, \sigma_{1}\right)\right)$, and the loop counter of $s_{1}$ is 1 in any states in the execution $\left(S_{1} ;\right.$ while $\left._{\left\langle n_{1}\right\rangle}(e) \quad\left\{S_{1}\right\}, m_{1}\left(\operatorname{loop}_{c}^{1}\left[i /\left(n_{1}\right)\right], \sigma_{1}\right)\right) \stackrel{*}{\rightarrow}$ $\left(s_{1}, m_{1_{1}}\left(\operatorname{loop}_{c}^{1_{1}}, \sigma_{1_{1}}\right)\right)$.

- In any state in the executions $\left(s_{2}, m_{2}\right) \stackrel{*}{\rightarrow}\left(s_{2}, m_{2_{1}}\left(\operatorname{loop}_{c}^{22_{1}}, \sigma_{2_{1}}\right)\right)$, the loop counter of $s_{2}$ is less than or equal to 1 .

By similar argument above.

- The value stores $\sigma_{1_{1}}$ and $\sigma_{2_{1}}$ agree on values of the termination deciding variables in $s_{1}$ and $s_{2}: \forall x \in$ $\mathrm{T} \operatorname{Var}(s), \sigma_{1_{1}}(x)=\sigma_{2_{1}}(x)$.

We show that the imported variables in $S_{1}$ relative to those in $\operatorname{LVar}(s)$ are a subset of $\operatorname{LVar}(s)$ and the imported variables in $S_{1}$ relative to those in $\mathrm{CVar}(s)$ are a subset of $C \operatorname{Var}(s)$.

$\operatorname{LVar}\left(s_{1}\right)$

$=\bigcup_{j \geq 0} \operatorname{Imp}\left(S_{1}^{j}, \operatorname{LVar}\left(S_{1}\right) \cup \operatorname{Use}(e)\right)(1)$

by the definition of loop variables.

$\operatorname{Imp}\left(S_{1}, \operatorname{LVar}(s)\right)=\operatorname{Imp}\left(S_{1}, \operatorname{LVar}\left(s_{1}\right)\right)$

$=\operatorname{Imp}\left(S_{1}, \operatorname{Imp}\left(s_{1}, \operatorname{Use}(e) \cup \operatorname{LVar}\left(S_{1}\right)\right)\right)$

by the definition of $\operatorname{LVar}(s)$

$=\operatorname{Imp}\left(S_{1}, \bigcup_{j>0} \operatorname{Imp}\left(S_{1}^{j}, \operatorname{LVar}\left(S_{1}\right) \cup \operatorname{Use}(e)\right)\right)$ by (1)

$=\bigcup_{j>0} \operatorname{Imp}\left(S_{1}, \operatorname{Imp}\left(S_{1}^{j}, \operatorname{LVar}\left(S_{1}\right) \cup \operatorname{Use}(e)\right)\right)$ by LemmaC.2

$=\bigcup_{j>0} \operatorname{Imp}\left(S_{1}^{j}, \operatorname{LVar}\left(S_{1}\right) \cup \operatorname{Use}(e)\right)$ by Lemma C. 1

$\subseteq \bigcup_{j>0} \operatorname{Imp}\left(S_{1}^{j}, \operatorname{LVar}\left(S_{1}\right) \cup \operatorname{Use}(e)\right)$

$=\operatorname{Imp}\left(s_{1}, \operatorname{LVar}\left(S_{1}\right) \cup \operatorname{Use}(e)\right)=\operatorname{LVar}\left(s_{1}\right)=\operatorname{LVar}(s)$.

Similarly, $\operatorname{Imp}\left(S_{1}, \mathrm{C} \operatorname{Var}(s)\right) \subseteq \mathrm{CV} \operatorname{Var}(s)$. Hence,

$\operatorname{Imp}\left(S_{1}, \mathrm{~T} \operatorname{Var}(s)\right) \subseteq \mathrm{T} \operatorname{Var}(s)$. In the same way, we can show that $\operatorname{Imp}\left(S_{2}, \mathrm{TVar}(s)\right) \subseteq \operatorname{TVar}(s)$. Consequently, the value stores $\sigma_{1_{1}}$ and $\sigma_{2_{1}}$ agree on the values of the imported variables in $S_{1}$ and $S_{2}$ relative to those in $\operatorname{TVar}(s), \forall x \in \operatorname{Imp}\left(S_{1}, \mathrm{~T} \operatorname{Var}(s)\right) \cup$ $\operatorname{Imp}\left(S_{2}, \operatorname{TVar}(s)\right), \sigma_{1}(x)=,\sigma_{2}(x)$. Because $S_{1}$ and $S_{2}$ have equivalent computation of every variable in $\mathrm{T} \operatorname{Var}(s)$ when started in states agreeing on the values of the imported variables relative to $\mathrm{TVar}(s)$, by Theorem 1 value stores $\sigma_{1_{1}}$ and $\sigma_{2_{1}}$ agree on the values of the variables $\operatorname{TVar}(s), \forall x \in \operatorname{TVar}(s), \sigma_{1_{1}}(x)=$ $\sigma_{2_{1}}(x)$.

It follows that, by Corollary E.1

$\left(S_{1}\right.$; while $\left.\left\langle n_{1}\right\rangle(e)\left\{S_{1}\right\}, m_{1}\left(\operatorname{loop}_{c}^{1}\left[1 /\left(n_{1}\right)\right], \sigma_{1}\right)\right) \stackrel{*}{\rightarrow}$ $\left(\right.$ while $\left._{\left\langle n_{1}\right\rangle}(e)\left\{S_{1}\right\}, m_{1_{1}}\left(\operatorname{loop}_{c}^{1_{1}}, \sigma_{1_{1}}\right)\right)=\left(s_{1}, m_{1_{1}}\left(\operatorname{loop}_{c}^{1_{1}}, \sigma_{1_{1}}\right)\right)$ and

$\left(S_{2} ;\right.$ while $\left._{\left\langle n_{2}\right\rangle}(e)\left\{S_{2}\right\}, m_{2}\left(\operatorname{loop}_{c}^{2}\left[1 /\left(n_{2}\right)\right], \sigma_{2}\right)\right) \stackrel{*}{\rightarrow}$

$\left(\right.$ while $\left._{\left\langle n_{2}\right\rangle}(e)\left\{S_{2}\right\}, m_{2_{1}}\left(\operatorname{loop}_{c}^{2_{1}}, \sigma_{2_{1}}\right)\right)=\left(s_{2}, m_{2_{1}}\left(\operatorname{loop}_{c}^{2_{1}}, \sigma_{2_{1}}\right)\right)$.

(b) $S_{1}$ and $S_{2}$ do not terminate when started in states $m_{1}\left(\operatorname{loop}_{c}^{1}\left[1 /\left(n_{1}\right)\right], \sigma_{1}\right)$ and

$m_{2}\left(\operatorname{loop}_{c}^{2}\left[1 /\left(n_{2}\right)\right], \sigma_{2}\right)$ respectively:

$\forall k>0,\left(S_{1}, m_{1}\left(\operatorname{loop}_{c}^{1}\left[1 /\left(n_{1}\right)\right], \sigma_{1}\right)\right) \stackrel{k}{\rightarrow}$

$\left(S_{1_{k}}, m_{1_{1_{k}}}\left(\operatorname{loop}_{c}{ }_{1_{k}}, \sigma_{1_{1_{k}}}\right)\right)$ and

$\left(S_{2}, m_{2}\left(\operatorname{loop}_{c}^{2}\left[1 /\left(n_{2}\right)\right], \sigma_{2}\right)\right) \stackrel{k}{\rightarrow}$

$\left(S_{2_{k}}, m_{2_{1_{k}}}\left(\operatorname{loop}_{c}^{21_{k}}, \sigma_{2_{1_{k}}}\right)\right)$ in which $S_{1_{k}} \neq$ skip, $S_{2_{k}} \neq$ skip.

By our assumption of unique loop labels, $s_{1} \notin S_{1}$. Then, $\forall k>0, \operatorname{loop}_{c}{ }^{1_{k}}\left(n_{1}\right)=\operatorname{loop}_{c}^{1}\left[1 /\left(n_{1}\right)\right]\left(n_{1}\right)=1$. Similarly, $\forall k>0, \operatorname{loop}_{c}^{2_{1}}{ }^{2}\left(n_{2}\right)$

$=\operatorname{loop}_{c}^{2}\left[1 /\left(n_{2}\right)\right]\left(n_{2}\right)=1$. In addition, by LemmaE.2.

$\forall k>0,\left(S_{1} ; s_{1}, m_{1}\left(\operatorname{loop}_{c}^{1}\left[1 /\left(n_{1}\right)\right], \sigma_{1}\right)\right) \stackrel{k}{\rightarrow}$

$\left(S_{k} ; s_{1}, m_{1_{k}}\left(\operatorname{loop}_{c}^{1_{k}}, \sigma_{1_{k}}\right)\right)$ and

$\left(S_{2} ; s_{2}, m_{2}\left(\operatorname{loop}_{c}^{2}\left[1 /\left(n_{2}\right)\right], \sigma_{2}\right)\right) \stackrel{k}{\rightarrow}\left(S_{2_{k}} ; s_{2}, m_{2_{k}}\left(\operatorname{loop}_{c}^{2 k}, \sigma_{2_{k}}\right)\right)$

in which $S_{1_{k}} \neq$ skip, $S_{2_{k}} \neq$ skip.

In summary, loop counters of $s_{1}$ and $s_{2}$ are less than or equal to 1 , and $s_{1}$ and $s_{2}$ do not terminate such that there are no configurations $\left(s_{1}, m_{1_{1}}\right)$ and $\left(s_{2}, m_{2_{1}}\right)$ reachable from $\left(s_{1}, m_{1}\right)$ and $\left(s_{2}, m_{2}\right)$, respectively, in which crash flags are not set, the loop counters of $s_{1}$ and $s_{2}$ are equal to 1 and value stores agree on the values of variables in $\operatorname{TVar}(s)$.

\section{Induction Step.}

The induction hypothesis $\mathrm{IH}$ is that, for a positive integer $i$, one of the following holds:

1. The loop counters for $s_{1}$ and $s_{2}$ are less than $i$, and $s_{1}$ and $s_{2}$ both terminate in the same way:

$\forall m_{1}^{\prime} m_{2}^{\prime}$ such that $\left(s_{1}, m_{1}\right) \stackrel{*}{\rightarrow}\left(S_{1}^{\prime}, m_{1}^{\prime}\left(\operatorname{loop}_{c}^{1^{\prime}}\right)\right)$ and $\left(s_{2}, m_{2}\right) \stackrel{*}{\rightarrow}$ $\left(S_{2}^{\prime}, m_{2}^{\prime}\left(\operatorname{loop}_{c}^{2^{\prime}}\right)\right)$,

$\operatorname{loop}_{c}^{1^{\prime}}\left(n_{1}\right)<i$ and $\operatorname{loop}_{c}^{2^{\prime}}\left(n_{2}\right)<i$ and one of the following holds:

(a) $s_{1}$ and $s_{2}$ both terminate:

$\left(s_{1}, m_{1}\right) \stackrel{*}{\rightarrow}\left(\right.$ skip, $\left.m_{1}^{\prime \prime}\right)$ and $\left(s_{2}, m_{2}\right) \stackrel{*}{\rightarrow}\left(\right.$ skip, $\left.m_{2}^{\prime \prime}\right)$.

(b) $s_{1}$ and $s_{2}$ both do not terminate:

$\forall k>0,\left(s_{1}, m_{1}\right) \stackrel{k}{\rightarrow}\left(S_{1_{k}}, m_{1_{k}}\right)$ and $\left(s_{2}, m_{2}\right) \stackrel{k}{\rightarrow}\left(S_{2_{k}}, m_{2_{k}}\right)$ in which $S_{1_{k}} \neq$ skip, $S_{2_{k}} \neq$ skip.

2. The loop counters for $s_{1}$ and $s_{2}$ are less than or equal to $i$, and $s_{1}$ and $s_{2}$ do not terminate such that there are no configurations $\left(s_{1}, m_{1_{i}}\right)$ and $\left(s_{2}, m_{2_{i}}\right)$ reachable from $\left(s_{1}, m_{1}\right)$ and $\left(s_{2}, m_{2}\right)$, respectively, in which crash flags are not set, the loop counters of $s_{1}$ and $s_{2}$ are equal to $i$ and value stores agree on the values of variables in $\operatorname{TVar}(s)$ :

- $\forall m_{1}^{\prime} m_{2}^{\prime}:\left(s_{1}, m_{1}\right) \stackrel{*}{\rightarrow}\left(S_{1}, m_{1}^{\prime}\left(\operatorname{loop}_{c}^{1^{\prime}}\right)\right),\left(s_{2}, m_{2}\right) \stackrel{*}{\rightarrow}$ $\left(S_{2}, m_{2}^{\prime}\left(\operatorname{loop}_{c}^{2^{\prime}}\right)\right)$ where $\operatorname{loop}_{c}^{1^{\prime}}\left(n_{1}\right) \leq i, \operatorname{loop}_{c}^{2^{\prime}}\left(n_{2}\right) \leq i$;

- $\forall k>0$ : $\left(s_{1}, m_{1}\right) \stackrel{k}{\rightarrow}\left(S_{1_{k}}, m_{1_{k}}\right),\left(s_{2}, m_{2}\right) \stackrel{k}{\rightarrow}\left(S_{2_{k}}, m_{2_{k}}\right)$ where $S_{1_{k}} \neq$ skip, $S_{2_{k}} \neq$ skip; and

- $\nexists\left(s_{1}, m_{1_{i}}\right),\left(s_{2}, m_{2_{i}}\right):\left(s_{1}, m_{1}\right) \stackrel{*}{\rightarrow}\left(s_{1}, m_{1_{i}}\left(\mathfrak{f}_{1}, \operatorname{loop}_{c}^{1_{i}}, \sigma_{1_{i}}\right)\right) \wedge$ $\left(s_{2}, m_{2}\right) \stackrel{*}{\rightarrow}\left(s_{2}, m_{2_{i}}\left(\mathfrak{f}_{2}, \operatorname{loop}_{c}^{2_{i}}, \sigma_{2_{i}}\right)\right)$ where

- $\mathfrak{f}_{1}=\mathfrak{f}_{2}=0$; and

- $\operatorname{loop}_{c}^{1_{i}}\left(n_{1}\right)=\operatorname{loop}_{c}^{2_{i}}\left(n_{2}\right)=i$; and

- $\forall x \in \operatorname{TVar}(s): \sigma_{1_{i}}(x)=\sigma_{2_{i}}(x)$.

3. There are two configurations $\left(s_{1}, m_{1_{i}}\right)$ and $\left(s_{2}, m_{2_{i}}\right)$ reachable from $\left(s_{1}, m_{1}\right)$ and $\left(s_{2}, m_{2}\right)$, respectively, in which crash flags are not set, the loop counters of $s_{1}$ and $s_{2}$ are equal to $i$ and value stores agree on the values of variables in $\operatorname{TVar}(s)$ and, for every state in execution, $\left(s_{1}, m_{1}\right) \stackrel{*}{\rightarrow}\left(s_{1}, m_{1_{i}}\right)$ or $\left(s_{2}, m_{2}\right) \stackrel{*}{\rightarrow}\left(s_{2}, m_{2_{i}}\right)$ the loop counters for $s_{1}$ and $s_{2}$ are less than or equal to $i$ respectively: 


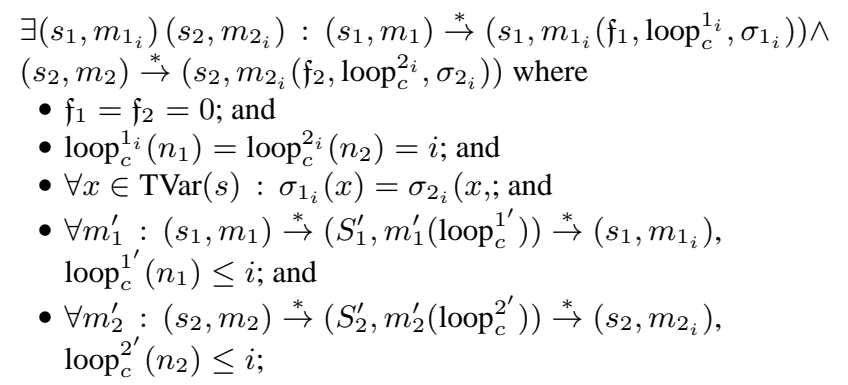

Then we show that, for the positive integer $i+1$, one of the following holds:

1. The loop counters for $s_{1}$ and $s_{2}$ are less than $i+1$, and $s_{1}$ and $s_{2}$ both terminate in the same way:

$\forall m_{1}^{\prime} m_{2}^{\prime}$ such that $\left(s_{1}, m_{1}\right) \stackrel{*}{\rightarrow}\left(S_{1}^{\prime}, m_{1}^{\prime}\left(\operatorname{loop}_{c}^{1^{\prime}}\right)\right)$ and $\left(s_{2}, m_{2}\right) \stackrel{*}{\rightarrow}$ $\left(S_{2}^{\prime}, m_{2}^{\prime}\left(\operatorname{loop}_{c}^{2^{\prime}}\right)\right)$,

$\operatorname{loop}_{c}^{1^{\prime}}\left(n_{1}\right)<i+1$ and $\operatorname{loop}_{c}^{2^{\prime}}\left(n_{2}\right)<i+1$ and one of the following holds:

(a) $s_{1}$ and $s_{2}$ both terminate:

$\left(s_{1}, m_{1}\right) \stackrel{*}{\rightarrow}\left(\right.$ skip, $\left.m_{1}^{\prime \prime}\right)$ and $\left(s_{2}, m_{2}\right) \stackrel{*}{\rightarrow}\left(\right.$ skip, $\left.m_{2}^{\prime \prime}\right)$.

(b) $s_{1}$ and $s_{2}$ both do not terminate:

$\forall k>0,\left(s_{1}, m_{1}\right) \stackrel{k}{\rightarrow}\left(S_{1_{k}}, m_{1_{k}}\right)$ and $\left(s_{2}, m_{2}\right) \stackrel{k}{\rightarrow}\left(S_{2_{k}}, m_{2_{k}}\right)$

in which $S_{1_{k}} \neq$ skip, $S_{2_{k}} \neq$ skip.

2. The loop counters for $s_{1}$ and $s_{2}$ are less than or equal to $i+1$, and $s_{1}$ and $s_{2}$ do not terminate such that there are no configurations $\left(s_{1}, m_{1_{i+1}}\right)$ and $\left(s_{2}, m_{2_{i+1}}\right)$ reachable from $\left(s_{1}, m_{1}\right)$ and $\left(s_{2}, m_{2}\right)$, respectively, in which crash flags are set, the loop counters of $s_{1}$ and $s_{2}$ are equal to $i+1$ and value stores agree on the values of variables in $\operatorname{TVar}(s)$ :

- $\forall m_{1}^{\prime} m_{2}^{\prime} \quad: \quad\left(s_{1}, m_{1}\right) \stackrel{*}{\rightarrow}\left(S_{1}, m_{1}^{\prime}\left(\operatorname{loop}_{c}^{1^{\prime}}\right)\right),\left(s_{2}, m_{2}\right) \stackrel{*}{\rightarrow}$ $\left(S_{2}, m_{2}^{\prime}\left(\operatorname{loop}_{c}^{2^{\prime}}\right)\right)$ where

$\operatorname{loop}_{c}^{1^{\prime}}\left(n_{1}\right) \leq i+1, \operatorname{loop}_{c}^{2^{\prime}}\left(n_{2}\right) \leq i+1$;

- $\forall k>0:\left(s_{1}, m_{1}\right) \stackrel{k}{\rightarrow}\left(S_{1_{k}}, m_{1_{k}}\right),\left(s_{2}, m_{2}\right) \stackrel{k}{\rightarrow}\left(S_{2_{k}}, m_{2_{k}}\right)$ where $S_{1_{k}} \neq$ skip, $S_{2_{k}} \neq$ skip; and

- $\nexists\left(s_{1}, m_{1_{i+1}}\right),\left(s_{2}, m_{2_{i+1}}\right)$ :

$\left(s_{1}, m_{1}\right) \stackrel{*}{\rightarrow}\left(s_{1}, m_{1_{i+1}}\left(\mathfrak{f}_{1}, \operatorname{loop}_{c}^{1_{i+1}}, \sigma_{1_{i+1}}\right)\right) \wedge\left(s_{2}, m_{2}\right) \stackrel{*}{\rightarrow}$ $\left(s_{2}, m_{2_{i+1}}\left(\mathfrak{f}_{2}, \operatorname{loop}_{c}^{2_{i+1}}, \sigma_{2_{i+1}}\right)\right)$ where

- $\mathfrak{f}_{1}=\mathfrak{f}_{2}=0$; and

- $\operatorname{loop}_{c}^{1_{i+1}}\left(n_{1}\right)=\operatorname{loop}_{c}^{2_{i+1}}\left(n_{2}\right)=i+1$; and

- $\forall x \in \operatorname{TVar}(s): \sigma_{1_{i+1}}(x)=\sigma_{2_{i+1}}(x)$.

3. There are two configurations $\left(s_{1}, m_{1_{i+1}}\right)$ and $\left(s_{2}, m_{2_{i+1}}\right)$ reachable from $\left(s_{1}, m_{1}\right)$ and $\left(s_{2}, m_{2}\right)$, respectively, in which the loop counters of $s_{1}$ and $s_{2}$ are equal to $i+1$ and value stores agree on the values of variables in $\operatorname{TVar}(s)$ and, for every state in execution, $\left(s_{1}, m_{1}\right) \stackrel{*}{\rightarrow}\left(s_{1}, m_{1_{i+1}}\right)$ or $\left(s_{2}, m_{2}\right) \stackrel{*}{\rightarrow}$ $\left(s_{2}, m_{2_{i+1}}\right)$ the loop counters for $s_{1}$ and $s_{2}$ are less than or equal to $i+1$ respectively:

$\exists\left(s_{1}, m_{1_{i+1}}\right)\left(s_{2}, m_{2_{i+1}}\right)$ :

$\left(s_{1}, m_{1}\right) \stackrel{*}{\rightarrow}\left(s_{1}, m_{1_{i+1}}\left(\operatorname{loop}_{c}^{1_{i+1}}, \sigma_{1_{i+1}}\right)\right) \wedge\left(s_{2}, m_{2}\right) \stackrel{*}{\rightarrow}$ $\left(s_{2}, m_{2_{i+1}}\left(\operatorname{loop}_{c}^{2_{i+1}}, \sigma_{2_{i+1}}\right)\right)$ where

- $\operatorname{loop}_{c}^{1_{i+1}}\left(n_{1}\right)=\operatorname{loop}_{c}^{2_{i+1}}\left(n_{2}\right)=i+1$; and

- $\forall x \in \operatorname{TVar}(s): \sigma_{1_{i+1}}(x)=\sigma_{2_{i+1}}(x)$; and

- $\forall m_{1}^{\prime}:\left(s_{1}, m_{1}\right) \stackrel{*}{\rightarrow}\left(S_{1}^{\prime}, m_{1}^{\prime}\left(\operatorname{loop}_{c}^{1^{\prime}}\right)\right) \stackrel{*}{\rightarrow}\left(s_{1}, m_{1_{i}}\right)$, $\operatorname{loop}_{c}^{1^{\prime}}\left(n_{1}\right) \leq i+1$; and

- $\forall m_{2}^{\prime}:\left(s_{2}, m_{2}\right) \stackrel{*}{\rightarrow}\left(S_{2}^{\prime}, m_{2}^{\prime}\left(\operatorname{loop}_{c}^{2^{\prime}}\right)\right) \stackrel{*}{\rightarrow}\left(s_{2}, m_{2_{i}}\right)$, $\operatorname{loop}_{c}^{2^{\prime}}\left(n_{2}\right) \leq i+1$;

By the hypothesis $\mathrm{IH}$, one of the following holds:
1. The loop counters for $s_{1}$ and $s_{2}$ are less than $i$ :

$\forall m_{1}^{\prime} m_{2}^{\prime}$ such that $\left(s_{1}, m_{1}\right) \stackrel{*}{\rightarrow}\left(S_{1}^{\prime}, m_{1}^{\prime}\left(\operatorname{loop}_{c}^{1^{\prime}}\right)\right)$ and $\left(s_{2}, m_{2}\right) \stackrel{*}{\rightarrow}$ $\left(S_{2}^{\prime}, m_{2}^{\prime}\left(\operatorname{loop}_{c}^{2^{\prime}}\right)\right)$,

$\operatorname{loop}_{c}^{1^{\prime}}\left(n_{1}\right)<i$ and $\operatorname{loop}_{c}^{2^{\prime}}\left(n_{2}\right)<i$ and one of the following holds:

(a) $s_{1}$ and $s_{2}$ both terminate: $\left(s_{1}, m_{1}\right) \stackrel{*}{\rightarrow}\left(\right.$ skip, $\left.m_{1}^{\prime \prime}\right)$ and $\left(s_{2}, m_{2}\right) \stackrel{*}{\rightarrow}\left(\right.$ skip, $\left.m_{2}^{\prime \prime}\right)$.

(b) $s_{1}$ and $s_{2}$ both do not terminate:

$\forall k>0,\left(s_{1}, m_{1}\right) \stackrel{k}{\rightarrow}\left(S_{1_{k}}, m_{1_{k}}\right)$ and $\left(s_{2}, m_{2}\right) \stackrel{k}{\rightarrow}\left(S_{2_{k}}, m_{2_{k}}\right)$ in which $S_{1_{k}} \neq$ skip, $S_{2_{k}} \neq$ skip.

When this case holds, then we have the loop counters for $s_{1}$ and $s_{2}$ are less than $i+1$, and $s_{1}$ and $s_{2}$ both terminate in the same way:

$\forall m_{1}^{\prime} m_{2}^{\prime}$ such that $\left(s_{1}, m_{1}\right) \stackrel{*}{\rightarrow}\left(S_{1}^{\prime}, m_{1}^{\prime}\left(\operatorname{loop}_{c}^{1^{\prime}}\right)\right)$ and $\left(s_{2}, m_{2}\right) \stackrel{*}{\rightarrow}$ $\left(S_{2}^{\prime}, m_{2}^{\prime}\left(\operatorname{loop}_{c}^{2^{\prime}}\right)\right)$,

$\operatorname{loop}_{c}^{1^{\prime}}\left(n_{1}\right)<i+1$ and $\operatorname{loop}_{c}^{2^{\prime}}\left(n_{2}\right)<i+1$ and one of the following holds:

(a) $s_{1}$ and $s_{2}$ both terminate:

$\left(s_{1}, m_{1}\right) \stackrel{*}{\rightarrow}\left(\right.$ skip, $\left.m_{1}^{\prime \prime}\right)$ and $\left(s_{2}, m_{2}\right) \stackrel{*}{\rightarrow}\left(\right.$ skip, $\left.m_{2}^{\prime \prime}\right)$.

(b) $s_{1}$ and $s_{2}$ both do not terminate:

$\forall k>0,\left(s_{1}, m_{1}\right) \stackrel{k}{\rightarrow}\left(S_{1_{k}}, m_{1_{k}}\right)$ and $\left(s_{2}, m_{2}\right) \stackrel{k}{\rightarrow}\left(S_{2_{k}}, m_{2_{k}}\right)$ in which $S_{1_{k}} \neq$ skip, $S_{2_{k}} \neq$ skip.

2. The loop counters for $s_{1}$ and $s_{2}$ are less than or equal to $i$, and $s_{1}$ and $s_{2}$ both do not terminate such that there are no configurations $\left(s_{1}, m_{1_{i}}\right)$ and $\left(s_{2}, m_{2_{i}}\right)$ reachable from $\left(s_{1}, m_{1}\right)$ and $\left(s_{2}, m_{2}\right)$, respectively, in which the loop counters of $s_{1}$ and $s_{2}$ are equal to $i$ and value stores agree on the values of variables in $\operatorname{TVar}(s)$ :

- $\forall m_{1}^{\prime} m_{2}^{\prime}:\left(s_{1}, m_{1}\right) \stackrel{*}{\rightarrow}\left(S_{1}, m_{1}^{\prime}\left(\operatorname{loop}_{c}^{1^{\prime}}\right)\right),\left(s_{2}, m_{2}\right) \stackrel{*}{\rightarrow}$ $\left(S_{2}, m_{2}^{\prime}\left(\operatorname{loop}_{c}^{2^{\prime}}\right)\right)$ where $\operatorname{loop}_{c}^{1^{\prime}}\left(n_{1}\right) \leq i, \operatorname{loop}_{c}^{2^{\prime}}\left(n_{2}\right) \leq i$;

- $\forall k>0:\left(s_{1}, m_{1}\right) \stackrel{k}{\rightarrow}\left(S_{1_{k}}, m_{1_{k}}\right),\left(s_{2}, m_{2}\right) \stackrel{k}{\rightarrow}\left(S_{2_{k}}, m_{2_{k}}\right)$ where $S_{1_{k}} \neq$ skip, $S_{2_{k}} \neq$ skip; and

$\nexists\left(s_{1}, m_{1_{i}}\right),\left(s_{2}, m_{2_{i}}\right):\left(s_{1}, m_{1}\right) \stackrel{*}{\rightarrow}\left(s_{1}, m_{1_{i}}\left(\mathfrak{f}_{1}, \operatorname{loop}_{c}^{1_{i}}, \sigma_{1_{i}}\right)\right) \wedge$

$\left(s_{2}, m_{2}\right) \stackrel{*}{\rightarrow}\left(s_{2}, m_{2_{i}}\left(\mathfrak{f}_{2}, \operatorname{loop}_{c}^{2_{i}}, \sigma_{2_{i}}\right)\right)$ where

- $\mathfrak{f}_{1}=\mathfrak{f}_{2}=0$; and

- $\operatorname{loop}_{c}^{1_{i}}\left(n_{1}\right)=\operatorname{loop}_{c}^{2_{i}}\left(n_{2}\right)=i$; and

- $\forall x \in \operatorname{TVar}(s): \sigma_{1_{i}}(x)=\sigma_{2_{i}}(x)$.

When this case holds, we have the loop counter of $s_{1}$ and $s_{2}$ are less than $i+1$, and $s_{1}$ and $s_{2}$ both do not terminate:

$\forall m_{1}^{\prime} m_{2}^{\prime}$ such that $\left(s_{1}, m_{1}\right) \stackrel{*}{\rightarrow}\left(S_{1}^{\prime}, m_{1}^{\prime}\left(\operatorname{loop}_{c}^{1^{\prime}}\right)\right)$ and $\left(s_{2}, m_{2}\right) \stackrel{*}{\rightarrow}$ $\left(S_{2}^{\prime}, m_{2}^{\prime}\left(\operatorname{loop}_{c}^{2^{\prime}}\right)\right)$,

$\operatorname{loop}_{c}^{1^{\prime}}\left(n_{1}\right)<i+1$ and $\operatorname{loop}_{c}^{2^{\prime}}\left(n_{2}\right)<i+1$ and $s_{1}$ and $s_{2}$ both do not terminate:

$\forall k>0,\left(s_{1}, m_{1}\right) \stackrel{k}{\rightarrow}\left(S_{1_{k}}, m_{1_{k}}\right)$ and $\left(s_{2}, m_{2}\right) \stackrel{k}{\rightarrow}\left(S_{2_{k}}, m_{2_{k}}\right)$ in which $S_{1_{k}} \neq$ skip, $S_{2_{k}} \neq$ skip.

3. There are two configurations $\left(s_{1}, m_{1_{i}}\right)$ and $\left(s_{2}, m_{2_{i}}\right)$ reachable from $\left(s_{1}, m_{1}\right)$ and $\left(s_{2}, m_{2}\right)$, respectively, in which crash flags are not set, the loop counters of $s_{1}$ and $s_{2}$ are equal to $i$ and value stores agree on the values of variables in $\operatorname{TVar}(s)$ and, for every state in executions $\left(s_{1}, m_{1}\right) \stackrel{*}{\rightarrow}\left(s_{1}, m_{1_{i}}\right)$ and $\left(s_{2}, m_{2}\right) \stackrel{*}{\rightarrow}\left(s_{2}, m_{2_{i}}\right)$ the loop counters for $s_{1}$ and $s_{2}$ are less than or equal to $i$ respectively:

$\exists\left(s_{1}, m_{1_{i}}\right)\left(s_{2}, m_{2_{i}}\right):\left(s_{1}, m_{1}\right) \stackrel{*}{\rightarrow}\left(s_{1}, m_{1_{i}}\left(\mathfrak{f}_{1}, \operatorname{loop}_{c}^{1_{i}}, \sigma_{1_{i}}\right)\right) \wedge$

$\left(s_{2}, m_{2}\right) \stackrel{*}{\rightarrow}\left(s_{2}, m_{2_{i}}\left(\mathfrak{f}_{2}, \operatorname{loop}_{c}^{2_{i}}, \sigma_{2_{i}}\right)\right)$ where

- $\mathfrak{f}_{1}=\mathfrak{f}_{2}=0$; and

- $\operatorname{loop}_{c}^{1_{i}}\left(n_{1}\right)=\operatorname{loop}_{c}^{2_{i}}\left(n_{2}\right)=i$; and

- $\forall x \in \operatorname{TVar}(s), \sigma_{1_{i}}(x)=\sigma_{2_{i}}(x)$; and 
- $\forall m_{1}^{\prime}:\left(s_{1}, m_{1}\right) \stackrel{*}{\rightarrow}\left(S_{1}^{\prime}, m_{1}^{\prime}\left(\operatorname{loop}_{c}^{1^{\prime}}\right)\right) \stackrel{*}{\rightarrow}\left(s_{1}, m_{1_{i}}\right)$, $\operatorname{loop}_{c}^{1^{\prime}}\left(n_{1}\right) \leq i$; and

- $\forall m_{2}^{\prime}:\left(s_{2}, m_{2}\right) \stackrel{*}{\rightarrow}\left(S_{2}^{\prime}, m_{2}^{\prime}\left(\operatorname{loop}_{c}^{2^{\prime}}\right)\right) \stackrel{*}{\rightarrow}\left(s_{2}, m_{2_{i}}\right)$, $\operatorname{loop}_{c}^{2^{\prime}}\left(n_{2}\right) \leq i$.

By similar argument in base case, evaluations of the predicate expression of $s_{1}$ and $s_{2}$ w.r.t value stores $\sigma_{1_{i}}$ and $\sigma_{2_{i}}$ produce same value. Then there are three possibilities:

(a) $\mathcal{E}^{\prime} \llbracket e \rrbracket \sigma_{1_{i}}=\mathcal{E}^{\prime} \llbracket e \rrbracket \sigma_{2_{i}}=$ (error, $*$ ).

Then the execution of $s_{1}$ proceeds as follows.

$\left(s_{1}, m_{1_{i}}\left(\mathfrak{f}_{1}, \sigma_{1_{i}}\right)\right)$

$=\left(\right.$ while $\left._{\left\langle n_{1}\right\rangle}(e)\left\{S_{1}\right\}, m_{1_{i}}\left(\mathfrak{f}_{1}, \sigma_{1_{i}}\right)\right)$

$\rightarrow\left(\right.$ while $\left\langle n_{1}\right\rangle(($ error, $\left.*))\left\{S_{1}\right\}, m_{1_{i}}\left(\mathfrak{f}_{1}, \sigma_{1_{i}}\right)\right)$ by the EEval' rule

$\rightarrow\left(\right.$ while $\left._{\left\langle n_{1}\right\rangle}(0)\left\{S_{1}\right\}, m_{1_{i}}\left(1 / \mathfrak{f}_{1}\right)\right)$ by the ECrash rule

$\stackrel{k}{\rightarrow}\left(\right.$ while $\left._{\left\langle n_{1}\right\rangle}(0)\left\{S_{1}\right\}, m_{1_{i}}\left(1 / \mathfrak{f}_{1}\right)\right)$, for any $k \geq 0$, by the Crash rule.

Similarly, the execution of $s_{2}$ started in the state $m_{2_{i}}\left(\sigma_{2_{i}}\right)$

does not terminate.

The loop counters for $s_{1}$ and $s_{2}$ are less than $i+1$ :

$\forall m_{1}^{\prime} m_{2}^{\prime}$ such that $\left(s_{1}, m_{1}\right) \stackrel{*}{\rightarrow}\left(S_{1}^{\prime}, m_{1}^{\prime}\left(\operatorname{loop}_{c}^{1^{\prime}}\right)\right)$ and $\left(s_{2}, m_{2}\right) \stackrel{*}{\rightarrow}\left(S_{2}^{\prime}, m_{2}^{\prime}\left(\right.\right.$ loop $\left.\left._{c}^{2^{\prime}}\right)\right)$,

$\operatorname{loop}_{c}^{1^{\prime}}\left(n_{1}\right)<i+1$ and $\operatorname{loop}_{c}^{2^{\prime}}\left(n_{2}\right)<i+1$.

Besides, $s_{1}$ and $s_{2}$ both do not terminate when started in states $m_{1}$ and $m_{2}$,

$\forall k>0,\left(s_{1}, m_{1}\right) \stackrel{k}{\rightarrow}\left(S_{1_{k}}, m_{1_{k}}\right)$ and $\left(s_{2}, m_{2}\right) \stackrel{k}{\rightarrow}\left(S_{2_{k}}, m_{2_{k}}\right)$ in which $S_{1_{k}} \neq$ skip, $S_{2_{k}} \neq$ skip.

(b) $\mathcal{E}^{\prime} \llbracket e \rrbracket \sigma_{1_{i}}=\mathcal{E}^{\prime} \llbracket e \rrbracket \sigma_{2_{i}}=\left(0, v_{\mathfrak{o f}}\right)$

The execution from $\left(s_{1}, m_{1_{i}}\left(\operatorname{loop}_{c}^{1_{i}}, \sigma_{1_{i}}\right)\right)$ proceeds as follows.

$$
\begin{aligned}
& \left(s_{1}, m_{1_{i}}\left(\operatorname{loop}_{c}^{1_{i}}, \sigma_{1_{i}}\right)\right) \\
= & \left(\text { while }_{\left\langle n_{1}\right\rangle}(e)\left\{S_{1}\right\}, m_{1_{i}}\left(\operatorname{loop}_{c}^{1_{i}}, \sigma_{1_{i}}\right)\right) \\
\rightarrow & \left(\text { while }_{\left\langle n_{1}\right\rangle}\left(\left(0, v_{\mathfrak{o f}}\right)\right)\left\{S_{1}\right\}, m_{1_{i}}\left(\operatorname{loop}_{c}^{1_{i}}, \sigma_{1_{i}}\right)\right) \text { by rule EEval' } \\
\rightarrow & \left(\text { while }_{\left\langle n_{1}\right\rangle}(0)\left\{S_{1}\right\}, m_{1_{i}}\left(\operatorname{loop}_{c}^{1_{i}}, \sigma_{1_{i}}\right)\right) \\
& \text { by rule E-Oflow1 or E-Oflow2 } \\
\rightarrow & \left(\text { skip }, m_{1_{i}}\left(\operatorname{loop}_{c}^{1_{i}}\left[0 /\left(n_{1}\right)\right], \sigma_{1_{i}}\right)\right) \text { by the Wh-F2 rule. }
\end{aligned}
$$

By the hypothesis $\mathrm{IH}$, the loop counter of $s_{1}$ and $s_{2}$ in any configuration in executions $\left(s_{1}, m_{1}\right) \stackrel{*}{\rightarrow}\left(s_{1}, m_{1_{i}}\left(\operatorname{loop}_{c}^{1_{i}}, \sigma_{1_{i}}\right)\right)$ and $\left(s_{2}, m_{2}\right) \stackrel{*}{\rightarrow}\left(s_{2}, m_{2_{i}}\left(\operatorname{loop}_{c}^{2_{i}}, \sigma_{2_{i}}\right)\right)$ respectively are less than or equal to $i$,

$\forall m_{1}^{\prime}:\left(s_{1}, m_{1}\right) \stackrel{*}{\rightarrow}\left(S_{1}^{\prime}, m_{1}^{\prime}\left(\operatorname{loop}_{c}^{1^{\prime}}\right)\right) \stackrel{*}{\rightarrow}\left(s_{1}, m_{1_{i}}\left(\operatorname{loop}_{c}^{1_{i}}\right.\right.$, $\left.\left.\sigma_{1_{i}}\right)\right)$, $\operatorname{loop}_{c}^{1^{\prime}}\left(n_{1}\right) \leq i$; and

$\forall m_{2}^{\prime}:\left(s_{2}, m_{2}\right) \stackrel{*}{\rightarrow}\left(S_{2}^{\prime}, m_{2}^{\prime}\left(\operatorname{loop}_{c}^{2^{\prime}}\right)\right) \stackrel{*}{\rightarrow}\left(s_{2}, m_{2_{i}}\left(\operatorname{loop}_{c}^{2_{i}}\right.\right.$, $\left.\left.\sigma_{2_{i}}\right)\right), \operatorname{loop}_{c}^{2^{\prime}}\left(n_{2}\right) \leq i$.

Therefore, $s_{1}$ and $s_{2}$ both terminate and the loop counter of $s_{1}$ and $s_{2}$ in any state in executions respectively are less than $i+1$.

(c) $\mathcal{E}^{\prime} \llbracket e \rrbracket \sigma_{1_{i}}=\mathcal{E}^{\prime} \llbracket e \rrbracket \sigma_{2_{i}}=\left(v, v_{\mathfrak{o f}}\right)$ where $v \notin\{0$, error $\}$;

The execution from $\left(s_{1}, m_{1_{i}}\left(\operatorname{loop}_{c}^{1_{i}}, \sigma_{1_{i}}\right)\right)$ proceeds as follows.

$$
\begin{aligned}
& \left(s_{1}, m_{1_{i}}\left(\operatorname{loop}_{c}^{1_{i}}, \sigma_{1_{i}}\right)\right) \\
= & \left(\text { while }_{\left\langle n_{1}\right\rangle}(e)\left\{S_{1}\right\}, m_{1_{i}}\left(\operatorname{loop}_{c}^{1_{i}}, \sigma_{1_{i}}\right)\right) \\
\rightarrow & \left(\text { while }_{\left\langle n_{1}\right\rangle}\left(\left(v, v_{\mathfrak{o f}}\right)\right)\left\{S_{1}\right\}, m_{1_{i}}\left(\operatorname{loop}_{c}^{1_{i}}, \sigma_{1_{i}}\right)\right) \text { by rule EEval'} \\
\rightarrow & \left(\text { while }_{\left\langle n_{1}\right\rangle}\left(\left(v, v_{\mathfrak{o f}}\right)\right)\left\{S_{1}\right\}, m_{1_{i}}\left(\operatorname{loop}_{c}^{1_{i}}, \sigma_{1_{i}}\right)\right) \text { by rule EEval' } \\
\rightarrow & (\text { while } \\
& \text { by rule E-Oflow1 or E-Oflow2 } \\
\rightarrow & \left(S_{1} ; S_{1} ; \text { while }_{\left\langle n_{1}\right\rangle}(e)\left\{S_{1}\right\}, m_{1_{i}}\left(\operatorname{loop}_{c}^{1_{i}}\left[(i+1) /\left(n_{1}\right)\right],\right.\right. \\
& \left.\left.\sigma_{1_{i}}\right)\right) \text { by rule Wh-T. }
\end{aligned}
$$

Similarly, $\left(s_{2}, m_{2_{i}}\left(\operatorname{loop}_{c}^{2_{i}}, \sigma_{2_{i}}\right)\right) \stackrel{2}{\rightarrow}\left(S_{2} ;\right.$ while $_{\left\langle n_{2}\right\rangle}(e)\left\{S_{2}\right\}$, $\left.m_{2_{i}}\left(\operatorname{loop}_{c}^{2_{i}}\left[(i+1) /\left(n_{2}\right)\right], \sigma_{2_{i}}\right)\right)$.

By similar argument in base case, the executions of $S_{1}$ and $S_{2}$ terminate in the same way when started in states $m_{1_{i}}\left(\operatorname{loop}_{c}^{1_{i}}\left[(i+1) /\left(n_{1}\right)\right], \sigma_{1_{i}}\right)$ and $m_{2_{i}}\left(\operatorname{loop}_{c}^{2_{i}}\left[(i+1) /\left(n_{2}\right)\right], \sigma_{2_{i}}\right)$ respectively. Then there are two possibilities.

i. $S_{1}$ and $S_{2}$ terminate when started in states $m_{1_{i}}\left(\operatorname{loop}_{c}^{1_{i}}[(i+\right.$

1) $\left.\left./\left(n_{1}\right)\right], \sigma_{1_{i}}\right)$ and

$m_{2_{i}}\left(\operatorname{loop}_{c}^{2_{i}}\left[(i+1) /\left(n_{2}\right)\right], \sigma_{2_{i}}\right)$ respectively

$\left(S_{1} ; s_{1}, m_{1_{i}}\left(\operatorname{loop}_{c}^{1_{i}}\left[(i+1) /\left(n_{1}\right)\right], \sigma_{1_{i}}\right)\right) \stackrel{*}{\rightarrow}$

$\left(s_{1}, m_{1_{i+1}}\left(\mathfrak{f}_{1}, \operatorname{loop}_{c}^{1_{i+1}}, \sigma_{1_{i+1}}\right)\right)$ and

$\left(S_{2} ; s_{1}, m_{2_{i}}\left(\operatorname{loop}_{c}^{2_{i}}\left[(i+1) /\left(n_{2}\right)\right], \sigma_{2_{i}}\right)\right) \stackrel{*}{\rightarrow}$

$\left(s_{2}, m_{2_{i+1}}\left(\mathfrak{f}_{2}\right.\right.$, loop $\left.\left._{c}^{2_{i+1}}, \sigma_{2_{i+1}}\right)\right)$ such that all of the following holds:

- $\mathfrak{f}_{1}=\mathfrak{f}_{2}=0$; and

- $\operatorname{loop}_{c}^{1_{i+1}}\left(n_{1}\right)=\operatorname{loop}_{c}^{2_{i+1}}\left(n_{2}\right)=i+1$; and

- $\forall y \in \operatorname{TVar}(s), \sigma_{1_{i+1}}(y)=\sigma_{2_{i+1}}(y)$, and

- in any state in the execution $\left(s_{1}, m_{1_{i}}\right) \stackrel{*}{\rightarrow}\left(s_{1}, m_{1_{i+1}}\left(\operatorname{loop}_{c}^{1_{i+1}}, \sigma_{1_{i+1}}\right)\right)$, the loop counter of $s_{1}$ is less than or equal to $i+1$.

- in any state in the executions

$\left(s_{2}, m_{2_{i}}\right) \stackrel{*}{\rightarrow}\left(s_{2}, m_{2_{i+1}}\left(\operatorname{loop}_{c}^{2_{i+1}}, \sigma_{2_{i+1}}\right)\right)$, the loop counter of $s_{2}$ is less than or equal to $i+1$.

With the hypothesis $\mathrm{IH}$, there are two configurations $\left(s_{1}, m_{1_{i+1}}\right)$ and $\left(s_{2}, m_{2_{i+1}}\right)$ reachable from $\left(s_{1}, m_{1}\right)$ and $\left(s_{2}, m_{2}\right)$, respectively, in which crash flags are not set, the loop counters of $s_{1}$ and $s_{2}$ are equal to $i+1$ and value stores agree on the values of $\operatorname{TVar}(s)$ and, for every state in executions $\left(s_{1}, m_{1}\right) \stackrel{*}{\rightarrow}\left(s_{1}, m_{1_{i+1}}\right)$ and $\left(s_{2}, m_{2}\right) \stackrel{*}{\rightarrow}\left(s_{2}, m_{2_{i+1}}\right)$ the loop counters for $s_{1}$ and $s_{2}$ are less than or equal to $i+1$ respectively:

$\exists\left(s_{1}, m_{1_{i+1}}\right)\left(s_{2}, m_{2_{i+1}}\right)$ :

$\left(s_{1}, m_{1}\right) \stackrel{*}{\rightarrow}\left(s_{1}, m_{1_{i+1}}\left(\mathfrak{f}_{1}, \operatorname{loop}_{c}^{1_{i+1}}, \sigma_{1_{i+1}}\right)\right) \wedge\left(s_{2}, m_{2}\right) \stackrel{*}{\rightarrow}$

$\left(s_{2}, m_{2_{i+1}}\left(\mathfrak{f}_{2}, \operatorname{loop}_{c}^{2_{i+1}}, \sigma_{2_{i+1}}\right)\right)$ where

- $\mathfrak{f}_{1}=\mathfrak{f}_{2}=0$; and

- $\operatorname{loop}_{c}^{1_{i+1}}\left(n_{1}\right)=\operatorname{loop}_{c}^{2_{i+1}}\left(n_{2}\right)=i+1$; and

- $\forall x \in \operatorname{TV} \operatorname{Var}(s), \sigma_{1_{i+1}}(x)=\sigma_{2_{i+1}}(x)$; and

- $\forall m_{1}^{\prime}:\left(s_{1}, m_{1}\right) \stackrel{*}{\rightarrow}\left(S_{1}^{\prime}, m_{1}^{\prime}\left(\operatorname{loop}_{c}^{1^{\prime}}\right)\right) \stackrel{*}{\rightarrow}$

$\left(s_{1}, m_{1_{i+1}}\left(\operatorname{loop}_{c}^{1_{i+1}}, \sigma_{1_{i+1}}\right)\right), \operatorname{loop}_{c}^{1^{\prime}}\left(n_{1}\right) \leq i+1$; and

- $\forall m_{2}^{\prime}:\left(s_{2}, m_{2}\right) \stackrel{*}{\rightarrow}\left(S_{2}^{\prime}, m_{2}^{\prime}\left(\operatorname{loop}_{c}^{2^{\prime}}\right)\right) \stackrel{*}{\rightarrow}$

$\left(s_{2}, m_{2_{i+1}}\left(\operatorname{loop}_{c}^{2_{i+1}}, \sigma_{2_{i+1}}\right)\right)$, $\operatorname{loop}_{c}^{2^{\prime}}\left(n_{2}\right) \leq i+1$.

ii. $S_{1}$ and $S_{2}$ do not terminate when started in states

$m_{1_{i}}\left(\operatorname{loop}_{c}^{1_{i}}\left[(i+1) /\left(n_{1}\right)\right], \sigma_{1_{i}}\right)$ and $m_{2_{i}}\left(\operatorname{loop}_{c}^{2_{i}}[(i+\right.$ $\left.\left.1) /\left(n_{2}\right)\right], \sigma_{2_{i}}\right)$ respectively:

$\forall k>0,\left(S_{1}, m_{1_{i}}\left(\operatorname{loop}_{c}^{1_{i}}\left[(i+1) /\left(n_{1}\right)\right], \sigma_{1_{i}}\right)\right) \stackrel{k}{\rightarrow}$ $\left(S_{1_{k}}, m_{1_{1_{k}}}\left(\operatorname{loop}_{c}{ }^{1_{1}}, \sigma_{1_{1_{k}}}\right)\right)$ and

$\left(S_{2}, m_{2_{i}}\left(\operatorname{loop}_{c}^{2_{i}}\left[(i+1) /\left(n_{2}\right)\right], \sigma_{2_{i}}\right)\right) \stackrel{k}{\rightarrow}$

$\left(S_{2_{k}}, m_{2_{1_{k}}}\left(\operatorname{loop}_{c}^{2}, \sigma_{2_{1_{k}}}\right)\right)$ in which $S_{1_{k}} \neq$ skip, $S_{2_{k}} \neq$ skip.

By our assumption of unique loop labels, $s_{1} \notin S_{1}$.

Then, $\forall k>0$, $\operatorname{loop}_{c}{ }^{1_{1}} k\left(n_{1}\right)=$

$\operatorname{loop}_{c}^{1_{i}}\left[(i+1) /\left(n_{1}\right)\right]\left(n_{1}\right)=i+1$. Similarly, $\forall k>$

$0, \operatorname{loop}_{c} 2_{1_{k}}\left(n_{2}\right)=$

loop $_{c}^{2 i}\left[(i+1) /\left(n_{2}\right)\right]\left(n_{2}\right)=i+1$. In addition, by LemmaE.2.

$\forall k>0,\left(S_{1} ; s_{1}, m_{1_{i}}\left(\operatorname{loop}_{c}^{1_{i}}\left[(i+1) /\left(n_{1}\right)\right], \sigma_{1_{i}}\right)\right) \stackrel{k}{\rightarrow}$

$\left(S_{1_{k}} ; s_{1}, m_{1_{1_{k}}}\left(\operatorname{loop}_{c}{ }_{1}, \sigma_{1_{1_{k}}}\right)\right)$ and $\left(S_{2} ; s_{2}, m_{2}\left(\operatorname{loop}_{c}^{2}[(i+\right.\right.$

$\left.\left.\left.1) /\left(n_{2}\right)\right], \sigma_{2}\right)\right) \stackrel{k}{\rightarrow}\left(S_{2_{k}} ; s_{2}, m_{2_{1_{k}}}\left(\operatorname{loop}_{c}^{2_{1}}, \sigma_{2_{1_{k}}}\right)\right)$ in which $S_{1_{k}} \neq$ skip, $S_{2_{k}} \neq$ skip.

In summary, the loop counter of $s_{1}$ and $s_{2}$ are less than equal to $i+1$, and $s_{1}$ and $s_{2}$ do not terminate such that 
there are no configurations $\left(s_{1}, m_{1_{i+1}}\right)$ and $\left(s_{2}, m_{2_{i+1}}\right)$ reachable from $\left(s_{1}, m_{1}\right)$ and $\left(s_{2}, m_{2}\right)$, respectively, in which crash flags are not set, the loop counters of $s_{1}$ and $s_{2}$ are equal to $i+1$ and value stores agree on values of variables in $\operatorname{TVar}(s)$.

Corollary 5.3. Let $s_{1}=$ "while ${ }_{\left\langle n_{1}\right\rangle}(e)\left\{S_{1}\right\}$ " and $s_{2}=$ "while ${ }_{\left\langle n_{2}\right\rangle}(e)\left\{S_{2}\right\}$ " be two while statements respectively, with the same set of the termination deciding variables, $\operatorname{TVar}\left(s_{1}\right)=$ $T \operatorname{Var}\left(s_{2}\right)=T \operatorname{Var}(s)$, whose bodies $S_{1}$ and $S_{2}$ satisfy the proof rule of equivalently computation of variables in TVar $(s), \forall x \in$ $T \operatorname{Var}(s):\left(S_{1}\right) \equiv_{x}^{S}\left(S_{2}\right)$, and whose bodies $S_{1}$ and $S_{2}$ terminate in the same way when started in states with crash flags not set and agreeing on values of variables in $T \operatorname{Var}\left(S_{1}\right) \cup \operatorname{TVar}\left(S_{2}\right)$ :

$\forall m_{S_{1}}\left(\mathfrak{f}_{S_{1}}, \sigma_{S_{1}}\right), m_{S_{2}}\left(\mathfrak{f}_{S_{2}}, \sigma_{S_{2}}\right)$ :

$\left(\left(\left(\forall z \in \operatorname{TVar}\left(S_{1}\right) \cup \operatorname{TVar}\left(S_{2}\right)\right), \sigma_{S_{1}}(z)=\sigma_{S_{2}}(z)\right) \wedge\left(\mathfrak{f}_{S_{1}}=\mathfrak{f}_{S_{2}}=\right.\right.$ $0)) \Rightarrow\left(S_{1}, m_{S_{1}}\left(\mathfrak{f}_{S_{1}}, \sigma_{S_{1}}\right)\right) \equiv_{H}\left(S_{2}, m_{S_{2}}\left(\mathfrak{f}_{S_{2}}, \sigma_{S_{2}}\right)\right)$.

If $s_{1}$ and $s_{2}$ start in the state $m_{1}\left(\mathfrak{f}_{1}\right.$, loop $\left._{c}^{1}, \sigma_{1}\right)$ and $m_{2}\left(\mathfrak{f}_{2}\right.$, loop $_{c}^{2}$, ,$\left.\sigma_{2}\right)$ respectively in which crash flags are not set, $\mathfrak{f}_{1}=$

$\mathfrak{f}_{2}=0, s_{1}$ and $s_{2}$ have not already executed, $\operatorname{loop}_{c}^{1}\left(n_{1}\right)=$ $\operatorname{loop}_{c}^{2}\left(n_{2}\right)=0$, value stores $\sigma_{1}$ and $\sigma_{2}$ agree on values of variables in $T \operatorname{Var}(s), \forall x \in T \operatorname{Var}(s), \sigma_{1}(x)=\sigma_{2}(x)$, then $s_{1}$ and $s_{2}$ terminate in the same way:

1. $s_{1}$ and $s_{2}$ both terminate, $\left(s_{1}, m_{1}\right) \stackrel{*}{\rightarrow}\left(\right.$ skip, $\left.m_{1}^{\prime}\right),\left(s_{2}, m_{2}\right) \stackrel{*}{\rightarrow}$ (skip, $m_{2}^{\prime}$ ).

2. $s_{1}$ and $s_{2}$ both do not terminate, $\forall k>0,\left(s_{1}, m_{1}\right) \stackrel{k}{\rightarrow}$ $\left(S_{1_{k}}, m_{1_{k}}\right),\left(s_{2}, m_{2}\right) \stackrel{k}{\rightarrow}\left(S_{2_{k}}, m_{2_{k}}\right)$ where $S_{1_{k}} \neq$ skip, $S_{2_{k}} \neq$ skip.

This is from Lemma 5.15 immediately.

Lemm 5.16 is necessary only for showing the same $\mathrm{I} / \mathrm{O}$ sequence in the next section.

Lemma 5.16. Let $s_{1}=$ "while ${ }_{\left\langle n_{1}\right\rangle}(e)\left\{S_{1}\right\}$ " and $s_{2}=$ "while ${ }_{\left\langle n_{2}\right\rangle}(e)\left\{S_{2}\right\}$ " be two while statements in program $P_{1}$ and $P_{2}$ respectively with the same set of termination deciding variables, $T \operatorname{Var}\left(s_{1}\right)=T \operatorname{Var}\left(s_{2}\right)=T \operatorname{Var}(s)$, whose bodies $S_{1}$ and $S_{2}$ satisfy the proof rule of equivalently computation of variables in $T \operatorname{Var}(s), \forall x \in T \operatorname{Var}(s): S_{1} \equiv_{x}^{S} S_{2}$ and whose bodies $S_{1}$ and $S_{2}$ terminate in the same way in executions when started in states with crash flags not set and agreeing on values of variables in $T \operatorname{Var}\left(S_{1}\right) \cup T \operatorname{Var}\left(S_{2}\right)$ :

$\forall m_{S_{1}}\left(\mathfrak{f}_{S_{1}}, \sigma_{S_{1}}\right) m_{S_{2}}\left(\mathfrak{f}_{S_{2}}, \sigma_{S_{2}}\right)$ :

$\left(\left(\left(\forall z \in T \operatorname{Var}\left(S_{1}\right) \cup T \operatorname{Var}\left(S_{2}\right)\right), \sigma_{S_{1}}(z)=\sigma_{S_{2}}(z)\right) \wedge\left(\mathfrak{f}_{S_{1}}=\mathfrak{f}_{S_{2}}=\right.\right.$ $0)$

$\Rightarrow\left(S_{1}, m_{S_{1}}\left(\mathfrak{f}_{S_{1}}, \sigma_{S_{1}}\right)\right) \equiv_{H}\left(S_{2}, m_{S_{2}}\left(\mathfrak{f}_{S_{2}}, \sigma_{S_{2}}\right)\right)$.

If $s_{1}$ and $s_{2}$ start in the state $m_{1}\left(\mathfrak{f}_{1}\right.$, loop $\left._{c}^{1}, \sigma_{1}\right)$ and $m_{2}\left(\mathfrak{f}_{2}\right.$, loop $_{c}^{2}$, ,$\left.\sigma_{2}\right)$ respectively in which crash flags are not set, $\mathfrak{f}_{1}=\mathfrak{f}_{2}=0, s_{1}$ and $s_{2}$ have not already executed, $\operatorname{loop}_{c}^{1}\left(n_{1}\right)=\operatorname{loop}_{c}^{2}\left(n_{2}\right)=0$, value stores $\sigma_{1}$ and $\sigma_{2}$ agree on values of variables in TVar $(s)$, $\forall x \in T \operatorname{Var}(s), \sigma_{1}(x)=\sigma_{2}(x)$, one of the following holds:

1. $s_{1}$ and $s_{2}$ both terminate and the loop counters of $s_{1}$ and $s_{2}$ are less than a positive integer $i$ and less than or equal to $i-1$ : $\left(s_{1}, m_{1}\right) \stackrel{*}{\rightarrow}\left(\right.$ skip, $\left.m_{1}^{\prime}\right),\left(s_{2}, m_{2}\right) \stackrel{*}{\rightarrow}\left(\right.$ skip, $\left.m_{2}^{\prime}\right)$ where both of the following hold:

- The loop counters of $s_{1}$ and $s_{2}$ are less than a positive integer $i$ :

$\exists i>0 \forall m_{1}^{\prime} m_{2}^{\prime}$ :

$\left(s_{1}, m_{1}\right) \stackrel{*}{\rightarrow}\left(S_{1}^{\prime}, m_{1}^{\prime}\left(\operatorname{loop}_{c}^{1^{\prime}}\right)\right), \operatorname{loop}_{c}^{1^{\prime}}\left(n_{1}\right)<i$ and

$\left(s_{2}, m_{2}\right) \stackrel{*}{\rightarrow}\left(S_{2}^{\prime}, m_{2}^{\prime}\left(\right.\right.$ loop $\left.\left._{c}^{2^{\prime}}\right)\right)$, loop $_{c}^{2^{\prime}}\left(n_{2}\right)<i$.

- $\forall 0<j<i$, there are two configurations $\left(s_{1}, m_{1_{j}}\right)$ and $\left(s_{2}, m_{2_{j}}\right)$ reachable from $\left(s_{1}, m_{1}\right)$ and $\left(s_{2}, m_{2}\right)$, respec- tively, in which both crash flags are not set, the loop counters of $s_{1}$ and $s_{2}$ are equal to $j$ and value stores agree on the values of variables in $T \operatorname{Var}(s)$, and for every state in execution $\left(s_{1}, m_{1}\right) \stackrel{*}{\rightarrow}\left(s_{1}, m_{1_{j}}\right)$ or $\left(s_{2}, m_{2}\right) \stackrel{*}{\rightarrow}\left(s_{2}, m_{2_{j}}\right)$, the loop counters for $s_{1}$ and $s_{2}$ are less than or equal to $j$ respectively:

$\exists\left(s_{1}, m_{1_{j}}\right)\left(s_{2}, m_{2_{j}}\right)$ :

$\left(s_{1}, m_{1}\right) \stackrel{*}{\rightarrow}\left(s_{1}, m_{1_{j}}\left(\mathfrak{f}_{1}, \operatorname{loop}_{c}^{1_{j}}, \sigma_{1_{j}}\right)\right) \wedge$

$\left(s_{2}, m_{2}\right) \stackrel{*}{\rightarrow}\left(s_{2}, m_{2_{j}}\left(\mathfrak{f}_{2}\right.\right.$, loop $\left.\left._{c}^{2_{j}}, \sigma_{2_{j}}\right)\right)$ where

- $\mathfrak{f}_{1}=\mathfrak{f}_{2}=0$; and

- $\operatorname{loop}_{c}^{1_{j}}\left(n_{1}\right)=\operatorname{loop}_{c}^{2_{j}}\left(n_{2}\right)=j$; and

- $\forall x \in T \operatorname{Var}(s): \sigma_{1_{j}}(x)=\sigma_{2_{j}}(x)$; and

- $\forall m_{1}^{\prime}:\left(s_{1}, m_{1}\right) \stackrel{*}{\rightarrow}\left(S_{1}^{\prime}, m_{1}^{\prime}\left(\right.\right.$ loop $\left.\left._{c}^{1^{\prime}}\right)\right) \stackrel{*}{\rightarrow}\left(s_{1}, m_{1_{j}}\right)$, $\operatorname{loop}_{c}^{1^{\prime}}\left(n_{1}\right) \leq j$; and

- $\forall m_{2}^{\prime}:\left(s_{2}, m_{2}\right) \stackrel{*}{\rightarrow}\left(S_{2}^{\prime}, m_{2}^{\prime}\left(\operatorname{loop}_{c}^{2^{\prime}}\right)\right) \stackrel{*}{\rightarrow}\left(s_{2}, m_{2_{j}}\right)$, $\operatorname{loop}_{c}^{2^{\prime}}\left(n_{2}\right) \leq j$.

2. $s_{1}$ and $s_{2}$ both do not terminate, $\forall k>0,\left(s_{1}, m_{1}\right) \stackrel{k}{\rightarrow}$ $\left(S_{1_{k}}, m_{1_{k}}\right),\left(s_{2}, m_{2}\right) \stackrel{k}{\rightarrow}\left(S_{2_{k}}, m_{2_{k}}\right)$ where $S_{1_{k}} \neq$ skip, $S_{2_{k}} \neq$ skip such that one of the following holds:

(a) For any positive integer $i$, there are two configurations $\left(s_{1}, m_{1_{i}}\right)$ and $\left(s_{2}, m_{2_{i}}\right)$ reachable from $\left(s_{1}, m_{1}\right)$ and $\left(s_{2}, m_{2}\right)$, respectively, in which both crash flags are not set, the loop counters of $s_{1}$ and $s_{2}$ are equal to $i$ and value stores agree on the values of variables in TVar $(s)$, and for every state in execution $\left(s_{1}, m_{1}\right) \stackrel{*}{\rightarrow}\left(s_{1}, m_{1_{i}}\right)$ or $\left(s_{2}, m_{2}\right) \stackrel{*}{\rightarrow}\left(s_{2}, m_{2_{i}}\right)$, the loop counters for $s_{1}$ and $s_{2}$ are less than or equal to $i$ respectively:

$\forall i>0 \exists\left(s_{1}, m_{1_{i}}\right)\left(s_{2}, m_{2_{i}}\right)$ :

$\left(s_{1}, m_{1}\right) \stackrel{*}{\rightarrow}\left(s_{1}, m_{1_{i}}\left(\mathfrak{f}_{1}, \operatorname{loop}_{c}^{1_{i}}, \sigma_{1_{i}}\right)\right) \wedge$

$\left(s_{2}, m_{2}\right) \stackrel{*}{\rightarrow}\left(s_{2}, m_{2_{i}}\left(\mathfrak{f}_{2}\right.\right.$, loop $\left.\left._{c}^{2_{i}}, \sigma_{2_{i}}\right)\right)$ where

- $\mathfrak{f}_{1}=\mathfrak{f}_{2}=0$; and

- $\operatorname{loop}_{c}^{1_{i}}\left(n_{1}\right)=\operatorname{loop}_{c}^{2_{i}}\left(n_{2}\right)=i$; and

- $\forall x \in \operatorname{TVar}(s): \sigma_{1_{i}}(x)=\sigma_{2_{i}}(x)$; and

- $\forall m_{1}^{\prime}:\left(s_{1}, m_{1}\right) \stackrel{*}{\rightarrow}\left(S_{1}^{\prime}, m_{1}^{\prime}\left(\operatorname{loop}_{c}^{1^{\prime}}\right)\right) \stackrel{*}{\rightarrow}\left(s_{1}, m_{1_{i}}\right)$, $\operatorname{loop}_{c}^{1^{\prime}}\left(n_{1}\right) \leq i$; and

- $\forall m_{2}^{\prime}:\left(s_{2}, m_{2}\right) \stackrel{*}{\rightarrow}\left(S_{2}^{\prime}, m_{2}^{\prime}\left(\right.\right.$ loop $\left.\left._{c}^{2^{\prime}}\right)\right) \stackrel{*}{\rightarrow}\left(s_{2}, m_{2_{i}}\right)$, $\operatorname{loop}_{c}^{2^{\prime}}\left(n_{2}\right) \leq i$;

(b) The loop counters for $s_{1}$ and $s_{2}$ are less than a positive integer $i$ and less than or equal to $i-1$ such that all of the following hold:

- $\exists i>0, \forall m_{1}^{\prime} m_{2}^{\prime}:\left(s_{1}, m_{1}\right) \stackrel{*}{\rightarrow}\left(S_{1}, m_{1}^{\prime}\left(\operatorname{loop}_{c}^{1^{\prime}}\right)\right)$, $\left(s_{2}, m_{2}\right) \stackrel{*}{\rightarrow}\left(S_{2}, m_{2}^{\prime}\left(\right.\right.$ loop $\left.\left._{c}^{2^{\prime}}\right)\right)$ where loop $_{c}^{1^{\prime}}\left(n_{1}\right)<i$, $\operatorname{loop}_{c}^{2^{\prime}}\left(n_{2}\right)<i$;

- $\forall 0<j<i$, there are two configurations $\left(s_{1}, m_{1_{j}}\right)$ and $\left(s_{2}, m_{2_{j}}\right)$ reachable from $\left(s_{1}, m_{1}\right)$ and $\left(s_{2}, m_{2}\right)$, respectively, in which both crash flags are not set, the loop counters of $s_{1}$ and $s_{2}$ are equal to $j$ and value stores agree on the values of variables in TVar $(s)$, and for every state in execution $\left(s_{1}, m_{1}\right) \stackrel{*}{\rightarrow}\left(s_{1}, m_{1_{j}}\right)$ or $\left(s_{2}, m_{2}\right) \stackrel{*}{\rightarrow}\left(s_{2}, m_{2_{j}}\right)$, the loop counters for $s_{1}$ and $s_{2}$ are less than or equal to $j$ respectively:

$\exists\left(s_{1}, m_{1_{j}}\right)\left(s_{2}, m_{2_{j}}\right)$ :

$\left(s_{1}, m_{1}\right) \stackrel{*}{\rightarrow}\left(s_{1}, m_{1_{j}}\left(\mathfrak{f}_{1}, \operatorname{loop}_{c}{ }_{1_{j}}, \sigma_{1_{j}}\right)\right) \wedge$

$\left(s_{2}, m_{2}\right) \stackrel{*}{\rightarrow}\left(s_{2}, m_{2_{j}}\left(\mathfrak{f}_{2}, \operatorname{loop}_{c}^{2_{j}}, \sigma_{2_{j}}\right)\right)$ where

- $\mathfrak{f}_{1}=\mathfrak{f}_{2}=0$; and

- $\operatorname{loop}_{c}^{1_{j}}\left(n_{1}\right)=\operatorname{loop}_{c}^{2_{j}}\left(n_{2}\right)=j$; and

- $\forall x \in T \operatorname{Var}(s): \sigma_{1_{j}}(x)=\sigma_{2_{j}}(x)$; and 


$$
\begin{aligned}
& \text { - } \forall m_{1}^{\prime}:\left(s_{1}, m_{1}\right) \stackrel{*}{\rightarrow}\left(S_{1}^{\prime}, m_{1}^{\prime}\left(\operatorname{loop}_{c}^{1^{\prime}}\right)\right) \stackrel{*}{\rightarrow}\left(s_{1}, m_{1_{j}}\right), \\
& \quad \operatorname{loop}_{c}^{1^{\prime}}\left(n_{1}\right) \leq j ; \text { and } \\
& \text { - } \forall m_{2}^{\prime}:\left(s_{2}, m_{2}\right) \stackrel{*}{\rightarrow}\left(S_{2}^{\prime}, m_{2}^{\prime}\left(\operatorname{loop}_{c}^{2^{\prime}}\right)\right) \stackrel{*}{\rightarrow}\left(s_{2}, m_{2_{j}}\right), \\
& \quad \operatorname{loop}_{c}^{2^{\prime}}\left(n_{2}\right) \leq j ;
\end{aligned}
$$

(c) The loop counters for $s_{1}$ and $s_{2}$ are less than or equal to some positive integer $i$ such that all of the following hold:

- $\exists i>0 \forall m_{1}^{\prime} m_{2}^{\prime}:\left(s_{1}, m_{1}\right) \stackrel{*}{\rightarrow}\left(S_{1}, m_{1}^{\prime}\left(\operatorname{loop}_{c}^{1^{\prime}}\right)\right)$, $\left(s_{2}, m_{2}\right) \stackrel{*}{\rightarrow}\left(S_{2}, m_{2}^{\prime}\left(\right.\right.$ loop $\left.\left._{c}^{2^{\prime}}\right)\right)$ where loop ${ }_{c}^{1^{\prime}}\left(n_{1}\right) \leq i$, loop $2_{c}^{2^{\prime}}\left(n_{2}\right) \leq i$;

- $\forall 0<j<i$, there are two configurations $\left(s_{1}, m_{1_{j}}\right)$ and $\left(s_{2}, m_{2_{j}}\right)$ reachable from $\left(s_{1}, m_{1}\right)$ and $\left(s_{2}, m_{2}\right)$, respectively, in which both crash flags are not set, the loop counters of $s_{1}$ and $s_{2}$ are equal to $j$ and value stores agree on the values of variables in TVar $(s)$, and for every state in execution $\left(s_{1}, m_{1}\right) \stackrel{*}{\rightarrow}\left(s_{1}, m_{1_{j}}\right)$ or $\left(s_{2}, m_{2}\right) \stackrel{*}{\rightarrow}\left(s_{2}, m_{2_{j}}\right)$, the loop counters for $s_{1}$ and $s_{2}$ are less than or equal to $j$ respectively:

$\exists\left(s_{1}, m_{1_{j}}\right)\left(s_{2}, m_{2_{j}}\right)$ :

$\left(s_{1}, m_{1}\right) \stackrel{*}{\rightarrow}\left(s_{1}, m_{1_{j}}\left(\mathfrak{f}_{1}, \operatorname{loop}_{c}^{1_{j}}, \sigma_{1_{j}}\right)\right) \wedge$

$\left(s_{2}, m_{2}\right) \stackrel{*}{\rightarrow}\left(s_{2}, m_{2_{j}}\left(\mathfrak{f}_{2}\right.\right.$, loop $\left.\left._{c}{ }^{2_{j}}, \sigma_{2_{j}}\right)\right)$ where

- $\mathfrak{f}_{1}=\mathfrak{f}_{2}=0$; and

- $\operatorname{loop}_{c}^{1_{j}}\left(n_{1}\right)=\operatorname{loop}_{c}^{2_{j}}\left(n_{2}\right)=j$; and

- $\forall x \in \operatorname{TVar}(s): \sigma_{1_{j}}(x)=\sigma_{2_{j}}(x)$; and

- $\forall m_{1}^{\prime}:\left(s_{1}, m_{1}\right) \stackrel{*}{\rightarrow}\left(S_{1}^{\prime}, m_{1}^{\prime}\left(\operatorname{loop}_{c}^{1^{\prime}}\right)\right) \stackrel{*}{\rightarrow}\left(s_{1}, m_{1_{j}}\right)$, $\operatorname{loop}_{c}^{1^{\prime}}\left(n_{1}\right) \leq j$; and

- $\forall m_{2}^{\prime}:\left(s_{2}, m_{2}\right) \stackrel{*}{\rightarrow}\left(S_{2}^{\prime}, m_{2}^{\prime}\left(\operatorname{loop}_{c}^{2^{\prime}}\right)\right) \stackrel{*}{\rightarrow}\left(s_{2}, m_{2_{j}}\right)$, $\operatorname{loop}_{c}^{2^{\prime}}\left(n_{2}\right) \leq j$;

- There are no configurations $\left(s_{1}, m_{1_{i}}\right)$ and $\left(s_{2}, m_{2_{i}}\right)$ reachable from $\left(s_{1}, m_{1}\right)$ and $\left(s_{2}, m_{2}\right)$, respectively, in which crash flags are not set, the loop counters of $s_{1}$ and $s_{2}$ are equal to $i$, and value stores agree on the values of variables in TVar $(s)$ :

$\nexists\left(s_{1}, m_{1_{i}}\right)\left(s_{2}, m_{2_{i}}\right)$ :

$\left(s_{1}, m_{1}\right) \stackrel{*}{\rightarrow}\left(s_{1}, m_{1_{i}}\left(\mathfrak{f}_{1}, \operatorname{loop}_{c}^{1_{i}}, \sigma_{1_{i}}\right)\right) \wedge$

$\left(s_{2}, m_{2}\right) \stackrel{*}{\rightarrow}\left(s_{2}, m_{2_{i}}\left(\mathfrak{f}_{2}\right.\right.$, loop $\left.\left._{c}^{2_{i}}, \sigma_{2_{i}}\right)\right)$ where

- $\mathfrak{f}_{1}=\mathfrak{f}_{2}=0$; and

- $\operatorname{loop}_{c}^{1_{i}}\left(n_{1}\right)=\operatorname{loop}_{c}^{2_{i}}\left(n_{2}\right)=i$; and

- $\forall x \in \operatorname{TVar}(s): \sigma_{1_{i}}(x)=\sigma_{2_{i}}(x)$.

Proof. From lemma 5.15, we have $s_{1}$ and $s_{2}$ terminate in the same way when started in states $m_{1}$ and $m_{2}$ respectively. Then there are two big cases.

1. $s_{1}$ and $s_{2}$ both terminate.

Let $i$ be the smallest integer such that the loop counters of $s_{1}$ and $s_{2}$ are less than $i$ in the executions. Then there are two possibilities.

(a) $i=1$.

In the proof of Lemma 5.15 the evaluation of the loop predicate of $s_{1}$ and $s_{2}$ produce zero w.r.t value stores, $\sigma_{1}$ and $\sigma_{2}$. Then the execution of $s_{1}$ proceeds as follows:

$$
\left(s_{1}, m_{1}\left(\operatorname{loop}_{c}^{1}, \sigma_{1}\right)\right)
$$

$=\left(\right.$ while $_{\left\langle n_{1}\right\rangle}(e)\left\{S_{1}\right\}, m_{1}\left(\right.$ loop $\left.\left._{c}^{1}\right)\right)$

$\rightarrow\left(\right.$ while $\left.\left._{\left\langle n_{1}\right\rangle}\right\rangle\left(\left(0, v_{\mathfrak{o f}}\right)\right)\left\{S_{1}\right\}, m_{1}\left(\operatorname{loop}_{c}^{1}\right)\right)$ by rule EEval'

$\rightarrow\left(\right.$ while $_{\left\langle n_{1}\right\rangle}(0)\left\{S_{1}\right\}, m_{1}\left(\right.$ loop $\left.\left._{c}^{1}\right)\right)$

by rule E-Oflow1 or E-Oflow2

$\rightarrow\left(\right.$ skip, $m_{1}\left(\right.$ loop $\left.\left._{c}^{1} \backslash\left\{\left(n_{1}, *\right)\right\}\right)\right)$

by rule Wh-F1 or Wh-F2.
Similarly, $\left(s_{2}, m_{2}\left(\operatorname{loop}_{c}^{2}, \sigma_{2}\right)\right) \stackrel{2}{\rightarrow}\left(\right.$ skip, $m_{2}\left(\right.$ loop $_{c}^{2} \backslash\left\{\left(n_{2}\right.\right.$, $, *)\}))$. Then the lemma holds because of the initial configuration $\left(s_{1}, m_{1}\left(\operatorname{loop}_{c}^{1}, \sigma_{1}\right)\right)$ and $\left(s_{2}, m_{2}\left(\operatorname{loop}_{c}^{2}, \sigma_{2}\right)\right)$.

(b) $i>1$.

Because $s_{1}$ and $s_{2}$ terminate, $i$ is the smallest positive integer such that the loop counters of $s_{1}$ and $s_{2}$ are less than $i$, by Lemma 5.15 $\forall 0<j<i$, there are two configurations $\left(s_{1}, m_{1_{j}}\right)$ and $\left(s_{2}, m_{2_{j}}\right)$ reachable from $\left(s_{1}, m_{1}\right)$ and $\left(s_{2}, m_{2}\right)$, respectively, in which both crash flags are not set, the loop counters of $s_{1}$ and $s_{2}$ are equal to $j$ and value stores agree on the values of variables in $\operatorname{TVar}(s)$, and for every state in execution, $\left(s_{1}, m_{1}\right) \stackrel{*}{\rightarrow}\left(s_{1}, m_{1_{j}}\right)$ or $\left(s_{2}, m_{2}\right) \stackrel{*}{\rightarrow}\left(s_{2}, m_{2_{j}}\right)$ the loop counters for $s_{1}$ and $s_{2}$ are less than or equal to $j$ respectively. With the initial configuration $\left(s_{1}, m_{1}\right)$ and $\left(s_{2}, m_{2}\right)$, the lemma holds.

2. $s_{1}$ and $s_{2}$ both do not terminate. There are three possibilities.

(a) $\forall i>0$, there are two configurations $\left(s_{1}, m_{1_{i}}\right)$ and $\left(s_{2}, m_{2_{i}}\right)$ reachable from $\left(s_{1}, m_{1}\right)$ and $\left(s_{2}, m_{2}\right)$, respectively, in which both crash flags are not set, the loop counters of $s_{1}$ and $s_{2}$ are equal to $i$ and value stores agree on the values of variables in $\operatorname{TVar}(s)$, and for every state in execution, $\left(s_{1}, m_{1}\right) \stackrel{*}{\rightarrow}\left(s_{1}, m_{1_{i}}\right)$ or $\left(s_{2}, m_{2}\right) \stackrel{*}{\rightarrow}\left(s_{2}, m_{2_{i}}\right)$ the loop counters for $s_{1}$ and $s_{2}$ are less than or equal to $i$ respectively.

(b) The loop counters of $s_{1}$ and $s_{2}$ are less than a positive integer $i$.

Let $i$ be the smallest positive integer such that there is no positive integer $j<i$ that the loop counters of $s_{1}$ and $s_{2}$ are less than the positive integer $j$. This case occurs when $s_{1}$ and $s_{2}$ finish the full $(i-1)$ th iterations and both executions raise an exception in the evaluation of the loop predicate of $s_{1}$ and $s_{2}$ for the $i$ th time. There are further two possibilities.

i. $i=1$.

In proof of Lemma 5.15 evaluations of the predicate expression of $s_{1}$ and $s_{2}$ raise an exception w.r.t value stores $\sigma_{1}$ and $\sigma_{2}$. The lemma holds.

ii. $i>1$. By the assumption of initial states $\left(s_{1}, m_{1}\right)$ and $\left(s_{2}, m_{2}\right)$, when $j=0$, initial states $\left(s_{1}, m_{1}\right)$ and $\left(s_{2}, m_{2}\right)$ have crash flags not set, the loop counters of $s_{1}$ and $s_{2}$ are zero and value stores agree on values of variables of $s_{1}$ and $s_{2}$.

By Lemma 5.15, $\forall 0<j<i$, there are two configurations $\left(s_{1}, m_{1_{j}}\right)$ and $\left(s_{2}, m_{2_{j}}\right)$ reachable from $\left(s_{1}, m_{1}\right)$ and $\left(s_{2}, m_{2}\right)$, respectively, in which both crash flags are not set, the loop counters of $s_{1}$ and $s_{2}$ are equal to $j$ and value stores agree on the values of variables in $\operatorname{TVar}(s)$, and for every state in execution, $\left(s_{1}, m_{1}\right) \stackrel{*}{\rightarrow}\left(s_{1}, m_{1_{j}}\right)$ or $\left(s_{2}, m_{2}\right) \stackrel{*}{\rightarrow}\left(s_{2}, m_{2_{j}}\right)$ the loop counters for $s_{1}$ and $s_{2}$ are less than or equal to $j$ respectively. With the initial configuration $\left(s_{1}, m_{1}\right)$ and $\left(s_{2}, m_{2}\right)$, the lemma holds.

(c) The loop counters of $s_{1}$ and $s_{2}$ are less than or equal to a positive integer $i$.

Let $i$ be the smallest positive integer such that the loop counters of $s_{1}$ and $s_{2}$ are less than or equal to the positive integer $i$. There are two possibilities.

i. $i=1$.

In the proof of Lemma 5.15 the execution of $s_{1}$ proceeds as follows:

$$
\begin{aligned}
& \left(s_{1}, m_{1}\left(\operatorname{loop}_{c}^{1}, \sigma_{1}\right)\right) \\
= & \left(\text { while }_{\left\langle n_{1}\right\rangle}(e)\left\{S_{1}\right\}, m_{1}\left(\operatorname{loop}_{c}^{1}, \sigma_{1}\right)\right) \\
\rightarrow & \left(\text { while }_{\left\langle n_{1}\right\rangle}\left(\left(v, v_{\mathfrak{o f}}\right)\right)\left\{S_{1}\right\}, m_{1}\left(\operatorname{loop}_{c}^{1}, \sigma_{1}\right)\right) \text { where } v \neq 0 \\
& \text { by rule EEval } \\
\rightarrow & \left(\text { while }_{\left\langle n_{1}\right\rangle}(v)\left\{S_{1}\right\}, m_{1}\left(\operatorname{loop}_{c}^{1}, \sigma_{1}\right)\right) \\
& \text { by rule E-Oflow1 or E-Oflow2 }
\end{aligned}
$$




$$
\rightarrow\left(S_{1} ; \text { while }_{\left\langle n_{1}\right\rangle}(e)\left\{S_{1}\right\}, m_{1}\left(\operatorname{loop}_{c}^{1}\left[1 /\left(n_{1}\right)\right], \sigma_{1}\right)\right)
$$
by rule $\mathrm{Wh}-\mathrm{T}$.

The execution of $s_{2}$ proceeds to $\left(S_{2} ;\right.$ while $\left._{\left\langle n_{2}\right\rangle}(e) \quad\left\{S_{2}\right\}, m_{2}\left(\operatorname{loop}_{c}^{2}\left[1 /\left(n_{2}\right)\right], \sigma_{2}\right)\right)$. In addition, executions of $S_{1}$ and $S_{2}$ do not terminate when started in states $m_{1}\left(\operatorname{loop}_{c}^{1}\left[1 /\left(n_{1}\right)\right], \sigma_{1}\right)$ and $m_{2}\left(\operatorname{loop}_{c}^{2}\left[1 /\left(n_{2}\right)\right], \sigma_{2}\right)$. By Lemma E.2 executions of $s_{1}$ and $s_{2}$ do not terminate.

ii. $i>1$.

In the proof of Lemma 5.15, when started in the state $\left(s_{1}, m_{1_{i-1}}\left(\mathfrak{f}_{1}, \operatorname{loop}_{c}^{1_{i-1}}, \sigma_{1_{i-1}}\right)\right)$ the execution of $s_{1}$ proceeds as follows:

$$
\begin{aligned}
& \left(s_{1}, m_{1_{i-1}}\left(\mathfrak{f}_{1}, \operatorname{loop}_{c}^{1_{i-1}}, \sigma_{1_{i-1}}\right)\right) \\
= & \left(\text { while }_{\left\langle n_{1}\right\rangle}(e)\left\{S_{1}\right\}, m_{1_{i-1}}\left(\mathfrak{f}_{1}, \operatorname{loop}_{c}^{1_{i-1}}, \sigma_{1_{i-1}}\right)\right) \\
\rightarrow & \left(\text { while }_{\left\langle n_{1}\right\rangle}\left(\left(v, v_{\mathfrak{o f}}\right)\right)\left\{S_{1}\right\}, m_{1_{i-1}}\left(\mathfrak{f}_{1}, \operatorname{loop}_{c}^{1_{i-1}}, \sigma_{1_{i-1}}\right)\right) \\
& \text { by rule EEval' } \\
\rightarrow & (\text { while } \\
& \text { by rule E-Oflow } 1 \text { or E-Oflow2 } \\
\rightarrow & \left(S_{1} ;\right. \text { while } \\
& \left.m_{1_{i-1}}\left(\mathfrak{f}_{1}, \operatorname{loop}_{1}\right\rangle(e)\left\{S_{1}\right\}, m_{1_{i-1}}\left(\mathfrak{f}_{1}, \operatorname{loop}_{c}^{1_{i-1}}, \sigma_{1_{i-1}}\right)\right) \\
& \text { by rule Wh-T1 or Wh-T2. }
\end{aligned}
$$

The execution of $s_{2}$ proceeds to $\left(S_{2} ;\right.$ while ${ }_{\left\langle n_{2}\right\rangle}(e)\left\{S_{2}\right\}$, $\left.m_{2_{i-1}}\left(\mathfrak{f}_{2}, \operatorname{loop}_{c}^{2_{i-1}}\left[i /\left(n_{2}\right)\right], \sigma_{2_{i-1}}\right)\right)$. In addition, executions of $S_{1}$ and $S_{2}$ do not terminate when started in states $m_{1_{i-1}}\left(\mathfrak{f}_{1}, \operatorname{loop}_{c}^{1_{i-1}}\left[i /\left(n_{1}\right)\right], \sigma_{1_{i-1}}\right)$ and $m_{2_{i-1}}\left(\mathfrak{f}_{2}, \operatorname{loop}_{c}^{2_{i-1}}\left[i /\left(n_{2}\right)\right], \sigma_{2_{i-1}}\right)$. By Lemma E.2 executions of $s_{1}$ and $s_{2}$ do not terminate.

\subsection{Behavioral equivalence}

We now propose a proof rule under which two programs produce the same output sequence, namely the same I/O sequence till any $i$ th output value. We care about the I/O sequence due to the possible crash from the lack of input. We start by giving the definition of the same output sequence, then we describe the proof rule under which two programs produce the same output sequence, finally we show that our proof rule ensures same output together with the necessary auxiliary lemmas. We use the notation "Out $(\sigma)$ " to represent the output sequence in value store $\sigma$, the I/O sequence $\sigma\left(i d_{I O}\right)$ till the rightmost output value. Particularly, when there is no output value in the $\mathrm{I} / \mathrm{O}$ sequence $\sigma\left(i d_{I O}\right)$, $\operatorname{Out}(\sigma)=\varnothing$.

Definition 20. (Same output sequence) Two statement sequences $S_{1}$ and $S_{2}$ produce the same output sequence when started in states $m_{1}$ and $m_{2}$ respectively, written $\left(S_{1}, m_{1}\right) \equiv_{O}\left(S_{2}, m_{2}\right)$, iff $\forall m_{1}^{\prime} m_{2}^{\prime}$ such that $\left(S_{1}, m_{1}\right) \stackrel{*}{\rightarrow}\left(S_{1}^{\prime}, m_{1}^{\prime}\left(\sigma_{1}^{\prime}\right)\right)$ and $\left(S_{2}, m_{2}\right) \stackrel{*}{\rightarrow}$ $\left(S_{2}^{\prime}, m_{2}^{\prime}\left(\sigma_{2}^{\prime}\right)\right)$, there are states $m_{1}^{\prime \prime} m_{2}^{\prime \prime}$ reachable from initial states $m_{1}$ and $m_{2},\left(S_{1}, m_{1}\right) \stackrel{*}{\rightarrow}\left(S_{1}^{\prime \prime}, m_{1}^{\prime \prime}\left(\sigma_{1}^{\prime \prime}\right)\right)$ and $\left(S_{2}, m_{2}\right) \stackrel{*}{\rightarrow}$ $\left(S_{2}^{\prime \prime}, m_{2}^{\prime \prime}\left(\sigma_{2}^{\prime \prime}\right)\right)$ so that Out $\left(\sigma_{2}^{\prime \prime}\right)=\operatorname{Out}\left(\sigma_{1}^{\prime}\right)$ and $\operatorname{Out}\left(\sigma_{1}^{\prime \prime}\right)=\operatorname{Out}\left(\sigma_{2}^{\prime}\right)$.

\subsubsection{Proof rule for behavioral equivalence}

We show the proof rules of the behavioral equivalence. The output sequence produced in executions of a statement sequence $S$ depends on values of a set of variables in the program, the output deciding variables $\operatorname{OVar}(S)$. The output deciding variables are of two parts: $\operatorname{TVar}_{o}(S)$ are variables affecting the termination of executions of a statement sequence; $\operatorname{Imp}_{o}(S)$ are variables affecting values of the I/O sequence produced in executions of a statement sequence. The definitions of $\operatorname{TV} \operatorname{Var}_{o}(S)$ and $\operatorname{Imp}_{o}(S)$ are shown in Definition 21 and 22

Definition 21. (Imported variables relative to output) The imported variables in one program $S$ relative to output, written $\operatorname{Imp}_{o}(S)$, are listed as follows.
1. $\operatorname{Imp}_{o}(S)=\left\{i d_{I O}\right\}$, if $(\forall e$ : "output $e " \notin S)$;

2. Imp $_{o}$ ("output $\left.e "\right)=\left\{i d_{I O}\right\} \cup \operatorname{Use}(e)$;

3. $\operatorname{Imp}_{o}\left(" I f(e)\right.$ then $\left\{S_{t}\right\}$ else $\left.\left\{S_{f}\right\} "\right)=U$ se $(e) \cup \operatorname{Imp}_{o}\left(S_{t}\right) \cup$ $\operatorname{Imp} p_{o}\left(S_{f}\right)$ if $(\exists e:$ : $\exists$ output $e " \in S)$;

4. $\operatorname{Imp}_{o}\left("\right.$ while $\left._{\langle n\rangle}(e)\left\{S^{\prime \prime}\right\} "\right)=\operatorname{Imp}\left(\right.$ "while $\left.{ }_{\langle n\rangle}(e)\left\{S^{\prime \prime}\right\} ",\left\{i d_{I O}\right\}\right)$ if $\left(\exists e:\right.$ "output e" $\left.\in S^{\prime \prime}\right)$;

5. For $k>0, \operatorname{Imp}_{o}\left(s_{1} ; \ldots ; s_{k} ; s_{k+1}\right)=\operatorname{Imp}\left(s_{1} ; \ldots ; s_{k}, \operatorname{Imp} p_{o}\left(s_{k+1}\right)\right)$ if $\left(\exists e:\right.$ "output $e$ " $\left.\in s_{k+1}\right)$;

6. For $k>0, \operatorname{Imp}_{o}\left(s_{1} ; \ldots ; s_{k} ; s_{k+1}\right)=\operatorname{Imp}_{o}\left(s_{1} ; \ldots ; s_{k}\right)$ if $(\forall e$ : "output $\left.e \notin s_{k+1}\right)$;

Definition 22. (Termination deciding variables relative to output) The termination deciding variables in a statement sequence $S$ relative to output, written $\operatorname{TVar}_{o}(S)$, are listed as follows:

1. $\operatorname{TVar}_{o}(S)=\emptyset$ if $(\forall e$ : "output $e " \notin S)$;

2. VVar $_{o}$ ("output $"$ ") $=\operatorname{Err}(e)$;

3. $\operatorname{TVar}_{o}\left(" I f(e)\right.$ then $\left\{S_{t}\right\}$ else $\left.\left\{S_{f}\right\} "\right)=\operatorname{Use}(e) \cup \operatorname{TVar}_{o}\left(S_{t}\right) \cup$ $\operatorname{TVar}_{o}\left(S_{f}\right)$ if $(\exists e:$ : "output e" $\in S)$;

4. $\operatorname{TVar}_{o}\left("\right.$ while $\left._{\langle n\rangle}(e)\left\{S^{\prime \prime}\right\} "\right)=\operatorname{TVar}\left(" w_{h i l e}\langle n\rangle(e)\left\{S^{\prime \prime}\right\} "\right)$ if $\left(\exists e:\right.$ "output e" $\left.\in S^{\prime \prime}\right)$;

5. For $k>0, T \operatorname{Var}_{o}\left(s_{1} ; \ldots ; s_{k} ; s_{k+1}\right)=\operatorname{TVar}\left(s_{1} ; \ldots ; s_{k}\right)$ $\cup \operatorname{Imp}\left(s_{1} ; \ldots ; s_{k}, \operatorname{TVar}_{o}\left(s_{k+1}\right)\right)$ if $\left(\exists e:\right.$ "output $\left.e " \in s_{k+1}\right)$;

6. For $k>0$, $\operatorname{TVar}_{o}\left(s_{1} ; \ldots ; s_{k} ; s_{k+1}\right)=\operatorname{TVar}_{o}\left(s_{1} ; \ldots ; s_{k}\right)$ if ( $\forall e$ : "output $\left.e " \notin s_{k+1}\right)$;

Definition 23. (Output deciding variables) The output deciding variables in a statement sequence $S$ are $\operatorname{Imp}_{o}(S) \cup \operatorname{TVar}_{o}(S)$, written $O \operatorname{Var}(S)$.

The condition of the behavioral equivalence is defined recursively. The base case is for two same output statements or two statements where the output sequence variable is not defined. The inductive cases are syntax directed considering the syntax of compound statements and statement sequences.

Definition 24. (proof rule of behavioral equivalence) Two statement sequences $S_{1}$ and $S_{2}$ satisfy the proof rule of behavioral equivalence, written $S_{1} \equiv \underset{O}{S} S_{2}$, iff one of the following holds:

1. $S_{1}$ and $S_{2}$ are one statement and one of the following holds:

(a) $S_{1}$ and $S_{2}$ are simple statement and one of the following holds:

i. $S_{1}$ and $S_{2}$ are not output statement, $\forall e_{1} e_{2}$ :

("output $e_{1}$ " $\left.\neq S_{1}\right) \wedge\left(\right.$ (output $e_{2}$ " $\left.\neq S_{2}\right)$; or

ii. $S_{1}=S_{2}=$ "output e".

(b) $S_{1}=$ "If $(e)$ then $\left\{S_{1}^{t}\right\}$ else $\left\{S_{1}^{f}\right\}$ ", $S_{2}=$ "If $(e)$ then $\left\{S_{2}^{t}\right\}$ else $\left\{S_{2}^{f}\right\}$ " and all of the following hold:

- There is an output statement in $S_{1}$ and $S_{2}$,

$\exists e_{1} e_{2}$ : ("output $e_{1}$ " $\left.\in S_{1}\right) \wedge$ ("output $e_{2}$ " $\in S_{2}$ );

- $\left(S_{1}^{t} \equiv_{O}^{S} S_{2}^{t}\right) \wedge\left(S_{1}^{f} \equiv \stackrel{S}{S} S_{2}^{f}\right)$;

(c) $S_{1}=$ "while ${ }_{\left\langle n_{1}\right\rangle}(e)\left\{S_{1}^{\prime \prime}\right\} "$ and $S_{2}=$ "while ${ }_{\left\langle n_{2}\right\rangle}(e)\left\{S_{2}^{\prime \prime}\right\} "$ and all of the following hold:

- There is an output statement in $S_{1}$ and $S_{2}$,

$\exists e_{1} e_{2}$ : ("output $e_{1}$ " $\left.\in S_{1}\right) \wedge$ ("output $e_{2}$ " $\in S_{2}$ );

- $S_{1}^{\prime \prime} \equiv{ }_{O}^{S} S_{2}^{\prime \prime}$;

- $S_{1}^{\prime \prime}$ and $S_{2}^{\prime \prime}$ have equivalent computation of $O \operatorname{Var}\left(S_{1}\right) \cup$ $O \operatorname{Var}\left(S_{2}\right)$;

- $S_{1}^{\prime \prime}$ and $S_{2}^{\prime \prime}$ satisfy the proof rule of termination in the same way, $S_{1}^{\prime \prime} \equiv_{H}^{S} S_{2}^{\prime \prime}$;

(d) Output statements are not in both $S_{1}$ and $S_{2}$, $\forall e_{1} e_{2}$ : ("output $e_{1}$ " $\left.\notin S_{1}\right) \wedge$ ("output $e_{2}$ " $\notin S_{2}$ ).

2. $S_{1}$ and $S_{2}$ are not both one statement and one of the following holds: 
(a) $S_{1}=S_{1}^{\prime} ; s_{1}$ and $S_{2}=S_{2}^{\prime} ; s_{2}$, and all of the following hold:

- $S_{1}^{\prime} \equiv \stackrel{S}{S} S_{2}^{\prime}$;

- $S_{1}^{\prime}$ and $S_{2}^{\prime}$ have equivalent computation of $O \operatorname{Var}\left(s_{1}\right) \cup$ $O \operatorname{Var}\left(s_{2}\right)$;

- $S_{1}^{\prime}$ and $S_{2}^{\prime}$ satisfy the proof rule of termination in the same way: $S_{1}^{\prime} \equiv{ }_{H}^{S} S_{2}^{\prime}$;

- There is an output statement in both $s_{1}$ and $s_{2}$, $\exists e_{1} e_{2}:$ : "output $e_{1}$ " $\left.\in s_{1}\right) \wedge$ ("output $e_{2}$ " $\left.\in s_{2}\right)$; - $s_{1} \equiv \stackrel{S}{O} s_{2}$;

(b) There is no output statement in the last statement in $S_{1}$ or $S_{2}$ :

$\left(\left(S_{1}=S_{1}^{\prime} ; s_{1}\right) \wedge\left(S_{1}^{\prime} \equiv \stackrel{S}{S} S_{2}\right) \wedge\left(\forall e\right.\right.$ : "output e" $\left.\left.\notin s_{1}\right)\right)$ $\vee\left(\left(S_{2}=S_{2}^{\prime} ; s_{2}\right) \wedge\left(S_{1} \equiv \stackrel{S}{O} S_{2}^{\prime}\right) \wedge\left(\forall e\right.\right.$ : “output e" $\left.\left.\notin s_{2}\right)\right)$;

\subsubsection{Soundness of the proof rule for behavioral equivalence}

We show that two statement sequences satisfy the proof rule of the behavioral equivalence and their initial states agree on values of their output deciding variables, then the two statement sequences produce the same output sequence when started in their initial states.

Theorem 5. Two statement sequences $S_{1}$ and $S_{2}$ satisfy the proof rule of the behavioral equivalence, $S_{1} \equiv S_{O}^{S} S_{2}$. If $S_{1}$ and $S_{2}$ start in states $m_{1}\left(\mathfrak{f}_{1}, \sigma_{1}\right)$ and $m_{2}\left(\mathfrak{f}_{2}, \sigma_{2}\right)$ where both of the following hold:

- Crash flags are not set, $\mathfrak{f}_{1}=\mathfrak{f}_{2}=0$;

- Value stores $\sigma_{1}$ and $\sigma_{2}$ agree on values of the output deciding variables of $S_{1}$ and $S_{2}, \forall i d \in \operatorname{OVar}\left(S_{1}\right) \cup \operatorname{OVar}\left(S_{2}\right)$ : $\sigma_{1}(i d)=\sigma_{2}(i d)$;

then $S_{1}$ and $S_{2}$ produce the same output sequence,

$\left(S_{1}, m_{1}\right) \equiv_{O}\left(S_{2}, m_{2}\right)$.

The proof is by induction on the sum of program size of $S_{1}$ and $S_{2}, \operatorname{size}\left(S_{1}\right)+\operatorname{size}\left(S_{2}\right)$ and is a case analysis based on $S_{1} \equiv \stackrel{S}{O} S_{2}$.

Proof. The proof is by induction on the sum of program size of $S_{1}$ and $S_{2}, \operatorname{size}\left(S_{1}\right)+\operatorname{size}\left(S_{2}\right)$ and is a case analysis based on $S_{1} \equiv{ }_{O}^{S} S_{2}$.

Base case.

$S_{1}$ and $S_{2}$ are simple statement. There are two cases according to the proof rule of behavioral equivalence because stacks are not changed in executions of $S_{1}$ and $S_{2}$.

1. $S_{1}$ and $S_{2}$ are not output statement, $\forall e_{1} e_{2}$ : ("output $e_{1}$ " $\neq$ $\left.S_{1}\right) \wedge\left(\right.$ "output $e_{2}$ " $\neq S_{2}$ );

By the definition of imported variables relative to output, $\operatorname{Imp}_{o}\left(S_{1}\right)=\operatorname{Imp}_{o}\left(S_{2}\right)=\left\{i d_{I O}\right\}$. By assumption, initial value stores $\sigma_{1}$ and $\sigma_{2}$ agree on the value of the I/O sequence variable, $\sigma_{1}\left(i d_{I O}\right)=\sigma_{2}\left(i d_{I O}\right)$. By definition, $\operatorname{Out}\left(\sigma_{1}\right)=\operatorname{Out}\left(\sigma_{2}\right)$.

By Lemma 5.23, in any state $m_{1}^{\prime}$ reachable from $m_{1}$, the output sequence in $m_{1}^{\prime}$ is same as that in $m_{1}, \forall m_{1}^{\prime}:\left(\left(S_{1}, m_{1}\left(\sigma_{1}\right)\right) \stackrel{*}{\rightarrow}\right.$ $\left.\left(S_{1}^{\prime}, m_{1}^{\prime}\left(\sigma_{1}^{\prime}\right)\right)\right) \Rightarrow\left(\operatorname{Out}\left(\sigma_{1}^{\prime}\right)=\operatorname{Out}\left(\sigma_{1}\right)\right)$. Similarly, for any state $m_{2}^{\prime}$ reachable from $m_{2}$, the output sequence in $m_{2}^{\prime}$ is same as that in $m_{2}$. The theorem holds.

2. $S_{1}=S_{2}=$ "output $e$ ".

We show that the expression $e$ evaluates to the same value w.r.t value stores, $\sigma_{1}, \sigma_{2}$. By the definition of imported variables relative to output, $\operatorname{Imp}_{o}\left(S_{1}\right)=\operatorname{Imp}_{o}\left(S_{2}\right)=\mathrm{Use}(e) \cup\left\{i d_{I O}\right\}$. Then $\forall x \in \operatorname{Use}(e) \cup\left\{i d_{I O}\right\} \quad: \quad \sigma_{1}(x)=\sigma_{2}(x)$ by assumption. By Lemma D.1 $\mathcal{E} \llbracket e \rrbracket \sigma_{1}=\mathcal{E} \llbracket e \rrbracket \sigma_{2}$. Then, there are two possibilities.

(a) $\mathcal{E} \llbracket e \rrbracket \sigma_{1}=\mathcal{E} \llbracket e \rrbracket \sigma_{2}=\left(\right.$ error, $\left.v_{\mathfrak{o f}}\right)$.

The execution of $S_{1}$ proceeds as follows. (output $e, m_{1}\left(\sigma_{1}\right)$ )

$=\left(\right.$ output $\left(\right.$ error, $\left.\left.v_{\mathfrak{o f}}\right), m_{1}\left(\sigma_{1}\right)\right)$ by the rule EEval' $\rightarrow\left(\right.$ output $\left.0, m_{1}(1 / \mathfrak{f})\right)$ by the ECrash rule

$\stackrel{i}{\rightarrow}$ (output $\left.0, m_{1}(1 / \mathfrak{f})\right)$ for any $i>0$ by the Crash rule.

Similarly, the execution of $S_{2}$ does not terminate and there is no change to $\mathrm{I} / \mathrm{O}$ sequence in execution. Because $\sigma_{1}\left(i d_{I O}\right)=\sigma_{2}\left(i d_{I O}\right)$, then the output sequence in value stores $\sigma_{1}$ and $\sigma_{2}$ are same, $\operatorname{Out}\left(\sigma_{1}\right)=\operatorname{Out}\left(\sigma_{2}\right)$, the theorem holds.

(b) $\mathcal{E} \llbracket e \rrbracket \sigma_{1}=\mathcal{E} \llbracket e \rrbracket \sigma_{2} \neq\left(\right.$ error, $\left.v_{\text {of }}\right)$

$S_{1}$ and $S_{2}$ satisfy the proof rule of equivalent computation of I/O sequence variable and their initial states agree on the values of the imported variables relative to I/O sequence variable. By Theorem 2, $S_{1}$ and $S_{2}$ produce the same output sequence after terminating execution when started in state $m_{1}\left(\sigma_{1}\right)$ and $m_{2}\left(\sigma_{2}\right)$ respectively. The theorem holds.

\section{Induction step.}

The hypothesis IH is that Theorem 5 holds when $\operatorname{size}\left(S_{1}\right)+$ $\operatorname{size}\left(S_{2}\right)=k \geq 2$.

We show Theorem 5 holds when $\operatorname{size}\left(S_{1}\right)+\operatorname{size}\left(S_{2}\right)=k+1$. The proof is a case analysis according to the cases in the definition of the proof rule of behavioral equivalence.

1. $S_{1}$ and $S_{2}$ are one statement and one of the following holds:

(a) $S_{1}=$ "If $(e)$ then $\left\{S_{1}^{t}\right\}$ else $\left\{S_{1}^{f}\right\} "$ and $S_{2}=$ "If $(e)$ then $\left\{S_{2}^{t}\right\}$ else $\left\{S_{2}^{f}\right\}$ " and all of the following hold:

- There is an output statement in $S_{1}$ and $S_{2}: \exists e_{1} e_{2}$ : ("output $e_{1}$ " $\left.\in S_{1}\right) \wedge$ ("output $e_{2}$ " $\in S_{2}$ );

- $S_{1}^{t} \equiv \stackrel{S}{S} S_{2}^{t}$;

- $S_{1}^{f} \equiv S_{O}^{S} S_{2}^{f}$;

By Lemma 5.17 $\left\{i d_{I O}\right\} \in \operatorname{Imp}_{o}\left(S_{1}\right)$. By assumption, value stores $\sigma_{1}$ and $\sigma_{2}$ agree on the value of the $\mathrm{I} / \mathrm{O}$ sequence variable and the I/O sequence variable, $\sigma_{1}\left(i d_{I O}\right)=$ $\sigma_{2}\left(i d_{I O}\right)$.

We show that the evaluations of the predicate expression of $S_{1}$ and $S_{2}$ w.r.t. initial value store $\sigma_{1}$ and $\sigma_{2}$ produce the same value. We need to show that value stores $\sigma_{1}$ and $\sigma_{2}$ agree on values of variables used in the predicate expression $e$ of $S_{1}$ and $S_{2}$. Because the output sequence is defined in $S_{1}$, by the definition of imported variables relative to output, $\operatorname{Imp}_{o}\left(S_{1}\right)=\mathrm{Use}(e) \cup \operatorname{Imp}_{o}\left(S_{1}^{t}\right) \cup \operatorname{Imp}_{o}\left(S_{1}^{f}\right)$. Thus, Use $(e) \subseteq \operatorname{OVar}\left(S_{1}\right)$. By assumption, value stores $\sigma_{1}$ and $\sigma_{2}$ agree on values of variables used in the predicate expression $e$ of $S_{1}$ and $S_{2}, \forall x \in \operatorname{Use}(e): \sigma_{1}(x)=\sigma_{2}(x)$. By Lemma D.1 the evaluations of the predicate expression of $S_{1}$ and $S_{2}$ w.r.t. pairs value stores, $\sigma_{1}$ and $\sigma_{2}$ generate the same value, $\mathcal{E}^{\prime} \llbracket e \rrbracket \sigma_{1}=\mathcal{E}^{\prime} \llbracket e \rrbracket \sigma_{2}$. Then there are two possibilities.

i. $\mathcal{E}^{\prime} \llbracket e \rrbracket \sigma_{1}=\mathcal{E}^{\prime} \llbracket e \rrbracket \sigma_{2}=\left(\right.$ error, $\left.v_{\mathfrak{o f}}\right)$.

Then the execution of $S_{1}$ proceeds as follows:

$$
\begin{aligned}
& \text { (If } \left.(e) \text { then }\left\{S_{1}^{t}\right\} \text { else }\left\{S_{1}^{f}\right\}, m_{1}\left(\sigma_{1}\right)\right) \\
& \rightarrow\left(\operatorname{If}\left(\left(\text { error, } v_{\mathfrak{o f}}\right)\right) \text { then }\left\{S_{1}^{t}\right\} \text { else }\left\{S_{1}^{f}\right\}, m_{1}\left(\sigma_{1}\right)\right) \\
& \text { by the EEval' rule } \\
& \rightarrow\left(\operatorname{If}(0) \text { then }\left\{S_{1}^{t}\right\} \text { else }\left\{S_{1}^{f}\right\}, m_{1}(1 / \mathfrak{f})\right) \\
& \text { by the ECrash rule } \\
& \stackrel{i}{\rightarrow}\left(\operatorname{If}(0) \text { then }\left\{S_{1}^{t}\right\} \text { else }\left\{S_{1}^{f}\right\}, m_{1}(1 / \mathfrak{f})\right) \\
& \text { for any } i>0 \text {, by the Crash rule. }
\end{aligned}
$$

Similarly, the execution of $S_{2}$ does not terminate and does not redefine I/O sequence. Because $\sigma_{1}\left(i d_{I O}\right)=$ $\sigma_{2}\left(i d_{I O}\right)$, the theorem holds.

ii. $\mathcal{E}^{\prime} \llbracket e \rrbracket \sigma_{1}=\mathcal{E}^{\prime} \llbracket e \rrbracket \sigma_{2} \neq$ (error, $\left.v_{\mathfrak{o f}}\right)$.

W.1.o.g., $\mathcal{E}^{\prime} \llbracket e \rrbracket \sigma_{1}=\mathcal{E}^{\prime} \llbracket e \rrbracket \sigma_{2}=\left(0, v_{\text {of }}\right)$. The execution of $S_{1}$ proceeds as follows. 
(If $(e)$ then $\left\{S_{1}^{t}\right\}$ else $\left.\left\{S_{1}^{f}\right\}, m_{1}\left(\sigma_{1}\right)\right)$

$\rightarrow\left(\operatorname{If}\left(\left(0, v_{\mathfrak{o f}}\right)\right)\right.$ then $\left\{S_{1}^{t}\right\}$ else $\left.\left\{S_{1}^{f}\right\}, m_{1}\left(\sigma_{1}\right)\right)$

by the EEval rule

$\rightarrow\left(\operatorname{If}(0)\right.$ then $\left\{S_{1}^{t}\right\}$ else $\left.\left\{S_{1}^{f}\right\}, m_{1}\left(\sigma_{1}\right)\right)$

by the E-Oflow1 or E-Oflow2 rule

$\rightarrow\left(S_{1}^{f}, m_{1}\left(\sigma_{1}\right)\right)$ by the If-F rule.

Similarly, the execution of $S_{2}$ proceeds to $\left(S_{2}^{f}, m_{2}\left(\sigma_{2}\right)\right)$ after two steps. By the hypothesis IH, we show that $S_{1}^{f}$ and $S_{2}^{f}$ produce the same output sequence when started in states $m_{1}\left(\sigma_{1}\right)$ and $m_{2}\left(\sigma_{2}\right)$. We need to show that all required conditions are satisfied.

- $\operatorname{size}\left(S_{1}^{f}\right)+\operatorname{size}\left(S_{2}^{f}\right) \leq k$.

By definition, $\operatorname{size}\left(S_{1}\right)=1+\operatorname{size}\left(S_{1}^{t}\right)+\operatorname{size}\left(S_{1}^{f}\right)$. Therefore, $\operatorname{size}\left(S_{1}^{f}\right)+\operatorname{size}\left(S_{2}^{f}\right)<k$.

- Value stores $\sigma_{1}$ and $\sigma_{2}$ agree on values of the outdeciding variables of $S_{1}^{f}$ and $S_{2}^{f}, \forall x \in \operatorname{OVar}\left(S_{1}^{f}\right) \cup$ $\operatorname{OVar}\left(S_{2}^{f}\right): \sigma_{1}(x)=\sigma_{2}(x)$.

By the definition of imported variables relative to output, $\operatorname{Imp}_{o}\left(S_{1}^{f}\right) \subseteq \operatorname{Imp}_{o}\left(S_{1}\right)$. Besides, by the definition of $\operatorname{TVar}_{o}\left(S_{1}\right), \operatorname{TVar}_{o}\left(S_{1}^{f}\right) \subseteq \operatorname{TVar}_{o}\left(S_{1}\right)$. Then $\operatorname{OVar}\left(S_{1}^{f}\right) \subseteq \operatorname{OVar}\left(S_{1}\right)$. Similarly, OVar $\left(S_{2}^{f}\right) \subseteq$ $\operatorname{OVar}\left(S_{2}\right)$.By assumption, the value stores $\sigma_{1}$ and $\sigma_{2}$ agree on the values of the out-deciding variables of $S_{1}^{f}$ and $S_{2}^{f}$.

By the hypothesis $\mathrm{IH}, S_{1}^{f}$ and $S_{2}^{f}$ produce the same output sequence when started from state $m_{1}\left(\sigma_{1}\right)$ and $m_{2}\left(\sigma_{2}\right)$ respectively. The theorem holds.

(b) $S_{1}=$ "while $\left\langle n_{1}\right\rangle(e)\left\{S_{1}^{\prime \prime}\right\} "$ and $S_{2}=$ "while ${ }_{\left\langle n_{2}\right\rangle}(e)\left\{S_{2}^{\prime \prime}\right\} "$ and all of the following hold:

- There is an output statement in $S_{1}$ and $S_{2}: \exists e_{1} e_{2}$ : ("output $e_{1}$ " $\left.\in S_{1}\right) \wedge$ ("output $e_{2}$ " $\in S_{2}$ );

- $S_{1}^{\prime \prime} \equiv_{O}^{S} S_{2}^{\prime \prime}$;

- Both loop bodies satisfy the proof rule of termination in the same way: $S_{1}^{\prime \prime} \equiv_{H}^{S} S_{2}^{\prime \prime}$;

- $S_{1}^{\prime \prime}$ and $S_{2}^{\prime \prime}$ have equivalent computation of $\operatorname{OVar}\left(S_{1}\right) \cup$ $\operatorname{OVar}\left(S_{2}\right)$;

By Corollary 5.5 we show that $S_{1}$ and $S_{2}$ produce the same output sequence when started in states $m_{1}\left(\sigma_{1}\right)$ and $m_{2}\left(\sigma_{2}\right)$ respectively. We need to show that the required conditions are satisfied.

- Crash flags are not set, $\mathfrak{f}_{1}=\mathfrak{f}_{2}=0$.

- Value stores $\sigma_{1}$ and $\sigma_{2}$ agree on the values of the outdeciding variables of $S_{1}$ and $S_{2}, \forall x \in \operatorname{OVar}\left(S_{1}\right) \cup$ $\operatorname{OVar}\left(S_{2}\right): \sigma_{1}(x)=\sigma_{2}(x)$.

- The loop counter value of $S_{1}$ and $S_{2}$ are zero in initial loop counter, $\operatorname{loop}_{c}^{1}\left(n_{1}\right)=\operatorname{loop}_{c}^{2}\left(n_{2}\right)=0$.

- The loop body of $S_{1}$ and $S_{2}$ satisfy the proof rule of termination in the same way, $S_{1}^{\prime \prime} \equiv_{H}^{S} S_{2}^{\prime \prime}$.

- The loop body of $S_{1}$ and $S_{2}$ satisfy the proof rule of equivalent computation of $\operatorname{OVar}\left(S_{1}\right) \cup \operatorname{OVar}\left(S_{2}\right)$. The above five conditions are from assumption.

- $S_{1}$ and $S_{2}$ have same set of termination deciding variables, $\mathrm{T} \operatorname{Var}\left(S_{1}\right)=\mathrm{T} \operatorname{Var}\left(S_{2}\right)$.

By the definition of $\operatorname{TVar}_{o}\left(S_{1}\right), \operatorname{TVar}_{o}\left(S_{1}\right)=\operatorname{TVar}\left(S_{1}\right)$ and $\operatorname{TVar}_{o}\left(S_{2}\right)=\operatorname{TVar}\left(S_{2}\right)$. By Lemma5.21. $\operatorname{TVar}_{o}\left(S_{1}\right)=$ $\operatorname{TVar}_{o}\left(S_{2}\right)$. Thus, $\operatorname{TVar}\left(S_{1}\right)=\mathrm{TVar}\left(S_{2}\right)$.

- $S_{1}$ and $S_{2}$ have same set of imported variables relative to the I/O sequence variable, $\operatorname{Imp}\left(S_{1},\left\{i d_{I O}\right\}\right)=\operatorname{Imp}\left(S_{2},\left\{i d_{I O}\right\}\right)$.
By Lemma 5.19. $\operatorname{Imp}_{o}\left(S_{1}\right)=\operatorname{Imp}_{o}\left(S_{2}\right)$. By definition, $\operatorname{Imp}_{o}\left(S_{1}\right)=\operatorname{Imp}\left(S_{1},\left\{i d_{I O}\right\}\right)$ and $\operatorname{Imp}_{o}\left(S_{2}\right)=$ $\operatorname{Imp}\left(S_{2},\left\{i d_{I O}\right\}\right)$. Thus, $\operatorname{Imp}\left(S_{1},\left\{i d_{I O}\right\}\right)=\operatorname{Imp}\left(S_{2},\left\{i d_{I O}\right\}\right)$.

- The loop body of $S_{1}$ and $S_{2}$ produce the same output sequence when started in states with crash flags not set and whose value stores agree on values of the out-deciding variables of $S_{1}^{\prime \prime}$ and $S_{2}^{\prime \prime}, \forall m_{S_{1}^{\prime \prime}}\left(\mathfrak{f}_{1}^{\prime \prime}, \sigma_{1}^{\prime \prime}\right) m_{S_{2}^{\prime \prime}}\left(\mathfrak{f}_{2}^{\prime \prime}, \sigma_{2}^{\prime \prime}\right)$ : $\left(\left(\forall x \in \operatorname{OVar}\left(S_{1}^{\prime \prime}\right) \cup \operatorname{OVar}\left(S_{2}^{\prime \prime}\right): \sigma_{1}^{\prime \prime}(x)=\sigma_{2}^{\prime \prime}(x)\right) \wedge\right.$ $\left.\left(\mathfrak{f}_{1}^{\prime \prime}=\mathfrak{f}_{2}^{\prime \prime}=0\right)\right) \Rightarrow\left(S_{1}^{\prime \prime}, m_{S_{1}^{\prime \prime}}\left(\mathfrak{f}_{1}^{\prime \prime}, \sigma_{1}^{\prime \prime}\right)\right) \equiv_{O}\left(S_{2}^{\prime \prime}, m_{S_{2}^{\prime \prime}}\left(\mathfrak{f}_{2}^{\prime \prime}, \sigma_{2}^{\prime \prime}\right)\right)$. Because size $\left(S_{1}\right)=1+\operatorname{size}\left(S_{1}^{\prime \prime}\right), \operatorname{size}\left(S_{2}\right)=1+$ $\operatorname{size}\left(S_{2}^{\prime \prime}\right)$, then size $\left(S_{1}^{\prime \prime}\right)+\operatorname{size}\left(S_{2}^{\prime \prime}\right)<k$. By the hypothesis IH, the condition is satisfied.

By Corollary 5.5 $S_{1}$ and $S_{2}$ produce the same output sequence when started in states $m_{1}\left(\sigma_{1}\right)$ and $m_{2}\left(\sigma_{2}\right)$ respectively. The theorem holds.

(c) Output statements are not in both $S_{1}$ and $S_{2}, \forall e_{1} e_{2}$ : ("output $e_{1}$ " $\left.\notin S_{1}\right) \wedge$ ("output $e_{2}$ " $\notin S_{2}$ ).

By Lemma 5.17, $\left\{i d_{I O}\right\} \subseteq \operatorname{Imp}_{o}\left(S_{1}\right)$. By assumption, value stores in initial states $m_{1}, m_{2}$ agree on values of the $\mathrm{I} / \mathrm{O}$ sequence variable, $\sigma_{1}\left(i d_{I O}\right)=\sigma_{2}\left(i d_{I O}\right)$. By Lemma 5.23, the value of output sequence is same in $m_{1}$ and any state reachable from $m_{1}, \forall m_{1}^{\prime} m_{2}^{\prime}:\left(S_{1}, m_{1}\left(\sigma_{1}\right)\right) \stackrel{*}{\rightarrow}$ $\left(S_{1}^{\prime}, m_{1}^{\prime}\left(\sigma_{1}^{\prime}\right)\right)$ and $\left(S_{2}, m_{2}\left(\sigma_{2}\right)\right) \stackrel{*}{\rightarrow}\left(S_{2}^{\prime}, m_{2}^{\prime}\left(\sigma_{2}^{\prime}\right)\right)$, Out $\left(\sigma_{1}^{\prime}\right)=$ $\operatorname{Out}\left(\sigma_{1}\right)=\operatorname{Out}\left(\sigma_{2}\right)=\operatorname{Out}\left(\sigma_{2}^{\prime}\right)$. The theorem holds.

2. $S_{1}=S_{1}^{\prime} ; s_{1}$ and $S_{2}=S_{2}^{\prime} ; s_{2}$ are not both one statement and one of the following holds:

(a) There is an output statement in both $s_{1}$ and $s_{2}, \exists e_{1} e_{2}$ : ("output $e_{1}$ " $\left.\in s_{1}\right) \wedge$ ("output $e_{2}$ " $\in s_{2}$ ), and all of the following hold:

- $S_{1}^{\prime} \equiv \stackrel{S}{S} S_{2}^{\prime}$;

- $S_{1}^{\prime}$ and $S_{2}^{\prime}$ satisfy the proof rule of termination in the same way: $S_{1}^{\prime} \equiv_{H}^{S} S_{2}^{\prime}$;

- $S_{1}^{\prime}$ and $S_{2}^{\prime}$ have equivalent computation of $\operatorname{OVar}\left(s_{1}\right) \cup$ $\operatorname{OVar}\left(s_{2}\right)$;

- $s_{1} \equiv \stackrel{S}{S} s_{2}$;

By the hypothesis IH, we show $S_{1}^{\prime}$ and $S_{2}^{\prime}$ produce the same output sequence when started in states $m_{1}\left(\sigma_{1}\right)$ and $m_{2}\left(\sigma_{2}\right)$ respectively. We need to show that all required conditions are satisfied.

- $\operatorname{size}\left(S_{1}^{\prime}\right)+\operatorname{size}\left(S_{2}^{\prime}\right)<k$.

By the definition of program size, $\operatorname{size}\left(s_{1}\right) \geq 1, \operatorname{size}\left(s_{2}\right) \geq$ 1. Then $\operatorname{size}\left(S_{1}^{\prime}\right)+\operatorname{size}\left(S_{2}^{\prime}\right)<k$.

- Value stores $\sigma_{1}$ and $\sigma_{2}$ agree on values of the outdeciding variables of $S_{1}^{\prime}$ and $S_{2}^{\prime}, \forall x \in \operatorname{OVar}\left(S_{1}^{\prime}\right) \cup$ $\operatorname{OVar}\left(S_{2}^{\prime}\right): \sigma_{1}(x)=\sigma_{2}(x)$.

We show that $\operatorname{TVar}_{o}\left(S_{1}^{\prime}\right) \subseteq \operatorname{TVar}_{o}\left(S_{1}\right)$. $\operatorname{TVar}_{o}\left(S_{1}^{\prime}\right)$

$\subseteq \mathrm{TVar}\left(S_{1}^{\prime}\right)$ by Lemma 5.20

$\subseteq \operatorname{TVar}_{o}\left(S_{1}\right)$ by the definition of $\operatorname{TVar}_{o}\left(S_{1}\right)$

We show that $\operatorname{Imp}_{o}\left(S_{1}^{\prime}\right) \subseteq \operatorname{Imp}_{o}\left(S_{1}\right)$. $\operatorname{Imp}_{o}\left(S_{1}^{\prime}\right)$

$\subseteq \operatorname{Imp}\left(S_{1}^{\prime},\left\{i d_{I O}\right\}\right)(1)$ by Lemma 5.18

$\left\{i d_{I O}\right\} \subseteq \operatorname{Imp}_{o}\left(s_{k+1}\right)$ (2) by Lemma 5.17

Combining (1) + (2)

$\operatorname{Imp}\left(S_{1}^{\prime},\left\{i d_{I O}\right\}\right)$

$\subseteq \operatorname{Imp}\left(S_{1}^{\prime}, \operatorname{Imp}_{o}\left(s_{1}\right)\right)$ by LemmaC.2

$=\operatorname{Imp}_{o}\left(S_{1}\right)$ by the definition of $\operatorname{Imp}_{o}(S)$. 
Similarly, $\operatorname{OVar}\left(S_{2}^{\prime}\right) \subseteq \mathrm{OVar}\left(S_{2}\right)$. By assumption, value stores $\sigma_{1}$ and $\sigma_{2}$ agree on values of out-deciding variables of $S_{1}^{\prime}$ and $S_{2}^{\prime}$.

By the hypothesis IH, $S_{1}^{\prime}$ and $S_{2}^{\prime}$ produce the same output sequence when started in state $m_{1}\left(\sigma_{1}\right)$ and $m_{2}\left(\sigma_{2}\right)$ respectively.

We show that $S_{1}$ and $S_{2}$ produce the same output sequence if $s_{1}$ and $s_{2}$ execute. We need to show that $S_{1}^{\prime}$ and $S_{2}^{\prime}$ terminate in the same way when started in states $m_{1}\left(\sigma_{1}\right)$ and $m_{2}\left(\sigma_{2}\right)$ respectively. Specifically, we prove that the value stores $\sigma_{1}$ and $\sigma_{2}$ agree on the values of termination deciding variables of $S_{1}^{\prime}$ and $S_{2}^{\prime}$. By definition, the termination deciding variables in $S_{1}^{\prime}$ are a subset of the termination deciding variables relative to output, $\mathrm{TV} \operatorname{Var}\left(S_{1}^{\prime}\right) \subseteq \mathrm{TVar}_{o}\left(S_{1}\right)$. Similarly, $\operatorname{TVar}\left(S_{2}^{\prime}\right) \subseteq \operatorname{TVar}_{o}\left(S_{2}\right)$. By assumption, the value stores $\sigma_{1}$ and $\sigma_{2}$ agree on the values of the termination deciding variables of $S_{1}^{\prime}$ and $S_{2}^{\prime}, \forall x \in \mathrm{T} \operatorname{Var}\left(S_{1}^{\prime}\right) \cup \mathrm{T} \operatorname{Var}\left(S_{2}^{\prime}\right)$ : $\sigma_{1}(x)=\sigma_{2}(x)$. By Theorem $4 S_{1}^{\prime}$ and $S_{2}^{\prime}$ terminate in the same way when started in state $m_{1}\left(\sigma_{1}\right)$ and $m_{2}\left(\sigma_{2}\right)$ respectively.

If $S_{1}^{\prime}$ and $S_{2}^{\prime}$ terminate when started in states $m_{1}\left(\sigma_{1}\right)$ and $m_{2}\left(\sigma_{2}\right)$, by Lemma 5.14. $S_{1}^{\prime}$ and $S_{2}^{\prime}$ consume same amount of input values. In addition, we show that value stores agree on values of the out-deciding variables of $s_{1}$ and $s_{2}$ by Theorem 2 We need to show that $S_{1}^{\prime}$ and $S_{2}^{\prime}$ start execution in states agreeing on values of the imported variables in $S_{1}^{\prime}$ and $S_{2}^{\prime}$ relative to the out-deciding variables of $s_{1}$ and $s_{2}$.

- $\operatorname{Imp}\left(\operatorname{TVar}_{o}\left(s_{1}\right), S_{1}^{\prime}\right) \subseteq \operatorname{TVar}_{o}\left(S_{1}\right)$.

This is by the definition of $\operatorname{TVar}_{o}\left(S_{1}\right)$.

- $\operatorname{Imp}\left(\operatorname{Imp}_{o}\left(s_{1}\right), S_{1}^{\prime}\right)=\operatorname{Imp}_{o}\left(S_{1}\right)$.

This is by the definition of $\operatorname{Imp}_{o}\left(S_{1}\right)$.

Thus, the imported variables in $S_{1}^{\prime}$ relative to the outdeciding variables of $s_{1}$ are a subset of the out-deciding variables of $S_{1}, \operatorname{Imp}\left(S_{1}^{\prime}, \operatorname{OVar}\left(s_{1}\right)\right) \subseteq \operatorname{OVar}\left(S_{1}\right)$. Similarly, $\operatorname{Imp}\left(S_{2}^{\prime}, \mathrm{OVar}\left(s_{2}\right)\right) \subseteq \operatorname{OVar}\left(S_{2}\right)$. By Corollary 5.4 $s_{1}$ and $s_{2}$ have same out-deciding variables, $\operatorname{OVar}\left(s_{1}\right)=$ $\operatorname{OVar}\left(s_{2}\right)$. By assumption, $S_{1}^{\prime}$ and $S_{2}^{\prime}$ terminate when started in states $m_{1}\left(\sigma_{1}\right)$ and $m_{2}\left(\sigma_{2}\right),\left(S_{1}^{\prime}, m_{1}\left(\sigma_{1}\right)\right) \stackrel{*}{\rightarrow}$ (skip, $\left.m_{1}^{\prime}\left(\sigma_{1}^{\prime}\right)\right),\left(S_{2}^{\prime}, m_{1}\left(\sigma_{2}\right)\right) \stackrel{*}{\rightarrow}$ (skip, $\left.m_{2}^{\prime}\left(\sigma_{2}^{\prime}\right)\right)$. By Theorem 2, value stores $\sigma_{1}^{\prime}$ and $\sigma_{2}^{\prime}$ agree on values of the outdeciding variables of $s_{1}$ and $s_{2}$.

By the hypothesis IH again, $s_{1}$ and $s_{2}$ produce the same output sequence when started in states $m_{1}^{\prime}\left(\sigma_{1}^{\prime}\right)$ and $m_{2}^{\prime}\left(\sigma_{2}^{\prime}\right)$ respectively. The theorem holds.

(b) There is no output statement in the last statement in $S_{1}$ or $S_{2}$ : W.l.o.g., ( $\forall e$ : "output $e$ " $\left.\notin s_{1}\right) \wedge\left(\left(S_{1}^{\prime}\right) \equiv_{O}^{S}\left(S_{2}\right)\right)$. By the hypothesis IH, we show that $S_{1}^{\prime}$ and $S_{2}$ produce the same output sequence when started in states $m_{1}\left(\sigma_{1}\right)$ and $m_{2}\left(\sigma_{2}\right)$ respectively. We need to show that two required conditions are satisfied.

- $\operatorname{size}\left(S_{1}^{\prime}\right)+\operatorname{size}\left(S_{2}\right) \leq k$.

$\operatorname{size}\left(s_{1}\right) \geq 1$ by definition. Then $\operatorname{size}\left(S_{1}^{\prime}\right)+\operatorname{size}\left(S_{2}\right) \leq$ $k$.

- $\forall x \in \operatorname{OVar}\left(S_{1}^{\prime}\right) \cup \operatorname{OVar}\left(S_{2}\right): \sigma_{1}(x)=\sigma_{2}(x)$

By definition of $\operatorname{TVar}_{o}(S) / \operatorname{Imp}_{o}(S), \operatorname{TVar}_{o}\left(S_{1}^{\prime}\right)=$ $\operatorname{TVar}_{o}\left(S_{1}\right)$, and $\operatorname{Imp}_{o}\left(S_{1}^{\prime}\right)=\operatorname{Imp}_{o}\left(S_{1}\right)$ Hence, $\forall x \in$ $\operatorname{OVar}\left(S_{1}^{\prime}\right) \cup \operatorname{OVar}\left(S_{2}\right): \sigma_{1}(x)=\sigma_{2}(x)$.

Therefore, $S_{1}^{\prime}$ and $S_{2}$ produce the same output sequence when started in state $m_{1}\left(\sigma_{1}\right)$ and $m_{2}\left(\sigma_{2}\right)$ respectively, $\left(S_{1}^{\prime}, m_{1}\right) \equiv_{O}\left(S_{2}, m_{2}\right)$ by the hypothesis IH.

When the execution of $S_{1}^{\prime}$ terminates, then the output sequence is not changed in the execution of $s_{1}$ by Lemma 5.23 The theorem holds.

\subsubsection{Supporting lemmas for the soundness proof of behavioral equivalence}

We listed the lemmas and corollaries used in the proof of Theorem 5 below. The supporting lemmas are of two parts. One part is various properties related to the out-deciding variables. The other part is the proof that two loop statements produce the same output sequence.

Lemma 5.17. For any statement sequence $S$, the $I / O$ sequence variable is in imported variable in $S$ relative to output, $i d_{I O} \in$ $\operatorname{Imp}_{o}(S)$.

Proof. By structure induction on abstract syntax of $S$.

Lemma 5.18. For any statement sequence $S$, the imported variables in $S$ relative to output are a subset of the imported variables in $S$ relative to the $I / O$ sequence variable, $\operatorname{Imp}_{o}(S) \subseteq$ $\operatorname{Imp}\left(S,\left\{i d_{I O}\right\}\right)$.

Proof. By induction on abstract syntax of $S$. In every case, there are subcases based on if there is output statement in the statement sequence $S$ or not if necessary.

Lemma 5.19. If two statement sequences $S_{1}$ and $S_{2}$ satisfy the proof rule of behavioral equivalence, then $S_{1}$ and $S_{2}$ have the same set of imported variables relative to output, $\left(S_{1} \equiv{ }_{O}^{S} S_{2}\right) \Rightarrow$ $\left(\operatorname{Imp}_{o}\left(S_{1}\right)=\operatorname{Imp}_{o}\left(S_{2}\right)\right)$.

Proof. By induction on $\operatorname{size}\left(S_{1}\right)+\operatorname{size}\left(S_{2}\right)$.

Lemma 5.20. For any statement sequence $S$ and any variable $x$, the termination deciding variables in $S$ relative to output is a subset of the termination deciding variables in $S, \operatorname{TVar}_{o}(S) \subseteq T \operatorname{Var}(S)$.

Proof. By induction on abstract syntax of $S$. In every case, there are subcases based on if there is output statement in the statement sequence $S$ or not if necessary.

Lemma 5.21. If two statement sequences $S_{1}$ and $S_{2}$ satisfy the proof rule of behavioral equivalence, then $S_{1}$ and $S_{2}$ have the same set of termination deciding variables relative to output, $\left(S_{1} \equiv{ }_{O}^{S}\right.$ $\left.S_{2}\right) \Rightarrow\left(\operatorname{TVar}_{o}\left(S_{1}\right)=\operatorname{TVar}_{o}\left(S_{2}\right)\right)$.

Proof. By induction on size $\left(S_{1}\right)+\operatorname{size}\left(S_{2}\right)$.

Corollary 5.4. If two statement sequences $S_{1}$ and $S_{2}$ satisfy the proof rule of behavioral equivalence, then $S_{1}$ and $S_{2}$ have the same set of out-deciding variables, $\left(S_{1} \equiv \stackrel{S}{S} S_{2}\right) \Rightarrow \operatorname{OVar}\left(S_{1}\right)=$ $O \operatorname{Var}\left(S_{2}\right)$.

Proof. By Lemma 5.19. $\operatorname{Imp}_{o}\left(S_{1}\right)=\operatorname{Imp}_{o}\left(S_{2}\right)$. By Lemma 5.21 $\operatorname{TVar}_{o}\left(S_{1}\right)=\operatorname{TVar}_{o}\left(S_{2}\right)$.

Lemma 5.22. In one step execution $(S, m(\sigma)) \rightarrow\left(S^{\prime}, m^{\prime}\left(\sigma^{\prime}\right)\right)$, if there is no output statement in $S$, then the output sequence is same in value store $\sigma$ and $\sigma^{\prime}$, Out $\left(\sigma^{\prime}\right)=\operatorname{Out}(\sigma)$.

Proof. By induction on abstract syntax of $S$ and crash flag $\mathfrak{f}$ in state $m$.

Lemma 5.23. If there is no output statement in $S$, then, after the execution $(S, m(\sigma)) \stackrel{*}{\rightarrow}\left(S^{\prime}, m^{\prime}\left(\sigma^{\prime}\right)\right)$, the output sequence is same in value store $\sigma$ and $\sigma^{\prime}$, Out $\left(\sigma^{\prime}\right)=\operatorname{Out}(\sigma)$. 
Proof. By induction on number $k$ of execution steps in the execution $(S, m(\sigma)) \stackrel{k}{\rightarrow}\left(S^{\prime}, m^{\prime}\left(\sigma^{\prime}\right)\right)$. The proof also relies on the fact that if $s \notin S$, then $s \notin S^{\prime}$.

Lemma 5.24. One while statement $s=$ "while ${ }_{\langle n\rangle}(e)\{S\} "$ starts in a state $m\left(\mathfrak{f}\right.$, loop $\left._{c}\right)$ in which the loop counter of $s$ is zero, $\operatorname{loop}_{c}(n)=0$ and the crash flag is not set, $\mathfrak{f}=0$. For any positive integer $i$, if there is a state $m^{\prime}\left(m_{c}^{\prime}\right)$ reachable from $m$ in which the loop counter is $i, \operatorname{loop}_{c}^{\prime}(n)=i$, then there is a configuration $\left(S ; s, m^{\prime \prime}\left(\mathfrak{f}^{\prime \prime}\right.\right.$, loop $\left.\left._{c}^{\prime \prime}\right)\right)$ reachable from the configuration $(s, m)$ in which loop counter of $s$ is $i, \operatorname{loop}_{c}^{\prime \prime}(n)=i$ and the crash flag is not set, $\mathfrak{f}^{\prime \prime}=0$ :

$\forall i>0:\left(\left(\left(s, m\left(\mathfrak{f}, m_{c}\right)\right) \stackrel{*}{\rightarrow}\left(S^{\prime}, m^{\prime}\left(\mathfrak{f}^{\prime}, \operatorname{loop}_{c}^{\prime}\right)\right)\right) \wedge\left(\operatorname{loop}_{c}(n)=\right.\right.$ 0) $\left.\wedge(\mathfrak{f}=0) \wedge\left(\operatorname{loop}_{c}^{\prime}(n)=i\right)\right) \Rightarrow$

$\left(s, m\left(\mathfrak{f}\right.\right.$, loop $\left.\left._{c}\right)\right) \stackrel{*}{\rightarrow}\left(S ; s, m^{\prime \prime}\left(\mathfrak{f}\right.\right.$, loop $\left.\left._{c}^{\prime \prime}\right)\right)$ where $\mathfrak{f}=0$ and $\operatorname{loop}_{c}^{\prime \prime}(n)=i$.

Proof. The proof is by induction on $i$.

Base case $i=1$.

We show that the evaluation of the loop predicate of $s$ w.r.t the value store $\sigma$ in the state $m\left(\operatorname{loop}_{c}, \sigma\right)$ produces an nonzero integer value. By our semantic rule, if the evaluation of the predicate expression of $s$ raises an exception, the execution of $s$ proceeds as follows:

$\left(s, m\left(\mathfrak{f}, \operatorname{loop}_{c}, \sigma\right)\right)$

$=\left(\right.$ while $_{\langle n\rangle}(e)\{S\}, m\left(\right.$ loop $\left.\left._{c}, \sigma\right)\right)$

$\rightarrow\left(\right.$ while $_{\langle n\rangle}($ error $)\{S\}, m\left(\right.$ loop $\left.\left._{c}, \sigma\right)\right)$ by the EEval rule

$\rightarrow\left(\right.$ while $_{\langle n\rangle}(0)\{S\}, m\left(1 / \mathfrak{f}\right.$, loop $\left.\left._{c}, \sigma\right)\right)$ by the ECrash rule

$\stackrel{k}{\rightarrow}\left(\right.$ while $_{\langle n\rangle}(0)\{S\}, m\left(1 / \mathfrak{f}\right.$, loop $\left.\left._{c}\right)\right)$ for any $k>0$, by the Crash rule.

Hence, we have a contradiction that there is no configuration in which the loop counter of $s$ is 1 .

When the evaluation of the loop predicate expression of $s$ produce zero, the execution of $s$ proceeds as follows:

$\left(s, m\left(\mathfrak{f}, \operatorname{loop}_{c}, \sigma\right)\right)$

$=\left(\right.$ while $\left._{\langle n\rangle}(e)\{S\}, m\left(\operatorname{loop}_{c}, \sigma\right)\right)$

$\rightarrow\left(\right.$ while $_{\langle n\rangle}(0)\{S\}, m\left(\right.$ loop $\left.\left._{c}, \sigma\right)\right)$ by the EEval rule

$\rightarrow\left(\right.$ skip, $\left.m\left(\operatorname{loop}_{c}\left[0 / n_{1}\right]\right)\right)$ by the Wh-F rule.

Hence, we have a contradiction that there is no configuration in which the loop counter of $s$ is 1 . The evaluation of the predicate expression of $s$ w.r.t value store $\sigma$ produce nonzero value. The execution of $s$ proceeds as follows:

$$
\begin{aligned}
& \left(s, m\left(\mathfrak{f}, \operatorname{loop}_{c}, \sigma\right)\right) \\
= & \left(\text { while }_{\langle n\rangle}(e)\{S\}, m\left(\mathfrak{f}, \operatorname{loop}_{c}, \sigma\right)\right) \\
\rightarrow & \left(\text { while }_{\langle n\rangle}(\mathcal{E} \llbracket e \rrbracket \sigma)\{S\}, m\left(\mathfrak{f}, \text { loop }_{c}, \sigma\right)\right) \text { by the EEval rule } \\
\rightarrow & \left(S ; \text { while }_{\langle n\rangle}(e)\{S\}, m\left(\mathfrak{f}, \operatorname{loop}_{c}\left[1 / n_{1}\right], \sigma\right)\right) \text { by the Wh-T rule. }
\end{aligned}
$$

The lemma holds.

\section{Induction step.}

The hypothesis IH is that, if there is a configuration $\left(S^{\prime}, m_{i}\left(\operatorname{loop}_{c}^{i}\right)\right)$ reachable from $(s, m)$ in which the loop counter of $s$ is $i, \operatorname{loop}_{c}^{i}(n)=$ $i>0$, then there is a reachable configuration $\left(S ; s, m_{i}\left(\mathfrak{f}, \operatorname{loop}_{c}^{i}\right)\right)$ from $(s, m)$ where the loop counter of $s$ is $i$ and the crash flag is not set.

Then we show that, if there is a configuration $\left(S^{\prime}, m_{i+1}\left(\operatorname{loop}_{c}^{i+1}\right)\right)$ reachable from $(s, m)$ in which the loop counter of $s$ is $i+1$, then there is a reachable configuration $\left(S ; s, m_{i+1}\left(\mathfrak{f}, \operatorname{loop}_{c}^{i+1}\right)\right)$ from $(s, m)$ where the loop counter of $s$ is $i+1$ and the crash flag $\mathfrak{f}$ is not set.

By Lemma E.8 the loop counter of $s$ is increasing by one in one step. Hence, there must be one configuration reachable from $(s, m)$ in which the loop counter of $s$ is $i$. By hypothesis, there is the configuration $\left(S ; s, m_{i}\left(\mathfrak{f}, \operatorname{loop}_{c}^{i}, \sigma_{i}\right)\right)$ reachable from $(s, m)$ in which the loop counter is $i, \operatorname{loop}_{c}^{i}(n)=i$, and the crash flag is not set, $\mathfrak{f}=0$. By the assumption of unique loop labels, $s \notin S$. Then the loop counter of $s$ is not redefined in the execution of $S$ started in state $m_{i}\left(\mathfrak{f}, \operatorname{loop}_{c}^{i}, \sigma_{i}\right)$. Because there is a configuration in which the loop counter of $s$ is $i+1$, then the execution of $S$ when started in the state $m_{i}\left(\mathfrak{f}, \operatorname{loop}_{c}^{i}, \sigma_{i}\right)$ terminates, $\left(S, m_{i}\left(\mathfrak{f}, \operatorname{loop}_{c}^{i}, \sigma_{i}\right)\right) \stackrel{*}{\rightarrow}$ (skip, $\left.m_{i+1}\left(\mathfrak{f}, \operatorname{loop}_{c}^{i+1}, \sigma_{i+1}\right)\right)$ where $\mathfrak{f}=0$ and $\operatorname{loop}_{c}^{i+1}(n)=i$. By Corollary E.1 $\left(S ; s, m_{i}\left(\mathfrak{f}, \operatorname{loop}_{c}^{i}, \sigma_{i}\right)\right) \stackrel{*}{\rightarrow}\left(s, m_{i+1}\left(\mathfrak{f}, \operatorname{loop}_{c}^{i+1}, \sigma_{i+1}\right)\right)$. By similar argument in base case, the evaluation of the predicate expression w.r.t the value store $\sigma_{i+1}$ produce nonzero integer value. The execution of $s$ proceeds as follows:

$$
\begin{aligned}
& \left(s, m_{i+1}\left(\mathfrak{f}, \operatorname{loop}_{c}^{i+1}, \sigma_{i+1}\right)\right) \\
= & \left(\text { while }_{\langle n\rangle}(e)\{S\}, m_{i+1}\left(\mathfrak{f}, \operatorname{loop}_{c}^{i+1}, \sigma_{i+1}\right)\right) \\
\rightarrow & \left(\text { while }_{\langle n\rangle}\left(\mathcal{E} \llbracket e \rrbracket \sigma_{i+1}\right)\{S\}, m_{i+1}\left(\mathfrak{f}, \operatorname{loop}_{c}^{i+1}, \sigma_{i+1}\right)\right) \\
& \text { by the EEval rule } \\
\rightarrow & (S ; \text { while } \\
& \text { by the Wh-T rule. }
\end{aligned}
$$

The lemma holds.

Lemma 5.25. Let $s_{1}="$ while ${ }_{\left\langle n_{1}\right\rangle}(e)\left\{S_{1}\right\} "$ and $s_{2}="$ while $e_{\left\langle n_{2}\right\rangle}(e)$ $\left\{S_{2}\right\}$ " be two while statements and all of the followings hold:

- There are output statements in $s_{1}$ and $s_{2}, \exists e_{1} e_{2}$ : ("output $e_{1}$ " $\in$ $\left.s_{1}\right) \wedge\left(\right.$ "output $e_{2}$ " $\left.\in s_{2}\right)$;

- $s_{1}$ and $s_{2}$ have the same set of termination deciding variables relative to output, and the same set of imported variables relative to output, $\left(\operatorname{TVar}_{o}\left(s_{1}\right)=\operatorname{TVar}_{o}\left(s_{2}\right)=\operatorname{TVar}(s)\right) \wedge$ $\left(\operatorname{Imp} p_{o}\left(s_{1}\right)=\operatorname{Imp} p_{o}\left(s_{2}\right)=\operatorname{Imp}(i o)\right)$;

- Loop bodies $S_{1}$ and $S_{2}$ satisfy the proof rule of equivalent computation of the out-deciding variables of $s_{1}$ and $s_{2}, \forall x \in$ $O \operatorname{Var}(s)=\operatorname{TVar}(s) \cup \operatorname{Imp}(i o): S_{1} \equiv_{x}^{S} S_{2}$;

- Loop bodies $S_{1}$ and $S_{2}$ satisfy the proof rule of termination in the same way, $S_{1} \equiv_{H}^{S} S_{2}$;

- Loop bodies $S_{1}$ and $S_{2}$ produce the same output sequence when started in states with crash flags not set and whose value stores agree on values of variables in $\operatorname{OVar}\left(S_{1}\right) \cup \operatorname{OVar}\left(S_{2}\right)$, $\forall m_{S_{1}}\left(\mathfrak{f}_{1}, \sigma_{S_{1}}\right) m_{S_{2}}\left(\mathfrak{f}_{2}, \sigma_{S_{2}}\right):$

$\left(\left(\mathfrak{f}_{1}=\mathfrak{f}_{2}=0\right) \wedge\left(\forall x \in O \operatorname{Var}\left(S_{1}\right) \cup \operatorname{OVar}\left(S_{2}\right): \sigma_{S_{1}}(x)=\right.\right.$ $\left.\left.\sigma_{S_{2}}(x)\right)\right) \Rightarrow$

$\left(\left(S_{1}, m_{S_{1}}\left(\mathfrak{f}_{1}, \sigma_{S_{1}}\right)\right) \equiv_{O}\left(S_{2}, m_{S_{2}}\left(\mathfrak{f}_{2}, \sigma_{S_{2}}\right)\right)\right)$.

If $s_{1}$ and $s_{2}$ start in states $m_{1}\left(\mathfrak{f}_{1}\right.$, loop $\left._{c}^{1}, \sigma_{1}\right), m_{2}\left(\mathfrak{f}_{2}, \operatorname{loop}_{c}^{2}, \sigma_{2}\right)$ respectively with crash flags not set $\mathfrak{f}_{1}=\mathfrak{f}_{2}=0$ and in which $s_{1}$ and $s_{2}$ have not started execution $\left(\operatorname{loop}_{c}^{1}\left(n_{1}\right)=\operatorname{loop}_{c}^{2}\left(n_{2}\right)=0\right)$, value stores $\sigma_{1}$ and $\sigma_{2}$ agree on values of variables in OVar $(s)$, $\forall x \in O \operatorname{Var}(s): \sigma_{1}(x)=\sigma_{2}(x)$, then one of the followings holds:

1. $s_{1}$ and $s_{2}$ both terminate and produce the same output sequence:

$\left(s_{1}, m_{1}\right) \stackrel{*}{\rightarrow}\left(\right.$ skip, $\left.m_{1}^{\prime}\left(\sigma_{1}^{\prime}\right)\right),\left(s_{2}, m_{2}\right) \stackrel{*}{\rightarrow}\left(\right.$ skip, $\left.m_{2}^{\prime}\left(\sigma_{2}^{\prime}\right)\right)$ where $\sigma_{1}^{\prime}\left(i d_{I O}\right)=\sigma_{2}^{\prime}\left(i d_{I O}\right)$.

2. $s_{1}$ and $s_{2}$ both do not terminate, $\forall k>0,\left(s_{1}, m_{1}\right) \stackrel{k}{\rightarrow}$ $\left(S_{1_{k}}, m_{1_{k}}\right),\left(s_{2}, m_{2}\right) \stackrel{k}{\rightarrow}\left(S_{2_{k}}, m_{2_{k}}\right)$ where $S_{1_{k}} \neq$ skip, $S_{2_{k}} \neq$ skip and one of the followings holds:

(a) For any positive integer $i$, there are two configurations $\left(s_{1}, m_{1_{i}}\right)$ and $\left(s_{2}, m_{2_{i}}\right)$ reachable from $\left(s_{1}, m_{1}\right)$ and $\left(s_{2}, m_{2}\right)$, respectively, in which both crash flags are not set, the loop counters of $s_{1}$ and $s_{2}$ are equal to $i$ and value stores agree on values of variables in $\operatorname{OVar}(s)$, and for every state in execution, $\left(s_{1}, m_{1}\right) \stackrel{*}{\rightarrow}\left(s_{1}, m_{1_{i}}\right)$ or $\left(s_{2}, m_{2}\right) \stackrel{*}{\rightarrow}\left(s_{2}, m_{2_{i}}\right)$, loop counters for $s_{1}$ and $s_{2}$ are less than or equal to $i$ respectively: 
$\forall i>0 \exists\left(s_{1}, m_{1_{i}}\right)\left(s_{2}, m_{2_{i}}\right):\left(s_{1}, m_{1}\right) \stackrel{*}{\rightarrow}\left(s_{1}, m_{1_{i}}\left(\mathfrak{f}_{1}\right.\right.$, loop $\left.\left._{c}^{1_{i}}, \sigma_{1_{i}}\right)\right) \wedge\left(s_{2}, m_{2}\right) \stackrel{*}{\rightarrow}\left(s_{2}, m_{2_{i}}\left(\mathfrak{f}_{2}\right.\right.$, loop $\left.\left._{c}^{2_{i}}, \sigma_{2_{i}}\right)\right)$ where

- $\mathfrak{f}_{1}=\mathfrak{f}_{2}=0$; and

- $\operatorname{loop}_{c}^{1_{i}}\left(n_{1}\right)=\operatorname{loop}_{c}^{2_{i}}\left(n_{2}\right)=i$; and

- $\forall x \in \operatorname{OVar}(s): \sigma_{1_{i}}(x)=\sigma_{2_{i}}(x)$.

- $\forall m_{1}^{\prime}:\left(s_{1}, m_{1}\right) \stackrel{*}{\rightarrow}\left(S_{1}^{\prime}, m_{1}^{\prime}\left(\operatorname{loop}_{c}^{1^{\prime}}\right)\right) \stackrel{*}{\rightarrow}\left(s_{1}, m_{1_{i}}\left(\operatorname{loop}_{c}^{1_{i}}\right.\right.$, $\left.\left.\sigma_{1_{i}}\right)\right)$, $\operatorname{loop}_{c}^{1^{\prime}}\left(n_{1}\right) \leq i$; and

- $\forall m_{2}^{\prime}:\left(s_{2}, m_{2}\right) \stackrel{*}{\rightarrow}\left(S_{2}^{\prime}, m_{2}^{\prime}\left(\operatorname{loop}_{c}^{2^{\prime}}\right)\right) \stackrel{*}{\rightarrow}\left(s_{2}, m_{2_{i}}\left(\operatorname{loop}_{c}^{2_{i}}\right.\right.$, $\left.\left.\sigma_{2_{i}}\right)\right)$, $\operatorname{loop}_{c}^{2^{\prime}}\left(n_{2}\right) \leq i$;

(b) The loop counters for $s_{1}$ and $s_{2}$ are less than a smallest positive integer $i$ and all of the followings hold:

- $\exists i>0 \forall m_{1}^{\prime}, m_{2}^{\prime}:\left(s_{1}, m_{1}\right) \stackrel{*}{\rightarrow}\left(S_{1}^{\prime}, m_{1}^{\prime}\left(\operatorname{loop}_{c}^{1^{\prime}}\right)\right)$, $\left(s_{2}, m_{2}\right) \stackrel{*}{\rightarrow}\left(S_{2}^{\prime}, m_{2}^{\prime}\left(\right.\right.$ loop $\left.\left._{c}^{2^{\prime}}\right)\right)$ where loop $_{c}^{1^{\prime}}\left(n_{1}\right)<i$, $\operatorname{loop}_{c}^{2^{\prime}}\left(n_{2}\right)<i$;

- $\forall 0<j<i$, there are two configurations $\left(s_{1}, m_{1_{j}}\right)$ and $\left(s_{2}, m_{2_{j}}\right)$ reachable from $\left(s_{1}, m_{1}\right)$ and $\left(s_{2}, m_{2}\right)$, respectively, in which both crash flags are not set, loop counters of $s_{1}$ and $s_{2}$ are equal to $j$ and value stores agree on values of variables in $O \operatorname{Var}(s)$ :

$\exists\left(s_{1}, m_{1_{j}}\right),\left(s_{2}, m_{2_{j}}\right):\left(s_{1}, m_{1}\right) \stackrel{*}{\rightarrow}\left(s_{1}, m_{1_{j}}\left(\mathfrak{f}_{1}, \operatorname{loop}_{c}^{1_{j}}\right.\right.$, $\left.\left.\sigma_{1_{j}}\right)\right) \wedge\left(s_{2}, m_{2}\right) \stackrel{*}{\rightarrow}\left(s_{2}, m_{2_{j}}\left(\mathfrak{f}_{2}\right.\right.$, loop $\left.\left._{c}^{2_{j}}, \sigma_{2_{j}}\right)\right)$ where

- $\mathfrak{f}_{1}=\mathfrak{f}_{2}=0$; and

- $\operatorname{loop}_{c}^{1_{j}}\left(n_{1}\right)=\operatorname{loop}_{c}^{2_{j}}\left(n_{2}\right)=j$; and

- $\forall x \in O \operatorname{Var}(s): \sigma_{1_{j}}(x)=\sigma_{2_{j}}(x)$.

- If $i=1$, then the I/O sequence is not redefined in any states reachable from $\left(s_{1}, m_{1}\right)$ and $\left(s_{2}, m_{2}\right)$.

- $\forall m_{1}^{\prime \prime} \quad: \quad\left(s_{1}, m_{1}\left(\right.\right.$ loop $\left.\left._{c}^{1}, \sigma_{1}\right)\right) \stackrel{*}{\rightarrow}\left(S_{1}^{\prime \prime}, m_{1}^{\prime \prime}\left(\sigma_{1}^{\prime \prime}\right)\right)$ where $\sigma_{1}^{\prime \prime}\left(i d_{I O}\right)=\sigma_{1}\left(i d_{I O}\right)$.

- $\forall m_{2}^{\prime \prime} \quad: \quad\left(s_{2}, m_{2}\left(\right.\right.$ loop $\left.\left._{c}^{2}, \sigma_{2}\right)\right) \stackrel{*}{\rightarrow}\left(S_{2}^{\prime \prime}, m_{2}^{\prime \prime}\left(\sigma_{2}^{\prime \prime}\right)\right)$ where $\sigma_{2}^{\prime \prime}\left(i d_{I O}\right)=\sigma_{2}\left(i d_{I O}\right)$.

- If $i>1$, then the I/O sequence is not redefined in any states reachable from $\left(s_{1}, m_{1_{i-1}}\right)$ and $\left(s_{2}, m_{2_{i-1}}\right)$.

- $\forall m_{1}^{\prime \prime}:\left(s_{1}, m_{1_{i-1}}\left(\operatorname{loop}_{c}^{1_{i-1}}, \sigma_{1_{i-1}}\right)\right) \stackrel{*}{\rightarrow}\left(S_{1}^{\prime \prime}, m_{1}^{\prime \prime}\left(\sigma_{1}^{\prime \prime}\right)\right)$ where $\sigma_{1}^{\prime \prime}\left(i d_{I O}\right)=\sigma_{1_{i-1}}\left(i d_{I O}\right)$.

- $\forall m_{2}^{\prime \prime}:\left(s_{2}, m_{2_{i-1}}\left(\operatorname{loop}_{c}^{2_{i-1}}, \sigma_{2_{i-1}}\right)\right) \stackrel{*}{\rightarrow}\left(S_{2}^{\prime \prime}, m_{2}^{\prime \prime}\left(\sigma_{2}^{\prime \prime}\right)\right)$ where $\sigma_{2}^{\prime \prime}\left(i d_{I O}\right)=\sigma_{2_{i-1}}\left(i d_{I O}\right)$.

(c) The loop counters for $s_{1}$ and $s_{2}$ are less than or equal to a smallest positive integer $i$ and all of the followings hold:

- $\exists i>0 \forall m_{1}^{\prime}, m_{2}^{\prime}:\left(s_{1}, m_{1}\right) \stackrel{*}{\rightarrow}\left(S_{1}^{\prime}, m_{1}^{\prime}\left(\operatorname{loop}_{c}^{1^{\prime}}\right)\right),\left(s_{2}\right.$, $\left.m_{2}\right) \stackrel{*}{\rightarrow}\left(S_{2}^{\prime}, m_{2}^{\prime}\left(\right.\right.$ loop $\left.\left._{c}^{2^{\prime}}\right)\right)$ where loop ${ }_{c}^{1^{\prime}}\left(n_{1}\right) \leq i$, $\operatorname{loop}_{c}^{2^{\prime}}\left(n_{2}\right) \leq i$;

- There are no configurations $\left(s_{1}, m_{1_{i}}\right)$ and $\left(s_{2}, m_{2_{i}}\right)$ reachable from $\left(s_{1}, m_{1}\right)$ and $\left(s_{2}, m_{2}\right)$, respectively, in which crash flags are not set, the loop counters of $s_{1}$ and $s_{2}$ are equal to $i$, and value stores agree on values of variables in $O \operatorname{Var}(s)$ :

$\nexists\left(s_{1}, m_{1_{i}}\right),\left(s_{2}, m_{2_{i}}\right):\left(s_{1}, m_{1}\right) \stackrel{*}{\rightarrow}\left(s_{1}, m_{1_{i}}\left(\mathfrak{f}_{1}\right.\right.$, loop $_{c}^{1_{i}}$, $\left.\left.\sigma_{1_{i}}\right)\right) \wedge\left(s_{2}, m_{2}\right) \stackrel{*}{\rightarrow}\left(s_{2}, m_{2_{i}}\left(\mathfrak{f}_{2}\right.\right.$, loop $\left.\left._{c}^{2_{i}}, \sigma_{2_{i}}\right)\right)$ where

- $\mathfrak{f}_{1}=\mathfrak{f}_{2}=0$; and

- $\operatorname{loop}_{c}^{1_{i}}\left(n_{1}\right)=\operatorname{loop}_{c}^{2_{i}}\left(n_{2}\right)=i$; and

- $\forall x \in \operatorname{OVar}(s): \sigma_{1_{i}}(x)=\sigma_{2_{i}}(x)$.

- $\forall 0<j<i$, there are two configurations $\left(s_{1}, m_{1_{j}}\right)$ and $\left(s_{2}, m_{2_{j}}\right)$ reachable from $\left(s_{1}, m_{1}\right)$ and $\left(s_{2}, m_{2}\right)$, respectively, in which crash flags are not set, the loop counters of $s_{1}$ and $s_{2}$ are equal to $j$ and value stores agree on values of variables in $\operatorname{OVar}(s)$ : $\exists\left(s_{1}, m_{1_{j}}\right),\left(s_{2}, m_{2_{j}}\right):\left(s_{1}, m_{1}\right) \stackrel{*}{\rightarrow}\left(s_{1}, m_{1_{j}}\left(\mathfrak{f}_{1}\right.\right.$, loop $_{c}^{1_{j}}$, $\left.\left.\sigma_{1_{j}}\right)\right) \wedge\left(s_{2}, m_{2}\right) \stackrel{*}{\rightarrow}\left(s_{2}, m_{2_{j}}\left(\mathfrak{f}_{2}, \operatorname{loop}_{c}^{2_{j}}, \sigma_{2_{j}}\right)\right)$ where

- $\mathfrak{f}_{1}=\mathfrak{f}_{2}=0$; and

- $\operatorname{loop}_{c}^{1_{j}}\left(n_{1}\right)=\operatorname{loop}_{c}^{2_{j}}\left(n_{2}\right)=j$; and

- $\forall x \in \operatorname{OVar}(s): \sigma_{1_{j}}(x)=\sigma_{2_{j}}(x)$.

- If $i=1$, then executions from $\left(s_{1}, m_{1}\right)$ and $\left(s_{2}, m_{2}\right)$ produce the same output sequence: $\left(s_{1}, m_{1}\left(\operatorname{loop}_{c}^{1}, \sigma_{1}\right)\right) \equiv_{O}\left(s_{2}, m_{2}\left(\right.\right.$ loop $\left.\left._{c}^{2}, \sigma_{2}\right)\right)$.

- If $i>1$, then executions from $\left(s_{1}, m_{1_{i-1}}\right)$ and $\left(s_{2}, m_{2_{i-1}}\right)$ produce the same output sequence:

$\left(s_{1}, m_{1_{i-1}}\left(\operatorname{loop}_{c}^{1_{i-1}}, \sigma_{1_{i-1}}\right)\right) \equiv_{O}\left(s_{2}, m_{2_{i-1}}\left(\operatorname{loop}_{c}^{2_{i-1}}\right.\right.$, $\left.\left.\sigma_{2_{i-1}}\right)\right)$.

Proof. We show that $s_{1}$ and $s_{2}$ terminate in the same way when started in states $m_{1}\left(\mathfrak{f}_{1}, \operatorname{loop}_{c}^{1}, \sigma_{1}\right)$ and $m_{2}\left(\mathfrak{f}_{2}, \operatorname{loop}_{c}^{2}, \sigma_{2}\right)$ respectively, $\left(s_{1}, m_{1}\right) \equiv_{H}\left(s_{2}, m_{2}\right)$. In addition, we show that $s_{1}$ and $s_{2}$ produce the same output sequence in every possibilities of termination in the same way, $\left(s_{1}, m_{1}\right) \equiv_{O}\left(s_{2}, m_{2}\right)$.

By definition, $s_{1}$ and $s_{2}$ satisfy the proof rule of termination in the same way because

- Loop bodies $S_{1}$ and $S_{2}$ satisfy the proof rule of termination in the same way;

By assumption.

- Loop bodies $S_{1}$ and $S_{2}$ satisfy the proof rule of equivalent computation of those in the termination deciding variables of $s_{1}$ and $s_{2}, \forall x \in \operatorname{TVar}\left(s_{1}\right) \cup \operatorname{TVar}\left(s_{2}\right): S_{1} \equiv_{x}^{S} S_{2}$;

By the definition of $\operatorname{OVar}(s), \operatorname{TVar}_{o}\left(s_{1}\right) \subseteq \operatorname{OVar}\left(s_{1}\right)$ and $\operatorname{TVar}_{o}\left(s_{2}\right) \subseteq \operatorname{OVar}\left(s_{2}\right)$. By the definition of $\operatorname{TVar}_{o}, \operatorname{TVar}_{o}\left(s_{1}\right)=$ $\operatorname{TVar}\left(s_{1}\right)$ and $\operatorname{TVar}_{o}\left(s_{2}\right)=\mathrm{TVar}\left(s_{2}\right)$.

By Lemma 5.16 we show $s_{1}$ and $s_{2}$ terminate in the same way when started in states $m_{1}\left(\mathfrak{f}_{1}, \operatorname{loop}_{c}^{1}, \sigma_{1}\right)$ and $m_{2}\left(\mathfrak{f}_{2}\right.$, loop $\left._{c}^{2}, \sigma_{2}\right)$. We need to show that all the required conditions are satisfied.

- Crash flags are not set, $\mathfrak{f}_{1}=\mathfrak{f}_{2}=0$;

- Loop counters of $s_{1}$ and $s_{2}$ are initially zero, $\operatorname{loop}_{c}^{1}\left(n_{1}\right)=$ $\operatorname{loop}_{c}^{2}\left(n_{2}\right)=0$

- $s_{1}$ and $s_{2}$ have same set of termination deciding variables, $\mathrm{T} \operatorname{Var}\left(s_{1}\right)=\mathrm{T} \operatorname{Var}\left(s_{2}\right)=\mathrm{T} \operatorname{Var}(s)$;

- Value stores $\sigma_{1}$ and $\sigma_{2}$ agree on values of variables in $\operatorname{TVar}\left(s_{1}\right)=$ $\operatorname{TVar}\left(s_{2}\right), \forall x \in \operatorname{TVar}(s): \sigma_{1}(x)=\sigma_{2}(x)$;

The above four conditions are from assumption.

- Loop bodies $S_{1}$ and $S_{2}$ terminate in the same way when started in states with crash flags not set and whose value stores agree on values of variables in $\operatorname{TVar}\left(S_{1}\right) \cup \mathrm{T} \operatorname{Var}\left(S_{2}\right)$;

By Theorem 4

Therefore, by Lemma 5.16 we have one of the followings holds:

1. $s_{1}$ and $s_{2}$ both terminate and the loop counters of $s_{1}$ and $s_{2}$ are less than a positive integer $i$ such that the loop counters of $s_{1}$ and $s_{2}$ are less than or equal to $i-1$ :

$\left(s_{1}, m_{1}\right) \stackrel{*}{\rightarrow}$ (skip, $\left.m_{1}^{\prime}\right),\left(s_{2}, m_{2}\right) \stackrel{*}{\rightarrow}$ (skip, $\left.m_{2}^{\prime}\right)$.

We show that, when $s_{1}$ and $s_{2}$ terminate, value stores of $s_{1}$ and $s_{2}$ agree on the value of the I/O sequence variable by Lemma5.2 We need to show all the required conditions hold.

- $\forall x \in \operatorname{Imp}(i o): \sigma_{1}(x)=\sigma_{2}(x)$

- $\operatorname{loop}_{c}^{1}\left(n_{1}\right)=\operatorname{loop}_{c}^{2}\left(n_{2}\right)=0$;

The above two conditions are from assumption.

- $i d_{I O} \in \operatorname{Def}\left(s_{1}\right) \cap \operatorname{Def}\left(s_{2}\right)$;

Because there are output statements in $s_{1}$ and $s_{2}$. By the definition of $\operatorname{Def}(\cdot)$, the I/O sequence variable is defined in $s_{1}$ and $s_{2}$. 
- $\operatorname{Imp}\left(s_{1},\left\{i d_{I O}\right\}\right)=\operatorname{Imp}\left(s_{2},\left\{i d_{I O}\right\}\right)=\operatorname{Imp}(i o)$; By the definition of $\operatorname{Imp}_{o}(\cdot), \operatorname{Imp}_{o}\left(s_{1}\right)=\operatorname{Imp}\left(s_{1},\left\{i d_{I O}\right\}\right)$, $\operatorname{Imp}_{o}\left(s_{2}\right)=\operatorname{Imp}\left(s_{2},\left\{i d_{I O}\right\}\right)$.

- $\forall y \in \operatorname{Imp}(i o), \forall m_{S_{1}}\left(\sigma_{S_{1}}\right) m_{S_{2}}\left(\sigma_{S_{2}}\right)$ :

$\left(\left(\forall z \in \operatorname{Imp}\left(S_{1}, \operatorname{Imp}(i o)\right) \cup \operatorname{Imp}\left(S_{2}, \operatorname{Imp}(i o)\right), \sigma_{S_{1}}(z)=\right.\right.$ $\left.\left.\sigma_{S_{2}}(z)\right) \Rightarrow\left(S_{1}, m_{S_{1}}\left(\sigma_{S_{1}}\right)\right) \equiv_{y}\left(S_{2}, m_{S_{2}}\left(\sigma_{S_{2}}\right)\right)\right)$.

By Theorem 2

In addition, by the semantic rules, the I/O sequence is appended at most by one value in one step. Hence, $s_{1}$ and $s_{2}$ produce the same output sequence when started in states $m_{1}$ and $m_{2}$ respectively.

2. $s_{1}$ and $s_{2}$ both do not terminate, $\forall k>0$, $\left(s_{1}, m_{1}\right) \stackrel{k}{\rightarrow}$ $\left(S_{1_{k}}, m_{1_{k}}\right),\left(s_{2}, m_{2}\right) \stackrel{k}{\rightarrow}\left(S_{2_{k}}, m_{2_{k}}\right)$ where $S_{1_{k}} \neq$ skip, $S_{2_{k}} \neq$ skip and one of the followings holds:

(a) $\forall i>0$, there are two configurations $\left(s_{1}, m_{1_{i}}\right)$ and $\left(s_{2}, m_{2_{i}}\right)$ reachable from $\left(s_{1}, m_{1}\right)$ and $\left(s_{2}, m_{2}\right)$, respectively, in which both crash flags are not set, the loop counters of $s_{1}$ and $s_{2}$ are equal to $i$ and value stores agree on the values of variables in $\operatorname{TVar}(s)$ :

$\forall i>0 \exists\left(s_{1}, m_{1_{i}}\right)\left(s_{2}, m_{2_{i}}\right):\left(s_{1}, m_{1}\right) \stackrel{*}{\rightarrow}\left(s_{1}, m_{1_{i}}\left(\mathfrak{f}_{1}\right.\right.$, $\left.\left.\operatorname{loop}_{c}^{1_{i}}, \sigma_{1_{i}}\right)\right) \wedge\left(s_{2}, m_{2}\right) \stackrel{*}{\rightarrow}\left(s_{2}, m_{2_{i}}\left(\mathfrak{f}_{2}, \operatorname{loop}_{c}^{2_{i}}, \sigma_{2_{i}}\right)\right)$ where

- $\mathfrak{f}_{1}=\mathfrak{f}_{2}=0$; and

- $\operatorname{loop}_{c}^{1_{i}}\left(n_{1}\right)=\operatorname{loop}_{c}^{2_{i}}\left(n_{2}\right)=i$; and

- $\forall x \in \operatorname{TVar}(s): \sigma_{1_{i}}(x)=\sigma_{2_{i}}(x)$.

- $\forall m_{1}^{\prime}:\left(s_{1}, m_{1}\right) \stackrel{*}{\rightarrow}\left(S_{1}^{\prime}, m_{1}^{\prime}\left(\operatorname{loop}_{c}^{1^{\prime}}\right)\right) \stackrel{*}{\rightarrow}\left(s_{1}, m_{1_{i}}\left(\operatorname{loop}_{c}^{1_{i}}\right.\right.$, $\left.\left.\sigma_{1_{i}}\right)\right), \operatorname{loop}_{c}^{1^{\prime}}\left(n_{1}\right) \leq i$; and

- $\forall m_{2}^{\prime}:\left(s_{2}, m_{2}\right) \stackrel{*}{\rightarrow}\left(S_{2}^{\prime}, m_{2}^{\prime}\left(\operatorname{loop}_{c}^{2^{\prime}}\right)\right) \stackrel{*}{\rightarrow}\left(s_{2}, m_{2_{i}}\left(\right.\right.$ loop $_{c}^{2_{i}}$, $\left.\left.\sigma_{2_{i}}\right)\right), \operatorname{loop}_{c}^{2^{\prime}}\left(n_{2}\right) \leq i$

We show that, for any positive integer $i$, value stores $\sigma_{1_{i}}$ and $\sigma_{2_{i}}$ agree on values of variables in $\operatorname{Imp}(i o)$ by the proof of Lemma5.1 We need to show that all the required conditions are satisfied.

- $\forall x \in \operatorname{Imp}(i o): \sigma_{1}(x)=\sigma_{2}(x)$;

- $\operatorname{loop}_{c}^{1}\left(n_{1}\right)=\operatorname{loop}_{c}^{2}\left(n_{2}\right)=0$;

The above two conditions are by assumption.

- $i d_{I O} \in \operatorname{Def}\left(s_{1}\right) \cap \operatorname{Def}\left(s_{2}\right)$;

- $\operatorname{Imp}\left(s_{1},\left\{i d_{I O}\right\}\right)=\operatorname{Imp}\left(s_{2},\left\{i d_{I O}\right\}\right)=\operatorname{Imp}(i o)$;

The above two conditions are obtained by similar argument in the case that $s_{1}$ and $s_{2}$ both terminate.

- $\forall y \in \operatorname{Imp}(i o), \forall m_{S_{1}}\left(\sigma_{S_{1}}\right) m_{S_{2}}\left(\sigma_{S_{2}}\right)$ :

$\left(\left(\forall z \in \operatorname{Imp}\left(S_{1}, \operatorname{Imp}(i o)\right) \cup \operatorname{Imp}\left(S_{2}, \operatorname{Imp}(i o)\right), \sigma_{S_{1}}(z)=\right.\right.$ $\left.\sigma_{S_{2}}(z)\right) \Rightarrow$

$\left.\left(S_{1}, m_{S_{1}}\left(\sigma_{S_{1}}\right)\right) \equiv_{y}\left(S_{2}, m_{S_{2}}\left(\sigma_{S_{2}}\right)\right)\right)$.

By Theorem 2 .

We cannot apply Lemma 5.1 directly because $s_{1}$ and $s_{2}$ do not terminate. But we can still have the proof closely similar to that of Lemma 5.1 by using the fact that there exists a configuration of arbitrarily large loop counters of $s_{1}$ and $s_{2}$ and in which crash flags are not set.

Then, $\forall i>0, \forall x \in \operatorname{Imp}(i o): \sigma_{1_{i}}(x)=\sigma_{2_{i}}(x)$. In addition, by the semantic rules, the $\mathrm{I} / \mathrm{O}$ sequence is appended at most by one value in one step. The lemma holds.

(b) The loop counters for $s_{1}$ and $s_{2}$ are less than a smallest positive integer $i$ and all of the followings hold:

- $\exists i>0 \forall m_{1}^{\prime}, m_{2}^{\prime}:\left(s_{1}, m_{1}\right) \stackrel{*}{\rightarrow}\left(S_{1}^{\prime}, m_{1}^{\prime}\left(\operatorname{loop}_{c}^{1^{\prime}}\right)\right),\left(s_{2}\right.$, $\left.m_{2}\right) \stackrel{*}{\rightarrow}\left(S_{2}^{\prime}, m_{2}^{\prime}\left(\operatorname{loop}_{c}^{2^{\prime}}\right)\right)$ where $\operatorname{loop}_{c}^{1^{\prime}}\left(n_{1}\right)<i$, $\operatorname{loop}_{c}^{2^{\prime}}\left(n_{2}\right)<i$;

- $\forall 0<j<i$, there are two configurations $\left(s_{1}, m_{1_{j}}\right)$ and $\left(s_{2}, m_{2_{j}}\right)$ reachable from $\left(s_{1}, m_{1}\right)$ and $\left(s_{2}, m_{2}\right)$, respectively, in which both crash flags are not set, the loop counters of $s_{1}$ and $s_{2}$ are equal to $j$ and value stores agree on the values of variables in $\operatorname{TVar}(s)$ :

$\exists\left(s_{1}, m_{1_{j}}\right),\left(s_{2}, m_{2_{j}}\right):\left(s_{1}, m_{1}\right) \stackrel{*}{\rightarrow}\left(s_{1}, m_{1_{j}}\left(\mathfrak{f}_{1}, \operatorname{loop}_{c}^{1_{j}}\right.\right.$,

$\left.\left.\sigma_{1_{j}}\right)\right) \wedge\left(s_{2}, m_{2}\right) \stackrel{*}{\rightarrow}\left(s_{2}, m_{2_{j}}\left(\mathfrak{f}_{2}, \operatorname{loop}_{c}^{2_{j}}, \sigma_{2_{j}}\right)\right)$ where

- $\mathfrak{f}_{1}=\mathfrak{f}_{2}=0$; and

- $\operatorname{loop}_{c}{ }^{1_{j}}\left(n_{1}\right)=\operatorname{loop}_{c}^{2_{j}}\left(n_{2}\right)=j$; and

- $\forall x \in \operatorname{T} \operatorname{Var}(s): \sigma_{1_{j}}(x)=\sigma_{2_{j}}(x)$.

This case corresponds to the situation that the $i$ th evaluations of the predicate expression of $s_{1}$ and $s_{2}$ raise an exception. There are two possibilities regarding the value of $i$.

i. $i=1$.

$s_{1}$ and $s_{2}$ raise an exception in the 1st evaluation of their predicate expression because loop counters of $s_{1}$ and $s_{2}$ are less than 1 . In the proof of Lemma 5.16 value stores $\sigma_{1}$ and $\sigma_{2}$ in states $m_{1}$ and $m_{2}$ respectively are not modified in the 1 st evaluation of the predicate expression of $s_{1}$ and $s_{2}$. In addition, value stores $\sigma_{1}$ and $\sigma_{2}$ are not modified after $s_{1}$ and $s_{2}$ both crash according to the rule Crash. We have the corresponding initial state in which value stores $\sigma_{1}$ and $\sigma_{2}$ agree on values of variables in $\operatorname{OVar}(s)$. Thus, $\sigma_{1}\left(i d_{I O}\right)=\sigma_{2}\left(i d_{I O}\right)$. The lemma holds.

ii. $i>1$.

We show that, for any positive integer $0<j<i$, value stores $\sigma_{1_{j}}$ and $\sigma_{2}$ agree on values of variables in $\operatorname{Imp}(s)$ by the proof of Lemma 5.1 We need to show that all the required conditions are satisfied.

- $\forall x \in \operatorname{Imp}(i o): \sigma_{1}(x)=\sigma_{2}(x)$;

- $\operatorname{loop}_{c}^{1}\left(n_{1}\right)=\operatorname{loop}_{c}^{2}\left(n_{2}\right)=0$;

The above two conditions are from assumption.

- $i d_{I O} \in \operatorname{Def}\left(s_{1}\right) \cap \operatorname{Def}\left(s_{2}\right)$;

- $\operatorname{Imp}\left(s_{1},\left\{i d_{I O}\right\}\right)=\operatorname{Imp}\left(s_{2},\left\{i d_{I O}\right\}\right)=\operatorname{Imp}(i o)$;

The above two conditions are obtained by the same argument in the case that $s_{1}$ and $s_{2}$ terminate.

- $\forall y \in \operatorname{Imp}(i o), \forall m_{S_{1}}\left(\sigma_{S_{1}}\right) m_{S_{2}}\left(\sigma_{S_{2}}\right)$ :

$\left(\left(\forall z \in \operatorname{Imp}\left(S_{1}, \operatorname{Imp}(i o)\right) \cup \operatorname{Imp}\left(S_{2}, \operatorname{Imp}(i o)\right), \sigma_{S_{1}}(z)=\right.\right.$ $\left.\sigma_{S_{2}}(z)\right) \Rightarrow$

$\left.\left(S_{1}, m_{S_{1}}\left(\sigma_{S_{1}}\right)\right) \equiv_{y}\left(S_{2}, m_{S_{2}}\left(\sigma_{S_{2}}\right)\right)\right)$.

By Theorem 2

We cannot apply Lemma 5.1 directly because $s_{1}$ and $s_{2}$ do not terminate. But we can still have the proof closely similar to that of Lemma 5.1 by using the fact that there are reachable configurations $\left(s_{1}, m_{1_{i-1}}\right)$ and $\left(s_{2}, m_{2_{i-1}}\right)$ with the loop counters of $s_{1}$ and $s_{2}$ of value $i-1$ and crash flags not set.

By assumption, there is configuration $\left(s_{1}, m_{1_{i-1}}\left(\mathfrak{f}_{1}\right.\right.$, loop $\left.\left._{c}^{1_{i-1}}, \sigma_{1_{i-1}}\right)\right)$ reachable from $\left(s_{1}, m_{1}\right)$ in which the loop counter of $s_{1}$ is $i-1$ and the crash flag is not set; there is also a configuration $\left(s_{2}, m_{2_{i-1}}\left(\mathfrak{f}_{2}, \operatorname{loop}_{c}^{2_{i-1}}, \sigma_{2_{i-1}}\right)\right)$ of $s_{2}$ reachable from $\left(s_{2}, m_{2}\right)$ in which the loop counter is $i-1$ and the crash flag is not set. In addition, value stores $\sigma_{1_{i-1}}$ and $\sigma_{2_{i-1}}$ agree on values of variables in $\operatorname{Imp}(i o)$. In the proof of Lemma 5.15 the $i$ th evaluations of the predicate expression of $s_{1}$ and $s_{2}$ must raise an exception because loop counters of $s_{1}$ and $s_{2}$ are less than $i$. Then the $\mathrm{I} / \mathrm{O}$ sequence is not redefined in any state reachable from $\left(s_{1}, m_{1_{i-1}}\left(\mathfrak{f}_{1}, \operatorname{loop}_{c}^{1_{i-1}}, \sigma_{1_{i-1}}\right)\right)$ and $\left(s_{2}, m_{2_{i-1}}\left(\mathfrak{f}_{2}, \operatorname{loop}_{c}^{2_{i-1}}, \sigma_{2_{i-1}}\right)\right)$ respectively. In addition, by the semantic rules, the $\mathrm{I} / \mathrm{O}$ sequence is appended at most by one value in one step. The lemma holds. 
(c) The loop counters for $s_{1}$ and $s_{2}$ are less than or equal to a smallest positive integer $i$ and all of the followings hold:

- $\exists i>0 \forall m_{1}^{\prime}, m_{2}^{\prime}:\left(s_{1}, m_{1}\right) \stackrel{*}{\rightarrow}\left(S_{1}^{\prime}, m_{1}^{\prime}\left(\operatorname{loop}_{c}^{1^{\prime}}\right)\right),\left(s_{2}, m_{2}\right) \stackrel{*}{\rightarrow}$ $\left(S_{2}^{\prime}, m_{2}^{\prime}\left(\operatorname{loop}_{c}^{2^{\prime}}\right)\right)$ where $\operatorname{loop}_{c}^{1^{\prime}}\left(n_{1}\right) \leq i$, $\operatorname{loop}_{c}^{2^{\prime}}\left(n_{2}\right) \leq i$

- There are no configurations $\left(s_{1}, m_{1_{i}}\right)$ and $\left(s_{2}, m_{2_{i}}\right)$ reachable from $\left(s_{1}, m_{1}\right)$ and $\left(s_{2}, m_{2}\right)$, respectively, in which crash flags are not set, the loop counters of $s_{1}$ and $s_{2}$ are equal to $i$, and value stores agree on values of variables in $\operatorname{TVar}(s)$ :

$\nexists\left(s_{1}, m_{1_{i}}\right),\left(s_{2}, m_{2_{i}}\right):\left(s_{1}, m_{1}\right) \stackrel{*}{\rightarrow}\left(s_{1}, m_{1_{i}}\left(\mathfrak{f}_{1}\right.\right.$, loop $\left.\left._{c}^{1_{i}}, \sigma_{1_{i}}\right)\right) \wedge$

$\left(s_{2}, m_{2}\right) \stackrel{*}{\rightarrow}\left(s_{2}, m_{2_{i}}\left(\mathfrak{f}_{2}, \operatorname{loop}_{c}^{2_{i}}, \sigma_{2_{i}}\right)\right)$ where

- $\mathfrak{f}_{1}=\mathfrak{f}_{2}=0$; and

- $\operatorname{loop}_{c}^{1_{i}}\left(n_{1}\right)=\operatorname{loop}_{c}^{2_{i}}\left(n_{2}\right)=i$; and

- $\forall x \in \operatorname{TVar}(s): \sigma_{1_{i}}(x)=\sigma_{2_{i}}(x)$.

- $\forall 0<j<i$, there are two configurations $\left(s_{1}, m_{1_{j}}\right)$ and $\left(s_{2}, m_{2_{j}}\right)$ reachable from $\left(s_{1}, m_{1}\right)$ and $\left(s_{2}, m_{2}\right)$, respectively, in which both crash flags are not set, the loop counters of $s_{1}$ and $s_{2}$ are equal to $j$ and value stores agree on values of variables in $\operatorname{TVar}(s)$ :

$$
\begin{aligned}
& \exists\left(s_{1}, m_{1_{j}}\right),\left(s_{2}, m_{2_{j}}\right):\left(s_{1}, m_{1}\right) \stackrel{*}{\rightarrow}\left(s_{1}, m_{1_{j}}\left(\mathfrak{f}_{1}, \operatorname{loop}_{c}{ }^{1_{j}}, \sigma_{1_{j}}\right)\right) \wedge \\
& \left(s_{2}, m_{2}\right) \stackrel{*}{\rightarrow}\left(s_{2}, m_{2_{j}}\left(\mathfrak{f}_{2}, \operatorname{loop}_{c}^{2_{j}}, \sigma_{2_{j}}\right)\right) \text { where } \\
& \quad \text { - } \mathfrak{f}_{1}=\mathfrak{f}_{2}=0 ; \text { and } \\
& \text { - } \operatorname{loop}_{c}{ }^{j}\left(n_{1}\right)=\operatorname{loop}_{c}{ }^{2_{j}}\left(n_{2}\right)=j \text {; and } \\
& \text { - } \forall x \in \operatorname{TVar}(s): \sigma_{1_{j}}(x)=\sigma_{2_{j}}(x) .
\end{aligned}
$$

This case corresponds to the situation that, the $i$ th evaluation of the predicate expression of $s_{1}$ and $s_{2}$ produce same nonzero integer value and loop bodies $S_{1}$ and $S_{2}$ do not terminate after the $i$ th evaluation of the predicate expression of $s_{1}$ and $s_{2}$. There are two possibilities regarding the value of $i$.

i. $i=1$.

By assumption, we have the initial value stores $\sigma_{1}$ and $\sigma_{2}$ agree on values of variables in $\operatorname{OVar}(s)$. In the proof of Lemma 5.15, the execution of $s_{1}$ proceeds as follows:

$$
\left(s_{1}, m_{1}\left(\operatorname{loop}_{c}^{1}, \sigma_{1}\right)\right)
$$

$=\left(\right.$ while $\left._{\left\langle n_{1}\right\rangle}(e)\left\{S_{1}\right\}, m_{1}\left(\operatorname{loop}_{c}^{1}, \sigma_{1}\right)\right)$

$\rightarrow\left(\right.$ while $\left._{\left\langle n_{1}\right\rangle}(v)\left\{S_{1}\right\}, m_{1}\left(\operatorname{loop}_{c}^{1}, \sigma_{1}\right)\right)$ by the EEval rule

$\rightarrow\left(S_{1} ;\right.$ while $\left._{\left\langle n_{1}\right\rangle}(e)\left\{S_{1}\right\}, m_{1}\left(\operatorname{loop}_{c}^{1}\left[1 / n_{1}\right], \sigma_{1}\right)\right)$ by the Wh-T rule.

The execution of $s_{2}$ proceeds to $\left(S_{2} ;\right.$ while ${ }_{\left\langle n_{2}\right\rangle}(e)\left\{S_{2}\right\}$, $\left.m_{2}\left(\operatorname{loop}_{c}^{2}\left[1 / n_{2}\right], \sigma_{2}\right)\right)$. Then the execution of $S_{1}$ and $S_{2}$ do not terminate when started in states $m_{1}\left(\operatorname{loop}_{c}^{1}\left[1 / n_{1}\right], \sigma_{1}\right)$ and $m_{2}\left(\operatorname{loop}_{c}^{2}\left[1 / n_{2}\right], \sigma_{2}\right)$. By assumption, value stores $\sigma_{1}$ and $\sigma_{2}$ agree on values of the out-deciding variables of $s_{1}$ and $s_{2}, \forall x \in \operatorname{OVar}\left(s_{1}\right) \cup \operatorname{OVar}\left(s_{2}\right)$ : $\sigma_{1}(x)=\sigma_{2}(x)$. By definition, $\operatorname{Imp}\left(S_{1},\left\{i d_{I O}\right\}\right) \subseteq$ $\operatorname{Imp}\left(s_{1},\left\{i d_{I O}\right\}\right), C \operatorname{Var}\left(S_{1}\right) \subseteq \operatorname{CVar}\left(s_{1}\right)$ and $\operatorname{LVar}\left(S_{1}\right) \subseteq$ LVar $\left(s_{1}\right)$. Thus, TVar $\left(S_{1}\right) \subseteq \mathrm{T} \operatorname{Var}\left(s_{1}\right)$. By Lemma 5.20 $\operatorname{TVar}_{o}\left(S_{1}\right) \subseteq \mathrm{TVar}\left(S_{1}\right)$. By Lemma 5.18. $\operatorname{Imp}_{o}\left(S_{1}\right) \subseteq$ $\operatorname{Imp}\left(S_{1}, i d_{I O}\right)$. In conclusion, $\operatorname{OVar}\left(S_{1}\right) \subseteq \operatorname{OVar}\left(s_{1}\right)$. Similarly, $\operatorname{OV} \operatorname{ar}\left(S_{2}\right) \subseteq \operatorname{OV} \operatorname{Var}\left(s_{2}\right)$. Thus, $\forall x \in \operatorname{OVar}\left(S_{1}\right) \cup$ $\operatorname{OVar}\left(S_{2}\right): \sigma_{1}(x)=\sigma_{2}(x)$. Then executions of $S_{1}$ and $S_{2}$ when started from states $m_{1}\left(\operatorname{loop}_{c}^{1}\left[1 / n_{1}\right], \sigma_{1}\right)$ and $m_{2}\left(\operatorname{loop}_{c}^{2}\left[1 / n_{2}\right], \sigma_{2}\right)$ produce the same output sequence:

$\left(S_{1}, m_{1}\left(\operatorname{loop}_{c}^{1}\left[1 / n_{1}\right], \sigma_{1}\right)\right) \equiv_{O}\left(S_{2}, m_{2}\left(\operatorname{loop}_{c}^{2}\left[1 / n_{2}\right], \sigma_{2}\right)\right)$. In addition, by the semantic rules, the $\mathrm{I} / \mathrm{O}$ sequence is appended at most by one value in one step. The lemma holds.

ii. $i>1$
We show that, for any positive integer $0<j<i$, value stores $\sigma_{1 j}$ and $\sigma_{2 j}$ agree on values of variables in $\operatorname{Imp}(i o)$ by the proof of Lemma 5.1 We need to show that all the required conditions are satisfied.

- $\forall x \in \operatorname{Imp}(i o): \sigma_{1}(x)=\sigma_{2}(x)$;

- $\operatorname{loop}_{c}^{1}\left(n_{1}\right)=\operatorname{loop}_{c}^{2}\left(n_{2}\right)=0$;

The above two conditions are from assumption.

- $i d_{I O} \in \operatorname{Def}\left(s_{1}\right) \cap \operatorname{Def}\left(s_{2}\right)$;

- $\operatorname{Imp}\left(s_{1},\left\{i d_{I O}\right\}\right)=\operatorname{Imp}\left(s_{2},\left\{i d_{I O}\right\}\right)=\operatorname{Imp}(i o)$;

The above two conditions are obtained by the same argument in the case that $s_{1}$ and $s_{2}$ terminate.

- $\forall y \in \operatorname{Imp}(i o), \forall m_{S_{1}}\left(\sigma_{S_{1}}\right) m_{S_{2}}\left(\sigma_{S_{2}}\right)$ :

$\left(\left(\forall z \in \operatorname{Imp}\left(S_{1}, \operatorname{Imp}(i o)\right) \cup \operatorname{Imp}\left(S_{2}, \operatorname{Imp}(i o)\right), \sigma_{S_{1}}(z)=\right.\right.$ $\left.\sigma_{S_{2}}(z)\right) \Rightarrow$

$\left.\left(S_{1}, m_{S_{1}}\left(\sigma_{S_{1}}\right)\right) \equiv_{y}\left(S_{2}, m_{S_{2}}\left(\sigma_{S_{2}}\right)\right)\right)$.

By Theorem 2

We cannot apply Lemma 5.1 directly because $s_{1}$ and $s_{2}$ do not terminate. But we can still have the proof closely similar to that of Lemma 5.1. The reason is that there are reachable configurations $\left(S_{1} ; s_{1}, m_{1}^{\prime}\right)$ and $\left(S_{2} ; s_{2}, m_{2}^{\prime}\right)$ with loop counters of $s_{1}$ and $s_{2}$ of value $i$ and crash flags not set. This is by Lemma 5.24 because there are configurations reachable from $\left(s_{1}, m_{1}\right)$ and $\left(s_{2}, m_{2}\right)$ respectively with loop counters of $s_{1}$ and $s_{2}$ of $i$.

There are configurations $\left(s_{1}, m_{1_{i-1}}\right)$ reachable from $\left(s_{1}, m_{1}\right)$ and $\left(s_{2}, m_{2_{i-1}}\right)$ reachable from $\left(s_{2}, m_{2}\right)$ in which loop counters of $s_{1}$ and $s_{2}$ are $i-1$ and crash flags are not set and value stores agree on values of variables in $\operatorname{Imp}(i o)$. Because loop counters of $s_{1}$ and $s_{2}$ are less than or equal to $i$. Then the execution of $s_{1}$ proceeds as follows:

$$
\begin{aligned}
& \left(s_{1}, m_{1_{i-1}}\left(\operatorname{loop}_{c}^{1_{i-1}}, \sigma_{1_{i-1}}\right)\right) \\
= & \left(\text { while }_{\left\langle n_{1}\right\rangle}(e)\left\{S_{1}\right\}, m_{1_{i-1}}\left(\operatorname{loop}_{c}^{1_{i-1}}, \sigma_{1_{i-1}}\right)\right) \\
\rightarrow & (\text { while } \\
& \text { by the EEval rule } \\
\rightarrow & \left.\left(S_{1}\right\}, m_{1_{i-1}}\left(\operatorname{loop}_{c}^{1_{i-1}}, \sigma_{1_{i-1}}\right)\right) \\
\rightarrow & \left(S_{1} ;\right. \text { while } \\
& \left.\left.\sigma_{1_{i-1}}\right)\right) \text { by the Wh-T rule. }
\end{aligned}
$$

The execution of $s_{2}$ proceeds to $\left(S_{2} ;\right.$ while $_{\left\langle n_{2}\right\rangle}(e)\left\{S_{2}\right\}$, $\left.m_{2_{i-1}}\left(\operatorname{loop}_{c}^{2_{i-1}}\left[i / n_{2}\right], \sigma_{2_{i-1}}\right)\right)$. By similar argument in the case $i=1, S_{2}$ and $S_{1}$ produce the same output sequence when started in states $m_{1_{i-1}}\left(\operatorname{loop}_{c}^{1_{i-1}}\left[i / n_{1}\right], \sigma_{1_{i-1}}\right)$ and $m_{2_{i-1}}\left(\operatorname{loop}_{c}^{2_{i-1}}\left[i / n_{2}\right], \sigma_{2_{i-1}}\right)$ respectively. In addition, by the semantic rules, the I/O sequence is appended at most by one value in one step. The lemma holds.

Corollary 5.5. Let $s_{1}=$ "while ${ }_{\left\langle n_{1}\right\rangle}(e) \quad\left\{S_{1}\right\}$ " and $s_{2}=$ "while ${ }_{\left\langle n_{2}\right\rangle}(e)\left\{S_{2}\right\}$ " be two while statements such that all of the followings hold

- There are output statements in $s_{1}$ and $s_{2}, \exists e_{1} e_{2}$ : ("output $e_{1}$ " $\in$ $\left.s_{1}\right) \wedge\left(\right.$ "output $e_{2}$ " $\left.\in s_{2}\right)$;

- $s_{1}$ and $s_{2}$ have same set of termination deciding variables and same set of imported variables relative to the $I / O$ sequence variable, $\left(T \operatorname{Var}\left(s_{1}\right)=T \operatorname{Var}\left(s_{2}\right)=T \operatorname{Var}(s)\right) \wedge$ $\left(\operatorname{Imp}\left(s_{1},\left\{i d_{I O}\right\}\right)=\operatorname{Imp}\left(s_{2},\left\{i d_{I O}\right\}\right)=\operatorname{Imp}(i o)\right)$;

- Loop bodies $S_{1}$ and $S_{2}$ satisfy the proof rule of equivalent computation of those in out-deciding variables of $s_{1}$ and $s_{2}$, $\forall x \in O \operatorname{Var}(s)=T \operatorname{Var}(s) \cup \operatorname{Imp}(i o): S_{1} \equiv_{x}^{S} S_{2}$;

- Loop bodies $S_{1}$ and $S_{2}$ satisfy the proof rule of termination in the same way, $S_{1} \equiv{ }_{H}^{S} S_{2}$; 
- Loop bodies $S_{1}$ and $S_{2}$ produce the same output sequence when started in states with crash flags not set and agreeing on values of variables in $O \operatorname{Var}\left(S_{1}\right) \cup \operatorname{OVar}\left(S_{2}\right), \forall m_{S_{1}}\left(\mathfrak{f}_{1}, \sigma_{S_{1}}\right) m_{S_{2}}\left(\mathfrak{f}_{2}\right.$, $\left.\sigma_{S_{2}}\right)$ :

$\left(\left(\mathfrak{f}_{1}=\mathfrak{f}_{2}=0\right) \wedge\left(\forall x \in O \operatorname{Var}\left(S_{1}\right) \cup \operatorname{OVar}\left(S_{2}\right): \sigma_{S_{1}}(x)=\right.\right.$ $\left.\left.\sigma_{S_{2}}(x)\right)\right) \Rightarrow\left(\left(S_{1}, m_{S_{1}}\left(\mathfrak{f}_{1}, \sigma_{S_{1}}\right)\right) \equiv_{O}\left(S_{2}, m_{S_{2}}\left(\mathfrak{f}_{2}, \sigma_{S_{2}}\right)\right)\right)$.

If $s_{1}$ and $s_{2}$ start in states $m_{1}\left(\mathfrak{f}_{1}\right.$, loop $\left._{c}^{1}, \sigma_{1}\right), m_{2}\left(\mathfrak{f}_{2}\right.$, loop $\left._{c}^{2}, \sigma_{2}\right)$ respectively with crash flags not set $\mathfrak{f}_{1}=\mathfrak{f}_{2}=0$ and in which $s_{1}$ and $s_{2}$ have not started execution ( $\operatorname{loop}_{c}^{1}\left(n_{1}\right)=\operatorname{loop}_{c}^{2}\left(n_{2}\right)=0$ ), value stores $\sigma_{1}$ and $\sigma_{2}$ agree on values of variables in OVar $(s)$, $\forall x \in O \operatorname{Var}(s): \sigma_{1}(x)=\sigma_{2}(x)$, then $s_{1}$ and $s_{2}$ produce the same output sequence: $\left(s_{1}, m_{1}\right) \equiv_{O}\left(s_{2}, m_{2}\right)$.

This is from lemma 5.25

\subsection{Backward compatible DSU based on program equivalence}

Based on the equivalence result above, we show that there exists backward compatible DSU. We need to show there exists a mapping of old program configurations and new program configurations and the hybrid execution obtained from the configuration mapping is backward compatible. We do not provide a practical algorithm to calculate the state mapping. Instead we only show that there exists new program configurations corresponding to some old program configurations via a simulation. The treatment in this section is informal.

The idea is to map a configuration just before an output is produced to a corresponding configuration. Based on the proof rule of same output sequences, not every statement of the old program can correspond to a statement of the new program, but every output statemet of the old program should correspond to an output statement of the new program. Consider configuration $C_{1}$ of the old program where the leftmost statement (next statement to execute) is an output statement. We can define a corresponding statement of the new program by simulating the execution of the new program on the input consumed so far in $C_{1}$. There are two cases. When the leftmost statement in $C_{1}$ is not included in a loop statement, then it is easy to know when to stop simulation. Otherwise, we have the bijection of loop statements including output statements based on the condition of same output sequences. Therefore, it is easy to know how many iterations of the loop statements including the output statement shall be carried out based on the loop counters in the old program configuration $C_{1}$. Based on Theorem 5 , there must be a configuration $C_{2}$ corresponding to $C_{1}$. Moreover, the executions starting from configurations $C_{1}$ and $C_{2}$ produce the same output sequence based on Theorem 5 In conclusion, we obtain a backward compatible hybrid execution where the state mapping is from $C_{1}$ to $C_{2}$.

\section{Real world backward compatible update classes: proof rules}

We propose our formal treatment for real world update classes. For each update class, we show how the old program and new program produce the same I/O sequence which guarantees backward compatible DSU.

\subsection{Proof rule for specializing new configuration variables}

New configuration variables can be introduced to generalize functionality. Figure 15 shows an example of how a new configuration variable introduces new code. The two statement sequences in Figure 15 are equivalent when the new variable $b$ is specialized to 0 .

Our generalized formal definition of "specializing new configuration variables" is defined as follows.

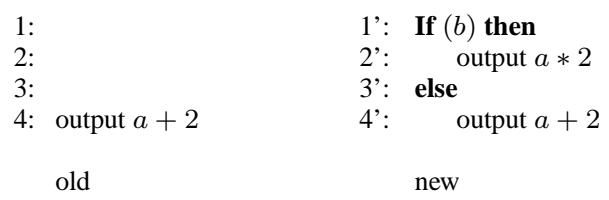

Figure 15: Specializing new configuration variables

Definition 25. (Specializing new configuration variables) $A$ statement sequence $S_{2}$ includes updates of specializing new configuration variables compared with $S_{1}$ w.r.t a mapping $\rho$ of new configuration variables in $S_{2}, \rho:\{i d\} \mapsto\{0,1\}$, denoted $S_{2} \approx_{\rho}^{S} S_{1}$, iff one of the following holds:

1. $S_{2}=$ "If $(i d)$ then $\left\{S_{2}^{t}\right\}$ else $\left\{S_{2}^{f}\right\} "$ where one of the following holds:

(a) $(\rho(i d)=0) \wedge\left(S_{2}^{f} \approx_{\rho}^{S} S_{1}\right)$;

(b) $(\rho(i d)=1) \wedge\left(S_{2}^{t} \approx_{\rho}^{S} S_{1}\right)$;

2. $S_{1}$ and $S_{2}$ produce the same output sequence, $S_{1} \approx_{O}^{S} S_{2}$;

3. $S_{1}=$ "If $(e)$ then $\left\{S_{1}^{t}\right\}$ else $\left\{S_{1}^{f}\right\} ", S_{2}=$ "If $(e) \operatorname{then}\left\{S_{2}^{t}\right\}$ else $\left\{S_{2}^{f}\right\} "$ where $\left(S_{2}^{t} \approx_{\rho}^{S} S_{1}^{t}\right) \wedge\left(S_{2}^{f} \approx_{\rho}^{S} S_{1}^{f}\right)$;

4. $S_{1}=$ "while $\left\langle n_{1}\right\rangle(e)\left\{S_{1}^{\prime}\right\} ", S_{2}=$ "while ${ }_{\left\langle n_{2}\right\rangle}(e)\left\{S_{2}^{\prime}\right\} "$ where $S_{2}^{\prime} \approx_{\rho}^{S} S_{1}^{\prime}$

5. $S_{1}=S_{1}^{\prime} ; s_{1}$ and $S_{2}=S_{2}^{\prime} ; s_{2}$ where $\left(S_{2}^{\prime} \approx_{\rho}^{S} S_{1}^{\prime}\right) \wedge\left(S_{2}^{\prime} \approx_{H}^{S}\right.$ $\left.S_{1}^{\prime}\right) \wedge\left(\forall x \in \operatorname{Imp}\left(s_{1}, i d_{I O}\right) \cup \operatorname{Imp}\left(s_{1}, i d_{I O}\right):\left(S_{2}^{\prime} \approx_{x}^{S}\right.\right.$ $\left.S_{1}^{\prime}\right) \wedge\left(s_{2} \approx_{\rho}^{S} s_{1}\right)$.

Then we show that executions of two statement sequences produce the same I/O sequence if there are updates of specializing new configuration variables between the two.

Lemma 6.1. Let $S_{1}$ and $S_{2}$ be two different statement sequences where there are updates of "specializing new configuration variables" in $S_{2}$ compared with $S_{1}$ w.r.t a mapping of new configuration variables $\rho, S_{2} \approx_{\rho}^{S} S_{1}$. If executions of $S_{2}$ and $S_{1}$ start in states $m_{2}\left(\mathfrak{f}_{2}, \sigma_{2}\right)$ and $m_{1}\left(\mathfrak{f}_{1}, \sigma_{1}\right)$ respectively where all of the following hold:

- Crash flags $\mathfrak{f}_{2}, \mathfrak{f}_{1}$ are not set, $\mathfrak{f}_{2}=\mathfrak{f}_{1}=0$;

- Value stores $\sigma_{1}$ and $\sigma_{2}$ agree on output deciding variables in both $S_{1}$ and $S_{2}$ including the input and I/O sequence variable, $\forall i d \in\left(O \operatorname{Var}\left(S_{1}\right) \cap \operatorname{OVar}\left(S_{2}\right)\right) \cup\left\{i d_{I}, i d_{I O}\right\}: \sigma_{1}(i d)=$ $\sigma_{2}(i d)$;

- Values of new configuration variables in the value store $\sigma_{2}$ are matching those in $\rho, \forall i d \in \operatorname{Dom}(\rho): \rho(i d)=\sigma_{2}(i d)$;

- Values of new configuration variables are not defined in the statement sequence $S_{2}, \operatorname{Dom}(\rho) \cap \operatorname{Def}\left(S_{2}\right)=\emptyset$;

then $S_{2}$ and $S_{1}$ satisfy all of the following:

- $\left(S_{1}, m_{1}\right) \equiv_{H}\left(S_{2}, m_{2}\right)$;

- $\left(S_{1}, m_{1}\right) \equiv_{O}\left(S_{2}, m_{2}\right)$;

- $\forall x \in\left\{i d_{I}, i d_{I O}\right\}:\left(S_{1}, m_{1}\right) \equiv_{x}\left(S_{2}, m_{2}\right)$;

Proof. The proof of Lemma 6.1 is by induction on the sum of program sizes of $S_{1}$ and $S_{2}$ and is a case analysis based on Definition 25

Base case.

$S_{1}$ is a simple statement $s, S_{2}=\operatorname{IIf}(i d)$ then $\left\{s_{2}^{t}\right\} \operatorname{else}\left\{s_{2}^{f}\right\} "$ where $s_{2}^{t}, s_{2}^{f}$ are simple statement and one of the following holds:

1. $(\rho(i d)=0) \wedge\left(s_{2}^{f}=s\right)$;

2. $(\rho(i d)=1) \wedge\left(s_{2}^{t}=s\right)$; 
W.1.o.g., we assume that $\rho(i d)=0$. By assumption, $\sigma_{2}(i d)=$ $\rho(i d)=0$. Then the execution of $S_{2}$ proceeds as follows:

$$
\begin{aligned}
& \left(\operatorname{If}(i d) \text { then }\left\{s_{2}^{t}\right\} \text { else }\left\{s_{2}^{f}\right\}, m_{2}\left(\sigma_{2}\right)\right) \\
\rightarrow & \left(\operatorname{If}(0) \text { then }\left\{s_{2}^{t}\right\} \operatorname{else}\left\{s_{2}^{f}\right\}, m_{2}\left(\sigma_{2}\right)\right) \\
& \text { by the rule Var } \\
\rightarrow & \left(s_{2}^{f}, m_{2}\left(\sigma_{2}\right)\right) \text { by the If-F rule. }
\end{aligned}
$$

By Theorem 5 and Theorem 4 this lemma holds. Induction step.

The induction hypothesis $(\mathrm{IH})$ is that Lemma 6.1 holds when the sum of the program size of $S_{1}$ and $S_{2}$ is at least $4, \operatorname{size}\left(S_{1}\right)+$ $\operatorname{size}\left(S_{2}\right)=k \geq 4$.

Then we show that the lemma holds when $\operatorname{size}\left(S_{1}\right)+\operatorname{size}\left(S_{2}\right)=$ $k+1$. There are cases to consider.

1. $S_{1}$ and $S_{2}$ satisfy the condition of same output sequence, $S_{1} \equiv \stackrel{S}{S} S_{2}$.

By Theorem 5 the lemma 6.1 holds.

2. $S_{1}$ and $S_{2}$ are both "If" statement:

$S_{1}=$ "If $(e)$ then $\left\{S_{1}^{t}\right\} \operatorname{else}\left\{S_{1}^{f}\right\} ", S_{2}=" \operatorname{If}(e) \operatorname{then}\left\{S_{2}^{t}\right\} \operatorname{else}\left\{S_{2}^{f}\right\} "$

where both of the following hold

- $S_{2}^{t} \approx_{\rho}^{S} S_{1}^{t}$;

- $S_{2}^{f} \approx_{\rho}^{S} S_{1}^{f}$;

By the definition of Use $\left(S_{1}\right)$, variables used in the predicate expression $e$ are a subset of used variables in $S_{1}$ and $S_{2}, \mathrm{Use}(e) \subseteq$ $\operatorname{Use}\left(S_{1}\right) \cap \operatorname{Use}\left(S_{2}\right)$. By assumption, corresponding variables used in $e$ are of same value in value stores $\sigma_{1}$ and $\sigma_{2}$. By Lemma D.1 the expression evaluates to the same value w.r.t value stores $\sigma_{1}$ and $\sigma_{2}$. There are three possibilities.

(a) The evaluation of $e$ crashes, $\mathcal{E}^{\prime} \llbracket e \rrbracket \sigma_{1}=\mathcal{E}^{\prime} \llbracket e \rrbracket \sigma_{2}=$ (error, $\left.v_{\mathfrak{o f}}\right)$.

The execution of $S_{1}$ continues as follows:

(If $(e)$ then $\left\{S_{1}^{t}\right\}$ else $\left.\left\{S_{1}^{f}\right\}, m_{1}\left(\sigma_{1}\right)\right)$

$\rightarrow\left(\operatorname{If}\left(\left(\right.\right.\right.$ error, $\left.\left.v_{\text {of }}\right)\right)$ then $\left\{S_{1}^{t}\right\}$ else $\left.\left\{S_{1}^{f}\right\}, m_{1}\left(\sigma_{1}\right)\right)$

by the rule EEval'

$\rightarrow\left(\operatorname{If}(0)\right.$ then $\left\{S_{1}^{t}\right\}$ else $\left.\left\{S_{1}^{f}\right\}, m_{1}(1 / \mathfrak{f})\right)$

by the ECrash rule

$\stackrel{i}{\rightarrow}\left(\operatorname{If}(0)\right.$ then $\left\{S_{1}^{t}\right\}$ else $\left.\left\{S_{1}^{f}\right\}, m_{1}(1 / \mathfrak{f})\right)$ for any $i>0$

by the Crash rule.

Similarly, the execution of $S_{2}$ started from the state $m_{2}\left(\sigma_{2}\right)$ crashes. The lemma holds.

(b) The evaluation of $e$ reduces to zero, $\mathcal{E}^{\prime} \llbracket e \rrbracket \sigma_{1}=\mathcal{E}^{\prime} \llbracket e \rrbracket \sigma_{2}=$ $\left(0, v_{\mathfrak{o f}}\right)$.

The execution of $S_{1}$ continues as follows.

$\left(\operatorname{If}(e)\right.$ then $\left.\left\{S_{1}^{t}\right\} \operatorname{else}\left\{S_{1}^{f}\right\}, m_{1}\left(\sigma_{1}\right)\right)$

$=\left(\operatorname{If}\left(\left(0, v_{\text {of }}\right)\right)\right.$ then $\left.\left\{S_{1}^{t}\right\} \operatorname{else}\left\{S_{1}^{f}\right\}, m_{1}\left(\sigma_{1}\right)\right)$ by the rule EEval'

$\rightarrow\left(\operatorname{If}(0)\right.$ then $\left.\left\{S_{1}^{t}\right\} \operatorname{else}\left\{S_{1}^{f}\right\}, m_{1}\left(\sigma_{1}\right)\right)$

by the E-Oflow1 or E-Oflow2 rule

$\rightarrow\left(S_{1}^{f}, m_{1}\left(\sigma_{1}\right)\right)$ by the If-F rule.

Similarly, the execution of $S_{2}$ gets to the configuration $\left(S_{2}^{f}, m_{2}\left(\sigma_{2}\right)\right)$.

By the hypothesis IH, we show the lemma holds. We need to show that all conditions are satisfied for the application of the hypothesis IH.

- $\left(S_{2}^{f} \approx_{\rho}^{S} S_{1}^{f}\right)$ By assumption.

- The sum of the program size of $S_{1}^{f}$ and $S_{2}^{f}$ is less than $k, \operatorname{size}\left(S_{1}^{f}\right)+\operatorname{size}\left(S_{2}^{f}\right)<k$.

By definition, $\operatorname{size}\left(S_{1}\right)=1+\operatorname{size}\left(S_{1}^{t}\right)+\operatorname{size}\left(S_{1}^{f}\right)$. Then, $\operatorname{size}\left(S_{1}^{f}\right)+\operatorname{size}\left(S_{2}^{f}\right)<k+1-2=k-1$.
- Value stores $\sigma_{1}$ and $\sigma_{2}$ agree on values of used variables in $S_{1}^{f}$ and $S_{2}^{f}$ as well as the input, I/O sequence variable. By definition, $\operatorname{Use}\left(S_{1}^{f}\right) \subseteq \operatorname{Use}\left(S_{1}\right)$. So are the cases to $S_{2}^{f}$ and $S_{2}$. In addition, value stores $\sigma_{1}$ and $\sigma_{2}$ are not changed in the evaluation of the predicate expression $e$. The condition holds.

- Values of new configuration variables are consistent in the value store $\sigma_{2}$ and the specialization $\rho, \forall i d \in$ $\operatorname{Dom}(\rho): \sigma_{2}(i d)=\rho(i d)$.

By assumption.

By the hypothesis $\mathrm{IH}$, the lemma holds.

(c) The evaluation of $e$ reduces to the same nonzero integer value, $\mathcal{E}^{\prime} \llbracket e \rrbracket \sigma_{1}=\mathcal{E}^{\prime} \llbracket e \rrbracket \sigma_{2}=\left(v, v_{\text {of }}\right)$ where $v \neq 0$.

By arguments similar to the second subcase above.

3. $S_{1}$ and $S_{2}$ are both "while" statements:

$S_{1}=$ "while $\langle n\rangle(e)\left\{S_{1}^{\prime}\right\} ", S_{2}=$ "while ${ }_{\langle n\rangle}(e)\left\{S_{2}^{\prime}\right\} "$ where $S_{2}^{\prime} \approx_{\rho}^{S} S_{1}^{\prime}$

By Lemma 6.3 we show this lemma holds. We need to show that all required conditions are satisfied for the application of Lemma 6.3

- $S_{1}$ and $S_{2}$ have same set of output deciding variables, $\operatorname{OVar}\left(S_{1}\right)=\mathrm{OVar}\left(S_{2}\right)=\mathrm{OVar}(S)$;

By Lemma 6.2 and Corollary 5.1

- When started in states $m_{1}^{\prime}\left(\sigma_{1}^{\prime}\right), m_{2}^{\prime}\left(\sigma_{1}^{\prime}\right)$ where value stores $\sigma_{1}^{\prime}$ and $\sigma_{2}^{\prime}$ agree on values of output deciding variables in both $S_{1}$ and $S_{2}$ as well as the input sequence variable and the I/O sequence variable, then $S_{1}^{\prime}$ and $S_{2}^{\prime}$ terminate in the same way, produce the same output sequence, and have equivalent computation of defined variables in both $S_{1}$ and $S_{2}$.

By the induction hypothesis IH. This is because the sum of the program size of $S_{1}^{\prime}$ and $S_{2}^{\prime}$ is less than $k$. By definition, $\operatorname{size}\left(S_{1}\right)=1+\operatorname{size}\left(S_{1}^{\prime}\right)$.

By Lemma 6.3 this lemma holds.

4. $S_{2}=$ "If $(i d)$ then $\left\{S_{2}^{t}\right\}$ else $\left\{S_{2}^{f}\right\}$ " where one of the following holds:

(a) $(\rho(i d)=0) \wedge\left(S_{2}^{f} \approx_{\rho}^{S} S_{1}\right)$;

(b) $(\rho(i d)=1) \wedge\left(S_{2}^{t} \approx_{\rho}^{S} S_{1}\right)$;

W.1.o.g, we assume $(\rho(i d)=0) \wedge\left(S_{2}^{f} \approx_{\rho}^{S} S_{1}\right)$;

Then the execution of $S_{2}$ proceeds as follows:

$$
\begin{aligned}
& \left(\operatorname{If}(i d) \text { then }\left\{S_{2}^{t}\right\} \text { else }\left\{S_{2}^{f}\right\}, m_{2}\left(\sigma_{2}\right)\right) \\
= & \left(\operatorname{If}(0) \text { then }\left\{S_{2}^{t}\right\} \operatorname{else}\left\{S_{2}^{f}\right\}, m_{2}\left(\sigma_{2}\right)\right) \\
& \text { by the Var rule } \\
\rightarrow & \left(S_{2}^{f}, m_{2}\left(\sigma_{2}\right)\right) \\
& \text { by the If-F rule }
\end{aligned}
$$

By the induction hypothesis, we show that the lemma holds. We need to show the required conditions are satisfied for the application of the hypothesis.

- $S_{2}^{f} \approx_{\rho}^{S} S_{1}$

By assumption.

- The sum of the program size of $S_{1}^{f}$ and $S_{2}^{f}$ is less than $k$, $\operatorname{size}\left(S_{1}^{f}\right)+\operatorname{size}\left(S_{2}^{f}\right)<k$.

By definition, $\operatorname{size}\left(S_{2}\right)=1+\operatorname{size}\left(S_{2}^{t}\right)+\operatorname{size}\left(S_{2}^{f}\right)$. Then, $\operatorname{size}\left(S_{2}^{f}\right)+\operatorname{size}\left(S_{1}\right)<k+1-1-\operatorname{size}\left(S_{2}^{t}\right)<k$.

- Value stores $\sigma_{1}$ and $\sigma_{2}$ agree on values of used variables in $S_{2}^{f}$ and $S_{1}$ as well as the input, I/O sequence variable.

By definition, Use $\left(S_{2}^{f}\right) \subseteq \operatorname{Use}\left(S_{2}\right)$. In addition, the value store $\sigma_{2}$ is not changed in the evaluation of the predicate expression $e$. The condition holds.

By the hypothesis IH, the lemma holds. 
5. $S_{1}$ and $S_{2}$ are same, $S_{1}=S_{2}$

By definition, used variables in $S_{1}$ and $S_{2}$ are same; defined variables in $S_{1}$ and $S_{2}$ are same. By semantic rules, $S_{1}$ and $S_{2}$ terminate in the same way, produce the same output sequence and have equivalent computation of defined variables in $S_{1}$ and $S_{2}$. This lemma holds.

6. $S_{1}=S_{1}^{\prime} ; s_{1}$ and $S_{2}=S_{2}^{\prime} ; s_{2}$ where both of the following hold:

- $S_{2}^{\prime} \approx_{\rho}^{S} S_{1}^{\prime}$;

- $s_{2} \approx_{\rho}^{S} s_{1}$;

By Theorem 4 and the hypothesis $\mathrm{IH}$, we show $S_{2}^{\prime}$ and $S_{1}^{\prime}$ terminate in the same way and produce the same output sequence and when $S_{2}^{\prime}$ and $S_{1}^{\prime}$ both terminate, $S_{2}^{\prime}$ and $S_{1}^{\prime}$ have equivalent terminating computation of variables used or defined in $S_{2}^{\prime}$ and $S_{1}^{\prime}$.

We show all the required conditions are satisfied for the application of the hypothesis IH.

- $S_{2}^{\prime} \approx_{\rho}^{S} S_{1}^{\prime}$.

By assumption.

- The sum of the program size of $S_{1}^{\prime}$ and $S_{2}^{\prime}$ is less than $k$, $\operatorname{size}\left(S_{1}^{\prime}\right)+\operatorname{size}\left(S_{2}^{\prime}\right)<k$.

By definition, $\operatorname{size}\left(S_{2}\right)=\operatorname{size}\left(s_{2}\right)+\operatorname{size}\left(S_{2}^{\prime}\right)$ where $\operatorname{size}\left(s_{2}\right)<1$. Then, $\operatorname{size}\left(S_{2}^{\prime}\right)+\operatorname{size}\left(S_{1}^{\prime}\right)<k+1-$ $\operatorname{size}\left(s_{2}\right)-\operatorname{size}\left(s_{1}\right)<k$.

- Value stores $\sigma_{1}$ and $\sigma_{2}$ agree on values of output deciding variables in $S_{2}^{\prime}$ and $S_{1}^{\prime}$ including the input, I/O sequence variable.

By definition of $\mathrm{TVar}_{o}$ and $\operatorname{Imp}{ }_{o}, \operatorname{OVar}\left(S_{2}^{\prime}\right) \subseteq \operatorname{OVar}\left(S_{2}\right)$. The condition holds.

- Values of new configuration variables are consistent in the value store $\sigma_{2}$ and the specialization $\rho, \forall i d \in \operatorname{Dom}(\rho)$ : $\sigma_{2}(i d)=\rho(i d)$.

By assumption.

By the hypothesis IH, one of the following holds:

(a) $S_{1}^{\prime}$ and $S_{2}^{\prime}$ both do not terminate.

By Lemma E.2. executions of $S_{1}=S_{1}^{\prime} ; s_{1}$ and $S_{2}=$ $S_{2}^{\prime} ; s_{2}$ both do not terminate and produce the same output sequence.

(b) $S_{1}^{\prime}$ and $S_{2}^{\prime}$ both terminate.

By assumption, $\left(S_{2}^{\prime}, m_{2}\left(\sigma_{2}\right)\right) \stackrel{*}{\rightarrow}\left(\right.$ skip, $\left.m_{2}^{\prime}\left(\sigma_{2}^{\prime}\right)\right)$,

$\left(S_{1}^{\prime}, m_{1}\left(\sigma_{1}\right)\right) \stackrel{*}{\rightarrow}$ (skip, $\left.m_{1}^{\prime}\left(\sigma_{1}^{\prime}\right)\right)$.

By Corollary E.1 $\left(S_{2}^{\prime} ; s_{2}, m_{2}\left(\sigma_{2}\right)\right) \stackrel{*}{\rightarrow}\left(s_{2}, m_{2}^{\prime}\left(\sigma_{2}^{\prime}\right)\right)$, $\left(S_{1}^{\prime} ; s_{1}, m_{1}\left(\sigma_{1}\right)\right) \stackrel{*}{\rightarrow}\left(s_{1}, m_{1}^{\prime}\left(\sigma_{1}^{\prime}\right)\right)$.

By the hypothesis $\mathrm{IH}$, we show that $s_{2}$ and $s_{1}$ terminate in the same way, produce the same output sequence and when $s_{2}$ and $s_{1}$ both terminate, $s_{2}$ and $s_{1}$ have equivalent computation of variables used or defined in $s_{1}$ and $s_{2}$ and the input, and I/O sequence variables.

We need to show that all conditions are satisfied for the application of the hypothesis IH.

- There are updates of "new configuration variables" between $s_{2}$ and $s_{1}$;

By assumption, $s_{2} \approx_{\rho}^{S} s_{1}$.

- The sum of the program size $s_{2}$ and $s_{1}$ is less than or equals to $k$;

By definition, $\operatorname{size}\left(S_{2}^{\prime}\right) \geq 1, \operatorname{size}\left(S_{1}^{\prime}\right) \geq 1$. Therefore, $\operatorname{size}\left(s_{2}\right)+\operatorname{size}\left(s_{1}\right)<k+1-\operatorname{size}\left(S_{2}^{\prime}\right)-\operatorname{size}\left(S_{1}^{\prime}\right) \leq k$.

- Value stores $\sigma_{1}^{\prime}$ and $\sigma_{2}^{\prime}$ agree on values of output deciding variables in $s_{2}$ and $s_{1}$ as well as the input, I/O sequence variable.

By induction hypothesis IH, OVar $\left(s_{1}\right) \subseteq \operatorname{OVar}\left(s_{2}\right)$, then $\operatorname{Use}\left(s_{2}\right) \cap \operatorname{Use}\left(s_{1}\right)=\operatorname{Use}\left(s_{1}\right)$. For any variable $i d$ in $\operatorname{OVar}\left(s_{1}\right)$, if $i d$ is in $\operatorname{OVar}\left(S_{1}^{\prime}\right)$, then the value of $i d$ is same after the execution of $S_{1}^{\prime}$ and $S_{2}^{\prime}, \sigma_{1}^{\prime}(i d)=$ $\sigma_{1}(i d)=\sigma_{2}(i d)=\sigma_{2}^{\prime}(i d)$. Otherwise, the variable $i d$ is defined in the execution of $S_{1}^{\prime}$ and $S_{2}^{\prime}$, by assumption, $\sigma_{1}^{\prime}(i d)=\sigma_{2}^{\prime}(i d)$. The condition holds.

- Values of new configuration variables are consistent in the value store $\sigma_{2}^{\prime}$ and the specialization $\rho, \forall i d \in$ $\operatorname{Dom}(\rho): \sigma_{2}^{\prime}(i d)=\rho(i d)$.

By assumption, $\operatorname{Dom}(\rho) \cap \operatorname{Def}\left(S_{2}\right)$. By Corollary E.2 values of new configuration variables are not changed in the execution of $S_{2}^{\prime}, \forall i d \in \operatorname{Dom}(\rho): \sigma_{2}^{\prime}(i d)=$ $\sigma_{2}(i d)=\rho(i d)$.

By the hypothesis $\mathrm{IH}$, the lemma holds.

We list properties of the update of new configuration variables and the proof of backward compatibility for the case of loop statement as follows. We present one auxiliary lemma used in the proof of Lemma6.1

Lemma 6.2. Let $S_{2}$ be a statement sequence and $S_{1}$ where there are updates of "specializing new configuration variables" w.r.t a mapping of new configuration variables $\rho, S_{2} \approx{ }_{\rho}^{S} S_{1}$. Then the output deciding variables in $S_{1}$ are a subset of the union of those in $S_{2}, O \operatorname{Var}\left(S_{1}\right) \subseteq O \operatorname{Var}\left(S_{2}\right)$.

Proof. By induction on the sum of the program size of $S_{1}$ and $S_{2}$.

Lemma 6.3. Let $S_{1}=$ while $_{\left\langle n_{1}\right\rangle}(e)\left\{S_{1}^{\prime}\right\}$ and $S_{2}=$ while $_{\left\langle n_{2}\right\rangle}(e)$ $\left\{S_{2}^{\prime}\right\}$ be two loop statements where all of the following hold:

- $S_{2}^{\prime}$ includes updates of "specializing new configuration variables" compared to $S_{1}^{\prime}, S_{2}^{\prime} \approx_{\rho}^{S} S_{1}^{\prime}$ where $\operatorname{Dom}(\rho) \cap \operatorname{Def}\left(S_{2}^{\prime}\right)=$ $\emptyset$.

- the output deciding variables in $S_{1}$ are a subset of those in $S_{2}$, $O \operatorname{Var}\left(S_{1}\right) \subseteq O \operatorname{Var}\left(S_{2}\right)$;

- When started in states agreeing on values of output deciding variables in $S_{1}$ and $S_{2}$ including the input sequence variable and the I/O sequence variable, $\forall x \in \operatorname{OVar}\left(S_{1}\right) \cup \operatorname{OVar}\left(S_{2}\right) \cup$ $\left\{i d_{I}, i d_{I O}\right\} \forall m_{1}^{\prime}\left(\sigma_{1}^{\prime}\right) m_{2}^{\prime}\left(\sigma_{2}^{\prime}\right):\left(\sigma_{1}^{\prime}(x)=\sigma_{2}^{\prime}(x)\right), S_{1}^{\prime}$ and $S_{2}^{\prime}$ terminate in the same way, produce the same output sequence, and have equivalent computation of defined variables in $S_{1}^{\prime}$ and $S_{2}^{\prime}$ as well as the input sequence variable and the I/O sequence variable $\left(\left(S_{1}^{\prime}, m_{1}\right) \equiv_{H}\left(S_{2}^{\prime}, m_{2}\right)\right) \wedge\left(\left(S_{1}^{\prime}, m_{1}\right) \equiv_{O}\right.$ $\left.\left(S_{2}^{\prime}, m_{2}\right)\right) \wedge\left(\forall x \in O \operatorname{Var}\left(S_{1}\right) \cup \operatorname{OVar}\left(S_{2}\right) \cup\left\{i d_{I}, i d_{I O}\right\} \quad:\right.$ $\left.\left(S_{1}^{\prime}, m_{1}\right) \equiv_{x}\left(S_{2}^{\prime}, m_{2}\right)\right)$;

If $S_{1}$ and $S_{2}$ start in states $m_{1}\left(\right.$ loop $\left._{c}^{1}, \sigma_{1}\right), m_{2}\left(\right.$ loop $\left._{c}^{2}, \sigma_{2}\right)$ respectively, with loop counters of $S_{1}$ and $S_{2}$ not initialized $\left(S_{1}, S_{2}\right.$ have not executed yet), value stores agree on values of output deciding variables in $S_{1}$ and $S_{2}$, then, for any positive integer $i$, one of the following holds:

1. Loop counters for $S_{1}$ and $S_{2}$ are always less than $i$ if any is present, $\forall m_{1}^{\prime}\left(\operatorname{loop}_{c}^{1^{\prime}}\right) m_{2}^{\prime}\left(\operatorname{loop}_{c}^{2^{\prime}}\right):\left(S_{1}, m_{1}\left(\operatorname{loop}_{c}^{1}, \sigma_{1}\right)\right) \stackrel{*}{\rightarrow}$ $\left(S_{1}^{\prime \prime}, m_{1}^{\prime}\left(\right.\right.$ loop $\left.\left._{c}^{1^{\prime}}\right)\right), \operatorname{loop}_{c}^{1^{\prime}}\left(n_{1}\right)<i,\left(S_{2}, m_{2}\left(\right.\right.$ loop $\left.\left._{c}^{2}, \sigma_{2}\right)\right) \stackrel{*}{\rightarrow}$ $\left(S_{2}^{\prime \prime}, m_{2}^{\prime}\left(\right.\right.$ loop $\left.\left._{c}^{2^{\prime}}\right)\right)$, loop lo $_{c}^{\prime}\left(n_{2}\right)<i, S_{1}$ and $S_{2}$ terminate in the same way, produce the same output sequence, and have equivalent computation of output deciding variables in both $S_{1}$ and $S_{2}$ and the input sequence variable, the I/O sequence variable, $\left(S_{1}, m_{1}\right) \equiv_{H}\left(S_{2}, m_{2}\right)$ and $\left(S_{1}, m_{1}\right) \equiv_{O}\left(S_{2}, m_{2}\right)$ and $\forall x \in\left(O \operatorname{Var}\left(S_{1}\right) \cap \operatorname{OVar}\left(S_{2}\right)\right) \cup\left\{i d_{I}, i d_{I O}\right\}:\left(S_{1}, m_{1}\right) \equiv_{x}$ $\left(S_{2}, m_{2}\right)$;

2. The loop counter of $S_{1}$ and $S_{2}$ are of value less than or equal to $i$, and there are no reachable configurations $\left(S_{1}, m_{1}\right.$ (loop ${ }_{c}^{1_{i}}$, $\left.\left.\sigma_{1_{i}}\right)\right)$ from $\left(S_{1}, m_{1}\left(\sigma_{1}\right)\right)$, $\left(S_{2}, m_{2}\left(\operatorname{loop}_{c}^{2}, \sigma_{2_{i}}\right)\right)$ from $\left(S_{2}\right.$, $\left.m_{2}\left(\sigma_{2}\right)\right)$ where all of the following hold: 
- The loop counters of $S_{1}$ and $S_{2}$ are of value $i$, loop ${ }_{c}^{1_{i}}\left(n_{1}\right)$ $=\operatorname{loop}_{c}^{2_{i}}\left(n_{2}\right)=i$.

- Value stores $\sigma_{1_{i}}$ and $\sigma_{2_{i}}$ agree on values of output deciding variables in both $S_{1}$ and $S_{2}$ as well as the input sequence variable and the I/O sequence variable, $\forall x \in\left(\operatorname{OVar}\left(S_{1}\right) \cap\right.$ $\left.O \operatorname{Var}\left(S_{2}\right)\right) \cup\left\{i d_{I}, i d_{I O}\right\}: \sigma_{1_{i}}(x)=\sigma_{2_{i}}(x)$.

3. There are reachable configurations $\left(S_{1}, m_{1}\left(\right.\right.$ loop $\left.\left._{c}^{1_{i}}, \sigma_{1_{i}}\right)\right)$ from $\left(S_{1}, m_{1}\left(\sigma_{1}\right)\right),\left(S_{2}, m_{2}\left(\operatorname{loop}_{c}^{2_{i}}, \sigma_{2_{i}}\right)\right)$ from $\left(S_{2}, m_{2}\left(\sigma_{2}\right)\right)$ where all of the following hold:

- The loop counter of $S_{1}$ and $S_{2}$ are of value $i, \operatorname{loop}_{c}^{1_{i}}\left(n_{1}\right)$ $=\operatorname{loop}_{c}^{2_{i}}\left(n_{2}\right)=i$.

- Value stores $\sigma_{1_{i}}$ and $\sigma_{2_{i}}$ agree on values of output deciding variables in both $S_{1}$ and $S_{2}$ including the input sequence variable and the I/O sequence variable, $\forall x \in\left(O \operatorname{Var}\left(S_{1}\right) \cap\right.$ $\left.O \operatorname{Var}\left(S_{2}\right)\right) \cup\left\{i d_{I}, i d_{I O}\right\}: \sigma_{1_{i}}(x)=\sigma_{2_{i}}(x)$.

Proof. By induction on $i$.

Base case.

We show that, when $i=1$, one of the following holds:

1. Loop counters for $S_{1}$ and $S_{2}$ are always less than 1 if any is present, $\forall m_{1}^{\prime}\left(\operatorname{loop}_{c}^{1^{\prime}}\right) m_{2}^{\prime}\left(\operatorname{loop}_{c}^{2^{\prime}}\right):\left(S_{1}, m_{1}\left(\operatorname{loop}_{c}^{1}, \sigma_{1}\right)\right) \stackrel{*}{\rightarrow}$ $\left(S_{1}^{\prime \prime}, m_{1}^{\prime}\left(\operatorname{loop}_{c}^{1^{\prime}}\right)\right), \operatorname{loop}_{c}^{1^{\prime}}\left(n_{1}\right)<i,\left(S_{2}, m_{2}\left(\operatorname{loop}_{c}^{2}, \sigma_{2}\right)\right) \stackrel{*}{\rightarrow}$ $\left(S_{2}^{\prime \prime}, m_{2}^{\prime}\left(\operatorname{loop}_{c}^{2^{\prime}}\right)\right), \operatorname{loop}_{c}^{2^{\prime}}\left(n_{2}\right)<i, S_{1}$ and $S_{2}$ terminate in the same way, produce the same output sequence, and have equivalent computation of used/defined variables in both $S_{1}$ and $S_{2}$ and the input sequence variable, the I/O sequence variable, $\left(S_{1}, m_{1}\right) \equiv_{H}\left(S_{2}, m_{2}\right)$ and $\left(S_{1}, m_{1}\right) \equiv_{O}\left(S_{2}, m_{2}\right)$ and $\forall x \in\left(\mathrm{OVar}\left(S_{1}\right) \cap \operatorname{OVar}\left(S_{2}\right)\right) \cup\left\{i d_{I}, i d_{I O}\right\}:\left(S_{1}, m_{1}\right) \equiv_{x}$ $\left(S_{2}, m_{2}\right)$;

2. Loop counters of $S_{1}$ and $S_{2}$ are of value less than or equal to 1 but there are no reachable configurations $\left(S_{1}, m_{1}\left(\operatorname{loop}_{c}^{1_{1}}, \sigma_{1_{i}}\right)\right)$ from $\left(S_{1}, m_{1}\left(\sigma_{1}\right)\right),\left(S_{2}, m_{2}\left(\operatorname{loop}_{c}^{21}, \sigma_{2_{i}}\right)\right)$ from $\left(S_{2}, m_{2}\left(\sigma_{2}\right)\right)$ where all of the following hold:

- The loop counter of $S_{1}$ and $S_{2}$ are of value $1, \operatorname{loop}_{c}^{1_{1}}\left(n_{1}\right)$ $=\operatorname{loop}_{c}^{2_{1}}\left(n_{2}\right)=1$.

- Value stores $\sigma_{1_{1}}$ and $\sigma_{2_{1}}$ agree on values of used variables in both $S_{1}$ and $S_{2}$ as well as the input sequence variable and the I/O sequence variable, $\forall x \in\left(\operatorname{OVar}\left(S_{1}\right) \cap \operatorname{OVar}\left(S_{2}\right)\right) \cup$ $\left\{i d_{I}, i d_{I O}\right\}: \sigma_{1_{1}}(x)=\sigma_{2_{1}}(x)$.

3. There are reachable configuration $\left(S_{1}, m_{1}\left(\operatorname{loop}_{c}^{1_{1}}, \sigma_{1_{i}}\right)\right)$ from $\left(S_{1}, m_{1}\left(\sigma_{1}\right)\right),\left(S_{2}, m_{2}\left(\operatorname{loop}_{c}^{21}, \sigma_{2_{i}}\right)\right)$ from $\left(S_{2}, m_{2}\left(\sigma_{2}\right)\right)$ where all of the following hold:

- The loop counter of $S_{1}$ and $S_{2}$ are of value $1, \operatorname{loop}_{c}^{1_{1}}\left(n_{1}\right)$ $=\operatorname{loop}_{c}^{2_{1}}\left(n_{2}\right)=1$.

- Value stores $\sigma_{1_{1}}$ and $\sigma_{21}$ agree on values of used variables in both $S_{1}$ and $S_{2}$ as well as the input sequence variable and the I/O sequence variable, $\forall x \in\left(\operatorname{OVar}\left(S_{1}\right) \cap \operatorname{OVar}\left(S_{2}\right)\right) \cup$ $\left\{i d_{I}, i d_{I O}\right\}: \sigma_{1_{1}}(x)=\sigma_{2_{1}}(x)$.

By definition, variables used in the predicate expression $e$ of $S_{1}$ and $S_{2}$ are used in $S_{1}$ and $S_{2}$, Use $(e) \subseteq \operatorname{OVar}\left(S_{1}\right) \cap \operatorname{OVar}\left(S_{2}\right)$. By assumption, value stores $\sigma_{1}$ and $\sigma_{2}$ agree on values of variables in Use $(e)$, the predicate expression $e$ evaluates to the same value w.r.t value stores $\sigma_{1}$ and $\sigma_{2}$. There are three possibilities.

1. The evaluation of $e$ crashes,

$\mathcal{E}^{\prime} \llbracket e \rrbracket \sigma_{1}=\mathcal{E}^{\prime} \llbracket e \rrbracket \sigma_{2}=$ (error, $\left.v_{\mathfrak{o f}}\right)$.

The execution of $S_{1}$ continues as follows:

$\left(\right.$ while $\left._{\left\langle n_{1}\right\rangle}(e)\left\{S_{1}^{\prime}\right\}, m_{1}\left(\sigma_{1}\right)\right)$

$\rightarrow\left(\right.$ while $\left._{\left\langle n_{1}\right\rangle}\right\rangle\left(\right.$ error, $\left.\left.\left.v_{\mathfrak{o f}}\right)\right)\left\{S_{1}^{\prime}\right\}, m_{1}\left(\sigma_{1}\right)\right)$

by the rule EEval'

$\rightarrow\left(\right.$ while $\left._{\left\langle n_{1}\right\rangle}(0)\left\{S_{1}^{\prime}\right\}, m_{1}(1 / \mathfrak{f})\right)$

by the ECrash rule $\stackrel{i}{\rightarrow}\left(\right.$ while $\left._{\left\langle n_{1}\right\rangle}(0)\left\{S_{1}^{\prime}\right\}, m_{1}(1 / \mathfrak{f})\right)$ for any $i>0$ by the Crash rule.

Similarly, the execution of $S_{2}$ started from the state $m_{2}\left(\sigma_{2}\right)$ crashes. Therefore $S_{1}$ and $S_{2}$ terminate in the same way when started from $m_{1}$ and $m_{2}$ respectively. Because $\sigma_{1}\left(i d_{I O}\right)=$ $\sigma_{2}\left(i d_{I O}\right)$, the lemma holds.

2. The evaluation of $e$ reduces to zero, $\mathcal{E}^{\prime} \llbracket e \rrbracket \sigma_{1}=\mathcal{E}^{\prime} \llbracket e \rrbracket \sigma_{2}=$ $\left(0, v_{\mathfrak{o f}}\right)$.

The execution of $S_{1}$ continues as follows.

$$
\begin{aligned}
& \left(\text { while }_{\left\langle n_{1}\right\rangle}(e)\left\{S_{1}^{\prime}\right\}, m_{1}\left(\sigma_{1}\right)\right) \\
= & (\text { while } \\
\left\langle n_{1}\right\rangle & \left.\left(\left(0, v_{\text {of }}\right)\right)\left\{S_{1}^{\prime}\right\}, m_{1}\left(\sigma_{1}\right)\right) \\
& \text { by the rule EEval' } \\
\rightarrow & (\text { while } \\
& \text { by the E-Oflow } 1 \text { or E-Oflow } 2 \text { rule } \\
\rightarrow & \text { (skip, } \left.m_{1}\left(\sigma_{1}\right)\right) \text { by the Wh-F rule. }
\end{aligned}
$$

Similarly, the execution of $S_{2}$ gets to the configuration (skip, $m_{2}\left(\sigma_{2}\right)$ ). Loop counters of $S_{1}$ and $S_{2}$ are less than 1 and value stores agree on values of used/defined variables in both $S_{1}$ and $S_{2}$ as well as the input sequence variable and the I/O sequence variable.

3. The evaluation of $e$ reduces to the same nonzero integer value, $\mathcal{E}^{\prime} \llbracket e \rrbracket \sigma_{1}=\mathcal{E}^{\prime} \llbracket e \rrbracket \sigma_{2}=\left(0, v_{\text {of }}\right)$.

Then the execution of $S_{1}$ proceeds as follows:

$$
\begin{aligned}
& \left(\text { while }_{\left\langle n_{1}\right\rangle}(e)\left\{S_{1}^{\prime}\right\}, m_{1}\left(\sigma_{1}\right)\right) \\
& =\left(\text { while }_{\left\langle n_{1}\right\rangle}\left(\left(v, v_{\mathfrak{o f}}\right)\right)\left\{S_{1}^{\prime}\right\}, m_{1}\left(\sigma_{1}\right)\right) \\
& \text { by the rule EEval' } \\
& \rightarrow\left(\text { while }_{\left\langle n_{1}\right\rangle}(v)\left\{S_{1}^{\prime}\right\}, m_{1}\left(\sigma_{1}\right)\right) \\
& \text { by the E-Oflow1 or E-Oflow2 rule } \\
& \rightarrow\left(S_{1}^{\prime} ; \text { while }_{\left\langle n_{1}\right\rangle}(e)\left\{S_{1}^{\prime}\right\}, m_{1}(\right. \\
& \text { loop } \left.\left._{c}^{1} \cup\left\{\left(n_{1}\right) \mapsto 1\right\}, \sigma_{1}\right)\right) \text { by the Wh-T rule. }
\end{aligned}
$$

Similarly, the execution of $S_{2}$ proceeds to the configuration $\left(S_{2}^{\prime}\right.$; while $\left\langle n_{2}\right\rangle(e)\left\{S_{2}^{\prime}\right\}, m_{2}\left(\right.$ loop $\left.\left._{c}^{2} \cup\left\{n_{2} \mapsto 1\right\}, \sigma_{2}\right)\right)$.

By the hypothesis IH, we show that $S_{1}^{\prime}$ and $S_{2}^{\prime}$ terminate in the same way and produce the same output sequence when started in the state $m_{1}\left(\operatorname{loop}_{c}^{1_{1}}, \sigma_{1}\right)$ and $m_{2}\left(\operatorname{loop}_{c}^{2_{1}}, \sigma_{2}\right)$, and $S_{1}^{\prime}$ and $S_{2}^{\prime}$ have equivalent computation of variables used or defined in both statement sequences if both terminate. We need to show that all conditions are satisfied for the application of the hypothesis IH.

- variables in the domain of $\rho$ are not redefined in the execution of $S_{2}^{\prime}$.

The above three conditions are by assumption.

By definition, $\operatorname{size}\left(S_{1}\right)=1+\operatorname{size}\left(S_{1}^{\prime}\right)$. Then, $\operatorname{size}\left(S_{1}^{\prime}\right)+$ $\operatorname{size}\left(S_{2}^{\prime}\right)=k+1-2=k-1$.

- Value stores $\sigma_{1}$ and $\sigma_{2}$ agree on values of used variables in $S_{1}^{\prime}$ and $S_{2}^{\prime}$ as well as the input, I/O sequence variable.

By definition, $\operatorname{OVar}\left(S_{1}^{\prime}\right) \subseteq \operatorname{OVar}\left(S_{1}\right)$. So are the cases to $S_{2}^{\prime}$ and $S_{2}$. In addition, value stores $\sigma_{1}$ and $\sigma_{2}$ are not changed in the evaluation of the predicate expression $e$. The condition holds.

- Values of new configuration variables are consistent in the value store $\sigma_{2}$ and the specialization $\rho, \forall i d \in \operatorname{Dom}(\rho)$ : $\sigma_{2}(i d)=\rho(i d)$.

By assumption.

By assumption, $S_{1}^{\prime}$ and $S_{2}^{\prime}$ terminate in the same way and produce the same output sequence when started in states $m_{1}\left(\operatorname{loop}_{c}^{\prime}, \sigma_{1}\right)$ and $m_{2}\left(\operatorname{loop}_{c}^{\prime}, \sigma_{2}\right)$. In addition, $S_{1}^{\prime}$ and $S_{2}^{\prime}$ have equivalent computation of variables used or defined in $S_{1}^{\prime}$ and $S_{2}^{\prime}$ when started in states $m_{1}\left(\operatorname{loop}_{c}^{\prime}, \sigma_{1}\right)$ and $m_{2}\left(\operatorname{loop}_{c}^{\prime}, \sigma_{2}\right)$.

Then there are two cases. 
(a) $S_{1}^{\prime}$ and $S_{2}^{\prime}$ both do not terminate and produce the same output sequence.

By Lemma E.2 $S_{1}^{\prime} ; S_{1}$ and $S_{2}^{\prime} ; S_{2}$ both do not terminate and produce the same output sequence.

(b) $S_{1}^{\prime}$ and $S_{2}^{\prime}$ both terminate and have equivalent computation of variables used or defined in $S_{1}^{\prime}$ and $S_{2}^{\prime}$.

By assumption, $\left(S_{1}^{\prime}, m_{1}\left(\operatorname{loop}_{c}^{\prime}, \sigma_{1}\right)\right) \stackrel{*}{\rightarrow}\left(\right.$ skip, $\left.m_{1}^{\prime}\left(\operatorname{loop}_{c}^{\prime \prime}, \sigma_{1}^{\prime}\right)\right)$; $\left(S_{2}^{\prime}, m_{2}\left(\operatorname{loop}_{c}^{\prime}, \sigma_{2}\right)\right) \stackrel{*}{\rightarrow}$ (skip, $\left.m_{2}^{\prime}\left(\operatorname{loop}_{c}^{\prime \prime}, \sigma_{2}^{\prime}\right)\right)$ where $\forall x \in$ $\left(\operatorname{OVar}\left(S_{1}^{\prime}\right) \cap \operatorname{OVar}\left(S_{2}^{\prime}\right)\right) \cup\left\{i d_{I}, i d_{I O}\right\}, \sigma_{1}^{\prime}(x)=\sigma_{2}^{\prime}(x)$.

Because $S_{1}$ and $S_{2}$ have the same predicate expression, variables used in the predicate expression of $S_{1}$ and $S_{2}$ are not in the domain of $\rho$. By assumption, $\operatorname{OVar}\left(S_{1}^{\prime}\right) \subseteq$ $\operatorname{OVar}\left(S_{2}^{\prime}\right) \subseteq \operatorname{OVar}\left(S_{2}^{\prime}\right) \cup \operatorname{Dom}(\rho)$ and $\operatorname{OVar}\left(S_{1}^{\prime}\right) \subseteq$ $\operatorname{OVar}\left(S_{2}^{\prime}\right)$. Then variables used in the predicate expression of $S_{1}$ and $S_{2}$ are either in variables used or defined in both $S_{1}^{\prime}$ and $S_{2}^{\prime}$ or not. Therefore value stores $\sigma_{2}^{\prime}$ and $\sigma_{1}^{\prime}$ agree on values of variables used in the expression $e$ and even variables used or defined in $S_{1}$ and $S_{2}$.

Induction step on iterations

The induction hypothesis $(\mathrm{IH})$ is that, when $i \geq 1$, one of the following holds:

1. Loop counters for $S_{1}$ and $S_{2}$ are always less than $i$ if any is present, $\forall m_{1}^{\prime}\left(\operatorname{loop}_{c}^{1^{\prime}}\right) m_{2}^{\prime}\left(\operatorname{loop}_{c}^{2^{\prime}}\right):\left(S_{1}, m_{1}\left(\operatorname{loop}_{c}^{1}, \sigma_{1}\right)\right) \stackrel{*}{\rightarrow}$ $\left(S_{1}^{\prime \prime}, m_{1}^{\prime}\left(\operatorname{loop}_{c}^{1^{\prime}}\right)\right), \operatorname{loop}_{c}^{1^{\prime}}\left(n_{1}\right)<i,\left(S_{2}, m_{2}\left(\operatorname{loop}_{c}^{2}, \sigma_{2}\right)\right) \stackrel{*}{\rightarrow}$ $\left(S_{2}^{\prime \prime}, m_{2}^{\prime}\left(\operatorname{loop}_{c}^{2^{\prime}}\right)\right), \operatorname{loop}_{c}^{2^{\prime}}\left(n_{2}\right)<i, S_{1}$ and $S_{2}$ terminate in the same way, produce the same output sequence, and have equivalent computation of used/defined variables in both $S_{1}$ and $S_{2}$ and the input sequence variable, the I/O sequence variable, $\left(S_{1}, m_{1}\right) \equiv_{H}\left(S_{2}, m_{2}\right)$ and $\left(S_{1}, m_{1}\right) \equiv_{O}\left(S_{2}, m_{2}\right)$ and $\forall x \in\left(\mathrm{OVar}\left(S_{1}\right) \cap \operatorname{OV} \operatorname{Var}\left(S_{2}\right)\right) \cup\left\{i d_{I}, i d_{I O}\right\}:\left(S_{1}, m_{1}\right) \equiv_{x}$ $\left(S_{2}, m_{2}\right)$;

2. The loop counter of $S_{1}$ and $S_{2}$ are of value less than or equal to $i$, and there are no reachable configurations $\left(S_{1}, m_{1}\left(\operatorname{loop}_{c}^{1_{i}}, \sigma_{1_{i}}\right)\right)$ from $\left(S_{1}, m_{1}\left(\sigma_{1}\right)\right),\left(S_{2}, m_{2}\left(\operatorname{loop}_{c}^{2_{i}}, \sigma_{2_{i}}\right)\right)$ from $\left(S_{2}, m_{2}\left(\sigma_{2}\right)\right)$ where all of the following hold:

- The loop counters of $S_{1}$ and $S_{2}$ are of value $i$, loop ${ }_{c}^{1_{i}}\left(n_{1}\right)$ $=\operatorname{loop}_{c}^{2 i}\left(n_{2}\right)=i$.

- Value stores $\sigma_{1_{i}}$ and $\sigma_{2_{i}}$ agree on values of used variables in both $S_{1}$ and $S_{2}$ as well as the input sequence variable and the I/O sequence variable, $\forall x \in\left(\operatorname{OVar}\left(S_{1}\right) \cap \operatorname{OVar}\left(S_{2}\right)\right) \cup$ $\left\{i d_{I}, i d_{I O}\right\}: \sigma_{1_{i}}(x)=\sigma_{2_{i}}(x)$.

3. There are reachable configurations $\left(S_{1}, m_{1}\left(\operatorname{loop}_{c}^{1_{i}}, \sigma_{1_{i}}\right)\right)$ from $\left(S_{1}, m_{1}\left(\sigma_{1}\right)\right),\left(S_{2}, m_{2}\left(\operatorname{loop}_{c}^{2_{i}}, \sigma_{2_{i}}\right)\right)$ from $\left(S_{2}, m_{2}\left(\sigma_{2}\right)\right)$ where all of the following hold:

- The loop counter of $S_{1}$ and $S_{2}$ are of value $i, \operatorname{loop}_{c}^{1_{i}}\left(n_{1}\right)$ $=\operatorname{loop}_{c}^{2_{i}}\left(n_{2}\right)=i$.

- Value stores $\sigma_{1_{i}}$ and $\sigma_{2_{i}}$ agree on values of used variables in both $S_{1}$ and $S_{2}$ as well as the input sequence variable and the I/O sequence variable, $\forall x \in\left(\mathrm{OVar}\left(S_{1}\right) \cap \operatorname{OV} \operatorname{Var}\left(S_{2}\right)\right) \cup$ $\left\{i d_{I}, i d_{I O}\right\}: \sigma_{1_{i}}(x)=\sigma_{2_{i}}(x)$.

Then we show that, when $i+1$, one of the following holds: The induction hypothesis $(\mathrm{IH})$ is that, when $i \geq 1$, one of the following holds:

1. Loop counters for $S_{1}$ and $S_{2}$ are always less than $i+1$ if any is present, $\forall m_{1}^{\prime}\left(\operatorname{loop}_{c}^{1^{\prime}}\right) m_{2}^{\prime}\left(\operatorname{loop}_{c}^{2^{\prime}}\right):\left(S_{1}, m_{1}\left(\operatorname{loop}_{c}^{1}, \sigma_{1}\right)\right) \stackrel{*}{\rightarrow}$ $\left(S_{1}^{\prime \prime}, m_{1}^{\prime}\left(\operatorname{loop}_{c}^{1^{\prime}}\right)\right), \operatorname{loop}_{c}^{1^{\prime}}\left(n_{1}\right)<i+1,\left(S_{2}, m_{2}\left(\operatorname{loop}_{c}^{2}, \sigma_{2}\right)\right) \stackrel{*}{\rightarrow}$ $\left(S_{2}^{\prime \prime}, m_{2}^{\prime}\left(\operatorname{loop}_{c}^{2^{\prime}}\right)\right), \operatorname{loop}_{c}^{2^{\prime}}\left(n_{2}\right)<i+1, S_{1}$ and $S_{2}$ terminate in the same way, produce the same output sequence, and have equivalent computation of used/defined variables in both $S_{1}$ and $S_{2}$ and the input sequence variable, the I/O sequence variable,
$\left(S_{1}, m_{1}\right) \equiv_{H} \quad\left(S_{2}, m_{2}\right)$ and $\left(S_{1}, m_{1}\right) \equiv_{O} \quad\left(S_{2}, m_{2}\right)$ and $\forall x \in\left(\operatorname{OVar}\left(S_{1}\right) \cap \operatorname{OVar}\left(S_{2}\right)\right) \cup\left\{i d_{I}, i d_{I O}\right\}:\left(S_{1}, m_{1}\right) \equiv_{x}$ $\left(S_{2}, m_{2}\right)$;

2. The loop counter of $S_{1}$ and $S_{2}$ are of value less than or equal to $i+1$, and there are no reachable configurations $\left(S_{1}, m_{1}\left(\operatorname{loop}_{c}^{1_{i+1}}, \sigma_{1_{i+1}}\right)\right)$ from $\left(S_{1}, m_{1}\left(\sigma_{1}\right)\right),\left(S_{2}, m_{2}\left(\operatorname{loop}_{c}^{2_{i+1}}, \sigma_{2_{i+1}}\right)\right)$ from $\left(S_{2}, m_{2}\left(\sigma_{2}\right)\right)$ where all of the following hold:

- The loop counters of $S_{1}$ and $S_{2}$ are of value $i+1$, $\operatorname{loop}_{c}^{1_{i+1}}\left(n_{1}\right)=\operatorname{loop}_{c}^{2_{i+1}}\left(n_{2}\right)=i+1$.

- Value stores $\sigma_{1_{i+1}}$ and $\sigma_{2_{i+1}}$ agree on values of used variables in both $S_{1}$ and $S_{2}$ as well as the input sequence variable and the I/O sequence variable, $\forall x \in\left(\operatorname{OVar}\left(S_{1}\right) \cap\right.$ $\left.\operatorname{OVar}\left(S_{2}\right)\right) \cup\left\{i d_{I}, i d_{I O}\right\}: \sigma_{1_{i+1}}(x)=\sigma_{2_{i+1}}(x)$.

3. There are reachable configurations $\left(S_{1}, m_{1}\left(\operatorname{loop}_{c}^{1_{i+1}}, \sigma_{1_{i}}\right)\right)$ from $\left(S_{1}, m_{1}\left(\sigma_{1}\right)\right),\left(S_{2}, m_{2}\left(\operatorname{loop}_{c}^{2 i+1}, \sigma_{2_{i}}\right)\right)$ from $\left(S_{2}, m_{2}\left(\sigma_{2}\right)\right)$ where all of the following hold:

- The loop counter of $S_{1}$ and $S_{2}$ are of value $i, \operatorname{loop}_{c}^{1_{i+1}}\left(n_{1}\right)$ $=\operatorname{loop}_{c}^{2 i+1}\left(n_{2}\right)=i+1$.

- Value stores $\sigma_{1_{i+1}}$ and $\sigma_{2_{i+1}}$ agree on values of used variables in both $S_{1}$ and $S_{2}$ as well as the input sequence variable and the I/O sequence variable, $\forall x \in\left(\operatorname{OVar}\left(S_{1}\right) \cap\right.$ $\left.\operatorname{OVar}\left(S_{2}\right)\right) \cup\left\{i d_{I}, i d_{I O}\right\}: \sigma_{1_{i+1}}(x)=\sigma_{2_{i+1}}(x)$.

By hypothesis $\mathrm{IH}$, there is no configuration where loop counters of $S_{1}$ and $S_{2}$ are of value $i+1$ when any of the following holds:

1. Loop counters for $S_{1}$ and $S_{2}$ are always less than $i$ if any is present, $\forall m_{1}^{\prime}\left(\operatorname{loop}_{c}^{1^{\prime}}\right) m_{2}^{\prime}\left(\operatorname{loop}_{c}^{2^{\prime}}\right):\left(S_{1}, m_{1}\left(\operatorname{loop}_{c}^{1}, \sigma_{1}\right)\right) \stackrel{*}{\rightarrow}$ $\left(S_{1}^{\prime \prime}, m_{1}^{\prime}\left(\operatorname{loop}_{c}^{1^{\prime}}\right)\right), \operatorname{loop}_{c}^{1^{\prime}}\left(n_{1}\right)<i,\left(S_{2}, m_{2}\left(\operatorname{loop}_{c}^{2}, \sigma_{2}\right)\right) \stackrel{*}{\rightarrow}$ $\left(S_{2}^{\prime \prime}, m_{2}^{\prime}\left(\operatorname{loop}_{c}^{2^{\prime}}\right)\right), \operatorname{loop}_{c}^{2^{\prime}}\left(n_{2}\right)<i, S_{1}$ and $S_{2}$ terminate in the same way, produce the same output sequence, and have equivalent computation of used/defined variables in both $S_{1}$ and $S_{2}$ and the input sequence variable, the I/O sequence variable, $\left(S_{1}, m_{1}\right) \equiv_{H}\left(S_{2}, m_{2}\right)$ and $\left(S_{1}, m_{1}\right) \equiv_{O}\left(S_{2}, m_{2}\right)$ and $\forall x \in\left(\mathrm{OVar}\left(S_{1}\right) \cap \operatorname{OVar}\left(S_{2}\right)\right) \cup\left\{i d_{I}, i d_{I O}\right\}:\left(S_{1}, m_{1}\right) \equiv_{x}$ $\left(S_{2}, m_{2}\right)$;

2. The loop counter of $S_{1}$ and $S_{2}$ are of value less than or equal to $i$, and there are no reachable configurations $\left(S_{1}, m_{1}\left(\operatorname{loop}_{c}^{1_{i}}, \sigma_{1_{i}}\right)\right)$ from $\left(S_{1}, m_{1}\left(\sigma_{1}\right)\right),\left(S_{2}, m_{2}\left(\operatorname{loop}_{c}^{2_{i}}, \sigma_{2_{i}}\right)\right)$ from $\left(S_{2}, m_{2}\left(\sigma_{2}\right)\right)$ where all of the following hold:

- The loop counters of $S_{1}$ and $S_{2}$ are of value $i, \operatorname{loop}_{c}^{1_{i}}\left(n_{1}\right)$ $=\operatorname{loop}_{c}^{2 i}\left(n_{2}\right)=i$.

- Value stores $\sigma_{1_{i}}$ and $\sigma_{2_{i}}$ agree on values of used variables in both $S_{1}$ and $S_{2}$ as well as the input sequence variable, and the I/O sequence variable, $\forall x \in\left(\mathrm{OV} \operatorname{Var}\left(S_{1}\right) \cap \operatorname{OV} \operatorname{Var}\left(S_{2}\right)\right) \cup$ $\left\{i d_{I}, i d_{I O}\right\}: \sigma_{1_{i}}(x)=\sigma_{2_{i}}(x)$.

When there are reachable configurations $\left(S_{1}, m_{1}\left(\operatorname{loop}_{c}^{1_{i}}, \sigma_{1_{i}}\right)\right)$ from $\left(S_{1}, m_{1}\left(\sigma_{1}\right)\right),\left(S_{2}, m_{2}\left(\operatorname{loop}_{c}^{2_{i}}, \sigma_{2_{i}}\right)\right)$ from $\left(S_{2}, m_{2}\left(\sigma_{2}\right)\right)$ where all of the following hold:

- The loop counter of $S_{1}$ and $S_{2}$ are of value $i, \operatorname{loop}_{c}^{1_{i}}\left(n_{1}\right)$ $=\operatorname{loop}_{c}^{2}\left(n_{2}\right)=i$.

- The loop counter of $S_{1}$ and $S_{2}$ are of value $i$, $\operatorname{loop}_{c}^{1_{i}}\left(n_{1}\right)$ $=\operatorname{loop}_{c}^{2 i}\left(n_{2}\right)=i$.

- Value stores $\sigma_{1_{i}}$ and $\sigma_{2_{i}}$ agree on values of used variables in both $S_{1}$ and $S_{2}$ as well as the input sequence variable and the I/O sequence variable, $\forall x \in\left(\operatorname{OVar}\left(S_{1}\right) \cap \operatorname{OVar}\left(S_{2}\right)\right) \cup$ $\left\{i d_{I}, i d_{I O}\right\}: \sigma_{1_{i}}(x)=\sigma_{2_{i}}(x)$. holds:

By similar argument in base case, we have one of the following

1. Loop counters for $S_{1}$ and $S_{2}$ are always less than $i+1$ if any is present, $\forall m_{1}^{\prime}\left(\operatorname{loop}_{c}^{1^{\prime}}\right) m_{2}^{\prime}\left(\operatorname{loop}_{c}^{2^{\prime}}\right):\left(S_{1}, m_{1}\left(\operatorname{loop}_{c}^{1}, \sigma_{1}\right)\right) \stackrel{*}{\rightarrow}$ 


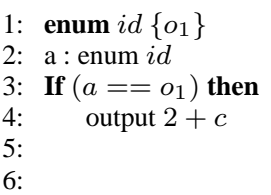

old

Figure 16: Enumeration type extension

$\left(S_{1}^{\prime \prime}, m_{1}^{\prime}\left(\operatorname{loop}_{c}^{1^{\prime}}\right)\right), \operatorname{loop}_{c}^{1^{\prime}}\left(n_{1}\right)<i+1,\left(S_{2}, m_{2}\left(\operatorname{loop}_{c}^{2}, \sigma_{2}\right)\right) \stackrel{*}{\rightarrow}$ $\left(S_{2}^{\prime \prime}, m_{2}^{\prime}\left(\operatorname{loop}_{c}^{2^{\prime}}\right)\right), \operatorname{loop}_{c}^{2^{\prime}}\left(n_{2}\right)<i+1, S_{1}$ and $S_{2}$ terminate in the same way, produce the same output sequence, and have equivalent computation of used/defined variables in both $S_{1}$ and $S_{2}$ and the input sequence variable, the I/O sequence variable, $\left(S_{1}, m_{1}\right) \equiv_{H}\left(S_{2}, m_{2}\right)$ and $\left(S_{1}, m_{1}\right) \equiv_{O}\left(S_{2}, m_{2}\right)$ and $\forall x \in\left(\mathrm{OVar}\left(S_{1}\right) \cap \operatorname{OVar}\left(S_{2}\right)\right) \cup\left\{i d_{I}, i d_{I O}\right\}:\left(S_{1}, m_{1}\right) \equiv_{x}$ $\left(S_{2}, m_{2}\right)$;

2. The loop counter of $S_{1}$ and $S_{2}$ are of value less than or equal to $i+1$, and there are no reachable configurations $\left(S_{1}, m_{1}\left(\operatorname{loop}_{c}^{1_{i+1}}, \sigma_{1_{i+1}}\right)\right)$ from $\left(S_{1}, m_{1}\left(\sigma_{1}\right)\right),\left(S_{2}, m_{2}\left(\operatorname{loop}_{c}^{2_{i+1}}\right.\right.$, $\left.\left.\sigma_{2_{i+1}}\right)\right)$ from $\left(S_{2}, m_{2}\left(\sigma_{2}\right)\right)$ where all of the following hold:

- The loop counters of $S_{1}$ and $S_{2}$ are of value $i, \operatorname{loop}_{c}^{1_{i+1}}\left(n_{1}\right)$ $=\operatorname{loop}_{c}^{2_{i+1}}\left(n_{2}\right)=i$.

- Value stores $\sigma_{1_{i+1}}$ and $\sigma_{2_{i+1}}$ agree on values of used variables in both $S_{1}$ and $S_{2}$ as well as the input sequence variable and the I/O sequence variable, $\forall x \in\left(\operatorname{OVar}\left(S_{1}\right) \cap\right.$ $\left.\operatorname{OVar}\left(S_{2}\right)\right) \cup\left\{i d_{I+1}, i d_{I O}\right\}: \sigma_{1_{i+1}}(x)=\sigma_{2_{i+1}}(x)$.

3. There are reachable configurations $\left(S_{1}, m_{1}\left(\operatorname{loop}_{c}^{1_{i+1}}, \sigma_{1_{i+1}}\right)\right)$ from $\left(S_{1}, m_{1}\left(\sigma_{1}\right)\right),\left(S_{2}, m_{2}\left(\operatorname{loop}_{c}^{2 i+1}, \sigma_{2_{i+1}}\right)\right)$ from $\left(S_{2}\right.$, $\left.m_{2}\left(\sigma_{2}\right)\right)$ where all of the following hold:

- The loop counter of $S_{1}$ and $S_{2}$ are of value $i, \operatorname{loop}_{c}^{1_{i+1}}\left(n_{1}\right)$ $=\operatorname{loop}_{c}^{2 i+1}\left(n_{2}\right)=i+1$.

- Value stores $\sigma_{1_{i+1}}$ and $\sigma_{2_{i+1}}$ agree on values of used variables in both $S_{1}$ and $S_{2}$ as well as the input sequence variable and the I/O sequence variable, $\forall x \in\left(\mathrm{OVar}\left(S_{1}\right) \cap\right.$ $\left.\operatorname{OVar}\left(S_{2}\right)\right) \cup\left\{i d_{I+1}, i d_{I O}\right\}: \sigma_{1_{i+1}}(x)=\sigma_{2_{i+1}}(x)$.

\subsection{Proof rule for enumeration type extension}

Enumeration types allow developers to list similar items. New code is usually accompanied with the introduction of new enumeration labels. Figure 16 shows an example of the update. The new enum label $o_{2}$ gives a new option for matching the value of the variable $a$, which introduce the new code $b:=3+c$. To show updates "enumeration type extension" to be backward compatible, we assume that values of enum variables, used in the If-predicate introducing the new code, are only from inputs that cannot be translated to new enum labels.

In order to have a general definition of the update class, we show a relation between two sequences of enumeration type definitions, called proper subset.

Definition 26. (Extension relation of enumeration types) Let $E N_{1}, E N_{2}$ be two different sequences of enumeration type definitions. $E N_{1}$ is a subset of $E N_{2}$, written $E N_{1} \subset E N_{2}$, iff one of the following holds:

1. $E N_{1}=$ "enum id $\left\{e l_{1}\right\} ", E N_{2}=$ "enumid $\left\{e l_{2}\right\} "$ where labels in type "enum id" in $E N_{1}$ are a subset of those in $E N_{2}$, $e l_{2}=e l_{1}$, el and $e l \neq \varnothing ;$
2. $E N_{1}, E N_{2}$ include more than one enumeration type definitions $E N_{1}=$ "enumid $\left\{e l_{1}\right\}, E N_{1}^{\prime}$ ", $E N_{2}=$ "enum id $\left\{e l_{2}\right\}, E N_{2}^{\prime}$ " where one of the following holds:

(a) $\left(E N_{1}^{\prime} \subset E N_{2}^{\prime}\right)$ and $\left(e l_{1}=e l_{2}\right) \vee\left(e l_{2}=e l_{1}, e l\right)$;

(b) $\left(E N_{1}^{\prime} \subset E N_{2}^{\prime}\right) \vee\left(E N_{1}^{\prime}=E N_{2}^{\prime}\right)$ and "enum id $\left\{e l_{1}\right\} " \subset$ "enum id $\left\{\mathrm{el}_{2}\right\}$ ".

Definition 27. (Enumeration type extension) Let $P_{1}, P_{2}$ be two programs where enumeration type definitions $E N_{1}$ in $P_{1}$ are a subset of $E N_{2}$ in $P_{2}, E N_{1} \subset E N_{2}$ and $E$ are new enum labels in $P_{2}$. A statement sequence $S_{2}$ in a program $P_{2}$ includes updates of enumeration type extension compared with a statement sequence $S_{1}$ in $P_{1}$, written $S_{2} \approx_{E}^{S} S_{1}$, iff one of the following holds:

1. $S_{2}=$ "If $(i d==l)$ then $\left\{S_{2}^{t}\right\}$ else $\left\{S_{2}^{f}\right\} "$ and all of the following hold:

- $l \in E$;

- The variable id is not lvalue in an assignment statement, "id:=e" $\notin P_{2}$;

- $S_{2}^{f} \approx_{E}^{S} S_{1}$;

2. $S_{1}=$ "If $(e)$ then $\left\{S_{1}^{t}\right\}$ else $\left\{S_{1}^{f}\right\} ", S_{2}=$ "If $(e)$ then $\left\{S_{2}^{t}\right\}$ else $\left\{S_{2}^{f}\right\}$ " where $\left(S_{2}^{t} \approx_{E}^{S} S_{1}^{t}\right) \wedge\left(S_{2}^{f} \approx_{E}^{S} S_{1}^{f}\right)$;

3. $S_{1}=$ "while $\left\langle n_{\left.n_{1}\right\rangle}(e)\left\{S_{1}^{\prime}\right\}\right.$ ", $S_{2}=$ "while $\left\langle n_{2}\right\rangle(e)\left\{S_{2}^{\prime}\right\}$ " where $S_{2}^{\prime} \approx_{E}^{S} S_{1}^{\prime}$

4. $S_{1} \approx{ }_{O}^{S} S_{2}$;

5. $S_{1}=S_{1}^{\prime} ; s_{1}$ and $S_{2}=S_{2}^{\prime} ; s_{2}$ where $\left(S_{2}^{\prime} \approx_{E}^{S} S_{1}^{\prime}\right) \wedge\left(S_{2}^{\prime} \approx_{H}^{S}\right.$ $\left.S_{1}^{\prime}\right) \wedge\left(\forall x \in \operatorname{Imp}\left(s_{1}, i d_{I O}\right) \cup \operatorname{Imp}\left(s_{1}, i d_{I O}\right):\left(S_{2}^{\prime} \approx_{x}^{S}\right.\right.$

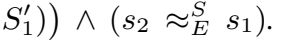

We show that two programs terminate in the same way, produce the same output sequence, and have equivalent computation of variables defined in both of them in executions if there are updates of enumeration type extension between them.

Lemma 6.4. Let $S_{1}$ and $S_{2}$ be two statement sequences in programs $P_{1}$ and $P_{2}$ respectively where there are updates of enumeration type extensions in $S_{2}$ of $P_{2}$ compared with $S_{1}$ of $P_{1}, S_{2} \approx_{E}^{S} S_{1}$. If $S_{1}$ and $S_{2}$ start in states $m_{1}\left(\sigma_{1}\right)$ and $m_{2}\left(\sigma_{2}\right)$ such that both of the following hold:

- Value stores $\sigma_{1}$ and $\sigma_{2}$ agree on values of output deciding variables in both $S_{1}$ and $S_{2}$ including the input sequence variable and the I/O sequence variable, $\forall x \in\left(\operatorname{OVar}\left(S_{1}\right) \cup \operatorname{OVar}\left(S_{2}\right)\right) \cup$ $\left\{i d_{I}, i d_{I O}\right\}: \sigma_{1}(x)=\sigma_{2}(x)$;

- No variables used in $S_{2}$ are of initial value of enum labels in $E, \forall x \in U \operatorname{se}\left(S_{2}\right):\left(\sigma_{2}(x) \notin E\right)$;

- No inputs are translated to any label in $E$ during the execution of $S_{2}$;

then $S_{1}$ and $S_{2}$ terminate in the same way, produce the same output sequence, and when $S_{1}$ and $S_{2}$ both terminate, they have equivalent computation of used variables and defined variables,

- $\left(S_{1}, m_{1}\right) \equiv_{H}\left(S_{2}, m_{2}\right)$;

- $\left(S_{1}, m_{1}\right) \equiv_{O}\left(S_{2}, m_{2}\right)$;

- $\forall x \in \operatorname{OVar}\left(S_{1}\right) \cup \operatorname{OVar}\left(S_{2}\right):\left(S_{1}, m_{1}\right) \equiv_{x}\left(S_{2}, m_{2}\right)$;

Proof. By induction on the sum of the program size of $S_{1}$ and $S_{2}$, $\operatorname{size}\left(S_{1}\right)+\operatorname{size}\left(S_{1}\right)$.

Base case. $S_{1}$ is a simple statement $s$, and $S_{2}=" \operatorname{If}(i d==l) \operatorname{then}\left\{s_{2}^{t}\right\}$ else $\left\{s_{2}^{f}\right\}$ " where all of the following hold:

- $l \in E$;

- $s_{2}^{t}, s_{2}^{f}$ are two simple statements;

- $s_{2}^{f}=s$; 
We informally argue that the value of the variable $i d$ in the predicate expression of $S_{2}$ only coming from an input value or the initial value. There are three ways a scalar variable is defined: the execution of an assignment statement, the execution of an input statement or the initial value. Because $i d$ is not lvalue in an assignment statement, then the value of $i d$ is only from the execution of an input statement or the initial value.

In addition, by assumption, any output deciding variable is not of the initial value of enum label in $E$; no input values are translated into an enum label in $E$. Then the execution of $S_{2}$ proceeds as follows:

$$
\begin{aligned}
& \left(\operatorname{If}(i d==l) \text { then }\left\{s_{2}^{t}\right\} \text { else }\left\{s_{2}^{f}\right\}, m_{2}\left(\sigma_{2}\right)\right) \\
\rightarrow & \left(\operatorname{If}(0) \text { then }\left\{s_{2}^{t}\right\} \text { else }\left\{s_{2}^{f}\right\}, m_{2}\left(\sigma_{2}\right)\right) \\
& \text { by the rule Eq-F } \\
\rightarrow & \left(s_{2}^{f}, m_{2}\left(\sigma_{2}\right)\right) \text { by the If-F rule. }
\end{aligned}
$$

The value store $\sigma_{2}$ is not updated in the execution of $S_{2}$ so far. By assumption, value stores $\sigma_{1}$ and $\sigma_{2}$ agree on values of output deciding variables in both $S_{1}$ and $S_{2}$.

By Theorem 2 and $4, S_{1}$ and $S_{2}$ terminate in the same way, produce the same I/O sequence. The lemma holds.

Induction step.

The hypothesis is that this lemma holds when the sum $k$ of the program size of $S_{1}$ and $S_{2}$ are great than or equal to $4, k \geq 4$.

We then show that this lemma holds when the sum of the program size of $S_{1}$ and $S_{2}$ is $k+1$. There are cases regarding $S_{2} \approx_{E}^{S} S_{1}$.

1. $S_{1}$ and $S_{2}$ are both "If" statement:

$S_{1}=$ "If $(e)$ then $\left\{S_{1}^{t}\right\}$ else $\left\{S_{1}^{f}\right\} ", S_{2}=$ "If $(e)$ then $\left\{S_{2}^{t}\right\} \operatorname{else}\left\{S_{2}^{f}\right\} "$ where both of the following hold

- $S_{2}^{t} \approx_{E}^{S} S_{1}^{t}$;

- $S_{2}^{f} \approx_{E}^{S} S_{1}^{f}$;

By the definition of $\operatorname{Imp}_{o}\left(S_{1}\right)$, variables used in the predicate expression $e$ are a subset of output deciding variables in $S_{1}$ and $S_{2}$, Use $(e) \subseteq \operatorname{OVar}\left(S_{1}\right) \cap \operatorname{OVar}\left(S_{2}\right)$. By assumption, corresponding variables used in $e$ are of same value in value stores $\sigma_{1}$ and $\sigma_{2}$. By Lemma D.1 the expression evaluates to the same value w.r.t value stores $\sigma_{1}$ and $\sigma_{2}$. There are three possibilities.

(a) The evaluation of $e$ crashes, $\mathcal{E}^{\prime} \llbracket e \rrbracket \sigma_{1}=\mathcal{E}^{\prime} \llbracket e \rrbracket \sigma_{2}=$ (error, $v_{\mathfrak{o f}}$ ).

The execution of $S_{1}$ continues as follows:

$\left(\operatorname{If}(e)\right.$ then $\left\{S_{1}^{t}\right\}$ else $\left.\left\{S_{1}^{f}\right\}, m_{1}\left(\sigma_{1}\right)\right)$

$\rightarrow\left(\operatorname{If}\left(\left(\right.\right.\right.$ error, $\left.\left.v_{\text {of }}\right)\right)$ then $\left\{S_{1}^{t}\right\}$ else $\left.\left\{S_{1}^{f}\right\}, m_{1}\left(\sigma_{1}\right)\right)$

by the rule EEval'

$\rightarrow\left(\operatorname{If}(0)\right.$ then $\left\{S_{1}^{t}\right\}$ else $\left.\left\{S_{1}^{f}\right\}, m_{1}(1 / \mathfrak{f})\right)$

by the ECrash rule

$\stackrel{i}{\rightarrow}\left(\operatorname{If}(0)\right.$ then $\left\{S_{1}^{t}\right\}$ else $\left.\left\{S_{1}^{f}\right\}, m_{1}(1 / \mathfrak{f})\right)$ for any $i>0$ by the Crash rule.

Similarly, the execution of $S_{2}$ started from the state $m_{2}\left(\sigma_{2}\right)$ crashes. The lemma holds.

(b) The evaluation of $e$ reduces to zero, $\mathcal{E}^{\prime} \llbracket e \rrbracket \sigma_{1}=\mathcal{E}^{\prime} \llbracket e \rrbracket \sigma_{2}=$ $\left(0, v_{\mathfrak{o f}}\right)$.

The execution of $S_{1}$ continues as follows.

$\left(\right.$ If $(e)$ then $\left\{S_{1}^{t}\right\}$ else $\left.\left\{S_{1}^{f}\right\}, m_{1}\left(\sigma_{1}\right)\right)$

$=\left(\operatorname{If}\left(\left(0, v_{\text {of }}\right)\right)\right.$ then $\left.\left\{S_{1}^{t}\right\} \operatorname{else}\left\{S_{1}^{f}\right\}, m_{1}\left(\sigma_{1}\right)\right)$

by the rule EEval'

$\rightarrow\left(\right.$ If $(0)$ then $\left\{S_{1}^{t}\right\}$ else $\left.\left\{S_{1}^{f}\right\}, m_{1}\left(\sigma_{1}\right)\right)$

by the E-Oflow1 or E-Oflow2 rule

$\rightarrow\left(S_{1}^{f}, m_{1}\left(\sigma_{1}\right)\right)$ by the If-F rule.

Similarly, the execution of $S_{2}$ gets to the configuration $\left(S_{2}^{f}, m_{2}\left(\sigma_{2}\right)\right)$.
By the hypothesis IH, we show the lemma holds. We need to show that all conditions are satisfied for the application of the hypothesis IH.

- $S_{2}^{f} \approx_{E}^{S} S_{1}^{f}$

By assumption.

- The sum of the program size of $S_{1}^{f}$ and $S_{2}^{f}$ is less than $k, \operatorname{size}\left(S_{1}^{f}\right)+\operatorname{size}\left(S_{2}^{f}\right)<k$.

By definition, $\operatorname{size}\left(S_{1}\right)=1+\operatorname{size}\left(S_{1}^{t}\right)+\operatorname{size}\left(S_{1}^{f}\right)$. Then, $\operatorname{size}\left(S_{1}^{f}\right)+\operatorname{size}\left(S_{2}^{f}\right)<k+1-2=k-1$.

- Value stores $\sigma_{1}$ and $\sigma_{2}$ agree on values of output deciding variables in $S_{1}^{f}$ and $S_{2}^{f}$ including the input, I/O sequence variable.

By definition, $\operatorname{OVar}\left(S_{1}^{f}\right) \subseteq \operatorname{OVar}\left(S_{1}\right)$. So are the cases to $S_{2}^{f}$ and $S_{2}$. In addition, value stores $\sigma_{1}$ and $\sigma_{2}$ are not changed in the evaluation of the predicate expression $e$. The condition holds.

- There are no inputs translated to enum labels in $E$ in $S_{2}^{f}$ 's execution.

By assumption.

By the hypothesis $\mathrm{IH}$, the lemma holds.

(c) The evaluation of $e$ reduces to the same nonzero integer value, $\mathcal{E}^{\prime} \llbracket e \rrbracket \sigma_{1}=\mathcal{E}^{\prime} \llbracket e \rrbracket \sigma_{2}=\left(0, v_{\text {of }}\right)$.

By similar to the second subcase above.

2. $S_{1}$ and $S_{2}$ are both "while" statements:

$S_{1}=$ "while $\left\langle n_{1}\right\rangle(e)\left\{S_{1}^{\prime}\right\} ", S_{2}=$ "while ${ }_{\left\langle n_{2}\right\rangle}(e)\left\{S_{2}^{\prime}\right\}$ " where $S_{2}^{\prime} \approx_{E}^{S} S_{1}^{\prime}$

By Lemma6.6 we show the lemma holds. We need to show all the required conditions for the application of Lemma 6.6 holds

(a) No variables are of initial values as new enum labels in $E$;

(b) Value stores $\sigma_{1}$ and $\sigma_{2}$ agree on values of variables used in both $S_{1}$ and $S_{2}$;

(c) Enumeration types in $P_{1}$ are a subset of those in $P_{2}$; The above three conditions are by assumption.

(d) The output deciding variables in $S_{1}^{\prime}$ are a subset of those in $S_{2}^{\prime}$;

The above condition is by Lemma 6.5

(e) $S_{1}^{\prime}$ and $S_{2}^{\prime}$ produce the same output sequence, terminate in the same way and have equivalent computation of defined variables in both $S_{1}^{\prime}$ and $S_{2}^{\prime}$ when started in states agreeing on values of variables used in both $S_{1}^{\prime}$ and $S_{2}^{\prime}$;

Because size $\left(S_{1}\right)=\operatorname{size}\left(S_{1}^{\prime}\right)+1$, then the condition holds by the induction hypothesis.

By Lemma 6.6 the lemma holds.

3. $S_{2}=$ "If $(i d==l)$ then $\left\{S_{2}^{t}\right\}$ else $\left\{S_{2}^{f}\right\}$ " such that both of the following hold:

- The label $l$ is in $E, l \in E$;

- The variable $i d$ is not lvalue in an assignment statement, $\nexists$ \# $i d:=\mathrm{e}$ " in $S_{2}$;

- There are updates of enumeration type extension from $S_{2}^{f}$ to $S_{1}, S_{2}^{f} \approx_{E}^{S} S_{1}$;

By Lemma 6.6 we show this lemma holds. We need to show all the conditions are satisfied for the application of Lemma 6.6

- $S_{1}^{\prime}$ and $S_{2}^{\prime}$ have same set of output deciding variables, OVar $\left(S_{1}^{\prime}\right)=\operatorname{OVar}\left(S_{2}^{\prime}\right)=\operatorname{OVar}(S)$;

- The output deciding variables in $S_{1}^{\prime}$ are a subset of those in $S_{2}^{\prime}, \operatorname{OVar}\left(S_{1}^{\prime}\right) \subseteq \operatorname{OVar}\left(S_{2}^{\prime}\right)$;

By Lemma 6.5

- There are no inputs translated to enum labels in the set $E$. By assumption.

- When started in states $m_{1}^{\prime}\left(\sigma_{1}^{\prime}\right), m_{2}^{\prime}\left(\sigma_{1}^{\prime}\right)$ where value stores $\sigma_{1}^{\prime}$ and $\sigma_{2}^{\prime}$ agree on values of output deciding variables in both $S_{1}^{\prime}$ and $S_{2}^{\prime}$ as well as the input sequence variable and 
the I/O sequence variable, and there are no inputs translated to enum labels in $E$, then $S_{1}^{\prime}$ and $S_{2}^{\prime}$ produce the same output sequence.

By the induction hypothesis IH. This is because the sum of the program size of $S_{1}^{\prime}$ and $S_{2}^{\prime}$ is less than $k$. By definition, $\operatorname{size}\left(S_{1}\right)=1+\operatorname{size}\left(S_{1}^{\prime}\right)$.

4. $S_{2}=$ "If $(i d==l)$ then $\left\{S_{2}^{t}\right\}$ else $\left\{S_{2}^{f}\right\} "$ such that both of the following hold:

- The label $l$ is in $E, l \in E$;

- The variable $i d$ is not lvalue in an assignment statement, "id :=e" $\notin S_{2}$;

- There are updates of enumeration type extension from $S_{2}^{f}$ to $S_{1}, S_{2}^{f} \approx_{E}^{S} S_{1}$

We informally argue that the value of the variable $i d$ in the predicate expression of $S_{2}$ only coming from an input value or the initial value. There are several ways a scalar variable is defined: the execution of an assignment statement, the execution of an input statement or the initial value. Because $i d$ is not lvalue in an assignment statement, then the value of $i d$ is only from the execution of an input statement or initial value. In addition, by assumption, any used variable is not of initial value of enum label in $E$; no input values are translated into an enum label in E.

Then the expression $i d==l$ evaluates to 0 . The execution of $S_{2}$ proceeds as follows.

$$
\begin{aligned}
& \left(\operatorname{If}(i d==l) \text { then }\left\{S_{2}^{t}\right\} \operatorname{else}\left\{S_{2}^{f}\right\}, m_{2}\left(\sigma_{2}\right)\right) \\
\rightarrow & \left(\operatorname{If}(v==l) \text { then }\left\{S_{2}^{t}\right\} \text { else }\left\{S_{2}^{f}\right\}, m_{2}\left(\sigma_{2}\right)\right) \text { where } v \neq l \\
& \text { by the rule Var } \\
\rightarrow & \left(\operatorname{If}(0) \text { then }\left\{S_{2}^{t}\right\} \text { else }\left\{S_{2}^{f}\right\}, m_{1}\left(\sigma_{2}\right)\right) \\
& \text { by the Eq-F rule } \\
\rightarrow & \left(S_{2}^{f}, m_{2}\left(\sigma_{2}\right)\right) \text { by the If-F rule. }
\end{aligned}
$$

By the hypothesis IH, we show the lemma holds. We need to show the conditions are satisfied for the application of the hypothesis IH.

- $S_{2}^{f} \approx_{E}^{S} S_{1}$

- There are no inputs translated to enum labels in $E$ in $S_{2}^{f}$,s execution.

- Initial values of used variables in $S_{2}$ are not enum labels in E.

The above three conditions are by assumption.

- The sum of the program size of $S_{1}$ and $S_{2}^{f}$ is less than $k$, $\operatorname{size}\left(S_{1}\right)+\operatorname{size}\left(S_{2}^{f}\right)<k$.

By definition, $\operatorname{size}\left(S_{1}\right)=1+\operatorname{size}\left(S_{1}^{t}\right)+\operatorname{size}\left(S_{1}^{f}\right)$. Then, $\operatorname{size}\left(S_{1}\right)+\operatorname{size}\left(S_{2}^{f}\right)<k+1-1-\operatorname{size}\left(S_{2}^{t}\right)<k$.

- Value stores $\sigma_{1}$ and $\sigma_{2}$ agree on values of used variables in both $S_{1}$ and $S_{2}^{f}$ as well as the input, I/O sequence variable. By definition, $\operatorname{OVar}\left(S_{2}^{f}\right) \subseteq \operatorname{OVar}\left(S_{2}\right)$. In addition, value stores $\sigma_{1}$ and $\sigma_{2}$ are not changed in the evaluation of the predicate expression $e$. The condition holds.

By the hypothesis IH, this lemma holds.

5. $S_{1}=S_{1}^{\prime} ; s_{1}$ and $S_{2}=S_{2}^{\prime} ; s_{2}$ such that both of the following hold:

- $S_{2}^{\prime} \approx_{E}^{S} S_{1}^{\prime}$

- $s_{2} \approx_{E}^{S} s_{1}$;

By the hypothesis IH, we show $S_{2}^{\prime}$ and $S_{1}^{\prime}$ terminate in the same way, produce the same output sequence, and have equivalent computation of defined variables in $S_{1}$ and $S_{2}$. We need to show that all the conditions are satisfied for the application of the hypothesis IH.

- $S_{2}^{\prime} \approx_{E}^{S} S_{1}^{\prime}$
- There are no inputs translated to enum labels in $E$ in $S_{2}^{\prime}$ 's execution.

- Initial values of used variables in $S_{2}^{\prime}$ are not enum labels in E.

The above three conditions are by assumption.

- The sum of the program size of $S_{1}^{\prime}$ and $S_{2}^{\prime}$ is less than $k$, $\operatorname{size}\left(S_{1}^{\prime}\right)+\operatorname{size}\left(S_{2}^{\prime}\right)<k$.

By definition, $\operatorname{size}\left(S_{1}\right)=\operatorname{size}\left(S_{1}^{\prime}\right)+\operatorname{size}\left(s_{1}\right)$. Then, $\operatorname{size}\left(S_{1}^{\prime}\right)+\operatorname{size}\left(S_{2}^{\prime}\right)<k+1-\operatorname{size}\left(s_{2}\right)-\operatorname{size}\left(s_{1}\right)<k$.

- Value stores $\sigma_{1}$ and $\sigma_{2}$ agree on values of used variables in both $S_{1}^{\prime}$ and $S_{2}^{\prime}$ as well as the input, output, I/O sequence variable.

By definition, $\mathrm{OVar}\left(S_{2}^{\prime}\right) \subseteq \operatorname{OVar}\left(S_{2}\right), \operatorname{OVar}\left(S_{1}^{\prime}\right) \subseteq \operatorname{OVar}\left(S_{1}\right)$. In addition, value stores $\sigma_{1}$ and $\sigma_{2}$ are not changed in the evaluation of the predicate expression $e$. The condition holds.

By the hypothesis IH, one of the following holds:

(a) $S_{1}^{\prime}$ and $S_{2}^{\prime}$ both do not terminate.

By Lemma E.2 executions of $S_{1}=S_{1}^{\prime} ; s_{1}$ and $S_{2}=$ $S_{2}^{\prime} ; s_{2}$ both do not terminate and produce the same output sequence.

(b) $S_{1}^{\prime}$ and $S_{2}^{\prime}$ both terminate.

By assumption, $\left(S_{2}^{\prime}, m_{2}\left(\sigma_{2}\right)\right) \stackrel{*}{\rightarrow}\left(\right.$ skip,$\left.m_{2}^{\prime}\left(\sigma_{2}^{\prime}\right)\right),\left(S_{1}^{\prime}, m_{1}\left(\sigma_{1}\right)\right) \stackrel{*}{\rightarrow}$ (skip, $\left.m_{1}^{\prime}\left(\sigma_{1}^{\prime}\right)\right)$.

By Corollary E.1 $\left(S_{2}^{\prime} ; s_{2}, m_{2}\left(\sigma_{2}\right)\right) \stackrel{*}{\rightarrow}\left(s_{2}, m_{2}^{\prime}\left(\sigma_{2}^{\prime}\right)\right)$, $\left(S_{1}^{\prime} ; s_{1}, m_{1}\left(\sigma_{1}\right)\right) \stackrel{*}{\rightarrow}\left(s_{1}, m_{1}^{\prime}\left(\sigma_{1}^{\prime}\right)\right)$.

By the hypothesis IH, we show that $s_{2}$ and $s_{1}$ terminate in the same way, produce the same output sequence and when $s_{2}$ and $s_{1}$ both terminate, $s_{2}$ and $s_{1}$ have equivalent computation of variables used or defined in $s_{1}$ and $s_{2}$ and the input, output, and I/O sequence variables.

We need to show that all conditions are satisfied for the application of the hypothesis IH.

- There are updates of "enumeration type extension" between $s_{2}$ and $s_{1}$;

- There are no input values translated into enum labels in $E$ in the execution of $s_{2}$;

The above two conditions are by assumption.

- The sum of the program size $s_{2}$ and $s_{1}$ is less than or equals to $k$; By definition, $\operatorname{size}\left(S_{2}^{\prime}\right) \geq 1, \operatorname{size}\left(S_{1}^{\prime}\right) \geq 1$. Therefore, $\operatorname{size}\left(s_{2}\right)+\operatorname{size}\left(s_{1}\right)<k+1-\operatorname{size}\left(S_{2}^{\prime}\right)-\operatorname{size}\left(S_{1}^{\prime}\right) \leq k$.

- Value stores $\sigma_{1}^{\prime}$ and $\sigma_{2}^{\prime}$ agree on values of used variables in $s_{2}$ and $s_{1}$ as well as the input, output, I/O sequence variable.

By Lemma 6.5 OVar $\left(s_{1}\right) \subseteq \operatorname{OVar}\left(s_{2}\right)$, then $\operatorname{OVar}\left(s_{2}\right)$ $\cap \operatorname{OVar}\left(s_{1}\right)=\operatorname{OVar}\left(s_{1}\right)$. Similarly, by Lemma 6.5 $\operatorname{OVar}\left(S_{1}^{\prime}\right) \subseteq \operatorname{OVar}\left(S_{2}^{\prime}\right)$. For any variable $i d$ in $\operatorname{OVar}\left(s_{1}\right)$, if $i d$ is not in $O \operatorname{Var}\left(S_{1}^{\prime}\right)$, then the value of $i d$ is not changed in the execution of $S_{1}^{\prime}$ and $S_{2}^{\prime}, \sigma_{1}^{\prime}(i d)=$ $\sigma_{1}(i d)=\sigma_{2}(i d)=\sigma_{2}^{\prime}(i d)$. Otherwise, the variable $i d$ is defined in the execution of $S_{1}^{\prime}$ and $S_{2}^{\prime}$, by assumption, $\sigma_{1}^{\prime}(i d)=\sigma_{2}^{\prime}(i d)$. The condition holds.

- Values of used variables in $s_{2}$ are not of value as enum labels in $E, \forall i d \in \operatorname{OVar}\left(s_{2}\right): \sigma_{2}^{\prime}(i d) \in E$.

By assumption, initial values of used variables in $s_{2}$ are not of values as enum labels in $E . S_{2}^{\prime}$ and $S_{1}^{\prime}$ have equivalent computation of defined variables in $S_{2}^{\prime}$ and $S_{1}^{\prime}$. Because enum labels are not defined in $P_{1}$, defined variables in the execution of $S_{2}^{\prime}$ and $S_{1}^{\prime}$ are not of values as enum labels in $E$.

By the hypothesis IH, the lemma holds. 
We show a auxiliary lemma telling that the two programs with updates of enumeration type extension have same set of used variables and the same set of defined variables.

Lemma 6.5. If there are updates of enumeration type extension in a statement sequence $S_{2}$ against a statement sequence $S_{1}$, $S_{2} \approx_{E}^{S} S_{1}$, then the output deciding variables in $S_{1}$ are a subset of those in $S_{2}, O \operatorname{Var}\left(S_{1}\right) \subseteq O \operatorname{Var}\left(S_{2}\right)$.

Proof. By induction on the sum of the program size of $S_{1}$ and $S_{2}$.

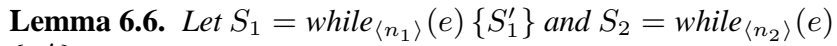
$\left\{S_{2}^{\prime}\right\}$ be two loop statements in programs $P_{1}$ and $P_{2}$ respectively where all of the following hold:

- Enumeration types $E N_{1}$ in $P_{1}$ are a proper subset of $E N_{2}$ in $P_{2}, E N_{1} \subset E N_{2}$, such that there are a set of enum labels $E$ only defined in $P_{2}$;

- When started in states agreeing on values of output deciding variables in both $S_{1}^{\prime}$ and $S_{2}^{\prime}$ as well as the input sequence variable and the $I / O$ sequence variable, initial values of used variables in $S_{2}^{\prime}$ are not enum labels in $E$, and there are no inputs in $S_{2}$ 's execution translated into any label in $E, \forall x \in$ $O \operatorname{Var}\left(S_{1}^{\prime}\right) \cup\left\{i d_{I}, i d_{I O}\right\} \forall m_{1}\left(\sigma_{1}\right) m_{2}\left(\sigma_{2}\right): \sigma_{1}(x)=\sigma_{2}(x)$, and $S_{1}^{\prime}$ and $S_{2}^{\prime}$ terminate in the same way, produce the same output sequence, and have equivalent computation of defined variables in $S_{1}^{\prime}$ and $S_{2}^{\prime}$ as well as the input sequence variable and the I/O sequence variable $\left(\left(S_{1}^{\prime}, m_{1}\right) \equiv_{H}\left(S_{2}^{\prime}, m_{2}\right)\right) \wedge$ $\left(\left(S_{1}^{\prime}, m_{1}\right) \equiv_{O}\left(S_{2}^{\prime}, m_{2}\right)\right) \wedge\left(\forall x \in \operatorname{OVar}\left(S_{1}\right) \cup \operatorname{OVar}\left(S_{2}\right) \cup\right.$ $\left\{i d_{I}, i d_{I O}\right\}$ :

$\left.\left(S_{1}^{\prime}, m_{1}\right) \equiv_{x}\left(S_{2}^{\prime}, m_{2}\right)\right)$;

If $S_{1}$ and $S_{2}$ start in states $m_{1}\left(\operatorname{loop}_{c}^{1}, \sigma_{1}\right), m_{2}\left(\operatorname{loop}_{c}^{2}, \sigma_{2}\right)$, with loop counters of $S_{1}$ and $S_{2}$ not initialized $\left(S_{1}, S_{2}\right.$ have not executed yet), value stores agree on values of output deciding variables in $S_{1}$ and $S_{2}$ as well as the input sequence variable, the I/O sequence variable, initial values of used variables in $S_{2}$ are not of values as enum labels in $E$, no inputs are translated into enum labels in $E$, then, for any positive integer $i$, one of the following holds:

1. Loop counters for $S_{1}$ and $S_{2}$ are always less than $i$ if any is present, $\forall m_{1}^{\prime}\left(\operatorname{loop}_{c}^{1^{\prime}}\right) m_{2}^{\prime}\left(\operatorname{loop}_{c}^{2^{\prime}}\right):\left(S_{1}, m_{1}\left(\operatorname{loop}_{c}^{1}, \sigma_{1}\right)\right) \stackrel{*}{\rightarrow}$ $\left(S_{1}^{\prime \prime}, m_{1}^{\prime}\left(\operatorname{loop}_{c}^{1^{\prime}}\right)\right), \operatorname{loop}_{c}^{1^{\prime}}\left(n_{1}\right)<i,\left(S_{2}, m_{2}\left(\operatorname{loop}_{c}^{2}, \sigma_{2}\right)\right) \stackrel{*}{\rightarrow}$ $\left(S_{2}^{\prime \prime}, m_{2}^{\prime}\left(\right.\right.$ loop $\left.\left._{c}^{2^{\prime}}\right)\right)$, loop $p_{c}^{2^{\prime}}\left(n_{2}\right)<i, S_{1}$ and $S_{2}$ terminate in the same way, produce the same output sequence, and have equivalent computation of output deciding variables in both $S_{1}$ and $S_{2}$ and the input sequence variable, the $I / O$ sequence variable, $\left(S_{1}, m_{1}\right) \equiv_{H}\left(S_{2}, m_{2}\right)$ and $\left(S_{1}, m_{1}\right) \equiv_{O}\left(S_{2}, m_{2}\right)$ and $\forall x \in\left(O \operatorname{Var}\left(S_{1}\right) \cup \operatorname{OVar}\left(S_{2}\right)\right) \cup\left\{i d_{I}, i d_{I O}\right\}:\left(S_{1}, m_{1}\right) \equiv_{x}$ $\left(S_{2}, m_{2}\right)$;

2. The loop counter of $S_{1}$ and $S_{2}$ are of value less than or equal to $i$, and there are no reachable configurations $\left(S_{1}, m_{1}\left(\operatorname{loop}_{c}^{1_{i}}, \sigma_{1_{i}}\right)\right)$ from $\left(S_{1}, m_{1}\left(\sigma_{1}\right)\right),\left(S_{2}, m_{2}\left(\operatorname{loop}_{c}^{2_{i}}, \sigma_{2_{i}}\right)\right)$ from $\left(S_{2}, m_{2}\left(\sigma_{2}\right)\right)$ where all of the following hold:

- The loop counters of $S_{1}$ and $S_{2}$ are of value $i$, $\operatorname{loop}_{c}^{1_{i}}\left(n_{1}\right)$ $=\operatorname{loop}_{c}^{2_{i}}\left(n_{2}\right)=i$.

- Value stores $\sigma_{1_{i}}$ and $\sigma_{2_{i}}$ agree on values of output deciding variables in both $S_{1}$ and $S_{2}$ as well as the input sequence variable and the I/O sequence variable, $\forall x \in\left(\operatorname{OVar}\left(S_{1}\right) \cap\right.$ $\left.O \operatorname{Var}\left(S_{2}\right)\right) \cup\left\{i d_{I}, i d_{I O}\right\}: \sigma_{1_{i}}(x)=\sigma_{2_{i}}(x)$.

3. There are reachable configurations $\left(S_{1}, m_{1}\left(\right.\right.$ loop $\left.\left._{c}^{1_{i}}, \sigma_{1_{i}}\right)\right)$ from $\left(S_{1}, m_{1}\left(\sigma_{1}\right)\right),\left(S_{2}, m_{2}\left(\operatorname{loop}_{c}^{2_{i}}, \sigma_{2_{i}}\right)\right)$ from $\left(S_{2}, m_{2}\left(\sigma_{2}\right)\right)$ where all of the following hold:

- The loop counter of $S_{1}$ and $S_{2}$ are of value $i$, $\operatorname{loop}_{c}^{1_{i}}\left(n_{1}\right)$ $=\operatorname{loop}_{c}^{2_{i}}\left(n_{2}\right)=i$.
- Value stores $\sigma_{1_{i}}$ and $\sigma_{2_{i}}$ agree on values of output deciding variables in both $S_{1}$ and $S_{2}$ as well as the input sequence variable and the I/O sequence variable, $\forall x \in\left(\operatorname{OVar}\left(S_{1}\right) \cap\right.$ $\left.O \operatorname{Var}\left(S_{2}\right)\right) \cup\left\{i d_{I}, i d_{I O}\right\}: \sigma_{1_{i}}(x)=\sigma_{2_{i}}(x)$.

Proof. By induction on $i$.

Base case.

We show that, when $i=1$, one of the following holds:

1. Loop counters for $S_{1}$ and $S_{2}$ are always less than 1 if any is present, $\forall m_{1}^{\prime}\left(\operatorname{loop}_{c}^{1^{\prime}}\right) m_{2}^{\prime}\left(\operatorname{loop}_{c}^{2^{\prime}}\right):\left(S_{1}, m_{1}\left(\operatorname{loop}_{c}^{1}, \sigma_{1}\right)\right) \stackrel{*}{\rightarrow}$ $\left(S_{1}^{\prime \prime}, m_{1}^{\prime}\left(\operatorname{loop}_{c}^{1^{\prime}}\right)\right), \operatorname{loop}_{c}^{1^{\prime}}\left(n_{1}\right)<i,\left(S_{2}, m_{2}\left(\operatorname{loop}_{c}^{2}, \sigma_{2}\right)\right) \stackrel{*}{\rightarrow}$ $\left(S_{2}^{\prime \prime}, m_{2}^{\prime}\left(\operatorname{loop}_{c}^{2^{\prime}}\right)\right)$, $\operatorname{loop}_{c}^{2^{\prime}}\left(n_{2}\right)<i, S_{1}$ and $S_{2}$ terminate in the same way, produce the same output sequence, and have equivalent computation of defined variables in both $S_{1}$ and $S_{2}$ and the input sequence variable, the I/O sequence variable, $\left(S_{1}, m_{1}\right) \equiv_{H}\left(S_{2}, m_{2}\right)$ and $\left(S_{1}, m_{1}\right) \equiv_{O}\left(S_{2}, m_{2}\right)$ and $\forall x \in$ $\left(\operatorname{Def}\left(S_{1}\right) \cap \operatorname{Def}\left(S_{2}\right)\right) \cup\left\{i d_{I}, i d_{I O}\right\}:\left(S_{1}, m_{1}\right) \equiv_{x}\left(S_{2}, m_{2}\right)$;

2. Loop counters of $S_{1}$ and $S_{2}$ are of value less than or equal to 1 but there are no reachable configurations $\left(S_{1}, m_{1}\left(\operatorname{loop}_{c}^{1_{1}}, \sigma_{1_{i}}\right)\right)$ from $\left(S_{1}, m_{1}\left(\sigma_{1}\right)\right),\left(S_{2}, m_{2}\left(\operatorname{loop}_{c}^{21}, \sigma_{2_{i}}\right)\right)$ from $\left(S_{2}, m_{2}\left(\sigma_{2}\right)\right)$ where all of the following hold:

- The loop counter of $S_{1}$ and $S_{2}$ are of value 1 , $\operatorname{loop}_{c}^{1_{1}}\left(n_{1}\right)$ $=\operatorname{loop}_{c}^{2_{1}}\left(n_{2}\right)=1$.

- Value stores $\sigma_{1_{1}}$ and $\sigma_{2_{1}}$ agree on values of used variables in both $S_{1}$ and $S_{2}$ as well as the input sequence variable, and the I/O sequence variable, $\forall x \in\left(\operatorname{Use}\left(S_{1}\right) \cap \operatorname{Use}\left(S_{2}\right)\right) \cup$ $\left\{i d_{I}, i d_{I O}\right\}: \sigma_{1_{1}}(x)=\sigma_{2_{1}}(x)$.

3. There are reachable configuration $\left(S_{1}, m_{1}\left(\operatorname{loop}_{c}^{1_{1}}, \sigma_{1_{i}}\right)\right)$ from $\left(S_{1}, m_{1}\left(\sigma_{1}\right)\right),\left(S_{2}, m_{2}\left(\operatorname{loop}_{c}^{21}, \sigma_{2_{i}}\right)\right)$ from $\left(S_{2}, m_{2}\left(\sigma_{2}\right)\right)$ where all of the following hold:

- The loop counter of $S_{1}$ and $S_{2}$ are of value $1, \operatorname{loop}_{c}^{1_{1}}\left(n_{1}\right)$ $=\operatorname{loop}_{c}^{2_{1}}\left(n_{2}\right)=1$.

- Value stores $\sigma_{1_{1}}$ and $\sigma_{2_{1}}$ agree on values of used variables in both $S_{1}$ and $S_{2}$ as well as the input sequence variable, and the I/O sequence variable, $\forall x \in\left(\operatorname{Use}\left(S_{1}\right) \cap \operatorname{Use}\left(S_{2}\right)\right) \cup$ $\left\{i d_{I}, i d_{I O}\right\}: \sigma_{1_{1}}(x)=\sigma_{2_{1}}(x)$.

By definition, variables used in the predicate expression $e$ of $S_{1}$ and $S_{2}$ are used in $S_{1}$ and $S_{2}$, Use $(e) \subseteq \operatorname{Use}\left(S_{1}\right) \cap \operatorname{Use}\left(S_{2}\right)$. By assumption, value stores $\sigma_{1}$ and $\sigma_{2}$ agree on values of variables in $\mathrm{Use}(e)$, the predicate expression $e$ evaluates to the same value w.r.t value stores $\sigma_{1}$ and $\sigma_{2}$ by LemmaD.2 There are three possibilities.

1. The evaluation of $e$ crashes,

$\mathcal{E}^{\prime} \llbracket e \rrbracket \sigma_{1}=\mathcal{E}^{\prime} \llbracket e \rrbracket \sigma_{2}=\left(\right.$ error, $\left.v_{\mathfrak{o f}}\right)$.

The execution of $S_{1}$ continues as follows:

$$
\begin{aligned}
& \left(\text { while }_{\left\langle n_{1}\right\rangle}(e)\left\{S_{1}^{\prime}\right\}, m_{1}\left(\sigma_{1}\right)\right) \\
\rightarrow & (\text { while } \\
\left\langle n_{1}\right\rangle & \left.\left(\left(\text { error, } v_{\mathfrak{o f}}\right)\right)\left\{S_{1}^{\prime}\right\}, m_{1}\left(\sigma_{1}\right)\right) \\
& \text { by the rule EEval' } \\
\rightarrow & (\text { while } \\
& \text { by the ECrash rule } \\
& \left.(0)\left\{S_{1}^{\prime}\right\}, m_{1}(1 / \mathfrak{f})\right) \\
\rightarrow & (\text { while } \\
& \text { by the Crash rule. }
\end{aligned}
$$

Similarly, the execution of $S_{2}$ started from the state $m_{2}\left(\sigma_{2}\right)$ crashes. Therefore $S_{1}$ and $S_{2}$ terminate in the same way when started from $m_{1}$ and $m_{2}$ respectively. Because $\sigma_{1}\left(i d_{I O}\right)=$ $\sigma_{2}\left(i d_{I O}\right)$, the lemma holds.

2. The evaluation of $e$ reduces to zero, $\mathcal{E}^{\prime} \llbracket e \rrbracket \sigma_{1}=\mathcal{E}^{\prime} \llbracket e \rrbracket \sigma_{2}=$ $\left(0, v_{\mathfrak{o f}}\right)$.

The execution of $S_{1}$ continues as follows. 


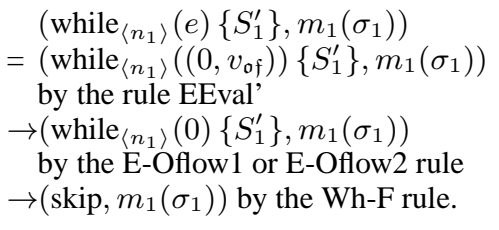

Similarly, the execution of $S_{2}$ gets to the configuration (skip, $m_{2}\left(\sigma_{2}\right)$ ). Loop counters of $S_{1}$ and $S_{2}$ are less than 1 and value stores agree on values of used/defined variables in both $S_{1}$ and $S_{2}$ as well as the input sequence variable, and the $\mathrm{I} / \mathrm{O}$ sequence variable.

3. The evaluation of $e$ reduces to the same nonzero integer value, $\mathcal{E}^{\prime} \llbracket e \rrbracket \sigma_{1}=\mathcal{E}^{\prime} \llbracket e \rrbracket \sigma_{2}=\left(0, v_{\mathfrak{o f}}\right)$.

Then the execution of $S_{1}$ proceeds as follows:

$$
\begin{aligned}
& \left(\text { while }_{\left\langle n_{1}\right\rangle}(e)\left\{S_{1}^{\prime}\right\}, m_{1}\left(\sigma_{1}\right)\right) \\
= & \left(\text { while }_{\left\langle n_{1}\right\rangle}\left(\left(v, v_{\text {of }}\right)\right)\left\{S_{1}^{\prime}\right\}, m_{1}\left(\sigma_{1}\right)\right) \\
& \text { by the rule EEval' } \\
\rightarrow & (\text { while } \\
& \text { by the E-Oflow } 1 \text { or E-Oflow } 2 \text { rule } \\
\rightarrow & \left.\left(S_{1}^{\prime} ; S_{1}^{\prime}\right\}, m_{1}\left(\sigma_{1}\right)\right) \\
& \left.\left.\operatorname{loop}_{c}^{1}\left[1 / n_{1}\right], \sigma_{1}\right)\right) \text { by the Wh-T rule. }
\end{aligned}
$$

Similarly, the execution of $S_{2}$ proceeds to the configuration $\left(S_{2}^{\prime} ;\right.$ while $\left._{\left\langle n_{2}\right\rangle}(e)\left\{S_{2}^{\prime}\right\}, m_{2}\left(\operatorname{loop}_{c}^{2}\left[1 / n_{1}\right], \sigma_{2}\right)\right)$.

By the assumption, we show that $S_{1}^{\prime}$ and $S_{2}^{\prime}$ terminate in the same way and produce the same output sequence when started in the state $m_{1}\left(\operatorname{loop}_{c}^{1_{1}}, \sigma_{1}\right)$ and $m_{2}\left(\operatorname{loop}_{c}^{21}, \sigma_{2}\right)$ respectively, and $S_{1}^{\prime}$ and $S_{2}^{\prime}$ have equivalent computation of variables defined in both statement sequences if both terminate. We need to show that all conditions are satisfied for the application of the assumption.

- There are no inputs translated into enum labels in $E$ in the execution of $S_{2}^{\prime}$.

The above condition is by assumption.

- Initial values of used variables in $S_{2}^{\prime}$ are not enum labels in E.

By the definition of used variables, Use $\left(S_{2}^{\prime}\right) \subseteq \operatorname{Use}\left(S_{2}\right)$. By assumption, initial values of used variables in $S_{2}$ are not enum labels in $E$. The condition holds.

- Value stores $\sigma_{1}$ and $\sigma_{2}$ agree on values of used variables in $S_{1}^{\prime}$ and $S_{2}^{\prime}$ as well as the input, output, I/O sequence variable.

By definition, $\operatorname{Use}\left(S_{1}^{\prime}\right) \subseteq \operatorname{Use}\left(S_{1}\right)$. So are the cases to $S_{2}^{\prime}$ and $S_{2}$. In addition, value stores $\sigma_{1}$ and $\sigma_{2}$ are not changed in the evaluation of the predicate expression $e$. The condition holds.

By assumption, $S_{1}^{\prime}$ and $S_{2}^{\prime}$ terminate in the same way and produce the same output sequence when started in states $m_{1}\left(\operatorname{loop}_{c}^{\prime}, \sigma_{1}\right)$ and $m_{2}\left(\operatorname{loop}_{c}^{\prime}, \sigma_{2}\right)$. In addition, $S_{1}^{\prime}$ and $S_{2}^{\prime}$ have equivalent computation of variables used or defined in $S_{1}^{\prime}$ and $S_{2}^{\prime}$ when started in states $m_{1}\left(\operatorname{loop}_{c}^{\prime}, \sigma_{1}\right)$ and $m_{2}\left(\operatorname{loop}_{c}^{\prime}, \sigma_{2}\right)$.

Then there are two cases.

(a) $S_{1}^{\prime}$ and $S_{2}^{\prime}$ both do not terminate and produce the same output sequence.

By Lemma E.2. $S_{1}^{\prime} ; S_{1}$ and $S_{2}^{\prime} ; S_{2}$ both do not terminate and produce the same output sequence.

(b) $S_{1}^{\prime}$ and $S_{2}^{\prime}$ both terminate and have equivalent computation of variables defined in $S_{1}^{\prime}$ and $S_{2}^{\prime}$.

By assumption, $\left(S_{1}^{\prime}, m_{1}\left(\operatorname{loop}_{c}^{\prime}, \sigma_{1}\right)\right) \stackrel{*}{\rightarrow}\left(\right.$ skip, $\left.m_{1}^{\prime}\left(\operatorname{loop}_{c}^{\prime \prime}, \sigma_{1}^{\prime}\right)\right)$;

$\left(S_{2}^{\prime}, m_{2}\left(\operatorname{loop}_{c}^{\prime}, \sigma_{2}\right)\right) \stackrel{*}{\rightarrow}$ (skip, $\left.m_{2}^{\prime}\left(\operatorname{loop}_{c}^{\prime \prime}, \sigma_{2}^{\prime}\right)\right)$ where $\forall x \in$ $\left(\operatorname{Def}\left(S_{1}^{\prime}\right) \cap \operatorname{Def}\left(S_{2}^{\prime}\right)\right) \cup\left\{i d_{I}, i d_{I O}\right\}, \sigma_{1}^{\prime}(x)=\sigma_{2}^{\prime}(x)$. By assumption, $\operatorname{Use}\left(S_{1}^{\prime}\right) \subseteq \operatorname{Use}\left(S_{2}^{\prime}\right)$ and $\operatorname{Def}\left(S_{1}^{\prime}\right)=$ $\operatorname{Def}\left(S_{2}^{\prime}\right)$. Then variables used in the predicate expression of $S_{1}$ and $S_{2}$ are either in variables used or defined in both $S_{1}^{\prime}$ and $S_{2}^{\prime}$ or not. Therefore value stores $\sigma_{2}^{\prime}$ and $\sigma_{1}^{\prime}$ agree on values of variables used in the expression $e$ and even variables used or defined in $S_{1}$ and $S_{2}$.

Induction step on iterations

The induction hypothesis $(\mathrm{IH})$ is that, when $i \geq 1$, one of the following holds:

1. Loop counters for $S_{1}$ and $S_{2}$ are always less than $i$ if any is present, $\forall m_{1}^{\prime}\left(\operatorname{loop}_{c}^{1^{\prime}}\right) m_{2}^{\prime}\left(\operatorname{loop}_{c}^{2^{\prime}}\right):\left(S_{1}, m_{1}\left(\operatorname{loop}_{c}^{1}, \sigma_{1}\right)\right) \stackrel{*}{\rightarrow}$ $\left(S_{1}^{\prime \prime}, m_{1}^{\prime}\left(\operatorname{loop}_{c}^{1^{\prime}}\right)\right), \operatorname{loop}_{c}^{1^{\prime}}\left(n_{1}\right)<i,\left(S_{2}, m_{2}\left(\operatorname{loop}_{c}^{2}, \sigma_{2}\right)\right) \stackrel{*}{\rightarrow}$ $\left(S_{2}^{\prime \prime}, m_{2}^{\prime}\left(\operatorname{loop}_{c}^{2^{\prime}}\right)\right), \operatorname{loop}_{c}^{2^{\prime}}\left(n_{2}\right)<i, S_{1}$ and $S_{2}$ terminate in the same way, produce the same output sequence, and have equivalent computation of used/defined variables in both $S_{1}$ and $S_{2}$ and the input sequence variable, the I/O sequence variable, $\left(S_{1}, m_{1}\right) \equiv_{H}\left(S_{2}, m_{2}\right)$ and $\left(S_{1}, m_{1}\right) \equiv_{O}\left(S_{2}, m_{2}\right)$ and $\forall x \in$ $\left(\operatorname{Def}\left(S_{1}\right) \cap \operatorname{Def}\left(S_{2}\right)\right) \cup\left\{i d_{I}, i d_{I O}\right\}:\left(S_{1}, m_{1}\right) \equiv_{x}\left(S_{2}, m_{2}\right)$;

2. The loop counter of $S_{1}$ and $S_{2}$ are of value less than or equal to $i$, and there are no reachable configurations $\left(S_{1}, m_{1}\left(\operatorname{loop}_{c}^{1_{i}}, \sigma_{1_{i}}\right)\right)$ from $\left(S_{1}, m_{1}\left(\sigma_{1}\right)\right),\left(S_{2}, m_{2}\left(\operatorname{loop}_{c}^{2_{i}}, \sigma_{2_{i}}\right)\right)$ from $\left(S_{2}, m_{2}\left(\sigma_{2}\right)\right)$ where all of the following hold:

- The loop counters of $S_{1}$ and $S_{2}$ are of value $i, \operatorname{loop}_{c}^{1_{i}}\left(n_{1}\right)$ $=\operatorname{loop}_{c}^{2_{i}}\left(n_{2}\right)=i$.

- Value stores $\sigma_{1_{i}}$ and $\sigma_{2_{i}}$ agree on values of used variables in both $S_{1}$ and $S_{2}$ as well as the input sequence variable, and the I/O sequence variable, $\forall x \in\left(\operatorname{Use}\left(S_{1}\right) \cap \operatorname{Use}\left(S_{2}\right)\right) \cup$ $\left\{i d_{I}, i d_{I O}\right\}: \sigma_{1_{i}}(x)=\sigma_{2_{i}}(x)$.

3. There are reachable configurations $\left(S_{1}, m_{1}\left(\operatorname{loop}_{c}^{1_{i}}, \sigma_{1_{i}}\right)\right)$ from $\left(S_{1}, m_{1}\left(\sigma_{1}\right)\right),\left(S_{2}, m_{2}\left(\operatorname{loop}_{c}^{2 i}, \sigma_{2_{i}}\right)\right)$ from $\left(S_{2}, m_{2}\left(\sigma_{2}\right)\right)$ where all of the following hold:

- The loop counter of $S_{1}$ and $S_{2}$ are of value $i, \operatorname{loop}_{c}^{1_{i}}\left(n_{1}\right)$ $=\operatorname{loop}_{c}^{2_{i}}\left(n_{2}\right)=i$.

- Value stores $\sigma_{1_{i}}$ and $\sigma_{2_{i}}$ agree on values of used variables in both $S_{1}$ and $S_{2}$ as well as the input sequence variable, and the I/O sequence variable, $\forall x \in\left(\operatorname{Use}\left(S_{1}\right) \cap \operatorname{Use}\left(S_{2}\right)\right) \cup$ $\left\{i d_{I}, i d_{I O}\right\}: \sigma_{1_{i}}(x)=\sigma_{2_{i}}(x)$.

Then we show that, when $i+1$, one of the following holds: The induction hypothesis (IH) is that, when $i \geq 1$, one of the following holds:

1. Loop counters for $S_{1}$ and $S_{2}$ are always less than $i+1$ if any is present, $\forall m_{1}^{\prime}\left(\operatorname{loop}_{c}^{1^{\prime}}\right) m_{2}^{\prime}\left(\operatorname{loop}_{c}^{2^{\prime}}\right):\left(S_{1}, m_{1}\left(\operatorname{loop}_{c}^{1}, \sigma_{1}\right)\right) \stackrel{*}{\rightarrow}$ $\left(S_{1}^{\prime \prime}, m_{1}^{\prime}\left(\operatorname{loop}_{c}^{1^{\prime}}\right)\right), \operatorname{loop}_{c}^{1^{\prime}}\left(n_{1}\right)<i+1,\left(S_{2}, m_{2}\left(\operatorname{loop}_{c}^{2}, \sigma_{2}\right)\right) \stackrel{*}{\rightarrow}$ $\left(S_{2}^{\prime \prime}, m_{2}^{\prime}\left(\operatorname{loop}_{c}^{2^{\prime}}\right)\right), \operatorname{loop}_{c}^{2^{\prime}}\left(n_{2}\right)<i+1, S_{1}$ and $S_{2}$ terminate in the same way, produce the same output sequence, and have equivalent computation of used/defined variables in both $S_{1}$ and $S_{2}$ and the input sequence variable, the I/O sequence variable, $\left(S_{1}, m_{1}\right) \equiv_{H}\left(S_{2}, m_{2}\right)$ and $\left(S_{1}, m_{1}\right) \equiv_{O}\left(S_{2}, m_{2}\right)$ and $\forall x \in$ $\left(\operatorname{Def}\left(S_{1}\right) \cap \operatorname{Def}\left(S_{2}\right)\right) \cup\left\{i d_{I}, i d_{I O}\right\}:\left(S_{1}, m_{1}\right) \equiv_{x}\left(S_{2}, m_{2}\right)$;

2. The loop counter of $S_{1}$ and $S_{2}$ are of value less than or equal to $i+1$, and there are no reachable configurations $\left(S_{1}, m_{1}\left(\operatorname{loop}_{c}^{1_{i+1}}, \sigma_{1_{i+1}}\right)\right)$ from $\left(S_{1}, m_{1}\left(\sigma_{1}\right)\right)$,

$\left(S_{2}, m_{2}\left(\operatorname{loop}_{c}^{2_{i+1}}, \sigma_{2_{i+1}}\right)\right)$ from $\left(S_{2}, m_{2}\left(\sigma_{2}\right)\right)$ where all of the following hold:

- The loop counters of $S_{1}$ and $S_{2}$ are of value $i+1$, $\operatorname{loop}_{c}^{1_{i+1}}\left(n_{1}\right)=\operatorname{loop}_{c}^{2_{i+1}}\left(n_{2}\right)=i+1$.

- Value stores $\sigma_{1_{i+1}}$ and $\sigma_{2_{i+1}}$ agree on values of used variables in both $S_{1}$ and $S_{2}$ as well as the input sequence variable, and the I/O sequence variable, $\forall x \in\left(\operatorname{Use}\left(S_{1}\right) \cap\right.$ $\left.\operatorname{Use}\left(S_{2}\right)\right) \cup\left\{i d_{I}, i d_{I O}\right\}: \sigma_{1_{i+1}}(x)=\sigma_{2_{i+1}}(x)$. 
3. There are reachable configurations $\left(S_{1}, m_{1}\left(\operatorname{loop}_{c}^{1_{i+1}}, \sigma_{1_{i}}\right)\right)$ from $\left(S_{1}, m_{1}\left(\sigma_{1}\right)\right),\left(S_{2}, m_{2}\left(\operatorname{loop}_{c}^{2 i+1}, \sigma_{2_{i}}\right)\right)$ from $\left(S_{2}, m_{2}\left(\sigma_{2}\right)\right)$ where all of the following hold:

- The loop counter of $S_{1}$ and $S_{2}$ are of value $i$, loop ${ }_{c}^{1_{i+1}}\left(n_{1}\right)$ $=\operatorname{loop}_{c}^{2 i+1}\left(n_{2}\right)=i+1$.

- Value stores $\sigma_{1_{i+1}}$ and $\sigma_{2_{i+1}}$ agree on values of used variables in both $S_{1}$ and $S_{2}$ as well as the input sequence variable, and the I/O sequence variable, $\forall x \in\left(\operatorname{Use}\left(S_{1}\right) \cap\right.$ $\left.\operatorname{Use}\left(S_{2}\right)\right) \cup\left\{i d_{I}, i d_{I O}\right\}: \sigma_{1_{i+1}}(x)=\sigma_{2_{i+1}}(x)$.

By hypothesis $\mathrm{IH}$, there is no configuration where loop counters of $S_{1}$ and $S_{2}$ are of value $i+1$ when any of the following holds:

1. Loop counters for $S_{1}$ and $S_{2}$ are always less than $i$ if any is present, $\forall m_{1}^{\prime}\left(\operatorname{loop}_{c}^{1^{\prime}}\right) m_{2}^{\prime}\left(\operatorname{loop}_{c}^{2^{\prime}}\right):\left(S_{1}, m_{1}\left(\operatorname{loop}_{c}^{1}, \sigma_{1}\right)\right) \stackrel{*}{\rightarrow}$ $\left(S_{1}^{\prime \prime}, m_{1}^{\prime}\left(\operatorname{loop}_{c}^{1^{\prime}}\right)\right), \operatorname{loop}_{c}^{1^{\prime}}\left(n_{1}\right)<i,\left(S_{2}, m_{2}\left(\operatorname{loop}_{c}^{2}, \sigma_{2}\right)\right) \stackrel{*}{\rightarrow}$ $\left(S_{2}^{\prime \prime}, m_{2}^{\prime}\left(\operatorname{loop}_{c}^{2^{\prime}}\right)\right), \operatorname{loop}_{c}^{2^{\prime}}\left(n_{2}\right)<i, S_{1}$ and $S_{2}$ terminate in the same way, produce the same output sequence, and have equivalent computation of used/defined variables in both $S_{1}$ and $S_{2}$ and the input sequence variable, the I/O sequence variable, $\left(S_{1}, m_{1}\right) \equiv_{H}\left(S_{2}, m_{2}\right)$ and $\left(S_{1}, m_{1}\right) \equiv_{O}\left(S_{2}, m_{2}\right)$ and $\forall x \in$ $\left(\operatorname{Def}\left(S_{1}\right) \cap \operatorname{Def}\left(S_{2}\right)\right) \cup\left\{i d_{I}, i d_{I O}\right\}:\left(S_{1}, m_{1}\right) \equiv_{x}\left(S_{2}, m_{2}\right)$;

2. The loop counter of $S_{1}$ and $S_{2}$ are of value less than or equal to $i$, and there are no reachable configurations $\left(S_{1}, m_{1}\left(\operatorname{loop}_{c}^{1_{i}}, \sigma_{1_{i}}\right)\right)$ from $\left(S_{1}, m_{1}\left(\sigma_{1}\right)\right),\left(S_{2}, m_{2}\left(\operatorname{loop}_{c}^{2_{i}}, \sigma_{2_{i}}\right)\right)$ from $\left(S_{2}, m_{2}\left(\sigma_{2}\right)\right)$ where all of the following hold:

- The loop counters of $S_{1}$ and $S_{2}$ are of value $i, \operatorname{loop}_{c}^{1_{i}}\left(n_{1}\right)$ $=\operatorname{loop}_{c}^{2_{i}}\left(n_{2}\right)=i$.

- Value stores $\sigma_{1_{i}}$ and $\sigma_{2_{i}}$ agree on values of used variables in both $S_{1}$ and $S_{2}$ as well as the input sequence variable, and the I/O sequence variable, $\forall x \in\left(\mathrm{Use}\left(S_{1}\right) \cap \mathrm{Use}\left(S_{2}\right)\right) \cup$ $\left\{i d_{I}, i d_{I O}\right\}: \sigma_{1_{i}}(x)=\sigma_{2_{i}}(x)$.

When there are reachable configurations $\left(S_{1}, m_{1}\left(\operatorname{loop}_{c}^{1_{i}}, \sigma_{1_{i}}\right)\right)$ from $\left(S_{1}, m_{1}\left(\sigma_{1}\right)\right),\left(S_{2}, m_{2}\left(\operatorname{loop}_{c}^{2_{i}}, \sigma_{2_{i}}\right)\right)$ from $\left(S_{2}, m_{2}\left(\sigma_{2}\right)\right)$ where all of the following hold:

- The loop counter of $S_{1}$ and $S_{2}$ are of value $i$, $\operatorname{loop}_{c}^{1_{i}}\left(n_{1}\right)$ $=\operatorname{loop}_{c}^{2 i}\left(n_{2}\right)=i$.

- Value stores $\sigma_{1_{i}}$ and $\sigma_{2_{i}}$ agree on values of used variables in both $S_{1}$ and $S_{2}$ as well as the input sequence variable, and the I/O sequence variable, $\forall x \in\left(\mathrm{Use}\left(S_{1}\right) \cap \operatorname{Use}\left(S_{2}\right)\right) \cup$ $\left\{i d_{I}, i d_{I O}\right\}: \sigma_{1_{i}}(x)=\sigma_{2_{i}}(x)$. holds:

By similar argument in base case, we have one of the following

1. Loop counters for $S_{1}$ and $S_{2}$ are always less than $i+1$ if any is present, $\forall m_{1}^{\prime}\left(\operatorname{loop}_{c}^{1^{\prime}}\right) m_{2}^{\prime}\left(\operatorname{loop}_{c}^{2^{\prime}}\right):\left(S_{1}, m_{1}\left(\operatorname{loop}_{c}^{1}, \sigma_{1}\right)\right) \stackrel{*}{\rightarrow}$ $\left(S_{1}^{\prime \prime}, m_{1}^{\prime}\left(\operatorname{loop}_{c}^{1^{\prime}}\right)\right), \operatorname{loop}_{c}^{1^{\prime}}\left(n_{1}\right)<i+1,\left(S_{2}, m_{2}\left(\operatorname{loop}_{c}^{2}, \sigma_{2}\right)\right) \stackrel{*}{\rightarrow}$ $\left(S_{2}^{\prime \prime}, m_{2}^{\prime}\left(\operatorname{loop}_{c}^{2^{\prime}}\right)\right), \operatorname{loop}_{c}^{2^{\prime}}\left(n_{2}\right)<i, S_{1}$ and $S_{2}$ terminate in the same way, produce the same output sequence, and have equivalent computation of used/defined variables in both $S_{1}$ and $S_{2}$ and the input sequence variable, the I/O sequence variable, $\left(S_{1}, m_{1}\right) \equiv_{H}\left(S_{2}, m_{2}\right)$ and $\left(S_{1}, m_{1}\right) \equiv_{O}\left(S_{2}, m_{2}\right)$ and $\forall x \in$ $\left(\operatorname{Def}\left(S_{1}\right) \cap \operatorname{Def}\left(S_{2}\right)\right) \cup\left\{i d_{I}, i d_{I O}\right\}:\left(S_{1}, m_{1}\right) \equiv_{x}\left(S_{2}, m_{2}\right)$;

2. The loop counter of $S_{1}$ and $S_{2}$ are of value less than or equal to $i+1$, and there are no reachable configurations $\left(S_{1}, m_{1}\left(\operatorname{loop}_{c}^{1_{i}}, \sigma_{1_{i}}\right)\right)$ from $\left(S_{1}, m_{1}\left(\sigma_{1}\right)\right),\left(S_{2}, m_{2}\left(\operatorname{loop}_{c}^{2_{i}}, \sigma_{2_{i}}\right)\right)$ from $\left(S_{2}, m_{2}\left(\sigma_{2}\right)\right)$ where all of the following hold:

- The loop counters of $S_{1}$ and $S_{2}$ are of value $i$, loop ${ }_{c}^{1_{i+1}}\left(n_{1}\right)$ $=\operatorname{loop}_{c}^{2_{i+1}}\left(n_{2}\right)=i+1$.
- Value stores $\sigma_{1_{i+1}}$ and $\sigma_{2_{i+1}}$ agree on values of used variables in both $S_{1}$ and $S_{2}$ as well as the input sequence variable, and the I/O sequence variable, $\forall x \in\left(\operatorname{Use}\left(S_{1}\right) \cap\right.$ $\left.\operatorname{Use}\left(S_{2}\right)\right) \cup\left\{i d_{I}, i d_{I O}\right\}: \sigma_{1_{i+1}}(x)=\sigma_{2_{i+1}}(x)$.

3. There are reachable configurations $\left(S_{1}, m_{1}\left(\operatorname{loop}_{c}^{1_{i+1}}, \sigma_{1_{i+1}}\right)\right)$ from $\left(S_{1}, m_{1}\left(\sigma_{1}\right)\right),\left(S_{2}, m_{2}\left(\operatorname{loop}_{c}^{2 i+1}, \sigma_{2_{i+1}}\right)\right)$ from $\left(S_{2}\right.$, $\left.m_{2}\left(\sigma_{2}\right)\right)$ where all of the following hold:

- The loop counter of $S_{1}$ and $S_{2}$ are of value $i, \operatorname{loop}_{c}^{1_{i+1}}\left(n_{1}\right)$ $=\operatorname{loop}_{c}^{2_{i+1}}\left(n_{2}\right)=i$.

- The loop counter of $S_{1}$ and $S_{2}$ are of value $i$, $\operatorname{loop}_{c}^{1_{i+1}}\left(n_{1}\right)$ $=\operatorname{loop}_{c}^{2 i+1}\left(n_{2}\right)=i$.

- Value stores $\sigma_{1_{i+1}}$ and $\sigma_{2_{i+1}}$ agree on values of used variables in both $S_{1}$ and $S_{2}$ as well as the input sequence variable and the I/O sequence variable, $\forall x \in\left(\mathrm{Use}\left(S_{1}\right) \cap\right.$ $\left.\operatorname{Use}\left(S_{2}\right)\right) \cup\left\{i d_{I+1}, i d_{I O}\right\}: \sigma_{1_{i+1}}(x)=\sigma_{2_{i+1}}(x)$.

\subsection{Proof rule for variable type weakening}

In programs, variable types are changed either to allow for larger ranges (weakening). For example, an integer variable might be changed to become a long variable to avoid integer overflow. Adding a new enumeration value can is also type weakening. Increasing array size is another example of weakening. Allowing for type weakening is essentially an assumption about the intent behind the update. The kinds of weakening that should be allowed are application dependent and would need to be defined by the user in general. The type weakening considered are either changes of type Int to Long or increase of array size. These updates fix integer overflow or array index out of bound. In order to prove the update of variable type weakening to be backward compatible, we assume that there are no integer overflow and array index out of bound in execution of the old program and the updated program. In conclusion, the old program and the new program produce the same output sequence because the integer overflow and index out of bound errors fixed by the new program do not occur.

We formalize the update of variable type weakening, then we show that the updated program produce the same output sequence as the old program in executions if there are no integer overflow or index out of bound exceptions related to variables with type changes. First, we define a relation between variable definitions showing the type weakening.

Definition 28. (Cases of type weakening) We say there is type weakening from a sequence of variable definitions $V_{1}$ to $V_{2}$, written $V_{1} \nearrow_{\tau} V_{2}$, iff one of the following holds:

1. $V_{1}=$ "Int id", $V_{2}=$ "Long $i d "$;

2. $V_{1}=$ " $\tau i d\left[n_{2}\right]$ ", $V_{2}=$ " $\tau$ id $\left[n_{1}\right]$ " where $n_{2}>n_{1}$;

3. $V_{1}=V_{1}^{\prime}$, " $\tau_{1} i d_{1} ", V_{2}=V_{2}^{\prime}$, " $\tau_{2} i d_{2}$ " where $\left(V_{1}^{\prime} \nearrow_{\tau} V_{2}^{\prime}\right) \wedge$ ( " $\tau_{1} i d_{1} " \nearrow_{\tau}$ " $\left.\tau_{2} i d_{2} "\right)$;

4. $V_{1}=V_{1}^{\prime}$, " $\tau_{1} i d_{1}\left[n_{1}\right] ", V_{2}=V_{2}^{\prime}$, " $\tau_{2} i d_{2}\left[n_{2}\right]$ " where $\left(V_{1}^{\prime} \nearrow_{\tau}\right.$ $\left.V_{2}^{\prime}\right) \wedge\left(" \tau_{1} i d_{1}\left[n_{1}\right] " \nearrow_{\tau}\right.$ " $\left.\tau_{2} i d_{2}\left[n_{2}\right] "\right)$;

The following is the generalized definition of variable type weakening.

Definition 29. (Variable type weakening) We say that there are updates of variable type weakening in the program $P_{2}=$ Pmpt; EN; $V_{2} ; S_{\text {entry }}$ compared with the program $P_{1}=$ Pmpt; $E N ; V_{1} ; S_{\text {entry }}$, written $P_{2} \approx_{\tau}^{S} P_{1}$, iff $V_{1} \nearrow_{\tau} V_{2}$.

We show that two programs terminate in the same way, produce the same output sequence, and have equivalent computation of defined variables in both programs in valid executions if there are updates of variable type weakening between them. 
Lemma 6.7. Let $P_{1}=E N ; V_{1} ; S_{\text {entry }}$ and $P_{2}=E N ; V_{2} ; S_{\text {entry }}$ be two programs where there are updates of variable type weakening, $P_{2} \approx_{\tau}^{S} P_{1}$. If the programs $P_{1}$ and $P_{2}$ start in states $m_{1}\left(\sigma_{1}\right)$ and $m_{2}\left(\sigma_{2}\right)$ such that both of the following hold:

- Value stores $\sigma_{1}$ and $\sigma_{2}$ agree on values of variables used in $S_{\text {entry }}$ as well as the input sequence variable, the I/O sequence variable, $\forall x \in U$ Use $\left(S_{\text {entry }}\right) \cup\left\{i d_{I}, i d_{I O}\right\}: \sigma_{1}(x)=\sigma_{2}(x)$;

- There is no integer overflow or index out of bound exceptions related to variables of type change;

then $S_{\text {entry }}$ in the program $P_{1}$ and $P_{2}$ terminate in the same way, produce the same output sequence, and when $S_{\text {entry }}$ both terminate, they have equivalent computation of defined variables in $S_{\text {entry }}$ in both programs as well as the input sequence variable, the I/O sequence variable,

- $\left(S_{\text {entry }}, m_{1}\right) \equiv_{H}\left(S_{\text {entry }}, m_{2}\right)$;

- $\left(S_{\text {entry }}, m_{1}\right) \equiv_{O}\left(S_{\text {entry }}, m_{2}\right)$;

- $\forall x \in \operatorname{Def}(S) \cup\left\{i d_{I}, i d_{I O}\right\}$ :

$\left(S_{\text {entry }}, m_{1}\right) \equiv_{x}\left(S_{\text {entry }}, m_{2}\right)$;

Because $S_{\text {entry }}$ are the exactly same in both programs $P_{1}$ and $P_{2}$, we omit the straightforward proof. Instead, we show that, if there is no array index out of bound and integer overflow in executions of the old program, then there is no array index out of bound or integer overflow in executions of updated program due to the increase of array index and change of type Int to Long.

Proof. The proof is straightforward because the statement sequence $S$ is same in programs $P_{1}$ and $P_{2}$. The only point is that if there is array index out of bound or integer overflow in execution of $S$ in $P_{1}$, then there is no array index out of bound or integer overflow in execution of $S$ in $P_{2}$. To show the point, we present the argument for the array index out of bound and integer overflow separately.

1. We show that, as to one expression $i d_{1}\left[i d_{2}\right]$, there is no array index out of bound in $P_{2}$ if there is no array index out of bound in $P_{1}$ when $P_{1}$ and $P_{2}$ are in states agreeing on values of used variables in $P_{1}$ and $P_{2}$;

$$
\begin{aligned}
& \left(i d_{1}\left[i d_{2}\right], m_{1}\left(\sigma_{1}\right)\right) \\
\rightarrow & \left(i d_{1}[v], m_{1}\left(\sigma_{1}\right)\right) \text { by the rule Var }
\end{aligned}
$$

Similarly, $\left(i d_{1}\left[i d_{2}\right], m_{2}\left(\sigma_{2}\right)\right) \rightarrow\left(i d_{1}[v], m_{2}\left(\sigma_{2}\right)\right)$. By Definition 28 the array bound of $i d_{1}$ in $P_{2}$ is no less than that in $P_{1}$, then there is no array out of bound exception in evaluation of $i d_{1}\left[i d_{2}\right]$ in $P_{2}$ if there is no array out of bound exception in evaluation of $i d_{1}\left[i d_{2}\right]$ in $P_{1}$.

2. We show that, as to one expression $e$, there is no integer overflow in evaluation of $e$ in $P_{2}$ if there is no integer overflow in evaluation of $e$ in $P_{1}$;

$$
\begin{aligned}
& \left(e, m_{1}\left(\sigma_{1}\right)\right) \\
\rightarrow & \left(\left(v_{e}, v_{\mathfrak{o f}}\right), m_{1}\left(\sigma_{1}\right)\right) \text { by the rule EEval' }
\end{aligned}
$$

When every used variable in the expression $e$ is of same type in $P_{1}$ and $P_{2}$, then the evaluation of the expression $e$ in $P_{2}$ is of the same result $\left(v_{e}, v_{\mathfrak{o f}}\right)$ in $P_{1}$. When every used variable in the expression $e$ is of type Int in $P_{1}$ and of type Long $P_{2}$, then there is no integer overflow in the evaluation of the expression $e$ in $P_{2}$ if there is no integer overflow in the evaluation of the expression $e$ in $P_{1}$. This is because the values of type Long are a superset of those of type Int.

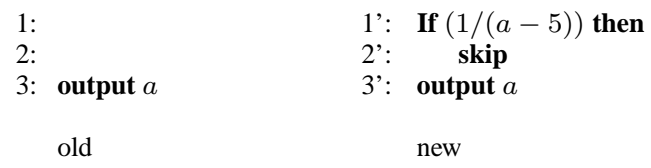

Figure 17: Exit-on-error

\subsection{Proof rule for exit on errors}

Another bugfix is called "exit-on-error", which causes the program to exit in observation of application-semantic-dependent errors. Figure 17 shows an example of exit-on-error update. In the example, the fixed bugs refer to the program semantic error that $a=5$. Instead of using an "exit" statement, we rely on the crash from expression evaluations to formalize the update class. In order to prove the update of exit-on-error to be backward compatible, we assume that there are no application related error in executions of the old program. Therefore, the two programs produce the same output sequence because the extra check does not cause the new program's execution to crash.

The following is the generalized definition of the update class "exit-on-error".

Definition 30. (Exit on error) We say a statement sequence $S_{2}$ includes updates of exit-on-err from a statement sequence $S_{1}$, written $S_{2} \approx_{\text {Exit }}^{S} S_{1}$, iff one of the following holds:

1. $S_{2}=$ "If(e) then $\{$ skip $\}$ else $\{$ skip $\} " ; S_{1}$;

2. $S_{1}=$ "If(e) then $\left\{S_{1}^{t}\right\}$ else $\left\{S_{1}^{f}\right\} ", S_{2}=$ "If $(e)$ then $\left\{S_{2}^{t}\right\}$ else $\left\{S_{2}^{f}\right\}$ " where both of the following hold

- $S_{2}^{t} \approx_{E x i t}^{S} S_{1}^{t}$;

- $S_{2}^{f} \approx_{\text {Exit }}^{S} S_{1}^{f}$;

3. $S_{1}=$ "while $\left\langle n_{1}\right\rangle(e)\left\{S_{1}^{\prime}\right\}$ ", $S_{2}=$ "while ${ }_{\left\langle n_{2}\right\rangle}(e)\left\{S_{2}^{\prime}\right\}$ " where $S_{2}^{\prime} \approx{ }_{\text {Exit }}^{S} S_{1}^{\prime}$

4. $S_{1} \approx S_{O}^{S} S_{2}$;

5. $S_{1}=S_{1}^{\prime} ; s_{1}$ and $S_{2}=S_{2}^{\prime} ; s_{2}$ such that both of the following hold:

- $S_{2}^{\prime} \approx_{\text {Exit }}^{S} S_{1}^{\prime}$;

- $S_{2}^{\prime} \approx_{H}^{S} S_{1}^{\prime}$;

- $\forall x \in \operatorname{Imp}\left(s_{1}, i d_{I O}\right) \cup \operatorname{Imp}\left(s_{1}, i d_{I O}\right): S_{2}^{\prime} \approx_{x}^{S} S_{1}^{\prime}$;

- $s_{2} \approx_{E x i t}^{S} s_{1}$;

Though the bugfix in Definition 30 is not in rare execution in the first case, the definition shows the basic form of bugfix clearly.

We show that two programs terminate in the same way, produce the same output sequence, and have equivalent computation of defined variables in both programs in valid executions if there are updates of exit-on-error between them.

Lemma 6.8. Let $S_{1}$ and $S_{2}$ be two statement sequences respectively where there are updates of exit-on-error in $S_{2}$ against $S_{1}$, $S_{2} \approx_{\text {Exit }}^{S} S_{1}$. If $S_{1}$ and $S_{2}$ start in states $m_{1}\left(\sigma_{1}\right)$ and $m_{2}\left(\sigma_{2}\right)$ such that both of the following hold:

- Value stores $\sigma_{1}$ and $\sigma_{2}$ agree on values of variables used in both $S_{1}$ and $S_{2}$ as well as the input sequence variable and the I/O sequence variable, $\forall x \in\left(\operatorname{Use}\left(S_{1}\right) \cap \operatorname{Use}\left(S_{2}\right)\right) \cup\left\{i d_{I}, i d_{I O}\right\}$ : $\sigma_{1}(x)=\sigma_{2}(x)$;

- There are no program semantic errors related to the extra check in the update of exit-on-error in the execution of $S_{1}$;

then $S_{1}$ and $S_{2}$ terminate in the same way, produce the same output sequence, and when $S_{1}$ and $S_{2}$ both terminate, they have equivalent computation of defined variables in both $S_{1}$ and $S_{2}$ as well as the input sequence variable and the I/O sequence variable,

- $\left(S_{1}, m_{1}\right) \equiv_{H}\left(S_{2}, m_{2}\right)$;

- $\left(S_{1}, m_{1}\right) \equiv_{O}\left(S_{2}, m_{2}\right)$; 
- $\forall x \in\left(\operatorname{Def}\left(S_{1}\right) \cap \operatorname{Def}\left(S_{2}\right)\right) \cup\left\{i d_{I}, i d_{I O}\right\}$ : $\left(S_{1}, m_{1}\right) \equiv_{x}\left(S_{2}, m_{2}\right)$;

Proof. By induction on the sum of the program size of $S_{1}$ and $S_{2}$, $\operatorname{size}\left(S_{1}\right)+\operatorname{size}\left(S_{2}\right)$.

Base case. $S_{1}=s$ and $S_{2}=$ "If $(e)$ then $\{\operatorname{skip}\}$ else $\{$ skip $\} " ; s$;

By assumption, there is no program semantic error related to the update of exit-on-error. Then the evaluation of the predicate expression $e$ in the first statement of $S_{2}$ does not crash. W.1.o.g., the expression $e$ evaluates to zero. Then the execution of $S_{2}$ proceeds as follows.

(If $(e)$ then $\{$ skip $\}$ else $\{$ skip $\left.\} ; s, m_{2}\left(\sigma_{2}\right)\right)$

$\rightarrow\left(\operatorname{If}\left(\left(0, v_{\text {of }}\right)\right)\right.$ then $\{$ skip $\}$ else $\{$ skip $\left.\} ; s, m_{2}\left(\sigma_{2}\right)\right)$

by the rule EEval'

$\rightarrow\left(\operatorname{If}(0)\right.$ then $\{$ skip $\}$ else $\{$ skip $\left.\} ; s, m_{2}\left(\sigma_{2}\right)\right)$

by the rule E-Oflow1 or E-Oflow2.

$\rightarrow$ (skip; $\left.s, m_{2}\left(\sigma_{2}\right)\right)$ by the rule If-F

$\rightarrow\left(s, m_{2}\left(\sigma_{2}\right)\right)$ by the rule Seq.

Value stores $\sigma_{2}$ are not changed in the execution of $\left(S_{2}, m_{2}\left(\sigma_{2}\right)\right) \stackrel{*}{\rightarrow}$ $\left(s, m_{2}\left(\sigma_{2}\right)\right)$. By assumption, $\sigma_{1}$ and $\sigma_{2}$ agree on values of used variables as well as the input sequence variable, and the I/O sequence variable, $\forall x \in \operatorname{Use}\left(S_{2}\right) \cap \operatorname{Use}\left(S_{1}\right) \cup\left\{i d_{I}, i d_{I O}\right\}$ : $\sigma_{1}(i d)=\sigma_{2}(i d)$. By semantics, $S_{1}$ and $S_{2}$ terminate in the same way, produce the same output sequence, and have equivalent computation of defined variables in both $S_{1}$ and $S_{2}$ as well as the input sequence variable, and the I/O sequence variable. Then this lemma holds.

Induction step.

The hypothesis is that this lemma holds when the sum $k$ of the program size of $S_{1}$ and $S_{2}$ are great than or equal to $4, k \geq 4$.

We then show that this lemma holds when the sum of the program size of $S_{1}$ and $S_{2}$ is $k+1$. There are cases to consider.

1. $S_{1}$ and $S_{2}$ are both "If" statement:

$S_{1}=$ "If $(e)$ then $\left\{S_{1}^{t}\right\}$ else $\left\{S_{1}^{f}\right\} ", S_{2}=" \operatorname{If}(e) \operatorname{then}\left\{S_{2}^{t}\right\} \operatorname{else}\left\{S_{2}^{f}\right\} "$

where both of the following hold

- $\left(S_{2}^{t} \approx \sim_{\text {Exit }}^{S} S_{1}^{t}\right)$;

- $\left(S_{2}^{f} \approx_{\mathrm{Exit}}^{S} S_{1}^{f}\right)$;

By the definition of Use $\left(S_{1}\right)$, variables used in the predicate expression $e$ are a subset of used variables in $S_{1}$ and $S_{2}, \mathrm{Use}(e) \subseteq$ Use $\left(S_{1}\right) \cap \operatorname{Use}\left(S_{2}\right)$. By assumption, corresponding variables used in $e$ are of same value in value stores $\sigma_{1}$ and $\sigma_{2}$. By Lemma D.1 the expression evaluates to the same value w.r.t value stores $\sigma_{1}$ and $\sigma_{2}$. There are three possibilities.

(a) The evaluation of $e$ crashes, $\mathcal{E}^{\prime} \llbracket e \rrbracket \sigma_{1}=\mathcal{E}^{\prime} \llbracket e \rrbracket \sigma_{2}=$ (error, $v_{\mathfrak{o f}}$ ).

The execution of $S_{1}$ continues as follows:

$\left(\right.$ If $(e)$ then $\left\{S_{1}^{t}\right\}$ else $\left.\left\{S_{1}^{f}\right\}, m_{1}\left(\sigma_{1}\right)\right)$

$\rightarrow\left(\operatorname{If}\left(\left(\right.\right.\right.$ error, $\left.\left.v_{\mathfrak{o f}}\right)\right)$ then $\left\{S_{1}^{t}\right\}$ else $\left.\left\{S_{1}^{f}\right\}, m_{1}\left(\sigma_{1}\right)\right)$

by the rule EEval'

$\rightarrow\left(\operatorname{If}(0)\right.$ then $\left\{S_{1}^{t}\right\}$ else $\left.\left\{S_{1}^{f}\right\}, m_{1}(1 / \mathfrak{f})\right)$

by the ECrash rule

$\stackrel{i}{\rightarrow}\left(\operatorname{If}(0)\right.$ then $\left.\left\{S_{1}^{t}\right\} \operatorname{else}\left\{S_{1}^{f}\right\}, m_{1}(1 / \mathfrak{f})\right)$ for any $i>0$

by the Crash rule.

Similarly, the execution of $S_{2}$ started from the state $m_{2}\left(\sigma_{2}\right)$ crashes. The lemma holds.

(b) The evaluation of $e$ reduces to zero, $\mathcal{E}^{\prime} \llbracket e \rrbracket \sigma_{1}=\mathcal{E}^{\prime} \llbracket e \rrbracket \sigma_{2}=$ $\left(0, v_{\mathfrak{o f}}\right)$.

The execution of $S_{1}$ continues as follows.

$\left(\operatorname{If}(e)\right.$ then $\left\{S_{1}^{t}\right\}$ else $\left.\left\{S_{1}^{f}\right\}, m_{1}\left(\sigma_{1}\right)\right)$

$=\left(\operatorname{If}\left(\left(0, v_{\mathfrak{o f}}\right)\right)\right.$ then $\left.\left\{S_{1}^{t}\right\} \operatorname{else}\left\{S_{1}^{f}\right\}, m_{1}\left(\sigma_{1}\right)\right)$

by the rule EEval'
$\rightarrow\left(\operatorname{If}(0)\right.$ then $\left\{S_{1}^{t}\right\}$ else $\left.\left\{S_{1}^{f}\right\}, m_{1}\left(\sigma_{1}\right)\right)$

by the E-Oflow1 or E-Oflow2 rule

$\rightarrow\left(S_{1}^{f}, m_{1}\left(\sigma_{1}\right)\right)$ by the If-F rule.

Similarly, the execution of $S_{2}$ gets to the configuration $\left(S_{2}^{f}, m_{2}\left(\sigma_{2}\right)\right)$.

By the hypothesis IH, we show the lemma holds. We need to show that all conditions are satisfied for the application of the hypothesis IH.

- $\left(S_{2}^{f} \approx_{\text {Exit }}^{S} S_{1}^{f}\right)$

By assumption.

- The sum of the program size of $S_{1}^{f}$ and $S_{2}^{f}$ is less than $k, \operatorname{size}\left(S_{1}^{f}\right)+\operatorname{size}\left(S_{2}^{f}\right)<k$.

By definition, $\operatorname{size}\left(S_{1}\right)=1+\operatorname{size}\left(S_{1}^{t}\right)+\operatorname{size}\left(S_{1}^{f}\right)$. Then, $\operatorname{size}\left(S_{1}^{f}\right)+\operatorname{size}\left(S_{2}^{f}\right)<k+1-2=k-1$.

- Value stores $\sigma_{1}$ and $\sigma_{2}$ agree on values of used variables in $S_{1}^{f}$ and $S_{2}^{f}$ as well as the input, I/O sequence variable. By definition, $\operatorname{Use}\left(S_{1}^{f}\right) \subseteq \operatorname{Use}\left(S_{1}\right)$. So are the cases to $S_{2}^{f}$ and $S_{2}$. In addition, value stores $\sigma_{1}$ and $\sigma_{2}$ are not changed in the evaluation of the predicate expression $e$. The condition holds.

- There are no program semantic error related to the extra check in the update of exit-on-error in the execution of $S_{2}$

By assumption.

By the hypothesis $\mathrm{IH}$, the lemma holds.

(c) The evaluation of $e$ reduces to the same nonzero integer value, $\mathcal{E}^{\prime} \llbracket e \rrbracket \sigma_{1}=\mathcal{E}^{\prime} \llbracket e \rrbracket \sigma_{2}=\left(v, v_{\text {of }}\right)$ where $v \neq 0$.

By similar to the second subcase above.

2. $S_{1}$ and $S_{2}$ are both "while" statements:

$S_{1}=$ "while $\langle n\rangle(e)\left\{S_{1}^{\prime}\right\} ", S_{2}=$ "while $\langle n\rangle(e)\left\{S_{2}^{\prime}\right\} "$ where $\left(S_{2}^{\prime} \approx_{\text {exit }}^{S} S_{1}^{\prime}\right)$;

By Lemma 6.10 we show this lemma holds. We need to show that all required conditions are satisfied for the application of Lemma 6.10

- The output deciding variables in $S_{1}^{\prime}$ are a subset of those in $S_{2}^{\prime}, \operatorname{OVar}\left(S_{1}^{\prime}\right)=\operatorname{OVar}\left(S_{2}^{\prime}\right)$;

By Lemma 6.9

- When started in states $m_{1}^{\prime}\left(\sigma_{1}^{\prime}\right), m_{2}^{\prime}\left(\sigma_{1}^{\prime}\right)$ where value stores $\sigma_{1}^{\prime}$ and $\sigma_{2}^{\prime}$ agree on values of used variables in both $S_{1}^{\prime}$ and $S_{2}^{\prime}$ as well as the input sequence variable, and the I/O sequence variable, then $S_{1}^{\prime}$ and $S_{2}^{\prime}$ terminate in the same way, produce the same output sequence, and have equivalent computation of defined variables in both $S_{1}$ and $S_{2}$ as well as the input sequence variable, and the I/O sequence variable.

By the induction hypothesis IH. This is because the sum of the program size of $S_{1}^{\prime}$ and $S_{2}^{\prime}$ is less than $k$. By definition, $\operatorname{size}\left(S_{1}\right)=1+\operatorname{size}\left(S_{1}^{\prime}\right)$.

By Lemma 6.10 this lemma holds.

3. $S_{1}=S_{1}^{\prime} ; s_{1}$ and $S_{2}=S_{2}^{\prime} ; s_{2}$ where both of the following hold:

- $\left(S_{2}^{\prime} \approx_{\text {Exit }}^{S} S_{1}^{\prime}\right)$

- $\left(s_{2} \approx_{\text {Exit }}^{S} s_{1}\right)$;

By the hypothesis $\mathrm{IH}$, we show $S_{2}^{\prime}$ and $S_{1}^{\prime}$ terminate in the same way and produce the same output sequence and when $S_{2}^{\prime}$ and $S_{1}^{\prime}$ both terminate, $S_{2}^{\prime}$ and $S_{1}^{\prime}$ have equivalent terminating computation of variables used or defined in $S_{2}^{\prime}$ and $S_{1}^{\prime}$ as well as the input sequence variable, and the I/O sequence variable. We show all the required conditions are satisfied for the application of the hypothesis IH.

- $\left(S_{2}^{\prime} \approx \approx_{\text {Exit }}^{S} S_{1}^{\prime}\right)$.

By assumption. 
- The sum of the program size of $S_{1}^{\prime}$ and $S_{2}^{\prime}$ is less than $k$, $\operatorname{size}\left(S_{1}^{\prime}\right)+\operatorname{size}\left(S_{2}^{\prime}\right)<k$.

By definition, $\operatorname{size}\left(S_{2}\right)=\operatorname{size}\left(s_{2}\right)+\operatorname{size}\left(S_{2}^{\prime}\right)$ where $\operatorname{size}\left(s_{2}\right)<1$. Then, $\operatorname{size}\left(S_{2}^{\prime}\right)+\operatorname{size}\left(S_{1}^{\prime}\right)<k+1-$ $\operatorname{size}\left(s_{2}\right)-\operatorname{size}\left(s_{1}\right)<k$.

- Value stores $\sigma_{1}$ and $\sigma_{2}$ agree on values of used variables in both $S_{2}^{\prime}$ and $S_{1}^{\prime}$ as well as the input, I/O sequence variable. By definition, $\operatorname{Use}\left(S_{2}^{\prime}\right) \subseteq \operatorname{Use}\left(S_{2}\right), \operatorname{Use}\left(S_{1}^{\prime}\right) \subseteq \operatorname{Use}\left(S_{1}\right)$. The condition holds.

By the hypothesis $\mathrm{IH}$, one of the following holds:

(a) $S_{1}^{\prime}$ and $S_{2}^{\prime}$ both do not terminate.

By Lemma E.2, executions of $S_{1}=S_{1}^{\prime} ; s_{1}$ and $S_{2}=$ $S_{2}^{\prime} ; s_{2}$ both do not terminate and produce the same output sequence.

(b) $S_{1}^{\prime}$ and $S_{2}^{\prime}$ both terminate.

By assumption, $\left(S_{2}^{\prime}, m_{2}\left(\sigma_{2}\right)\right) \stackrel{*}{\rightarrow}\left(\operatorname{skip}, m_{2}^{\prime}\left(\sigma_{2}^{\prime}\right)\right),\left(S_{1}^{\prime}, m_{1}\left(\sigma_{1}\right)\right) \stackrel{*}{\rightarrow}$ (skip, $\left.m_{1}^{\prime}\left(\sigma_{1}^{\prime}\right)\right)$.

By Corollary E.1 $\left(S_{2}^{\prime} ; s_{2}, m_{2}\left(\sigma_{2}\right)\right) \stackrel{*}{\rightarrow}\left(s_{2}, m_{2}^{\prime}\left(\sigma_{2}^{\prime}\right)\right)$, $\left(S_{1}^{\prime} ; s_{1}, m_{1}\left(\sigma_{1}\right)\right) \stackrel{*}{\rightarrow}\left(s_{1}, m_{1}^{\prime}\left(\sigma_{1}^{\prime}\right)\right)$.

By the hypothesis $\mathrm{IH}$, we show that $s_{2}$ and $s_{1}$ terminate in the same way, produce the same output sequence and when $s_{2}$ and $s_{1}$ both terminate, $s_{2}$ and $s_{1}$ have equivalent computation of variables defined in both $s_{1}$ and $s_{2}$ and the input, and $\mathrm{I} / \mathrm{O}$ sequence variables.

We need to show that all conditions are satisfied for the application of the hypothesis IH.

- There are updates of "exit-on-error" between $s_{2}$ and $s_{1}$, $s_{2} \approx_{\text {Exit }}^{S} s_{1}$;

By assumption, $s_{2} \approx_{\text {Exit }}^{S} s_{1}$.

- The sum of the program size $s_{2}$ and $s_{1}$ is less than or equals to $k$;

By definition, $\operatorname{size}\left(S_{2}^{\prime}\right) \geq 1, \operatorname{size}\left(S_{1}^{\prime}\right) \geq 1$. Therefore, $\operatorname{size}\left(s_{2}\right)+\operatorname{size}\left(s_{1}\right)<k+1-\operatorname{size}\left(S_{2}^{\prime}\right)-\operatorname{size}\left(S_{1}^{\prime}\right) \leq k$.

- Value stores $\sigma_{1}^{\prime}$ and $\sigma_{2}^{\prime}$ agree on values of output deciding variables in $s_{2}$ and $s_{1}$ as well as the input, I/O sequence variable.

By Lemma 6.9 OVar $\left(s_{1}\right) \subseteq \operatorname{OVar}\left(s_{2}\right)$, then $\operatorname{OVar}\left(s_{2}\right)$ $\cap \operatorname{OVar}\left(s_{1}\right)=\operatorname{OVar}\left(s_{1}\right)$. For any variable $i d$ in $\operatorname{Use}\left(s_{1}\right)$, if $i d$ is not in $\operatorname{OVar}\left(S_{1}^{\prime}\right)$, then the value of $i d$ is not changed in the execution of $S_{1}^{\prime}$ and $S_{2}^{\prime}, \sigma_{1}^{\prime}(i d)=$ $\sigma_{1}(i d)=\sigma_{2}(i d)=\sigma_{2}^{\prime}(i d)$. Otherwise, the variable $i d$ is defined in the execution of $S_{1}^{\prime}$ and $S_{2}^{\prime}$, by assumption, $\sigma_{1}^{\prime}(i d)=\sigma_{2}^{\prime}(i d)$. The condition holds.

- There are no program semantic errors related to the extra check in the update of exit-on-error in the execution of $S_{2}$.

By assumption.

By the hypothesis IH, the lemma holds.

We list the auxiliary lemmas below. One lemma shows that, if there are updates of exit-on-error between two statement sequences, then there are same set of defined variables in the two statement sequences, and the used variables in the update program are the superset of those in the old program.

Lemma 6.9. Let $S_{2}$ be a statement sequence and $S_{1}$ where there are updates of exit-on-error, $S_{2} \approx_{\text {Exit }}^{S} S_{1}$. Then output deciding variables in $S_{1}$ are a subset of those in $S_{2}, O \operatorname{Var}\left(S_{1}\right) \subseteq \operatorname{OVar}\left(S_{2}\right)$.

Proof. By induction on the sum of the program size of $S_{1}$ and $S_{2}$.
Lemma 6.10. Let $S_{1}=$ while $_{\left\langle n_{1}\right\rangle}(e)\left\{S_{1}^{\prime}\right\}$ and $S_{2}=$ while $_{\left\langle n_{2}\right\rangle}(e)$ $\left\{S_{2}^{\prime}\right\}$ be two loop statements where all of the following hold:

- the output deciding variables in $S_{1}^{\prime}$ are a subset of those in $S_{2}^{\prime}$, $O \operatorname{Var}\left(S_{1}^{\prime}\right) \subseteq \operatorname{OVar}\left(S_{2}^{\prime}\right)=\operatorname{OVar}(S)$;

- When started in states $m_{1}^{\prime}\left(\sigma_{1}^{\prime}\right), m_{2}^{\prime}\left(\sigma_{2}^{\prime}\right)$ where

- Value stores agree on values of output deciding variables in both $S_{1}^{\prime}$ and $S_{2}^{\prime}$ as well as the input sequence variable, and the I/O sequence variable, $\forall x \in \operatorname{OVar}\left(S_{2}^{\prime}\right) \cup$ $\left\{i d_{I}, i d_{I O}\right\} \forall m_{1}^{\prime}\left(\sigma_{1}^{\prime}\right) m_{2}^{\prime}\left(\sigma_{2}^{\prime}\right): \sigma_{1}^{\prime}(x)=\sigma_{2}^{\prime}(x)$;

- There are no program semantic errors related to the extra check in the update of exit-on-error in executions of $S_{1}^{\prime}$ and $S_{2}^{\prime}$;

then $S_{1}^{\prime}$ and $S_{2}^{\prime}$ terminate in the same way, produce the same output sequence, and have equivalent computation of defined variables in $S_{1}^{\prime}$ and $S_{2}^{\prime}$ as well as the input sequence variable, and the I/O sequence variable $\left(\left(S_{1}^{\prime}, m_{1}\right) \equiv_{H}\left(S_{2}^{\prime}, m_{2}\right)\right) \wedge$ $\left(\left(S_{1}^{\prime}, m_{1}\right) \equiv_{O}\left(S_{2}^{\prime}, m_{2}\right)\right) \wedge\left(\forall x \in \operatorname{OVar}(S) \cup\left\{i d_{I}, i d_{I O}\right\}:\right.$ $\left.\left(S_{1}^{\prime}, m_{1}\right) \equiv_{x}\left(S_{2}^{\prime}, m_{2}\right)\right)$;

If $S_{1}$ and $S_{2}$ start in states $m_{1}\left(\right.$ loop $\left._{c}^{1}, \sigma_{1}\right), m_{2}\left(\right.$ loop $\left._{c}^{2}, \sigma_{2}\right)$ respectively, with loop counters of $S_{1}$ and $S_{2}$ not initialized $\left(S_{1}, S_{2}\right.$ have not executed yet), value stores agree on values of used variables in $S_{1}$ and $S_{2}$, and there are no program semantic errors related to the extra check in the update of exit-on-error, then, for any positive integer $i$, one of the following holds:

1. Loop counters for $S_{1}$ and $S_{2}$ are always less than $i$ if any is present, $\forall m_{1}^{\prime}\left(\operatorname{loop}_{c}^{1^{\prime}}\right) m_{2}^{\prime}\left(\operatorname{loop}_{c}^{2^{\prime}}\right):\left(S_{1}, m_{1}\left(\operatorname{loop}_{c}^{1}, \sigma_{1}\right)\right) \stackrel{*}{\rightarrow}$ $\left(S_{1}^{\prime \prime}, m_{1}^{\prime}\left(\operatorname{loop}_{c}^{1^{\prime}}\right)\right)$, loop $_{c}^{1^{\prime}}\left(n_{1}\right)<i,\left(S_{2}, m_{2}\left(\operatorname{loop}_{c}^{2}, \sigma_{2}\right)\right) \stackrel{*}{\rightarrow}$ $\left(S_{2}^{\prime \prime}, m_{2}^{\prime}\left(\right.\right.$ loop $\left.\left._{c}^{2^{\prime}}\right)\right)$, loop 2. $_{c}^{\prime}\left(n_{2}\right)<i, S_{1}$ and $S_{2}$ terminate in the same way, produce the same output sequence, and have equivalent computation of output deciding variables in $S_{1}$ and $S_{2}$ and the input sequence variable, the I/O sequence variable, $\left(S_{1}, m_{1}\right) \equiv_{H}\left(S_{2}, m_{2}\right)$ and $\left(S_{1}, m_{1}\right) \equiv_{O}\left(S_{2}, m_{2}\right)$ and $\forall x \in\left(O \operatorname{Var}\left(S_{1}\right) \cup \operatorname{OVar}\left(S_{2}\right)\right) \cup\left\{i d_{I}, i d_{I O}\right\}:$ $\left(S_{1}, m_{1}\right) \equiv_{x}\left(S_{2}, m_{2}\right)$;

2. The loop counter of $S_{1}$ and $S_{2}$ are of value less than or equal to $i$, and there are no reachable configurations $\left(S_{1}, m_{1}\left(\right.\right.$ loop $_{c}^{1_{i}}$, $\left.\left.\sigma_{1_{i}}\right)\right)$ from $\left(S_{1}, m_{1}\left(\sigma_{1}\right)\right)$, $\left(S_{2}, m_{2}\left(\operatorname{loop}_{c}^{2}, \sigma_{2_{i}}\right)\right)$ from $\left(S_{2}\right.$, $\left.m_{2}\left(\sigma_{2}\right)\right)$ where all of the following hold:

- The loop counters of $S_{1}$ and $S_{2}$ are of value $i$, $\operatorname{loop}_{c}^{1_{i}}\left(n_{1}\right)$ $=\operatorname{loop}_{c}^{2 i}\left(n_{2}\right)=i$.

- Value stores $\sigma_{1_{i}}$ and $\sigma_{2_{i}}$ agree on values of output deciding variables in $S_{1}$ and $S_{2}$ as well as the input sequence variable, and the I/O sequence variable, $\forall x \in\left(\operatorname{OVar}\left(S_{1}\right) \cup\right.$ $\left.O \operatorname{Var}\left(S_{2}\right)\right) \cup\left\{i d_{I}, i d_{I O}\right\}: \sigma_{1_{i}}(x)=\sigma_{2_{i}}(x)$.

3. There are reachable configurations $\left(S_{1}, m_{1}\left(\right.\right.$ loop $\left.\left._{c}^{1_{i}}, \sigma_{1_{i}}\right)\right)$ from $\left(S_{1}, m_{1}\left(\sigma_{1}\right)\right),\left(S_{2}, m_{2}\left(\operatorname{loop}_{c}^{2_{i}}, \sigma_{2_{i}}\right)\right)$ from $\left(S_{2}, m_{2}\left(\sigma_{2}\right)\right)$ where all of the following hold:

- The loop counter of $S_{1}$ and $S_{2}$ are of value $i, \operatorname{loop}_{c}^{1_{i}}\left(n_{1}\right)$ $=\operatorname{loop}_{c}^{2_{i}}\left(n_{2}\right)=i$.

- Value stores $\sigma_{1_{i}}$ and $\sigma_{2_{i}}$ agree on values of output deciding variables in $S_{1}$ and $S_{2}$ including the input sequence variable and the I/O sequence variable, $\forall x \in\left(\operatorname{OVar}\left(S_{1}\right) \cup\right.$ $\left.O \operatorname{Var}\left(S_{2}\right)\right) \cup\left\{i d_{I}, i d_{I O}\right\}: \sigma_{1_{i}}(x)=\sigma_{2_{i}}(x)$.

Proof. By induction on $i$.

Base case.

We show that, when $i=1$, one of the following holds:

1. Loop counters for $S_{1}$ and $S_{2}$ are always less than 1 if any is present, $\forall m_{1}^{\prime}\left(\operatorname{loop}_{c}^{1^{\prime}}\right) m_{2}^{\prime}\left(\operatorname{loop}_{c}^{2^{\prime}}\right):\left(S_{1}, m_{1}\left(\operatorname{loop}_{c}^{1}, \sigma_{1}\right)\right) \stackrel{*}{\rightarrow}$ $\left(S_{1}^{\prime \prime}, m_{1}^{\prime}\left(\operatorname{loop}_{c}^{1^{\prime}}\right)\right), \operatorname{loop}_{c}^{1^{\prime}}\left(n_{1}\right)<i,\left(S_{2}, m_{2}\left(\operatorname{loop}_{c}^{2}, \sigma_{2}\right)\right) \stackrel{*}{\rightarrow}$ $\left(S_{2}^{\prime \prime}, m_{2}^{\prime}\left(\operatorname{loop}_{c}^{2^{\prime}}\right)\right)$, loop $2_{c}^{2^{\prime}}\left(n_{2}\right)<i, S_{1}$ and $S_{2}$ terminate in the 
same way, produce the same output sequence, and have equivalent computation of output deciding variables in $S_{1}$ and $S_{2}$ including the input sequence variable, the I/O sequence variable, $\left(S_{1}, m_{1}\right) \equiv_{H}\left(S_{2}, m_{2}\right)$ and $\left(S_{1}, m_{1}\right) \equiv_{O}\left(S_{2}, m_{2}\right)$ and $\forall x \in\left(\operatorname{OVar}\left(S_{1}\right) \cup \operatorname{OVar}\left(S_{2}\right)\right) \cup\left\{i d_{I}, i d_{I O}\right\}:\left(S_{1}, m_{1}\right) \equiv_{x}$ $\left(S_{2}, m_{2}\right)$

2. Loop counters of $S_{1}$ and $S_{2}$ are of values less than or equal to 1 but there are no reachable configurations $\left(S_{1}, m_{1}\left(\operatorname{loop}_{c}^{1_{1}}, \sigma_{1_{i}}\right)\right)$ from $\left(S_{1}, m_{1}\left(\sigma_{1}\right)\right),\left(S_{2}, m_{2}\left(\operatorname{loop}_{c}^{2_{1}}, \sigma_{2_{i}}\right)\right)$ from $\left(S_{2}, m_{2}\left(\sigma_{2}\right)\right)$ where all of the following hold:

- The loop counter of $S_{1}$ and $S_{2}$ are of value $1, \operatorname{loop}_{c}^{1_{1}}\left(n_{1}\right)$ $=\operatorname{loop}_{c}^{2_{1}}\left(n_{2}\right)=1$.

- Value stores $\sigma_{1_{1}}$ and $\sigma_{2_{1}}$ agree on values of output deciding variables in $S_{1}$ and $S_{2}$ including the input sequence variable and the I/O sequence variable, $\forall x \in\left(\operatorname{OVar}\left(S_{1}\right) \cup\right.$ $\left.\operatorname{OVar}\left(S_{2}\right)\right) \cup\left\{i d_{I}, i d_{I O}\right\}: \sigma_{1_{1}}(x)=\sigma_{2_{1}}(x)$.

3. There are reachable configuration $\left(S_{1}, m_{1}\left(\operatorname{loop}_{c}^{1_{1}}, \sigma_{1_{i}}\right)\right)$ from $\left(S_{1}, m_{1}\left(\sigma_{1}\right)\right),\left(S_{2}, m_{2}\left(\operatorname{loop}_{c}^{21}, \sigma_{2_{i}}\right)\right)$ from $\left(S_{2}, m_{2}\left(\sigma_{2}\right)\right)$ where all of the following hold:

- The loop counter of $S_{1}$ and $S_{2}$ are of value $1, \operatorname{loop}_{c}^{1_{1}}\left(n_{1}\right)$ $=\operatorname{loop}_{c}^{2_{1}}\left(n_{2}\right)=1$.

- Value stores $\sigma_{1_{1}}$ and $\sigma_{21}$ agree on values of output deciding variables in $S_{1}$ and $S_{2}$ including the input sequence variable and the I/O sequence variable, $\forall x \in\left(\mathrm{OV} \operatorname{Var}\left(S_{1}\right) \cup\right.$ $\left.\operatorname{OVar}\left(S_{2}\right)\right) \cup\left\{i d_{I}, i d_{I O}\right\}: \sigma_{1_{1}}(x)=\sigma_{2_{1}}(x)$.

By definition, variables used in the predicate expression $e$ of $S_{1}$ and $S_{2}$ are in output deciding variables in $S_{1}$ and $S_{2}, \operatorname{Use}(e) \subseteq$ $\operatorname{OVar}\left(S_{1}\right) \cup \operatorname{OVar}\left(S_{2}\right)$. By assumption, value stores $\sigma_{1}$ and $\overline{\sigma_{2}}$ agree on values of variables in $\operatorname{Use}(e)$, the predicate expression $e$ evaluates to the same value w.r.t value stores $\sigma_{1}$ and $\sigma_{2}$ by LemmaD.2 There are three possibilities.

1. The evaluation of $e$ crashes,

$\mathcal{E}^{\prime} \llbracket e \rrbracket \sigma_{1}=\mathcal{E}^{\prime} \llbracket e \rrbracket \sigma_{2}=\left(\right.$ error, $\left.v_{\mathfrak{o f}}\right)$.

The execution of $S_{1}$ continues as follows:

$$
\begin{aligned}
& \left(\text { while }_{\left\langle n_{1}\right\rangle}(e)\left\{S_{1}^{\prime}\right\}, m_{1}\left(\sigma_{1}\right)\right) \\
& \rightarrow\left(\text { while }_{\left\langle n_{1}\right\rangle}\left(\left(\text { error, } v_{\mathfrak{o f}}\right)\right)\left\{S_{1}^{\prime}\right\}, m_{1}\left(\sigma_{1}\right)\right) \\
& \text { by the rule EEval' } \\
& \rightarrow\left(\text { while }_{\left\langle n_{1}\right\rangle}(0)\left\{S_{1}^{\prime}\right\}, m_{1}(1 / \mathfrak{f})\right) \\
& \text { by the ECrash rule } \\
& \stackrel{i}{\rightarrow}\left(\text { while }_{\left\langle n_{1}\right\rangle}(0)\left\{S_{1}^{\prime}\right\}, m_{1}(1 / \mathfrak{f})\right) \text { for any } i>0
\end{aligned}
$$

Similarly, the execution of $S_{2}$ started from the state $m_{2}\left(\sigma_{2}\right)$ crashes. Therefore $S_{1}$ and $S_{2}$ terminate in the same way when started from $m_{1}$ and $m_{2}$ respectively. Because $\sigma_{1}\left(i d_{I O}\right)=$ $\sigma_{2}\left(i d_{I O}\right)$, the lemma holds.

2. The evaluation of $e$ reduces to zero, $\mathcal{E}^{\prime} \llbracket e \rrbracket \sigma_{1}=\mathcal{E}^{\prime} \llbracket e \rrbracket \sigma_{2}=$ $\left(0, v_{\text {of }}\right)$.

The execution of $S_{1}$ continues as follows.

$$
\begin{aligned}
& \left(\text { while }_{\left\langle n_{1}\right\rangle}(e)\left\{S_{1}^{\prime}\right\}, m_{1}\left(\sigma_{1}\right)\right) \\
= & (\text { while } \\
& \text { by the rule EEval } \\
\rightarrow & \left.\left(\left(0, v_{\text {of }}\right)\right)\left\{S_{1}^{\prime}\right\}, m_{1}\left(\sigma_{1}\right)\right) \\
\rightarrow & (\text { while } \\
& \text { by the E-Oflow1 or E-Oflow2 rule } \\
\rightarrow & \text { (skip, } \left.m_{1}\left(\sigma_{1}\right)\right) \text { by the Wh-F rule. }
\end{aligned}
$$

Similarly, the execution of $S_{2}$ gets to the configuration (skip, $m_{2}\left(\sigma_{2}\right)$ ). Loop counters of $S_{1}$ and $S_{2}$ are less than 1 and value stores agree on values of output deciding variables in $S_{1}$ and $S_{2}$ including the input sequence variable and the I/O sequence variable.
3. The evaluation of $e$ reduces to the same nonzero integer value, $\mathcal{E}^{\prime} \llbracket e \rrbracket \sigma_{1}=\mathcal{E}^{\prime} \llbracket e \rrbracket \sigma_{2}=\left(0, v_{\mathfrak{o f}}\right)$.

Then the execution of $S_{1}$ proceeds as follows:

$$
\begin{aligned}
& \left(\text { while }_{\left\langle n_{1}\right\rangle}(e)\left\{S_{1}^{\prime}\right\}, m_{1}\left(\sigma_{1}\right)\right) \\
= & \left(\text { while }_{\left\langle n_{1}\right\rangle}\left(\left(v, v_{\text {of }}\right)\right)\left\{S_{1}^{\prime}\right\}, m_{1}\left(\sigma_{1}\right)\right) \\
& \text { by the rule EEval' } \\
\rightarrow & (\text { while } \\
& \text { by the E-Oflow } 1 \text { or E-Oflow } 2 \text { rule } \\
\rightarrow & \left.\left(S_{1}^{\prime} ; S_{1}^{\prime}\right\}, m_{1}\left(\sigma_{1}\right)\right) \\
& \left.\left.\operatorname{loop}_{c}^{1} \cup\left\{\left(n_{1}\right) \mapsto 1\right\}, \sigma_{1}\right)\right) \text { by the Wh-T rule. }
\end{aligned}
$$

Similarly, the execution of $S_{2}$ proceeds to the configuration $\left(S_{2}^{\prime}\right.$; while ${\left.\text {, } n_{2}\right\rangle}_{\text {(e) }}(e) S_{2}^{\prime}\}, m_{2}\left(\right.$ loop $\left.\left._{c}^{2} \cup\left\{\left(n_{2}\right) \mapsto 1\right\}, \sigma_{2}\right)\right)$.

By the assumption, we show that $S_{1}^{\prime}$ and $S_{2}^{\prime}$ terminate in the same way and produce the same output sequence when started in the state $m_{1}\left(\operatorname{loop}_{c}^{1_{1}}, \sigma_{1}\right)$ and $m_{2}\left(\operatorname{loop}_{c}^{2{ }_{1}}, \sigma_{2}\right)$ respectively, and $S_{1}^{\prime}$ and $S_{2}^{\prime}$ have equivalent computation of variables defined in both statement sequences if both terminate. We need to show that all conditions are satisfied for the application of the assumption.

- There are no program semantic errors related to the extra check in the update of exit-on-error in executions of $S_{2}^{\prime}$ and $S_{1}^{\prime}$.

The above two conditions are by assumption.

- Value stores $\sigma_{1}$ and $\sigma_{2}$ agree on values of output deciding variables in $S_{1}^{\prime}$ and $S_{2}^{\prime}$ including the input, I/O sequence variable.

By definition, $\operatorname{OVar}\left(S_{1}^{\prime}\right) \subseteq \operatorname{OVar}\left(S_{1}\right)$. So are the cases to $S_{2}^{\prime}$ and $S_{2}$. In addition, value stores $\sigma_{1}$ and $\sigma_{2}$ are not changed in the evaluation of the predicate expression $e$. The condition holds.

By assumption, $S_{1}^{\prime}$ and $S_{2}^{\prime}$ terminate in the same way and produce the same output sequence when started in states $m_{1}\left(\operatorname{loop}_{c}^{\prime}, \sigma_{1}\right)$ and $m_{2}\left(\operatorname{loop}_{c}^{\prime}, \sigma_{2}\right)$. In addition, $S_{1}^{\prime}$ and $S_{2}^{\prime}$ have equivalent computation of output deciding variables in $S_{1}^{\prime}$ and $S_{2}^{\prime}$ when started in states $m_{1}\left(\operatorname{loop}_{c}^{\prime}, \sigma_{1}\right)$ and $m_{2}\left(\operatorname{loop}_{c}^{\prime}, \sigma_{2}\right)$. Then there are two cases.

(a) $S_{1}^{\prime}$ and $S_{2}^{\prime}$ both do not terminate and produce the same output sequence.

By Lemma E.2. $S_{1}^{\prime} ; S_{1}$ and $S_{2}^{\prime} ; S_{2}$ both do not terminate and produce the same output sequence.

(b) $S_{1}^{\prime}$ and $S_{2}^{\prime}$ both terminate and have equivalent computation of output deciding variables in $S_{1}^{\prime}$ and $S_{2}^{\prime}$.

By assumption, $\left(S_{1}^{\prime}, m_{1}\left(\right.\right.$ loop $\left.\left._{c}^{\prime}, \sigma_{1}\right)\right) \stackrel{*}{\rightarrow}\left(\right.$ skip, $m_{1}^{\prime}\left(\right.$ loop $\left.\left._{c}^{\prime \prime}, \sigma_{1}^{\prime}\right)\right)$; $\left(S_{2}^{\prime}, m_{2}\left(\operatorname{loop}_{c}^{\prime}, \sigma_{2}\right)\right) \stackrel{*}{\rightarrow}$ (skip, $\left.m_{2}^{\prime}\left(\operatorname{loop}_{c}^{\prime \prime}, \sigma_{2}^{\prime}\right)\right)$ where $\forall x \in$ $\left(\mathrm{OVar}\left(S_{1}^{\prime}\right) \cup \operatorname{OVar}\left(S_{2}^{\prime}\right)\right) \cup\left\{i d_{I}, i d_{I O}\right\}, \sigma_{1}^{\prime}(x)=\sigma_{2}^{\prime}(x)$. By Lemma 6.9 $\operatorname{OVar}\left(S_{1}^{\prime}\right) \subseteq \operatorname{OVar}\left(S_{2}^{\prime}\right)$. Then variables used in the predicate expression of $S_{1}$ and $S_{2}$ are either in output deciding variables in both $S_{1}^{\prime}$ and $S_{2}^{\prime}$ or not. Therefore value stores $\sigma_{2}^{\prime}$ and $\sigma_{1}^{\prime}$ agree on values of variables used in the expression $e$ and even output deciding variables in $S_{1}$ and $S_{2}$.

Induction step on iterations

The induction hypothesis (IH) is that, when $i \geq 1$, one of the following holds:

1. Loop counters for $S_{1}$ and $S_{2}$ are always less than $i$ if any is present, $\forall m_{1}^{\prime}\left(\operatorname{loop}_{c}^{1^{\prime}}\right) m_{2}^{\prime}\left(\operatorname{loop}_{c}^{2^{\prime}}\right):\left(S_{1}, m_{1}\left(\operatorname{loop}_{c}^{1}, \sigma_{1}\right)\right) \stackrel{*}{\rightarrow}$ $\left(S_{1}^{\prime \prime}, m_{1}^{\prime}\left(\operatorname{loop}_{c}^{1^{\prime}}\right)\right), \operatorname{loop}_{c}^{1^{\prime}}\left(n_{1}\right)<i,\left(S_{2}, m_{2}\left(\operatorname{loop}_{c}^{2}, \sigma_{2}\right)\right) \stackrel{*}{\rightarrow}$ $\left(S_{2}^{\prime \prime}, m_{2}^{\prime}\left(\operatorname{loop}_{c}^{2^{\prime}}\right)\right), \operatorname{loop}_{c}^{2^{\prime}}\left(n_{2}\right)<i, S_{1}$ and $S_{2}$ terminate in the same way, produce the same output sequence, and have equivalent computation of output deciding variables in both $S_{1}$ and $S_{2}$ 
as well as the input sequence variable, the I/O sequence variable, $\left(S_{1}, m_{1}\right) \equiv_{H}\left(S_{2}, m_{2}\right)$ and $\left(S_{1}, m_{1}\right) \equiv_{O}\left(S_{2}, m_{2}\right)$ and $\forall x \in\left(\mathrm{OVar}\left(S_{1}\right) \cup \operatorname{OVar}\left(S_{2}\right)\right) \cup\left\{i d_{I}, i d_{I O}\right\}:\left(S_{1}, m_{1}\right) \equiv_{x}$ $\left(S_{2}, m_{2}\right)$;

2. The loop counter of $S_{1}$ and $S_{2}$ are of value less than or equal to $i$, and there are no reachable configurations $\left(S_{1}, m_{1}\left(\operatorname{loop}_{c}^{1_{i}}, \sigma_{1_{i}}\right)\right)$ from $\left(S_{1}, m_{1}\left(\sigma_{1}\right)\right),\left(S_{2}, m_{2}\left(\operatorname{loop}_{c}^{2_{i}}, \sigma_{2_{i}}\right)\right)$ from $\left(S_{2}, m_{2}\left(\sigma_{2}\right)\right)$ where all of the followings hold:

- The loop counters of $S_{1}$ and $S_{2}$ are of value $i, \operatorname{loop}_{c}^{1_{i}}\left(n_{1}\right)$ $=\operatorname{loop}_{c}^{2_{i}}\left(n_{2}\right)=i$.

- Value stores $\sigma_{1_{i}}$ and $\sigma_{2_{i}}$ agree on values of output deciding variables in $S_{1}$ and $S_{2}$ including the input sequence variable, and the I/O sequence variable, $\forall x \in\left(\mathrm{OV} \operatorname{ar}\left(S_{1}\right) \cup\right.$ $\left.\operatorname{OVar}\left(S_{2}\right)\right) \cup\left\{i d_{I}, i d_{I O}\right\}: \sigma_{1_{i}}(x)=\sigma_{2_{i}}(x)$.

3. There are reachable configurations $\left(S_{1}, m_{1}\left(\operatorname{loop}_{c}^{1_{i}}, \sigma_{1_{i}}\right)\right)$ from $\left(S_{1}, m_{1}\left(\sigma_{1}\right)\right),\left(S_{2}, m_{2}\left(\operatorname{loop}_{c}^{2_{i}}, \sigma_{2_{i}}\right)\right)$ from $\left(S_{2}, m_{2}\left(\sigma_{2}\right)\right)$ where all of the following hold:

- The loop counter of $S_{1}$ and $S_{2}$ are of value $i, \operatorname{loop}_{c}^{1_{i}}\left(n_{1}\right)$ $=\operatorname{loop}_{c}^{2_{i}}\left(n_{2}\right)=i$.

- Value stores $\sigma_{1_{i}}$ and $\sigma_{2_{i}}$ agree on values of output deciding variables in $S_{1}$ and $S_{2}$ including the input sequence variable, and the I/O sequence variable, $\forall x \in\left(\operatorname{OVar}\left(S_{1}\right) \cup\right.$ $\left.\left.\operatorname{OVar}\left(S_{2}\right)\right) \cup\left\{i d_{I}, i d_{I O}\right\}: \sigma_{1_{i}}(x)=\sigma_{2_{i}}(x)\right)$.

Then we show that, when $i+1$, one of the following holds:

1. Loop counters for $S_{1}$ and $S_{2}$ are always less than $i+1$ if any is present, $\forall m_{1}^{\prime}\left(\operatorname{loop}_{c}^{1^{\prime}}\right) m_{2}^{\prime}\left(\operatorname{loop}_{c}^{2^{\prime}}\right):\left(S_{1}, m_{1}\left(\operatorname{loop}_{c}^{1}, \sigma_{1}\right)\right) \stackrel{*}{\rightarrow}$ $\left(S_{1}^{\prime \prime}, m_{1}^{\prime}\left(\operatorname{loop}_{c}^{1^{\prime}}\right)\right), \operatorname{loop}_{c}^{1^{\prime}}\left(n_{1}\right)<i+1,\left(S_{2}, m_{2}\left(\operatorname{loop}_{c}^{2}, \sigma_{2}\right)\right) \stackrel{*}{\rightarrow}$ $\left(S_{2}^{\prime \prime}, m_{2}^{\prime}\left(\operatorname{loop}_{c}^{2^{\prime}}\right)\right)$, loop $2_{c}^{2^{\prime}}\left(n_{2}\right)<i+1, S_{1}$ and $S_{2}$ terminate in the same way, produce the same output sequence, and have equivalent computation of output deciding variables in $S_{1}$ and $S_{2}$ including the input sequence variable and the I/O sequence variable, $\left(S_{1}, m_{1}\right) \equiv_{H}\left(S_{2}, m_{2}\right)$ and $\left(S_{1}, m_{1}\right) \equiv_{O}$ $\left(S_{2}, m_{2}\right)$ and $\forall x \in\left(\operatorname{OVar}\left(S_{1}\right) \cup \operatorname{OVar}\left(S_{2}\right)\right) \cup\left\{i d_{I}, i d_{I O}\right\}$ : $\left(S_{1}, m_{1}\right) \equiv_{x}\left(S_{2}, m_{2}\right)$;

2. The loop counter of $S_{1}$ and $S_{2}$ are of value less than or equal to $i+1$, and there are no reachable configurations $\left(S_{1}, m_{1}\left(\operatorname{loop}_{c}^{1_{i+1}}, \sigma_{1_{i+1}}\right)\right)$ from $\left(S_{1}, m_{1}\left(\sigma_{1}\right)\right),\left(S_{2}, m_{2}\left(\operatorname{loop}_{c}^{2_{i+1}}\right.\right.$, $\left.\left.\sigma_{2_{i+1}}\right)\right)$ from $\left(S_{2}, m_{2}\left(\sigma_{2}\right)\right)$ where all of the following hold:

- The loop counters of $S_{1}$ and $S_{2}$ are of value $i+1$, $\operatorname{loop}_{c}^{1_{i+1}}\left(n_{1}\right)=\operatorname{loop}_{c}^{2_{i+1}}\left(n_{2}\right)=i+1$.

- Value stores $\sigma_{1_{i+1}}$ and $\sigma_{2_{i+1}}$ agree on values of output deciding variables in $S_{1}$ and $S_{2}$ including the input sequence variable, and the I/O sequence variable, $\forall x \in\left(\operatorname{OV} \operatorname{ar}\left(S_{1}\right) \cup\right.$ $\left.\operatorname{OVar}\left(S_{2}\right)\right) \cup\left\{i d_{I}, i d_{I O}\right\}: \sigma_{1_{i+1}}(x)=\sigma_{2_{i+1}}(x)$.

3. There are reachable configurations $\left(S_{1}, m_{1}\left(\operatorname{loop}_{c}^{1_{i+1}}, \sigma_{1_{i}}\right)\right)$ from $\left(S_{1}, m_{1}\left(\sigma_{1}\right)\right),\left(S_{2}, m_{2}\left(\operatorname{loop}_{c}^{2_{i+1}}, \sigma_{2_{i}}\right)\right)$ from $\left(S_{2}, m_{2}\left(\sigma_{2}\right)\right)$ where all of the following hold:

- The loop counter of $S_{1}$ and $S_{2}$ are of value $i$, $\operatorname{loop}_{c}^{1_{i+1}}\left(n_{1}\right)$ $=\operatorname{loop}_{c}^{2_{i+1}}\left(n_{2}\right)=i+1$.

- Value stores $\sigma_{1_{i+1}}$ and $\sigma_{2_{i+1}}$ agree on values of output deciding variables in $S_{1}$ and $S_{2}$ including the input sequence variable, and the I/O sequence variable, $\forall x \in\left(\mathrm{OVar}\left(S_{1}\right) \cup\right.$ $\left.\operatorname{OVar}\left(S_{2}\right)\right) \cup\left\{i d_{I}, i d_{I O}\right\}: \sigma_{1_{i+1}}(x)=\sigma_{2_{i+1}}(x)$.

By hypothesis IH and theorem 4 and 5 there is no configuration where loop counters of $S_{1}$ and $S_{2}$ are of value $i+1$ when any of the following holds:

1. Loop counters for $S_{1}$ and $S_{2}$ are always less than $i$ if any is present, $\forall m_{1}^{\prime}\left(\operatorname{loop}_{c}^{1^{\prime}}\right) m_{2}^{\prime}\left(\operatorname{loop}_{c}^{2^{\prime}}\right):\left(S_{1}, m_{1}\left(\operatorname{loop}_{c}^{1}, \sigma_{1}\right)\right) \stackrel{*}{\rightarrow}$ $\left(S_{1}^{\prime \prime}, m_{1}^{\prime}\left(\operatorname{loop}_{c}^{1^{\prime}}\right)\right), \operatorname{loop}_{c}^{1^{\prime}}\left(n_{1}\right)<i,\left(S_{2}, m_{2}\left(\operatorname{loop}_{c}^{2}, \sigma_{2}\right)\right) \stackrel{*}{\rightarrow}$ $\left(S_{2}^{\prime \prime}, m_{2}^{\prime}\left(\operatorname{loop}_{c}^{2^{\prime}}\right)\right), \operatorname{loop}_{c}^{2^{\prime}}\left(n_{2}\right)<i, S_{1}$ and $S_{2}$ terminate in the same way, produce the same output sequence, and have equivalent computation of output deciding variables in $S_{1}$ and $S_{2}$ including the input sequence variable and the $\mathrm{I} / \mathrm{O}$ sequence variable, $\left(S_{1}, m_{1}\right) \equiv_{H}\left(S_{2}, m_{2}\right)$ and $\left(S_{1}, m_{1}\right) \equiv_{O}\left(S_{2}, m_{2}\right)$ and $\forall x \in\left(\mathrm{OVar}\left(S_{1}\right) \cup \operatorname{OVar}\left(S_{2}\right)\right) \cup\left\{i d_{I}, i d_{I O}\right\}:\left(S_{1}, m_{1}\right) \equiv_{x}$ $\left(S_{2}, m_{2}\right)$

2. The loop counter of $S_{1}$ and $S_{2}$ are of value less than or equal to $i$, and there are no reachable configurations $\left(S_{1}, m_{1}\left(\operatorname{loop}_{c}^{1_{i}}, \sigma_{1_{i}}\right)\right)$ from $\left(S_{1}, m_{1}\left(\sigma_{1}\right)\right),\left(S_{2}, m_{2}\left(\operatorname{loop}_{c}^{2_{i}}, \sigma_{2_{i}}\right)\right)$ from $\left(S_{2}, m_{2}\left(\sigma_{2}\right)\right)$ where all of the following hold:

- The loop counters of $S_{1}$ and $S_{2}$ are of value $i, \operatorname{loop}_{c}^{1_{i}}\left(n_{1}\right)$ $=\operatorname{loop}_{c}^{2_{i}}\left(n_{2}\right)=i$.

- Value stores $\sigma_{1_{i}}$ and $\sigma_{2_{i}}$ agree on values of output deciding variables in both $S_{1}$ and $S_{2}$ as well as the input sequence variable, and the I/O sequence variable, $\forall x \in\left(\operatorname{OVar}\left(S_{1}\right) \cup\right.$ $\left.\operatorname{OVar}\left(S_{2}\right)\right) \cup\left\{i d_{I}, i d_{I O}\right\}: \sigma_{1_{i}}(x)=\sigma_{2_{i}}(x)$.

When there are reachable configurations $\left(S_{1}, m_{1}\left(\operatorname{loop}_{c}^{1_{i}}, \sigma_{1_{i}}\right)\right)$ from $\left(S_{1}, m_{1}\left(\sigma_{1}\right)\right),\left(S_{2}, m_{2}\left(\operatorname{loop}_{c}^{2_{i}}, \sigma_{2_{i}}\right)\right)$ from $\left(S_{2}, m_{2}\left(\sigma_{2}\right)\right)$ where all of the following hold:

- The loop counter of $S_{1}$ and $S_{2}$ are of value $i$, $\operatorname{loop}_{c}^{1_{i}}\left(n_{1}\right)$ $=\operatorname{loop}_{c}^{2 i}\left(n_{2}\right)=i$.

- Value stores $\sigma_{1_{i}}$ and $\sigma_{2_{i}}$ agree on values of output deciding variables in $S_{1}$ and $S_{2}$ including the input sequence variable and the I/O sequence variable, $\forall x \in\left(\operatorname{OV} \operatorname{Var}\left(S_{1}\right) \cup \operatorname{OVar}\left(S_{2}\right)\right) \cup$ $\left\{i d_{I}, i d_{I O}\right\}: \sigma_{1_{i}}(x)=\sigma_{2_{i}}(x)$. holds:

By similar argument in base case, we have one of the following

1. Loop counters for $S_{1}$ and $S_{2}$ are always less than $i+1$ if any is present, $\forall m_{1}^{\prime}\left(\operatorname{loop}_{c}^{1^{\prime}}\right) m_{2}^{\prime}\left(\operatorname{loop}_{c}^{2^{\prime}}\right):\left(S_{1}, m_{1}\left(\operatorname{loop}_{c}^{1}, \sigma_{1}\right)\right) \stackrel{*}{\rightarrow}$ $\left(S_{1}^{\prime \prime}, m_{1}^{\prime}\left(\operatorname{loop}_{c}^{1^{\prime}}\right)\right), \operatorname{loop}_{c}^{1^{\prime}}\left(n_{1}\right)<i+1,\left(S_{2}, m_{2}\left(\operatorname{loop}_{c}^{2}, \sigma_{2}\right)\right) \stackrel{*}{\rightarrow}$ $\left(S_{2}^{\prime \prime}, m_{2}^{\prime}\left(\operatorname{loop}_{c}^{2^{\prime}}\right)\right), \operatorname{loop}_{c}^{2^{\prime}}\left(n_{2}\right)<i, S_{1}$ and $S_{2}$ terminate in the same way, produce the same output sequence, and have equivalent computation of output deciding variables in $S_{1}$ and $S_{2}$ including the input sequence variable, the I/O sequence variable, $\left(S_{1}, m_{1}\right) \equiv_{H}\left(S_{2}, m_{2}\right)$ and $\left(S_{1}, m_{1}\right) \equiv_{O}\left(S_{2}, m_{2}\right)$ and $\forall x \in\left(\operatorname{OVar}\left(S_{1}\right) \cup \operatorname{OVar}\left(S_{2}\right)\right) \cup\left\{i d_{I}, i d_{I O}\right\}:\left(S_{1}, m_{1}\right) \equiv_{x}$ $\left(S_{2}, m_{2}\right)$;

2. The loop counter of $S_{1}$ and $S_{2}$ are of value less than or equal to $i+1$, and there are no reachable configurations $\left(S_{1}, m_{1}\left(\operatorname{loop}_{c}^{1_{i}}, \sigma_{1_{i}}\right)\right)$ from $\left(S_{1}, m_{1}\left(\sigma_{1}\right)\right),\left(S_{2}, m_{2}\left(\operatorname{loop}_{c}^{2_{i}}, \sigma_{2_{i}}\right)\right)$ from $\left(S_{2}, m_{2}\left(\sigma_{2}\right)\right)$ where all of the following hold:

- The loop counters of $S_{1}$ and $S_{2}$ are of value $i$, loop ${ }_{c}^{1_{i+1}}\left(n_{1}\right)$ $=\operatorname{loop}_{c}^{2_{i+1}}\left(n_{2}\right)=i+1$.

- Value stores $\sigma_{1_{i+1}}$ and $\sigma_{2_{i+1}}$ agree on values of output deciding variables in $S_{1}$ and $S_{2}$ including the input sequence variable and the I/O sequence variable, $\forall x \in\left(\operatorname{OVar}\left(S_{1}\right) \cup\right.$ $\left.\operatorname{OVar}\left(S_{2}\right)\right) \cup\left\{i d_{I}, i d_{I O}\right\}: \sigma_{1_{i+1}}(x)=\sigma_{2_{i+1}}(x)$.

3. There are reachable configurations $\left(S_{1}, m_{1}\left(\operatorname{loop}_{c}^{1_{i+1}}, \sigma_{1_{i+1}}\right)\right)$ from $\left(S_{1}, m_{1}\left(\sigma_{1}\right)\right),\left(S_{2}, m_{2}\left(\operatorname{loop}_{c}^{2_{i+1}}, \sigma_{2_{i+1}}\right)\right)$ from $\left(S_{2}\right.$, $\left.m_{2}\left(\sigma_{2}\right)\right)$ where all of the following hold:

- The loop counter of $S_{1}$ and $S_{2}$ are of value $i$, $\operatorname{loop}_{c}^{1_{i+1}}\left(n_{1}\right)$ $=\operatorname{loop}_{c}^{2_{i+1}}\left(n_{2}\right)=i$.

- Value stores $\sigma_{1_{i+1}}$ and $\sigma_{2_{i+1}}$ agree on values of output deciding variables in $S_{1}$ and $S_{2}$ including the input sequence variable, and the I/O sequence variable, $\forall x \in\left(\mathrm{OVar}\left(S_{1}\right) \cup\right.$ $\left.\operatorname{OVar}\left(S_{2}\right)\right) \cup\left\{i d_{I+1}, i d_{I O}\right\}: \sigma_{1_{i+1}}(x)=\sigma_{2_{i+1}}(x)$. 


\subsection{Proof rule for improved prompt message}

If the only difference between two programs are the constant messages that the user receives, we consider that the two programs to be equivalent. We realize that in general it is possible to introduce new semantics even by changing constant strings. An old version might have incorrectly labeled output: "median value $=5$ " instead of "average value $=5$, for example. We rule out such possibilities because all non-constant values are guaranteed to be exactly same. In practice, outputs could be classified into prompt outputs and actual outputs. Prompt outputs are those asking clients for inputs, which are constants hardcoded in the output statement. Actual outputs are dynamic messages produced by evaluation of non-constant expression in execution. The changes of prompt outputs are equivalent only for interactions with human clients. In order to prove the update of improved prompt messages to be backward compatible, we assume that the different prompt outputs produced in executions of the old program and the updated program, due to the different constants in output statements, are equivalent. Because the old program and the new program are exactly same except some output statements with different constants as expression $e$, we could show two programs produce the "equivalent" output sequence under the assumption of equivalent prompt outputs.

We formalize the generalized update of improved prompt messages, then we show that the updated program produce the same $\mathrm{I} / \mathrm{O}$ sequence as the old program in executions without program semantic errors. The following is the definition of the update class of improved prompt messages.

Definition 31. (Improved user messages) $A$ program $P_{2}=$ $P_{m p t} ; E N ; V ; S_{\text {entry }}$ includes updates of improved prompt messages compared with a program $P_{1}=P m p t_{1} ; E N ; V ; S_{\text {entry }}$, written $P_{2} \approx_{\text {Out }}^{S} P_{1}$, iff Pmpt $t_{2} \neq$ Pmpt $_{1}$.

We give the lemma that two programs terminate in the same way, produce the equivalent output sequence, and have equivalent computation of defined variables in both programs in valid executions if there are updates of improved prompt messages between them.

Lemma 6.11. Let $P_{1}=P m p t_{1} ; E N ; V ; S_{\text {entry }}$ and $P_{2}=$ $\mathrm{Pmpt}_{2} ; E N ; V ; S_{\text {entry }}$ be two programs where there are updates of improved prompt messages in $P_{2}$ compared with $P_{1}$. If $S_{1}$ and $S_{2}$ start in states $m_{1}\left(\sigma_{1}\right)$ and $m_{2}\left(\sigma_{2}\right)$ such that both of the following hold:

- Value stores $\sigma_{1}$ and $\sigma_{2}$ agree on values of variables used in $S_{\text {entry }}$ in both programs as well as the input sequence variable, $\forall x \in U$ Use $\left(S_{\text {entry }}\right) \cup\left\{i d_{I}\right\}: \sigma_{1}(x)=\sigma_{2}(x)$;

- Value stores $\sigma_{1}$ and $\sigma_{2}$ have "equivalent" I/O sequence, $\sigma_{1}\left(i d_{I O}\right) \equiv \sigma_{2}\left(i d_{I O}\right)$;

- The different prompt outputs in the update of improved prompt messages are equivalent;

then $S_{1}$ and $S_{2}$ terminate in the same way, produce the equivalent output sequence, and when $S_{1}$ and $S_{2}$ both terminate, they have equivalent computation of defined variables in $S_{\text {entry }}$ in both programs as well as the input sequence variable, $S_{\text {entry }}$ in the two programs produce the equivalent I/O sequence variable,

- $\left(S_{\text {entry }}, m_{1}\right) \equiv_{H}\left(S_{\text {entry }}, m_{2}\right)$;

- $\forall x \in\left(\operatorname{Def}\left(S_{1}\right) \cap \operatorname{Def}\left(S_{2}\right)\right) \cup\left\{i d_{I}\right\} \quad:\left(S_{\text {entry }}, m_{1}\right) \equiv_{x}$ $\left(S_{\text {entry }}, m_{2}\right)$;

- The produced output sequences in executions of $S_{\text {entry }}$ in both programs are "equivalent", $\sigma_{1}\left(i d_{I O}\right) \equiv \sigma_{2}\left(i d_{I O}\right)$.

The difference between prompt types in $P_{1}$ and $P_{2}$ can be either addition/removal of labels as well as the change of the mapping of labels with constants. The proof is straightforward because programs $P_{1}$ and $P_{2}$ have the same entry statement sequence and we have the assumption that different prompt outputs due to the difference of the prompt type are equivalent.

Proof. By induction on the sum of the program size of $S_{1}$ and $S_{2}$, $\operatorname{size}\left(S_{1}\right)+\operatorname{size}\left(S_{2}\right)$.

Base case. $S_{1}=$ "output $v_{1}$ " and $S_{2}=$ "output $v_{2}$ ";

Then the execution of $S_{2}$ proceeds as follows.

$$
\begin{aligned}
& \left(\text { output } v_{2}, m_{2}\left(\sigma_{2}\right)\right) \\
\rightarrow\left(\operatorname{skip}, m_{2}\left(\sigma_{2}\left[" \sigma_{2}\left(i d_{I O}\right) \cdot \overline{v_{2}} " / i d_{I O}\right]\right)\right) &
\end{aligned}
$$$$
\text { by the rule Out- } 1 \text { or Out- } 2
$$

Similarly, (output $\left.v_{1}, m_{1}\left(\sigma_{1}\right)\right) \stackrel{*}{\rightarrow}\left(\operatorname{skip}, m_{1}\left(\sigma_{1}\left[“ \sigma_{1}\left(i d_{I O}\right) \cdot \overline{v_{1}} " / i d_{I O}\right]\right)\right)$. By assumption, $\sigma_{2}\left(i d_{I O}\right) \equiv \sigma_{1}\left(i d_{I O}\right)$. In addition, by assumption, $\overline{v_{2}} \equiv \overline{v_{1}}$. Therefore, $S_{1}$ and $S_{2}$ terminate in the same way, produce the same output sequence and have equivalent computation of defined variables in $S_{1}$ and $S_{2}$. This lemma holds.

Induction step.

The hypothesis is that this lemma holds when the sum $k$ of the program size of $S_{1}$ and $S_{2}$ are great than or equal to $2, k \geq 2$.

We then show that this lemma holds when the sum of the program size of $S_{1}$ and $S_{2}$ is $k+1$. There are cases to consider.

1. $S_{1}$ and $S_{2}$ are both "If" statement:

$S_{1}=$ "If $(e)$ then $\left\{S_{1}^{t}\right\}$ else $\left\{S_{1}^{f}\right\} ", S_{2}=" \operatorname{If}(e)$ then $\left\{S_{2}^{t}\right\} \operatorname{else}\left\{S_{2}^{f}\right\} "$ where both of the following hold

- $S_{2}^{t} \approx_{\text {Out }}^{S} S_{1}^{t}$;

- $S_{2}^{f} \approx_{\text {Out }}^{S} S_{1}^{f}$;

By the definition of Use $\left(S_{1}\right)$, variables used in the predicate expression $e$ are a subset of used variables in $S_{1}$ and $S_{2}, \mathrm{Use}(e) \subseteq$ Use $\left(S_{1}\right) \cap \operatorname{Use}\left(S_{2}\right)$. By assumption, corresponding variables used in $e$ are of same value in value stores $\sigma_{1}$ and $\sigma_{2}$. By Lemma D.1 the expression evaluates to the same value w.r.t value stores $\left(\sigma_{1}\right.$ and $\sigma_{2}$. There are three possibilities.

(a) The evaluation of $e$ crashes, $\mathcal{E}^{\prime} \llbracket e \rrbracket \sigma_{1}=\mathcal{E}^{\prime} \llbracket e \rrbracket \sigma_{2}=$ (error, $v_{\mathfrak{o f}}$ ).

The execution of $S_{1}$ continues as follows:

(If $(e)$ then $\left\{S_{1}^{t}\right\}$ else $\left.\left\{S_{1}^{f}\right\}, m_{1}\left(\sigma_{1}\right)\right)$

$\rightarrow\left(\operatorname{If}\left(\left(\right.\right.\right.$ error, $\left.\left.v_{\text {of }}\right)\right)$ then $\left\{S_{1}^{t}\right\}$ else $\left.\left\{S_{1}^{f}\right\}, m_{1}\left(\sigma_{1}\right)\right)$

by the rule EEval'

$\rightarrow\left(\operatorname{If}(0)\right.$ then $\left\{S_{1}^{t}\right\}$ else $\left.\left\{S_{1}^{f}\right\}, m_{1}(1 / \mathfrak{f})\right)$

by the ECrash rule

$\stackrel{i}{\rightarrow}\left(\operatorname{If}(0)\right.$ then $\left.\left\{S_{1}^{t}\right\} \operatorname{else}\left\{S_{1}^{f}\right\}, m_{1}(1 / \mathfrak{f})\right)$ for any $i>0$

by the Crash rule.

Similarly, the execution of $S_{2}$ started from the state $m_{2}\left(\sigma_{2}\right)$ crashes. The lemma holds.

(b) The evaluation of $e$ reduces to zero, $\mathcal{E}^{\prime} \llbracket e \rrbracket \sigma_{1}=\mathcal{E}^{\prime} \llbracket e \rrbracket \sigma_{2}=$ $\left(0, v_{\mathfrak{o f}}\right)$.

The execution of $S_{1}$ continues as follows.

$$
\begin{aligned}
& \left(\operatorname{If}(e) \text { then }\left\{S_{1}^{t}\right\} \text { else }\left\{S_{1}^{f}\right\}, m_{1}\left(\sigma_{1}\right)\right) \\
= & \left(\operatorname{If}\left(\left(0, v_{\text {of }}\right)\right) \text { then }\left\{S_{1}^{t}\right\} \operatorname{else}\left\{S_{1}^{f}\right\}, m_{1}\left(\sigma_{1}\right)\right) \\
& \text { by the rule EEval' } \\
\rightarrow & \left(\operatorname{If}(0) \text { then }\left\{S_{1}^{t}\right\} \text { else }\left\{S_{1}^{f}\right\}, m_{1}\left(\sigma_{1}\right)\right) \\
& \text { by the E-Oflow } 1 \text { or E-Oflow } 2 \text { rule } \\
\rightarrow & \left(S_{1}^{f}, m_{1}\left(\sigma_{1}\right)\right) \text { by the If-F rule. }
\end{aligned}
$$

Similarly, the execution of $S_{2}$ gets to the configuration $\left(S_{2}^{f}, m_{2}\left(\sigma_{2}\right)\right)$.

By the hypothesis IH, we show the lemma holds. We need to show that all conditions are satisfied for the application of the hypothesis IH.

- $S_{2}^{f} \approx_{\text {Out }}^{S} S_{1}^{f}$

By assumption. 
- The sum of the program size of $S_{1}^{f}$ and $S_{2}^{f}$ is less than $k, \operatorname{size}\left(S_{1}^{f}\right)+\operatorname{size}\left(S_{2}^{f}\right)<k$.

By definition, $\operatorname{size}\left(S_{1}\right)=1+\operatorname{size}\left(S_{1}^{t}\right)+\operatorname{size}\left(S_{1}^{f}\right)$. Then, $\operatorname{size}\left(S_{1}^{f}\right)+\operatorname{size}\left(S_{2}^{f}\right)<k+1-2=k-1$.

- Value stores $\sigma_{1}$ and $\sigma_{2}$ agree on values of used variables in $S_{1}^{f}$ and $S_{2}^{f}$ as well as the input, I/O sequence variable. By definition, $\operatorname{Use}\left(S_{1}^{f}\right) \subseteq \operatorname{Use}\left(S_{1}\right)$. So are the cases to $S_{2}^{f}$ and $S_{2}$. In addition, value stores $\sigma_{1}$ and $\sigma_{2}$ are not changed in the evaluation of the predicate expression $e$. The condition holds.

- Different constants used in output statements are equivalent as output values.

By assumption.

By the hypothesis IH, the lemma holds.

(c) The evaluation of $e$ reduces to the same nonzero integer value, $\mathcal{E}^{\prime} \llbracket e \rrbracket \sigma_{1}=\mathcal{E}^{\prime} \llbracket e \rrbracket \sigma_{2}=\left(v, v_{\mathfrak{o f}}\right)$ where $v \neq 0$

By argument similar to the second subcase above.

2. $S_{1}$ and $S_{2}$ are both "while" statements:

$S_{1}=$ "while $_{\langle n\rangle}(e)\left\{S_{1}^{\prime}\right\} ", S_{2}=$ "while $\langle n\rangle(e)\left\{S_{2}^{\prime}\right\} "$ where $S_{2}^{\prime} \approx{ }_{\text {Out }}^{S} S_{1}^{\prime}$

By Lemma 6.13, we show this lemma holds. We need to show that all required conditions are satisfied for the application of Lemma 6.13

- $S_{1}^{\prime}$ and $S_{2}^{\prime}$ have same set of defined variables, $\operatorname{Def}\left(S_{1}^{\prime}\right)=$ $\operatorname{Def}\left(S_{2}^{\prime}\right)=\operatorname{Def}(S)$

- The used variables in $S_{1}^{\prime}$ are a subset of those in $S_{2}^{\prime}$, $\operatorname{Use}\left(S_{1}^{\prime}\right)=\operatorname{Use}\left(S_{2}^{\prime}\right)$

By Lemma 6.12

- When started in states $m_{1}^{\prime}\left(\sigma_{1}^{\prime}\right), m_{2}^{\prime}\left(\sigma_{1}^{\prime}\right)$ where value stores $\sigma_{1}^{\prime}$ and $\sigma_{2}^{\prime}$ agree on values of used variables in both $S_{1}^{\prime}$ and $S_{2}^{\prime}$ as well as the input sequence variable, , and the I/O sequence variable, then $S_{1}^{\prime}$ and $S_{2}^{\prime}$ terminate in the same way, produce the same output sequence, and have equivalent computation of defined variables in both $S_{1}$ and $S_{2}$ as well as the input sequence variable and the I/O sequence variable.

By the induction hypothesis IH. This is because the sum of the program size of $S_{1}^{\prime}$ and $S_{2}^{\prime}$ is less than $k$. By definition, $\operatorname{size}\left(S_{1}\right)=1+\operatorname{size}\left(S_{1}^{\prime}\right)$.

By Lemma 6.13, this lemma holds.

3. $S_{1}=S_{1}^{\prime} ; s_{1}$ and $S_{2}=S_{2}^{\prime} ; s_{2}$ where both of the following hold:

- $S_{2}^{\prime} \approx_{\text {Out }}^{S} S_{1}^{\prime}$;

- $s_{2} \approx_{\text {Out }}^{S} s_{1}$

By the hypothesis IH, we show $S_{2}^{\prime}$ and $S_{1}^{\prime}$ terminate in the same way and produce the equivalent output sequence and when $S_{2}^{\prime}$ and $S_{1}^{\prime}$ both terminate, $S_{2}^{\prime}$ and $S_{1}^{\prime}$ have equivalent terminating computation of variables defined in $S_{2}^{\prime}$ and $S_{1}^{\prime}$ as well as the input sequence variable. By assumption, the different value of the I/O sequence in executions of $S_{1}$ and $S_{2}$ are equivalent.

We show all the required conditions are satisfied for the application of the hypothesis $\mathrm{IH}$.

- $S_{2}^{\prime} \approx_{\text {Out }}^{S} S_{1}^{\prime}$

- The I/O sequence variable in executions of $S_{1}$ and $S_{2}$ are equivalent, $\sigma_{1}\left(i d_{I O}\right) \equiv \sigma_{2}\left(i d_{I O}\right)$;

By assumption.

- The sum of the program size of $S_{1}^{\prime}$ and $S_{2}^{\prime}$ is less than $k$, $\operatorname{size}\left(S_{1}^{\prime}\right)+\operatorname{size}\left(S_{2}^{\prime}\right)<k$.

By definition, $\operatorname{size}\left(S_{2}\right)=\operatorname{size}\left(s_{2}\right)+\operatorname{size}\left(S_{2}^{\prime}\right)$ where $\operatorname{size}\left(s_{2}\right)<1$. Then, $\operatorname{size}\left(S_{2}^{\prime}\right)+\operatorname{size}\left(S_{1}^{\prime}\right)<k+1-$ $\operatorname{size}\left(s_{2}\right)-\operatorname{size}\left(s_{1}\right)<k$.

- Value stores $\sigma_{1}$ and $\sigma_{2}$ agree on values of used variables in both $S_{2}^{\prime}$ and $S_{1}^{\prime}$ as well as the input sequence variable.
By definition, $\operatorname{Use}\left(S_{2}^{\prime}\right) \subseteq \operatorname{Use}\left(S_{2}\right), \operatorname{Use}\left(S_{1}^{\prime}\right) \subseteq \operatorname{Use}\left(S_{1}\right)$. The condition holds.

By the hypothesis IH, one of the following holds:

(a) $S_{1}^{\prime}$ and $S_{2}^{\prime}$ both do not terminate.

By Lemma E.2 executions of $S_{1}=S_{1}^{\prime} ; s_{1}$ and $S_{2}=$ $S_{2}^{\prime} ; s_{2}$ both do not terminate and produce the same output sequence.

(b) $S_{1}^{\prime}$ and $S_{2}^{\prime}$ both terminate.

By assumption, $\left(S_{2}^{\prime}, m_{2}\left(\sigma_{2}\right)\right) \stackrel{*}{\rightarrow}\left(\right.$ skip, $\left.m_{2}^{\prime}\left(\sigma_{2}^{\prime}\right)\right),\left(S_{1}^{\prime}, m_{1}\left(\sigma_{1}\right)\right) \stackrel{*}{\rightarrow}$ (skip, $\left.m_{1}^{\prime}\left(\sigma_{1}^{\prime}\right)\right)$.

By Corollary E.1 $\left(S_{2}^{\prime} ; s_{2}, m_{2}\left(\sigma_{2}\right)\right) \stackrel{*}{\rightarrow}\left(s_{2}, m_{2}^{\prime}\left(\sigma_{2}^{\prime}\right)\right)$, $\left(S_{1}^{\prime} ; s_{1}, m_{1}\left(\sigma_{1}\right)\right) \stackrel{*}{\rightarrow}\left(s_{1}, m_{1}^{\prime}\left(\sigma_{1}^{\prime}\right)\right)$.

By the hypothesis $\mathrm{IH}$, we show that $s_{2}$ and $s_{1}$ terminate in the same way, produce the "equivalent" output sequence and when $s_{2}$ and $s_{1}$ both terminate, $s_{2}$ and $s_{1}$ have equivalent computation of variables defined in both $s_{1}$ and $s_{2}$ and the input sequence variable; $s_{2}$ and $s_{1}$ produce "equivalent" output sequence.

We need to show that all conditions are satisfied for the application of the hypothesis IH.

- There are updates of "improved prompt messages" in $s_{2}$ compared with $s_{1}, s_{2} \approx_{\text {Out }}^{S} s_{1}$;

By assumption, $s_{2} \approx_{\text {Out }}^{S} s_{1}$.

- The sum of the program size $s_{2}$ and $s_{1}$ is less than or equals to $k$;

By definition, $\operatorname{size}\left(S_{2}^{\prime}\right) \geq 1, \operatorname{size}\left(S_{1}^{\prime}\right) \geq 1$. Therefore, $\operatorname{size}\left(s_{2}\right)+\operatorname{size}\left(s_{1}\right)<k+1-\operatorname{size}\left(S_{2}^{\prime}\right)-\operatorname{size}\left(S_{1}^{\prime}\right) \leq k$.

- Value stores $\sigma_{1}^{\prime}$ and $\sigma_{2}^{\prime}$ agree on values of used variables in $s_{2}$ and $s_{1}$ as well as the input sequence variable; By Lemma 6.9 Use $\left(s_{1}\right)=\operatorname{Use}\left(s_{2}\right)$, then $\operatorname{Use}\left(s_{2}\right)$ $=\operatorname{Use}\left(s_{1}\right)=\operatorname{Use}(s)$. Similarly, by Lemma 6.9 $\operatorname{Def}\left(S_{1}^{\prime}\right)=\operatorname{Def}\left(S_{2}^{\prime}\right)$. For any variable $i d$ in Use $\left(s_{1}\right)$, if $i d$ is not in $\operatorname{Def}\left(S_{1}^{\prime}\right)$, then the value of $i d$ is not changed in the execution of $S_{1}^{\prime}$ and $S_{2}^{\prime}, \sigma_{1}^{\prime}(i d)=\sigma_{1}(i d)=$ $\sigma_{2}(i d)=\sigma_{2}^{\prime}(i d)$. Otherwise, the variable $i d$ is defined in the execution of $S_{1}^{\prime}$ and $S_{2}^{\prime}$, by assumption, $\sigma_{1}^{\prime}(i d)=\sigma_{2}^{\prime}(i d)$. The condition holds.

- Values of , the I/O sequence variable in value stores $\sigma_{1}^{\prime}$ and $\sigma_{2}^{\prime}$ are equivalent.

By assumption.

By the hypothesis IH, the lemma holds.

We list the auxiliary lemmas below. One lemma shows that, if there are updates of improved prompt messages between two statement sequences, then there are same set of defined variables and used variables in the two statement sequences. The second lemma shows that, if there are updates of improved prompt messages between two loop statements, then the two loop statement terminate in the same way, produce the equivalent output sequence, and have equivalent computation of defined variables in both the old and updated programs as well as the input sequence variable.

Lemma 6.12. Let $S_{2}$ be a statement sequence and $S_{1}$ where there are updates of "improved prompt messages", $S_{2} \approx_{\text {Out }}^{S} S_{1}$. Then used variables in $S_{2}$ are the same of used variables in $S_{1}$, Use $\left(S_{1}\right)=$ Use $\left(S_{2}\right)$, defined variables in $S_{2}$ are the same as used variables in $S_{1}, \operatorname{Def}\left(S_{1}\right)=\operatorname{Def}\left(S_{2}\right)$.

Proof. By induction on the sum of the program size of $S_{1}$ and $S_{2}$.

Lemma 6.13. Let $S_{1}=$ while $_{\left\langle n_{1}\right\rangle}(e)\left\{S_{1}^{\prime}\right\}$ and $S_{2}=$ while $_{\left\langle n_{2}\right\rangle}(e)$ $\left\{S_{2}^{\prime}\right\}$ be two loop statements where all of the following hold: 
- There are updates of improved prompt messages in $S_{2}^{\prime}$ compared with $S_{1}^{\prime}, S_{2}^{\prime} \approx{ }_{\text {Out }}^{S} S_{1}^{\prime}$;

- $S_{1}^{\prime}$ and $S_{2}^{\prime}$ have same set of defined variables,

$\operatorname{Def}\left(S_{1}^{\prime}\right)=\operatorname{Def}\left(S_{2}^{\prime}\right)=\operatorname{Def}(S)$;

- $S_{1}^{\prime}$ and $S_{2}^{\prime}$ have same set of used variables, Use $\left(S_{1}^{\prime}\right)=$ Use $\left(S_{2}^{\prime}\right)$;

- When started in states $m_{1}^{\prime}\left(\sigma_{1}^{\prime}\right), m_{2}^{\prime}\left(\sigma_{2}^{\prime}\right)$ where

- Value stores agree on values of used variables in both $S_{1}^{\prime}$ and $S_{2}^{\prime}$ as well as the input sequence variable, $\forall x \in$ Use $\left(S_{1}^{\prime}\right) \cup\left\{i d_{I}\right\} \forall m_{1}^{\prime}\left(\sigma_{1}^{\prime}\right) m_{2}^{\prime}\left(\sigma_{2}^{\prime}\right): \sigma_{1}^{\prime}(x)=\sigma_{2}^{\prime}(x)$;

- Values of the I/O sequence variable in value stores $\sigma_{1}^{\prime}, \sigma_{2}^{\prime}$ are equivalent, $\left.\sigma_{1}^{\prime}\left(i d_{I O}\right) \equiv \sigma_{2}^{\prime}\left(i d_{I O}\right)\right)$;

then $S_{1}^{\prime}$ and $S_{2}^{\prime}$ terminate in the same way, produce the "equivalent" output sequence, and have equivalent computation of defined variables in $S_{1}^{\prime}$ and $S_{2}^{\prime}$ as well as the input sequence variable, $\left(\left(S_{1}^{\prime}, m_{1}\right) \equiv_{H}\left(S_{2}^{\prime}, m_{2}\right)\right) \wedge\left(\forall x \in \operatorname{Def}(S) \cup\left\{i d_{I}\right\}\right.$ : $\left.\left(S_{1}^{\prime}, m_{1}\right) \equiv_{x}\left(S_{2}^{\prime}, m_{2}\right)\right)$;

If $S_{1}$ and $S_{2}$ start in states $m_{1}\left(\operatorname{loop}_{c}^{1}, \sigma_{1}\right), m_{2}\left(\right.$ loop $\left._{c}^{2}, \sigma_{2}\right)$ respectively, with loop counters of $S_{1}$ and $S_{2}$ not initialized $\left(S_{1}, S_{2}\right.$ have not executed yet), value stores agree on values of used variables in $S_{1}$ and $S_{2}$, and there are no program semantic errors, then, for any positive integer $i$, one of the following holds:

1. Loop counters for $S_{1}$ and $S_{2}$ are always less than $i$ if any is present, $\forall m_{1}^{\prime}\left(\right.$ loop $\left._{c}^{1^{\prime}}\right) m_{2}^{\prime}\left(\right.$ loop $\left._{c}^{2^{\prime}}\right):\left(S_{1}, m_{1}\left(\right.\right.$ loop $\left.\left._{c}^{1}, \sigma_{1}\right)\right) \stackrel{*}{\rightarrow}$ $\left(S_{1}^{\prime \prime}, m_{1}^{\prime}\left(\operatorname{loop}_{c}^{1^{\prime}}\right)\right), \operatorname{loop}_{c}^{1^{\prime}}\left(n_{1}\right)<i,\left(S_{2}, m_{2}\left(\operatorname{loop}_{c}^{2}, \sigma_{2}\right)\right) \stackrel{*}{\rightarrow}$ $\left(S_{2}^{\prime \prime}, m_{2}^{\prime}\left(\operatorname{loop}_{c}^{2^{\prime}}\right)\right)$, loop ${ }_{c}^{2^{\prime}}\left(n_{2}\right)<i, S_{1}$ and $S_{2}$ terminate in the same way, produce the equivalent output sequence, and have equivalent computation of defined variables in both $S_{1}$ and $S_{2}$ and the input sequence variable, $\left(S_{1}, m_{1}\right) \equiv_{H}\left(S_{2}, m_{2}\right)$ and $\forall x \in\left(\operatorname{Def}\left(S_{1}\right) \cap \operatorname{Def}\left(S_{2}\right)\right) \cup\left\{i d_{I}\right\} \quad:\left(S_{1}, m_{1}\right) \equiv_{x}$ $\left(S_{2}, m_{2}\right) ; S_{1}$ and $S_{2}$ produce the "equivalent" I/O sequence;

2. The loop counter of $S_{1}$ and $S_{2}$ are of value less than or equal to $i$, and there are no reachable configurations $\left(S_{1}, m_{1}\right.$ (loop $_{c}^{1_{i}}$, $\left.\left.\sigma_{1_{i}}\right)\right)$ from $\left(S_{1}, m_{1}\left(\sigma_{1}\right)\right)$, $\left(S_{2}, m_{2}\left(\operatorname{loop}_{c}^{2}, \sigma_{2_{i}}\right)\right)$ from $\left(S_{2}\right.$, $\left.m_{2}\left(\sigma_{2}\right)\right)$ where all of the following hold:

- The loop counters of $S_{1}$ and $S_{2}$ are of value $i, \operatorname{loop}_{c}^{1_{i}}\left(n_{1}\right)$ $=\operatorname{loop}_{c}^{2 i}\left(n_{2}\right)=i$.

- Value stores $\sigma_{1_{i}}$ and $\sigma_{2_{i}}$ agree on values of used variables in both $S_{1}$ and $S_{2}$ as well as the input sequence variable, $\forall x \in\left(\operatorname{Use}\left(S_{1}\right) \cap \operatorname{Use}\left(S_{2}\right)\right) \cup\left\{i d_{I}\right\}: \sigma_{1_{i}}(x)=\sigma_{2_{i}}(x)$.

- Values of the I/O sequence variable in value stores $\sigma_{1_{i}}\left(i d_{I O}\right) \equiv$ $\sigma_{2_{i}}\left(i d_{I O}\right)$;

3. There are reachable configurations $\left(S_{1}, m_{1}\left(\operatorname{loop}_{c}^{1_{i}}, \sigma_{1_{i}}\right)\right)$ from $\left(S_{1}, m_{1}\left(\sigma_{1}\right)\right),\left(S_{2}, m_{2}\left(\operatorname{loop}_{c}^{2 i}, \sigma_{2_{i}}\right)\right)$ from $\left(S_{2}, m_{2}\left(\sigma_{2}\right)\right)$ where all of the following hold:

- The loop counter of $S_{1}$ and $S_{2}$ are of value $i$, loop $p_{c}^{1_{i}}\left(n_{1}\right)$ $=\operatorname{loop}_{c}^{2_{i}}\left(n_{2}\right)=i$.

- Value stores $\sigma_{1_{i}}$ and $\sigma_{2_{i}}$ agree on values of used variables in both $S_{1}$ and $S_{2}$ as well as the input sequence variable, $\forall x \in\left(\operatorname{Use}\left(S_{1}\right) \cap \operatorname{Use}\left(S_{2}\right)\right) \cup\left\{i d_{I}\right\}: \sigma_{1_{i}}(x)=\sigma_{2_{i}}(x)$.

- Values of the I/O sequence variable in value stores $\sigma_{1_{i}}, \sigma_{2_{i}}$ are equivalent, $\sigma_{1_{i}}\left(i d_{I O}\right) \equiv \sigma_{2_{i}}\left(i d_{I O}\right)$;

Proof. By induction on $i$.

Base case.

We show that, when $i=1$, one of the followings holds:

1. Loop counters for $S_{1}$ and $S_{2}$ are always less than 1 if any is present, $\forall m_{1}^{\prime}\left(\operatorname{loop}_{c}^{1^{\prime}}\right) m_{2}^{\prime}\left(\operatorname{loop}_{c}^{2^{\prime}}\right):\left(S_{1}, m_{1}\left(\operatorname{loop}_{c}^{1}, \sigma_{1}\right)\right) \stackrel{*}{\rightarrow}$ $\left(S_{1}^{\prime \prime}, m_{1}^{\prime}\left(\operatorname{loop}_{c}^{1^{\prime}}\right)\right), \operatorname{loop}_{c}^{1^{\prime}}\left(n_{1}\right)<i,\left(S_{2}, m_{2}\left(\operatorname{loop}_{c}^{2}, \sigma_{2}\right)\right) \stackrel{*}{\rightarrow}$ $\left(S_{2}^{\prime \prime}, m_{2}^{\prime}\left(\operatorname{loop}_{c}^{2^{\prime}}\right)\right), \operatorname{loop}_{c}^{2^{\prime}}\left(n_{2}\right)<i, S_{1}$ and $S_{2}$ terminate in the same way, produce the equivalent I/O sequence, and have equivalent computation of defined variables in both $S_{1}$ and $S_{2}$ and the input sequence variable, the I/O sequence variable, $\left(S_{1}, m_{1}\right) \equiv_{H}\left(S_{2}, m_{2}\right)$ and $\forall x \in \operatorname{Def}(S) \cup\left\{i d_{I}\right\}$ : $\left(S_{1}, m_{1}\right) \equiv_{x}\left(S_{2}, m_{2}\right)$;

2. $S_{1}$ and $S_{2}$ produce the equivalent output sequence and the equivalent I/O sequence;

3. Loop counters of $S_{1}$ and $S_{2}$ are of values less than or equal to 1 but there are no reachable configurations $\left(S_{1}, m_{1}\left(\operatorname{loop}_{c}^{1_{1}}, \sigma_{1_{i}}\right)\right)$ from $\left(S_{1}, m_{1}\left(\sigma_{1}\right)\right),\left(S_{2}, m_{2}\left(\operatorname{loop}_{c}^{2_{1}}, \sigma_{2_{i}}\right)\right)$ from $\left(S_{2}, m_{2}\left(\sigma_{2}\right)\right)$ where all of the following hold:

- The loop counter of $S_{1}$ and $S_{2}$ are of value $1, \operatorname{loop}_{c}^{1_{1}}\left(n_{1}\right)$ $=\operatorname{loop}_{c}^{2_{1}}\left(n_{2}\right)=1$.

- Value stores $\sigma_{1_{1}}$ and $\sigma_{21}$ agree on values of used variables in both $S_{1}$ and $S_{2}$ as well as the input sequence variable, and the I/O sequence variable, $\forall x \in\left(\mathrm{Use}\left(S_{1}\right) \cap \mathrm{Use}\left(S_{2}\right)\right) \cup$ $\left\{i d_{I}\right\}: \sigma_{1_{1}}(x)=\sigma_{2_{1}}(x)$.

- Values of the I/O sequence variable in value stores $\sigma_{1_{1}}$ and $\sigma_{2_{1}}$ are equivalent, $\sigma_{1_{1}}\left(i d_{I O}\right) \equiv \sigma_{2_{1}}\left(i d_{I O}\right)$;

4. There are reachable configuration $\left(S_{1}, m_{1}\left(\operatorname{loop}_{c}^{1_{1}}, \sigma_{1_{i}}\right)\right)$ from $\left(S_{1}, m_{1}\left(\sigma_{1}\right)\right),\left(S_{2}, m_{2}\left(\operatorname{loop}_{c}^{2}, \sigma_{2_{i}}\right)\right)$ from $\left(S_{2}, m_{2}\left(\sigma_{2}\right)\right)$ where all of the following hold:

- The loop counter of $S_{1}$ and $S_{2}$ are of value 1 , $\operatorname{loop}_{c}^{1_{1}}\left(n_{1}\right)$ $=\operatorname{loop}_{c}^{2_{1}}\left(n_{2}\right)=1$.

- Value stores $\sigma_{1_{1}}$ and $\sigma_{2_{1}}$ agree on values of used variables in both $S_{1}$ and $S_{2}$ as well as the input sequence variable, and the I/O sequence variable, $\forall x \in\left(\operatorname{Use}\left(S_{1}\right) \cap \operatorname{Use}\left(S_{2}\right)\right) \cup$ $\left\{i d_{I}\right\}: \sigma_{1_{1}}(x)=\sigma_{2_{1}}(x)$.

- Values of the I/O sequence variable in value stores $\sigma_{1_{1}}$ and $\sigma_{2_{1}}$ are equivalent, $\sigma_{1_{1}}\left(i d_{I O}\right) \equiv \sigma_{2_{1}}\left(i d_{I O}\right)$;

By definition, variables used in the predicate expression $e$ of $S_{1}$ and $S_{2}$ are used in $S_{1}$ and $S_{2}$, Use $(e) \subseteq \operatorname{Use}\left(S_{1}\right) \cap \operatorname{Use}\left(S_{2}\right)$. By assumption, value stores $\sigma_{1}$ and $\sigma_{2}$ agree on values of variables in $\operatorname{Use}(e)$, the predicate expression $e$ evaluates to the same value w.r.t value stores $\sigma_{1}$ and $\sigma_{2}$ by LemmaD.2 There are three possibilities.

1. The evaluation of $e$ crashes,

$\mathcal{E}^{\prime} \llbracket e \rrbracket \sigma_{1}=\mathcal{E}^{\prime} \llbracket e \rrbracket \sigma_{2}=\left(\right.$ error, $\left.v_{\mathfrak{o f}}\right)$.

The execution of $S_{1}$ continues as follows:

$\left(\right.$ while $\left._{\left\langle n_{1}\right\rangle}(e)\left\{S_{1}^{\prime}\right\}, m_{1}\left(\sigma_{1}\right)\right)$

$\rightarrow\left(\right.$ while $\left\langle n_{1}\right\rangle\left(\left(\right.\right.$ error, $\left.\left.\left.v_{\mathfrak{o f}}\right)\right)\left\{S_{1}^{\prime}\right\}, m_{1}\left(\sigma_{1}\right)\right)$ by the rule EEval'

$\rightarrow\left(\right.$ while $\left._{\left\langle n_{1}\right\rangle}(0)\left\{S_{1}^{\prime}\right\}, m_{1}(1 / \mathfrak{f})\right)$ by the ECrash rule

$\stackrel{i}{\rightarrow}\left(\right.$ while $\left._{\left\langle n_{1}\right\rangle}(0)\left\{S_{1}^{\prime}\right\}, m_{1}(1 / \mathfrak{f})\right)$ for any $i>0$ by the Crash rule.

Similarly, the execution of $S_{2}$ started from the state $m_{2}\left(\sigma_{2}\right)$ crashes. Therefore $S_{1}$ and $S_{2}$ terminate in the same way when started from $m_{1}$ and $m_{2}$ respectively. Because $\sigma_{1}\left(i d_{I O}\right) \equiv$ $\sigma_{2}\left(i d_{I O}\right)$, the lemma holds.

2. The evaluation of $e$ reduces to zero, $\mathcal{E}^{\prime} \llbracket e \rrbracket \sigma_{1}=\mathcal{E}^{\prime} \llbracket e \rrbracket \sigma_{2}=$ $\left(0, v_{\mathfrak{o f}}\right)$.

The execution of $S_{1}$ continues as follows.

$$
\begin{aligned}
& \left(\text { while }_{\left\langle n_{1}\right\rangle}(e)\left\{S_{1}^{\prime}\right\}, m_{1}\left(\sigma_{1}\right)\right) \\
= & (\text { while } \\
\left\langle n_{1}\right\rangle & \left.\left(\left(0, v_{\mathfrak{o f}}\right)\right)\left\{S_{1}^{\prime}\right\}, m_{1}\left(\sigma_{1}\right)\right) \\
& \text { by the rule EEval' } \\
\rightarrow & (\text { while } \\
& \text { by the E-Oflow } 1 \text { or E-Oflow } 2 \text { rule } \\
\rightarrow & \left(\text { skip, } m_{1}\left(\sigma_{1}\right)\right) \text { by the Wh-F rule. }
\end{aligned}
$$

Similarly, the execution of $S_{2}$ gets to the configuration (skip, $m_{2}\left(\sigma_{2}\right)$ ). Loop counters of $S_{1}$ and $S_{2}$ are less than 1 and value stores agree on values of used/defined variables in both $S_{1}$ and $S_{2}$ 
as well as the input sequence variable and the I/O sequence variable.

3. The evaluation of $e$ reduces to the same nonzero integer value, $\mathcal{E}^{\prime} \llbracket e \rrbracket \sigma_{1}=\mathcal{E}^{\prime} \llbracket e \rrbracket \sigma_{2}=\left(v, v_{\mathfrak{o f}}\right)$ where $v \neq 0$.

Then the execution of $S_{1}$ proceeds as follows:

$$
\begin{aligned}
& \left(\text { while }_{\left\langle n_{1}\right\rangle}(e)\left\{S_{1}^{\prime}\right\}, m_{1}\left(\sigma_{1}\right)\right) \\
= & \left.\left(\text { while }_{\left\langle n_{1}\right\rangle}\right\rangle\left(\left(v, v_{\text {of }}\right)\right)\left\{S_{1}^{\prime}\right\}, m_{1}\left(\sigma_{1}\right)\right) \\
& \text { by the rule EEval } \\
\rightarrow & (\text { while } \\
& \text { by the E-Oflow }\rangle \text {-Oflow } 1 \text { or E-Oflow } 2 \text { rule } \\
\rightarrow & \left.\left(S_{1}^{\prime} ; S_{1}^{\prime}\right\}, m_{1}\left(\sigma_{1}\right)\right) \\
& \text { by the Wh-T rule. }
\end{aligned}
$$

Similarly, the execution of $S_{2}$ proceeds to the configuration $\left(S_{2}^{\prime}\right.$; while ${\left.\text {, } n_{2}\right\rangle}(e)\left\{S_{2}^{\prime}\right\}, m_{2}\left(\operatorname{loop}_{c}^{2}\left[1 / n_{2}\right], \sigma_{2}\right))$.

By the assumption, we show that $S_{1}^{\prime}$ and $S_{2}^{\prime}$ terminate in the same way and produce the equivalent I/O sequence when started in the state $m_{1}\left(\operatorname{loop}_{c}^{1_{1}}, \sigma_{1}\right)$ and $m_{2}\left(\operatorname{loop}_{c}^{21}, \sigma_{2}\right)$ respectively, and $S_{1}^{\prime}$ and $S_{2}^{\prime}$ have equivalent computation of variables defined in both statement sequences if both terminate. We need to show that all conditions are satisfied for the application of the assumption.

- Values of the I/O sequence variable in value stores $\sigma_{1}$ and $\sigma_{2}$ are equivalent, $\sigma_{1}\left(i d_{I O}\right) \equiv \sigma_{2}\left(i d_{I O}\right)$;

The above two conditions are by assumption.

- Value stores $\sigma_{1}$ and $\sigma_{2}$ agree on values of used variables in $S_{1}^{\prime}$ and $S_{2}^{\prime}$ as well as the input sequence variable.

By definition, Use $\left(S_{1}^{\prime}\right) \subseteq \operatorname{Use}\left(S_{1}\right)$. So are the cases to $S_{2}^{\prime}$ and $S_{2}$. In addition, value stores $\sigma_{1}$ and $\sigma_{2}$ are not changed in the evaluation of the predicate expression $e$. The condition holds.

By assumption, $S_{1}^{\prime}$ and $S_{2}^{\prime}$ terminate in the same way and produce the equivalent output sequence when started in states $m_{1}\left(\right.$ loop $\left._{c}^{\prime}, \sigma_{1}\right)$ and $m_{2}\left(\right.$ loop $\left._{c}^{\prime}, \sigma_{2}\right)$. In addition, $S_{1}^{\prime}$ and $S_{2}^{\prime}$ have equivalent computation of variables used or defined in $S_{1}^{\prime}$ and $S_{2}^{\prime}$ when started in states $m_{1}\left(\operatorname{loop}_{c}^{\prime}, \sigma_{1}\right)$ and $m_{2}\left(\operatorname{loop}_{c}^{\prime}, \sigma_{2}\right)$.

Then there are two cases.

(a) $S_{1}^{\prime}$ and $S_{2}^{\prime}$ both do not terminate and produce the equivalent I/O sequence.

By Lemma E.2 $S_{1}^{\prime} ; S_{1}$ and $S_{2}^{\prime} ; S_{2}$ both do not terminate and produce the equivalent I/O sequence.

(b) $S_{1}^{\prime}$ and $S_{2}^{\prime}$ both terminate and have equivalent computation of variables defined in $S_{1}^{\prime}$ and $S_{2}^{\prime}$.

By assumption, $\left(S_{1}^{\prime}, m_{1}\left(\operatorname{loop}_{c}^{\prime}, \sigma_{1}\right)\right) \stackrel{*}{\rightarrow}\left(\right.$ skip, $\left.m_{1}^{\prime}\left(\operatorname{loop}_{c}^{\prime \prime}, \sigma_{1}^{\prime}\right)\right)$; $\left(S_{2}^{\prime}, m_{2}\left(\operatorname{loop}_{c}^{\prime}, \sigma_{2}\right)\right) \stackrel{*}{\rightarrow}$ (skip, $\left.m_{2}^{\prime}\left(\operatorname{loop}_{c}^{\prime \prime}, \sigma_{2}^{\prime}\right)\right)$ where $\forall x \in$ $\left(\operatorname{Def}\left(S_{1}^{\prime}\right) \cap \operatorname{Def}\left(S_{2}^{\prime}\right)\right) \cup\left\{i d_{I}\right\}, \sigma_{1}^{\prime}(x)=\sigma_{2}^{\prime}(x)$.

By assumption, Use $\left(S_{1}^{\prime}\right)=\operatorname{Use}\left(S_{2}^{\prime}\right)$ and $\operatorname{Def}\left(S_{1}^{\prime}\right)=$ $\operatorname{Def}\left(S_{2}^{\prime}\right)$. Then variables used in the predicate expression of $S_{1}$ and $S_{2}$ are either in variables used or defined in both $S_{1}^{\prime}$ and $S_{2}^{\prime}$ or not. Therefore value stores $\sigma_{2}^{\prime}$ and $\sigma_{1}^{\prime}$ agree on values of variables used in the expression $e$ and even variables used or defined in $S_{1}$ and $S_{2}$.

By assumption, $S_{1}$ and $S_{2}$ produce the equivalent output sequence.

Induction step on iterations

The induction hypothesis $(\mathrm{IH})$ is that, when $i \geq 1$, one of the following holds:

1. Loop counters for $S_{1}$ and $S_{2}$ are always less than $i$ if any is present, $\forall m_{1}^{\prime}\left(\operatorname{loop}_{c}^{1^{\prime}}\right) m_{2}^{\prime}\left(\operatorname{loop}_{c}^{2^{\prime}}\right):\left(S_{1}, m_{1}\left(\operatorname{loop}_{c}^{1}, \sigma_{1}\right)\right) \stackrel{*}{\rightarrow}$ $\left(S_{1}^{\prime \prime}, m_{1}^{\prime}\left(\operatorname{loop}_{c}^{1^{\prime}}\right)\right), \operatorname{loop}_{c}^{1^{\prime}}\left(n_{1}\right)<i,\left(S_{2}, m_{2}\left(\operatorname{loop}_{c}^{2}, \sigma_{2}\right)\right) \stackrel{*}{\rightarrow}$ $\left(S_{2}^{\prime \prime}, m_{2}^{\prime}\left(\operatorname{loop}_{c}^{2^{\prime}}\right)\right), \operatorname{loop}_{c}^{2^{\prime}}\left(n_{2}\right)<i, S_{1}$ and $S_{2}$ terminate in the same way, and have equivalent computation of defined variables in both $S_{1}$ and $S_{2}$ and the input sequence variable, $\left(S_{1}, m_{1}\right)$ $\equiv_{H}\left(S_{2}, m_{2}\right)$ and $\forall x \in \operatorname{Def}(S) \cup\left\{i d_{I}\right\}:\left(S_{1}, m_{1}\right) \equiv_{x}$ $\left(S_{2}, m_{2}\right) ; S_{1}$ and $S_{2}$ produce the equivalent I/O sequence;

2. The loop counter of $S_{1}$ and $S_{2}$ are of value less than or equal to $i$, and there are no reachable configurations $\left(S_{1}, m_{1}\left(\operatorname{loop}_{c}^{1_{i}}, \sigma_{1_{i}}\right)\right)$ from $\left(S_{1}, m_{1}\left(\sigma_{1}\right)\right),\left(S_{2}, m_{2}\left(\operatorname{loop}_{c}^{2_{i}}, \sigma_{2_{i}}\right)\right)$ from $\left(S_{2}, m_{2}\left(\sigma_{2}\right)\right)$ where all of the following hold:

- The loop counters of $S_{1}$ and $S_{2}$ are of value $i, \operatorname{loop}_{c}^{1_{i}}\left(n_{1}\right)$ $=\operatorname{loop}_{c}^{2 i}\left(n_{2}\right)=i$.

- Value stores $\sigma_{1_{i}}$ and $\sigma_{2_{i}}$ agree on values of used variables in both $S_{1}$ and $S_{2}$ as well as the input sequence variable, $\forall x \in\left(\operatorname{Use}\left(S_{1}\right) \cap \operatorname{Use}\left(S_{2}\right)\right) \cup\left\{i d_{I}\right\}: \sigma_{1_{i}}(x)=\sigma_{2_{i}}(x)$.

- Values of the I/O sequence variable in value stores $\sigma_{1_{i}}$ and $\sigma_{2_{i}}, \sigma_{1_{i}}\left(i d_{I O}\right) \equiv \sigma_{2_{i}}\left(i d_{I O}\right)$;

3. There are reachable configurations $\left(S_{1}, m_{1}\left(\operatorname{loop}_{c}^{1_{i}}, \sigma_{1_{i}}\right)\right)$ from $\left(S_{1}, m_{1}\left(\sigma_{1}\right)\right),\left(S_{2}, m_{2}\left(\operatorname{loop}_{c}^{2_{i}}, \sigma_{2_{i}}\right)\right)$ from $\left(S_{2}, m_{2}\left(\sigma_{2}\right)\right)$ where all of the following hold:

- The loop counter of $S_{1}$ and $S_{2}$ are of value $i, \operatorname{loop}_{c}^{1_{i}}\left(n_{1}\right)$ $=\operatorname{loop}_{c}^{2_{i}}\left(n_{2}\right)=i$.

- Value stores $\sigma_{1_{i}}$ and $\sigma_{2 i}$ agree on values of used variables in both $S_{1}$ and $S_{2}$ as well as the input sequence variable, $\forall x \in\left(\operatorname{Use}\left(S_{1}\right) \cap \operatorname{Use}\left(S_{2}\right)\right) \cup\left\{i d_{I}\right\}: \sigma_{1_{i}}(x)=\sigma_{2_{i}}(x)$.

- Values of the I/O sequence variable in value stores $\sigma_{1_{i}}$ and $\sigma_{2_{i}}$ are equivalent, $\sigma_{1_{i}}\left(i d_{I O}\right) \equiv \sigma_{2_{i}}\left(i d_{I O}\right)$;

Then we show that, when $i+1$, one of the following holds: The induction hypothesis (IH) is that, when $i \geq 1$, one of the following holds:

1. Loop counters for $S_{1}$ and $S_{2}$ are always less than $i+1$ if any is present, $\forall m_{1}^{\prime}\left(\operatorname{loop}_{c}^{1^{\prime}}\right) m_{2}^{\prime}\left(\operatorname{loop}_{c}^{2^{\prime}}\right):\left(S_{1}, m_{1}\left(\operatorname{loop}_{c}^{1}, \sigma_{1}\right)\right) \stackrel{*}{\rightarrow}$ $\left(S_{1}^{\prime \prime}, m_{1}^{\prime}\left(\operatorname{loop}_{c}^{1^{\prime}}\right)\right), \operatorname{loop}_{c}^{1^{\prime}}\left(n_{1}\right)<i+1,\left(S_{2}, m_{2}\left(\operatorname{loop}_{c}^{2}, \sigma_{2}\right)\right) \stackrel{*}{\rightarrow}$ $\left(S_{2}^{\prime \prime}, m_{2}^{\prime}\left(\operatorname{loop}_{c}^{2^{\prime}}\right)\right)$, $\operatorname{loop}_{c}^{2^{\prime}}\left(n_{2}\right)<i+1, S_{1}$ and $S_{2}$ terminate in the same way, produce the equivalent $\mathrm{I} / \mathrm{O}$ sequence, and have equivalent computation of defined variables in both $S_{1}$ and $S_{2}$ and the input sequence variable, $\left(S_{1}, m_{1}\right) \equiv_{H}\left(S_{2}, m_{2}\right)$ and $\left(S_{1}, m_{1}\right) \equiv_{O}\left(S_{2}, m_{2}\right)$ and $\forall x \in\left(\operatorname{Def}(S) \cup\left\{i d_{I}\right\}\right.$ : $\left(S_{1}, m_{1}\right) \equiv_{x}\left(S_{2}, m_{2}\right) ; S_{1}$ and $S_{2}$ produce the "equivalent" I/O sequence variable;

2. The loop counter of $S_{1}$ and $S_{2}$ are of value less than or equal to $i+1$, and there are no reachable configurations $\left(S_{1}, m_{1}\left(\operatorname{loop}_{c}^{1_{i+1}}, \sigma_{1_{i+1}}\right)\right)$ from $\left(S_{1}, m_{1}\left(\sigma_{1}\right)\right),\left(S_{2}, m_{2}\left(\right.\right.$ loop $_{c}^{2 i+1}$, $\left.\left.\sigma_{2_{i+1}}\right)\right)$ from $\left(S_{2}, m_{2}\left(\sigma_{2}\right)\right)$ where all of the following hold:

- The loop counters of $S_{1}$ and $S_{2}$ are of value $i+1$, $\operatorname{loop}_{c}^{1_{i+1}}\left(n_{1}\right)=\operatorname{loop}_{c}^{2_{i+1}}\left(n_{2}\right)=i+1$.

- Value stores $\sigma_{1_{i+1}}$ and $\sigma_{2_{i+1}}$ agree on values of used variables in both $S_{1}$ and $S_{2}$ as well as the input sequence variable, $\forall x \in\left(\operatorname{Use}\left(S_{1}\right) \cap \operatorname{Use}\left(S_{2}\right)\right) \cup\left\{i d_{I}\right\}: \sigma_{1_{i+1}}(x)=$ $\sigma_{2_{i+1}}(x)$.

- Values of the I/O sequence variable in value stores $\sigma_{1_{i+1}}$ and $\sigma_{2_{i+1}}$ are equivalent, $\sigma_{1_{i+1}}\left(i d_{I O}\right) \equiv \sigma_{2_{i+1}}\left(i d_{I O}\right)$;

3. There are reachable configurations $\left(S_{1}, m_{1}\left(\operatorname{loop}_{c}^{1_{i+1}}, \sigma_{1_{i}}\right)\right)$ from $\left(S_{1}, m_{1}\left(\sigma_{1}\right)\right),\left(S_{2}, m_{2}\left(\operatorname{loop}_{c}^{2 i+1}, \sigma_{2_{i}}\right)\right)$ from $\left(S_{2}, m_{2}\left(\sigma_{2}\right)\right)$ where all of the following hold:

- The loop counter of $S_{1}$ and $S_{2}$ are of value $i, \operatorname{loop}_{c}^{1_{i+1}}\left(n_{1}\right)$ $=\operatorname{loop}_{c}^{2 i+1}\left(n_{2}\right)=i+1$.

- Value stores $\sigma_{1_{i+1}}$ and $\sigma_{2_{i+1}}$ agree on values of used variables in both $S_{1}$ and $S_{2}$ as well as the input sequence variable, $\forall x \in\left(\operatorname{Use}\left(S_{1}\right) \cap \operatorname{Use}\left(S_{2}\right)\right) \cup\left\{i d_{I}\right\}: \sigma_{1_{i+1}}(x)=$ $\sigma_{2_{i+1}}(x)$;

- Values of the I/O sequence variable in value stores $\sigma_{1_{i+1}}$ and $\sigma_{2_{i+1}}$ are equivalent, $\sigma_{1_{i+1}}\left(i d_{I O}\right) \equiv \sigma_{2_{i+1}}\left(i d_{I O}\right)$; 
By hypothesis $\mathrm{IH}$, there is no configuration where loop counters of $S_{1}$ and $S_{2}$ are of value $i+1$ when any of the following holds:

1. Loop counters for $S_{1}$ and $S_{2}$ are always less than $i$ if any is present, $\forall m_{1}^{\prime}\left(\operatorname{loop}_{c}^{1^{\prime}}\right) m_{2}^{\prime}\left(\operatorname{loop}_{c}^{2^{\prime}}\right):\left(S_{1}, m_{1}\left(\operatorname{loop}_{c}^{1}, \sigma_{1}\right)\right) \stackrel{*}{\rightarrow}$ $\left(S_{1}^{\prime \prime}, m_{1}^{\prime}\left(\operatorname{loop}_{c}^{1^{\prime}}\right)\right), \operatorname{loop}_{c}^{1^{\prime}}\left(n_{1}\right)<i,\left(S_{2}, m_{2}\left(\operatorname{loop}_{c}^{2}, \sigma_{2}\right)\right) \stackrel{*}{\rightarrow}$ $\left(S_{2}^{\prime \prime}, m_{2}^{\prime}\left(\operatorname{loop}_{c}^{2^{\prime}}\right)\right), \operatorname{loop}_{c}^{2^{\prime}}\left(n_{2}\right)<i, S_{1}$ and $S_{2}$ terminate in the same way, produce the equivalent I/O sequence, and have equivalent computation of used/defined variables in both $S_{1}$ and $S_{2}$ and the input sequence variable, , the I/O sequence variable, $\left(S_{1}, m_{1}\right) \equiv_{H}\left(S_{2}, m_{2}\right)$ and $\left(S_{1}, m_{1}\right) \equiv_{O}\left(S_{2}, m_{2}\right)$ and $\forall x \in\left(\operatorname{Def}\left(S_{1}\right) \cap \operatorname{Def}\left(S_{2}\right)\right) \cup\left\{i d_{I}\right\}:\left(S_{1}, m_{1}\right) \equiv_{x}\left(S_{2}, m_{2}\right)$; $S_{1}$ and $S_{2}$ produce , and the I/O sequence variable;

2. The loop counter of $S_{1}$ and $S_{2}$ are of value less than or equal to $i$, and there are no reachable configurations $\left(S_{1}, m_{1}\left(\operatorname{loop}_{c}^{1_{i}}, \sigma_{1_{i}}\right)\right)$ from $\left(S_{1}, m_{1}\left(\sigma_{1}\right)\right),\left(S_{2}, m_{2}\left(\operatorname{loop}_{c}^{2_{i}}, \sigma_{2_{i}}\right)\right)$ from $\left(S_{2}, m_{2}\left(\sigma_{2}\right)\right)$ where all of the following hold:

- The loop counters of $S_{1}$ and $S_{2}$ are of value $i$, $\operatorname{loop}_{c}^{1_{i}}\left(n_{1}\right)$ $=\operatorname{loop}_{c}^{2_{i}}\left(n_{2}\right)=i$.

- Value stores $\sigma_{1_{i}}$ and $\sigma_{2_{i}}$ agree on values of used variables in both $S_{1}$ and $S_{2}$ as well as the input sequence variable, $\forall x \in\left(\mathrm{Use}\left(S_{1}\right) \cap \operatorname{Use}\left(S_{2}\right)\right) \cup\left\{i d_{I},, i d_{I O}\right\}: \sigma_{1_{i}}(x)=$ $\sigma_{2_{i}}(x)$.

- Values of the $\mathrm{I} / \mathrm{O}$ sequence variable in value stores $\sigma_{1_{i}}$ and $\sigma_{2_{i}}$ are equivalent, $\sigma_{1_{i}}\left(i d_{I O}\right) \equiv \sigma_{2_{i}}\left(i d_{I O}\right)$;

When there are reachable configurations $\left(S_{1}, m_{1}\left(\operatorname{loop}_{c}^{1_{i}}, \sigma_{1_{i}}\right)\right)$ from $\left(S_{1}, m_{1}\left(\sigma_{1}\right)\right),\left(S_{2}, m_{2}\left(\operatorname{loop}_{c}^{2_{i}}, \sigma_{2_{i}}\right)\right)$ from $\left(S_{2}, m_{2}\left(\sigma_{2}\right)\right)$ where all of the following hold:

- The loop counter of $S_{1}$ and $S_{2}$ are of value $i$, $\operatorname{loop}_{c}^{1_{i}}\left(n_{1}\right)$ $=\operatorname{loop}_{c}^{2_{i}}\left(n_{2}\right)=i$.

- Value stores $\sigma_{1_{i}}$ and $\sigma_{2}$ agree on values of used variables in both $S_{1}$ and $S_{2}$ as well as the input sequence variable, $\forall x \in\left(\operatorname{Use}\left(S_{1}\right) \cap \operatorname{Use}\left(S_{2}\right)\right) \cup\left\{i d_{I}\right\}: \sigma_{1_{i}}(x)=\sigma_{2_{i}}(x)$.

- Values of the I/O sequence variable in value stores $\sigma_{1_{i}}$ and $\sigma_{2_{i}}$ are equivalent, $\sigma_{1_{i}}\left(i d_{I O}\right) \equiv \sigma_{2_{i}}\left(i d_{I O}\right)$; holds:

By similar argument in base case, we have one of the following

1. Loop counters for $S_{1}$ and $S_{2}$ are always less than $i+1$ if any is present, $\forall m_{1}^{\prime}\left(\operatorname{loop}_{c}^{1^{\prime}}\right) m_{2}^{\prime}\left(\operatorname{loop}_{c}^{2^{\prime}}\right):\left(S_{1}, m_{1}\left(\operatorname{loop}_{c}^{1}, \sigma_{1}\right)\right) \stackrel{*}{\rightarrow}$ $\left(S_{1}^{\prime \prime}, m_{1}^{\prime}\left(\operatorname{loop}_{c}^{1^{\prime}}\right)\right), \operatorname{loop}_{c}^{1^{\prime}}\left(n_{1}\right)<i+1,\left(S_{2}, m_{2}\left(\operatorname{loop}_{c}^{2}, \sigma_{2}\right)\right) \stackrel{*}{\rightarrow}$ $\left(S_{2}^{\prime \prime}, m_{2}^{\prime}\left(\operatorname{loop}_{c}^{2^{\prime}}\right)\right), \operatorname{loop}_{c}^{2^{\prime}}\left(n_{2}\right)<i, S_{1}$ and $S_{2}$ terminate in the same way, produce the equivalent I/O sequence, and have equivalent computation of defined variables in both $S_{1}$ and $S_{2}$ and the input sequence variable, $\left(S_{1}, m_{1}\right) \equiv_{H}\left(S_{2}, m_{2}\right)$ and $\forall x \in(\operatorname{Def}(S)) \cup\left\{i d_{I}\right\}:\left(S_{1}, m_{1}\right) \equiv_{x}\left(S_{2}, m_{2}\right)$;

2. The loop counter of $S_{1}$ and $S_{2}$ are of value less than or equal to $i+1$, and there are no reachable configurations $\left(S_{1}, m_{1}\left(\operatorname{loop}_{c}^{1_{i}}, \sigma_{1_{i}}\right)\right)$ from $\left(S_{1}, m_{1}\left(\sigma_{1}\right)\right),\left(S_{2}, m_{2}\left(\operatorname{loop}_{c}^{2_{i}}, \sigma_{2_{i}}\right)\right)$ from $\left(S_{2}, m_{2}\left(\sigma_{2}\right)\right)$ where all of the following hold:

- The loop counters of $S_{1}$ and $S_{2}$ are of value $i, \operatorname{loop}_{c}^{1_{i+1}}\left(n_{1}\right)$ $=\operatorname{loop}_{c}^{2_{i+1}}\left(n_{2}\right)=i+1$.

- Value stores $\sigma_{1_{i+1}}$ and $\sigma_{2_{i+1}}$ agree on values of used variables in both $S_{1}$ and $S_{2}$ as well as the input sequence variable, $\forall x \in\left(\operatorname{Use}\left(S_{1}\right) \cap \operatorname{Use}\left(S_{2}\right)\right) \cup\left\{i d_{I}\right\}: \sigma_{1_{i+1}}(x)=$ $\sigma_{2_{i+1}}(x)$.

- Values of the I/O sequence variable in value stores $\sigma_{1_{i+1}}$ and $\sigma_{2_{i+1}}$ are equivalent, $\sigma_{1_{i+1}}\left(i d_{I O}\right) \equiv \sigma_{2_{i+1}}\left(i d_{I O}\right)$;

3. There are reachable configurations $\left(S_{1}, m_{1}\left(\operatorname{loop}_{c}^{1_{i+1}}, \sigma_{1_{i+1}}\right)\right)$ from $\left(S_{1}, m_{1}\left(\sigma_{1}\right)\right),\left(S_{2}, m_{2}\left(\operatorname{loop}_{c}^{2 i+1}, \sigma_{2_{i+1}}\right)\right)$ from $\left(S_{2}\right.$, $\left.m_{2}\left(\sigma_{2}\right)\right)$ where all of the following hold:

$$
\begin{array}{lc}
\text { 1: } & \\
\text { 2: } & \text { If }(a>0) \text { then } \\
\text { 3: } & b:=c+1 \\
\text { 4: } & \text { output } b+c
\end{array}
$$

old

$$
\begin{array}{ll}
1^{\prime}: & b:=2 \\
2^{\prime}: & \text { If }(a>0) \text { then } \\
3^{\prime}: & b:=c+1 \\
4^{\prime}: & \text { output } b+c
\end{array}
$$

new
- The loop counter of $S_{1}$ and $S_{2}$ are of value $i$, $\operatorname{loop}_{c}^{1_{i+1}}\left(n_{1}\right)$ $=\operatorname{loop}_{c}^{2 i+1}\left(n_{2}\right)=i$.

- The loop counter of $S_{1}$ and $S_{2}$ are of value $i, \operatorname{loop}_{c}^{1_{i+1}}\left(, n_{1}\right)$ $=\operatorname{loop}_{c}^{2_{i+1}}\left(, n_{2}\right)=i$.

- Value stores $\sigma_{1_{i+1}}$ and $\sigma_{2_{i+1}}$ agree on values of used variables in both $S_{1}$ and $S_{2}$ as well as the input sequence variable, $\forall x \in\left(\operatorname{Use}\left(S_{1}\right) \cap \operatorname{Use}\left(S_{2}\right)\right) \cup\left\{i d_{I}\right\}: \sigma_{1_{i+1}}(x)=$ $\sigma_{2_{i+1}}(x)$;

- Values of the I/O sequence variable in value stores $\sigma_{1_{i+1}}$ and $\sigma_{2_{i+1}}$ are equivalent, $\sigma_{1_{i+1}}\left(i d_{I O}\right) \equiv \sigma_{2_{i+1}}\left(i d_{I O}\right)$;

\subsection{Proof rule for missing variable initializations}

A kind of bugfix we call missing-initialization includes variable initialization for those in the imported variables relative to the $\mathrm{I} / \mathrm{O}$ sequence variable in the old program. Figure 18 shows an example of missing-initializations. The initialization $b:=2$ ensures the value used in "output $b+c$ " is not to be undefined. In general, new variable initializations only affect rare buggy executions of the old program, where there are uses of undefined imported variables relative to the $\mathrm{I} / \mathrm{O}$ sequence variable in the program. Because DSU is not starting in error state, we assume that, in the proof of backward compatibility, there are no uses of variables with undefined variables in executions of the old program.

The following is the definition of the update class "missing initializations".

Definition 32. (Missing initializations) A statement sequence $S_{2}$ includes updates of missing initializations compared with a statement sequence $S_{1}$, written $S_{2} \approx_{\text {Init }}^{S} S_{1}$, iff $S_{2}=S_{\text {Init }} ; S_{1}$ where $S_{\text {Init }}$ is a sequence of assignment statements of form "lval $:=v$ " and $\operatorname{Def}\left(S_{\text {Init }}\right) \subseteq \operatorname{Imp}\left(S_{1},\left\{i d_{I O}\right\}\right)$;

Though the bugfix in the update of missing initializations are not in rare execution in the first case in Definition 32 , the definition shows the basic form of bugfix clearly.

We show that two statement sequences terminate in the same way, produce the same output sequence, and have equivalent computation of defined variables in both programs in valid executions if there are updates of missing initializations between them.

Lemma 6.14. Let $S_{1}$ and $S_{2}$ be two statement sequences respectively where there are updates of "missing initializations" in $S_{2}$ compared with $S_{1}, S_{2} \approx_{\text {Init }}^{S} S_{1}$. If $S_{1}$ and $S_{2}$ start in states $m_{1}\left(\sigma_{1}\right)$ and $m_{2}\left(\sigma_{2}\right)$ respectively such that both of the following hold:

- Value stores $\sigma_{1}$ and $\sigma_{2}$ agree on values of variables used in both $S_{1}$ and $S_{2}$ as well as the input sequence variable and the I/O sequence variable, $\forall i d \in\left(\operatorname{Use}\left(S_{1}\right) \cap U s e\left(S_{2}\right)\right) \cup\left\{i d_{I}, i d_{I O}\right\}$ : $\sigma_{1}(i d)=\sigma_{2}(i d)$

- defined variables in $S_{\text {Init }}$ are of undefined value in value stores $\sigma_{1}, \sigma_{2}, \forall i d \in \operatorname{Def}\left(S_{\text {Init }}\right): \sigma_{1}(i d)=\sigma_{2}(i d)=U d f \llbracket \tau \rrbracket$ where $\tau$ is the type of the variable $i d$;

- There are no use of variables with undefined values in the execution of $S_{1}$;

- There are no crash in execution of $S_{\text {Init }}$; 
then $S_{1}$ and $S_{2}$ terminate in the same way, produce the same output sequence, and when $S_{1}$ and $S_{2}$ both terminate, they have equivalent computation of used variables and defined variables in both $S_{1}$ and $S_{2}$ as well as the input sequence variable and the I/O sequence variable,

- $\left(S_{1}, m_{1}\right) \equiv_{H}\left(S_{2}, m_{2}\right)$;

- $\left(S_{1}, m_{1}\right) \equiv_{O}\left(S_{2}, m_{2}\right)$;

- $\forall x \in\left(\operatorname{Def}\left(S_{1}\right) \cup \operatorname{Def}\left(S_{2}\right)\right) \cup\left\{i d_{I}, i d_{I O}\right\}$ : $\left(S_{1}, m_{1}\right) \equiv_{x}$ $\left(S_{2}, m_{2}\right)$;

Proof. By induction on the sum of the program size of $S_{1}$ and $S_{2}$, $\operatorname{size}\left(S_{1}\right)+\operatorname{size}\left(S_{2}\right)$.

Base case. $S_{1}=s$ and $S_{2}=S_{\text {Init }} ; s$ where $S_{\text {Init }}=$ "lval $:=v "$ and $\operatorname{Def}($ lval $) \in \operatorname{Use}(s)$;

There are cases regarding lval in $S_{\text {Init }}$.

1. lval $=i d$.

Then the execution of $S_{2}$ proceeds as follows.

$$
\begin{aligned}
& \left(i d:=v ; s, m_{2}\left(\sigma_{2}\right)\right) \\
\rightarrow & \left(\text { skip; } s, m_{2}\left(\sigma_{2}[v /(i d)]\right)\right) \\
& \text { by the rule As-Scl } \\
\rightarrow & \left(s, m_{2}\left(\sigma_{2}[v /(i d)]\right)\right) \text { by the rule Seq. }
\end{aligned}
$$

By assumption, $i d \in \mathrm{Use}(e)$. By assumption, the value of $i d$ is undefined in value store $\sigma_{1}$. Then there is no valid execution of $S_{1}$. Then it holds that, in valid executions of $S_{1}, S_{1}$ and $S_{2}$ terminate in the same way, produce the same output sequence, and have equivalent computation of defined variables in both $S_{1}$ and $S_{2}$. Then this lemma holds.

2. lval $=i d[n]$.

Then the execution of $S_{2}$ proceeds as follows.

$$
\begin{aligned}
& \left(i d[n]:=v ; s, m_{2}\left(\sigma_{2}\right)\right) \\
\rightarrow & \left(\text { skip; } s, m_{2}\left(\sigma_{2}[v /(i d, n)]\right)\right) \\
& \text { by the rule As-Err } \\
\rightarrow & \left(s, m_{2}\left(\sigma_{2}[v /(i d, n)]\right)\right) \text { by the rule Seq. }
\end{aligned}
$$

By similar argument above, this lemma holds.

3. lval $=i d_{1}\left[i d_{2}\right]$.

Then the execution of $S_{2}$ proceeds as follows.

$$
\begin{aligned}
& \left(i d_{1}\left[i d_{2}\right]:=v ; s, m_{2}\left(\sigma_{2}\right)\right) \\
\rightarrow & \left(i d_{1}\left[v_{1}\right]:=v ; s, m_{2}\left(\sigma_{2}[v /(i d, n)]\right)\right) \\
& \text { by the rule Var } \\
\rightarrow & \left(\text { skip; } s, m_{2}\left(\sigma_{2}\left[v /\left(i d, v_{1}\right)\right]\right)\right) \text { by the rule As-Arr. } \\
\rightarrow & \left(s, m_{2}\left(\sigma_{2}\left[v /\left(i d, v_{1}\right)\right]\right)\right) \text { by the rule Seq. }
\end{aligned}
$$

By similar argument above, this lemma holds.

Induction step.

The hypothesis is that this lemma holds when the sum $k$ of the program size of $S_{1}$ and $S_{2}$ are great than or equal to $3, k \geq 3$.

We then show that this lemma holds when the sum of the program size of $S_{1}$ and $S_{2}$ is $k+1$.

$S_{2}=S_{\text {Init }} ; S_{1}$ where $S_{\text {Init }}$ is a sequence of assignment statements and $\operatorname{Def}\left(S_{\text {Init }}\right) \in \operatorname{Imp}\left(S_{1},\left\{i d_{I O}\right\}\right)$;

The proof is similar to that in the base case. By assumption, the execution of $S_{\text {Init }}$ does not crash, $\left(S_{\text {Init }}, m_{2}\left(\sigma_{2}\right)\right) \stackrel{*}{\rightarrow}$ (skip, $\left.m_{2}\left(\sigma_{2}^{\prime}\right)\right)$ where $\sigma_{2}^{\prime}=\sigma_{2}\left[v_{1} / x_{1}\right] \ldots\left[v_{k} / x_{k}\right]$ and $\forall 1 \leq i \leq$ $k: x_{k} \in \operatorname{Def}\left(S_{\text {Init }}\right)$.

By assumption, there are no use of variables with undefined values in the execution of $S_{1}$ by Theorem 5 and Theorem 4 this lemma holds.

\section{Related Work}

We discuss related work on DSU safety and program equivalence in order.

Existing studies on DSU safety could be roughly divided into high level studies and low level ones. There are a few studies on high level DSU safety. In [19], Kramer and Magee defined the DSU correctness that the updated system shall "operate as normal instead of progressing to an error state". This is covered by our requirement that hybrid executions conform to the old program's specification and our accommodation for bug fixes. Moreover, our backward compatibility includes I/O behavior, which is more concrete than the behavior in [19]. In [9], Bloom and Day proposed a DSU correctness which allows functionality extension that could not produce past behavior. This is probably because Bloom and Day considered updated environment. On the contrary, we assume that the environment is not updated. In addition, we explicitly present the error state, which is not mentioned in [9]. Panzica La Manna [28] presented a high level correctness only considering scenario-based specifications for controller systems instead of general programs.

There are also studies on low level DSU safety. Hayden et al. [15] discussed DSU correctness and concluded that there is only client-oriented correctness. Zhang et al. [32] asked the developers to ensure DSU correctness. Magill et al. [24] did ad-hoc program correlation without definitions of any correctness. We consider that there is general principle of DSU safety. The difference lies at the abstraction of the program behavior. We model program behavior by concrete I/O while others [15, 24, 32] consider a general program behavior.

We next discuss existing work on program equivalence. There is a rich literature on program equivalence and we compare our work only with most related work. Our study of program equivalence is inspired by original work of Horwitz et al. [17] on program dependence graphs, but we take a much more formal approach and we consider terminating as well as non-terminating programs with recurring I/O. In [13], Godlin and Strichman have a structured study of program equivalence similar to that of ours. Godlin and Strichman [13] restricted the equivalence to corresponding functions and therefore weakens the applicability to general transformations affecting loops such as loop fission, loop fusion and loop invariant code motion. However, our program equivalence allows loop optimizations such as loop fusion and loop fission. Furthermore, our syntactic conditions imply more program point mapping because we allow corresponding program point in arbitrary nested statements and in the middle of program that does not include function call.

\section{Conclusion}

In this paper, we propose a formal and practical general definition of DSU correction based on I/O sequences, backward compatibility. We devised a formal language and adapt the general definition of DSU correctness for executable programs based on our language. Based on the adapted backward compatibility, we proposed syntactic conditions that help guarantee correct DSUs for both terminating and nonterminating executions. In addition, we formalize typical program updates that are provably backward compatible, covering both new feature and bugfix.

In the future, we plan to identify more backward compatible update patterns by studying more open source programs. Though it is dubious if open source programs' evolution history includes typical update patterns, open source programs are the most important source of widely-used programs for our study of DSU. In addition, we plan to develop an algorithm for automatic state mapping based on our syntactic condition of program equivalence and definition of update classes. 


\section{References}

[1] Software Engineering - Software Life Cycle Processes - Maintenance. Technical Report ISO/IEC 14764:2006(E). 2.4

[2] Openssh users. http://www.openssh.com/users.html 2015. [Online; accessed 15-Jan-2015]. 3

[3] Vsftpd wikipage. http://en.wikipedia.org/wiki/Vsftpd 2015. [Online; accessed 15-Jan-2015]. [3]

[4] A. V. Aho, R. Sethi, and J. D. Ullman. Compilers: Principles, Techniques, and Tools. Addison-Wesley Longman Publishing Co., Inc., Boston, MA, USA, 1986. 5.2.1

[5] J. Arnold and M. F. Kaashoek. KSplice: Automatic Rebootless Kernel Updates. In EuroSys 2009, April 2009. 1

[6] N. Benton. Simple relational correctness proofs for static analyses and program transformations. SIGPLAN Not., 39(1):14-25, Jan. 2004. 1

[7] D. Binkley, S. Horwitz, and T. Reps. The multi-procedure equivalence theorem. Technical report, 1989. 1

[8] S. Blazy and X. Leroy. Mechanized semantics for the clight subset of the c language. Journal of Automated Reasoning, 43(3), 2009. 4.2

[9] T. Bloom and M. Day. Reconfiguration and module replacement in argus: theory and practice. Software Engineering Journal, Mar 1993. 17

[10] L. Cardelli. Type systems. ACM Computing Surveys, 28(1):263-264, 1996. A

[11] R. Cartwright and M. Felleisen. The semantics of program dependence. SIGPLAN Not., 24(7):13-27, 1989. 1

[12] W. Dietz, P. Li, J. Regehr, and V. Adve. Understanding integer overflow in c/c++. ICSE 2012, pages 760-770, 2012. 2

[13] B. Godlin and O. Strichman. Inference rules for proving the equivalence of recursive procedures. Acta Informatica, 45(6):403-439, 2008 17

[14] A. D. Gordon. Functional programming and input/output. Number 8. Cambridge University Press, 1994. 4.14.2

[15] C. M. Hayden, S. Magill, M. Hicks, N. Foster, and J. S. Foster Specifying and verifying the correctness of dynamic software updates. VSTTE'12, pages 278-293, Jan. 2012. 107

[16] M. Hicks. Dynamic Software Updating. PhD thesis, University of Pennsylvania, August 2001. 1

[17] S. Horwitz, J. Prins, and T. Reps. On the adequacy of program dependence graphs for representing programs. POPL '88, pages 146157. ACM, 1988. 1,7

[18] C. Karfa, K. Banerjee, D. Sarkar, and C. Mandal. Verification of loop and arithmetic transformations of array-intensive behaviors. Computer-Aided Design of Integrated Circuits and Systems, IEEE Transactions on, 32(11):1787-1800, Nov 2013. 1

[19] J. Kramer and J. Magee. The evolving philosophers problem: dynamic change management. Software Engineering, IEEE Transactions on, 16, Nov 1990. 17

[20] S. Kundu, Z. Tatlock, and S. Lerner. Proving optimizations correct using parameterized program equivalence. SIGPLAN Not., 44(6). 1

[21] D. Lacey, N. D. Jones, E. Van Wyk, and C. C. Frederiksen. Proving correctness of compiler optimizations by temporal logic. SIGPLAN Not., 37(1):283-294, Jan. 2002. ISSN 0362-1340. 1

[22] Y.-F. Lee and R.-C. Chang. Hotswapping linux kernel modules. Journal of Systems and Software, 79(2):163-175, February 2006. 1

[23] D. Lucanu and V. Rusu. Program equivalence by circular reasoning. In Integrated Formal Methods, volume 7940 of Lecture Notes in Computer Science, pages 362-377. Springer Berlin Heidelberg, 2013. 1

[24] S. Magill, M. Hicks, S. Subramanian, and K. S. McKinley. Automating object transformations for dynamic software updating. SIGPLAN Not., 47(10):265-280, Oct. 2012.117

[25] K. Makris. Whole-Program Dynamic Software Updating. PhD thesis, Arizona State University, December 2009. 1,3
[26] K. Makris and R. A. Bazzi. Immediate Multi-Threaded Dynamic Software Updates Using Stack Reconstruction. In Proceedings of the USENIX '09 Annual Technical Conference, June 2009. 3

[27] I. Neamtiu. Practical Dynamic Software Updating. PhD thesis, University of Maryland, August 2008. 3

[28] V. Panzica La Manna, J. Greenyer, C. Ghezzi, and C. Brenner. Formalizing correctness criteria of dynamic updates derived from specification changes. SEAMS '13, pages 63-72, 2013. 1,7

[29] D. L. Parnas. Software aging. ICSE '94, Los Alamitos, CA, USA IEEE Computer Society Press. 2.4

[30] B. C. Pierce. Types and programming languages. MIT press, 2002 4.2

[31] S. Verdoolaege, G. Janssens, and M. Bruynooghe. Equivalence checking of static affine programs using widening to handle recurrences. ACM Trans. Program. Lang. Syst., 34(3):11:1-11:35, Nov. 2012. 1

[32] M. Zhang, K. Ogata, and K. Futatsugi. Formalization and verification of behavioral correctness of dynamic software updates. Electronic Notes in Theoretical Computer Science, 294:12 - 23, 2013. 1]

\section{A. Type system}

Figure 19 shows an almost standard unsound and incomplete type system. The type system is unsound because of three reasons, (a) the possible value mismatch due to the subtype rule from hte type Int to Long, (b) the implicit subtype between enumeration types and the type Long allowed by our semantics and (c) the possible array index out of bound. The type system is incomplete due to the parameterized "other" expressions. The notation $\operatorname{Dom}(\Gamma)$ borrowed from Cardelli [10] in rules Tvar1, Tvar2, Tlabels an Tfundecl refers to the domain of the typing environment $\Gamma$, which are identifiers bound to a type in $\Gamma$.

\section{B. Syntactic definitions}

The syntax-directed definitions listed below make our argument independent of existing program analysis partially.

Definition 33. $(\operatorname{Idx}(l v a l))$ The used variables in index of a left value lval, written Idx(lval), are listed as follows:

1. $\operatorname{Idx}(i d)=\emptyset$;

2. $\operatorname{Idx}(i d[n])=\emptyset$;

3. $\operatorname{Idx}\left(i d_{1}\left[i d_{2}\right]\right)=\left\{i d_{2}\right\}$;

Definition 34. (Base(lval)) The base of a left value lval, written Base(lval), is listed as follows:

1. $\operatorname{Base}(i d)=\{i d\}$;

2. Base $(i d[n])=\{i d\}$;

3. $\operatorname{Base}\left(i d_{1}\left[i d_{2}\right]\right)=\left\{i d_{1}\right\}$;

Definition 35. (Use $(e))$ The set of used variables in an expression $e$, written Use $(e)$, are listed as follows:

1. Use $($ lval $)=$ Base $($ lval $) \cup I d x(l v a l)$;

2. Use $(i d==l)=\{i d\}$;

3. Use (other $)=$ Use(other) where function Use : other $\rightarrow\{i d\}$ is parameterized;

Definition 36. (Use $(S))$ The used variables in a sequence of statements $S$, written Use $(S)$, are listed as follows:

1. Use $($ skip $)=\emptyset$;

2. Use $($ lval $:=e)=U s e(e) \cup I d x(l v a l)$;

3. Use $($ output e $)=U s e(e) \cup\left\{i d_{I O}\right\}$;

4. Use (input $i d)=\left\{i d_{I}, i d_{I O}\right\}$;

5. Use $\left(I f(e)\right.$ then $\left\{S_{t}\right\}$ else $\left.\left\{S_{f}\right\}\right)=U$ se $(e) \cup U \operatorname{se}\left(S_{t}\right) \cup U \operatorname{se}\left(S_{f}\right)$;

6. Use $\left(\right.$ while $\left._{\langle n\rangle}(e)\left\{S^{\prime}\right\}\right)=\operatorname{Use}(e) \cup \operatorname{Use}\left(S^{\prime}\right)$; 


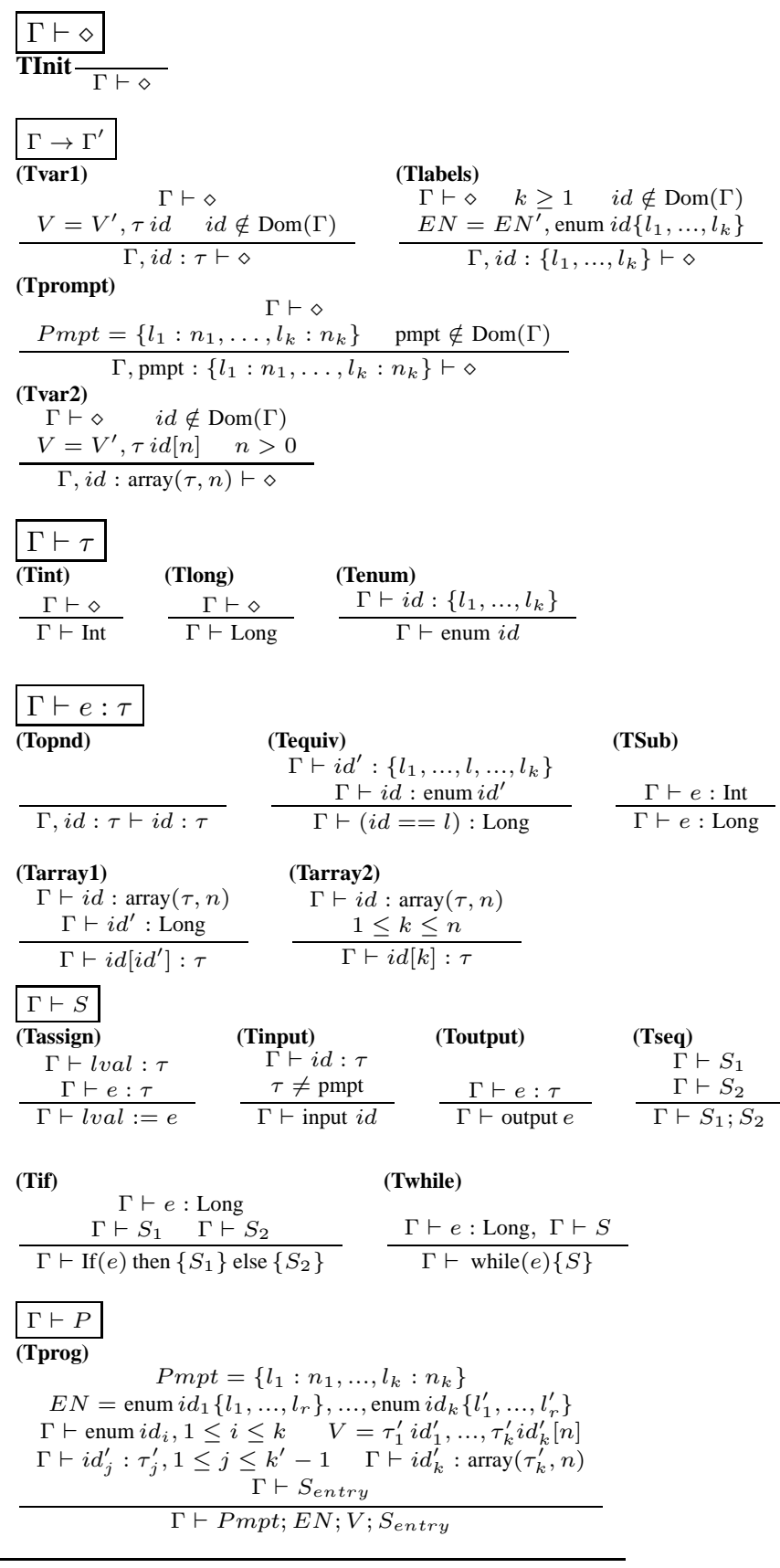

Figure 19: Typing rules

7. For $k>0$, Use $\left(s_{1} ; \ldots ; s_{k+1}\right)=U s e\left(s_{1} ; \ldots ; s_{k}\right) \cup U s e\left(s_{k+1}\right)$;

Definition 37. $(\operatorname{Def}(S))$ The defined variables in a sequence of statements $S$, written $\operatorname{Def}(S)$, are listed as follows:
1. $\operatorname{Def}($ skip $)=\emptyset$;
2. $\operatorname{Def}(i d:=e)=\{i d\}$;
3. Def(input $i d)=\left\{i d_{I}, i d_{I O}, i d\right\}$;
4. Def (output e) $=\left\{i d_{I O}\right\}$;
5. $\operatorname{Def}\left(I f(e)\right.$ then $\left\{S_{t}\right\}$ else $\left.\left\{S_{f}\right\}\right)=\operatorname{Def}\left(S_{t}\right) \cup \operatorname{Def}\left(S_{f}\right)$;
6. $\operatorname{Def}\left(\right.$ while $\left._{\langle n\rangle}(e)\{S\}\right)=\operatorname{Def}(S)$;
7. For $k>0, \operatorname{Def}\left(s_{1} ; \ldots ; s_{k+1}\right)=\operatorname{Def}\left(s_{1} ; \ldots ; s_{k}\right) \cup \operatorname{Def}\left(s_{k+1}\right)$;

Definition 38. $(s \in S)$ We say a statement $s$ is in a sequence of statements $S$ of a program $P$, written $s \in S$, if one of the following holds:

1. $S=s$;

2. If $S=$ "If $(e)$ then $\left\{S_{t}\right\}$ else $\left\{S_{f}\right\} ",\left(s \in S_{t}\right) \vee\left(s \in S_{f}\right)$;

3. If $S=$ "while(e) $\left\{S^{\prime}\right\} ", s \in S^{\prime}$;

4. For $k>0$, if $S=s_{1} ; \ldots ; s_{k} ; s_{k+1},\left(s \in s_{k+1}\right) \vee(s \in$ $\left.s_{1} ; \ldots ; s_{k}\right)$;

We write $s \notin S$ if $s \in S$ does not hold.

We show the definition of program size, which is based of our induction proof.

Definition 39. (size $(S))$ The program size of a statement sequence $S$, written size $(S)$, is listed as follows:

1. $\operatorname{size}(" s k i p ")=\operatorname{size}(" i d:=e ")=\operatorname{size}\left(" i d_{1}:=\operatorname{call}{ } d_{2}\left(e^{*}\right) "\right)$ $=\operatorname{size}\left(\right.$ "input id") = size ("output $\left.e^{\prime}\right)=1$;

2. size("If $(e)$ then $\left\{S_{t}\right\}$ else $\left\{S_{f}\right\}$ ") $=1+\operatorname{size}\left(S_{t}\right)+\operatorname{size}\left(S_{f}\right)$;

3. $\operatorname{size}\left(" w h i l e(e)\left\{S^{\prime}\right\} "\right)=1+\operatorname{size}\left(S^{\prime}\right)$;

4. For $k>0$, size $\left(s_{1} ; \ldots ; s_{k}=\sum_{i=1}^{k} \operatorname{size}\left(s_{i}\right)\right.$;

\section{Properties of imported variables}

Lemma C.1. $\operatorname{Imp}\left(S_{1} ; S_{2}, X\right)=\operatorname{Imp}\left(S_{1}, \operatorname{Imp}\left(S_{2}, X\right)\right)$.

Proof. Let statement sequence $S_{2}=s_{1} ; s_{2} ; \ldots ; s_{k}$ for some $k>0$. The proof is by induction on $k$.

Corollary C.1. $\forall i \in \mathbb{Z}_{+}, \operatorname{Imp}\left(S^{i+1}, X\right)=\operatorname{Imp}\left(S, \operatorname{Imp}\left(S^{i}, X\right)\right)$.

This is by lemma C.1

Lemma C.2. $\operatorname{Imp}(S, A \cup B)=\operatorname{Imp}(S, A) \cup \operatorname{Imp}(S, B)$.

Proof. By structural induction on abstract syntax of statement sequence $S$.

Lemma C.3. For statement $s=$ "while $(e)\{S\}$ " and a set of finite number of variables $X$ such that $X \cap \operatorname{Def}(s) \neq \emptyset$, there is $\beta>0$ such that $\bigcup_{0 \leq i \leq(\beta+1)} \operatorname{Imp}\left(S^{i}, X\right) \subseteq \bigcup_{1 \leq j \leq \beta} \operatorname{Imp}\left(S^{j}, X\right)$.

Proof. By contradiction against the fact that is finite number of variables redefined in statement $s$.

\section{Properties of expression evaluation}

We wrap the two properties of expression evaluation, which is based on the two properties of "other" expression evaluation. In the following, we use the notation $\mathcal{E}^{\prime}$ to expand the domain of the expression meaning function $\mathcal{E}^{\prime}: e \rightarrow \sigma \rightarrow\left(v_{\text {error }},\{0,1\}\right)$.

Lemma D.1. If every variable in Use(e) of an expression e has the same value w.r.t two value stores, the expression e evaluates to same value against the two value stores, $\left(\forall x \in U\right.$ Use $(e): \sigma_{1}(x)=$ $\left.\sigma_{2}(x)\right) \Rightarrow\left(\mathcal{E}^{\prime} \llbracket e \rrbracket \sigma_{1}=\mathcal{E}^{\prime} \llbracket e \rrbracket \sigma_{2}\right)$.

Proof. The proof is a case analysis of the expression $e$.

1. $e=l v a l$;

There are further cases regarding lval.

(a) $l v a l=i d$;

By definition, Use $(e)=\{i d\}$. Besides, there is no integer overflow in both evaluations. The lemma holds trivially.

(b) $l v a l=i d[n]$;

By definition, Use $(e)=\{i d\}$. Because the array has fixed size, by assumption, $\sigma_{1}(i d, n)=\sigma_{2}(i d, n)$ or $(i d, n, *) \notin$ $\sigma_{1},(i d, n, *) \notin \sigma_{2}$. Besides, there is no integer overflow in both evaluations. The lemma holds. 
(c) $l v a l=i d_{1}\left[i d_{2}\right]$;

By definition, Use $(e)=\left\{i d_{1}, i d_{2}\right\}$. By assumption, $\sigma_{1}\left(i d_{2}\right)=$ $\sigma_{2}\left(i d_{2}\right)=n$ By similar argument to the case lval $=i d[n]$, the lemma holds.

2. $e=$ "id $==l "$;

By definition, Use $(e)=\{i d\}$. W.l.o.g, $i d$ is a global variable. By assumption, $\sigma_{1}(i d)=\sigma_{2}(i d)=l^{\prime}$. If $l^{\prime}=l$, by rule Eq-T, $\left(l^{\prime}==l, m(\sigma)\right) \rightarrow(1, m)$. If $l^{\prime} \neq l$, by rule Eq$\mathrm{F},\left(l^{\prime}==l, m(\sigma)\right) \rightarrow(0, m)$. Besides, there is no integer overflow in both evaluations. The lemma holds.

3. $e=$ other;

By definition, $\operatorname{Use}(e)=\operatorname{Use}(e)$. By assumption, $\forall x \in$ Use $(e): \sigma_{1}(x)=\sigma_{2}(x) \vee \sigma_{1}(x)=\sigma_{2}(x)$. The lemma holds by parameterized expression meaning function for "other" expression.

Lemma D.2. If every variable in Err(e) of an expression e has same value w.r.t two pairs of (block, value store), $\forall x \in \operatorname{Err}(e)$ : $\sigma_{1}(x)=\sigma_{2}(x)$ then one of the following holds:

1. the expression evaluates to crash against the two value stores, $\left(\mathcal{E}^{\prime} \llbracket e \rrbracket \sigma_{1}=\left(\right.\right.$ error,$\left.\left.v_{\mathfrak{o f}}\right)\right) \wedge\left(\mathcal{E}^{\prime} \llbracket e \rrbracket \sigma_{2}=\left(\right.\right.$ error,$\left.\left.v_{\mathfrak{o f}}\right)\right) ;$

2. the expression evaluates to no crash against the two pairs of (block, value store $)\left(\mathcal{E}^{\prime} \llbracket e \rrbracket \sigma_{1} \neq\left(\right.\right.$ error,$\left.\left.v_{\mathfrak{o f}}^{1}\right)\right) \wedge\left(\mathcal{E}^{\prime} \llbracket e \rrbracket \sigma_{2} \neq\right.$ $\left(\right.$ error, $\left.\left.v_{\mathfrak{o f}}^{2}\right)\right)$.

Proof. The proof is a case analysis of the expression $e$.

1. $e=$ lval;

There are further cases regarding lval.

(a) $l v a l=i d$;

By definition, $\operatorname{Err}(e)=\operatorname{Idx}(e)=\emptyset$. By our semantic, the evaluation of $i d$ never crash. Besides, there is no integer overflow in both evaluations. The lemma holds.

(b) lval $=i d[n]$;

By definition, $\operatorname{Err}(e)=\operatorname{Idx}(e)=\emptyset$. Because the array $i d_{1}$ has a fixed array size, by assumption, either $\left(\left(i d_{1}, n, v_{1}\right) \in\right.$ $\left.\sigma_{1}\right) \wedge\left(\left(i d_{1}, n, v_{2}\right) \in \sigma_{2}\right)$ or $\left(\left(i d_{1}, n, v_{1}\right) \notin \sigma_{1}\right) \wedge$ $\left(\left(i d_{1}, n, v_{2}\right) \notin \sigma_{2}\right)$. Besides, there is no integer overflow in both evaluations. The lemma holds.

(c) $l v a l=i d_{1}\left[i d_{2}\right]$

By definition, $\operatorname{Err}(e)=\operatorname{Idx}(e)=\left\{i d_{2}\right\}$. By assumption, $\sigma_{1}\left(i d_{2}\right)=\sigma_{2}\left(i d_{2}\right)=n$ or $\sigma_{1}\left(i d_{2}\right)=\sigma_{2}\left(i d_{2}\right)=n$. By similar argument to the case lval $=i d[n]$, the lemma holds.

2. $e=$ "id $==l "$;

By definition, $\operatorname{Err}(e)=\emptyset$. W.l.o.g, $i d$ is a global variable. Let $\sigma_{1}(i d)=l_{1}, \sigma_{2}(i d)=l_{2}$. W.1.o.g., $l_{1}=l$ and $l_{2} \neq l$, by rule Eq-T, $\left(l_{1}==l, m(\sigma)\right) \rightarrow(1, m)$ and, by rule Eq$\mathrm{F},\left(l_{2}==l, m(\sigma)\right) \rightarrow(0, m)$. Besides, there is no integer overflow in both evaluations. The lemma holds.

3. $e=$ other;

By definition, $\operatorname{Err}(e)=\operatorname{Err}(e)$. By assumption, $\forall x \in \operatorname{Err}(e)$ : $\sigma_{1}(x)=\sigma_{2}(x)$. The lemma holds by the property of parameterized expression meaning function for "other" expression.

With respect to Lemma D.1 and LemmaD.2 we extend semantic rule for expression evaluation as follows.

\section{E. Properties of remaining execution}

We assume that crash flag $\mathfrak{f}=0$ in given execution state $m(\mathfrak{f})$.

Lemma E.1. $\left(S_{1}, m\right) \rightarrow\left(S_{1}^{\prime}, m^{\prime}\right) \Rightarrow\left(S_{1} ; S_{2}, m\right) \rightarrow\left(S_{1}^{\prime} ; S_{2}, m^{\prime}\right)$.

$$
\begin{aligned}
& (r, m) \rightarrow\left(r^{\prime}, m^{\prime}\right) \\
& \mathcal{E}^{\prime}: e \rightarrow \sigma \rightarrow\left(v_{\text {error }} \times\{0,1\}\right) \\
& \text { EEval' } \frac{\mathfrak{f}=0}{(e, m(\mathfrak{f}, \sigma)) \rightarrow\left(\mathcal{E}^{\prime} \llbracket e \rrbracket \sigma, m\right)}
\end{aligned}
$$

Figure 20: An extended SOS rule for expressions

Proof. The proof is by structural induction on abstract syntax of $S_{1}$.

Case 1. $S_{1}=$ "skip".

By rule Seq, $\left(\right.$ skip $\left.; S_{2}, m\right) \rightarrow\left(S_{2}, m\right)$ where $m=m^{\prime}$.

Case 2. $S_{1}=$ "id $:=e "$.

There are two subcases.

Case 2.1. $(e, m) \stackrel{*}{\rightarrow}(v, m)$ for some value $v$.

By rule Assign,

$(i d:=v, m) \rightarrow(\operatorname{skip}, m(\sigma[v / x]))$.

Then, by contextual (semantic) rule,

$\left(i d:=v ; S_{2}, m\right) \rightarrow\left(\right.$ skip; $\left.S_{2}, m(\sigma[v / x])\right)$.

Case 2.2. $(e, m) \stackrel{*}{\rightarrow}\left(e^{\prime}, m(1 / \mathfrak{f})\right)$ for some expression $e^{\prime}$.

Then, by rule crash,

$\left(i d:=e^{\prime}, m(1 / \mathfrak{f})\right) \rightarrow\left(i d:=e^{\prime}, m(1 / \mathfrak{f})\right)$.

Then, by contextual rule,

$\left(i d:=e^{\prime} ; S_{2}, m(1 / \mathfrak{f})\right) \rightarrow\left(i d:=e^{\prime} ; S_{2}, m(1 / \mathfrak{f})\right)$.

Case 3. $S_{1}=$ "output $e$ "

Case 4. $S_{1}=$ "input $i d$ "

By similar argument in Case 2, the lemma holds for case 3 and 4.

Case 5. $S_{1}=$ "If $(e)$ then $\left\{S_{t}\right\}$ else $\left\{S_{f}\right\}$ ".

Case 5.1. W.1.o.g., expression $e$ in predicate of $S_{1}$ evaluates to nonzero in state $m$, written $(e, m) \stackrel{*}{\rightarrow}(0, m)$.

By rule If-T, (If $(0)$ then $\left\{S_{t}\right\}$ else $\left.\left\{S_{f}\right\}, m\right) \rightarrow\left(S_{t}, m\right)$.

By contextual (semantic) rule,

(If $(0)$ then $\left\{S_{t}\right\}$ else $\left.\left\{S_{f}\right\} ; S_{2}, m\right) \rightarrow\left(S_{t} ; S_{2}, m\right)$.

Case 5.2. Evaluation of expression $e$ in predicate of $S_{1}$ crashes, written $(e, m) \stackrel{*}{\rightarrow}\left(e^{\prime}, m(1 / \mathfrak{f})\right)$.

Then, by rule crash,

(If $\left(e^{\prime}\right)$ then $\left\{S_{t}\right\}$ else $\left.\left\{S_{f}\right\}, m(1 / \mathfrak{f})\right) \rightarrow$

(If $\left(e^{\prime}\right)$ then $\left\{S_{t}\right\}$ else $\left\{S_{f}\right\}, m(1 / \mathfrak{f})$ ).

Then, by contextual rule,

(If $\left(e^{\prime}\right)$ then $\left\{S_{t}\right\}$ else $\left.\left\{S_{f}\right\} ; S_{2}, m(1 / \mathfrak{f})\right) \rightarrow$

(If $\left(e^{\prime}\right)$ then $\left\{S_{t}\right\}$ else $\left.\left\{S_{f}\right\} ; S_{2}, m(1 / \mathfrak{f})\right)$.

Case 6. $S_{1}=$ "while $\langle n\rangle(e)\{S\}$ ".

Case 6.1 When expression $e$ in predicate of $S_{1}$ evaluates to nonzero value, written $(e, m) \stackrel{*}{\rightarrow}(v, m)$ for some $v \neq 0$, then, by rule Wh- $\mathrm{T}$,

$\left(\right.$ while $\left._{\langle n\rangle}(e)\{S\}, m\right) \rightarrow\left(S ;\right.$ while $_{\langle n\rangle}(e)\{S\}, m\left(m_{c}[(k+\right.$ 1) $/ n]))$ for some nonnegative integer $k$.

Then, by contextual rule,

$\left(\right.$ while $\left._{\langle n\rangle}(e)\{S\} ; S_{2}, m\right) \rightarrow$

$\left(S ;\right.$ while $\left.\langle n\rangle(e)\{S\} ; S_{2}, m\left(m_{c}[(k+1) / n]\right)\right)$.

Case 6.2 When expression $e$ in predicate of $S_{1}$ evaluates to zero, written $(e, m) \stackrel{*}{\rightarrow}(0, m)$, then, by rule Wh-F,

$\left(\right.$ while $\left._{\langle n\rangle}(e)\{S\}, m\right) \rightarrow$ (skip, $\left.m\left(m_{c}[0 / n]\right)\right)$.

By contextual rule,

$\left(\right.$ while $\left._{\langle n\rangle}(e)\{S\} ; S_{2}, m\right) \rightarrow\left(\right.$ skip; $\left.S_{2}, m\left(m_{c}[0 / n]\right)\right)$.

Case 6.3 Evaluation of expression $e$ in predicate of $S_{1}$ crashes, written $(e, m) \stackrel{*}{\rightarrow}\left(e^{\prime}, m\right)$.

By rule crash,

$\left(\right.$ while $\left._{\langle n\rangle}\left(e^{\prime}\right)\{S\}, m(1 / \mathfrak{f})\right) \rightarrow\left(\right.$ while $\left._{\langle n\rangle}\left(e^{\prime}\right)\{S\}, m(1 / \mathfrak{f})\right)$.

Then, by contextual rule, 
$\left(\right.$ while $\left._{\langle n\rangle}\left(e^{\prime}\right)\{S\} ; S_{2}, m(1 / \mathfrak{f})\right) \rightarrow$

$\left(\right.$ while $\left._{\langle n\rangle}\left(e^{\prime}\right)\{S\} ; S_{2}, m(1 / \mathfrak{f})\right)$.

Lemma E.2. $\left(S_{1}, m\right) \stackrel{*}{\rightarrow}\left(S_{1}^{\prime}, m^{\prime}\right) \Rightarrow\left(S_{1} ; S_{2}, m\right) \stackrel{*}{\rightarrow}\left(S_{1}^{\prime} ; S_{2}, m^{\prime}\right)$.

Proof. By induction on number of steps $k$ in execution $\left(S_{1}, m\right) \stackrel{k}{\rightarrow}$ $\left(S_{1}^{\prime}, m^{\prime}\right)$.

Base case. $k=0$ and 1 .

By definition,

$\left(S_{1}, m\right) \stackrel{0}{\rightarrow}\left(S_{1}, m\right)$, and $\left(S_{1} ; S_{2}, m\right) \stackrel{0}{\rightarrow}\left(S_{1} ; S_{2}, m\right)$.

By lemma E.1.

$\left(S_{1}, m\right) \rightarrow\left(S_{1}^{\prime}, m^{\prime}\right) \Rightarrow\left(S_{1} ; S_{2}, m\right) \rightarrow\left(S_{1}^{\prime} ; S_{2}, m^{\prime}\right)$.

Induction step.

The induction hypothesis $\mathrm{IH}$ is that, for $k \geq 1$,

$\left(S_{1}, m\right) \stackrel{k}{\rightarrow}\left(S_{1}^{\prime}, m^{\prime}\right) \Rightarrow\left(S_{1} ; S_{2}, m\right) \stackrel{k}{\rightarrow}\left(S_{1}^{\prime} ; S_{2}, m^{\prime}\right)$.

Then we show that,

$\left(S_{1}, m\right) \stackrel{k+1}{\rightarrow}\left(S_{1}^{\prime}, m^{\prime}\right) \Rightarrow\left(S_{1} ; S_{2}, m\right) \stackrel{k+1}{\rightarrow}\left(S_{1}^{\prime} ; S_{2}, m^{\prime}\right)$.

We decompose the $\mathrm{k}+1$ step execution into

$\left(S_{1}, m\right) \rightarrow\left(S_{1}^{\prime \prime}, m^{\prime \prime}\right) \stackrel{k}{\rightarrow}\left(S_{1}^{\prime}, m^{\prime}\right)$.

By lemmaE.1

$\left(S_{1} ; S_{2}, m\right) \rightarrow\left(S_{1}^{\prime \prime} ; S_{2}, m^{\prime \prime}\right)$.

Next, by IH,

$\left(S_{1}^{\prime \prime} ; S_{2}, m^{\prime \prime}\right) \stackrel{k}{\rightarrow}\left(S_{1}^{\prime} ; S_{2}, m^{\prime}\right)$.

Corollary E.1. $\left(S_{1}, m\right) \stackrel{*}{\rightarrow}\left(\right.$ skip,$\left.m^{\prime}\right) \Rightarrow\left(S_{1} ; S_{2}, m\right) \stackrel{*}{\rightarrow}$ $\left(S_{2}, m^{\prime}\right)$.

Proof. By lemma E.2

$\left(S_{1}, m\right) \stackrel{*}{\rightarrow}\left(\right.$ skip,$\left.m^{\prime}\right) \Rightarrow\left(S_{1} ; S_{2}, m\right) \stackrel{*}{\rightarrow}\left(\right.$ skip $\left.; S_{2}, m^{\prime}\right)$.

Then, by rule Seq,

(skip; $\left.S_{2}, m^{\prime}\right) \rightarrow\left(S_{2}, m^{\prime}\right)$.

Lemma E.3. If one statement $s$ is not in $S$, then, after one step of execution $(S, m) \rightarrow\left(S^{\prime}, m^{\prime}\right), s$ is not in the $S^{\prime},(s \notin S) \wedge$ $\left((S, m) \rightarrow\left(S^{\prime}, m^{\prime}\right)\right) \Rightarrow\left(s \notin S^{\prime}\right)$.

Proof. By induction on abstract syntax of $S$.

Lemma E.4. If one statement $s$ is not in $S$, then, after the execution $(S, m) \stackrel{*}{\rightarrow}\left(S^{\prime}, m^{\prime}\right), s$ is not in the $S^{\prime},(s \notin S) \wedge((S, m) \stackrel{*}{\rightarrow}$ $\left.\left(S^{\prime}, m^{\prime}\right)\right) \Rightarrow\left(s \notin S^{\prime}\right)$.

Proof. By induction on the number $k$ of the steps in the execution $(S, m) \stackrel{k}{\rightarrow}\left(S^{\prime}, m^{\prime}\right)$.

Lemma E.5. If a variable $x$ is not defined in a statement sequence $S$, then, after one step execution of $S$, the value of $x$ is not redefined, $(x \notin \operatorname{Def}(S)) \wedge\left((S, m(\sigma)) \rightarrow\left(S^{\prime}, m^{\prime}\left(\sigma^{\prime}\right)\right)\right) \Rightarrow\left(x \notin \operatorname{Def}\left(S^{\prime}\right)\right) \wedge$ $\left(\sigma^{\prime}(x)=\sigma(x)\right)$

Proof. By structural induction on abstract syntax of statement sequence $S$, we show the lemma holds.

Case 1. $S=$ " $i d:=e$ ".

By definition, $\operatorname{Def}(S)=\{i d\}$. Then $i d \neq x$ by condition that $x \notin \operatorname{Def}(S)$.

Then there are two subcases.

Case 1.1 Expression $e$ evaluates to some value $v$, written $(e, m) \stackrel{*}{\rightarrow}(v, m)$.

Then, by rule Assign, $(S, m(\sigma)) \rightarrow($ skip, $m(\sigma[v / i d]))$ where $m^{\prime}=m(\sigma[v / i d])$.

Hence, $\sigma^{\prime}(x)=\sigma(x)$. Besides, $x \notin$ Def (skip) by definition.

Case 1.2 Evaluation of expression $e$ crashes, written $(e, m) \stackrel{*}{\rightarrow}$ $\left(e^{\prime}, m(1 / \mathfrak{f})\right)$.

Then, by rule crash, $\left(i d:=e^{\prime}, m(1 / \mathfrak{f}, \sigma)\right) \rightarrow\left(i d:=e^{\prime}, m(1 / \mathfrak{f}, \sigma)\right)$ where $m^{\prime}=$ $m(1 / \mathfrak{f}, \sigma)$.

Hence, $\sigma^{\prime}(x)=\sigma(x)$. Besides, $x \notin \operatorname{Def}\left(i d:=e^{\prime}\right)$ by definition.

Case 2. $S=$ " output $e$ ".

Case 3. $S=$ " input $i d$ ".

By similar argument in case 1 .

Case 4. $S=$ "If $(e)$ then $\left\{S_{t}\right\}$ else $\left\{S_{f}\right\}$ ".

$\operatorname{Def}(S)=\operatorname{Def}\left(S_{f}\right) \cup \operatorname{Def}\left(S_{t}\right)$ by definition. Then $x \notin$ $\operatorname{Def}\left(S_{f}\right) \cup \operatorname{Def}\left(S_{t}\right)$.

There are two subcases.

Case 4.1 W.l.o.g., expression $e$ in predicate of $S$ evaluates to nonzero value, written $(e, m) \stackrel{*}{\rightarrow}(v, m)$ where $v \neq 0$.

Then by rule If-T, (If $(v)$ then $\left\{S_{t}\right\}$ else $\left.\left\{S_{f}\right\}, m(\sigma)\right) \rightarrow\left(S_{t}, m(\sigma)\right)$ where $m^{\prime}=m$.

Therefore, $\sigma^{\prime}(x)=\sigma(x)$. By argument above, $x \notin \operatorname{Def}\left(S_{t}\right)$.

Case 4.2 Evaluation of expression $e$ in predicate of $S$ crashes, written $(e, m) \stackrel{*}{\rightarrow}\left(e^{\prime}, m(1 / \mathfrak{f})\right)$.

Then, by rule crash,

(If $\left(e^{\prime}\right)$ then $\left\{S_{t}\right\}$ else $\left.\left\{S_{f}\right\}, m(1 / \mathfrak{f}, \sigma)\right) \rightarrow$

(If $\left(e^{\prime}\right)$ then $\left\{S_{t}\right\}$ else $\left.\left\{S_{f}\right\}, m(1 / \mathfrak{f}, \sigma)\right)$ where $m^{\prime}=m(1 / \mathfrak{f}, \sigma)$.

Therefore, $\sigma^{\prime}(x)=\sigma(x)$.

Besides, $x \notin \operatorname{Def}\left(\right.$ If $\left(e^{\prime}\right)$ then $\left\{S_{t}\right\}$ else $\left.\left\{S_{f}\right\}\right)=\operatorname{Def}\left(S_{f}\right) \cup$ Def $\left(S_{t}\right)$.

Case 5. $S=$ "while $\langle n\rangle$ (e) $\left\{S^{\prime}\right\}$ ".

$\operatorname{Def}(S)=\operatorname{Def}\left(S^{\prime}\right)$ by definition. Then $x \notin \operatorname{Def}\left(S^{\prime}\right)$ by condition $x \notin \operatorname{Def}(S)$.

There are subcases.

Case 5.1 Expression $e$ evaluates to nonzero value, written $(e, m) \stackrel{*}{\rightarrow}(v, m)$ where $v \neq 0$.

By rule Wh-T, (while $\left.\langle n\rangle(v)\left\{S^{\prime}\right\}, m(\sigma)\right) \rightarrow$

$\left(S^{\prime} ;\right.$ while $\left._{\langle n\rangle}(e)\left\{S^{\prime}\right\}, m\left(m_{c}[(k+1) / n]\right), \sigma\right)$ for some nonnegative integer $k$.

Let $m^{\prime}=m\left(m_{c}[(k+1) / n], \sigma\right)$. Then $\sigma^{\prime}(x)=\sigma(x)$.

Besides, $x \notin \operatorname{Def}\left(S^{\prime} ;\right.$ while $\left.\langle n\rangle \quad(e) \quad\left\{S^{\prime}\right\}\right)=\operatorname{Def}\left(S^{\prime}\right) \cup$ Def $(S)$, because $x \notin \operatorname{Def}\left(S^{\prime}\right)$.

Case 5.2 Expression $e$ evaluates to zero in state $m$, written $(e, m) \stackrel{*}{\rightarrow}(0, m)$.

By rule Wh-F,

$\left(\right.$ while $\left._{\langle n\rangle}(0)\left\{S^{\prime}\right\}, m(\sigma)\right) \rightarrow\left(\right.$ skip, $\left.m\left(m_{c}[0 / n], \sigma\right)\right)$ where $m^{\prime}=m\left(m_{c}[0 / n], \sigma\right)$.

Therefore, $\sigma^{\prime}(x)=\sigma(x)$. Besides, $x \notin$ Def (skip).

Case 5.3 Evaluation of expression $e$ crashes, written $(e, m) \stackrel{*}{\rightarrow}$ $\left(e^{\prime}, m(1 / \mathfrak{f})\right)$.

By rule crash

$\left(\right.$ while $\left._{\langle n\rangle}\left(e^{\prime}\right)\left\{S^{\prime}\right\}, m(1 / \mathfrak{f}, \sigma)\right) \rightarrow$

(while $\left.\langle n\rangle\left(e^{\prime}\right)\left\{S^{\prime}\right\}, m(1 / \mathfrak{f}, \sigma)\right)$ where $m^{\prime}=m(1 / \mathfrak{f}, \sigma)$.

Therefore, $\sigma^{\prime}(x)=\sigma(x)$. Besides, $x \notin \operatorname{Def}\left(\right.$ while $\left._{\langle n\rangle}\left(e^{\prime}\right)\left\{S^{\prime}\right\}\right)$

$=\operatorname{Def}\left(S^{\prime}\right)$ by definition.

Case 6. $S=S_{1} ; S_{2}$.

By argument in Case 1 to 5, after one step execution $\left(\left(S_{1}, m(\sigma)\right) \rightarrow\right.$ $\left.\left(S^{\prime}, m^{\prime}(\sigma)\right)\right), \sigma^{\prime}(x)=\sigma(x)$.

By contextual rule, the lemma holds.

Corollary E.2. If a variable $x$ is not defined in a statement sequence $S$, then, after an execution of $S$, the value of $x$ is not redefined, $\left(x \notin \operatorname{Def}(S) \wedge(S, m(\sigma)) \stackrel{*}{\rightarrow}\left(S^{\prime}, m^{\prime}\left(\sigma^{\prime}\right)\right) \Rightarrow(x \notin\right.$ $\left.\left.\operatorname{Def}\left(S^{\prime}\right)\right) \wedge \sigma^{\prime}(x)=\sigma(x)\right)$.

Proof. Let $(S, m) \stackrel{k}{\rightarrow}\left(S^{\prime}, m^{\prime}\right)$. The proof is by induction on $k$ using lemmaE.5 
Based on Corollary E.2 we extend the result to array variable elements.

Corollary E.3. If an element in an array variable $x[i]$ is not defined in a statement sequence $S$ in a program $P=E N ; V ; S_{\text {entry }}$, then, after an execution of $S$, the value of $x[i]$ is not redefined, $(x \notin \operatorname{Def}(S)) \wedge((x, i, *) \in \sigma) \wedge(S, m(\sigma)) \stackrel{*}{\rightarrow}\left(S^{\prime}, m^{\prime}\left(\sigma^{\prime}\right)\right) \Rightarrow$ $\left.\left(x \notin \operatorname{Def}\left(S^{\prime}\right)\right) \wedge \sigma^{\prime}(x, i)=\sigma(x, i)\right)$.

Lemma E.6. If all of the following hold:

1. There is no loop of label $n$ in statements $S$, "while ${ }_{\langle n\rangle}(e)\left\{S^{\prime}\right\}$ " $\notin$ $S$;

2. The crash flag is not set, $\mathfrak{f}=0$;

3. There is an entry $n$ in loop counter, $(n, *) \in$ loop $_{c}$;

4. There is one step execution, $\left(S, m\left(\mathfrak{f}\right.\right.$, loop $\left.\left._{c}\right)\right) \rightarrow\left(S^{\prime}, m^{\prime}\left(\operatorname{loop}_{c}^{\prime}\right)\right)$;

then, $\operatorname{loop}_{c}^{\prime}(n)=\operatorname{loop}_{c}(n)$.

Proof. The proof is by induction on abstract syntax of $S$, similar to that for lemmaE.5

Corollary E.4. If all of the following hold:

1. There is no loop of label $n$ in statements $S$, "while ${ }_{\langle n\rangle}(e)\left\{S^{\prime}\right\}$ " $\notin$ $S$;

2. The crash flag is not set, $\mathfrak{f}=0$;

3. There is an entry $n$ in loop counter, $(n, *) \in$ loop $_{c}$;

4. There is multiple steps execution of stack depth $d=0$, $\left(S, m\left(\mathfrak{f}, \operatorname{loop}_{c}\right)\right) \stackrel{*}{\rightarrow}\left(S^{\prime}, m^{\prime}\left(\operatorname{loop}_{c}^{\prime}\right)\right) ;$

then, $\operatorname{loop}_{c}^{\prime}(n)=\operatorname{loop}_{c}(n)$.

Lemma E.7. If all of the following hold:

1. A non-skip statement $s$ is not in $S,(s \neq s k i p) \wedge(s \notin S)$;

2. There is one step execution of stack depth $d=0,(S, m) \rightarrow$ $\left(S^{\prime}, m^{\prime}\right)$,

then, $s \notin S^{\prime}$.

Proof. By structural induction on abstract syntax of statement sequence $S$, we show the lemma holds.

Case 1. $S=$ " $i d:=e$ ".

Then there are two subcases.

Case 1.1 Expression $e$ evaluates to some value $v$, written $(e, m) \stackrel{*}{\rightarrow}(v, m)$.

Then, by rule Assign, $(S, m) \rightarrow($ skip, $m(\sigma[v / i d]))$.

Hence, $s \notin$ skip by definition.

Case 1.2 Evaluation of expression $e$ crashes, written $(e, m) \stackrel{*}{\rightarrow}$ $\left(e^{\prime}, m(1 / \mathfrak{f})\right)$.

By parameterized type rule TExpr, $\Gamma \forall e^{\prime}$. Then, by type rule TAssign, $\Gamma \forall i d:=e^{\prime}$.

Then, by rule crash,

$\left(i d:=e^{\prime}, m(1 / \mathfrak{f})\right) \rightarrow\left(i d:=e^{\prime}, m(1 / \mathfrak{f})\right)$.

Because $\Gamma \vdash s$, then $s \neq i d:=e^{\prime}$. Hence, $s \notin i d:=e^{\prime}$ by definition.

Case 2. $S=$ " output $e$ ".

Case 3. $S=$ " input $i d$ ".

By similar argument in case 1.

Case 4. $S=$ "If $(e)$ then $\left\{S_{t}\right\}$ else $\left\{S_{f}\right\}$ ".

$s \notin S_{f}, s \notin S_{t}$ by definition. There are two subcases.

Case 4.1 W.1.o.g., expression $e$ in predicate of $S$ evaluates to nonzero value, written $(e, m) \stackrel{*}{\rightarrow}(v, m)$ where $v \neq 0$.

Then by rule If-T, (If $(v)$ then $\left\{S_{t}\right\}$ else $\left.\left\{S_{f}\right\}, m\right) \rightarrow\left(S_{t}, m\right)$. Therefore, $s \notin S_{t}$.

Case 4.2 Evaluation of expression $e$ in predicate of $S$ crashes, written $(e, m) \stackrel{*}{\rightarrow}\left(e^{\prime}, m(1 / \mathfrak{f})\right)$.

Then, by rule crash,
(If $\left(e^{\prime}\right)$ then $\left\{S_{t}\right\}$ else $\left.\left\{S_{f}\right\}, m(1 / \mathfrak{f})\right) \rightarrow$

(If $\left(e^{\prime}\right)$ then $\left\{S_{t}\right\}$ else $\left.\left\{S_{f}\right\}, m(1 / \mathfrak{f})\right)$.

By parameterized type rule TExpr, $\Gamma \nvdash e^{\prime}$. By type rule Tif,

$\Gamma \nvdash$ If $\left(e^{\prime}\right)$ then $\left\{S_{t}\right\}$ else $\left\{S_{f}\right\}$.

Because $\Gamma \vdash s$, then $s \neq$ If $\left(e^{\prime}\right)$ then $\left\{S_{t}\right\}$ else $\left\{S_{f}\right\}$.

Besides, $s \notin S_{t}, s \notin S_{f}$ by condition. Therefore, $s \notin$

If $\left(e^{\prime}\right)$ then $\left\{S_{t}\right\}$ else $\left\{S_{f}\right\}$.

Case 5. $S=$ "while $\langle n\rangle(e)\left\{S^{\prime}\right\}$ ".

$s \notin S^{\prime}$ by definition. There are subcases.

Case 5.1 Expression $e$ evaluates to nonzero value, written $(e, m) \stackrel{*}{\rightarrow}(v, m)$ where $v \neq 0$.

By rule Wh-T, $\left(\right.$ while $\left._{\langle n\rangle}(v)\left\{S^{\prime}\right\}, m\right) \rightarrow$

$\left(S^{\prime} ;\right.$ while $\left._{\langle n\rangle}(e)\left\{S^{\prime}\right\}, m\left(m_{c}[(k+1) / n]\right)\right)$ for some nonnegative integer $k$.

Then $s \notin S^{\prime}$; while $_{\langle n\rangle}(e)\left\{S^{\prime}\right\}$ by definition.

Case 5.2 Expression $e$ evaluates to zero in state $m$, written $(e, m) \stackrel{*}{\rightarrow}(0, m)$.

By rule Wh-F,

$\left(\right.$ while $\left._{\langle n\rangle}(0)\left\{S^{\prime}\right\}, m\right) \rightarrow\left(\operatorname{skip}, m\left(m_{c}[0 / n]\right)\right)$.

Therefore, $s \notin$ skip.

Case 5.3 Evaluation of expression $e$ crashes, written $(e, m) \stackrel{*}{\rightarrow}$ $\left(e^{\prime}, m(1 / \mathfrak{f})\right)$.

By rule crash,

$\left(\right.$ while $\left._{\langle n\rangle}\left(e^{\prime}\right)\left\{S^{\prime}\right\}, m(1 / \mathfrak{f})\right) \rightarrow$

(while $\left.\langle n\rangle\left(e^{\prime}\right)\left\{S^{\prime}\right\}, m(1 / \mathfrak{f})\right)$.

Then, by type rule Twhile, $\Gamma \forall$ while $_{\langle n\rangle}\left(e^{\prime}\right)\left\{S^{\prime}\right\}$. Because $\Gamma \vdash s$, then $s \neq$ while $_{\langle n\rangle}\left(e^{\prime}\right)\left\{S^{\prime}\right\}$.

Besides $s \notin S^{\prime}$, then $s \notin$ while $_{\langle n\rangle}\left(e^{\prime}\right)\left\{S^{\prime}\right\}$ by definition.

Case 6. $S=S_{1} ; S_{2}$.

By argument in Case 1 to 5 , after one step execution $\left(S_{1}, m\right) \rightarrow$ $\left(S^{\prime}, m^{\prime}\right), s \notin S^{\prime}$.

By contextual rule, $\left(S_{1} ; S_{2}, m\right) \rightarrow\left(S^{\prime} ; S_{2}, m^{\prime}\right)$.

By definition, $s \notin S_{2}$.

Then, by definition, $s \notin S^{\prime} ; S_{2}$

Lemma E.8. Let $s=$ "while $\langle n\rangle(e)\left\{S^{\prime \prime}\right\}$ ". If both of the following hold:

- $s \in S$;

- $\left(S, m\left(\operatorname{loop}_{c}\right)\right) \rightarrow\left(S^{\prime}, m^{\prime}\left(\operatorname{loop}_{c}^{\prime}\right)\right)$;

then one of the following holds:

1. The loop counter of label $n$ is incremented by one, $\operatorname{loop}_{c}^{\prime}(n)-$ $\operatorname{loop}_{c}(n)=1$;

2. There is no entry for label $n$ in loop counter, $(n, v) \notin$ loop $_{c}^{\prime}$;

3. The loop counter of label $n$ is not changed, $\operatorname{loop}_{c}^{\prime}(n)-\operatorname{loop}_{c}(n)$ $=0$;

Proof. Let $S=s^{\prime} ; S^{\prime \prime}$. The proof is by induction on abstract syntax of $s^{\prime}$. 